Copyright

by

Heesong Koh

2015 
The Dissertation Committee for Heesong Koh Certifies that this is the approved version of the following dissertation:

\section{Experimental Investigation of the Effect of Polymers on Residual Oil Saturation}

\section{Committee:}

Gary A. Pope, Supervisor

Chun Huh

Kishore Mohanty

Matthew Balhoff

Keith P. Johnston 


\title{
Experimental Investigation of the Effect of Polymers on Residual Oil Saturation
}

\author{
by \\ Heesong Koh, B.S.; M.S.E. \\ Dissertation \\ Presented to the Faculty of the Graduate School of \\ The University of Texas at Austin \\ in Partial Fulfillment \\ of the Requirements \\ for the Degree of
}

Doctor of Philosophy

The University of Texas at Austin

January 2015 


\section{Dedication}

To my family 


\section{Acknowledgements}

I would like to express my gratitude to Dr. Gary A. Pope for his guidance, support and patience. He gave me great opportunity and then guided and challenged me to constantly improve myself to become a better engineer and to always expand the limits of my knowledge. I am truly honored to have been granted the opportunity to work under his supervision. I would also like to thank Dr. Chun Huh whose wealth of knowledge and experience have been invaluable to me. His guidance and encouragement always pointed me in the right direction whenever I needed it. I would thank my committee members Dr. Kishore Mohanty, Dr. Matthew Balhoff, and Dr. Keith P. Johnston for their time and effort in reviewing and commenting on my dissertation.

The financial support of the industrial affiliates of the Chemical Enhanced Oil Recovery Research Program in the Center for Petroleum and Geosystems Engineering at the University of Texas at Austin that made this research and my graduate studies possible is gratefully acknowledged.

I want to thank Dr. Pope's current and former research staff including Dr. Do Hoon Kim, Chris Britton, Jith Liyanage, Erandimala Kulawardana, Pradeep Wickremasiri, Nadeeka Upamali, Gayani Pinnawala, Suneth Rajapaksha, Austin Lim and Arnob Bhyuan. I truly could not have finished without your help and insight. I would like to thank all of the undergraduate research assistants that helped me along the way, particularly James Choi, Yongdo Kim and Colin Beasley. I would like thank to my fellow graduate students Jun Lu, Vincent Lee, Nabi Nizamidin, Leonard Chang, and Sean Li for your valuable discussion, support and friendship. Thanks also to the research and departmental staff including Esther Barrientes, Joanna Castillo, Frankie Hart, Glen Baum, and Gary Miscoe for all of your support. 
I would like to thank my bible study group members Bernie and Sally Boudreaux, Terry \& JingJing Wang, Vincent \& Camilla Yu, Yulin Pan, Steven \& Nikki Chen, and Steven \& Ayou who have been my parents and brothers in Austin. You guys have been with me through the best and toughest times.

Finally, to mom, you have always been there to encourage me to achieve my full potential and to pick me up whenever I falter. I thank you from the bottom of my heart for everything you have done for me. 


\title{
Experimental Investigation of the Effect of Polymers on Residual Oil Saturation
}

\author{
Heesong Koh, Ph.D. \\ The University of Texas at Austin, 2015
}

Supervisor: Gary A. Pope

The main objective of this research was to better understand the effect of polymer flooding on the remaining oil saturation by conducting experiments and interpreting these experimental data in terms of measured polymer and rock characteristics. This is because one of the most important factors in chemical enhanced oil recovery (EOR) is mobility control, for which partially hydrolyzed polyacrylamide (HPAM) and other polymers are extensively used.

Rheological properties of the EOR polymer solutions depend on the various factors such as a polymer's molecular properties and concentration, salinity, hardness, shear rate and temperature. Therefore, rheological measurements with commonly employed EOR polymers under various conditions were made and the effect of these factors on the polymer's viscosity and mobility was quantified. In addition to the steady shear viscosities, the oscillatory rheological properties were measured to better define the polymer's viscoelastic behavior during flow in porous media.

Commonly used partially hydrolyzed polyacrylamides (HPAM) have been successfully used in the field for decades, but they hydrolyze at high temperature and eventually precipitate in the presence of high concentrations of divalent cations. New polymers that are stable in harsh environments (high salinity/hardness and high vii 
temperature) are in high demand because of the need for chemical EOR in oil reservoirs with these conditions. Both scleroglucan and NVP co- or ter-polymers show good filterability and transport properties in sandstone and carbonate cores at high temperature and in brine with high salinity and hardness. Therefore, both polymers are promising candidates for polymer flooding, surfactant-polymer flooding and alkali-surfactantpolymer flooding in hard brine at high temperature, and their rheological properties were also evaluated for some representative reservoir conditions.

Several polymer coreflood experiments have been carried out using both sandpacks and reservoir cores, starting at different water cuts to measure the effect of polymer on the remaining oil saturation. In order to interpret the polymer corefloods, fractional flow theory that incorporated non-Newtonian rheology was developed and applied.

The much higher oil recovery from polymer flooding compared to water flooding observed in numerous coreflood experiments is deemed to be mainly due to the improved microscopic or displacement sweep efficiency of the polymer. There is no clear evidence from these experiments that polymer floods reduce the residual oil saturation substantially when the experiments are done with low pressure gradients typical of the pressure gradients that are feasible under field conditions. 
Table of Contents

List of Tables .......................................................................................... xiv

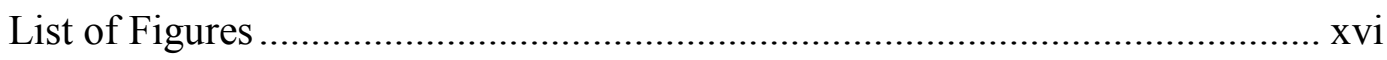

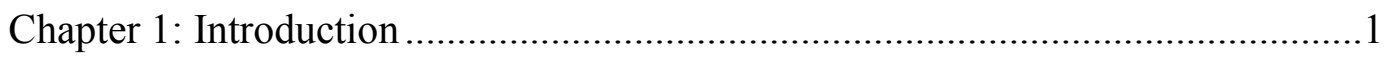

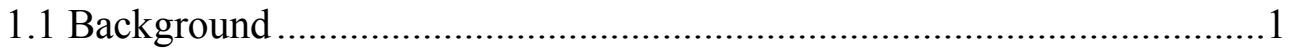

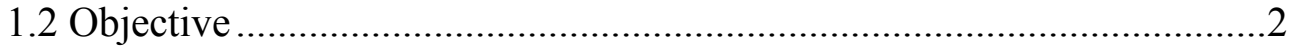

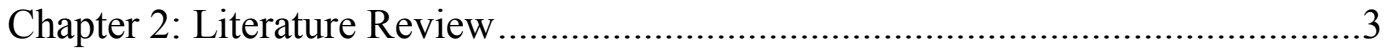

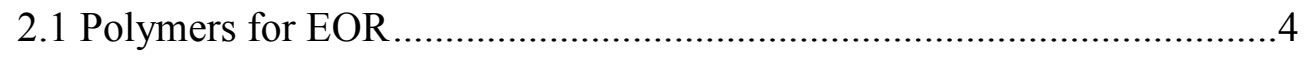

2.2 Polymer Rheology .................................................................... 9

Effect of polymer concentration and molecular weight on the viscosity .................................................................. 10

Effect of ions and $\mathrm{pH}$ on the viscosity ....................................11

2.2.1 Flow of polymer solutions in porous media ............................13

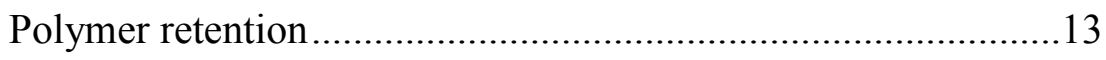

Permeability Reduction..................................................... 17

Apparent viscosity ............................................................. 18

Calculation of shear rate in porous media.................................19

2.2.2 Non-Newtonian properties.................................................26

Determination of Deborah number .........................................28

2.3 Reduction of residual oil saturation during polymer flood ....................30

Chapter 3: Experimental Materials, Methodology and Data Analysis ..................33

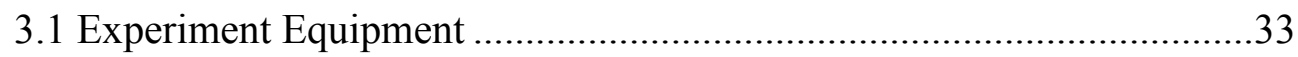

3.1.1 Polymer Preparation.......................................................... 33

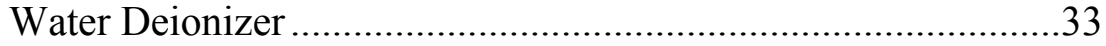

Convection Ovens ................................................................. 34

Dynamic Light Scattering (DLS) ........................................36

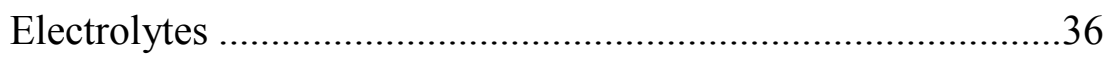

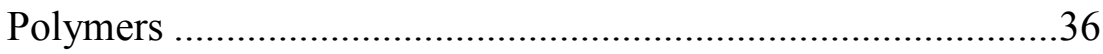


3.1.2 Rheological Properties Measurements...........................................37

3.1.3. Core Flood Experimental Equipment ……………………..........39

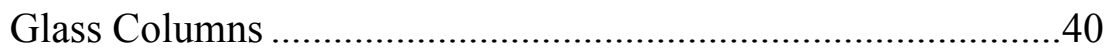

Stainless Steel Columns ..............................................................

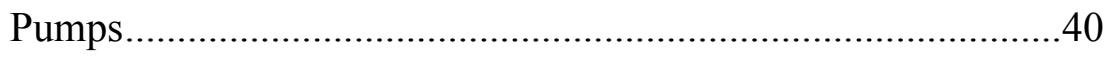

Pressure Transducer .................................................................. 40

Data Acquisition Recorder............................................................41

Fraction Collector …………………………………………....4

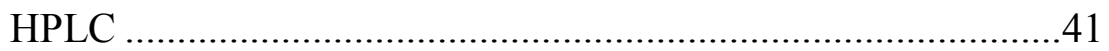

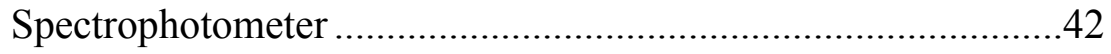

3.2 Core Flood Description and Methodology ……………………….......4

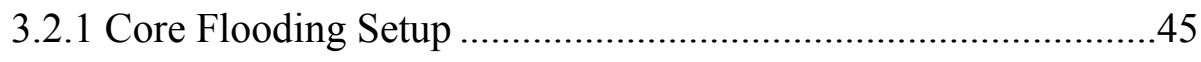

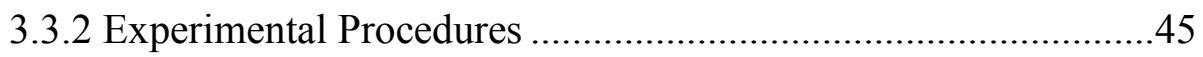

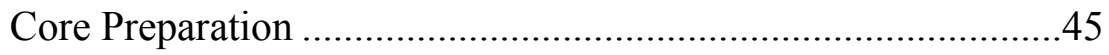

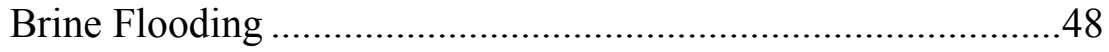

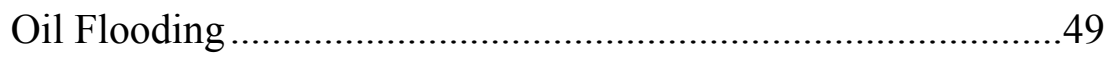

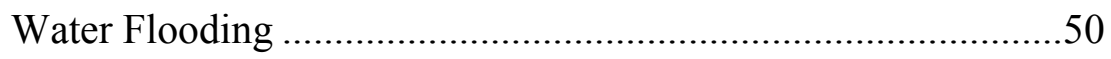

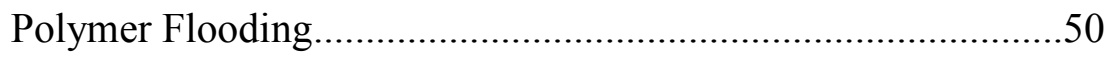

3.3.3 Core Flood Calculations …………………………………….....50

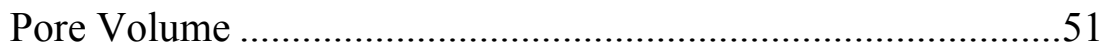

Bulk Volume and Porosity ............................................................51

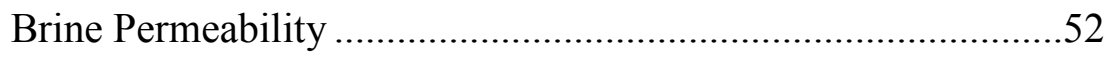

Effective Oil Permeability .........................................................52

Effective Water Permeability......................................................52

End Point Oil/Water Relative Permeability ..................................52

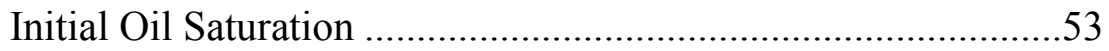

Residual Oil Saturation ..............................................................53

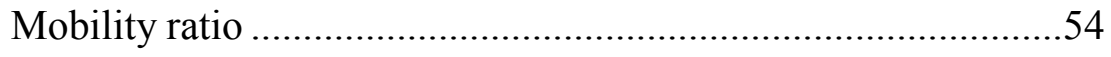

Polymer Resistance Factor.........................................................55

Polymer Permeability Reduction Factor.......................................56 


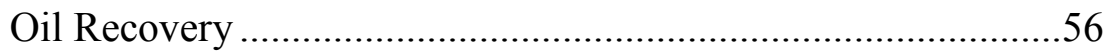

Chapter 4. Polymer Solution Characterization ...................................................58

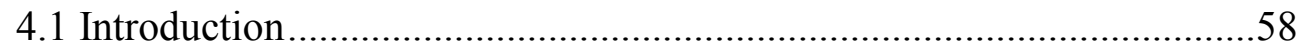

4.2 Experimental Material, Apparatus and Methodology .............................58

4.2.1 The Polymers .............................................................................

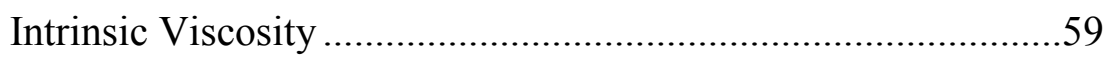

The Relaxation Time of a polymer solution .................................61

The Deborah Number ...................................................................61

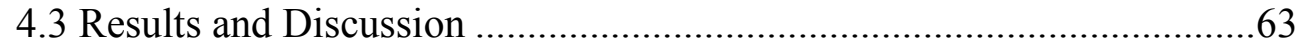

4.3.1 Bulk Rheological Results........................................................63

4.3.2 Trends of the $\mathrm{G}^{\prime}$ and $\mathrm{G}^{\prime \prime}$ Data for Different Polymers. ................78

Dependence on Polymer Concentration.......................................78

Dependence on Salinity and Hardness..........................................79

Dependence on Temperature ........................................................ 80

Dependence on Intrinsic viscosity …….....................................8

4.4 Summary and Conclusions ..................................................................... 84

Chapter 5. Transport and In-situ Rheology of EOR Polymers ................................86

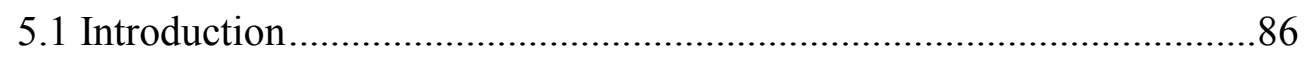

5.2 Materials and Experimental Methods ....................................................

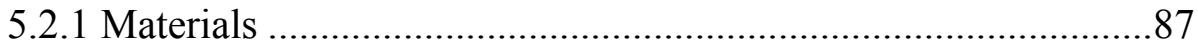

5.2.2 Preparation of Polymer Samples................................................... 88

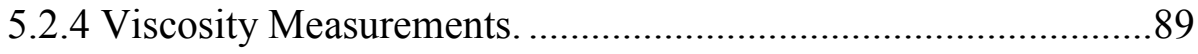

5.2.5 Thermal Stability Measurements. ..................................................8 89

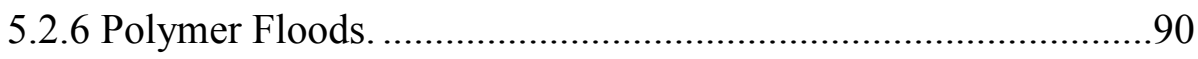

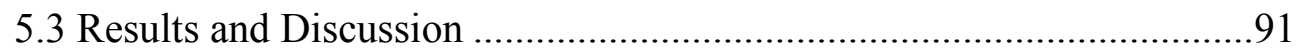

5.3.1 Filterability and Filterability Improvement Methods.................91

5.3.2 Viscosity measurements..............................................................95

5.3.3 Thermal Stability of Polymers...................................................105

Thermal Stability of SAV Polymers.........................................111

5.3.4 Polymer Transport and Retention..............................................112 
5.3.5 Viscoelastic Behavior of High Molecular Weight Polymers in Porous Media 129

5.4 Summary and Conclusions ………......................................................134

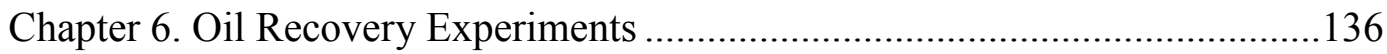

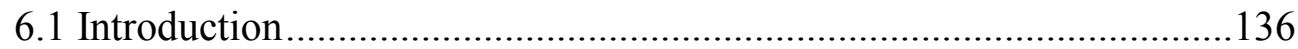

6.2 Coreflood Experimental Setup and Background ..................................136

6.2.1 Coreflood Experimental Setup.................................................136

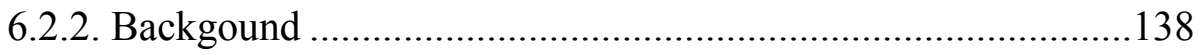

Mechanism of Oil Displacement ..............................................138

Mobility Ratio ........................................................................139

Capillary Number..................................................................141

6.3 Experiment Results USING HPAM POLYMER ....................................143

6.3.1 Coreflood experiments..............................................................143

6.3.2 Analysis of Coreflood Results ...................................................167

Polymer Retention ...............................................................170

Determination of polymer concentration to calculate polymer

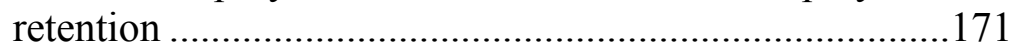

Fractional Flow Analysis .......................................................178

The shear correction factor ........................................................204

6.4 Experiment Results Using Modified HPAM Polymer...........................212

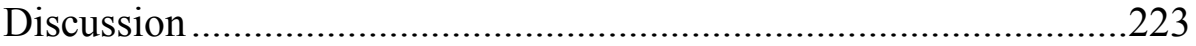

6.5 Summary and Conclusions ..........................................................230

Chapter 7. Summary, Conclusions and Recommendations .................................232

7.1 Rheological Properties of Polymer Solutions ......................................232

7.2 Transport of Polymer Solution in Cores .................................................2232

7.3 Oil Recovery Experiments ..............................................................234

7.4 Conclusions about Shear Correction Factor ………………………......235

7.5 Conclusions about Residual Oil Saturation .........................................237

7.6 Recommendations for Future Research .............................................240 


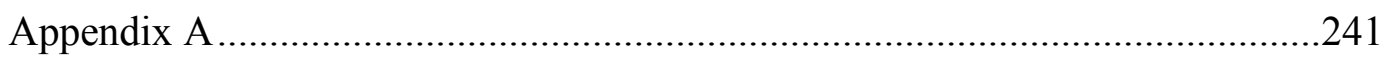

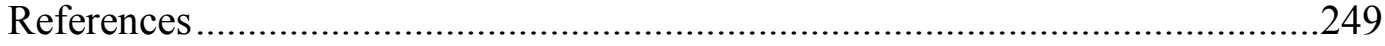




\section{List of Tables}

Table 4.1 The polymers tested in this study ....................................................59

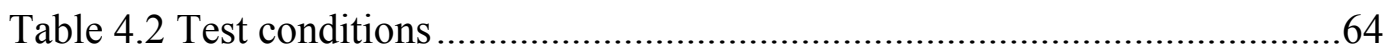

Table 4.3 Summary of bulk viscosity and relaxation times for 500 ppm FP 3630S65

Table 4.4 Summary of bulk viscosity and relaxation times for 1000 ppm FP 3630S .66

Table 4.5 Summary of bulk viscosity and relaxation times for 2000 ppm FP 3630S .67

Table 4.6 Summary of bulk viscosity and relaxation times for 3000 ppm FP 3630S

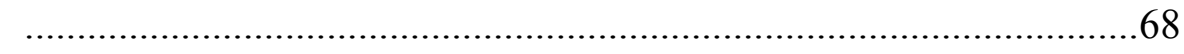

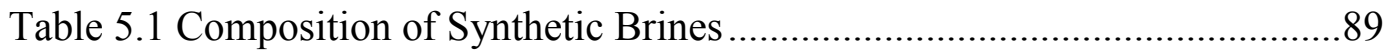

Table 5.2 Summary of HPAM Filtration ...................................................92

Table 5.3 Filterability Improvement of $1250 \mathrm{ppm}$ Scleroglucan in synthetic seawater by heat treatment ................................................................... 94

Table 5.4 Scleroglucan Filterability Improvement after Heat Treatment and Passing

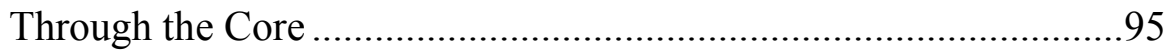

Table 5.5 Viscosity of FP 3630S in Soft Brine-1S at $62{ }^{\circ} \mathrm{C}$................................97

Table 5.6 Viscosity of FP 3630S in Hard Brine- $1 \mathrm{H}$ at $62{ }^{\circ} \mathrm{C}$.............................97

Table 5.7 Viscosity of H-EOR 63020 in Soft Brine-1S at $62{ }^{\circ} \mathrm{C}$.........................98

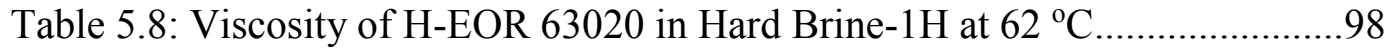

Table 5.9 Additive Package used with HPAM in Thermal Stability Experiments 107

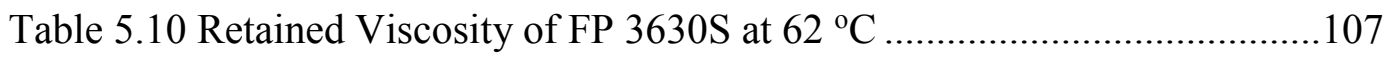

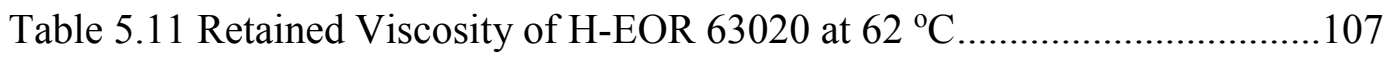

Table 5.12 Polymer Properties for HPAM Polymers in Bentheimer Cores ........114 
Table 5.13 Polymer Flood Properties for HPAM Polymers in Bentheimer Cores 115 Table 5.14 Polymer Flood Analysis for HPAM Polymers in Bentheimer Cores 116 Table 5.10 Raw data from FP 3630S polymer flood ............................................119

Table 5.11 Raw data from FP 3630S polymer flood ..............................................119

Table 5.15 Scleroglucan Transport in Berea Sandstone Cores..............................125

Table 5.16 SAV-301 Transport Summary in Estillades Limestone Core.............127

Table 5.17 Polymer solutions used for corefloods..............................................130

Table 5.18 Summary of core properties.............................................................131

Table 5.19 Apparent viscosity of FP3630S in experiment BV-1 ........................131

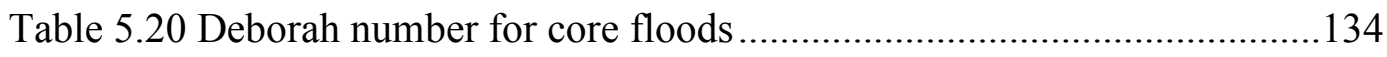

Table 6.1 Summary of Coreflood Results...........................................................144

Table 6.2 Core Properties for experiments \#1, \#2 and \#3 .................................145

Table 6.3 Relative water permeability for experiment \#4 ...................................156

Table 6.4 Brine Compositions ..........................................................................163

Table 6.5 Water flood results for experiments \#1, \#2, \#3 and \#7 ........................169

Table 6.6 Experimental data and adjusted Corey exponents for experiment \#3.182

Table 6.7 Experiment data and adjusted Corey exponents for experiment \#4 ....191

Table 6.8 Experiment data and adjusted parameters for Corey correlation..........195

Table 6.9 Experiment data and adjusted Corey exponents for experiment \#8 ....199

Table 6.10 Experiment data and adjusted Corey exponents for experiment \#9 ..202

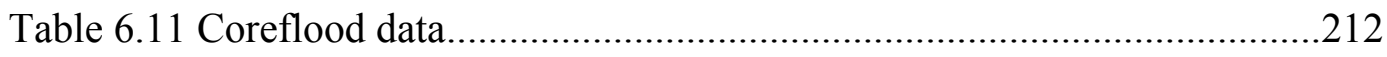

Table 6.12 Summary of core flood results..........................................................2215 


\section{List of Figures}

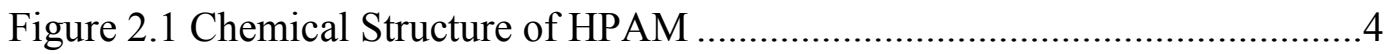

Figure 2.2 Chemical structure of NVP-AM copolymers.......................................

Figure 2.3 Chemical structure of AMPS..........................................................6

Figure 2.4 Chemical structure of xanthan gum....................................................

Figure 2.5 Chemical structure of Scleroglucan...................................................

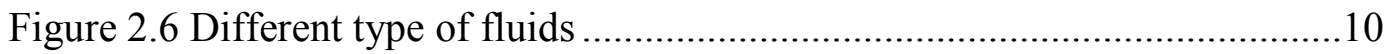

Figure 2.7 The long and flexible polymer backbone of HPAM coils- up when increasing the salinity/hardness of the solution (Sorbie, 1991)........13

Figure 2.8 Schematic diagram of polymer retention mechanisms in porous media

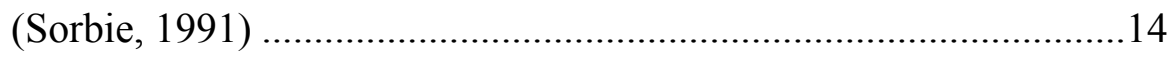

Figure 2.9 Schematic of shear rate dependence of polymer (HPAM) apparent

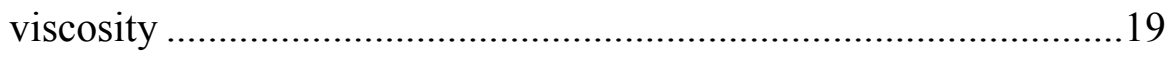

Figure 2.10 Schematic diagram of water invasion into porous medium ...............25

Figure 2.11 Typical water/oil relative permeability curves ...................................25

Figure 2.12 Illustration of (a) low and (b) high aspect ratio pores and the effect of aspect ratio in causing snap-off of phase $n$ during imbibition. ........30

Figure 3.1 The filter press unit used in this study ................................................42

Figure 3.2 TA Instruments' Advanced Rheometric Expansion System Low Shear-1

Figure 3.3 Typical result of dynamic strain sweep test ........................................43

Figure 3.4 Typical result of dynamic frequency sweep test ...................................43

Figure 3.5 Typical result of strain controlled step rate transient test......................44

Figure 3.6 Typical result of steady rate sweep test...............................................4 
Figure 3.7 Schematic setup of polymer flood

Figure 4.1 Effect of polymer concentration on crossover point for Flopaam ${ }^{\mathrm{TM}} 3630 \mathrm{~S}$ in $0.1 \% \mathrm{NaCl}$ at $25^{\circ} \mathrm{C}$ 69

Figure 4.2 Effect of polymer concentration on crossover point for Flopaam ${ }^{\mathrm{TM}} 3630 \mathrm{~S}$ in $1 \% \mathrm{NaCl}$ at $25^{\circ} \mathrm{C}$ 69

Figure 4.3 Effect of polymer concentration on crossover point for Flopaam ${ }^{\mathrm{TM}} 3630 \mathrm{~S}$ in $4 \% \mathrm{NaCl}$ at $25^{\circ} \mathrm{C}$ 70

Figure 4.4 Variation of relaxation time with polymer concentration and salinity for Flopaam $^{\mathrm{TM}} 3630 \mathrm{~S}$ at $25^{\circ} \mathrm{C}$ .70

Figure 4.5 Variation of relaxation time with polymer concentration and salinity for Flopaam ${ }^{\mathrm{TM}} 3630 \mathrm{~S}$ at $25^{\circ} \mathrm{C}$ in $\log -\log$ scale. 71

Figure 4.6 Variation of relaxation time with salinity and polymer concentration for Flopaam $^{\mathrm{TM}} 3630 \mathrm{~S}$ at $25^{\circ} \mathrm{C}$ 71

Figure 4.7 Variation of relaxation time with salinity and polymer concentration for Flopaam $^{\mathrm{TM}} 3630 \mathrm{~S}$ at $25^{\circ} \mathrm{C}, 0 \% \mathrm{Ca}^{2+}$ .72

Figure 4.8 Variation of relaxation time with salinity and polymer concentration for Flopaam ${ }^{\mathrm{TM}} 3630 \mathrm{~S}$ at $25^{\circ} \mathrm{C}, 0.05 \% \mathrm{Ca}^{2+}$ 72

Figure 4.9 Variation of relaxation time with hardness for Flopaam ${ }^{\mathrm{TM}} 3630 \mathrm{~S}$ in $0.1 \%$

$\mathrm{NaCl}$ at $25^{\circ} \mathrm{C}$ .73

Figure 4.10 Variation of relaxation time with hardness for Flopaam ${ }^{\mathrm{TM}} 3630 \mathrm{~S}$ in $1 \%$ $\mathrm{NaCl}$ at $25^{\circ} \mathrm{C}$ .73

Figure 4.11 Variation of relaxation time with hardness for Flopaam ${ }^{\mathrm{TM}} 3630 \mathrm{~S}$ in $4 \%$ $\mathrm{NaCl}$ at $25^{\circ} \mathrm{C}$ .74

Figure 4.12 The effect of temperature on relaxation time for 2000 ppm $^{\text {Flopaam }}{ }^{\mathrm{TM}}$ $3630 \mathrm{~S}$ in $1 \% \mathrm{NaCl}$ .74 xvii 
Figure 4.13 Variation of viscosity with salinity and polymer concentration for Flopaam ${ }^{\mathrm{TM}} 3630 \mathrm{~S}$ at $25^{\circ} \mathrm{C}$ in $\log -\log$ scale ......................................

Figure 4.14 Variation of viscosity with salinity and polymer concentration for Flopaam ${ }^{\mathrm{TM}} 3630 \mathrm{~S}$ at $25^{\circ} \mathrm{C}$ 75

Figure 4.15 Variation of relaxation time with polymer concentration in $1 \mathrm{wt} \% \mathrm{NaCl}$

brine 76

Figure 4.16 Variation of relaxation time with temperature for $2000 \mathrm{ppm}$ polymers in 1 wt $\% \mathrm{NaCl}$ brine. .76

Figure 4.17 Variation of viscosity @ $10 \mathrm{~s}^{-1}$ with $1 \% \mathrm{NaCl}$ and polymer concentration for polymers in log-log scale 77

Figure 4.18 Variation of viscosity @ $10 \mathrm{~s}^{-1}$ with $1 \% \mathrm{NaCl}$ and polymer concentration for $2000 \mathrm{ppm}$ polymer 77

Figure 4.20 Plot to estimate intrinsic viscosity of FP $3630 \mathrm{~S}$ in $0.5 \% \mathrm{NaCl} \ldots \ldots \ldots . . .81$

Figure 4.21 Plot to estimate intrinsic viscosity of FP $3630 \mathrm{~S}$ in $2 \% \mathrm{NaCl}$.............82

Figure 4.23 Plot to estimate intrinsic viscosity of AN-125 in $1 \% \mathrm{NaCl}$.................83

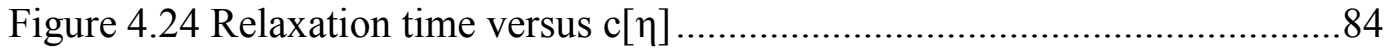

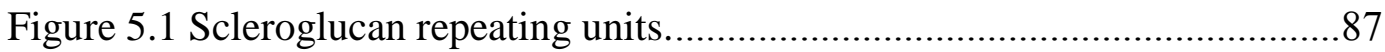

Figure 5.2 Molecular structure of NVP-AM co-polymers.....................................87

Figure 5.3 Filtration of $5000 \mathrm{ppm}$ scleroglucan in deionized water after four consecutive high-pressure filtrations. Filtration ratio $=1$ with 1.2 micron filter and 15 psi pressure gradient....................................................94

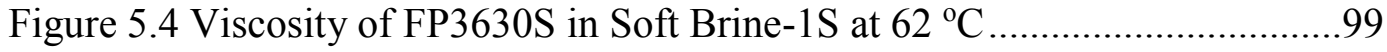

Figure 5.5 Viscosity of FP3630S in Soft Brine-1S + 10,000 ppm Sodium Carbonate at $62^{\circ} \mathrm{C}$ 99 
Figure 5.6 Viscosity of FP3630S in Soft Brine-1S + 30,000 ppm Sodium Carbonate at $62{ }^{\circ} \mathrm{C}$ 100

Figure 5.7 Viscosity of FP3630S in Hard Brine-1H at $62{ }^{\circ} \mathrm{C}$ 100

Figure 5.8 Viscosity of H-EOR 63020 in Soft Brine-1S at $62{ }^{\circ} \mathrm{C}$ 101

Figure 5.9 Viscosity of H-EOR 63020 in Soft Brine-1S $+10,000$ ppm Sodium

Carbonate at $62{ }^{\circ} \mathrm{C}$ 101

Figure 5.10 Viscosity of H-EOR 63020 in Soft Brine-1S + 30,000 ppm Sodium Carbonate at $62{ }^{\circ} \mathrm{C}$ 102

Figure 5.11 Viscosity of H-EOR 63020 in Hard Brine-1H at $62{ }^{\circ} \mathrm{C}$ 102

Figure 5.12 Relative viscosity of $1250 \mathrm{ppm}$ scleroglucan at $90{ }^{\circ} \mathrm{C}$ 103

Figure 5.13 Relative viscosity of 1250 ppm scleroglucan and SAV 301 solutions at 90

${ }^{\circ} \mathrm{C}$ (shear rate $\left.1 \mathrm{~s}^{-1}\right)$. 103

Figure 5.14 Viscosity of different polymers at $90{ }^{\circ} \mathrm{C}$ in synthetic seawater $(57,670$ ppm TDS) 104

Figure 5.16 Retained Viscosity of FP 3630S in Soft Brine-1S at $62{ }^{\circ} \mathrm{C}$ 108

Figure 5.17 Retained Viscosity of FP 3630S in Soft Brine-1S with 10,000 ppm

$\mathrm{Na}_{2} \mathrm{CO}_{3}$ at $62{ }^{\circ} \mathrm{C}$ 108

Figure 5.18: Retained Viscosity of FP $3630 \mathrm{~S}$ in Hard Brine- $1 \mathrm{H}$ at $62{ }^{\circ} \mathrm{C}$. 109

Figure 5.19 Retained Viscosity of H-EOR 63020 in Soft Brine-1S at $62{ }^{\circ} \mathrm{C}$......109 Figure 5.20 Retained Viscosity of H-EOR 63020 in Soft Brine-1S with 10,000 ppm $\mathrm{Na}_{2} \mathrm{CO}_{3}$ at $62{ }^{\circ} \mathrm{C}$. 110

Figure 5.21 Retained Viscosity of H-EOR 63020 in Hard Brine-1H at $62{ }^{\circ} \mathrm{C} \ldots . .110$ 
Figure 5.22 Thermal Stability of NVP polymers. Polymer Concentration- 3000 ppm, Additives: IPA $=1200 \mathrm{ppm}, \mathrm{TU}=600 \mathrm{ppm}$. Aged at $100{ }^{\circ} \mathrm{C}$ in synthetic seawater. (Viscosity was measured at shear rate $11 \mathrm{~s}^{-1}$ at $25^{\circ} \mathrm{C}$ ). $\mu_{\mathrm{t}}=$ viscosity of the polymer at day $\mathrm{t}$, and $\mu_{\mathrm{i}}=$ viscosity of the polymer before aging. 112

Figure 5.23 Viscosity of 1,500 ppm FP $3630 \mathrm{~S}$ in soft Brine-1S $(5,425 \mathrm{ppm})$ at $62{ }^{\circ} \mathrm{C}$.

Figure 5.24 Viscosity of $\quad 1,500$ ppm H-EOR 63020 in soft Brine-1S (5,425 ppm) at $62{ }^{\circ} \mathrm{C}$ 117

Figure 5.25 Pressure Drop across Bentheimer Core During FP 3630S injection at 62 ${ }^{\circ} \mathrm{C}$ at Multiple Flow Rates. 117

Figure 5.26 Pressure Drop across Bentheimer Core During injection of H-EOR 63020 at $62{ }^{\circ} \mathrm{C}$ at Multiple Flow Rates. 118

Figure 5.27: Apparent viscosity vs. uncorrected shear rate for FP 3630S polymer flood 120

Figure 5.28 Comparison of bulk viscosity and apparent viscosity with corrected shear rate for FP 3630 S polymer flood 121

Figure 5.29 Comparison of bulk viscosity and apparent viscosity with corrected shear rate for H-EOR 63020 polymer flood

Figure 5.30 Injected and effluent polymer viscosity for FP $3630 \mathrm{~S}$ coreflood at $62{ }^{\circ} \mathrm{C}$. 122

Figure 5.31 Injected and effluent polymer viscosity for H-EOR 63020 coreflood at 62 ${ }^{\circ} \mathrm{C}$. 122

Figure 5.32 The normalized viscosity at $10 \mathrm{~s}^{-1}$ and $\mathrm{pH}$ profile for FP $3630 \mathrm{~S}$ at $62{ }^{\circ} \mathrm{C}$. 
Figure 5.33 The normalized viscosity at $10 \mathrm{~s}^{-1}$ and $\mathrm{pH}$ data for H-EOR 63020 at 62 ${ }^{\circ} \mathrm{C}$. 123

Figure 5.34 Pressure Drop in Berea Sandstone Cores at Different Temperatures at 9 $\mathrm{ft} /$ day injection of 1,250 ppm scleroglucan in 20,185 ppm TDS Brine. 125

Figure 5.35 Normalized Viscosity of Effluent Samples during Scleroglucan Floods at Different Temperatures. 1,250 ppm scleroglucan in 20,185 ppm TDS Brine 126

Figure 5.36 Pressure drop for SAV-301 polymer flood in Estillades Limestone core. Polymer Concentration $=3000$ ppm, Salinity $=29000$ ppm TDS, velocity $=2 \mathrm{ft} /$ day. 127

Figure 5.37 SAV 301 viscosity retention. Polymer concentration=3000 ppm, salinity= 29000 ppm TDS 128

Figure 5.38 Unified apparent viscosity model fit for 1500 ppm FP 3630S in 2\% soften brine with velocity. 132

Figure 5.39 Unified apparent viscosity model fit for 1500 ppm FP 3630 S in $2 \%$ soften brine with shear rate 132

Figure 5.40 Comparison of the unified apparent viscosity model fit for all four polymers. 133

Figure 6.1 Schematic coreflood setup 137

Figure 6.2 Schematic of microscopic and macroscopic sweep efficiencies (Lyons and Plisga, 2005) 140

Figure 6.3 Capillary number curves for various sandstones (Chatzis and Morrow, 1984) .143

Figure 6.4 Experiment \#3 tracer test data 145 
Figure 6.5 Oil saturations for experiments \#1, \#2 and \#3 waterfloods

Figure 6.6 Viscosity of FP 3630s in 2000 ppm NaCl Brine at 68 oC .................148

Figure 6.7 Oil Saturations for experiment \#1 ................................................150

Figure 6.8 Pressure drop data for experiment $\# 1$...........................................150

Figure 6.9 Oil Saturations for experiment \#2 ................................................151

Figure 6.10 Pressure drop data for experiment $\# 2$.........................................151

Figure 6.11 Oil Saturations for experiment \#3 ............................................. 152

Figure 6.12 Pressure drop data for experiment \#3 …......................................152

Figure 6.13 Oil Saturations during polymer flood for experiments \#1,\#2 and \#3153

Figure 6.14 Experiment \#3 tracer test........................................................... 154

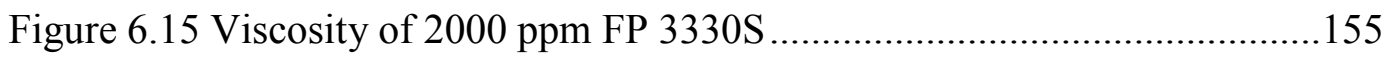

Figure 6.16 Oil Saturation for experiment \#4 …........................................... 157

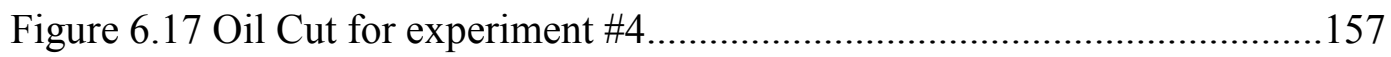

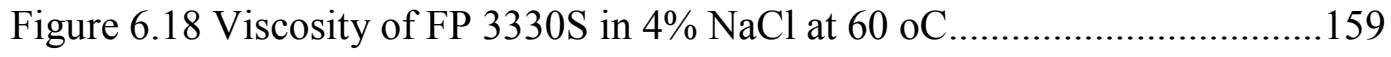

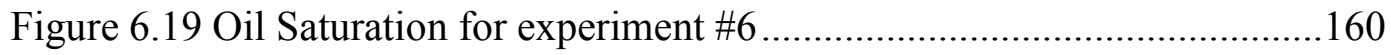

Figure 6.20 Oil Cut for experiment \#6.......................................................... 160

Figure 6.21 Oil Saturation for experiment \#7 f...............................................161

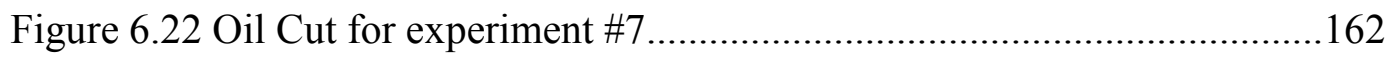

Figure 6.23 Pressure drop for experiment \#7 ..................................................162

Figure 6.24 Viscosity of FP $3630 \mathrm{~S}$ polymer solutions in Lake Water at $73^{\circ} \mathrm{C} \ldots 164$

Figure 6.25 Oil Recovery for experiment \#8 ................................................ 165

Figure 6.26 Pressure drop data for experiment \#8 ….................................... 165

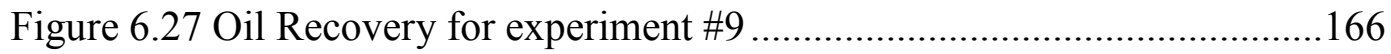

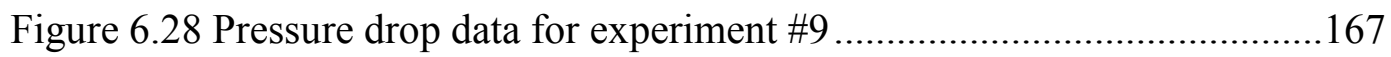


Figure 6.29 Comparison of oil saturations for experiments \#1, \#2, \#3 and \#7 water floods. 168

Figure 6.30 Comparison of oil saturation for experiments \#1, \#2, \#3 and \#7 water floods up to $99 \%$ water cut 168

Figure 6.31: Concentration profile at outlet in a core flood experiment. 171

Figure 6.32 Polyacrylamide reaction with bleach to produce chloramide insoluble suspension. 172

Figure 6.33: UV-visible spectra for different concentrations of FP $3330 \mathrm{~S}$ in $3 \% \mathrm{NaCl}$. .173

Figure 6.34 Calibration curve for FP 3330 polymer in $3 \% \mathrm{NaCl}$. 174

Figure 6.35 Calibration curve for FP 3630S polymer standard solutions in high salinity brine (56000 ppm TDS) at $520 \mathrm{~nm}$. 175

Figure 6.36 Calibration curve for ChemPam 8177 polymer standard solutions in high salinity brine (56000 ppm TDS) at $520 \mathrm{~nm}$. 175

Figure 6.37 Effluent polymer concentration for experiment \#7 ........................176

Figure 6.38 Effluent polymer concentration for experiment \#10......................177

Figure 6.39 Relative permeability curve for experiment \#3 ............................. 183

Figure 6.40 Fractional flow curve for experiment \#3 ...................................... 184

Figure 6.41 Pressure drop data for experiment \#3 .........................................186

Figure 6.42 Oil recovery by secondary polymer flood for experiment \#3 .........187

Figure 6.43 Oil recovery versus log of pore volumes for experiment \#3 ...........188

Figure 6.44 Oil cut for experiment $\# 3$ …..................................................... 189

Figure 6.45 Polymer flood pressure drop for experiment \#4 .............................190

Figure 6.46 Relative permeability curve for experiment \#4 ............................192

Figure 6.47 Fractional flow curve for experiment $\# 4$......................................192 xxiii 
Figure 6.48 Oil recovery for tertiary polymer flood experiment \#4 193

Figure 6.49 Oil cut for experiment \#4 193

Figure 6.50 Equivalent shear rates after polymer breakthrough for experiment \#7196 Figure 6.51 Viscosity after polymer breakthrough for experiment \#7 based on shear

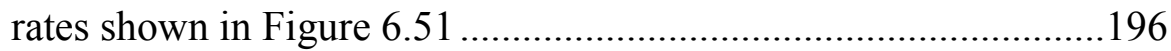

Figure 6.52 Relative permeability curve for experiment \#7 ..............................197

Figure 6.53 Fractional flow curve for experiment \#7 ….................................197

Figure 6.54 Oil cut for experiment \#7 .............................................................198

Figure 6.55 Oil recovery by tertiary polymer flood for experiment \#7 ..............198

Figure 6.56 Fractional flow curve for experiment \#8 …................................200

Figure 6.57: Oil recovery for experiment $\# 8$...............................................200

Figure 6.58 Oil cut for experiment $\# 8$......................................................201

Figure 6.59 Fractional flow curves for experiment $\# 9$.....................................202

Figure 6.60 Oil recovery for experiment \#9 ...................................................203

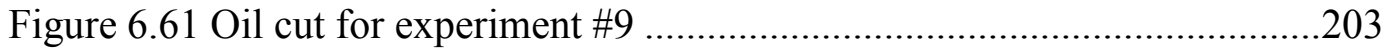

Figure 6.62 Shear rate correction factor for HPAM polymers taken from Wreath

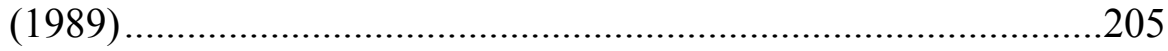

Figure 6.63 Shear rate correction factor for xanthan gum polymer...................205

Figure 6.64 Shear rate correction factor calculated from experiments using HPAM206

Figure 6.65 Oil Recovery for ASP experiment............................................208

Figure 6.66 Pressure drop data for ASP experiment......................................209

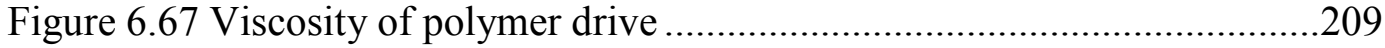

Figure 6.68 Cumulative Oil Recovery, Oil Saturation and Oil Cut for Experiment \#10 
Figure 6.69 Oil Recovery, Oil Saturation and Oil Cut for Experiment \#12 using ChemPam 8177 polymer... 217

Figure 6.70 Comparison for cumulative oil recovery and oil saturation for experiments $\# 7$ and $\# 12$ 217

Figure 6.71 Comparison of oil cuts for experiment \#7 and \#12 .......................218

Figure 6.72 Pressure drop data for experiment \#12 using ChemPam 8177 polymer219

Figure 6.73 Pressure drop for experiment \#7 ................................................219

Figure 6.74 Fractional flow curve for Experiment \#10 ....................................220

Figure 6.75 Effluent polymer concentration for experiment \#12 using ChemPam 8177 polymer 221

Figure 6.76 Oil Recovery, Oil Saturation and Oil Cut for Experiment \#12 _.......221

Figure 6.77 Oil Recovery, Oil Saturation and Oil Cut for Experiment \#12 _.......222

Figure 6.78 Pressure drop data for experiment \#12 _.........................................222

Figure 6.79 Schematic diagram of pushing force .........................................223

Figure 6.80 Schematic diagram of friction force ..........................................224

Figure 6.81 Tomlinson atom motion model ...............................................224

Figure 6.82 Schematic illustration of the probale structures of the adsorbed layer for HAPM (top) and hydrophobically modified HPAM (bottom) (Volpert et al., 1998) 227

Figure 6.83 Mobility reduction on section E for injection of the modified HPAM solution in 1, 2.5 and 11 Darcy sand packs (Dupuis et al., 2011) ..229 


\section{Chapter 1: Introduction}

\subsection{BACKGROUND}

Polymer flooding is a commercially proven technology to enhance oil recovery. Polymer is added to the water to increase the viscosity of the water. This results in a decrease in the mobility ratio between the water and oil. The reduced mobility ratio results in an increase in the sweep efficiency compared to water flooding. The increase in volumetric sweep efficiency is particularly significant in highly heterogeneous reservoirs and reservoirs with poor vertical sweep efficiency due to gravity. The increase in displacement sweep efficiency is the largest for viscous oils (typically greater than about $20 \mathrm{cp}$ ) with unfavorable fractional flow between water and oil. Until recent years, the conventional thinking was that polymer did not reduce the residual oil saturation to water at low capillary numbers typical of water floods. This understanding was based on the correlation between residual oil saturation and capillary number (Abrams, 1975; Stegemeier, 1974; Lake, 1989). Injection of polymers solutions under field conditions does not usually increase the capillary number enough to reduce the oil saturation because the injection rate is constrained by the maximum injection pressure of the well. The first published experimental study to clearly indicate that polymer solutions might reduce residual oil saturation under some conditions was the MS thesis study by Wreath (1989). Subsequently many other experimental studies have also shown a reduction under some conditions. This reduction is most often attributed to the viscoelasticity of polymer solutions (Afsharpoor and Balhoff, 2013; Sheng, 2010). However, despite many studies over the past 20 years, there is still a lot of uncertainty about both the reduction and its cause. 


\subsection{OBJECTIVE}

The main objective of this research was to better understand the effect of polymer flooding on the remaining oil saturation by conducting experiments and interpreting these experimental data in terms of measured polymer and rock characteristics. The experiments were done using a variety of permeable media including both sandpacks and reservoir cores under reservoir conditions. First the polymer solutions were characterized with respect to rheology and filtration. Next polymer corefloods were conducted both to further characterize the polymers and to determine their effect on residual oil saturation. These corefloods were done using a wide variety of cores and crude oils including light and viscous oils. The corefloods were done at low velocity (low capillary number) corresponding to reservoir conditions. Long cores (about $30 \mathrm{~cm}$ ) were used to avoid end effects as much as feasible. A variety of polymers with different structures and properties were used as well as some Newtonian fluids for control experiments. 


\section{Chapter 2: Literature Review}

Some of the early work on polymer flooding can be found in Sandiford (1964), Mungan et al., (1966), Gogarty (1967), Smith (1970), Hirasaki and Pope (1974), Szabo (1975), Thurston and Pope (1981), Cannella et al. (1988) and Wreath et al. (1990). Books by Littmann (1988), Lake (1989), Sorbie (1991), Green and Willhite (1998), Sheng (2011) and Lake et al. (2014) include additional background on polymer flooding and other related applications of polymers for enhanced oil recovery. Many of the commercial polymer floods are summarized in these books. Delamaide et al. (1994) and Chang et al. (2006) report some of the early field successes in China.

The number of papers on both polymer flooding research and polymer flooding field applications has significantly increased in the past ten years. No attempt will be made to review such a vast literature. A few of the more recent papers include those by Gaillard et al. (2010), Kim et al. (2010), Seright (2010), Levitt et al. (2011), Kulawardana et al. (2012), Seright et al. (2012), Skauge et al. (2012), Afsharpoor and Balhoff (2013b), Delaplace et al. (2013), Levitt et al. (2013), Yerramilli et al. (2013), Afsharpoor et al. (2014), Gaillard et al. (2014), Leonhardt et al. (2014), Prasad et al. (2014), and Wang et al. (2014).

As polymer flooding has become more widely and successfully applied worldwide, the upper bound of oil viscosity for its effective application has increased. A good example is the recent application of polymer flooding in the Pelican Lake field in Canada (Delamaide et al., 2013) where the oil viscosity is 1,000-2,500 cp. To displace such viscous oil, the polymer concentration needs to be quite high to achieve a sufficiently high polymer solution viscosity. To properly and efficiently design a polymer flood, laboratory corefloods must to be carried out. In carrying out such laboratory tests, 
preparation of a homogeneous polymer solution, its injection into the core, the measurement of the mobility data from the pressure taps along the core, and the interpretation of the effluent data, all require special methods and special care. The laboratory procedures used in this study to accomplish these challenging tasks are described in Chapter 3. A brief review of the most commonly used polymers and some of their most important properties related to this research is given below.

\subsection{POLYMERS FOR EOR}

Partially hydrolyzed polyacrylamide (HPAM) was the first polymer used as a thickening agent for aqueous solutions injected into oil reservoirs for enhanced oil recovery. In general, the its performance depends on its molecular weight and its degree of hydrolysis (Sorbie, 1991). The HPAM molecule is a flexible chain structure known as a random coil and since it is a polyelectrolyte, it interacts with ions in solution. The structure of HPAM is shown in Figure 2.1. For most commercial products, the degree of hydrolysis is between 25 and $35 \%$. The same structure is also made commercially as a co-polymer of acrylamide and acrylate monomers. The co-polymer is more uniform and has other advantages over the post-hydrolyzed polymer. For convenience, both will be designated as HPAM except when it is important to make a distinction between them.

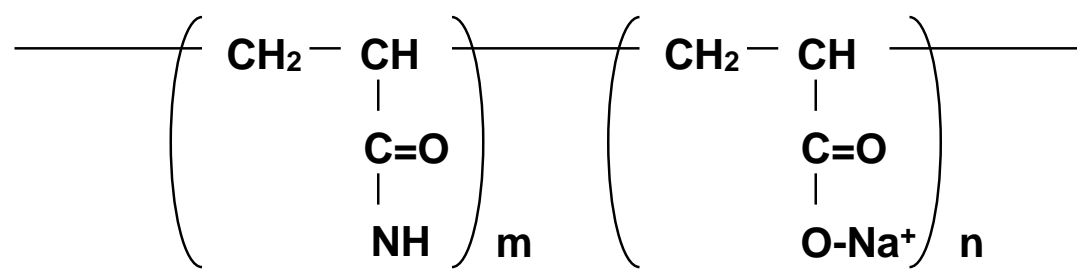

Figure 2.1 Chemical Structure of HPAM 
A wide variety of synthetic polymers besides HPAM have been evaluated for EOR applications under high temperature and high salinity conditions (Askinsat, 1980). Incorporating monomer groups that are more resistant to hydrolysis such as 2acrylamido-2-methylpropane sulfonic acid (AMPS), poly-vinyl pyrrolidones (PVP), or N-vinyl pyrrolidones (Doe et al., 1987; Vermolen et al., 2011) significantly increased their tolerance to divalent cations.

Incorporation of N-vinylpyrrolidone monomers (NVP) in the acrylamide (AM) polymer chain improves its stability at high temperature and high salinity (Doe et. al., 1987). The NVP monomer protects the acrylamide neighbors in the co-polymer against hydrolysis (Gaillard et al., 2014; Fernandez, 2005). The hydrolysis limit of the amide groups decreases as the content of NVP in the polymer increases. As the sequence lengths of acrylamide units are short, i.e., the higher the number of acrylamide groups surrounded by NVP units, the more the polymer is resistant to hydrolysis (Gaillard et al., 2014; Fernandez, 2005). Figure 2.2 represents a schematic structure of NVP-AM copolymer. The mechanism of amide group protection against the thermal hydrolysis could be steric hindrance or intramolecular hydrogen bonding in the NVP-AM copolymer (Doe et. al., 1987; Fernandez, 2005).

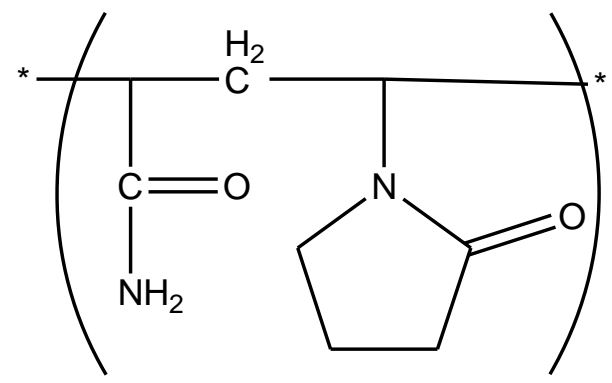

Figure 2.2 Chemical structure of NVP-AM copolymers. 


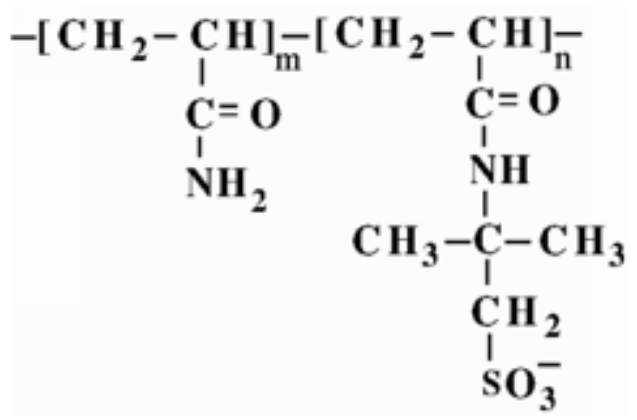

Figure 2.3 Chemical structure of AMPS

Recently, there are reports for thermal stability of NVP-AM polymers under harsh conditions (Gaillard et al., 2014; Vermolen et. al., 2011; Gaillard et. al., 2010). However, transport and retention data in both outcrop and reservoir cores is very limited for these types of polymer. There is a great need for a systematic investigation and comparison of these polymers under a range of harsh reservoir conditions to determine their limitations, and to develop a guideline for polymer selection for polymer flooding, surfactantpolymer flooding and alkali-surfactant-polymer flooding.

Xanthan gum has also been used in the past for enhanced oil recovery, but its current use is very small compared to HPAM in part due to its higher cost. It is a polysaccharide with the structure shown in Figure 2.4. Xanthan gum is a biopolymer produced during fermentation of glucose and is subject to biodegradation. It is also subject to free radical degradation at high temperature. It has good salinity tolerance and good mechanical stability i.e. it is not easily shear degraded. It is compatible with most surfactants and other injection fluid additives used in tertiary oil recovery formulations (Abidin et al., 2012). Disadvantages of xanthan are high cost, high susceptibility to biodegradation and potential for injectivity problems due to cellular debris remaining from the manufacturing process (Taylor and Nasr-El-Din, 1998). 


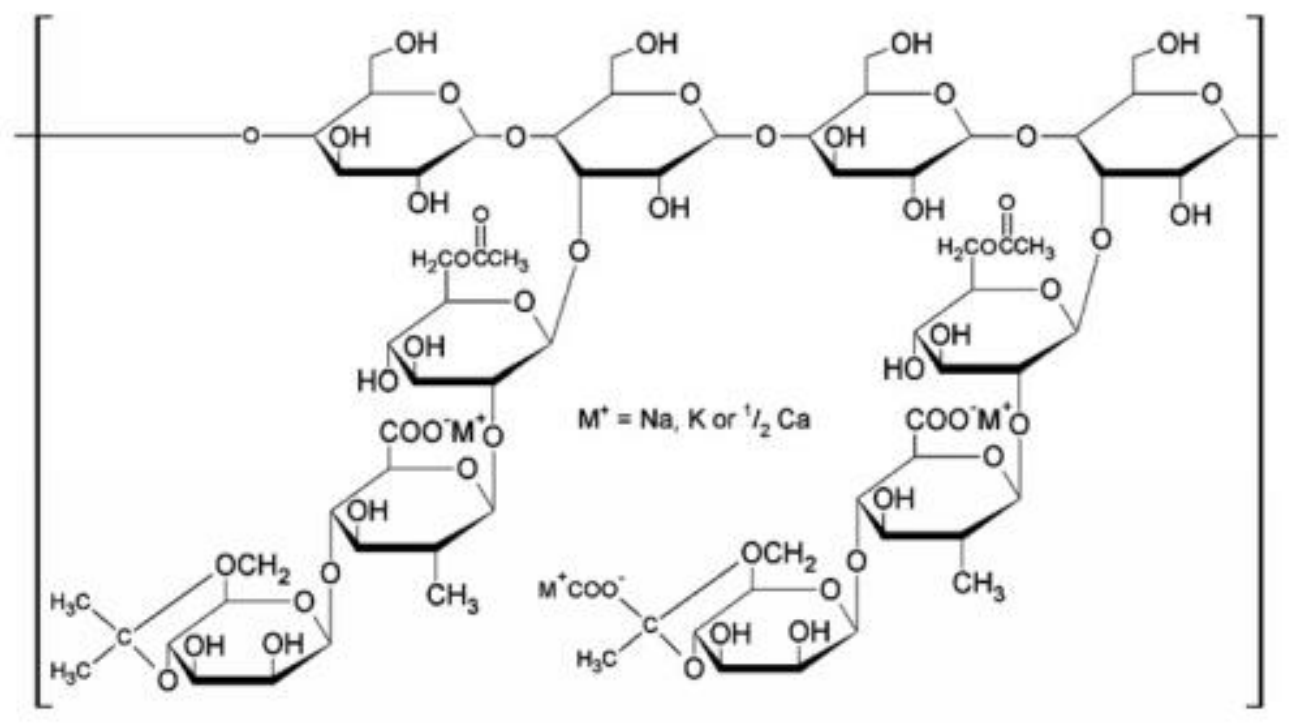

Figure 2.4 Chemical structure of xanthan gum

In addition to xanthan gum, polysaccharides such as scleroglucan, carboxymethylcellulose and guar gum, have also been extensively investigated for EOR. These biopolymers are less sensitive to salinity or hardness and to mechanical degradation due to their semi-rigid molecular structure (Kohler and Chauveteau, 1981). However, combinations of high temperature, high salinity and high divalent ion concentrations limit the performance of many of these polymers (Davison and Mentzer, 1982).

Scleroglucan showed the best performance at high temperature and high salinity among 140 polymers (Davison and Mentzor, 1982; Rivenq et al., 1992; Kalpacki et al., 1990). Scleroglucan is a non-ionic, branched homo-polysaccharide produced by fermentation of a plant pathogen fungus genus Sclerotium. The polymer backbone consists of linearly linked $\beta-1,3-\mathrm{D}$-glucose residues and $\beta-1,6-\mathrm{D}$ - glucose side chain attached to every third main chain residue in the backbone (Figure 2.3). Scleroglucan is 
insensitive to salinity because of its non-ionic character. Since the polymer exists as rodlike triple helix chains, they behave as semi-rigid molecules in aqueous solutions (Rivenq et al., 1992; Yanaki et al., 1981; Kalpakci and Jeans, 1990). This semi-rigid structure explains the high viscosifying power and shear resistance of the molecule. However, in contrast to the high performance of scleroglucan under harsh conditions, the major drawback noted is the poor filterability and limited transport data for this polymer.

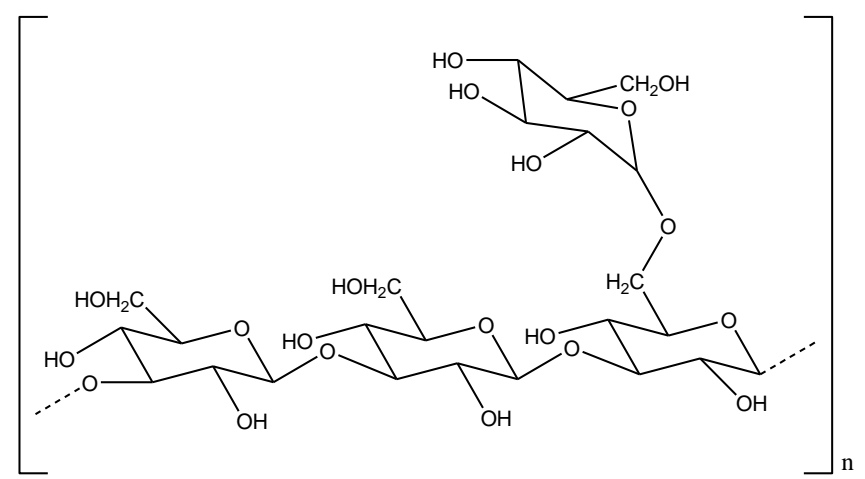

Figure 2.5 Chemical structure of Scleroglucan

Water-soluble hydrophobically associating polymers are water-soluble polymers that contain hydrophobic groups attached directly to the polymer backbone. In aqueous solutions, the hydrophobic groups can associate to minimize their exposure to the solvent, similar to the formation of micelles by a surfactant above its critical micelle concentration. This association results in an increase in the hydrodynamic size of the polymer that increases solution viscosity (Taylor and Nasr-El-Din, 1998). Another significant type of associating polymer is prepared by hydrophobically modifying hydroxyl ethyl cellulose HEC or hydroxypropyl cellulose (HPC) by reaction with alkyl halides, acid halides, acid anhydrides, isocyanates, or epoxides (Landoll, 1982). These polymers are claimed to have potential in IOR (Landoll, 1985; Sau and Landoll, 1989) . 
Evani (1984) and van Phung and.Evani (1986) claim that cellulosic associating thickeners have acceptable salt tolerance, but are ineffective at low concentrations and have poor thermal stability. They are also readily biodegraded. The synthesis, solution properties and rheology of associating cellulosic thickeners have been studied (Goodwin et al., 1989; Sau and Landoll, 1989; Dersch, 1994) and are not examined in further detail in this work (Taylor and Nasr-El-Din, 1998).

\subsection{Polymer Rheology}

The term rheology is defined as the study of the deformation and flow of different fluids in response to surface forces (Bird et al., 1987). The Newtonian relationship between stress and deformation rate (strain rate) is:

$$
\mathrm{F}=\mu \mathrm{A} \frac{d V}{d x}
$$

where $\mathrm{F}$ is force on the surface of the fluid, $\mathrm{A}$ is the contact area between two adjacent

layers in the fluid, $\frac{d V}{d x}$ is the velocity gradient between the two layers, and $\mu$ is the fluid viscosity (Bird et. al, 1987).

Based on the definition of the fluid viscosity as the fluid resistance to shear (Bird, et al., 1987), it is possible to formulate fluid viscosity as:

$$
\sigma=\mu \gamma
$$

where $\sigma(\mathrm{Pa})$ is shear stress on the fluid surface, $\mu(\mathrm{Pa} . \mathrm{s})$ is fluid viscosity and $\gamma\left(\mathrm{s}^{-1}\right)$ is shear rate.

Fluids can be classified as Newtonian or non-Newtonian. The viscosity of Newtonian fluids is not a function of shear rate while the viscosity of non-Newtonian 
changes with shear rate. This change in the viscosity as a function of shear rate is different for different fluids in Figure 2.6 (Bird, et al., 1987).

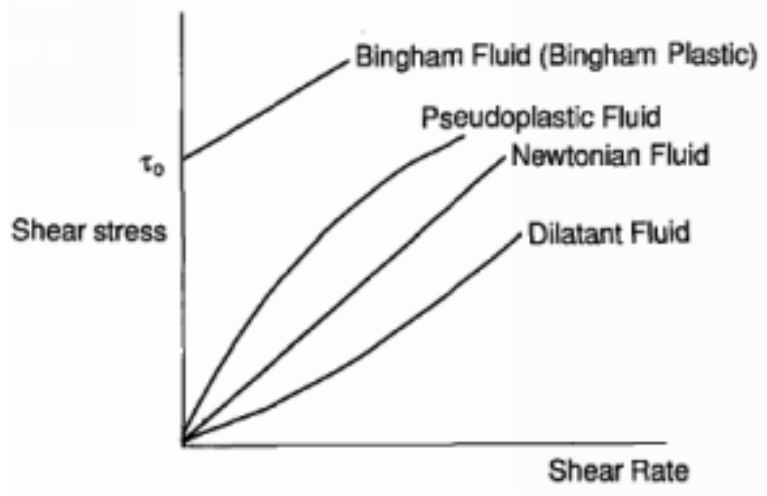

Figure 2.6 Different type of fluids

Pseudo plastic fluids shows shear thinning behavior which means the viscosity deceases as shear rate increases, and vast majority of polymer solutions are shear thinning in their nature. There are also several other parameters which may affect polymer viscosity behavior, e.g. molecular weight, polymer concentration, and the nature of polymer and solvent.

\section{Effect of polymer concentration and molecular weight on the viscosity}

Higher molecular weight and higher concentration gives a solution with a higher viscosity. This is because the polymer concentration changes the interactions among polymer molecules, and the molecular weight directly affects the chain size. While polymers with higher molecular weight gives a solution with a higher viscosity, they are more easily shear degradable. (Sorbie, 1991). Mechanical degradation (also known as shear degradation) of polymers can also cause loss of viscosity. Noik et al. (1994) studied the mechanical degradation of HPAM and of one sulfonated polyacrylamide with 25 mole $\%$ sulfonation degree. Their results show that due to the rigidity of sulfonate group, 
polymers with a sulfonate group have better resistance to mechanical degradation than HPAM. Degradation refers to any process that breaks down polymer molecular structures. Polymer degradation and loss of viscosity in particular must be either prevented or at least minimized for long periods of time for a polymer to be suitable for enhanced oil recovery. Viscosity may be degraded chemically, mechanically or biologically (Mungan, 1969; Gaillard et al., 2010; Sheng, 2010; Sorbie 1991).

\section{Effect of ions and $p H$ on the viscosity}

To understand the effect of monovalent and divalent ions on the viscosity behavior of polyelectrolyte solutions, the interaction of these ions with the charges on the polymer chain must be understood.

Molecules of anionic polymer in distilled water may be almost fully expanded due to the repulsion between charges distributed along the polymer chain. In aqueous solvents because of positive mobile ions, the charges on the negative polymer chain are screened and the degree of expansion decreases. Some theories have been developed to explain the effect of ions on the rheological behavior of polyelectrolyte solutions (Flory, 1953; Tanford, 1961).

The ions in the aqueous solution are divided into two categories; monovalent cations, e.g. $\mathrm{Na}^{+}$or $\mathrm{K}^{+}$and divalent cations, e.g. $\mathrm{Ca}^{2+}$ or $\mathrm{Mg}^{2+}$. HPAM molecules are more sensitive to divalent cations compare to monovalent cations. It has been generally accepted this is due to the strong binding between divalent cations and the carboxylate group $\left(\mathrm{COO}^{-}\right)$on the polymer chain (Szabo, 1979; Zaitoun and Potie, 1983; MoradiAraghi et al., 1995). 
When salts are added the aqueous solution, the anionic carboxyl side groups will react with monovalent and divalent cations. The Coulomb repulsion between the negative charged groups on the polymer are less effective as the salinity of the brine increases, which reduces the swelling in the macromolecule (Reichenbach-Klinke et al., 2011).

When the acrylamide and acrylic acid co-polymer is dissolved in water without any salt, the interactions occurring between the anionic side groups cause electrostatic repulsion (Dupuis and Rousseau, 2010), which induces a swelling in the macromolecule. When the salinity increases by addition of monovalent cations, screening of the repulsion between the negative charged carboxyl groups occurs. As a consequence of the interactions between the positive charged monovalent ions and the side groups, the polymer molecules will start to coil up. When the salinity and hardness of the brine by addition of divalent ions increase further, the macromolecules are no longer in a stretched state. The chains are considered to be in a coiled state. In the presence of divalent cations, a precipitation of the polymer may occur. Precipitation of HPAM macromolecules in hard brine is related to the degree of hydrolysis. Above a certain level of acrylic acid in the polymer, insoluble complexes between these anionic groups and divalent cations can be formed in the solution. When these segments precipitate out of the solution due to insolubility, the viscosity will drop (Dupuis and Rousseau, 2010). 


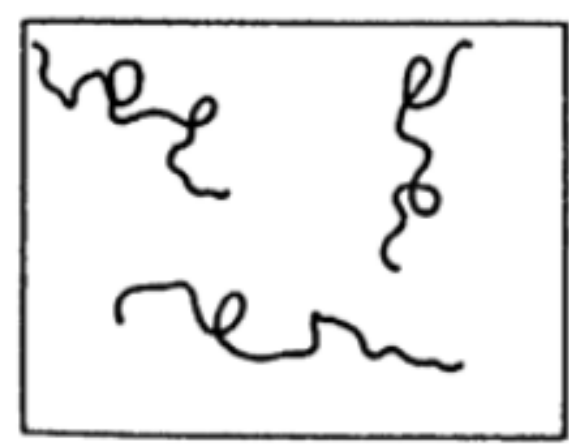

Low salinity polymer solution

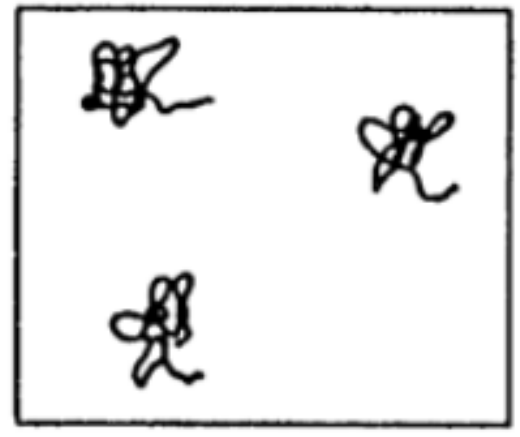

High salinity polymer solution

Figure 2.7 The long and flexible polymer backbone of HPAM coils- up when increasing the salinity/hardness of the solution (Sorbie, 1991).

The negatively charged carboxyl or AMPS groups at high $\mathrm{pH}$ conditions induce extension of the molecules through electrostatic repulsion among the groups, thereby increasing viscosity; conversely, low $\mathrm{pH}$ conditions cause the molecules to become coiled, resulting in low viscosity (Reichenbach-Klinke and Langlotz, 2011).

\subsubsection{Flow of polymer solutions in porous media}

The viscosity of a polymer solution is a function of flow rate since a dissolved polymer molecule can be easily deformed during flow.

\section{Polymer retention}

Polymer molecules can be retained in a porous medium by three mechanisms: physical adsorption, mechanical entrapment, and hydrodynamic retention (Sorbie, 1991).

Retention by mechanical entrapment occurs when larger polymer molecules are trapped in narrow flow channels (Huh et al., 1990; Dominguez and Willhite, 1977). Assuming a complex pore structure with large interconnected pore networks means the polymer has many possible routes to flow through the permeable medium. As polymer solution passes through this complex connected network, molecules may go through any 
available routes and if the route is narrow enough, polymer molecules will be trapped and block the route as well as probably cause more trapping upstream of the blockage. As a consequence of this process, the polymer concentration may not reach its injected value until after many pore volumes of polymer solution have been injected. And if the number of entrapment locations exceeds the critical number the core would block eventually. Mechanical entrapment is a more likely mechanism for polymer retention for lower permeability cores where the pore sizes are small and chance for polymer molecules to be trapped is very high. This has been studied by several workers. Only a very few workers have studied polymer retention in the presence of a residual oil phase and most of their experiments were done on water-wet cores.

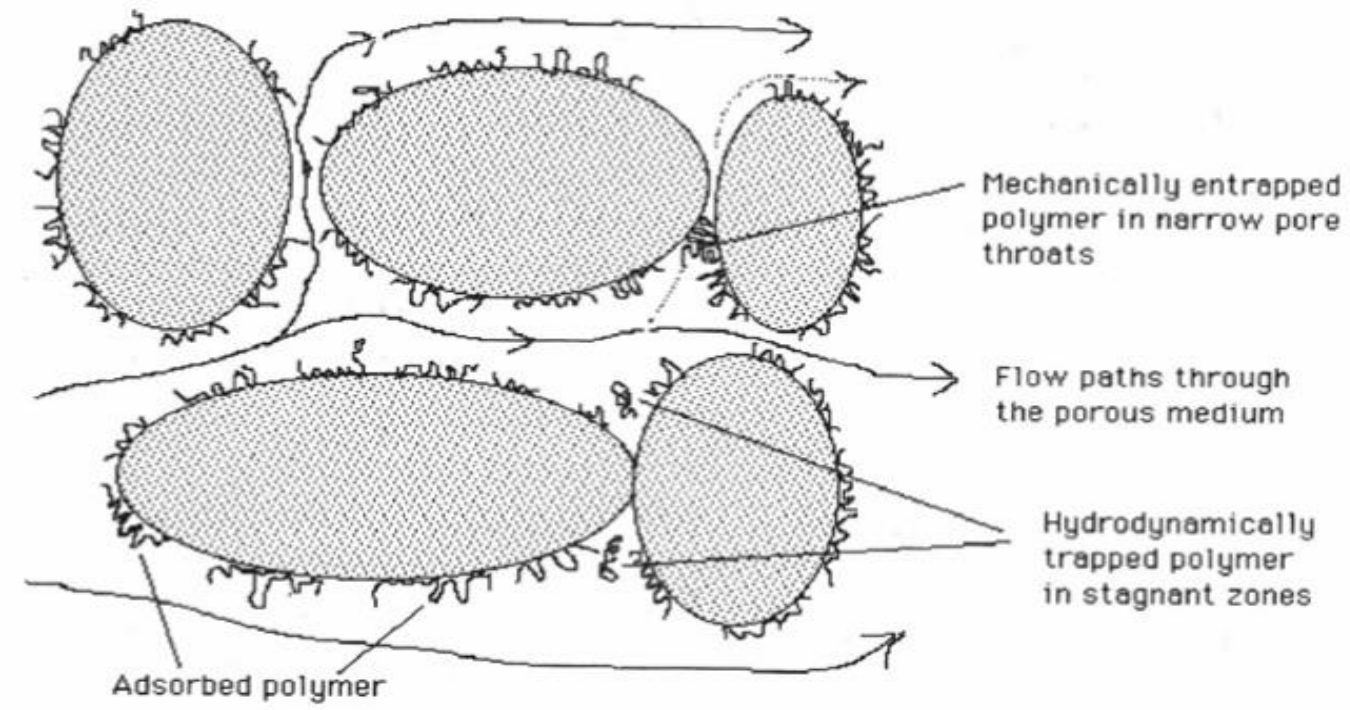

Figure 2.8 Schematic diagram of polymer retention mechanisms in porous media (Sorbie, 1991)

Chauveteau and Kohler (1974) observed that polymer retention changed when the fluid flow was adjusted to a new value. As illustrated in Figure 2.8, hydrodynamic drag force traps some of the polymer molecules temporarily in stagnant flow regions. In such 
region it may be possible to exceed the polymer stream concentration. When flow rate stops, these molecules may be introduced into main stream channels and increase the polymer concentration. When the flow starts again the effluent concentration shows a peak.

The interaction between polymer molecules and solid surfaces causes polymer molecules to be bound to the surface of the solid mainly by physical adsorption. Basically the polymer sits on the surface of the rock, and the larger the surface area available the higher the level of adsorption. Rocks with lower permeability have higher surface area available and generally higher adsorption. In rocks with very low permeability, the polymer may not be able to flow into the small pores and adsorption may be reduced.

Polymer retention varies with polymer type, polymer concentration, molecular weight, rock characteristics and composition, brine salinity, hardness, $\mathrm{pH}$, flow rate and temperature. At low polymer concentrations, polymer adsorption is directly proportional to polymer concentration in the absence of mechanical entrapment (Baijal, 1981). Higher polymer concentration increases the probability of polymer adsorption on rock surfaces. Laboratory data on the effect of molecular weight on polymer adsorption show conflicting results. Baijal (1981) and Gramain and Myard (1981) reported higher levels of adsorption with increasing molecular weight. However, other studies noted that polymer adsorption on silica sand is inversely proportional to the molecular weight of the polymer (Lakatos and Szabo, 2001).

Based on the experimental evidence in the literature, it is possible to define three different regimes by considering the permeability of the porous medium (Denys, 2003). At low permeability, mechanical entrapment is the main mechanism of polymer retention. A polymer coil can just enter a cylindrical pore, which has been pre-adsorbed. 
The latter means that due to adsorption an adsorbed polymer layer (thickness $2 \mathrm{R}_{\mathrm{h}}$ ) covers the pore walls. When assuming that this adsorbed layer reduces the pore size effectively, it follows that mechanical entrapment can occur as $R_{p}<3 R_{h}$. Some characteristics of mechanical entrapment are: 1) The retention occurs at any flow rate, 2) The retention rate increases with flow rate, 3) The level of retained polymer is largest close to the core entrance and 4) The mechanism can take place both under adsorption and non-adsorption conditions (Denys, 2003).

For high permeability porous media, the criterion of $R_{p}>50 R_{h}$ is based on experimental evidence (Sorbie, 1991). In this regime, the average pore diameter is large compared to the polymer coil size and retention by filtration on pore throats is negligible so polymer retention can only occur by adsorption. After complete saturation of the surface available by adsorption, polymer injection at a certain flow rate Q results in stationary flow conditions and a constant mobility reduction $\mathrm{R}_{\mathrm{m}}$, where

$$
R_{m}=\frac{\Delta P_{p}}{\Delta P_{b}}
$$

Here $\Delta \mathrm{P}_{\mathrm{p}}$ is the pressure drop during polymer solution flow at $\mathrm{Q}$ after adsorption. $\Delta \mathrm{P}_{\mathrm{b}}$ is the pressure drop during brine flow at $\mathrm{Q}$ before adsorption. In this regime, the mobility reduction $\mathrm{R}_{\mathrm{m}}$ is a function of the (in-situ) viscosity, which depends on the flow rate and the permeability reduction $\mathrm{R}_{\mathrm{k}}$ of the porous medium due to adsorption. The latter is defined by: where $\Delta \mathrm{P}_{\mathrm{b}}$ is the pressure drop during brine flow at $\mathrm{Q}$ after adsorption. For high permeability porous media, the permeability reduction $R_{k}$ is usually independent of Q. $R_{k}$ depends on the ratio between the adsorbed layer thickness and the pore radius. Since the (in-situ) rheological behavior is a function of the deformation rates in the medium, an estimation of these parameters would be valuable. This is however 
impossible though due to the unknown structure. Therefore a macroscopic description is often used, which describes the porous medium as a capillary bundle model.

Experimental evidence has been found for the existence of a retention mechanism specific to intermediate permeability cores (Zaitoun and Chauveteau, 1998; Zitha and Botermans, 1996; Zitha et al., 1995). Zitha et al.(1995) found a critical shear rate when injecting a dilute polyacrylamide/brine solution in $18 \mu \mathrm{m} \mathrm{SiC} \mathrm{grain} \mathrm{packs}(\mathrm{k}=$ $1.4 \times 10^{-13} \mathrm{~m}^{2}$ ). Below this rate only a small permeability reduction resulted due to polymer adsorption. Above this rate $\mathrm{R}_{\mathrm{m}}$ increased continuously in time during injection. The authors proposed the following mechanism. At the critical flow rate the polymer coils can stretch due to the fact that the elongational deformation rates have become high enough. The stretched chains can bridge pore throats by adsorption. This bridging process, resulting in a continuous permeability reduction, can last as long as still free adsorption sites around the pore throat are available. The retention mechanism was called bridging adsorption. Other experimental findings (Zitha et al., 1995) are: (1) The plugging rate is larger when injecting solutions of higher polymer concentration, (2) The plugging rate increases with decreasing core permeability, (3) The plugging phenomenon is absent under non-adsorbing conditions. (4) The polymer is usually retained in the first part of the core. Not only the retention mechanism, but also the rheology of polymer solutions in porous media is dependent on the permeability (Denys, 2003).

\section{Permeability Reduction}

Many investigators have reported that HPAM polymers reduce the brine permeability during and following injection of the polymer (Gogarty, 1967; Smith, 1970; Zaitoun and Chauveteau, 1998; Zitha and Botermans, 1996; Zitha et al., 1995; Bondor et al., 1972; Hirasaki and Pope, 1974; Sorbie, 1991; Zitha et al., 1995; Green and Willhite, 
1998). The mobility reduction is called permeability reduction factor when polymer is flowing in the porous medium and residual resistance factor after water has displaced the mobile polymer. This phenomenon has been modeled as a function of polymer retention, but there does not appear to be a simple relationship between retention and permeability reduction. Other variables that may affect permeability reduction factor are the size of the polymer molecule (molecular weight), the size of the pores (permeability/porosity), salinity and flow rate.

Many investigators have reported permeability reduction factors as high as 10 or even higher, especially for low permeability rocks. One of the most significant findings of this research is that the HPAM polymers used in this research did not reduce the permeability of the cores. These results are presented in Chapters 5 and 6. The higher quality HPAM polymer solutions used in this research compared to the polymers available in 1960s and 1970s when much of the early polymer flooding research was done may account for this important difference in behavior.

\section{Apparent viscosity}

Three flow regimes have been identified. Newtonian flow behavior was observed at low shear rates (no coil deformation), shear-thinning flow behavior at higher shear rates (coil deformation) and thickening behavior above a critical shear rate. In a conventional shear rheometer only the first two regimes can be measured. The thickening regime is a consequence of the extension of polymer coils to a stretched state, resulting in a large increase in polymer-solvent friction (Bird et al., 1987). This coil-stretch transition is induced by the elongational deformation gradients present during porous media flow. At large enough flow rates, also mechanical degradation i.e. chain breakage can occur in the thickening regime. 


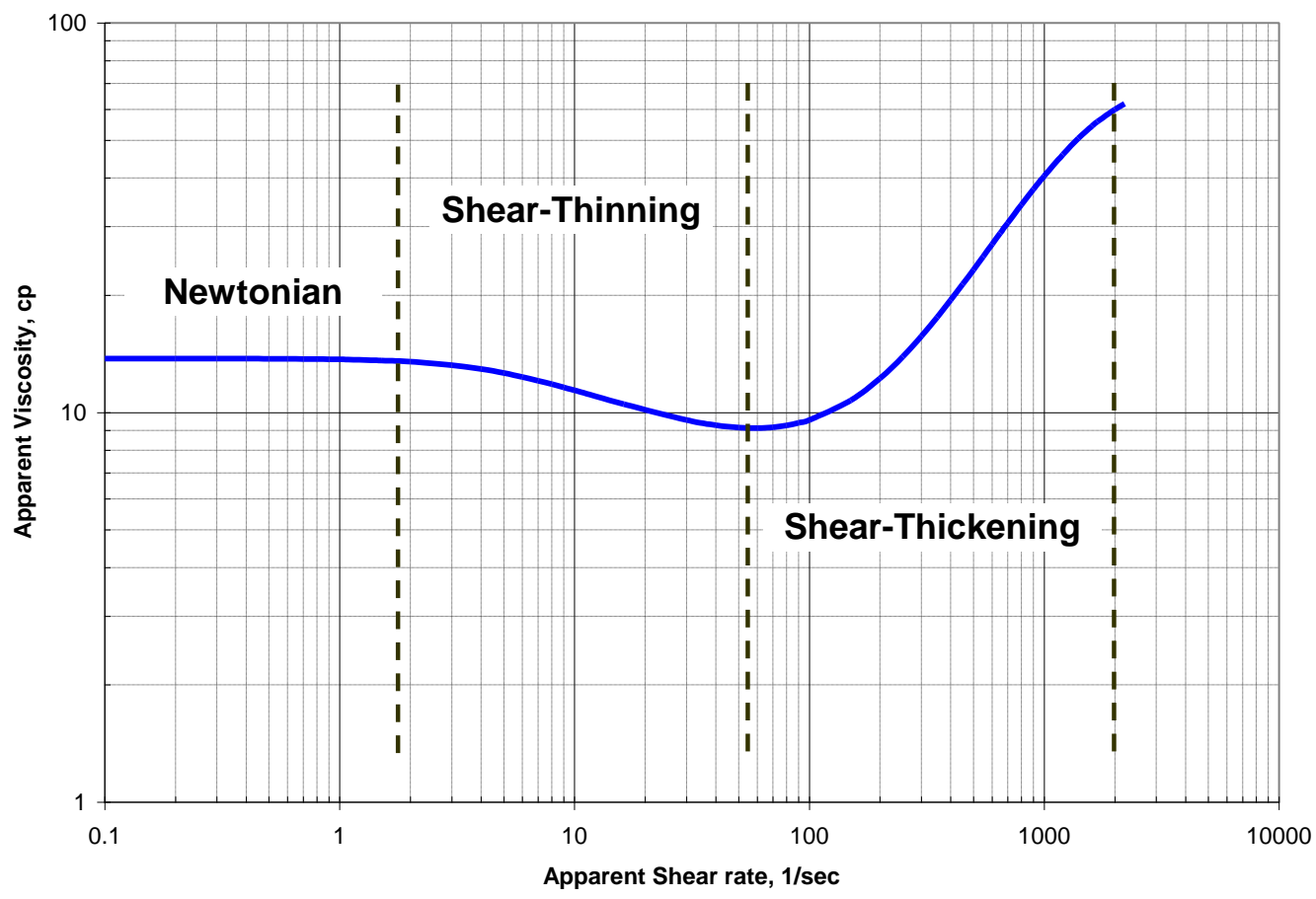

Figure 2.9 Schematic of shear rate dependence of polymer (HPAM) apparent viscosity

\section{Calculation of shear rate in porous media}

Since most polymer solutions are non-Newtonian fluids, their shear rate vs. viscosity is not a linear relationship. For a given viscosity, a shear rate should be defined in advance. The average shear rate for flow in a porous medium is related to its permeability, porosity and velocity. This leads to an expression of an apparent shear rate, $\gamma_{\mathrm{app}}$, to describe the interstitial shear rate in the porous medium.

The viscosity for a power-law fluid flowing in a uniform porous medium can be approximated using the following equation:

$$
\mu=K_{p l}\left(\dot{\gamma}_{a p p}\right)^{n-1}
$$

where $K_{p l}$ and $n$ are the power-law coefficient and exponent. For shear-thinning fluids, $0<n<1$; for Newtonian fluids, $n=1$. 
Carman (1937) approximated the shear rate of a Newtonian fluid in a porous medium as follows:

$$
\gamma=\frac{4 u}{\phi r} \frac{L_{e}}{L}
$$

where $\mathrm{u}$ is the superficial velocity, $\mathrm{cm} / \mathrm{s} ; \phi$ is porosity; $\mathrm{r}$ is the average pore radius, $\mathrm{cm} ; \mathrm{L}_{\mathrm{e}}$ is length of the tortuous flow path, $\mathrm{cm}$; $\mathrm{L}$ is the porous medium length, $\mathrm{cm}$. The porous medium is considered as a bundle of straight capillary tubes with a length that is greater than the macroscopic length by a tortuosity factor and the capillaries are of uniform size and not interconnected.

Christopher and Middleman (1965) used the following equation for the apparent shear rate of a power-law fluid in a porous medium:

$$
\gamma_{a p p}=\left(\frac{3 n+1}{4 n}\right) \frac{12 G}{\rho \sqrt{150 k \emptyset}}
$$

where $\mathrm{n}$ is the power law parameter; $\rho$ is fluid density, $\mathrm{g} / \mathrm{cm}^{3} ; \mathrm{k}$ is permeability, $\mathrm{cm} 2 ; \phi$ is porosity; $\mathrm{G}$ is mass velocity, $\mathrm{g} /(\mathrm{cm} 2 \cdot \mathrm{sec})$

$$
\mathrm{G}=\rho\left(\frac{k}{H} \frac{\Delta P}{L}\right)^{\frac{1}{n}}
$$

where $\Delta \mathrm{P}$ is the pressure drop, dyne $/ \mathrm{cm}^{2} ; \mathrm{k}$ is permeability measured by a Newtonian fluid, $\mathrm{cm}^{2}$; $\mathrm{L}$ is the length, $\mathrm{cm} ; \mathrm{H}$ is the non-Newtonian bed factor.

$$
\mathrm{H}=\frac{K}{12}\left(9+\frac{3}{n}\right)^{n}(150 k \varphi)^{\frac{1-n}{2}}
$$


where $\mathrm{K}$ is the power law coefficient (viscosity at $1 \mathrm{~s}^{-1}$ ).

Hirasaki and Pope (1974) modeled the apparent viscosity of a power-law fluid in a bundle of capillary tubes assuming a tortuosity of 25/12:

$$
\begin{gathered}
\mu_{a p p}=H_{p l} u^{n-1} \\
H_{p l}=K_{p l}\left(\frac{3 n+1}{n}\right)^{n-1}\left(8 k k_{r w} \phi_{w}\right)^{\frac{1-n}{2}} \\
\gamma_{a p p}=\left(\frac{3 n+1}{4 n}\right)^{\frac{n}{n-1}} \frac{12 u}{\sqrt{150 k_{p} \phi_{w}}}
\end{gathered}
$$

where $\mathrm{n}$ is the bulk power law exponent; $\mathrm{u}$ is the Darcy velocity, $\mathrm{cm} / \mathrm{s} ; \mathrm{k}_{\mathrm{p}}$ is polymer permeability (includes the effect of relative permeability and permeability reduction), $\mathrm{cm}^{2} ; \phi$ is porosity; $\phi_{w}$ is $\phi \mathrm{Sw}$.

Chauveteau and Zaitoun (1981) calculated the shear rate using:

$$
\dot{\gamma}=\alpha \frac{4 v}{r}
$$

Where $\mathrm{k}$ is permeability, $\mathrm{cm}^{2} ; \phi$ is porosity; $\alpha$ is a shape parameter characteristic of the pore structure, $\alpha=1.7$ for packs of large spheres with the same diameter, $\alpha=2.5$ for packed beds of angular grains; $v$ is the interstitial velocity, $\mathrm{cm} / \mathrm{s} ; \mathrm{r}$ is the average pore radius, $\mathrm{cm}$ and $r=\left(\frac{8 k}{\varphi}\right)^{\frac{1}{2}}$ was used to estimate of the average hydrodynamic pore radius in a homogeneous unconsolidated porous medium. 
Cannella et al. (1988) modeled the flow of a power-law fluid (xanthan gum polymer) in a permeable medium using the following equation for the apparent shear rate:

$$
\gamma_{a p p}=C\left(\frac{3 n+1}{4 n}\right)^{\frac{n}{n-1}} \frac{u}{\sqrt{k_{w} S_{w} \phi}}
$$

where $\mathrm{k}_{\mathrm{w}}$ is water permeability, $\mathrm{cm}^{2} ; S_{w}$ is water saturation; $\mathrm{n}$ is the bulk power law exponent; $\mathrm{C}$ is a shear rate correction factor, which is a function of the network parameters; $\phi$ is porosity; and $\mathrm{u}$ is Darcy velocity, $\mathrm{u}=\mathrm{Q} / \mathrm{A}, \mathrm{cm} / \mathrm{s}$. They showed good agreement between the measured and calculated apparent viscosity of the xanthan gum polymer flowing in rocks over a range of permeability and saturation using a correction factor of 6 .

Balhoff and Thompson (2006) modeled the flow of non-Newtonian fluids using physically representative network models instead of using a capillary tube model. A network model is a much more realistic model than a capillary tube model. They also presented an equation for the apparent shear rate with an adjustable parameter called $\beta$. Their equation is the same as the Cannella et al. (1988) equation if beta is replaced by C. They estimated the value of beta to be about 1.46 by fitting experimental data for flow of polymer solutions in bead packs with extremely high permeability.

The tortuosity of a uniform bead pack is close to 2 using the definition $\left(\mathrm{Le}_{\mathrm{e}} / \mathrm{L}\right)^{2}$ where $L_{e}$ is the effective path length through the permeable medium of length $L$ (Lake, 1989). The pore velocity is the interstitial velocity times the square root of the tortuosity, which is remarkably close to the beta value of 1.46 reported by Balhoff and Thompson (2006). For comparison, the tortuosity of Bentheimer sandstone from both diffusion experiments and formation resistivity factor data is about 4 .. Tortuosity is rarely reported 
for multiphase flow in porous media, but it can be estimated from the porosity and electrical resistivity factor for partially saturated rocks and increases roughly as the inverse of water saturation. This is in at least qualitative agreement with the results for two-phase flow polymer floods given in Chapter 6.

The shear rate during a polymer flood changes because the water saturation and the water relative permeability change during the displacement. Of course this means that the aqueous phase viscosity is changing as well even in a linear coreflood at constant flow rate. This change is usually neglected when analyzing coreflood data. However, in this study the shear rate was calculated as a function of water saturation and water relative permeability since it was found that this improved the agreement between the experimental data and the fractional flow calculations.

Darcy's law for two-phase flow in a linear porous medium can be written:

$$
\begin{aligned}
q_{w} & =-A \frac{k k_{r w}}{\mu_{w}} \frac{\Delta P}{L} \\
q_{n w} & =-A \frac{k k_{r n w}}{\mu_{n w}} \frac{\Delta P}{L}
\end{aligned}
$$

where $k_{r w}$ is the relative permeability of the wetting phase, and $k_{r n w}$ is the relative permeability of the non-wetting phase.

The relative permeability of the wetting phase is defined as:

$$
k_{r w}\left(S_{w}\right)=\frac{k_{w}\left(S_{w}\right)}{k} ; 0 \leq k_{r w}\left(S_{w}\right) \leq 1
$$

where $k_{w}$ is the effective permeability of the wetting phase. This definition is valid for $k_{\text {rnw }}$ also.

In this work, the water relative permeability was calculated as a function of saturation using a Corey-type model as described below. 


$$
\begin{gathered}
k_{r w}=k_{r w}^{o} S^{n} \\
k_{r o}=k_{r o}^{o}(1-S)^{m} \\
S=\frac{S_{w}-S_{w r}}{1-S_{o r}-S_{w r}}
\end{gathered}
$$

where

$$
\begin{aligned}
& \mathrm{k}_{\mathrm{rw}}=\text { water relative permeability } \\
& \mathrm{k}_{\mathrm{ro}}=\text { oil relative permeability } \\
& \mathrm{k}_{\mathrm{ro}}{ }^{\mathrm{o}}=\text { end-point oil relative permeability } \\
& \mathrm{k}_{\mathrm{rw}}{ }^{\mathrm{o}}=\text { end-point water relative permeability } \\
& \mathrm{S}_{\mathrm{w}}=\text { water saturation } \\
& \mathrm{S}_{\mathrm{wr}}=\text { residual water saturation, irreducible water saturation } \\
& \mathrm{S}_{\mathrm{or}}=\text { residual oil saturation } \\
& \mathrm{S}=\text { normalized water saturation } \\
& \mathrm{n}=\text { water exponent } \\
& \mathrm{m}=\text { oil exponent }
\end{aligned}
$$

The empirical parameters $\mathrm{n}$ and $\mathrm{m}$ are obtained by fitting measured data. The experimental values of the end-point relative permeability values $\mathrm{k}_{\mathrm{ro}}{ }^{\mathrm{o}}$ and $\mathrm{k}_{\mathrm{rw}}{ }^{\mathrm{o}}$ were used in the fractional flow calculations discussed in Chapter 6.

The relative permeability is not only a function of saturation but also depends on the saturation distribution as indicated in Figure 2.10 (in consequence of the structure of the pore channels and the wettability of the porous medium). 

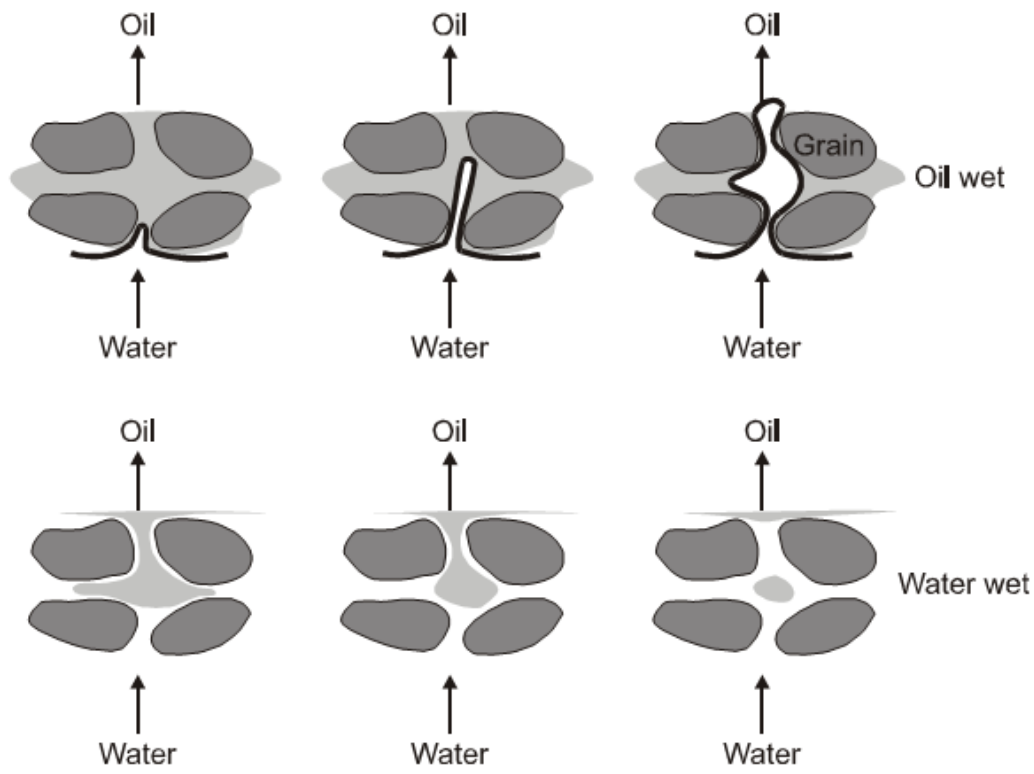

Figure 2.10 Schematic diagram of water invasion into porous medium

Figure 2.11 shows oil/water relative permeability curves for water wet and oil wet rocks
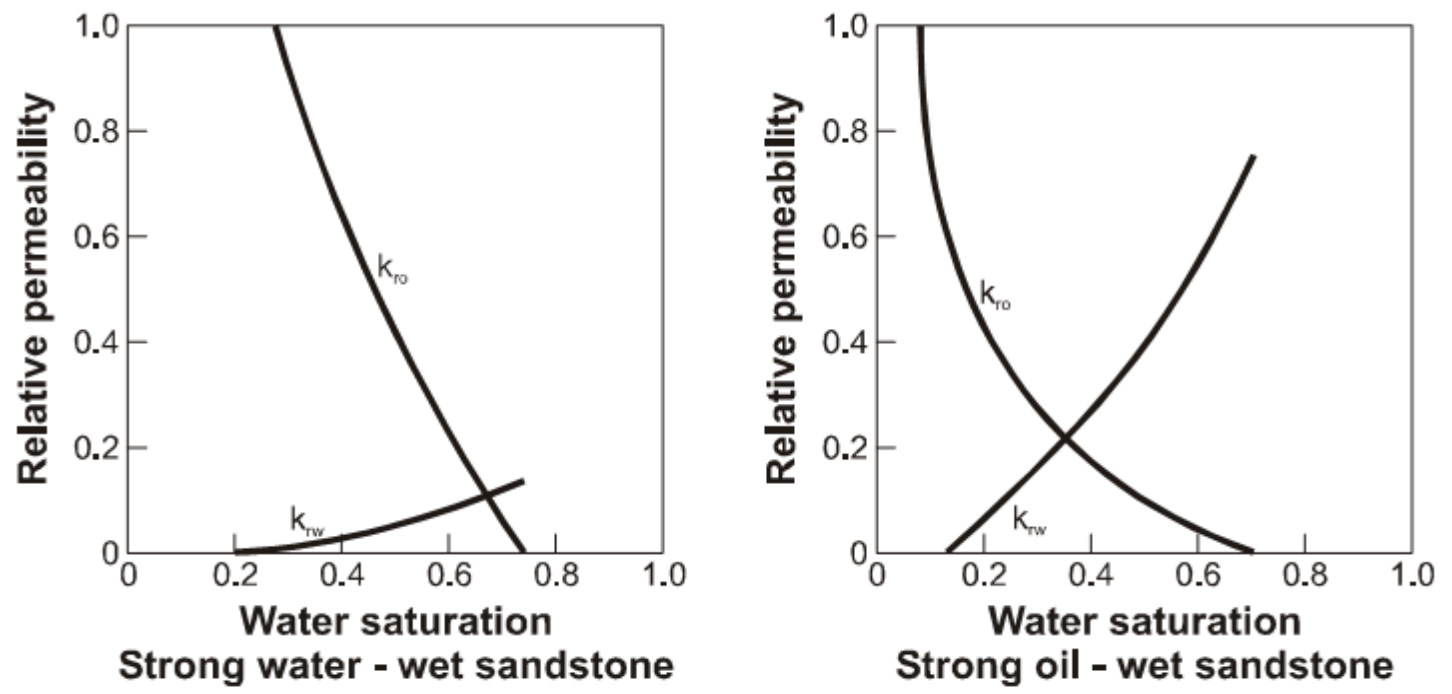

Figure 2.11 Typical water/oil relative permeability curves 


\subsubsection{Non-Newtonian properties}

The viscoelasticity of polymer solutions was not originally recognized as important in terms of microscopic sweep efficiency, but more recent publications show evidence that residual oil can be altered with the injection of polymer under certain conditions (Wreath 1989; Wang et al., 2000; Wu et al., 2007; Huh and Pope 2008). Kamaraj et al. (2011) discuss the impact that a reduction in the residual oil saturation might have on the recovery of heavy oils. Wu (2007) shows that the viscoelasticity of the polymer can have a large effect on oil recovery, including mobilization of residual oil. Huh and Pope (2008) indicate that the polymer viscoelasticity can reduce residual oil saturation in secondary polymer floods, but not in tertiary floods. Zhang et al. (2010) developed a model of viscoelastic behavior in flow through porous media and concluded that polymers can increase both the macroscopic and the microscopic sweep efficiencies.

The flow path of a fluid through a porous medium is tortuous. This results in alternate acceleration and deceleration of the fluid as the flow converges into and diverges out of a constrictions in the porous rock. As a consequence, a material element entering a converging section is not only sheared, but also stretched in the direction of flow. Clearly, the amount of shear thickening depends on the relative amounts of shearing and stretching deformation. An important example of the occurrence of extensional effects is the flow of polymer solutions in porous media in the enhanced oil recovery process, in which the fluid is stretched as the extent and shape of the flow passages change. The mode of extension affects the way in which the fluid resists deformation and this resistance can be referred to in terms of an elongational or extensional viscosity. The ratio is referred to as the Trouton ratio.

$$
T_{r}=\frac{\mu_{E}}{\mu}
$$


The value of 3 for Trouton ratio for an incompressible Newtonian fluid applies to all values of shear and elongation rates. The Trouton ratio for a non-Newtonian fluid is:

$$
T_{r}=\frac{\mu_{E}(\dot{\varepsilon})}{\mu(\dot{\gamma})}
$$

For a convenient estimate of behavior, (Jones et al., 1987) proposed the following definition of the Trouton ratio

$$
T_{r}=\frac{\mu_{E}(\dot{\varepsilon})}{\mu(\sqrt{3} \dot{\varepsilon})}
$$

i.e., the extensional viscosity is evaluated at $\quad \dot{\gamma}=\sqrt{3} \dot{\varepsilon}$. They also suggested that for inelastic isotropic fluids, the Trouton ratio is equal to 3 for all values of $\dot{\varepsilon}$ and $\dot{\gamma}$, and any departure from the value of 3 can be ascribed to viscoelasticity.

There are several ways of defining a relaxation time. One of them is combining shear stress and the first normal stress difference, e.g. the so-called Maxwellian relaxation time is given by:

$$
\begin{aligned}
& N_{1}=\sigma_{x x}-\sigma_{y y} \\
& \lambda \cong \frac{N_{1}}{2 \dot{\gamma} \tau}=\frac{N_{1}}{2 \eta \dot{\gamma}^{2}}
\end{aligned}
$$

Both shear stress and the first normal stress difference are functions of shear rate.

Sorbie (1990) suggested that if the transit time of a polymer solution through a porous medium is small compared to the relaxation time, then the elastic effects of the polymer would be observed. The Deborah number is used to characterize the viscoelasticity of a polymer solution (Jones et al., 1987). The definition of Deborah number is

$$
\text { De }=\frac{\text { Relaxation time of the liquid }}{\text { Characteristic time of the flow }}=\frac{\lambda}{t_{\text {flow }}}
$$


De is also interpreted as the ratio of elastic forces to viscous forces. The implication is such that if De is large, solid-like behavior (elastic effects dominate) results. A small Deborah number results in fluid-like behavior (viscous forces dominate).

A critical value of De exists beyond which viscoelasticity effects the flow and below which the flow is mainly viscous. However, (Metzner et al., 1966) indicated that the critical Deborah number can only be used to give a first estimate of the onset of shear thickening behavior because of the difficulty in evaluating the stretch rates that exists in a porous media. Gupta and Sridhar (1985) also concluded that viscoelastic behavior cannot be predicted by De alone but also by the ratio of maximum and minimum diameters of pore structure.

According to Heemskerk and Rosmalen (1984), the steep increase in pressure beyond a critical flow rate is a direct consequence of viscoelasticity with respect to the unsteadiness of the flow field in a porous medium. They also concluded that the onset of shear thickening in terms of critical flow rate shifts towards higher values with increasing permeability, temperature and salinity and with decreasing molecular weight and polymer concentration.

\section{Determination of Deborah number}

Many researchers use the longest relaxation time $\left(\lambda^{*}\right)$ for $\lambda$. Although the longest relaxation time $\left(\lambda^{*}\right)$ can be used to represent non-Newtonian behavior, the relaxation time varies with shear rate in a porous medium. Using the longest relaxation time can overestimate Deborah number at given shear rate. Normal stress difference is the evidence of viscoelastic behavior for polymer solution. The relaxation time can be estimated from normal stress difference (Choi and Kim, 1992; Choi, 1991; Rubinstein and Colby, 2003). 
i) If we consider capillary bundle model

$$
\begin{aligned}
& \operatorname{De}=\frac{N_{1}}{2 \eta \dot{\gamma}^{2}} \dot{\gamma}_{e f f}=\frac{N_{1}}{2 \eta \dot{\gamma}_{e f f}}=\frac{N_{1}}{2 \tau} \\
& \tau \text { is shear stress }
\end{aligned}
$$

ii) If we consider pore throat

$$
t_{\text {flow }}=\frac{\varepsilon}{\dot{\varepsilon}}
$$

$\varepsilon$ is stain

$\dot{\varepsilon}$ is stain rate

$$
\mathrm{De}=\frac{N_{1} \dot{\varepsilon}}{2 \eta \dot{\gamma}^{2} \varepsilon}
$$

By Jones et al. (1987)

$$
\begin{gathered}
\dot{\eta}=C_{1} \dot{\varepsilon} \\
\operatorname{De}=\frac{N_{1}}{2 C_{1} \eta \dot{\gamma} \varepsilon}=\frac{N_{1}}{2 C_{1} \varepsilon \tau}
\end{gathered}
$$

By using Hencky strain rate

$$
\varepsilon=\ln \left(\frac{r_{o}}{r_{p}}\right)^{2}
$$

Low shear rate region,

$$
C_{1}=\sqrt{3}
$$

The Trouton ratio can be used to estimate the onset point for non-Newtonian behavior for De. Since the Trouton ratio is 3 for Newtonian fluids and greater than 3 for non-Newtonian regime, De $>1 / 2$. If we consider $r_{p}=r_{o}$ i.e. capillary tube, De is 0.5 . If $3 r_{p}=r_{o}$, De is 0.39. If $2 r_{p}=r_{o}$, De is 0.62. If $1.5 r_{p}=r_{o}, \mathrm{De}=1$ 
(a)

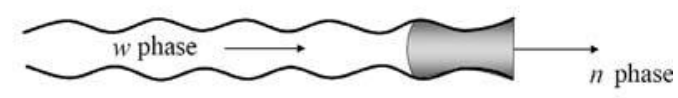

(b)

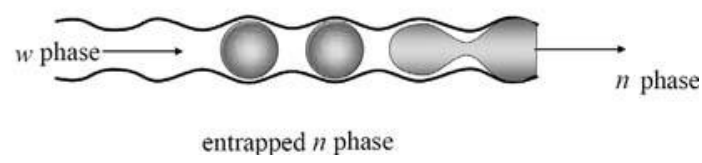

Figure 2.12 Illustration of (a) low and (b) high aspect ratio pores and the effect of aspect ratio in causing snap-off of phase $n$ during imbibition.

\subsection{REDUCTION OF RESIDUAL OIL SATURATION DURING POLYMER FLOOD}

Based on a review of both the experimental and theoretical polymer flooding literature, the following parameters seem to be important in terms of understanding the difference between residual oil saturation after water flooding and after polymer flooding:

1. Increase in the polymer viscosity compared to water (mobility ratio)

2. Initial oil saturation at start of polymer flood

3. Wettability of the core

4. Viscoelasticity and other characteristics of the polymer solution

5. Heterogeneity of the core on both the pore level and macroscopic level

6. Core flood variables such as pressure gradient and polymer concentration

7. Capillary number

The first systematic published studies of the effect of polymer on residual oil saturation were conducted at the University of Texas. Wreath (1989) and Wang (1995) measured the residual oil saturation to water and to polymer in both homogeneous Berea sandstone cores and in heterogeneous Antolini sandstone cores. For the latter, the distribution of permeability in the core samples was measured with a mini-permeameter 
and the distribution of porosity was measured by CAT scans. These and other data such as tracer data were employed as input for the history-matching simulations by Lu (1994). For both Berea and Antolini cores, a tertiary polymer flood did not reduce the waterflood residual oil saturation. For nine Antolini cores, a secondary polymer flood reduced the residual oil saturation below the waterflood value by $0.02-0.22$ in saturation units. To investigate whether the differences observed for the heterogeneous Antolini cores are due to the improved volumetric sweep or the reduction in residual oil saturation, historymatching simulations were carried out by Lu (1994). The secondary polymer flood results could be matched only when its residual oil saturation value was lower than that for the waterflood.

Wang et al. $(2000 ; 2001 b)$ reported that polymer injection after an extensive waterflood in Daqing sandstone cores resulted in an additional oil recovery of $7-14 \%$ OOIP above the waterflood recovery of $52-67 \%$ OOIP. They attributed this additional recovery to the improvement in microscopic displacement efficiency. Based on the coreflood results and the micromodel studies, they concluded that the viscoelasticity of the HPAM solution accelerated the drainage of oil films on rock surfaces; scoured oil in the dead-end pores; improved displacement of unswept oil due to micro-scale heterogeneity in the core; and even scraped off some of the trapped oil ganglia. While recognizing that the mobilization of the trapped oil ganglia by a polymer solution is unlikely due to exceeding the capillary force (capillary desaturation), they proposed a “dragging” mechanism, especially for mixed-wet and oil-wet rocks (Xia et al., 2004).

As another way of explaining the lower residual oil saturation obtained from polymer floods, (Xia et al., 2004)) is that the trapped oil may be pulled out by the viscoelastic polymer solution in the form of thin, stable "oil threads", which then flow 
downstream in the pore channel. To study the stability of oil threads, they derived relevant fluid flow equations, but no solutions to the problem were given. In order to model the scouring of oil from a dead-end pore by a viscoelastic polymer solution, Yin et al. (2006) solved the relevant flow equations numerically, and qualitatively showed the feasibility of the scouring mechanism. The above results are not completely consistent, but the overall trend is that polymer flooding reduces residual oil saturation under certain conditions. Xia et al. (2008) reported that HPAM polymers reduced the waterflood $\mathrm{S}_{\text {or }}$ by up to 15 saturation percentage points (i.e., a $S_{\text {or }}$ of $36.8 \%$ with waterflooding versus $21.75 \%$ for polymer flooding) using a constant capillary number of $5 \times 10^{-5}$. They observed reductions in $\mathrm{S}_{\text {or }}$ under oil-wet, weakly oil-wet, and mixed-wet conditions and attributed the reduction in oil saturation to the viscoelasticity of high molecular-weight HPAM solutions associated with a polymer solution's normal stress difference. They did not observe the effect with Newtonian glycerin solutions. Others have also reported reductions in $\mathrm{S}_{\text {or }}$ during polymer flooding in cores that were not water wet. Schneider and Owens (1982) found that HPAM floods resulted in $1 \%$ to $6 \%$ reductions in $\mathrm{S}_{\text {or }}$ in Berea sandstone treated with diesel oil to make the core oil wet. In Bentheimer sandstone, Pusch et al. (1987) reported $1 \%$ to $4 \%$ reductions in $\mathrm{S}_{\text {or }}$ with xanthan gum polymer and a Newtonian viscous sugar solution.

Recently, Ehrenfried (2013) conducted a series of coreflood experiments in several different sandstones using displacing fluids with a wide range of elasticity. Since the fluid rheology, core heterogeneity, initial oil saturation, relative permeability and viscosity varied from experiment to experiment, the isolated contribution of viscoelasticity was difficult to definitively sort out. 


\section{Chapter 3: Experimental Materials, Methodology and Data Analysis}

As polymer flooding is more widely applied worldwide with successful commercial outcomes, the upper bound for oil viscosity for its effective application has increased. The recent application of polymer flooding at the Pelican Lake field in Canada (Delamaid et al., 2013) is a good example, where the oil viscosity is 1,000-2,500 cp. To displace such a high viscosity oil, the polymer concentration needs to be high to achieve a sufficiently high polymer solution viscosity, and accordingly a sufficiently low mobility ratio. In order to design a polymer flood for such a reservoir, laboratory oil displacement corefloods need to be carried out. In carrying out such laboratory tests, preparation of a homogeneous polymer solution, its injection into the heavy oil-containing core, the measurement of the mobility data from the pressure taps, and the interpretation of the effluent data, require a special care. Because of the laboratory polymer flooding procedure for heavy oil displacement is described in detail in this chapter.

This chapter presents a description of the experimental apparatus and methodology used in this research. The first two segments detail general laboratory equipment and materials common to all experiments and all analytical equipment. This chapter describes experimental materials, procedures and conditions.

\subsection{EXPERIMENT EQUIPMENT}

\subsubsection{Polymer Preparation}

\section{Water Deionizer}

Deionized water (DI) was used for all experiments. A Nanopure ${ }^{\mathrm{TM}}$ filter system was used to de-ionize the water. This filter uses a recirculation pump and monitors the 
water resistivity to indicate whether the ions have been removed. Distilled water was fed into the system and deionized water was obtained at the outlet.

\section{Weight Measurements}

A weighing balance (P-603D) manufactured by Denver Instrument with readability of $0.001 \mathrm{~g}$ was used to measure the mass of all the components necessary for preparing solutions. When measuring all powder material such as polymer, $\mathrm{NaCl}$ and $\mathrm{CaCO}_{2}$, disposable weighing dishes from Fisher Scientific were used together.

\section{Magnetic Stir Plates and Stirrers}

In order to mix a polymer stock solution, $C O R N I N G \circledR P C-420 D$ stirring plates with Nalgene ${ }^{\circledR}$ floating magnetic stir bars was used. For small amount of polymer solution, IKA® RO 10 Power IKAMAG Magnetic Stirrer with PTFE disposable stir bars from Fisherbrand ${ }^{\circledR}$ was also used. Magnetic stir bars placed inside plastic containers and glass bottle rotate in response to the motor of the stir plate.

\section{Convection Ovens}

Blue $M{ }^{\circledR}$ and Tenney ${ }^{\circledR}$ convection ovens were set to the reservoir temperature to observe aqueous solubility and the microemulsion phase behavior. Core flood experiments are conducted in convection ovens to imitate reservoir temperature. A digital display on the convection ovens indicated the real-time temperature.

\section{Filter Press Unit}

To remove any particle or dust, all polymer stock and saline solution were filtered using FANN® ${ }^{\circledR}$ 12BL filter press with Millipore ${ }^{\mathrm{TM}}$ hydrophilic cellulose filter papers. The polymer stock solutions were filtered through $1.2 \mu \mathrm{m}$ sized filter papers and saline brine 
solutions were filtered through $0.45 \mu \mathrm{m}$ sized filter papers respectively. $15 \mathrm{psi}$ of compressed argon was used to supply the differential pressure. The filter press unit is shown in Figure 3.1.

\section{Polymer Hydration}

The aqueous polymer solution was prepared by slowly adding the dry powder onto the shoulder of the vortex of $\mathrm{NaCl}$ brine and, at the same time, stirring the mixture with a floating magnetic stir bar at 360-400 rpm. An argon gas blanket was used to minimize contact with oxygen. Polymer solution was mixed for at least 48 hours at room temperature to ensure full hydration of the polymer. As the polymer hydrates, the solution becomes more homogenous and clear. In this study, $500 \mathrm{~g}$ of stock solution was prepared for dilution and with a composition of $4000 \mathrm{ppm}$ polymer in $1000 \mathrm{ppm} \mathrm{NaCl}$ brine.

\section{Filtration}

To ensure that proper hydration of polymers has been achieved, a filtration test was carried out. Approximately $250 \mathrm{~mL}$ of aqueous polymer solution was filtered through 5-micron Millipore cellulose filter under 15-psi argon pressure; and the time was recorded to calculate the filtration ratio when $60,80,180$, and $200 \mathrm{~mL}$ of filtered fluid had been collected. The filtration ratio is calculated as follows:

$$
F . R .=\frac{t_{200 \mathrm{ml}}-t_{180 \mathrm{~m} l}}{t_{80 \mathrm{ml}}-t_{60 \mathrm{ml}}}
$$

After the test, the filter paper was inspected to see if any polymer micro-gel remains due to improper hydration. Only polymer solutions with a filtration ratio below 1.2 were used for the rheological testing. 


\section{Dynamic Light Scattering (DLS)}

DLS measurements of the average ds were obtained using Zetasizer Nano ZS (Malvern Instruments Limited) at $25 \mathrm{oC}$.

\section{Electrolytes}

Salts such as $\mathrm{NaCl}, \mathrm{CaCl}_{2}$, and $\mathrm{MgCl}_{2} * 6 \mathrm{H}_{2} \mathrm{O}$ were used to create synthetic brines . All of the salts were supplied by Fisher Scientific.

\section{Polymers}

Polymers used in this research were supplied by SNF Floerger and ChemPAM. The polymers are granular powders. All of the polymers except scleroglucan biopolymer are acrylamide-based (AM) and have molecular weights ranging from 8 million to over 20 million Daltons as reported by their respective manufacturers. Most are co- or ter-polymers with functional groups such as acrylate (AA) and n-vinyl pyrrolidone (NVP). Table 3.1 lists the polymers that were used in this research.

Table 3.1 Polymers

\begin{tabular}{|c|c|c|c|c|}
\hline Polymer type & Polymer & M.W. (Dalton) & D.H. & Manufacturer \\
\hline $\begin{array}{c}\text { HPAM } \\
\begin{array}{c}\text { Co-polymers of } \\
\text { acrylamide and } \\
\text { acrylate) }\end{array}\end{array}$ & Flopaam $^{\mathrm{tm}} 3630 \mathrm{~S}$ & $20 \times 10^{6}$ & $30 \%$ & SNF Floerger \\
\cline { 2 - 5 } & Flopaam $^{\mathrm{tm}} 3330 \mathrm{~S}$ & $8 \times 10^{6}$ & $30 \%$ & SNF Floerger \\
\hline Modified HPAM & ChemPam 8177 & $20 \times 10^{6}$ & $30 \%$ & ChemPam \\
\hline $\begin{array}{c}\text { ATBS-based Ter- } \\
\text { polymers }\end{array}$ & SAV 301 & $2-5 \times 10^{6}$ & High & SNF Floerger \\
\cline { 2 - 5 } & SAV 522 & $2-5 \times 10^{6}$ & medium & SNF Floerger \\
\hline Biopolymer & Scleroglucan & $2 \times 10^{6}$ & NA & \\
\hline
\end{tabular}




\subsubsection{Rheological Properties Measurements}

\section{The Rheometer}

For this study, all the bulk rheological properties were measured using the Advanced Rheometric Expansion System Low Shear-1 (ARES LS-1) from TA instruments ${ }^{\circledR}$ (Figure 3.2). The ARES is a mechanical spectrometer that subjects a sample to either a dynamic (sinusoidal) or steady shear strain deformation, and then measures the resultant torque of the sample in response to this shear strain. During the tests, shear strain is forced by motor and torque is detected by the transducer. Strain amplitude and frequency are set by the operator, with the actual sample deformation determined by the measured motor, and transducer, displacement. The LS following ARES means a type of motor. The LS is most commonly used for fluid testing. The motor shaft is supported axially by a precision air bearing. This minimizes axial runout, providing the smoothest normal force data. It also has a special controller that allows extremely low shear rates to be applied during steady shear test.

Dynamic properties of the fluid such as storage modulus, loss modulus and complex viscosity were obtained by the dynamic strain frequency sweep test. When in dynamic mode, the instrument measures strain and torque. Steady rate sweep test gives the shear viscosity of the solution as a function of the shear rate. When in steady mode, the instrument measures rotational rate, sample torque, and normal force. For both dynamic and steady test, double wall coquette geometry was used. Since the shear area is doubled in comparison to the plain cylinder geometry, it is usually used for very low viscous fluid and relatively small amount of sample. The cup has $34 \mathrm{~mm}$ OD and 27.95 $\mathrm{mm}$ ID and the BOB has $32 \mathrm{~mm}$ OD and $29.5 \mathrm{~mm}$ ID. 
To measure rheological properties of polymer solution, two oscillatory tests (strain and frequency sweep tests) and two steady rate tests (transient and steady shear sweep tests) were carried out. The details of each test are described in the following subsections. During the test, the temperature of the instrument is controlled by circulator.

\section{Dynamic Strain Sweep Test}

The purpose of dynamic strain sweep test is to determine the range of linear viscoelasticity and torque levels at constant frequency and temperature. For the polymer

solutions tested in this study, the sweeps were conducted over the range of strain from $1 \%$ to $200 \%$ at a fixed frequency of $10 \mathrm{rad} / \mathrm{s}$. Storage modulus, loss modulus and complex viscosity are measured with strain. Figure 3.3 shows a typical example of this test. As shown in the figure, the measurement becomes out of range of linear viscoelasticity after a strain value of about $30 \%$. Based on the results from this test, a strain value within the range of linear viscoelasticity is chosen for the frequency sweep test.

\section{Dynamic Frequency Sweep Test}

Dynamic frequency sweep test is carried out to measure the dynamic properties of the polymer solution such as the storage modulus, loss modulus and complex viscosity in the specified range of oscillatory frequencies. These measurements are performed at the strain value within the range of linear viscoelasticity from dynamic strain sweep test. This test was performed over the range of frequencies between 0.1 and $100 \mathrm{rad} / \mathrm{s}$ in this study. A typical result of dynamic frequency sweep test of polymer solution in shown in Figure 3.4. The relaxation time of the polymer solution is estimated from the data obtained from this test.

\section{Strain Controlled Step Rate Transient Test}


The aim of strain controlled step rate transient test is to determine the time for the stress response of the polymer solution to attain steady state. This test measures the steady state rheological properties of the fluid at a specific shear rate as a function of time. That ensures accurate steady shear viscosity measurement made for following steady rate sweep test. Four separate zones can be programmed to observe stress growth. Time is required to reach steady state behavior and relaxation before and after steady shear. Figure 3.5 shows a typical result of this test. In this test, 4 separate zones with $0,1,10$ and $0 \mathrm{~s}^{-1}$ for 5, 50, 50 and 20 seconds each were programmed. From the plot, it can be observed that before the stress or viscosity becomes steady, it initially overshoots the steady state value before relaxing and becoming steady. This overshoot indicates the elastic behavior of polymer solution.

\section{Steady Rate Sweep Test}

Steady rate sweep test gives the shear viscosity of the solution as a function of the shear rate at specific temperature. The range of shear rate for steady rate sweep test was from 0.01 to $800 \mathrm{~s}^{-1}$ in this study. In most reservoir polymer floods, shear rates on the order of 1 to $20 \mathrm{~s}^{-1}$ are expected except very close to wells. Figure 3.6 shows a typical plot of steady shear viscosity of polymer solutions. In the figure, Newtonian plateau and shear thinning regime of polymer solution can be easily observed.

\subsubsection{Core Flood Experimental Equipment}

Once a good formulation for specific crude is found, a core flood experiment is conducted to check the performance of the formulation. Schematic of the setup to be used for the core flood experiment is shown in Figure 3.7. It shows the location of the different 
pressure transducers as well as the different sections of the core. This section describes the experimental equipment used in core flood experiments.

\section{Glass Columns}

Kontes Chromaflex ${ }^{\circledR}$ columns were used to contain fluids to be injected for core floods experiments. These columns were 0.5 to 2 foot in length and 2 inch in outer diameter. The end pieces include a Vitron $\mathrm{O}$ ring and washer to prevent leaking when hand tightened. These columns can withstand up to $50 \mathrm{psi}$; but usually, $20 \mathrm{psi}$ is used as a safe guard.

\section{Stainless Steel Columns}

Whitey ${ }^{\circledR}$ stainless steel columns were used for fluid injection under higher pressures of up to 120 psi. These columns were 2 feet in length and 1 inch in outer diameter. The stainless steel columns were custom made from stainless steel tubing with Swagelok fittings used as end pieces. These steel columns were used in oil flood experiments involving high pressures.

\section{Pumps}

A Teledyne ISCO 5000 syringe pump was used to inject the fluids into the core at constant rate. The pump was filled with mineral oil to displace the fluid in the columns into core. Air bubbles should be purged prior to use and the flow rate should be checked by effluent volume.

\section{Pressure Transducer}

Pressure drop across sections of the core were measured using Validyne DP 15 pressure transducers. These transducers are made of a stainless steel diaphragm sandwiched between two stainless steel blocks. Having been calibrated, they measure the 
pressure difference between inlet and exit ports. Bleed ports located on the side of these transducers make it possible to clean the transducers. Pressure transducers that could measure $0-10 \mathrm{psi}, 0-100 \mathrm{psi}$ and $0-1000$ psi were used so that all the expected pressure can be measured accurately.

\section{Data Acquisition Recorder}

A National Instruments (NI) data acquisition card was used to capture signals from the pressure transducers and subsequently transferred to NI's LabView 8.0 software on the computer. Prior to recording the pressures, the transducers are zeroed by using an offset value in LabView.

\section{Fraction Collector}

An Instrument Specialties Company (ISCO) Retriever II fractional collector was used for collecting effluent samples. The collector can be programmed to collect the samples at fixed time intervals or fixed volume interval. System leaks can be detected by checking the volume of effluent in the fraction collector.

\section{HPLC}

HPLC is a chromatographic technique that can separate a mixture of compounds to identify the individual components of the mixture. Varian prostar $230 \AA$ used in this research is a ternary solvent delivery module with microborn-to-analytical flows and the column was Acclaim ${ }^{\circledR}$ surfactant having $250 \mathrm{~mm}$ in length and $4.6 \mathrm{~mm}$ in outer diameter. Effluent samples were measured on all even numbered samples by HPLC in order to determine surfactant retention in the porous medium. 


\section{Spectrophotometer}

Turbidity measured using a UV-Vis spectroscopy. Calibration curves were measured at two wavelengths $470 \mathrm{~nm}$ and $520 \mathrm{~nm}$. Absorbance increased linearly with increasing polymer concentration.

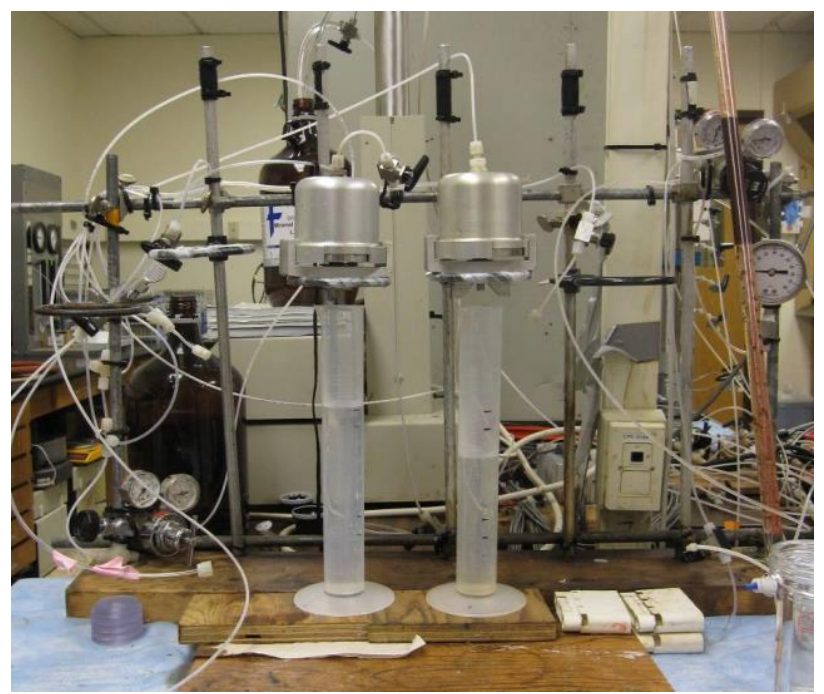

Figure 3.1 The filter press unit used in this study

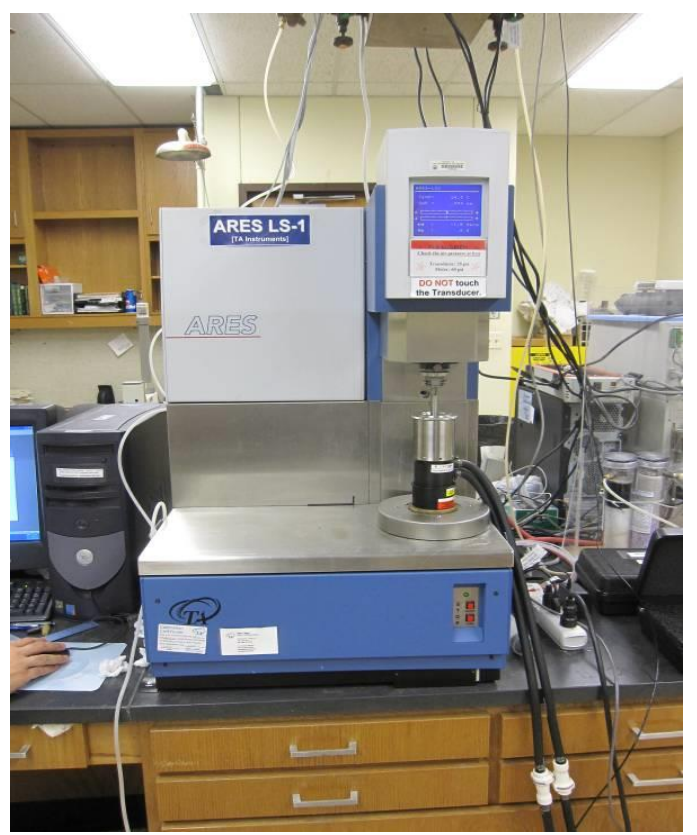

Figure 3.2 TA Instruments' Advanced Rheometric Expansion System Low Shear-1 


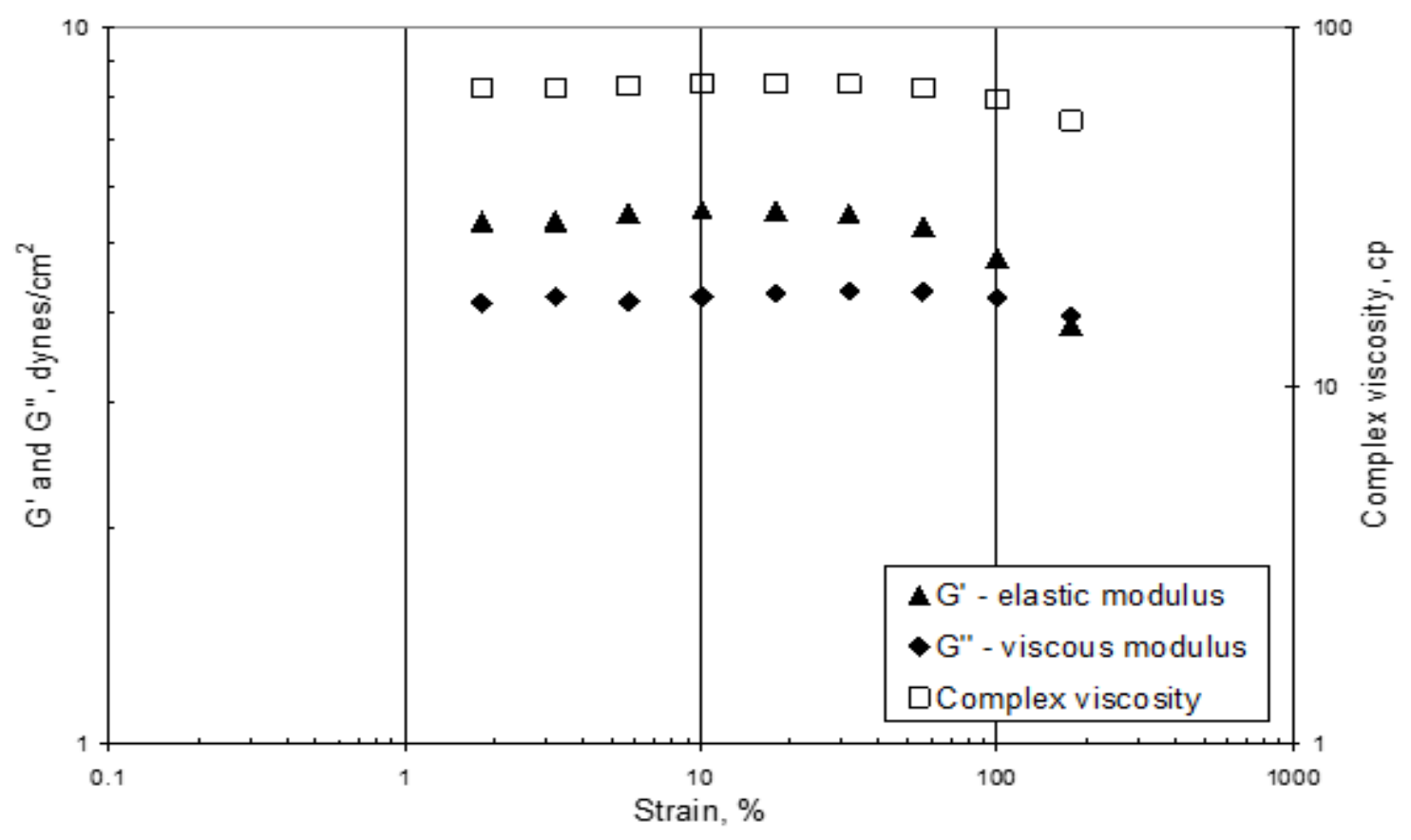

Figure 3.3 Typical result of dynamic strain sweep test

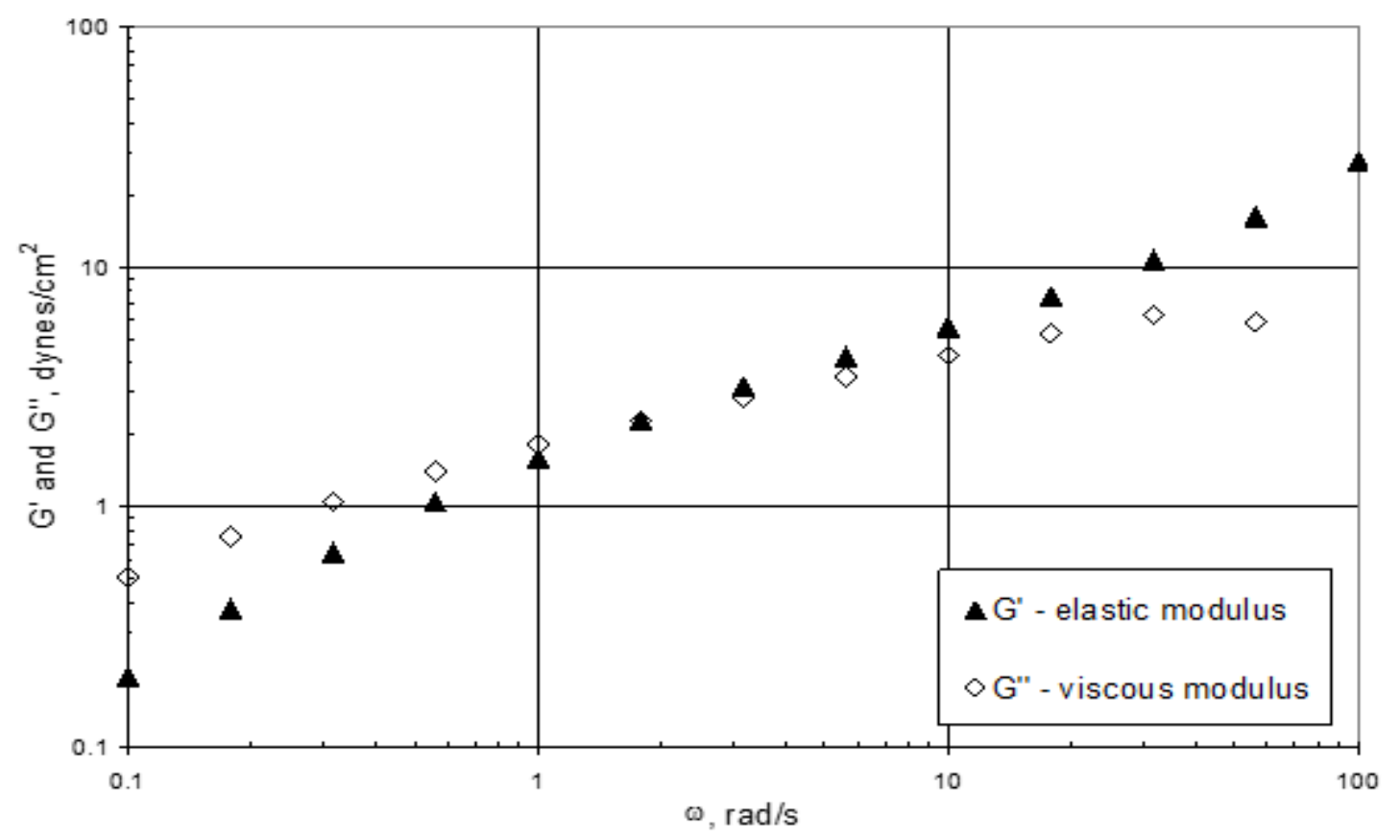

Figure 3.4 Typical result of dynamic frequency sweep test 


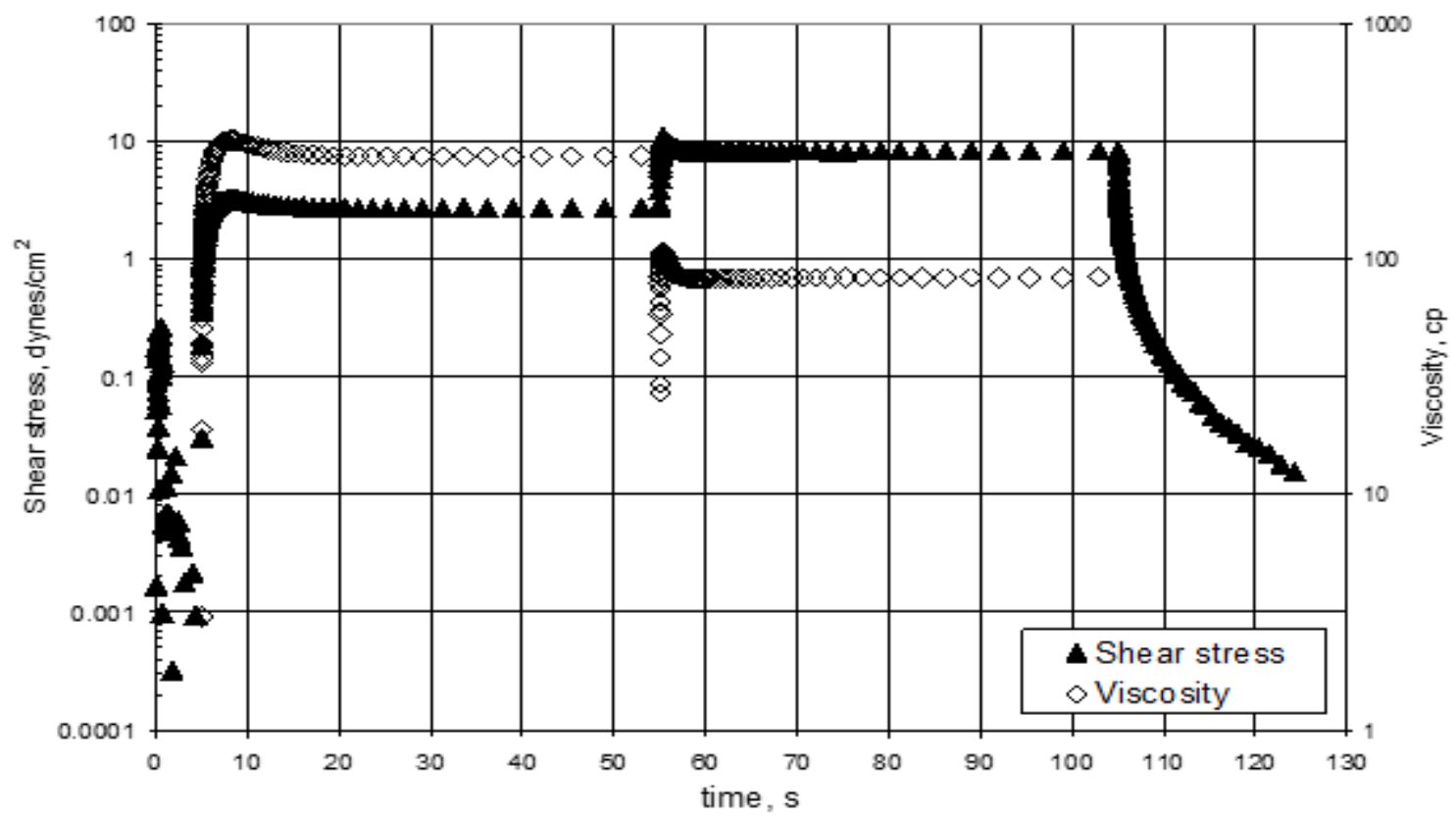

Figure 3.5 Typical result of strain controlled step rate transient test

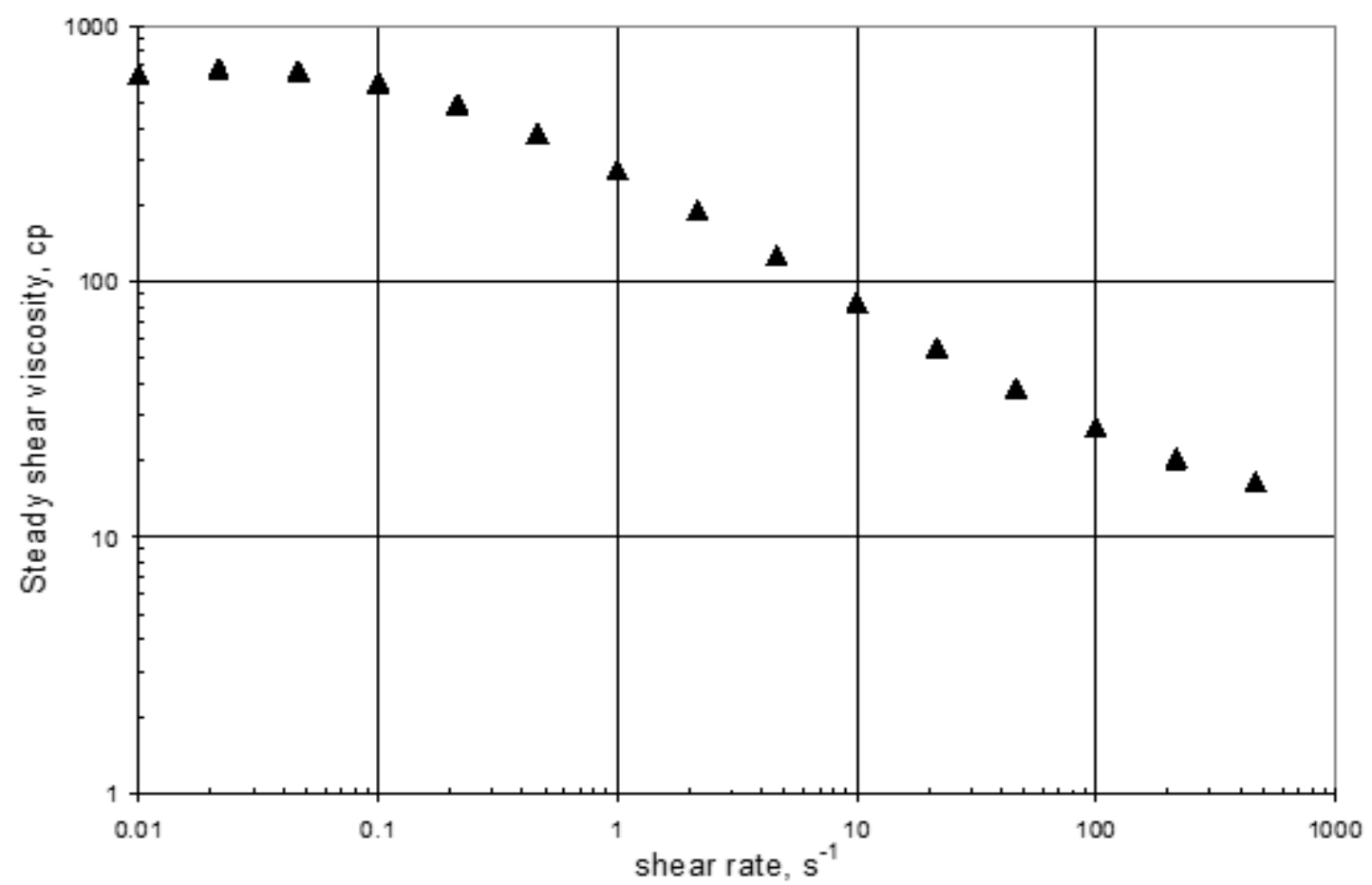

Figure 3.6 Typical result of steady rate sweep test 


\subsection{CORE FLOOD DESCRIPTION AND METHODOLOGY}

\subsubsection{Core Flooding Setup}

A schematic of the coreflood setup is shown in Figure 3.7. It consists of a syringe pump, fluid reservoir, core-holder, pressure transducers, tubing and fraction collectors. Using the pump, the brine or polymer solution in the fluid reservoir is injected into the core inside the core-holder. The pressure difference between sections of the core is measured by the transducers. Effluent samples are collected with the fraction collector and their viscosities measured and compared with the injected fluid.

\subsubsection{Experimental Procedures}

The core flood procedure includes the method of core preparation, core saturation, ageing with brine or crude oil, brine flooding, oil flooding, water flooding and polymer flooding, collecting and analyzing the effluent samples for cumulative oil recovery, $\mathrm{pH}$, and polymer concentration.

\section{Core Preparation}

The cores were 1.5 inch (or 2 inch) in diameter and 12 inches long. After being cut, the core was dried in an oven at reservoir temperature overnight or $12 \mathrm{hrs}$ and then weighed using a mass balance. In case of reservoir sand, short core plugs of 1.5 inch in diameter were stacked to make a composite core 12 inches long. The core was wrapped with Teflon tape and Teflon shrink sleeve to seal the core. The Teflon heat shrink sleeve was applied using a heat gun. The prepared core was placed inside the core-holder and firmly secured with an overburden pressure applied using an ENERPAC P-39 hydraulic hand pump. The pressure taps were accessed by drilling holes through the taps into the core. This enabled the fluids to flow to the inlet ports of the pressure transducers. 
The core preparation for outcrop cores is described in more detail below:

1. The core was drilled from a block of sandstone rock, dried in a convection oven overnight at reservoir temperature and its mass recorded.

2. Air permeability measurements were made on the dried core using the air permeameter.

3. It was wrapped in Teflon tape.

4. The core was placed inside a Teflon heat shrink sleeve an end pieces were put at both ends of the core. Using a heat gun, the sleeve was shrunk until it wrapped tightly around the core.

5. A vacuum pump was attached to the core using the end pieces whereby the entire the core was placed under vacuum. The shrinking of the sleeve with heat continued as the air in the core was being expelled. This ensured a complete seal around the core.

6. The core was then loaded into the stainless steel phoenix instruments coreholder. The end pieces were then put to ensure that the core was firmly in place.

7. A confining overburden pressure was applied on the core-holder using the ENERPAC P-39 hydraulic hand pump.

8. Holes were then drilled into the core through the pressure tap apertures using the appropriate drill bit size. Air was flowing through the core while that drilling was done. The drilling was stopped when air began to exit the pressure tap. 
9. The core now firmly secured in the core-holder was placed under vacuum by attaching the outlet of the core-holder to a vacuum pump. The vacuum was applied for approximately 6 hours. This was to remove air from the core.

10. While under vacuum, the inlet of the core-holder was connected to a burette containing the brine and the present level of the brine recorded. After vacuuming, the inlet valve to the core-holder was opened and the brine was allowed to saturate the core. The core was fully saturated when the level of brine in the burette remained constant. The final level of the burette was then recorded. This brine saturation was done to establish the pore volume of the core.

The coreflood experiments began only after preparing the core and placing it in the core-holder as described above.

The core preparation for sandpack is described in more detail below:

1. Ottawa sand (F-95) was sieved through stainless steel sieve with 250 mesh.

2. The rubber sleeve was then loaded into the stainless steel Phoenix instruments core-holder.

3. The bottom end piece was then put to ensure that the core was firmly in place.

4. Two different stainless steel mesh screens were put to hold the sand in place. The two screen sizes are 100 mesh and 250 mesh.

5. The rubber sleeve was packed with sieved sand.

6. A confining overburden pressure was applied on the core-holder using the ENERPAC P-39 hydraulic hand pump. 
7. Holes were then drilled into the core through the pressure tap apertures using the appropriate drill bit size. Air was flowing through the core while that drilling was done. The drilling was stopped when air began to exit the pressure tap.

8. The core now firmly secured in the core-holder was placed under vacuum by attaching the exit of the core-holder to a vacuum pump. The vacuum was applied for approximately 6 hours. This was to ensure that there was absolutely no air left in the pores of the core.

9. While under vacuum, the inlet of the core-holder was connected to a burette containing the synthetic formation brine and the present level of the brine recorded. After vacuuming, the inlet valve to the core-holder was opened and the synthetic formation brine was allowed to saturate the core. The core was fully saturated when the level of brine in the burette remained constant. The final level of the burette was then recorded. This brine saturation was done to establish the pore volume of the core.

\section{Brine Flooding}

Brine flooding was used to establish the brine permeability of the core and the effective particle diameter of the particles that make up the berea sandstone core. The brine was made by dissolving the salts into deionized water (DI) with the divalent ions added last. The solution was mixed on a magnetic stir plate. The prepared brine was then filtered through a $0.45 \mu \mathrm{m}$ Millipore filter paper using a vacuum operated filtration system. The filtered brine is then put into the glass column by vacuum suction; the column was vacuumed, then the brine was allowed to fill it up as a result of the pressure 
gradient created by the vacuum in the column. The brine in the column was then degassed; air bubbles in the valves and fittings were also purged.

The brine which is now in the fluid reservoir (i.e. glass column) was then injected into the core at a specific flow rate. The ISCO syringe pump was used to control the flow rate, which was also verified from the time duration and volume of effluent samples collected.

Pressure drops across sections of the core were measured using pressure transducers from the pressure taps located along the length of the core-holder. The pressure drop measured were recorded and displayed using NI's LabView 8.0 software on a computer monitor in real-time. The brine flood experiment was only stopped after a steady pressure was observed for about 2 pore volumes of injected fluid.

\section{Oil Flooding}

After brine flooding, oil flooding was conducted at high injection pressure at the reservoir temperature. The main purpose of the oil flooding is to determine initial oil saturation, residual water saturation, effective oil permeability, and relative oil permeability. Prior to oil flooding, the crude was filtered by $0.45 \mu \mathrm{m}$ filter paper at the reservoir temperature. Oil flooding was conducted under a constant pressure (80-120 psi) to saturate the pore volume with oil and obtain accurate residual water saturation. Approximately, 1.5 PV of the oil was injected into the top end to consider density effect of oil and water. The effluent fluids were collected in $100 \mathrm{ml}$ burettes and the volume of displaced water was the volume of saturated oil inside the core. Oil flooding was continued until water cut was less than $1 \%$ and pressure stabilized. The pressure drop was recorded during oil flooding in order to calculate the oil permeability. 


\section{Water Flooding}

Water flooding with filtered synthetic injection brine was followed by oil flooding. Water flooding was conducted in order to determine residual oil saturation, effective water permeability, and relative water permeability after water flooding. The effluent fluids were collected in a burette and water flooding was stopped when the oil cut was less $1 \%$ and pressure stabilized. The remaining oil saturation was estimated based on the volumes of oil in a burette and effective brine permeability was calculated by the pressure drop across the core.

\section{Polymer Flooding}

Polymer solution was injected after water flooding to recover oil in either secondary or tertiary mode. Polymer flooding was performed at a constant interstitial velocity of about $1 \mathrm{ft} /$ day and the flooding was performed until no more oil produced. The effluent fluids were collected by fractional collector for further analysis. Oil recovery and residual oil saturation were determined after polymer flooding by measuring volumes of oil produced. After polymer flooding, effluent fluids were placed in the convection oven at the reservoir temperature and tubes were centrifuged for 1-2 min at $1000 \mathrm{rpm}$. Then, polymer concentration, viscosity, and $\mathrm{pH}$ data for the effluent fluids were analyzed to evaluate the performance of the formulation.

\subsubsection{Core Flood Calculations}

Calculations for core flood experiments include pore volume, porosity, permeability, phase saturation, fluid mobility, polymer resistance factor, permeability 
reduction factor, and oil recovery. Calculations and parameters used are described in this section.

\section{Pore Volume}

The pore volume is the mass of the saturated core minus the mass of the dry core divided by the brine density:

$$
\mathrm{V}_{\mathrm{p}}=\frac{\mathrm{M}_{\mathrm{sat}}-\mathrm{M}_{\mathrm{dry}}}{\rho_{\mathrm{w}}}
$$

where

$$
\begin{array}{ll}
\mathrm{V}_{\mathrm{p}}= & \text { pore volume } \\
\mathrm{M}_{\text {sat }}= & \text { mass of brine saturated core } \\
\mathrm{M}_{\text {dry }}= & \text { mass of evacuated core }
\end{array}
$$

\section{Bulk Volume and Porosity}

The porosity of the core is pore volume divided by bulk volume as follows:

$$
\phi=\frac{V_{p}}{V_{b}}=\frac{V_{p}-V_{s}}{V_{b}}
$$

And the bulk volume is calculated by total volume of the bare core.

$$
\mathrm{V}_{\mathrm{b}}=\pi \mathrm{r}^{2} \mathrm{~L}
$$

where

$$
\begin{array}{lll}
\mathrm{V}_{\mathrm{b}} & = & \text { bulk volume } \\
\mathrm{r} & = & \text { radius of core } \\
\mathrm{L} & = & \text { length of core }
\end{array}
$$




\section{Brine Permeability}

After saturating the core with brine, a brine flood was conducted in order to measure pressure drop between inlet and outlet of the entire core. The brine permeability was calculated from Darcy's law for single phase, steady-state flow Jackson (2006).

\section{Effective Oil Permeability}

The effective oil permeability was calculated from the oil flood. The pressure drop was measured throughout the flooding process; the stabilized portion of the pressure data was used to calculate the effective oil permeability.

\section{Effective Water Permeability}

After the water flood, the effective water permeability was calculated. Again, the pressure drop was measured across the core. When pressure stabilized and the oil cut was less than $1 \%$, the pressure drop was recorded and used in Darcy's equation to calculate relative permeability. Flow at this end point was assumed to be steady state.

\section{End Point Oil/Water Relative Permeability}

After running the water flood, end-point relative permeability for both oil and water were calculated. Relative permeability was used for removing pore size effect and normalization by using a base permeability in multiphase flow. End-point oil permeability was calculated by dividing effective oil permeability by the brine permeability. End-point water permeability was calculated in same manner. 


$$
\begin{aligned}
& \mathrm{k}_{\text {ro }}{ }^{\mathrm{o}}=\frac{\mathrm{k}_{\mathrm{O}}}{\mathrm{k}_{\text {brine }}} \\
& \mathrm{k}_{\mathrm{rw}}{ }^{\mathrm{o}}=\frac{\mathrm{k}_{\mathrm{w}}}{\mathrm{k}_{\text {brine }}}
\end{aligned}
$$

\section{Initial Oil Saturation}

An oil flood was conducted in the brine saturated core until residual water saturation was reached. Mass balance was used to determine the initial oil saturation: the volume of produced water is the volume of oil saturated in the core. Initial oil saturation was estimated by dividing volume of produced water by the pore volume as follows:

$$
\mathrm{S}_{\mathrm{oi}}=\frac{\mathrm{V}_{\mathrm{w}}}{\mathrm{V}_{\mathrm{p}}}
$$

where

$$
\begin{array}{lll}
\mathrm{S}_{\mathrm{oi}} & = & \text { initial oil saturation } \\
\mathrm{V}_{\mathrm{w}} & = & \text { volume of produced water from oil flood }
\end{array}
$$

\section{Residual Oil Saturation}

The residual oil saturation from the water flood was calculated after the oil cut from the water flood was less than $1 \%$. The volume of oil produced during the water

flood was the mobile oil saturation. Residual oil saturation was estimated using the following equation:

$$
S_{\text {orw }}=\frac{\mathrm{V}_{\mathrm{w}}-\mathrm{V}_{\mathrm{o}}}{\mathrm{V}_{\mathrm{p}}}
$$


where

$$
\begin{array}{lll}
\mathrm{S}_{\text {orw }}= & \text { residual oil saturation after water flood } \\
\mathrm{V}_{\mathrm{w}}= & \text { volume of produced water from oil flood } \\
\mathrm{V}_{\mathrm{o}}= & \text { volume of produced oil from water flood }
\end{array}
$$

The residual oil saturation after the polymer flood is the difference between the volume of oil remaining after the water flood and the volume of oil produced by the polymer flood. Residual oil saturation after polymer flood can be calculated as follows:

$$
S_{\text {orp }}=\frac{S_{\text {orw }} V_{p}-V_{o}}{V_{p}}
$$

where

$$
\begin{array}{lll}
\mathrm{S}_{\text {orp }} & = & \text { residual oil saturation after polymer flood } \\
\mathrm{V}_{\mathrm{o}} & = & \text { volume of produced oil from chemical flood }
\end{array}
$$

\section{Mobility ratio}

Mobility control in a chemical flood is a crucial step to improve oil recovery. Gogarty suggested that minimum total mobility yield a favorable viscosity and improve sweep efficiency (Gogarty et al., 1968). Relative permeability of oil and water at different water saturations could be calculated using Corey's equation as follows:

$$
\begin{aligned}
& \mathrm{k}_{\mathrm{rw}}=\mathrm{k}_{\mathrm{rw}}{ }^{\mathrm{o}} \mathrm{S}^{\mathrm{n}} \\
& \mathrm{k}_{\mathrm{ro}}=\mathrm{k}_{\text {ro }}{ }^{\mathrm{o}} \mathrm{S}^{\mathrm{m}} \\
& \mathrm{S}=\frac{\mathrm{S}_{\mathrm{w}}-\mathrm{S}_{\text {wirr }}}{1-\mathrm{S}_{\text {wirr }}-\mathrm{S}_{\text {or }}}
\end{aligned}
$$


where
$\mathrm{k}_{\mathrm{rw}}{ }^{\mathrm{O}}=$ Water end point relative permeability
$\mathrm{k}_{\mathrm{ro}}{ }^{\mathrm{o}}=$ Oil end point relative permeability
$\mathrm{n} \quad=\quad$ Exponent of water relative permeability from water cut in oil flood
$\mathrm{m} \quad=\quad$ Exponent of oil relative permeability from oil cut in water flood

Total mobility is the sum of water mobility and oil mobility as below:

$$
\lambda_{\mathrm{t}}=\left(\frac{\mathrm{k}_{\mathrm{w}}}{\mu_{\mathrm{w}}}+\frac{\mathrm{k}_{\mathrm{o}}}{\mu_{\mathrm{o}}}\right)
$$

A plot of water saturation versus total relative mobility is made to find the minimum in the curve. For a stable displacement, the drive viscosity must be equal or greater than the inverse total relative mobility of the oil bank as follows:

$$
\mu s=\frac{1}{\lambda_{\mathrm{tmin}}}=\frac{1}{\left(\frac{\mathrm{k}_{\mathrm{w}}}{\mu_{\mathrm{w}}}+\frac{\mathrm{k}_{\mathrm{o}}}{\mu_{\mathrm{o}}}\right)_{\min }}
$$

The concentration of polymer required to have such a viscosity was estimated by plotting viscosity versus different polymer concentration at identical conditions such as salinity, hardness, and temperature.

\section{Polymer Resistance Factor}

The polymer resistance factor is defined as the ratio of water mobility over polymer mobility as follows: 


$$
\mathrm{R}_{\mathrm{f}}=\frac{\lambda_{\mathrm{w}}}{\lambda_{\mathrm{p}}}=\frac{\mathrm{k}_{\mathrm{w}} \mu_{\mathrm{p}}}{\mathrm{k}_{\mathrm{p}} \mu_{\mathrm{w}}}
$$

where

$\mathrm{R}_{\mathrm{f}}=$ polymer resistance factor

The resistance factor can be determined from the pressure drop data by dividing the steady state polymer pressure drop by the steady state water pressure drop provided the flow rate and saturation are the same for both floods:

$$
R_{f}=\left(\frac{\Delta P_{p}}{\Delta P_{w}}\right)_{q, s j}
$$

\section{Polymer Permeability Reduction Factor}

The polymer permeability reduction factor indicated the effect of trapped or adsorbed polymer. This factor was the ratio of effective brine permeability to effective polymer permeability as follows:

$$
\mathrm{R}_{\mathrm{k}}=\frac{\mathrm{k}_{\mathrm{w}}}{\mathrm{k}_{\mathrm{p}}}=\mathrm{R}_{\mathrm{f}} \frac{\mu_{\mathrm{w}}}{\mu_{\mathrm{p}}}
$$

where $\mathrm{R}_{\mathrm{k}}=$ polymer permeability reduction factor

\section{Oil Recovery}

Oil recovery can be estimated after polymer flood. Oil recovery was calculated by dividing the sum of oil recovered from polymer flood by volume of residual oil after water flood as follows 


$$
\mathrm{f}_{\text {op }}=\frac{\sum_{\mathrm{i}=1} \mathrm{~V}_{\text {opi }}}{\mathrm{V}_{\text {or }}}
$$

where

$$
\begin{array}{ll}
\mathrm{f}_{\mathrm{op}}= & \text { fraction of oil produced } \\
\mathrm{V}_{\text {opi }}= & \text { volume of free oil produced in tube } \mathrm{i} \\
\mathrm{V}_{\text {or }}= & \text { volume of residual oil after water flood }
\end{array}
$$

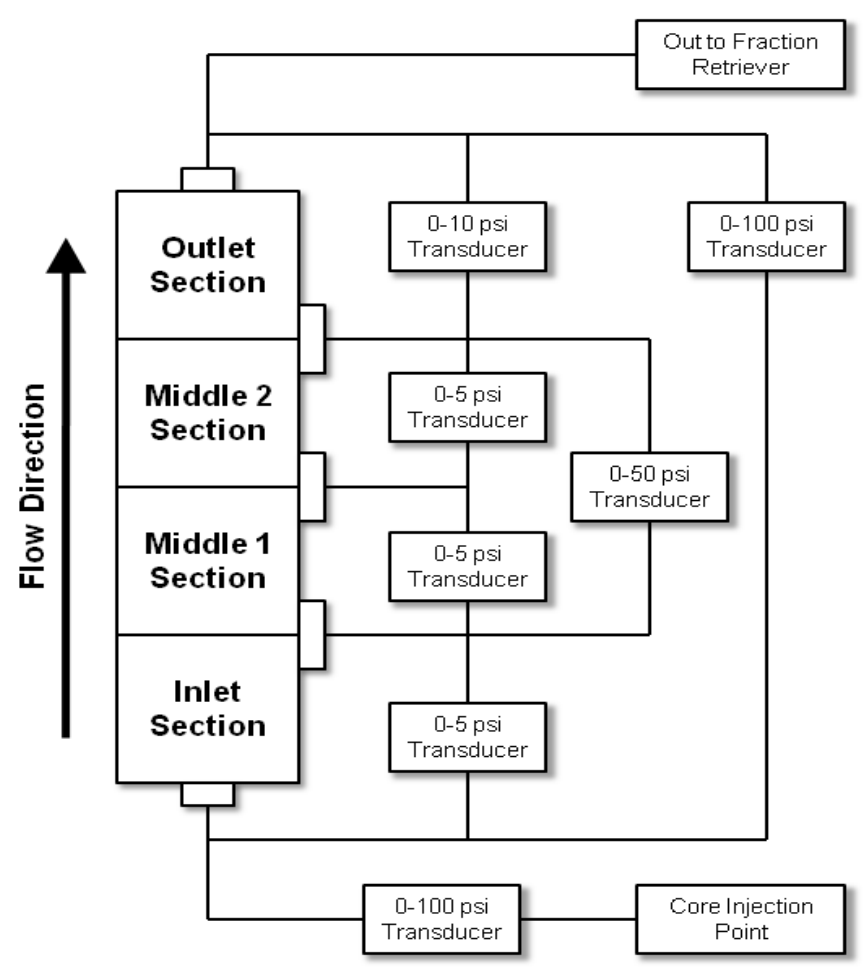

Figure 3.7 Schematic setup of polymer flood 


\section{Chapter 4. Polymer Solution Characterization}

\subsection{INTRODUCTION}

Because the main objective of this research was to understand the reduction of the residual or remaining oil saturation by polymer flooding, the rheology of the polymer solutions was systematically measured and analyzed. Rheological properties of polymer solutions depend on the various factors such as the polymer's molecular structure, polymer concentration, shear rate, temperature and the composition of the brine. Therefore, rheological measurements with commonly employed EOR polymers under various conditions were made and the effect of these factors on polymer viscosity was quantified.

\subsection{EXPERIMENTAl MATERIAL, APPARATUS AND METHOdOlogy}

This section describes the polymers tested, how the polymer solutions were prepared and the rheological methods used to characterize the polymers.

\subsubsection{The Polymers}

Three polymers were used in this study (Table 4.1). The polymers were manufactured and provided by SNF Floerger ${ }^{\circledR}$ (Cedex, France) in the form of a white powder. The commercial products are called Flopaam ${ }^{\circledR}$ 3330S, 3630S and AN-125. FP 3330S and FP 3630S are co-polymers of acrylamide (AM) and acrylic acid (about 25 to $30 \mathrm{~mol} \%$ ) with different molecular weights. Other polymer manufacturers hydrolyze polyacrylamide rather than co-polymerize it with acrylic acid (AA). Such products are commonly referred to as hydrolyzed polyacrylamide (HPAM). AN-125 is a co-polymer 
of acrylamide and 2-acrylamido-2-methyl propane sulfonate (AMPS) with an anionicity of about 25 to $30 \mathrm{~mol} \%$. The AMPS monomer has a higher tolerance to divalent cations such as $\mathrm{Ca}++$ than the AA momomer.

The degree of anionicity of a water-soluble polymer affects its properties such as water solubility, viscosity, shear stability, thermal stability and salinity and hardness tolerance. The molecular weight (MW) of a polymer is also important. It determines the thickening potential of the polymer. Higher MW is related to bigger molecular size which has a direct effect on its transport in porous media. If the molecular size of the polymer is close to the size of the pore throat in a porous medium, the polymer molecules may be trapped in the pore throat and slowly accumulate, which causes retention and pressure buildup (plugging).

Table 4.1 The polymers tested in this study

\begin{tabular}{|c|c|c|c|}
\hline Polymer & $\begin{array}{c}\text { Mw } \\
\times 10^{6}\end{array}$ & Anionicity (mol\%) & Description \\
\hline FloppamTM 3330S & 8 & $25 \sim 30$ & HPAM \\
\hline FloppamTM 3630S & 20 & $25 \sim 30$ & HPAM \\
\hline AN-125 & 8 & $25 \sim 30^{*}$ & Poly(AM-co-AMPS) \\
\hline
\end{tabular}

* indicates mol \% of AMPS.

\section{Intrinsic Viscosity}

Intrinsic viscosity is defined as:

$$
[\eta]=\lim _{C \rightarrow 0}\left(\frac{\eta_{s p}}{C}\right)=\lim _{C \rightarrow 0}\left(\frac{\eta_{\text {solution }}-\eta_{\text {solvent }}}{\eta_{\text {solvent }} C}\right)
$$

where $\eta_{s p}$ is the specific viscosity. It can also be estimated from: 


$$
[\eta]=\lim _{C \rightarrow 0}\left(\frac{\ln \eta_{r}}{C}\right)=\lim _{C \rightarrow 0}\left(\frac{1}{C} \ln \left(\frac{\eta_{\text {solution }}}{\eta_{\text {solvent }}}\right)\right)
$$

In Equation (4.2), $\eta_{r}$ is the relative viscosity. $\eta_{\text {solution }}$ and $\eta_{\text {solvent }}$ are the solution and solvent viscosities, respectively.

The intrinsic viscosity increases as the polymer molecular weight increases following the Mark-Houwink equation:

$$
[\eta]=K M_{w}^{a}
$$

where $\mathrm{K}$ and "a" are empirical constants at a given temperature for a polymer in a particular solvent. The value of exponent "a" usually ranges from 0.5 to 1 . Factors affecting the intrinsic viscosity of a polymer with a given molecular weight include but are not limited to the following: type of solvent, temperature, shear rate, charge, chain branching and chemical structure.

Intrinsic viscosity also provides a link between the average size of polymer molecules and rheology of solution. The hydrodynamic radius of a polymer molecule increases as the intrinsic viscosity increases. It is difficult to experimentally determine this parameter. However, a theoretical expression has been proposed and is known as the Flory-Fox equation (Flory, 1953). It is given as:

$$
\Omega_{p}=\left[\frac{[\eta] \cdot M}{\Phi}\right]^{1 / 3}
$$

where $\Omega_{\mathrm{p}}$ is the hydrodynamic radius of polymer molecule and $\Phi$ is a universal constant of value $2.1 \times 10^{21} \mathrm{dl} / \mathrm{g} \cdot \mathrm{mol} . \mathrm{cm}^{3}$. 


\section{The Relaxation Time of a polymer solution}

The relaxation time is defined as the time required for a property having been displaced from equilibrium to decay back to its original value. The relaxation time is of interest because it is used to quantify the viscoelasticity of polymer solutions. It is used to calculate Deborah the number, which determines the onset of the shear thickening behavior of polymer solutions flowing in permeable media. The relaxation time is a time scale for the relaxation of stress in a fluid and can be estimated from the storage and loss modulus, G' and G", measured with the dynamic frequency sweep test with a rheometer.

Munoz et al. (2003) proposed that the reciprocal of the frequency at which G' and G" cross each other is the characteristic relaxation time of the polymer solution. From the stress response to the oscillatory strain, the complex solution viscosity, $\mu^{*}=\mu_{\mathrm{V}-} i \mu_{\mathrm{E}}$, is obtained, where $\mu_{\mathrm{E}}=\mathrm{G}^{\prime} / \omega$ and $\mu_{\mathrm{V}}=\mathrm{G}^{\prime \prime} / \omega$; and $\omega$ is angular frequency. This intersection has been considered as an indication of the onset entanglement coupling. This phenomenon occurs by strong coupling of neighboring molecules to molecular motion along the chain and causes significant changes in the relaxation spectrum and hence affects the accuracy of relaxation time.

\section{The Deborah Number}

If the transit time of the polymer molecules through the pores of a porous medium is large compared to the relaxation time, then there would not be an elastic effect. On the other hand, if the transit time is small compared to the relaxation time, an elastic effect would be expected to exist (Sorbie, 1991).

The Deborah number is used for characterization of viscoelasticity of polymer solution. It is the ratio of the characteristic time, $\theta_{1}$, to the duration time of the process, $\theta_{\mathrm{p}}$; 


$$
N_{D e}=\frac{\theta_{l}}{\theta_{p}}
$$

and is often estimated as:

$$
N_{D e}=\frac{\tau_{r} v}{D_{P}}
$$

where $\tau_{\mathrm{r}}$ is the relaxation time, $\mathrm{v}$ is the interstitial velocity, and $\mathrm{D}_{\mathrm{p}}$ is the diameter of particle.

$\mathrm{N}_{\mathrm{De}}$ is also considered as the ratio of the elastic force to viscous force. If $\mathrm{N}_{\mathrm{De}}$ is large, then the elastic effect is dominant and the material behaves like a solid. However, if $\mathrm{N}_{\mathrm{De}}$ is small, then the viscous force is dominant and the material behaves like a fluid. Beyond a critical value of $\mathrm{N}_{\mathrm{De}}$, therefore, the viscoelasticity of the polymer solution has an effect on the flow, while the flow is viscous below the critical value.

However, Heemskerk et al. (1984) indicated that the critical Deborah number can only be used for a first estimation of the onset of dilatant behavior because it is difficult to evaluate the stretch rate which exists in a porous matrix. Gupta and Sridhar (1985) also indicated that viscoelastic behavior cannot be predicted by $\mathrm{N}_{\mathrm{De}}$ alone, but other factors such as the ratio of maximum and minimum diameters in a pore and pore throat affect the behavior.

The Deborah number has also been expressed as:

$$
N_{D e}=\dot{\varepsilon} \cdot \tau_{r}
$$

where $\dot{\varepsilon}$ is stretching rate and $\tau_{\mathrm{r}}$ is the relaxation time of the fluid.

Heemskerk et al. (1984) concluded that the steep increase in pressure beyond a critical flow rate is a result of viscoelasticity in recognition of the unsteady flow in a porous medium. It was also concluded that the onset of shear thickening in terms of 
critical flow rate increases with increasing permeability, salinity and temperature and decreasing molecular weight and polymer concentration.

\subsection{RESUlTS AND DisCUSSION}

The results of the two kinds of rheological measurements (oscillatory and steady shear test) of representative polymer solutions are presented in this chapter. The rheological data described here were obtained by using ARES LS-1 and employed to characterize the polymer solutions.

\subsubsection{Bulk Rheological Results}

The rheological characterization of polymer solutions is presented in this section. Dynamic frequency sweep test and steady rate sweep test were carried out under various conditions. In this study, Flopaam ${ }^{\mathrm{TM}} 3330 \mathrm{~S}$, Flopaam ${ }^{\mathrm{TM}} 3630 \mathrm{~S}$ and AN-125 were tested. The rheological measurements were made for a range of polymer concentration, salinity, hardness and temperature, as described in Table 4.2. The relaxation times of the polymer solutions were obtained with the $G^{\prime} / G^{\prime \prime}$ crossover point from the dynamic frequency sweep test. Table 4.3, 4.4, 4.5 and 4.6 are a summary of the relaxation times and viscosity for different $\mathrm{NaCl}$ salinity, the divalent ion concentration and polymer concentration. The samples with higher polymer concentration showed more physical elasticity during their preparation, thus suggesting that their relaxation times should be higher. Lee et al. (2009) and Kim et al. (2010) developed an empirical correlation to predict the relaxation time and viscosity. They compared the relaxation time by the crossover point method and by fitting the measured data using the generalized Maxwell model. 
Table 4.2 Test conditions

\begin{tabular}{|c|c|}
\hline Condition Factors & Range \\
\hline Polymer concentration & $500 \mathrm{ppm} \sim 3000 \mathrm{ppm}$ \\
\hline Salinity $(\mathrm{NaCl})$ & $1000 \mathrm{ppm} \sim 40000 \mathrm{ppm}$ \\
\hline Hardness $\left(\mathrm{Ca}^{2+}\right)$ & $0 \mathrm{ppm} \sim 2000 \mathrm{ppm}$ \\
\hline Temperature & $25^{\circ} \mathrm{C} \sim 90^{\circ} \mathrm{C}$ \\
\hline
\end{tabular}


Table 4.3 Summary of bulk viscosity and relaxation times for 500 ppm FP 3630S

\begin{tabular}{|c|c|c|c|}
\hline $\begin{array}{l}\mathrm{NaCl} \text { concentration } \\
(\mathrm{ppm})\end{array}$ & $\begin{array}{l}\mathrm{Ca}^{2+} \text { concentration } \\
(\mathrm{ppm})\end{array}$ & $\begin{array}{l}\text { Relaxation time } \\
\text { (s) }\end{array}$ & $\begin{array}{c}\text { viscosity }(\mathrm{cP}) \\
@ 10 \mathrm{~s}^{-1}\end{array}$ \\
\hline \multirow[t]{4}{*}{1000} & 0 & 0.1082 & 16.2 \\
\hline & 500 & 0.0672 & 2.8 \\
\hline & 1000 & 0.1314 & 2.1 \\
\hline & 2000 & 0.1000 & 1.9 \\
\hline \multirow[t]{4}{*}{5000} & 0 & 0.0643 & 7.2 \\
\hline & 500 & 0.0870 & 3.3 \\
\hline & 1000 & 0.3125 & 2.4 \\
\hline & 2000 & 0.1000 & 2.0 \\
\hline \multirow[t]{4}{*}{10000} & 0 & 0.0775 & 5.2 \\
\hline & 500 & 0.0633 & 3.1 \\
\hline & 1000 & 0.0873 & 2.9 \\
\hline & 2000 & 0.0835 & 2.2 \\
\hline \multirow[t]{4}{*}{20000} & 0 & 0.0653 & 4.4 \\
\hline & 500 & 0.1000 & 3.1 \\
\hline & 1000 & 0.1000 & 2.9 \\
\hline & 2000 & 0.0842 & 2.3 \\
\hline \multirow[t]{4}{*}{40000} & 0 & 0.0267 & 3.7 \\
\hline & 500 & 0.0930 & 3.1 \\
\hline & 1000 & 0.1000 & 2.9 \\
\hline & 2000 & 0.0849 & 2.5 \\
\hline
\end{tabular}


Table 4.4 Summary of bulk viscosity and relaxation times for 1000 ppm FP 3630 S

\begin{tabular}{|c|c|c|c|}
\hline $\begin{array}{l}\mathrm{NaCl} \text { concentration } \\
(\mathrm{ppm})\end{array}$ & $\begin{array}{l}\mathrm{Ca}^{2+} \text { concentration } \\
(\mathrm{ppm})\end{array}$ & $\begin{array}{l}\text { Relaxation time } \\
\text { (s) }\end{array}$ & $\begin{array}{c}\text { viscosity }(\mathrm{cP}) \\
@ 10 \mathrm{~s}^{-1}\end{array}$ \\
\hline \multirow[t]{4}{*}{1000} & 0 & 1.3545 & 41.0 \\
\hline & 500 & 0.0693 & 7.1 \\
\hline & 1000 & 0.0714 & 3.3 \\
\hline & 2000 & 0.0733 & 2.8 \\
\hline \multirow[t]{4}{*}{5000} & 0 & 0.0693 & 19.7 \\
\hline & 500 & 0.0653 & 4.8 \\
\hline & 1000 & 0.0712 & 4.0 \\
\hline & 2000 & 0.0710 & 3.1 \\
\hline \multirow[t]{4}{*}{10000} & 0 & 0.0822 & 13.9 \\
\hline & 500 & 0.0673 & 7.5 \\
\hline & 1000 & 0.0702 & 4.8 \\
\hline & 2000 & 0.0736 & 3.7 \\
\hline \multirow[t]{4}{*}{20000} & 0 & 0.0755 & 10.4 \\
\hline & 500 & 0.0657 & 7.3 \\
\hline & 1000 & 0.0667 & 5.0 \\
\hline & 2000 & 0.0696 & 4.1 \\
\hline \multirow[t]{4}{*}{40000} & 0 & 0.0859 & 8.5 \\
\hline & 500 & 0.0699 & 7.2 \\
\hline & 1000 & 0.0714 & 5.2 \\
\hline & 2000 & 0.0678 & 4.6 \\
\hline
\end{tabular}


Table 4.5 Summary of bulk viscosity and relaxation times for 2000 ppm FP 3630 S

\begin{tabular}{|c|c|c|c|}
\hline $\begin{array}{l}\mathrm{NaCl} \text { concentration } \\
(\mathrm{ppm})\end{array}$ & $\begin{array}{l}\mathrm{Ca}^{2+} \text { concentration } \\
(\mathrm{ppm})\end{array}$ & $\begin{array}{l}\text { Relaxation time } \\
\text { (s) }\end{array}$ & $\begin{array}{c}\text { viscosity }(\mathrm{cP}) \\
@ 10 \mathrm{~s}^{-1}\end{array}$ \\
\hline \multirow[t]{4}{*}{1000} & 0 & 7.7821 & 118.0 \\
\hline & 500 & 0.0584 & 22.0 \\
\hline & 1000 & 0.0474 & 12.4 \\
\hline & 2000 & 0.0498 & 9.6 \\
\hline \multirow[t]{4}{*}{5000} & 0 & 0.4827 & 53.9 \\
\hline & 500 & 0.0602 & 21.7 \\
\hline & 1000 & 0.0528 & 16.7 \\
\hline & 2000 & 0.0519 & 12.6 \\
\hline \multirow[t]{4}{*}{10000} & 0 & 0.1136 & 41.2 \\
\hline & 500 & 0.0640 & 23.3 \\
\hline & 1000 & 0.0516 & 18.4 \\
\hline & 2000 & 0.0501 & 14.6 \\
\hline \multirow[t]{4}{*}{20000} & 0 & 0.0622 & 31.7 \\
\hline & 500 & 0.0471 & 23.6 \\
\hline & 1000 & 0.0529 & 18.8 \\
\hline & 2000 & 0.0505 & 15.2 \\
\hline \multirow[t]{4}{*}{40000} & 0 & 0.0603 & 25.6 \\
\hline & 500 & 0.0463 & 21.3 \\
\hline & 1000 & 0.0548 & 20.6 \\
\hline & 2000 & 0.0528 & 16.8 \\
\hline
\end{tabular}


Table 4.6 Summary of bulk viscosity and relaxation times for 3000 ppm FP 3630S

\begin{tabular}{|c|c|c|c|}
\hline $\begin{array}{l}\mathrm{NaCl} \text { concentration } \\
(\mathrm{ppm})\end{array}$ & $\begin{array}{l}\mathrm{Ca}^{2+} \text { concentration } \\
(\mathrm{ppm})\end{array}$ & $\begin{array}{l}\text { Relaxation time } \\
\text { (s) }\end{array}$ & $\begin{array}{c}\text { viscosity }(\mathrm{cP}) \\
@ 10 \mathrm{~s}^{-1}\end{array}$ \\
\hline \multirow[t]{4}{*}{1000} & 0 & 19.0078 & 225.0 \\
\hline & 500 & 0.0797 & 51.9 \\
\hline & 1000 & 0.0430 & 21.3 \\
\hline & 2000 & 0.0420 & 14.4 \\
\hline \multirow[t]{4}{*}{5000} & 0 & 1.1617 & 111.0 \\
\hline & 500 & 0.0825 & 54.8 \\
\hline & 1000 & 0.0443 & 28.8 \\
\hline & 2000 & 0.0452 & 19.2 \\
\hline \multirow[t]{4}{*}{10000} & 0 & 0.4949 & 83.5 \\
\hline & 500 & 0.0904 & 54.0 \\
\hline & 1000 & 0.0493 & 33.0 \\
\hline & 2000 & 0.0457 & 23.9 \\
\hline \multirow[t]{4}{*}{20000} & 0 & 0.2564 & 64.4 \\
\hline & 500 & 0.0764 & 50.8 \\
\hline & 1000 & 0.0597 & 34.0 \\
\hline & 2000 & 0.0497 & 28.4 \\
\hline \multirow[t]{4}{*}{40000} & 0 & 0.0709 & 55.2 \\
\hline & 500 & 0.0739 & 48.9 \\
\hline & 1000 & 0.0712 & 44.5 \\
\hline & 2000 & 0.0537 & 32.7 \\
\hline
\end{tabular}




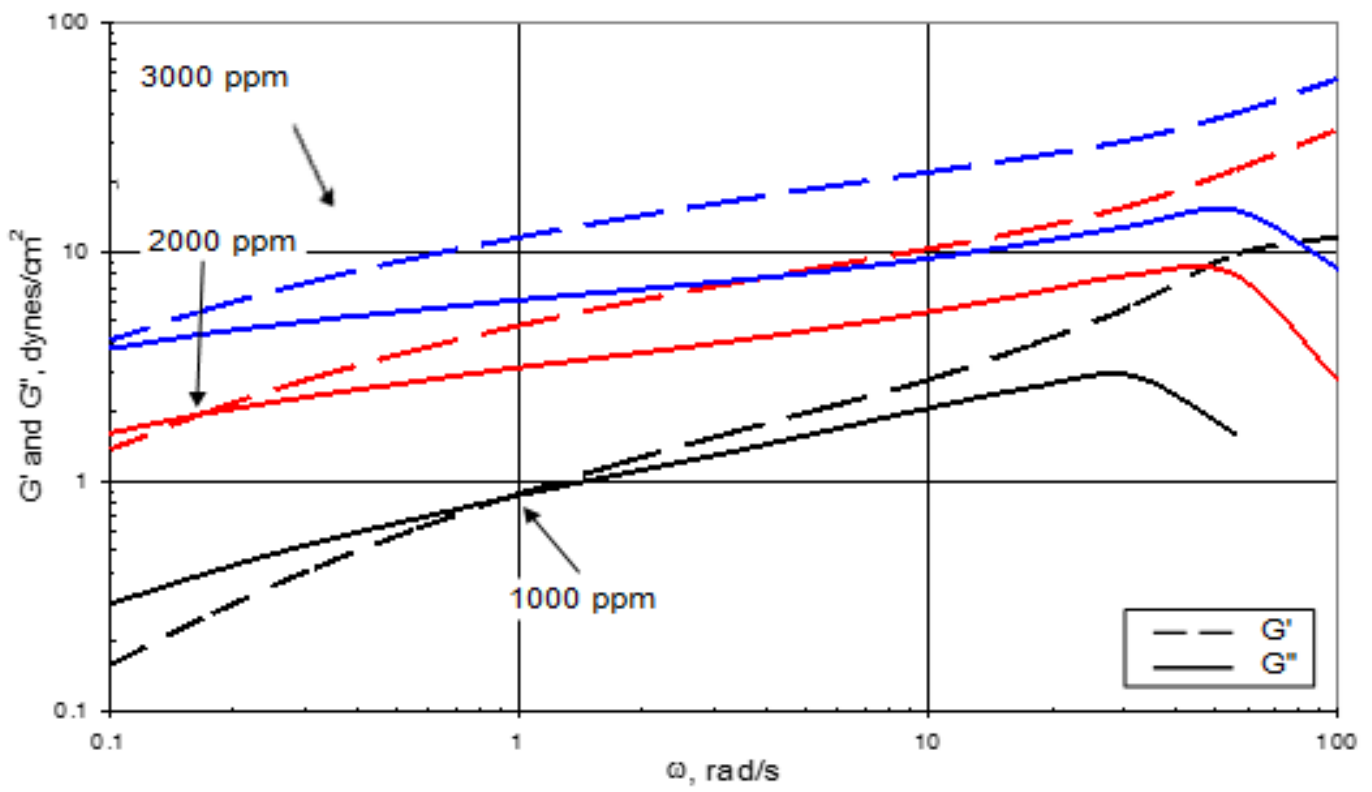

Figure 4.1 Effect of polymer concentration on crossover point for Flopaam ${ }^{\mathrm{TM}} 3630 \mathrm{~S}$ in $0.1 \% \mathrm{NaCl}$ at $25^{\circ} \mathrm{C}$

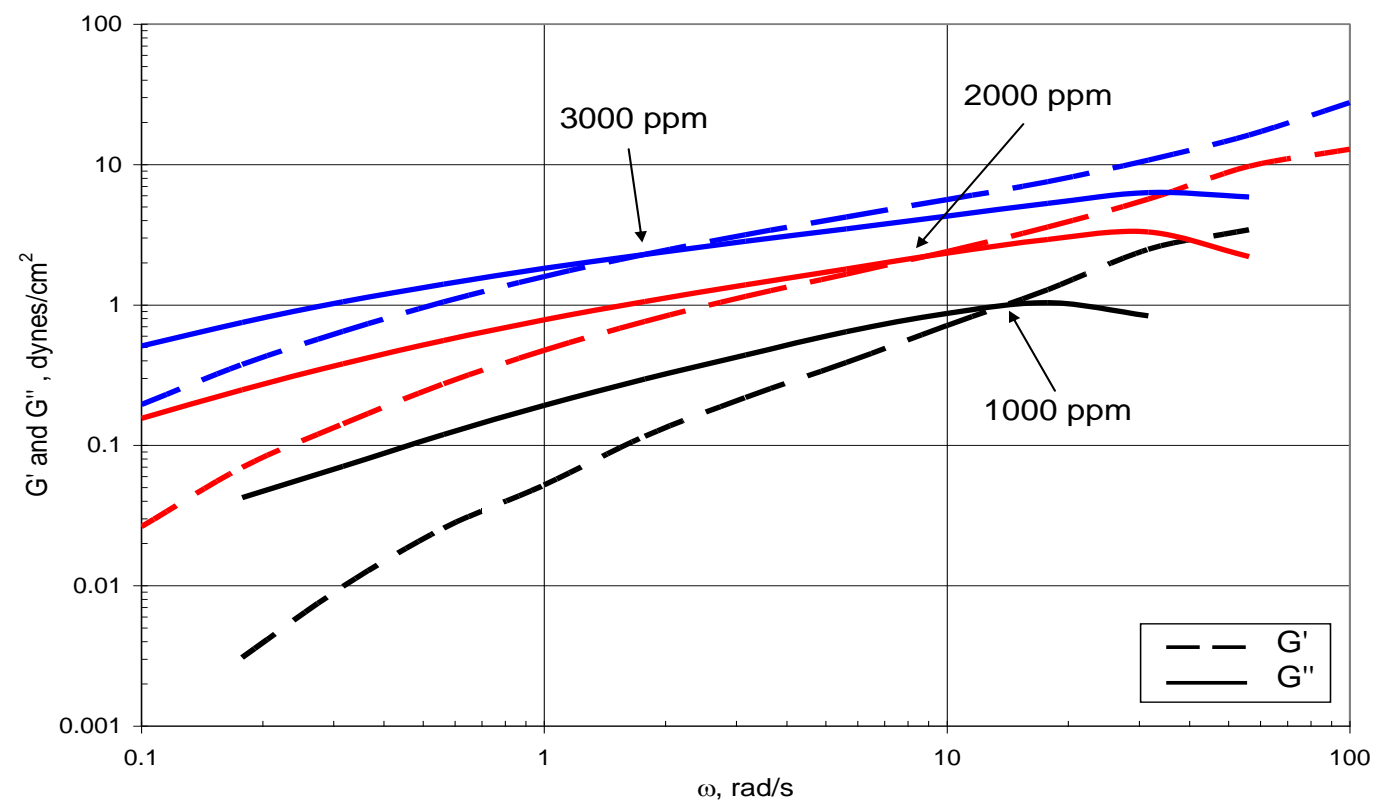

Figure 4.2 Effect of polymer concentration on crossover point for Flopaam ${ }^{\mathrm{TM}} 3630 \mathrm{~S}$ in $1 \% \mathrm{NaCl}$ at $25^{\circ} \mathrm{C}$ 


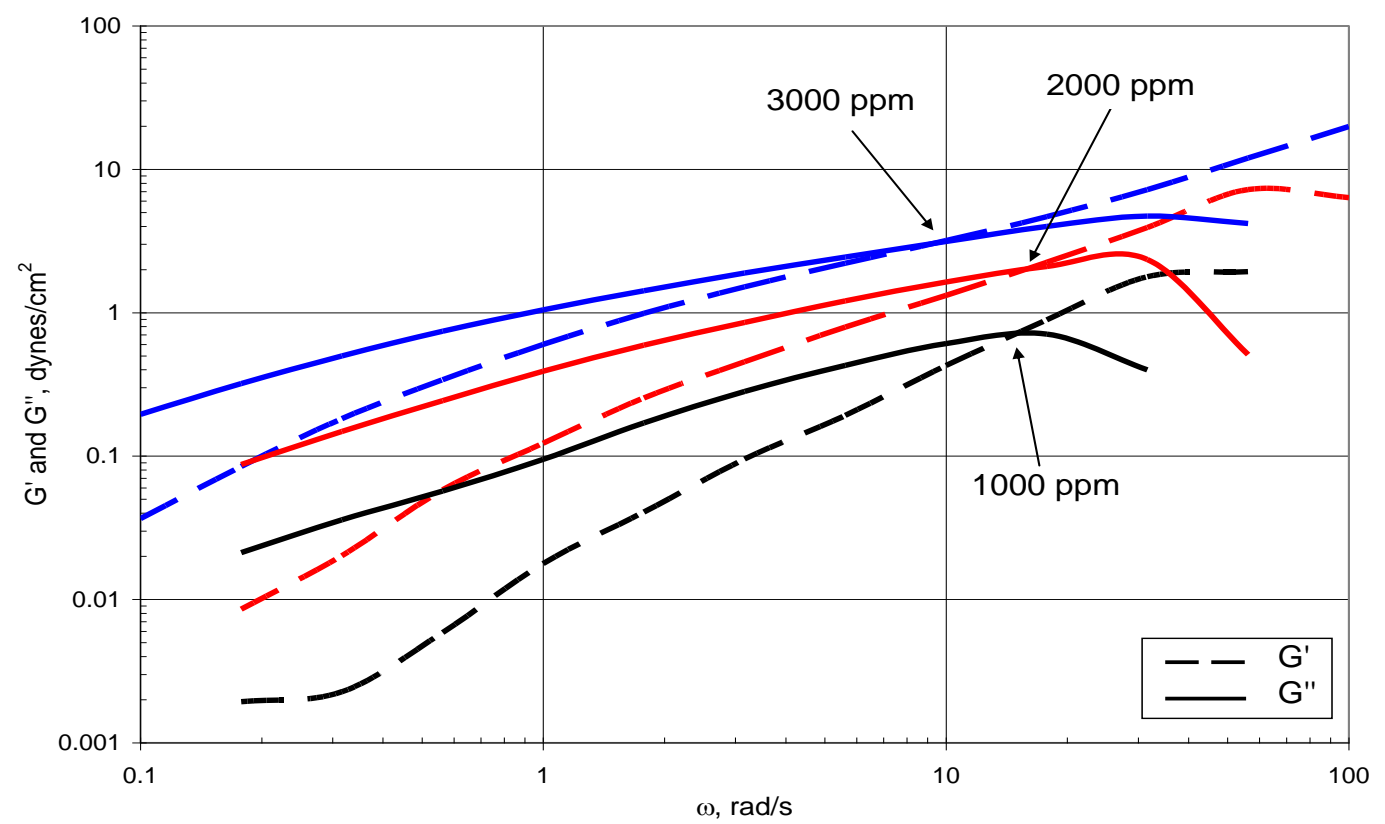

Figure 4.3 Effect of polymer concentration on crossover point for Flopaam ${ }^{\mathrm{TM}} 3630 \mathrm{~S}$ in $4 \% \mathrm{NaCl}$ at $25^{\circ} \mathrm{C}$

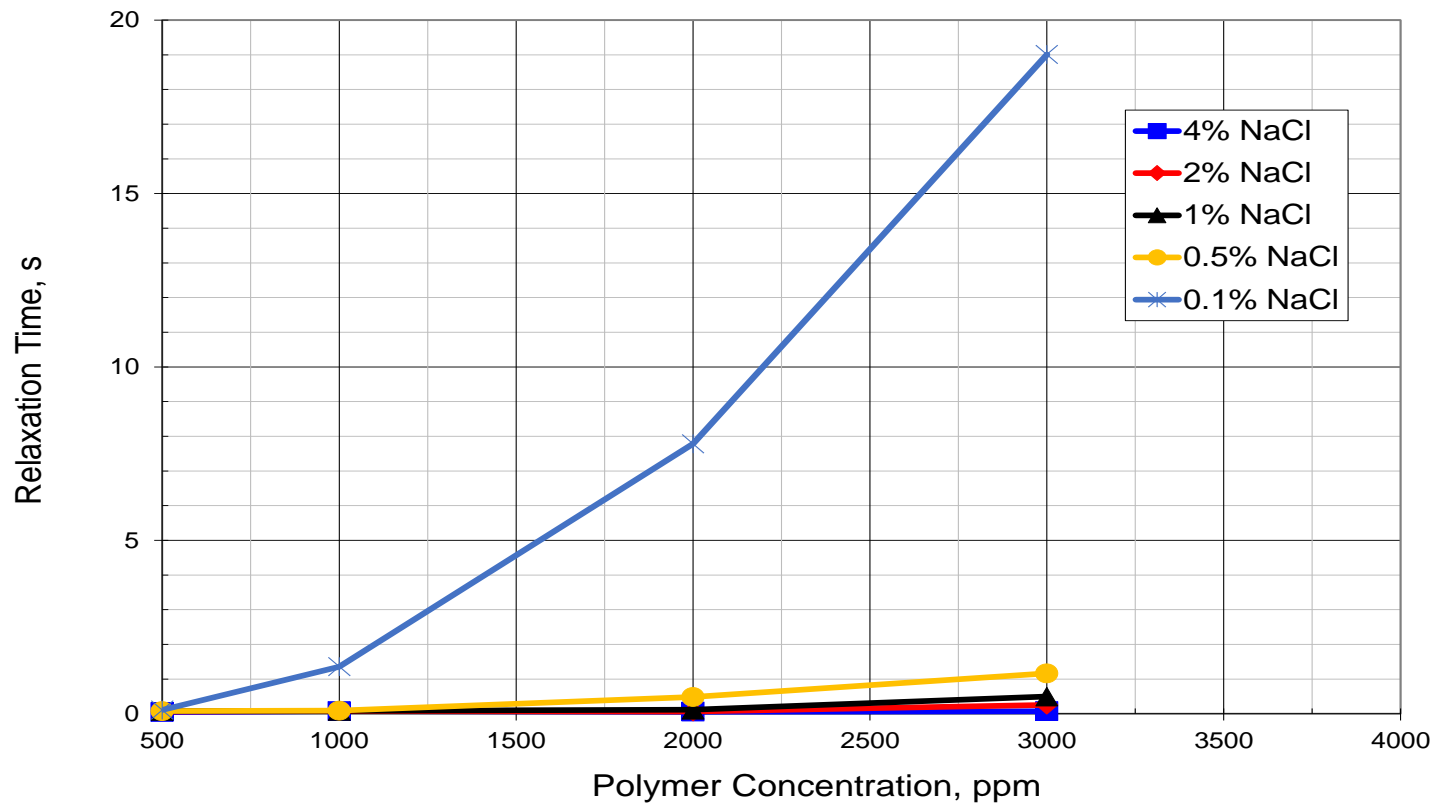

Figure 4.4 Variation of relaxation time with polymer concentration and salinity for Flopaam $^{\mathrm{TM}} 3630 \mathrm{~S}$ at $25^{\circ} \mathrm{C}$ 


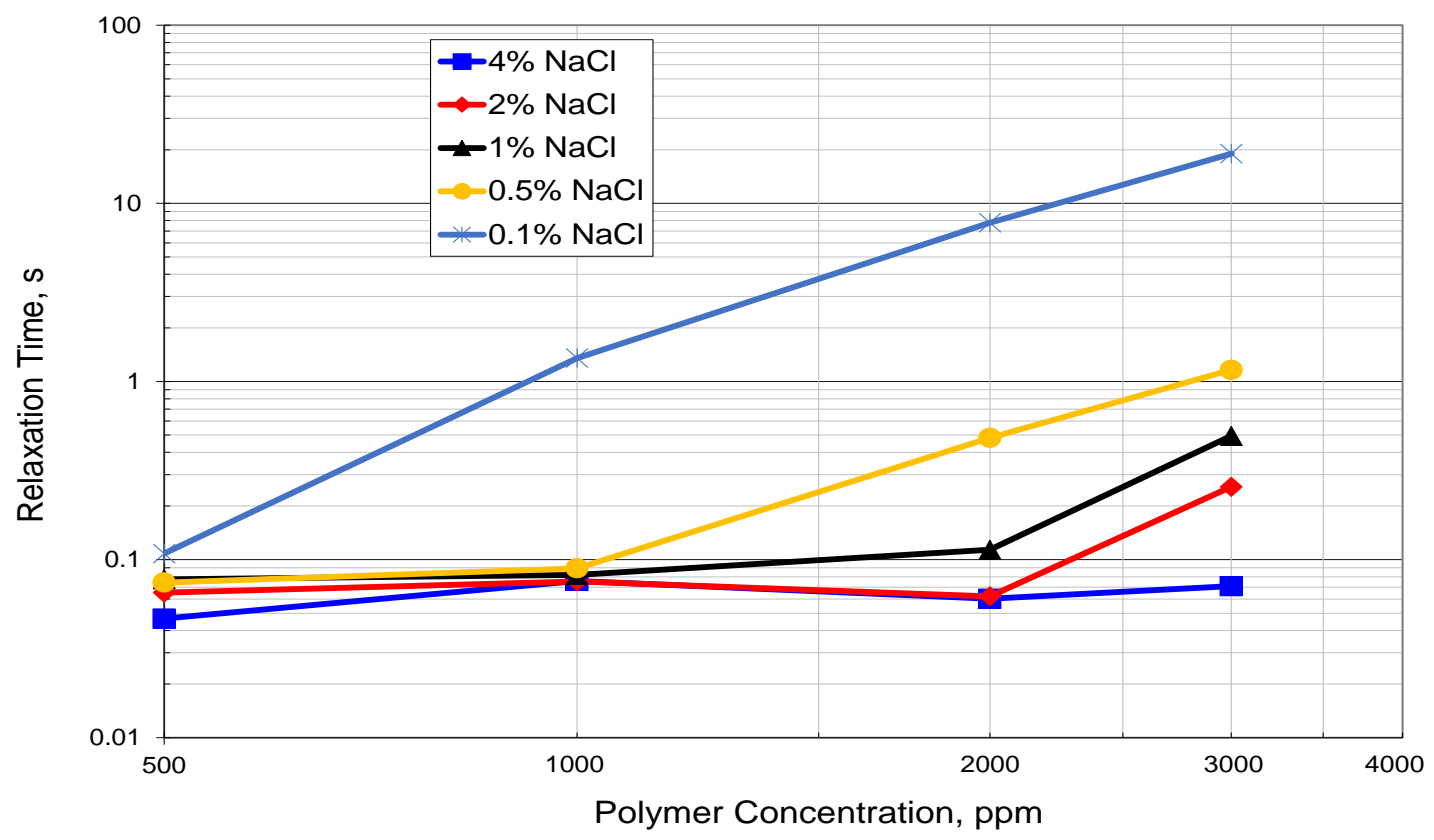

Figure 4.5 Variation of relaxation time with polymer concentration and salinity for Flopaam $^{\mathrm{TM}} 3630 \mathrm{~S}$ at $25^{\circ} \mathrm{C}$ in $\log -\log$ scale

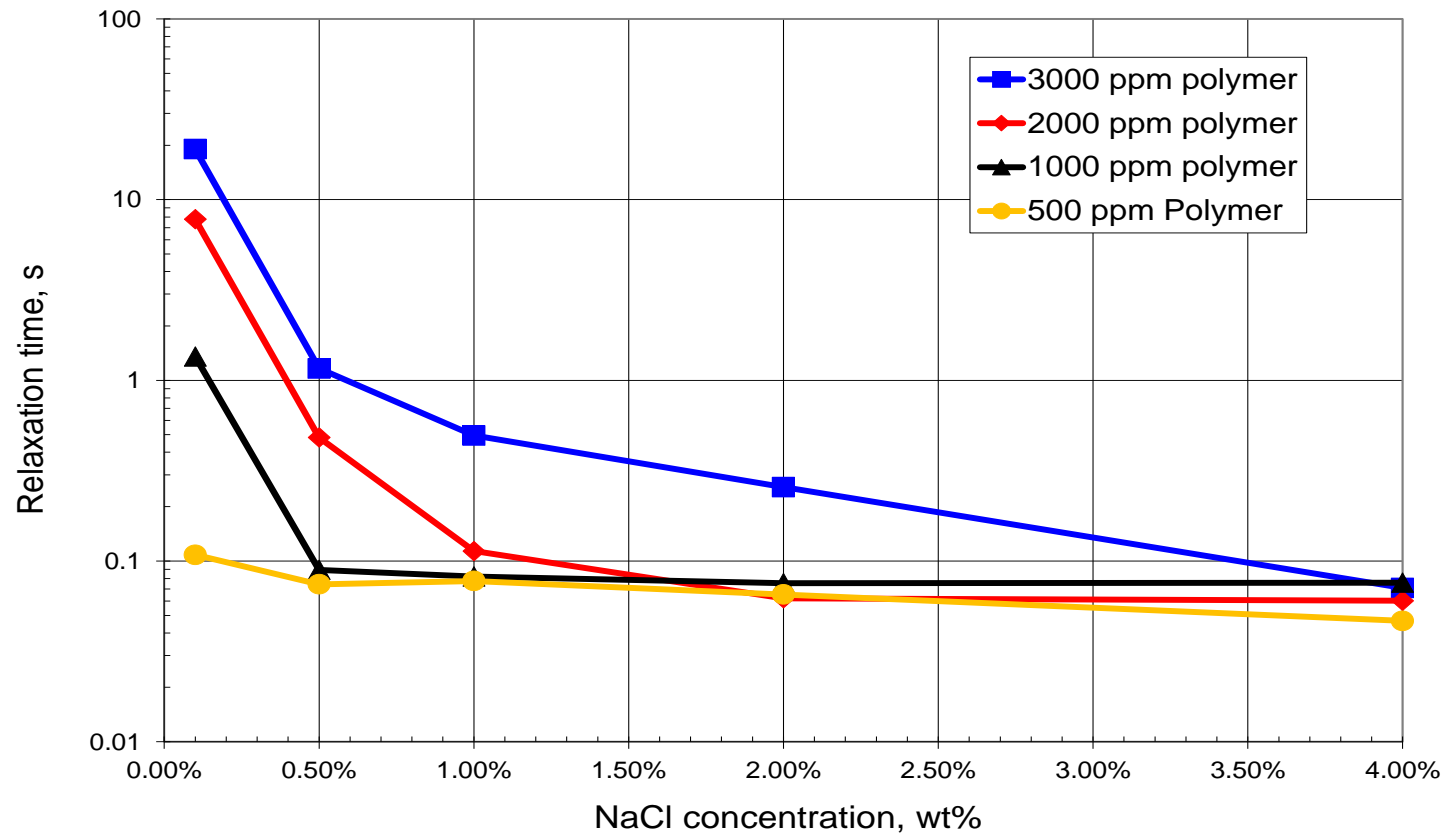

Figure 4.6 Variation of relaxation time with salinity and polymer concentration for Flopaam $^{\mathrm{TM}} 3630 \mathrm{~S}$ at $25^{\circ} \mathrm{C}$ 


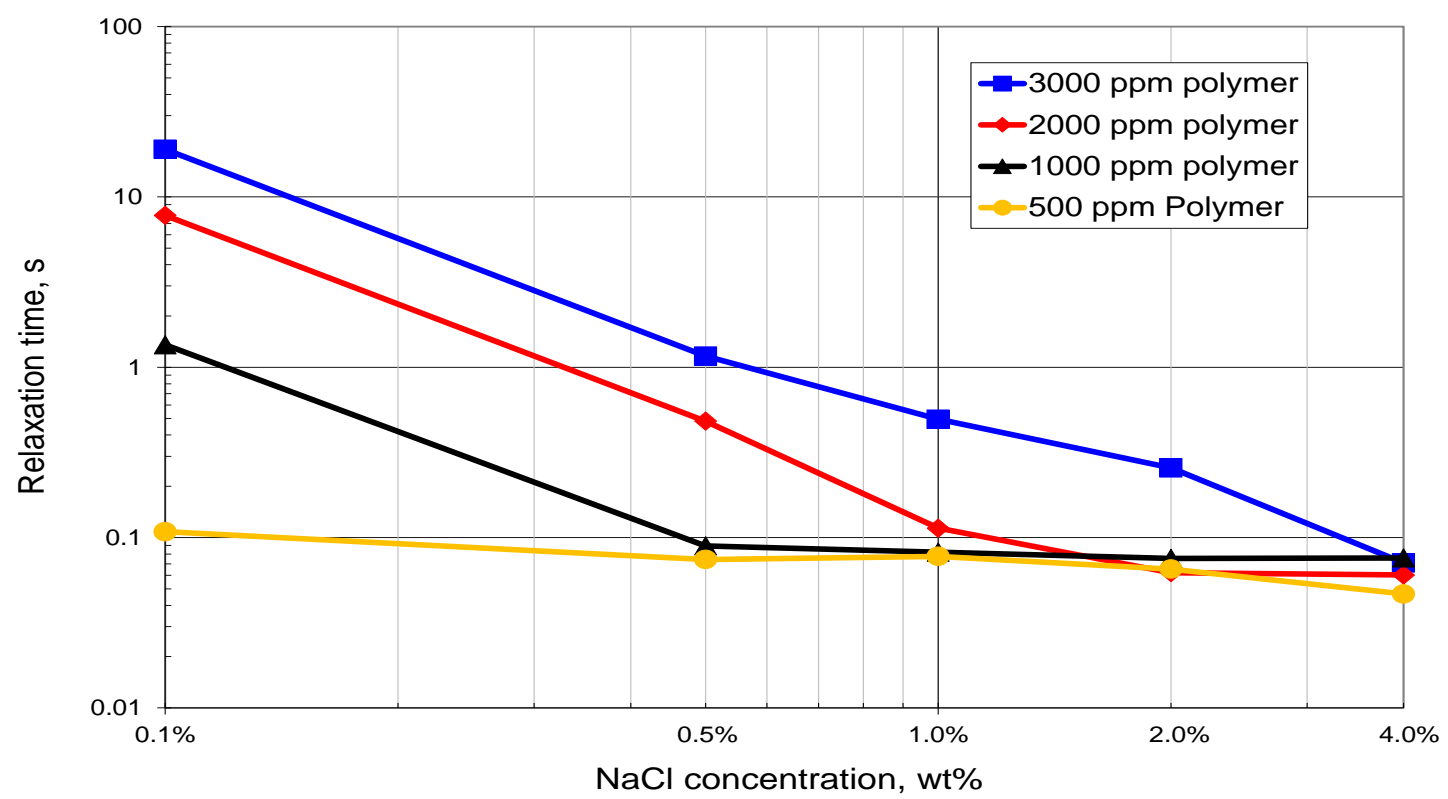

Figure 4.7 Variation of relaxation time with salinity and polymer concentration for Flopaam $^{\mathrm{TM}} 3630 \mathrm{~S}$ at $25^{\circ} \mathrm{C}, 0 \% \mathrm{Ca}^{2+}$

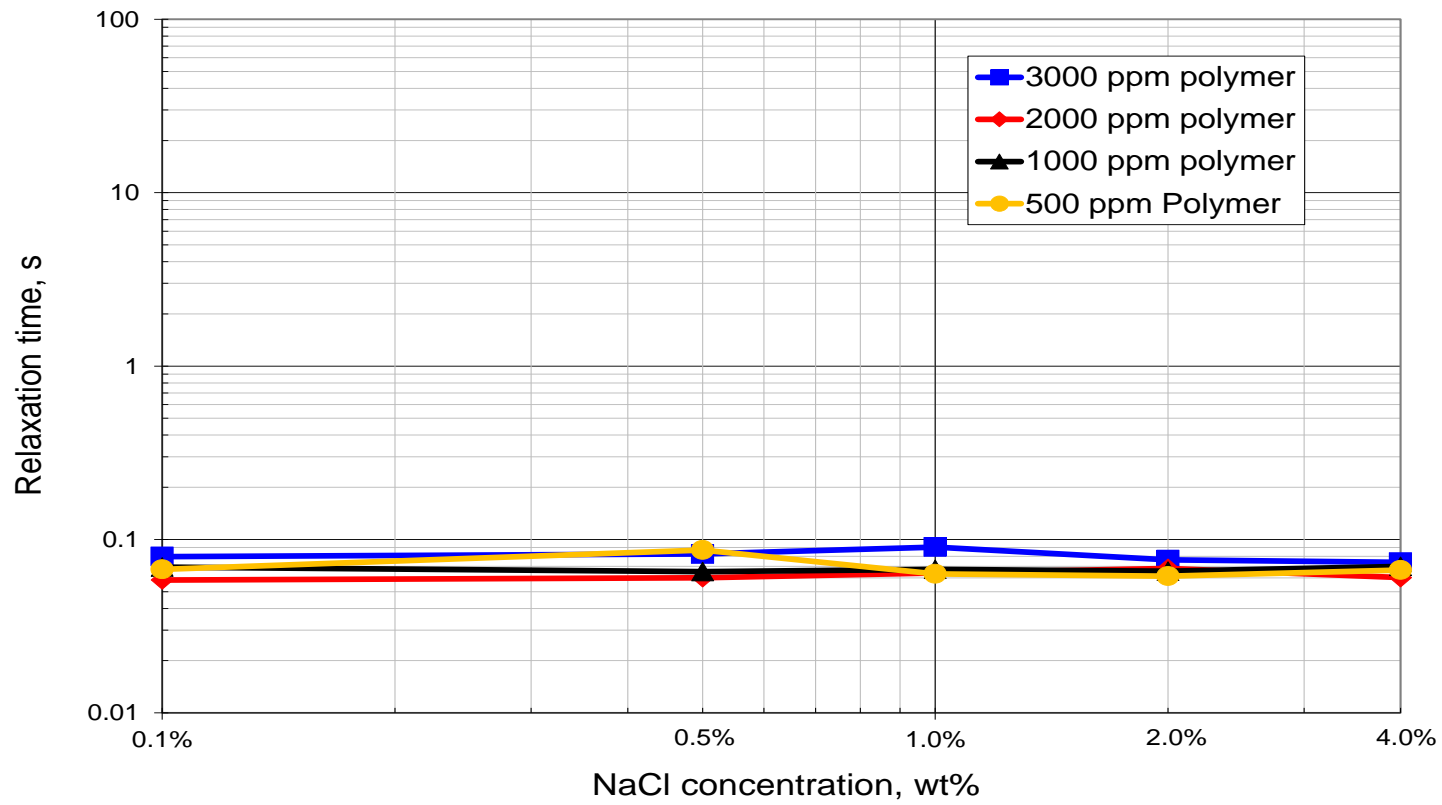

Figure 4.8 Variation of relaxation time with salinity and polymer concentration for Flopaam ${ }^{\mathrm{TM}} 3630 \mathrm{~S}$ at $25^{\circ} \mathrm{C}, 0.05 \% \mathrm{Ca}^{2+}$ 


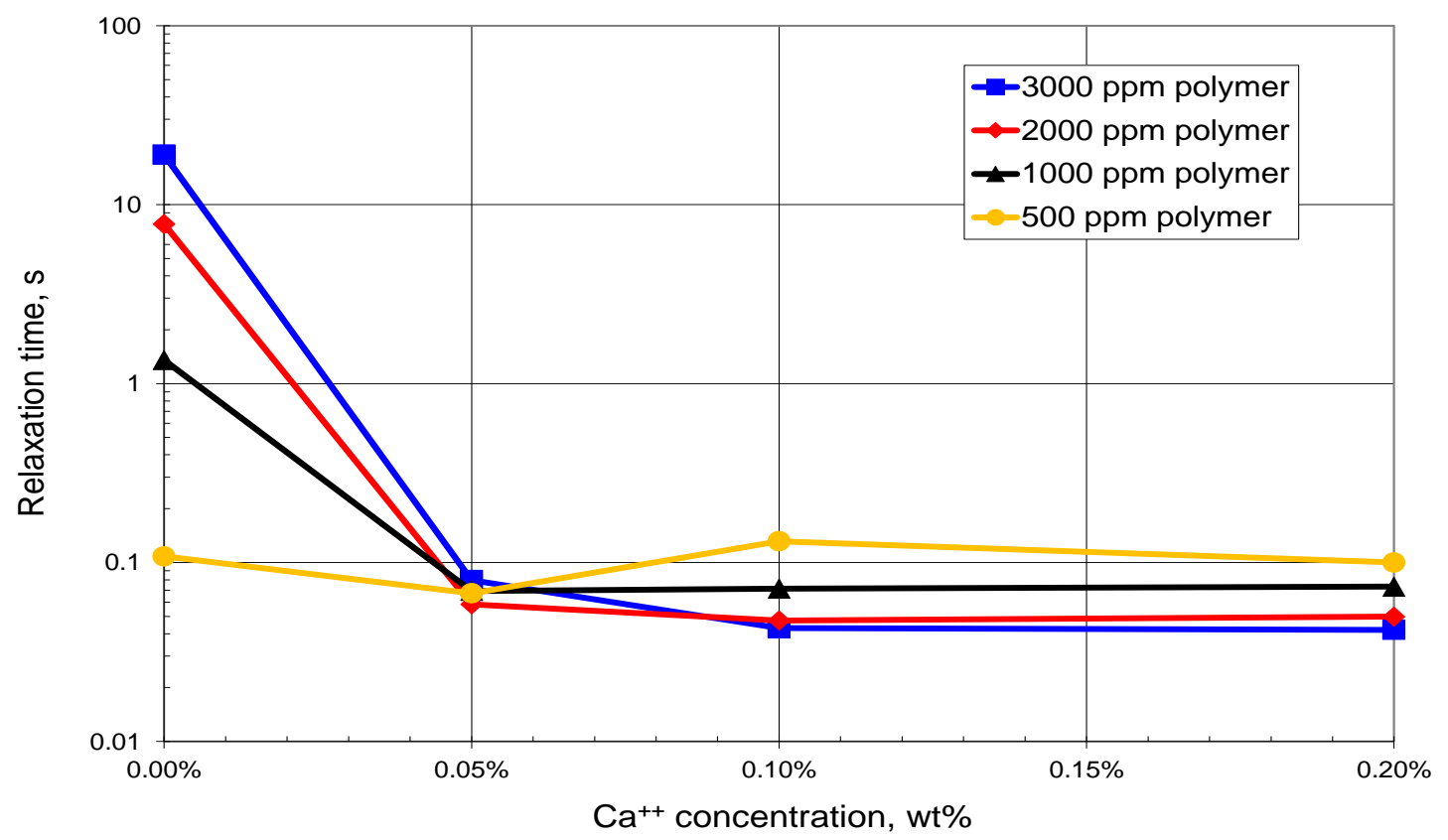

Figure 4.9 Variation of relaxation time with hardness for Flopaam ${ }^{\mathrm{TM}} 3630 \mathrm{~S}$ in $0.1 \% \mathrm{NaCl}$ at $25^{\circ} \mathrm{C}$

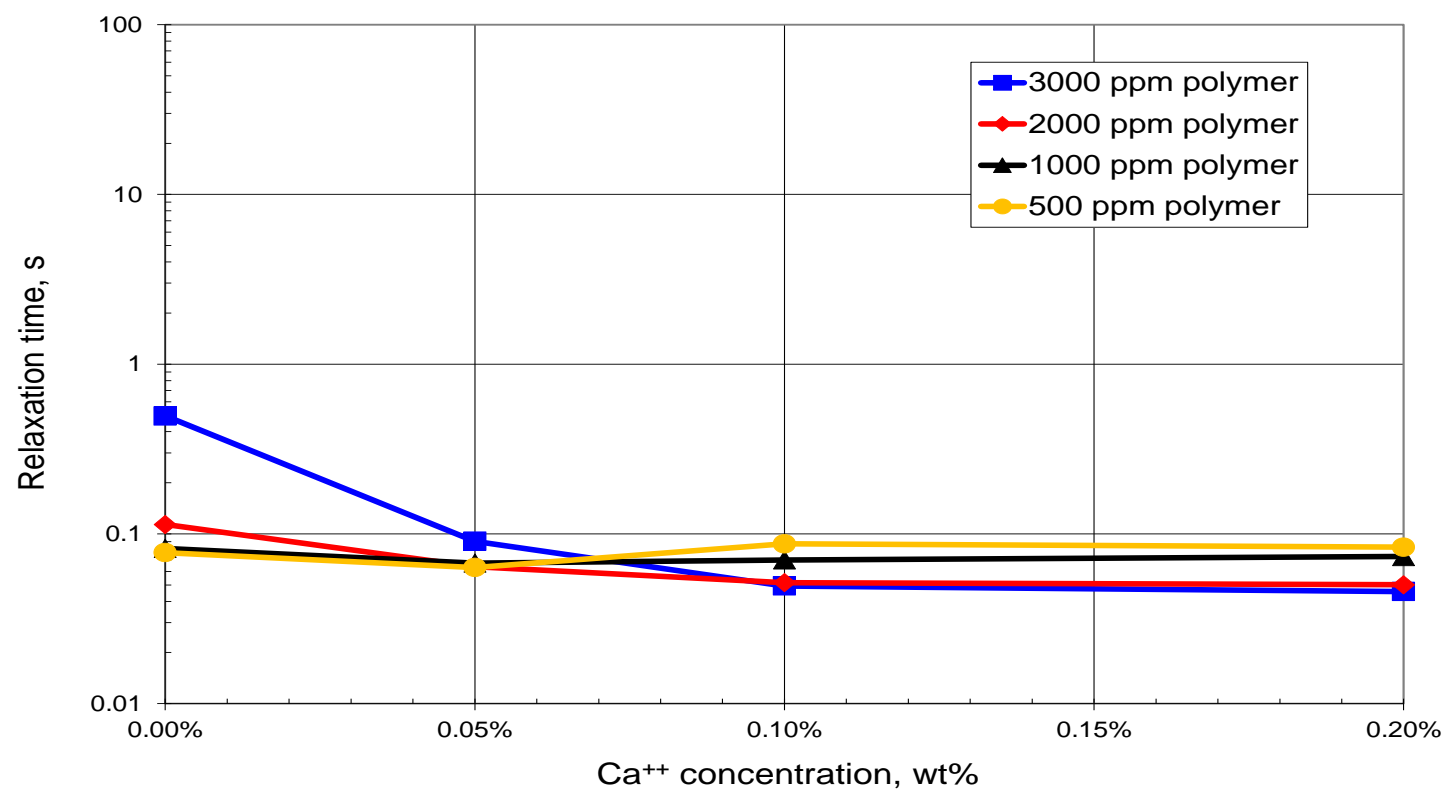

Figure 4.10 Variation of relaxation time with hardness for Flopaam ${ }^{\mathrm{TM}} 3630 \mathrm{~S}$ in $1 \% \mathrm{NaCl}$ at $25^{\circ} \mathrm{C}$ 


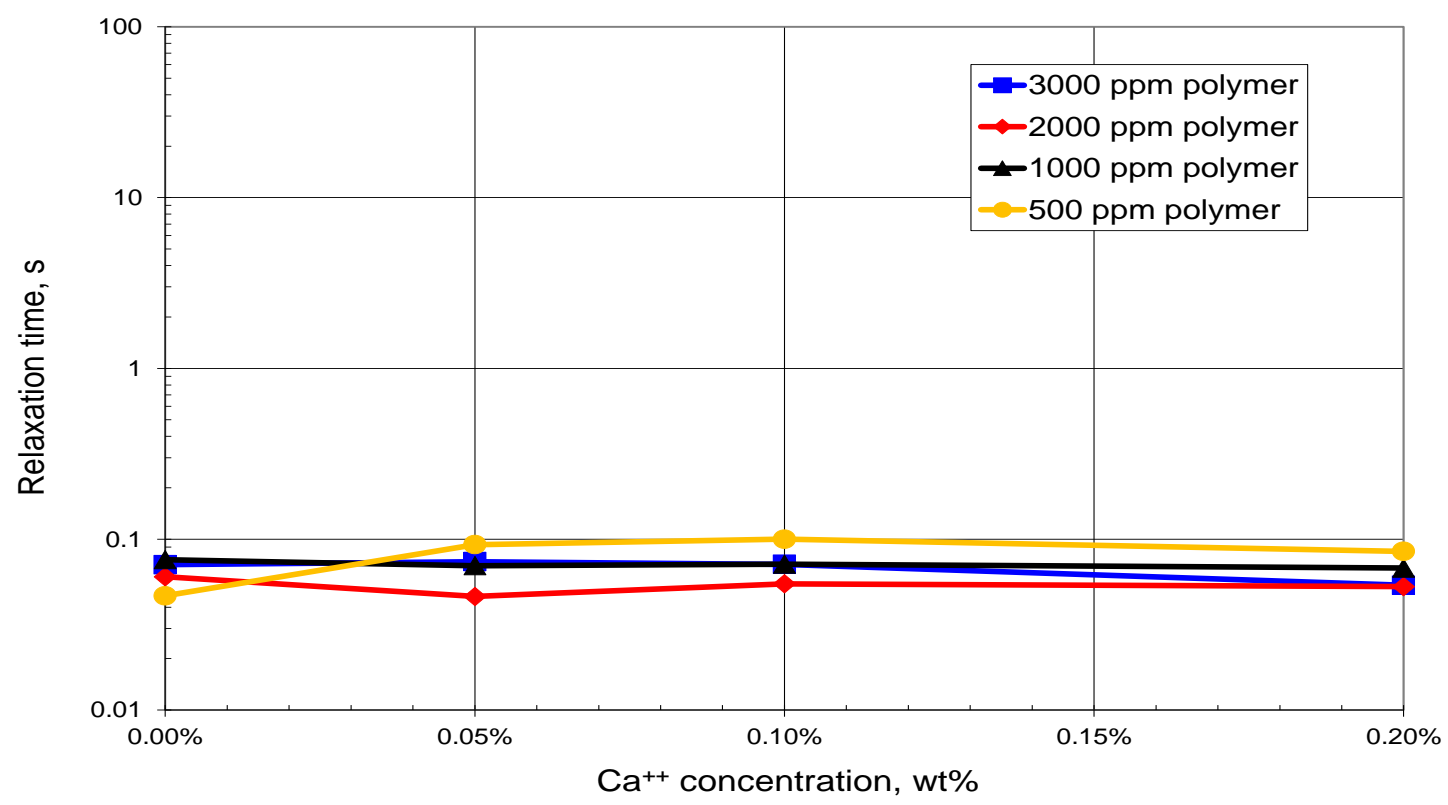

Figure 4.11 Variation of relaxation time with hardness for Flopaam ${ }^{\mathrm{TM}} 3630 \mathrm{~S}$ in $4 \% \mathrm{NaCl}$ at $25^{\circ} \mathrm{C}$

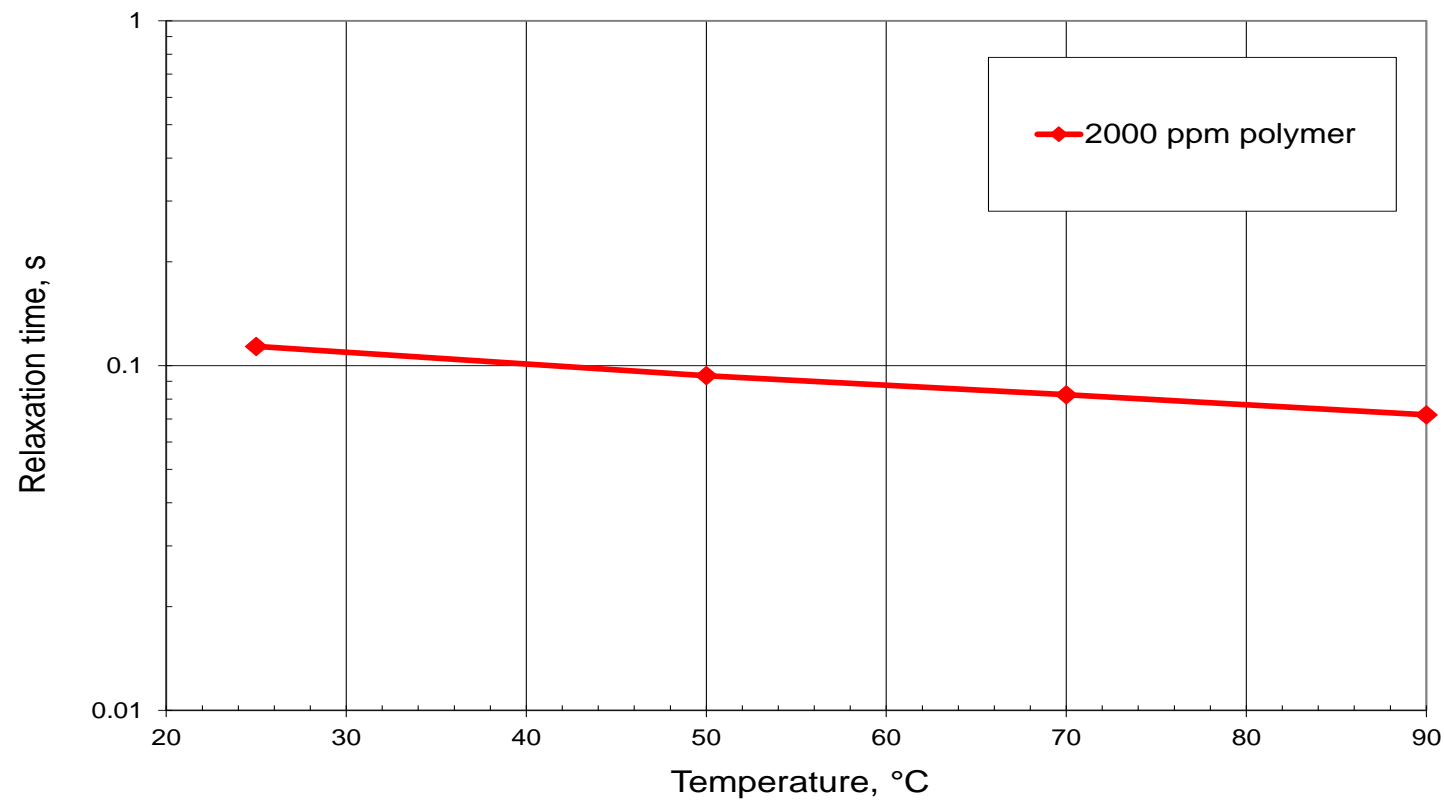

Figure 4.12 The effect of temperature on relaxation time for 2000 ppm $^{\text {Flopaam }}{ }^{\mathrm{TM}} 3630 \mathrm{~S}$ in $1 \% \mathrm{NaCl}$ 


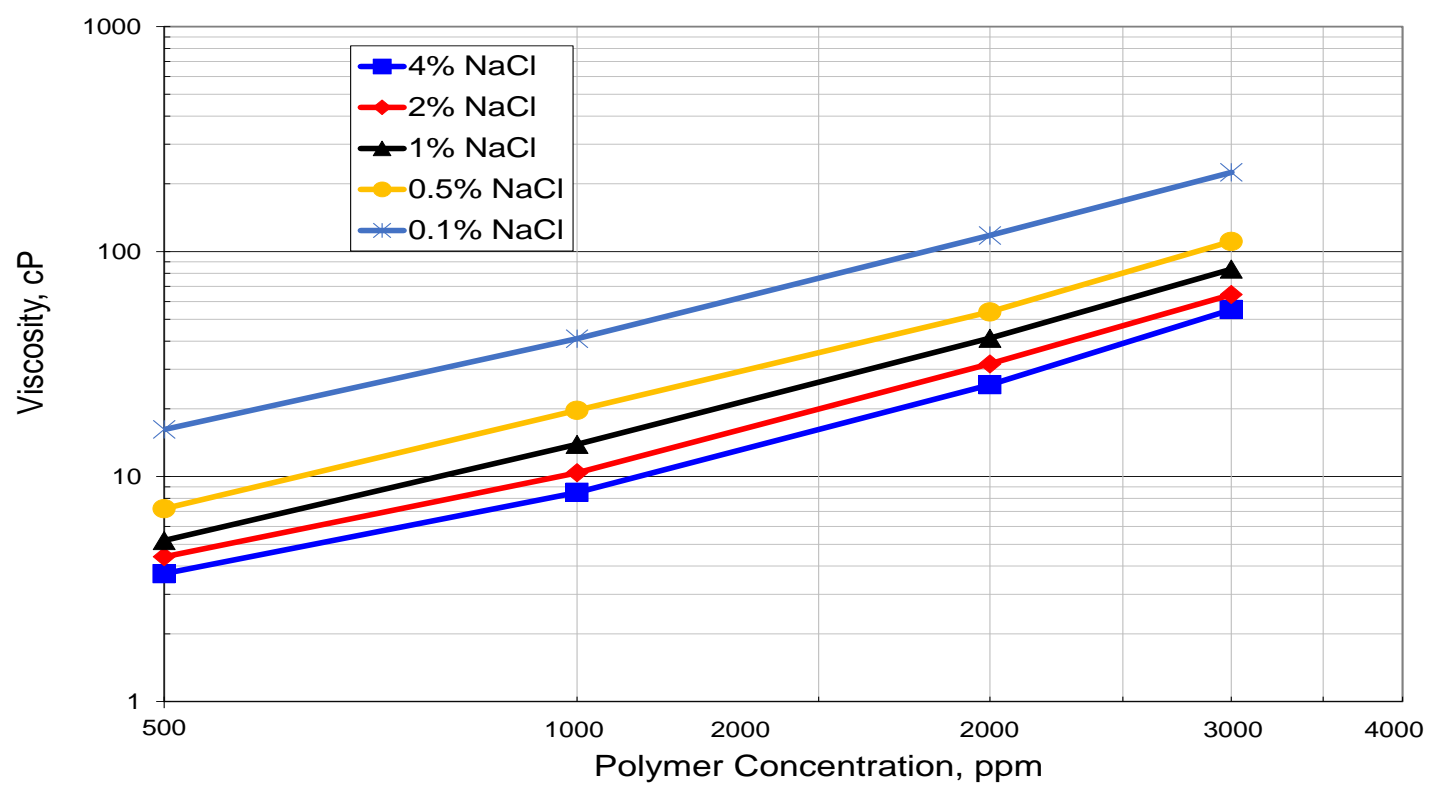

Figure 4.13 Variation of viscosity with salinity and polymer concentration for Flopaam ${ }^{\mathrm{TM}}$ $3630 \mathrm{~S}$ at $25^{\circ} \mathrm{C}$ in $\log -\log$ scale

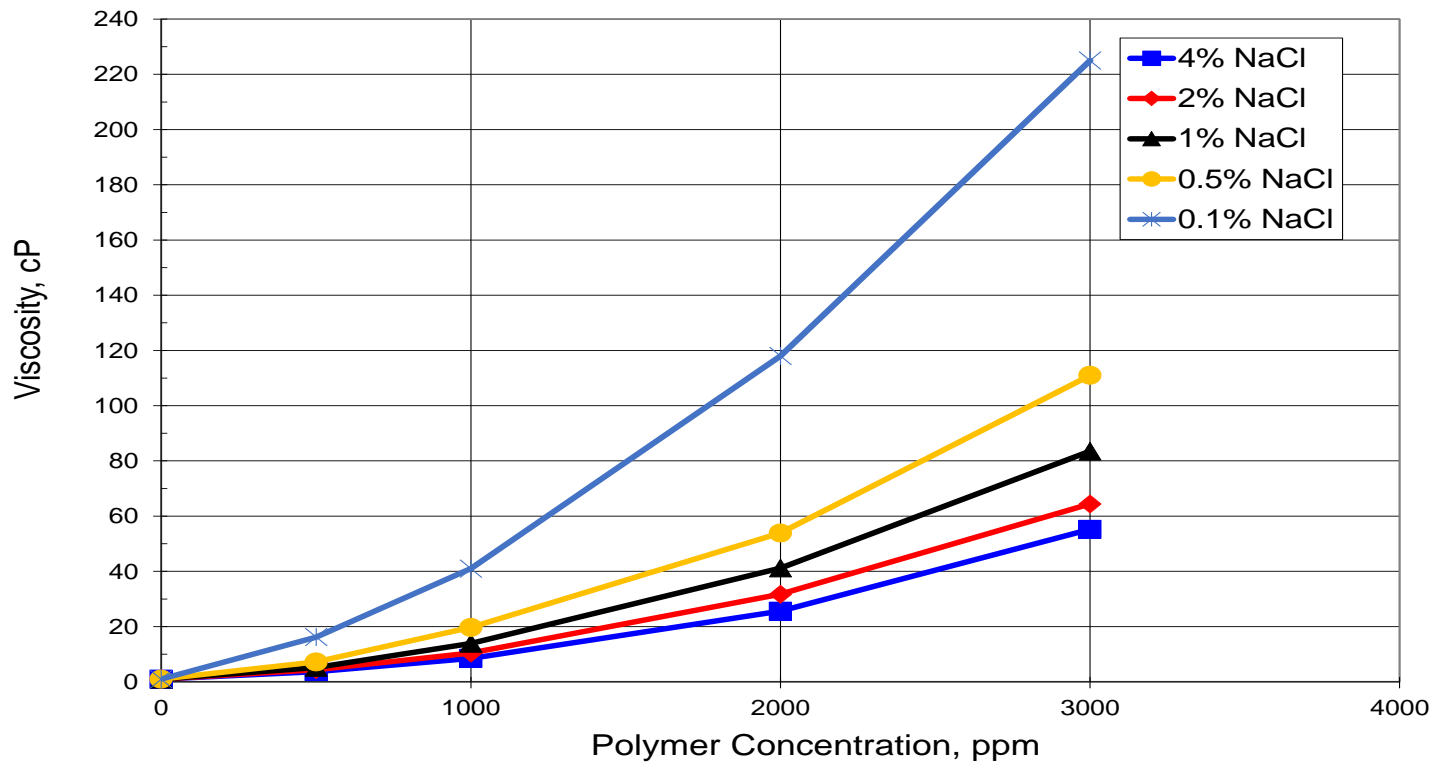

Figure 4.14 Variation of viscosity with salinity and polymer concentration for Flopaam ${ }^{\mathrm{TM}}$ $3630 \mathrm{~S}$ at $25^{\circ} \mathrm{C}$ 


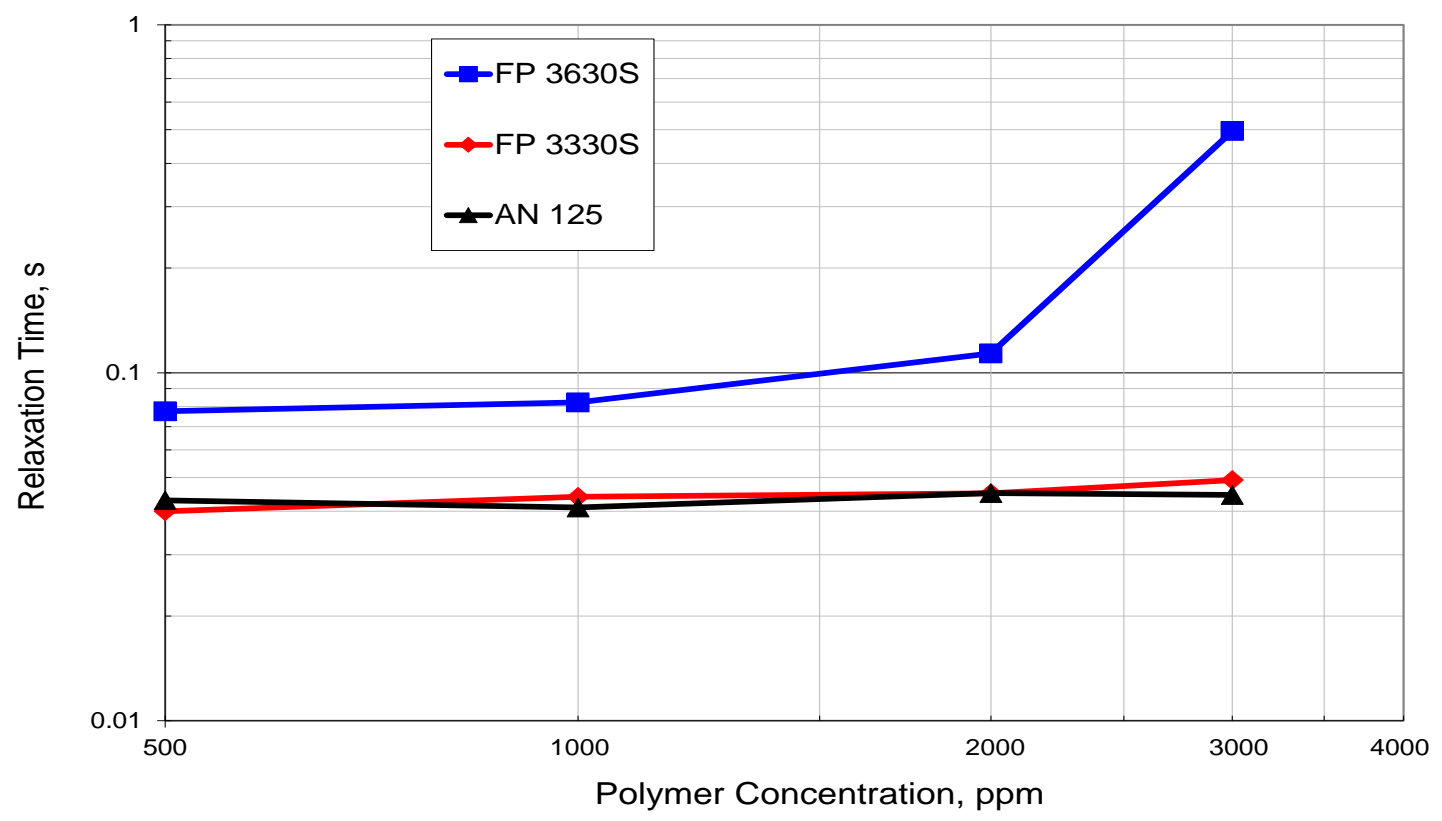

Figure 4.15 Variation of relaxation time with polymer concentration in $1 \mathrm{wt} \% \mathrm{NaCl}$ brine

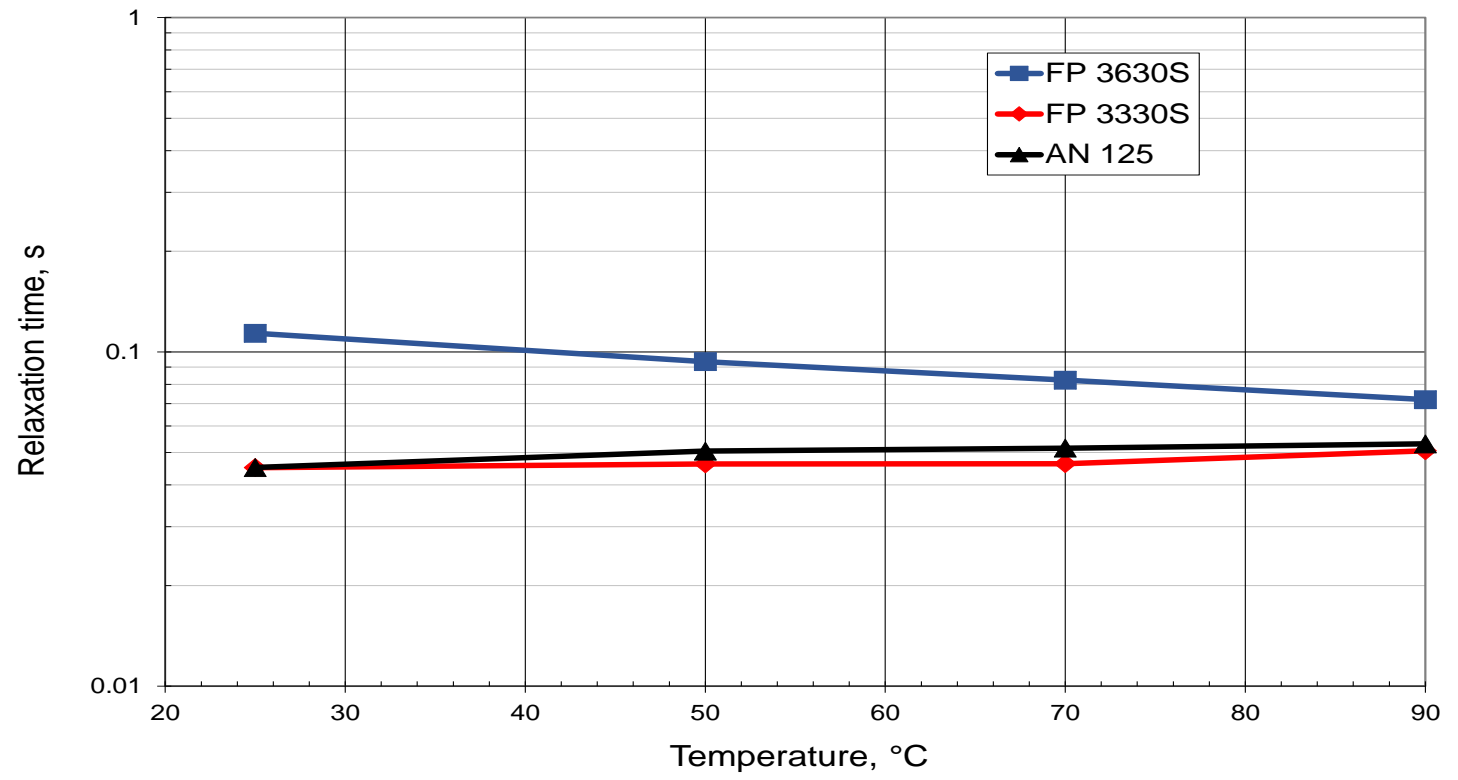

Figure 4.16 Variation of relaxation time with temperature for $2000 \mathrm{ppm}$ polymers in 1 $\mathrm{wt} \% \mathrm{NaCl}$ brine 


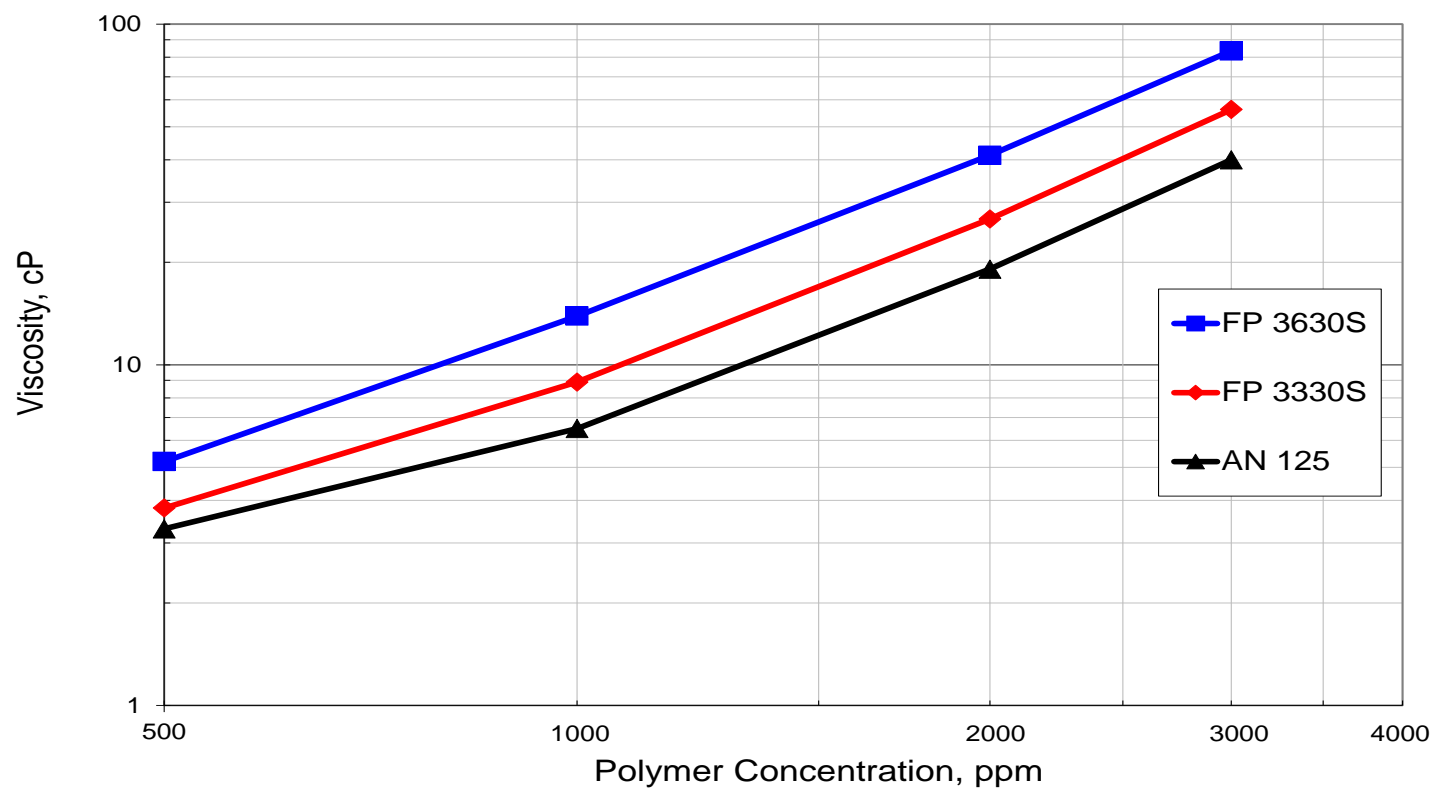

Figure 4.17 Variation of viscosity @ $10 \mathrm{~s}^{-1}$ with $1 \% \mathrm{NaCl}$ and polymer concentration for polymers in log-log scale

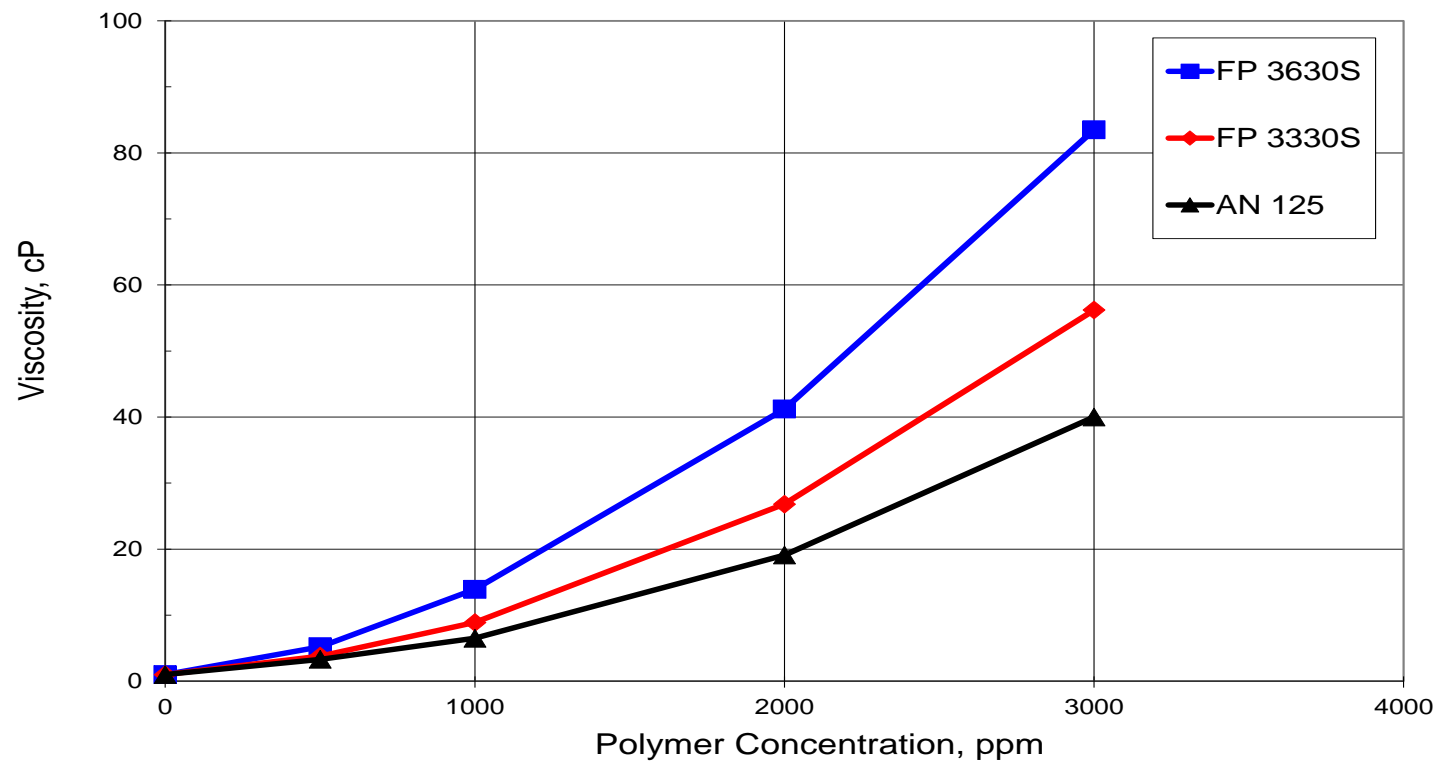

Figure 4.18 Variation of viscosity @ $10 \mathrm{~s}^{-1}$ with $1 \% \mathrm{NaCl}$ and polymer concentration for 2000 ppm polymer 


\subsubsection{Trends of the $G^{\prime}$ and $G^{\prime \prime}$ Data for Different Polymers.}

Figures 4.1, 4.2 and 4.3 show the storage modulus, G', and viscous modulus, G", for FP $3630 \mathrm{~S}$ as a function of frequency on polymer concentration and salinity respectively. Tables 4.3 to 4.6 show the dependence of relaxation time on polymer concentration, salinity, hardness. As shown in the figures, $G^{\prime}$ and $G^{\prime \prime}$ increase with increase in polymer concentration. However, the crossover point does not show a simple dependence on the polymer concentration. In contrast with the polymer concentration, the lower value of salinity gives the higher values of $\mathrm{G}^{\prime}$ and $\mathrm{G}^{\prime \prime}$ at constant polymer concentration. The effect of hardness on relaxation time shown in Table 4.3 reveals that the dependence on hardness is similar to that of salinity.

From the experimental data, the following major trend/differences are observed: (1) Increase in polymer concentration relates to increase in $\mathrm{G}^{\prime}$ and $\mathrm{G}^{\prime \prime}$ for the polymers tested. (2) Increase in salinity and hardness decreases the values of G' and G".

\section{Dependence on Polymer Concentration}

Figures 4.1 to 4.3 show the effect of polymer concentration of Flopaam ${ }^{\mathrm{TM}} 3630 \mathrm{~S}$ on the storage or elastic modulus, $\mathrm{G}^{\prime}$, loss or viscous modulus, $\mathrm{G}^{\prime \prime}$, and the crossover points of $\mathrm{G}^{\prime}$ and $\mathrm{G}^{\prime \prime}$ as a function of frequency for three different salinities. As shown in the figures, $\mathrm{G}^{\prime}$ and $\mathrm{G}^{\prime \prime}$ increase with increase in polymer concentration. However, the crossover point does not show a simple dependence on the polymer concentration.

Lower salinity gives higher values of $G^{\prime}$ and $G^{\prime \prime}$ at constant polymer concentration. Figures 4.4 and Figure 4.5 show the effect of polymer concentration of Flopaam $^{\mathrm{TM}} 3630 \mathrm{~S}$ on the relaxation time for different values of salinity with $0.1 \%$, $0.5 \%, 1 \%, 2 \%$ and $4 \% \mathrm{NaCl}$. The relaxation time increases as polymer concentration increases. As shown in the Figure 4.4, a strong polynomial relationship exists between 
polymer concentration and relaxation time $\tau_{\mathrm{r}}$, especially in case of $0.1 \% \mathrm{NaCl}$. Figure 4.5 in log-log scale shows clearer trend of the relaxation time on polymer concentration. The interesting result in Figure 4.12 was observed. If the minimum value of the relaxation time which FP 3630s has around $0.8 \mathrm{~s}$ in this range of measurement, each case of salinity has a different onset point of polymer concentration to begin to increase the relaxation time from minimum value of the relaxation time. In case of $4 \% \mathrm{NaCl}$, the relaxation time begins to increase from $500 \mathrm{ppm}$ of polymer concentration. In the case of $0.5 \% \mathrm{NaCl}$ and $1 \% \mathrm{NaCl}$, the onset points are $1000 \mathrm{ppm}$ and $2000 \mathrm{ppm}$ of polymer concentration, respectively. In case of $4 \% \mathrm{NaCl}$, the trend of the relaxation time is flat for the entire range of polymer concentration between $500 \mathrm{ppm}$ and $3000 \mathrm{ppm}$.

\section{Dependence on Salinity and Hardness}

Figures 4.6 to Figure 4.11 show the effect of salinity on the relaxation time. In contrast with polymer concentration, an increase in the salinity decreases in the relaxation time for constant polymer concentration. As Figure 4.7 shows, there is a specific $\mathrm{NaCl}$ concentration to drop the relaxation time to minimum value for each case of constant polymer concentration. For $500 \mathrm{ppm}$ polymer concentration, the relaxation time has minimum value of around $0.08 \mathrm{~s}$ over the entire range of salinity from $0.1 \%$ to $4 \% \mathrm{NaCl}$. However, for 1000 ppm, 2000 ppm, and 3000 ppm polymer concentration, the relaxation time has its minimum value after critical point of $0.5 \%, 1 \%$, and $4 \% \mathrm{NaCl}$ concentration. Figure 4.9 represents the effect of hardness on the relaxation. The effect of hardness is very severe on the relaxation time for FP 3630 S polymer. The value of relaxation time drop to the minimum value after just $500 \mathrm{ppm}$ of $\mathrm{Ca}^{2+}$. This is 80 times stronger effect than $\mathrm{NaCl}$ concentration. After the relaxation time reached the minimum value of the relaxation time for the FP 3630 S polymer solution, the increase of salinity doesn't 
decrease the value of the relaxation time. The relaxation time maintains the constant value.

\section{Dependence on Temperature}

As shown in Figure 4.12, higher temperature gives lower values of the relaxation time. Even though, the relaxation time shifts to a slightly lower value as the temperature increases for 2000 ppm FP $3630 \mathrm{~S}$ in $1 \% \mathrm{NaCl}$, the effect of temperature on the relaxation time is bigger than what Figure 4.12 shows. The chosen condition, 2000 ppm FP 3630S in $1 \% \mathrm{NaCl}$ was not proper condition to show dependence of temperature because the salinity of $1 \% \mathrm{NaCl}$ is already the critical point to drop the relaxation time to the minimum value.

\section{Dependence on Intrinsic viscosity}

Figure 4.13 and Figure 4.14 show the viscosity as a function of polymer concentration for FP 3630S. Figure 4.17 and Figure 4.18 show a comparison of the viscosity of AN-125, FP 3330 S and FP 3630 S as function of polymer concentration.

In Figures 4.20 and 4.21, intrinsic viscosity for FP $3630 \mathrm{~S}$ was determined by using Equation 4.1. Intrinsic viscosity for FP 3330S and AN-125 were obtained in the same manner. The intrinsic viscosity $[\eta]$ characterizes the hydrodynamic volume in solution. The intrinsic viscosity is affected by molecular weight and molecular weight distribution as well as the polymer-solvent interactions due to the equilibrium coil shape and size (Graessley, 2004; Doi and Edwards, 1986). The intrinsic viscosity, or hydrodynamic volume for $\mathrm{FP} 3630 \mathrm{~S}$ in $0.1 \% \mathrm{NaCl}$ is bigger than that in $2 \% \mathrm{NaCl}$ in Figures 4.20 and 4.21 since HPAM flexible chains are compressed in high salinity water where the charge repulsion of the carboxylic group is shielded. As shown in Figure 
4.21 and Figure 4.22, the intrinsic viscosity for FP $3630 \mathrm{~S}$ is greater than the intrinsic viscosity of FP 3330 S due to its higher molecular weight and this is true even when the salinity is higher for the FP 3630 S solution.

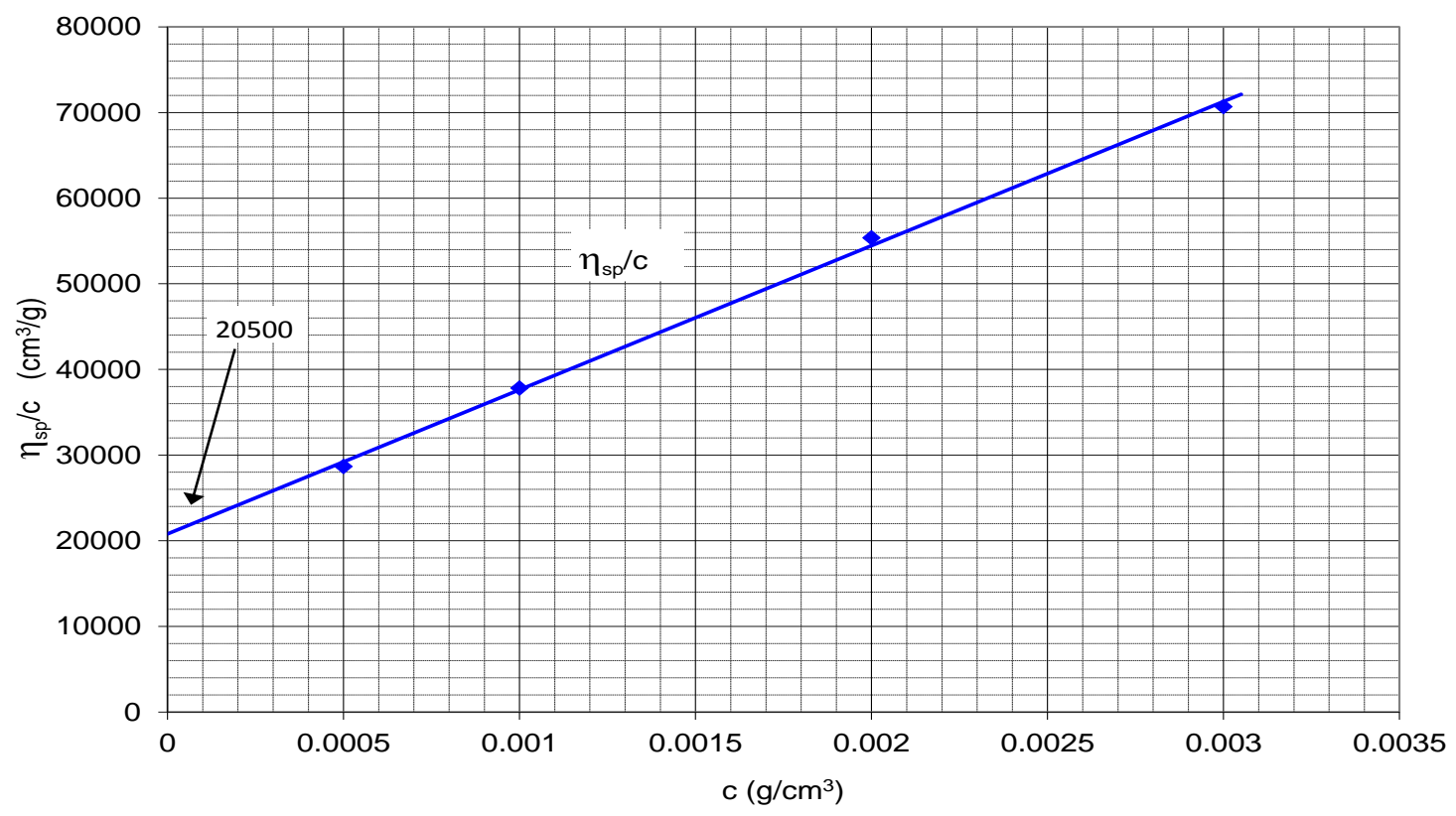

Figure 4.20 Plot to estimate intrinsic viscosity of FP $3630 \mathrm{~S}$ in $0.5 \% \mathrm{NaCl}$ 


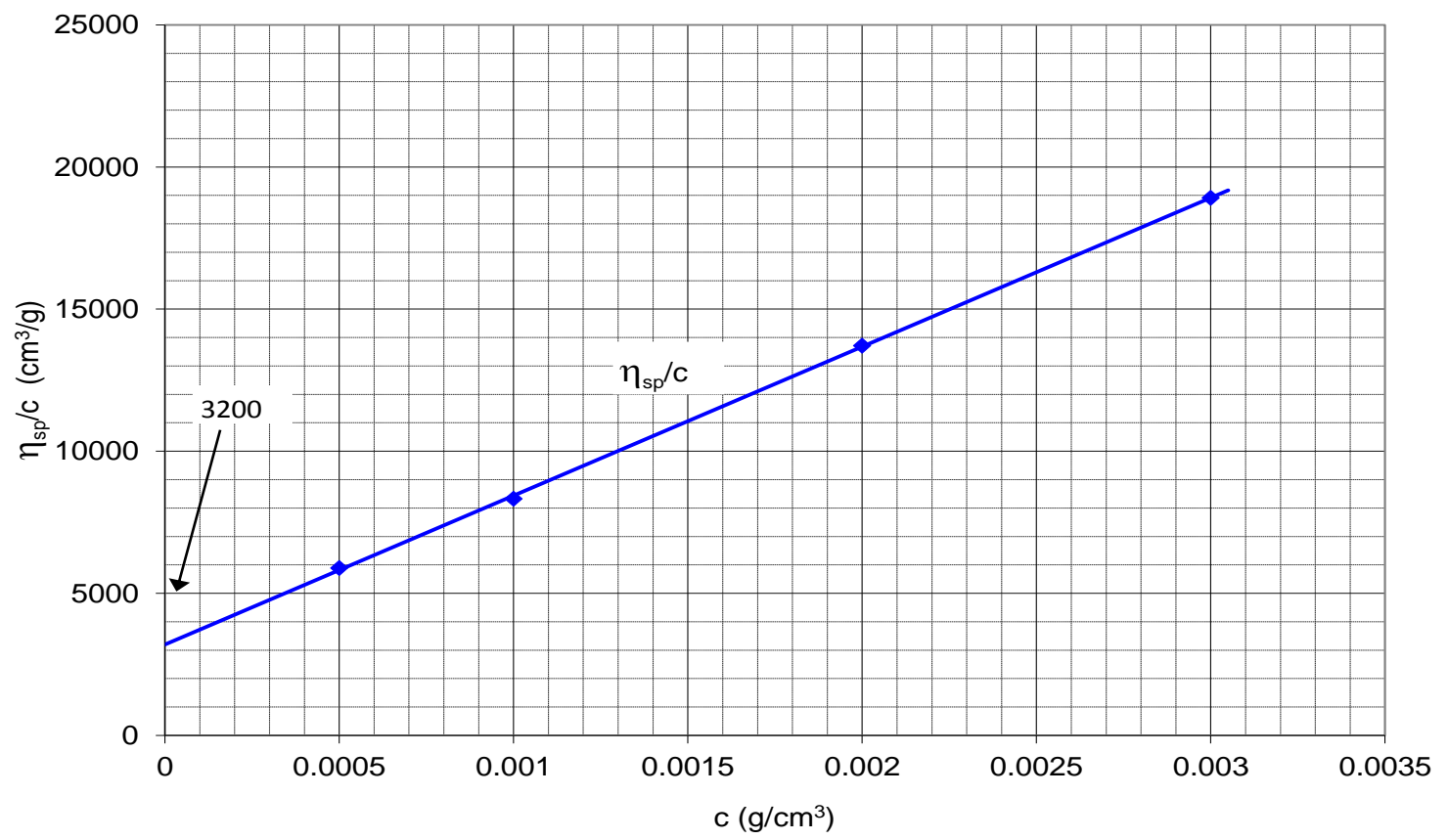

Figure 4.21 Plot to estimate intrinsic viscosity of FP $3630 \mathrm{~S}$ in $2 \% \mathrm{NaCl}$

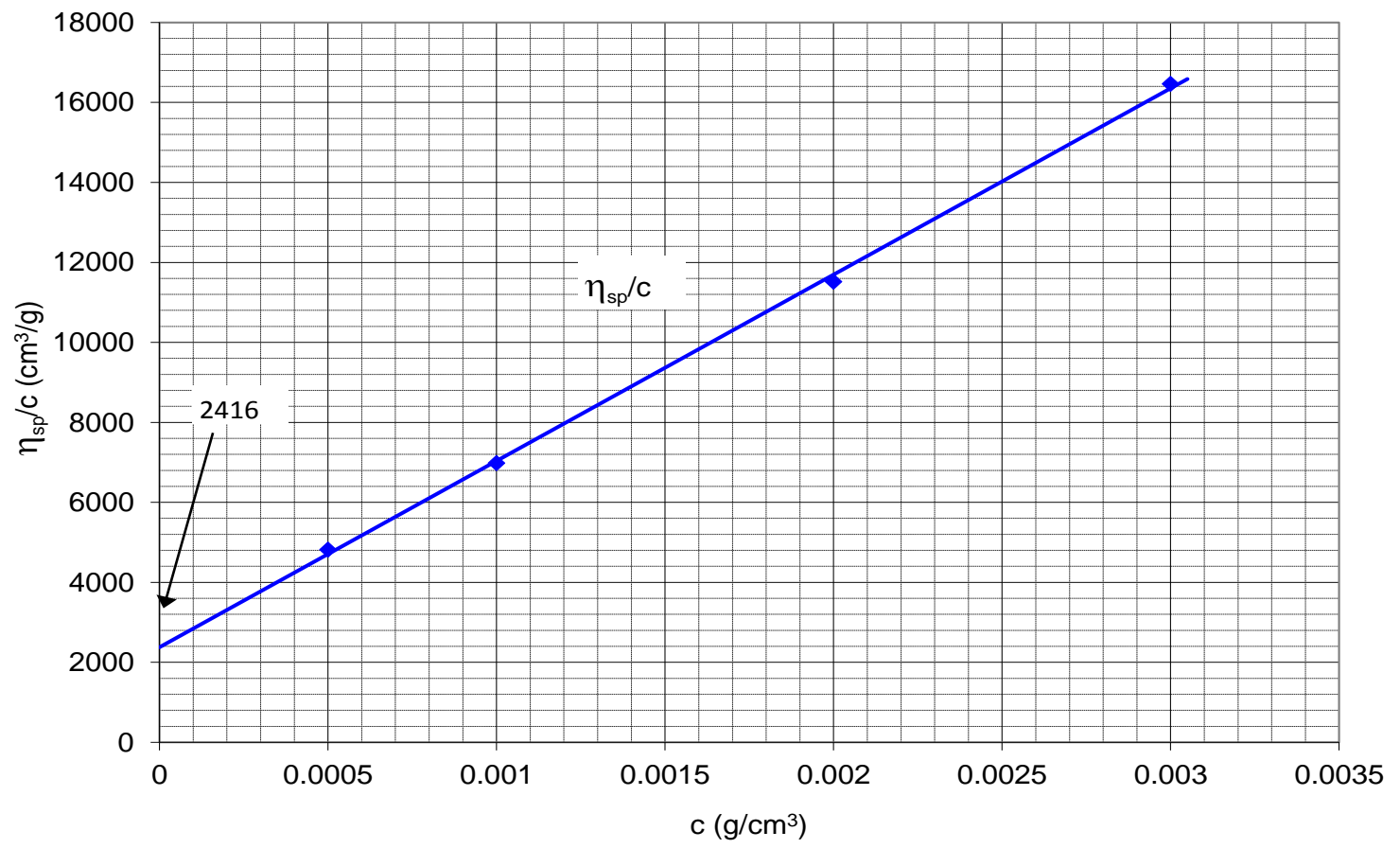

Figure 4.22 Plot to estimate intrinsic viscosity of FP $3330 \mathrm{~S}$ in $1 \% \mathrm{NaCl}$ 


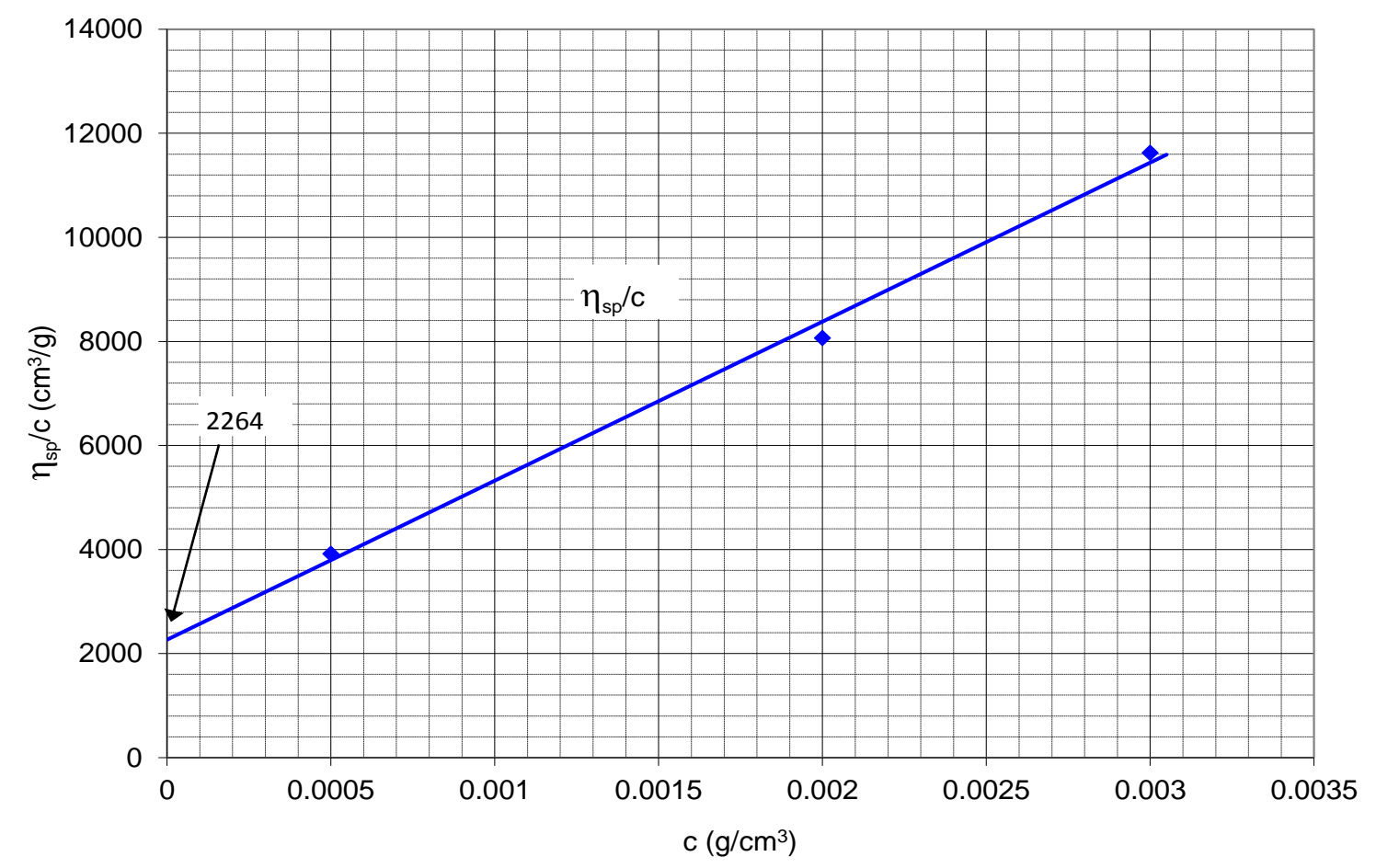

Figure 4.23 Plot to estimate intrinsic viscosity of AN-125 in $1 \% \mathrm{NaCl}$

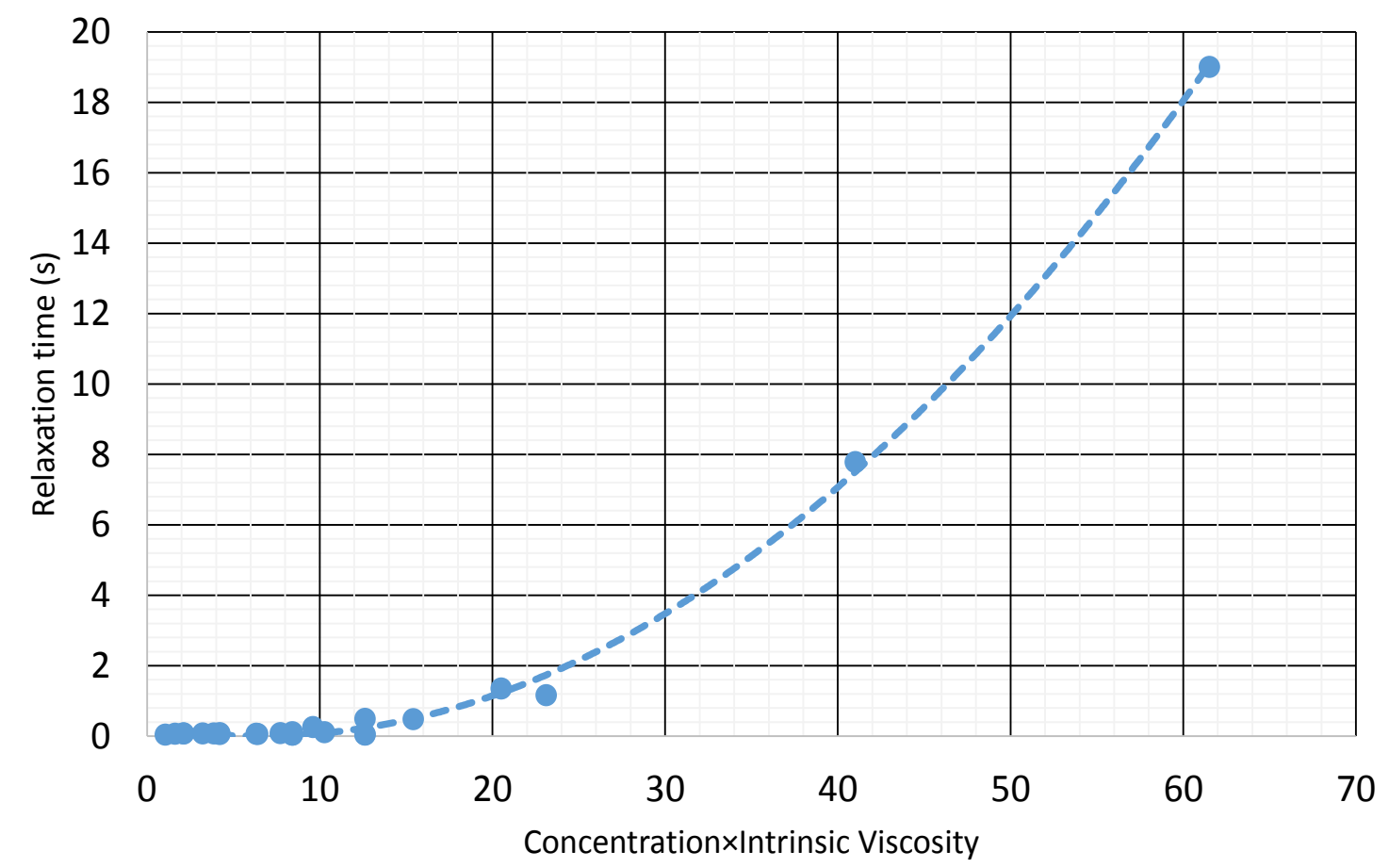


Figure 4.24 Relaxation time versus c[ $\eta]$

Stavland et al. (2010) found that their relaxation time data correlated with the product of intrinsic viscosity and polymer concentration. Figure 4.24 shows the values of relaxation time measured in this study also correlate well with $[\eta] c$. The relaxation times were used to determine the Deborah number defined by Equation 4.7 to quantify the viscoelasticity of polymer solutions in porous media as discussed in Chapter 5.

\subsection{SUMMARY AND CONCLUSIONS}

Two types of polymers were used in this study: two partially hydrolyzed polyacrylamide (HPAM) polymers with different molecular weights and a co-polymer of acrylamido-methyl-propane sulfonate (AMPS) and acrylamide. The rheological measurements were made to characterize the steady state and oscillatory viscosity of these polymers in terms of a function of polymer concentration, salinity, hardness, and temperature. The results can be summarized as follows:

1. Both $\mathrm{G}^{\prime}$ and $\mathrm{G}^{\prime \prime}$ increase with polymer concentration.

2. Both $\mathrm{G}^{\prime}$ and $\mathrm{G}^{\prime \prime}$ decrease with salinity and hardness.

3. There is a critical polymer concentration and a critical salinity concentration above which the relaxation time increases from its minimum value. .

4. The effect of divalent ion, $\mathrm{Ca}^{2+}$, on the relaxation time for Flopaam ${ }^{\mathrm{TM}} 3630 \mathrm{~S}$ polymer solution is about 800 times higher than the effect of monovalent ion, $\mathrm{Na}^{+}$. Addition of $500 \mathrm{ppm} \mathrm{Ca}^{2+}$ causes the relaxation time for $3000 \mathrm{ppm}$ Flopaam $^{\mathrm{TM}} 3630 \mathrm{~S}$ solution in $0.1 \% \mathrm{NaCl}$ to drop as much as one for $500 \mathrm{ppm}$ Flopaam $^{\mathrm{TM}} 3630 \mathrm{~S}$ solution in $0.5 \% \mathrm{NaCl}$. 
5. The relaxation time correlates well with the product of the intrinsic viscosity and polymer concentration. 


\section{Chapter 5. Transport and In-situ Rheology of EOR Polymers}

\subsection{INTRODUCTION}

While the rheological characterization of bulk polymer solutions is important and useful, their rheological characterization in cores is essential for EOR applications. Such tests are reported and analyzed in this chapter.

Polymers that are stable in harsh environments (high salinity/hardness and high temperature) are needed for chemical flooding under these conditions. Partially hydrolyzed polyacrylamides (HPAM) have been successfully used in the field for decades, and have been shown to be stable at moderately high temperatures under some conditions (Levitt and Pope, 2008). But, in general, they hydrolyze at high temperatures and eventually precipitate in the presence of high concentrations of divalent cations. Therefore, polymers with modified molecular structures have been developed to enhance their stability at high temperature and high salinity/hardness. Scleroglucan (biopolymer) and the new N-vinylpyrrolidone (NVP)-polyacrylamide (AM) co-polymer showed promising results in the laboratory. The molecular structures of scleroglucan and NVPAM copolymers are shown in Figures 5.1 and 5.2, respectively. The rigid, rod-like, triple helical structure of scleroglucan imparts exceptional stability and its non-ionic functionality makes it insensitive to salinity and hardness. NVP groups protect against hydrolysis by sterically hindering the amide groups. This chapter mainly focuses on the rheology and transport behavior of these three types of polymers under a wide range of conditions. 


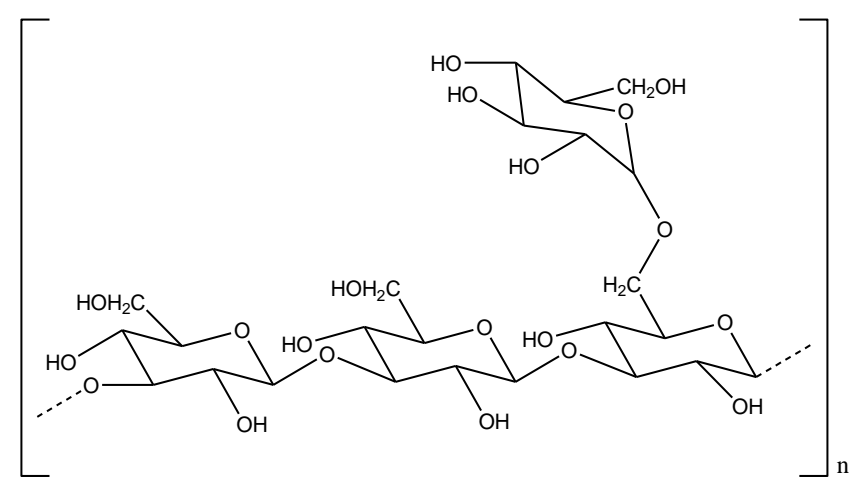

Figure 5.1 Scleroglucan repeating units.

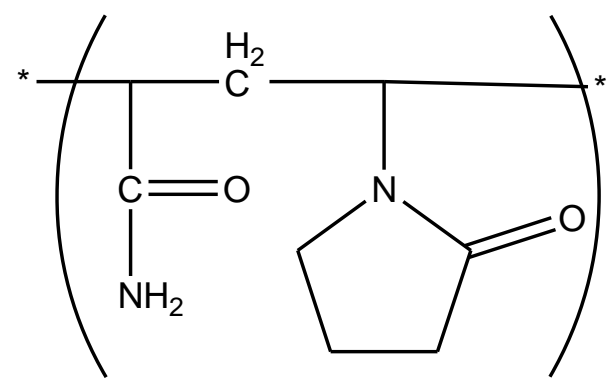

Figure 5.2 Molecular structure of NVP-AM co-polymers.

\subsection{Materials ANd EXPerimental Methods}

\subsubsection{Materials}

FP 3630S and H-EOR63020 polymers are manufactured by SNF Floeger and Beijing Hengju respectively, and supplied as powders. The scleroglucan (Actigum CS 11) was manufactured by Cargill Inc. and was supplied as a powder. The NVP terpolymers (Superpusher SAV 301, Superpusher SAV 522) and NVP co-polymer (Superpusher SAV 505) were supplied by SNF Floeger as powders. 


\subsubsection{Preparation of Polymer Samples.}

Stock solutions were prepared with both HPAM polymers to make a 5,000 ppm polymer solution in softened reservoir brine (5,425 ppm TDS). The standard polymer hydration protocol was used to prepare the stock solutions. Degassed synthetic soft brine (497.5 g) was placed in a shallow polycarbonate bottle and was equipped with a 3 inch long magnetic stir bar. A vortex was created by stirring the brine solution at 360-400 rpm, and polymer powder (2.5 g) was added to the shoulder of the vortex to swiftly and efficiently disperse the polymer in the matrix. The solution was covered by an argon blanket, sealed and mixed for 48-72 hrs. After thorough hydration, the polymer solution becomes clear and homogenous (no visible micro-gels). Dilute polymer solutions were prepared by mixing the filtered stock solution with various synthetic brines. The ionic compositions of soft Brine -1S and hard Brine -1H used in HPAM experiments are given in Table 5.1.

A concentrated solution (5000 ppm) of scleroglucan was prepared as follows. The polymer powder $(2.5 \mathrm{~g})$ was dispersed in DI water $(200 \mathrm{~g})$ in a Waring ${ }^{\circledR}$ blender at low speed for 90 seconds. The rest of deionized water (297.5 g) was added to the blender and mixed for another 210 seconds. The polymer solution was transferred to a glass jar and thermostated at $60{ }^{\circ} \mathrm{C}$ for 30 minutes with occasional stirring. The test polymer solutions were prepared from this stock solution by dilution with synthetic sea water. Table 5.1 shows the composition of synthetic seawater.

The NVP co-polymer solutions were prepared using similar protocol as the HPAM polymers. Mixing time was at least 48 hours at room temperature to ensure complete hydration of the polymer. Dilute polymer solutions were prepared by mixing the stock solution with synthetic seawater. 
Table 5.1 Composition of Synthetic Brines

\begin{tabular}{|c|c|c|c|}
\hline \multirow{2}{*}{$\begin{array}{c}\text { Ionic } \\
\text { Component }\end{array}$} & Brine -1S & Brine $-1 \mathrm{H}$ & Synthetic Seawater \\
\cline { 2 - 4 } & 2,045 & 1,558 & 18,300 \\
\hline $\mathrm{Na}^{+}$ & 0 & 227 & 650 \\
\hline $\mathrm{Ca}^{2+}$ & 0 & 115 & 2,110 \\
\hline $\mathrm{Mg}^{2+}$ & 2,680 & 2,680 & 32,200 \\
\hline $\mathrm{Cl}^{-}$ & 447 & 443 & 4,290 \\
\hline $\mathrm{SO}_{4}^{2-}$ & 253 & 253 & 0 \\
\hline $\mathrm{HCO}_{3}{ }^{-}$ & 5,425 & 5,267 & 57,670 \\
\hline $\mathrm{TDS}$ & & & \\
\hline
\end{tabular}

\subsubsection{Viscosity Measurements.}

The viscosities of polymer solutions were measured using an Ares LS-1 straincontrolled rheometer from TA Instruments ${ }^{\circledR}$. The double-wall couette geometry was a preferred option to measure polymer viscosities due to its higher accuracy at low viscosity range. The range of shear rates of the instrument was 0.1 to $800 \mathrm{~s}^{-1}$. The temperature of the instrument was controlled by an external water bath/circulator.

\subsubsection{Thermal Stability Measurements.}

Thermal stability experiments were carried out under strict argon environment, and the dissolved oxygen concentration was kept below $40 \mathrm{ppb}$ level at all times. In order to remove dissolved oxygen, the polymer solutions were degassed by bubbling argon for at least one hour. All brines and additives were also deoxygenated before diluting to prepare samples with target polymer concentration and salinity. The polymer concentration in hard Brine-1H was kept constant at 2,000 ppm and in soft Brine-1S at $2,500 \mathrm{ppm}$. The soft brine case was evaluated for both with and without $10,000 \mathrm{ppm}$ sodium carbonate as the alkali source. After mixing, the polymer solutions were held in 
thick walled ampules and was subjected to several cycles of vacuum/argon purging to remove any trace amounts of oxygen introduced in latter stages. The dissolved oxygen concentration in the final samples were undetectable $(<10 \mathrm{ppb})$. The ampules were then sealed by a methane-oxygen torch, while being cautious not to heat the polymer solution. Then, the samples were placed in a $62{ }^{\circ} \mathrm{C}$ oven and aged for 1, 2, 7 and 15 days. After samples were removed from the oven on the due dates, they were allowed to cool down to room temperature. They were cracked open under argon environment and the dissolved oxygen concentration was immediately measured. If the dissolved oxygen is higher than $40 \mathrm{ppb}$, the samples were discarded. The viscosity was measured under argon blanket. This method was described more completely by Levitt and Pope (2008).

\subsubsection{Polymer Floods.}

Berea sandstone cores with an initial permeability of 150 to $300 \mathrm{mD}$ were used for scleroglucan corefloods. Estillades limestone of $150 \mathrm{mD}$ was used for SAV 301polymer floods. Bentheimer sandstone cores with 1500 to $1750 \mathrm{mD}$ permeability range were used for FP 3630 floods.

All corefloods reported here were in vertical orientation with the injection from the bottom and the production at the top. High temperature $\left(>85^{\circ} \mathrm{C}\right)$ corefloods were done using stainless steel core holders under confining pressure (typically 1000 psi using mineral oil). Low temperature corefloods were done in epoxy molded cores. The injection flow rates were controlled by Teledyne Isco 500D syringe pumps, which were mainly operated in constant delivery rate mode. Three taps along the length of the core holder were used to measure pressure drop in different sections of the core using Rosemount ${ }^{\circledR}$ differential pressure transducers. 
Brine was injected before the polymer floods to determine the initial brine permeability of the core. Typically, a salinity tracer test was done to determine the pore volume of the cores held in stainless steel core holders and to check for heterogeneities. Pore volumes of epoxy molded cores were measured by mass balance. Injection polymer slugs were filtered and deoxygenated by bubbling argon for a minimum of one hour. The effluent produced at the outlet of the core was collected in graduated test tubes using an automatic fractional collector. Effluent samples were collected under argon blanket and analyzed for viscosity, $\mathrm{pH}$, polymer concentration etc.

\subsection{RESULTS AND DisCUSSION}

In this section, results are presented for filterability, rheological properties, thermal stability and transport behavior of HPAM, scleroglucan and SAV 301 polymers. Corefloods were done in order to understand the transport behavior of scleroglucan and NVP co-polymers as well as the HPAM polymers.

\subsubsection{Filterability and Filterability Improvement Methods.}

Both FP 3630S and H-EOR 63020 stock solutions showed good filterability through 5 micron Nitrocellulose membrane under 15 psi argon pressure drop across the filter. Further, these two polymers were diluted with synthetic hard brine and soft brines with respective polymer concentrations of 2,000 ppm and 2,500 ppm. The soft brine case contained additional $10,000 \mathrm{ppm}$ of sodium carbonate. The filtration ratios for all four cases were between 1 and 1.2, and none of them indicated any viscosity loss after filtration. The filtration results for HPAM polymers are summarized in Table 5.2. 
Table 5.2 Summary of HPAM Filtration

\begin{tabular}{|l|c|c|c|c|}
\hline \multirow{2}{*}{ Sample } & \multirow{2}{*}{ F.R } & \multicolumn{2}{|c|}{ Viscosity at $11 \mathrm{~s}^{-1}$ at RT (cP) } & Retained \\
\cline { 3 - 5 } & Before Filtration & After Filtration & viscosity \\
\hline $\begin{array}{l}2,000 \text { ppm FP3630S in } \\
\text { hard brine }\end{array}$ & 1.02 & 41.9 & 41.5 & 1.0 \\
\hline $\begin{array}{l}2,500 \text { ppm FP3630S in } \\
\text { soft brine + 10,000 ppm } \\
\text { sodium carbonate }\end{array}$ & 1.16 & 66.3 & 66.1 & 1.0 \\
\hline $\begin{array}{l}\text { 2000 ppm H-EOR63020 } \\
\text { in hard brine }\end{array}$ & 1.01 & 42.0 & 41.7 & 1.0 \\
\hline
\end{tabular}

The filterability of the scleroglucan biopolymer used in this study was poor because of cell debris, minute aggregates of incompletely dissolved polymers, and residual proteinaceous materials remaining from the fermentation process (Carter et. al., 1980). Many methods have been investigated and studied in this regard, such as filtering using Millipore filters (Kalpacki et. al., 1990; Fletcher et. al., 1991), filtering through diatomaceous earth (Yost et. al.,1975), mixing with surfactants (Pirri et. al., 1993), and enzyme treatment (Frohnwieser et. al., 2009). Each of these methods has their own limitations, such as the need to change the filters frequently and the high cost of processing with surfactants or enzymes. Improvement of filterability using a cost effective method was a main objective in this study.

Two filtration methods were developed to improve the filterability of scleroglucan. Filtration at a high pressure gradient is a known method for clarification of polysaccharides (Kohler and Chauveteau, 1981). By filtering the polymer solution through increasing smaller pores the larger particles are removed at the beginning stages filteration (Sparlin and Guidry, 1980; Davison and Mentzor, 1982). This method consists of four consecutive high pressure (50 psi) filtrations of the stock solution through 
Millipore filters of decreasing pore size: (i) 5-10 $\mu \mathrm{m}$, (ii) $5 \mu \mathrm{m}$, (iii) $3 \mu \mathrm{m}$, and (iv) 1.2 $\mu \mathrm{m}$. The polymer solutions were filtered in $250 \mathrm{~mL}$ batches. Figure 5.3 shows the filtration index for $5000 \mathrm{ppm}$ scleroglucan in deionized water after four high pressure filtrations. The final solution passed with a filtration ratio of unity in the laboratory standard filtration test (1.2 micron filter under 15 psi argon pressure drop).

In the second method, the filterability was improved by heating the scleroglucan solution to $85 \mathrm{C}$ and subsequently filtering it through 1.2 micron filter at higher (50 psi) pressure. Scleroglucan solution containing $1250 \mathrm{ppm}$ polymer in synthetic sea water was prepared by first deoxygenated by bubbling argon, and subsequently heating to $85^{\circ} \mathrm{C}$ for 4 hours with occasional stirring. Then, it was filtered directly through $1.2 \mu \mathrm{m}$ under 50 psi argon pressure at $85{ }^{\circ} \mathrm{C}$. The filtered scleroglucan solution was then subjected to the standard filtration test with a filtration ratio of unity. Later, the nitrocellulose filters were replaced by short Berea sandstone cores (1 inch in diameter and 7.5 inches in length). The core method is more desirable because it can be used at high temperature. Also, it's less expensive than multiple filtrations using membranes. Table 5.3 and 5.4 show the filterability improvement of $1250 \mathrm{ppm}$ scleroglucan in synthetic seawater by filter paper and the cores respectively. At higher temperature, the interactions between solventpolymer and polymer-polymer decreases, and the aggregation of polymer molecules decreases. Subsequently, the filterability of scleroglucan is improved (Rivenq et. al., 1992). 


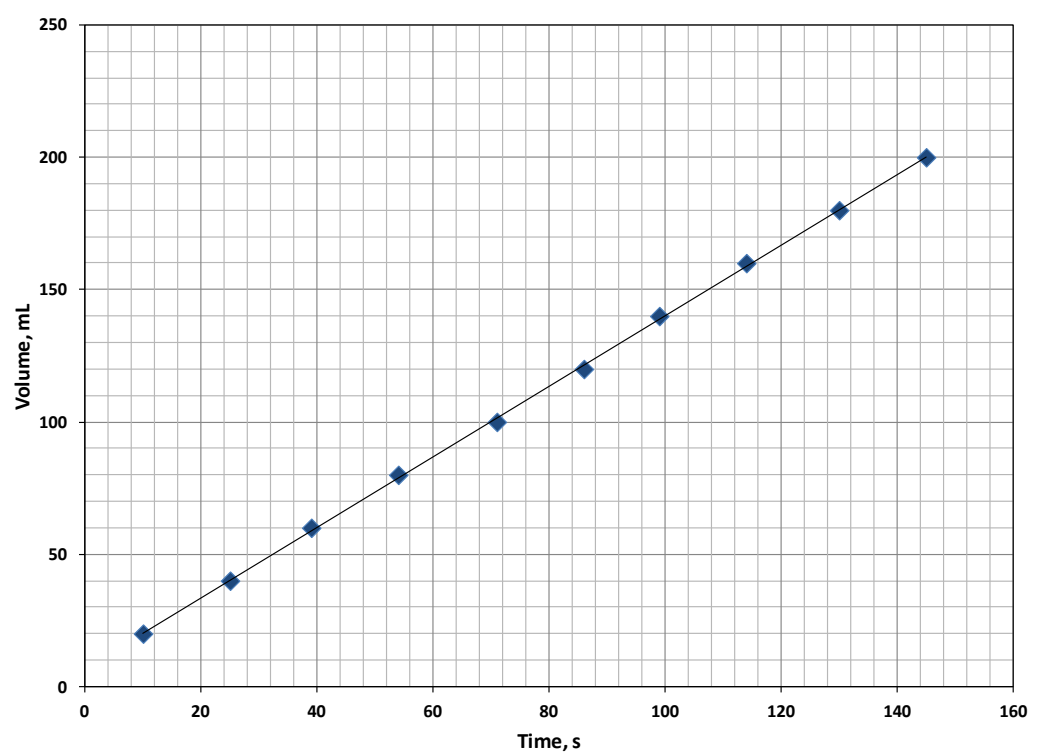

Figure 5.3 Filtration of 5000 ppm scleroglucan in deionized water after four consecutive high-pressure filtrations. Filtration ratio $=1$ with 1.2 micron filter and 15 psi pressure gradient.

Table 5.3 Filterability Improvement of $1250 \mathrm{ppm}$ Scleroglucan in synthetic seawater by heat treatment

\begin{tabular}{|c|c|c|c|}
\hline & Filtration Step & FR & Temperature $\left({ }^{\circ} \mathrm{C}\right)$ \\
\hline 1 & $1.2 \mu \mathrm{m}, 15 \mathrm{psi}$ & Plugging & 25 \\
\hline 2 & $1.2 \mu \mathrm{m}, 15 \mathrm{psi}$ & 4.33 & 85 \\
\hline 3 & (i) $3 \mu \mathrm{m}, 15 \mathrm{psi}$; (ii) $1.2 \mu \mathrm{m}, 15 \mathrm{psi}$ & 1.00 & 85 \\
\hline 4 & (i) $1.2 \mu \mathrm{m}, 50 \mathrm{psi}$; (ii) $1.2 \mu \mathrm{m}, 15 \mathrm{psi}$ & 1.00 & 85 \\
\hline
\end{tabular}


Table 5.4 Scleroglucan Filterability Improvement after Heat Treatment and Passing Through the Core

\begin{tabular}{|c|c|c|}
\hline Filtration ratio after passing through the core & Flood No. 3 & Flood No 4 \\
\hline $\begin{array}{c}\text { Viscosity of Initial polymer before heat treatment (cP) } \\
\text { the core) (cP) }\end{array}$ & 73 & 46.2 \\
\hline $\begin{array}{c}\text { Viscosity of heat treated polymer (Before sending through } \\
\text { Viscosity of Effluent (cP) }\end{array}$ & 63 & 46.2 \\
\hline $\begin{array}{c}\text { (c) After Filtration through } 1.2 \mu \mathrm{m}, 15 \\
\text { psi at } 85^{\circ} \mathrm{C}(\mathrm{cP})\end{array}$ & 59 & 46.2 \\
\hline $\begin{array}{c}\text { Viscosity of effluent } \\
\text { Viscosity loss during filtration }\end{array}$ & 19 & 2.8 \\
\hline
\end{tabular}

Of the two filtration methods described above, the second method, where the polymer was heated to $85^{\circ} \mathrm{C}$ and filtered through a 1.2 micron filter paper under 50 psi argon, is considered less expensive for filtering larger volumes of polymer solution. The filtered polymer solutions from both methods passed the standard and more rigorous filtration test through 1.2 micron filter paper at 15 psi and room temperature.

Scleroglucan showed improved filterability after subjected to a purification method developed in the lab. NVP co-polymers showed good filterability with filtration ratios less than 1.2 given the hydration is carefully achieved.

\subsubsection{Viscosity measurements.}

The viscosities of two HPAM polymer solutions were measured at different concentrations in both hard and soft Brine-1S with and without alkali. The results are summarized in Tables 5.5 through 5.8. These measurements were done at $62{ }^{\circ} \mathrm{C}$. The viscosity vs. shear rate data are given in Figures 5.4 through 5.11. 
The scleroglucan polymer was used in rheological measurements only after passing the standard filtration test. Target polymer solutions were prepared by diluting with synthetic seawater to a desired concentration and salinity. Figure 5.12 shows the relative viscosities (polymer viscosity divided by brine viscosity) of scleroglucan at different salinities as a function of shear rate at $90{ }^{\circ} \mathrm{C}$. Figure 5.13 shows the relative viscosity as a function of salinity at $90{ }^{\circ} \mathrm{C}$ for scleroglucan compared to NVP and AM copolymers. This clearly shows the insensitivity of scleroglucan to salinity because of its non-ionic nature (Rivenq et al., 1992; Kalpacki et al., 1990). On the contrary, NVP and AM co-polymers show decreasing viscosity with increased salinity. Figure 5.14 shows the viscosity of SAV 301, FP 3330S, FP 3630S and scleroglucan (CS-11) vs. concentration, which clearly shows the scleroglucan's superiority in its viscosifying power. SAV 301 has the lowest viscosity at a given concentration due to its low molecular weight. However, NVP-AM copolymers with low molecular weight may have the advantage of better transport in rocks with relatively low permeability because of their smaller molecular size as well as the advantage of better hydrolytic stability.

In Figure 5.15, the relative viscosity of $1250 \mathrm{ppm}$ scleroglucan in seawater is compared to the viscosities of 3000 ppm 8 M HPAM, SAV 301 and SAV 505 at temperatures ranging from 25 to $90{ }^{\circ} \mathrm{C}$. The relative viscosity of scleroglucan actually increases with temperature whereas the relative viscosity of the synthetic polymers decreases with temperature. The viscosifying power of the scleroglucan remains high because of its triple-helix molecular conformation (Rivenq et al., 1992; Kalpacki et al., 1990). Increasing the temperature of the solution leads to a reduction of the solvent viscosity and hence the mobility of the polymer chains is increased, but the interaction of the AM and NVP on the polymer chains decreases. 
Table 5.5 Viscosity of FP $3630 \mathrm{~S}$ in Soft Brine-1S at $62{ }^{\circ} \mathrm{C}$

\begin{tabular}{|c|c|c|c|c|c|c|c|c|c|}
\hline \multirow{2}{*}{ Exp\# } & \multirow{2}{*}{$\begin{array}{l}\text { Polymer } \\
\text { Conc. } \\
\text { (ppm) }\end{array}$} & \multirow{2}{*}{$\begin{array}{l}\text { TDS } \\
(\mathrm{ppm})\end{array}$} & \multirow{2}{*}{$\begin{array}{l}\text { Sodium } \\
\text { carbonate } \\
\text { conc. } \\
(\mathrm{ppm})\end{array}$} & \multicolumn{6}{|c|}{ Viscosity at shear rate $(\mathrm{cP})$} \\
\hline & & & & $\begin{array}{c}1 \\
\left(\mathrm{~s}^{-1}\right) \\
\end{array}$ & $\begin{array}{l}4.64 \\
\left(\mathrm{~s}^{-1}\right)\end{array}$ & $\begin{array}{c}10 \\
\left(\mathrm{~s}^{-1}\right)\end{array}$ & $\begin{array}{l}21.5 \\
\left(\mathrm{~s}^{-1}\right)\end{array}$ & $\begin{array}{l}46.4 \\
\left(\mathrm{~s}^{-1}\right)\end{array}$ & $\begin{array}{l}100 \\
\left(\mathrm{~s}^{-1}\right)\end{array}$ \\
\hline FP-1 & 1500 & 5425 & - & 58.8 & 34.4 & 24.5 & 17.2 & 12.0 & 8.7 \\
\hline FP-2 & 2000 & 5425 & - & 126.3 & 61.5 & 40.8 & 27.1 & 18.3 & 13.0 \\
\hline FP-3 & 2500 & 5425 & - & 217.5 & 94.5 & 60.1 & 38.5 & 25.5 & 17.5 \\
\hline FP-4 & 1500 & 15425 & 10000 & 43.1 & 32.4 & 25.7 & 19.8 & 14.8 & 11.1 \\
\hline FP-5 & 2000 & 15425 & 10000 & 89.3 & 58.0 & 43.2 & 31.2 & 22.1 & 15.8 \\
\hline FP-6 & 2500 & 15425 & 10000 & 166.9 & 95.8 & 67.1 & 46.0 & 31.3 & 22.0 \\
\hline FP-7 & 1500 & 35425 & 30000 & 27.4 & 23.3 & 19.7 & 16.0 & 12.6 & 9.7 \\
\hline FP-8 & 2000 & 35425 & 30000 & 68.3 & 47.0 & 36.1 & 26.9 & 19.7 & 14.6 \\
\hline FP-9 & 2500 & 35425 & 30000 & 120.4 & 74.6 & 54.3 & 38.6 & 27.1 & 19.6 \\
\hline
\end{tabular}

Table 5.6 Viscosity of FP $3630 \mathrm{~S}$ in Hard Brine- $1 \mathrm{H}$ at $62{ }^{\circ} \mathrm{C}$

\begin{tabular}{|c|c|c|c|c|c|c|c|c|c|}
\hline \multirow{2}{*}{ Exp\# } & \multirow{2}{*}{$\begin{array}{l}\text { Polymer } \\
\text { Conc. } \\
\text { (ppm) }\end{array}$} & \multirow{2}{*}{$\begin{array}{c}\text { TDS } \\
(\mathrm{ppm})\end{array}$} & \multirow{2}{*}{$\begin{array}{c}\text { Sodium } \\
\text { carbonate } \\
\text { conc.(ppm) }\end{array}$} & \multicolumn{6}{|c|}{ Viscosity at shear rate $(\mathrm{cP})$} \\
\hline & & & & $\begin{array}{c}1 \\
\left(\mathrm{~s}^{-1}\right)\end{array}$ & $\begin{array}{l}4.64 \\
\left(\mathrm{~s}^{-1}\right)\end{array}$ & $\begin{array}{c}10 \\
\left(\mathrm{~s}^{-1}\right)\end{array}$ & $\begin{array}{l}21.5 \\
\left(\mathrm{~s}^{-1}\right)\end{array}$ & $\begin{array}{l}46.4 \\
\left(\mathrm{~s}^{-1}\right)\end{array}$ & $\begin{array}{l}100 \\
\left(\mathrm{~s}^{-1}\right)\end{array}$ \\
\hline FP-10 & 1500 & 5267 & - & 8.1 & 7.1 & 6.2 & 5.3 & 4.3 & 3.5 \\
\hline FP-11 & 2000 & 5267 & - & 56.3 & 33.6 & 24.3 & 17.3 & 12.3 & 9.1 \\
\hline
\end{tabular}


Table 5.7 Viscosity of H-EOR 63020 in Soft Brine-1S at $62{ }^{\circ} \mathrm{C}$

\begin{tabular}{|l|c|c|c|c|c|c|c|c|c|}
\hline & \multirow{2}{*}{ Exp\# } & $\begin{array}{c}\text { Polymer } \\
\text { Conc. } \\
(\mathrm{ppm})\end{array}$ & \multirow{2}{*}{$\begin{array}{c}\text { TDS } \\
(\mathrm{ppm})\end{array}$} & $\begin{array}{c}\text { Sodium } \\
\text { carbonate } \\
\text { conc. } \\
(\mathrm{ppm})\end{array}$ & \multicolumn{6}{|c|}{$\begin{array}{c}1 \\
\left(\mathrm{~s}^{-1}\right)\end{array}$} & $\begin{array}{c}4.64 \\
\left(\mathrm{~s}^{-1}\right)\end{array}$ & $\begin{array}{c}10 \\
\left(\mathrm{~s}^{-1}\right)\end{array}$ & $\begin{array}{c}21.5 \\
\left(\mathrm{~s}^{-1}\right)\end{array}$ & $\begin{array}{c}46.4 \\
\left(\mathrm{~s}^{-1}\right)\end{array}$ & $\begin{array}{c}100 \\
\left(\mathrm{~s}^{-1}\right)\end{array}$ \\
\hline $\mathrm{H}-1$ & 1500 & 5425 & - & 76.5 & 43.2 & 30.7 & 21.5 & 15.0 & 11.0 \\
\hline H-2 & 2000 & 5425 & - & 152.3 & 75.3 & 50.5 & 33.6 & 23.0 & 16.0 \\
\hline H-3 & 2500 & 5425 & - & 262.2 & 116.7 & 75.3 & 49.0 & 32.7 & 21.9 \\
\hline H-4 & 1500 & 15425 & 10000 & 28.9 & 21.6 & 17.2 & 13.3 & 9.9 & 7.5 \\
\hline H-5 & 2000 & 15425 & 10000 & 62.8 & 39.7 & 29.4 & 21.3 & 15.2 & 11.2 \\
\hline H-6 & 2500 & 15425 & 10000 & 112.8 & 63.7 & 44.9 & 31.2 & 21.6 & 15.5 \\
\hline H-7 & 1500 & 35425 & 30000 & 17.9 & 15.1 & 12.8 & 10.4 & 8.2 & 6.4 \\
\hline H-8 & 2000 & 35425 & 30000 & 36.6 & 27.7 & 22.2 & 17.1 & 12.8 & 9.6 \\
\hline H-9 & 2500 & 35425 & 30000 & 68.7 & 45.6 & 34.4 & 25.1 & 18.0 & 13.1 \\
\hline
\end{tabular}

Table 5.8: Viscosity of H-EOR 63020 in Hard Brine-1H at $62{ }^{\circ} \mathrm{C}$

\begin{tabular}{|l|c|c|c|c|c|c|c|c|c|}
\hline \multirow{2}{*}{ Exp\# } & \multirow{2}{*}{$\begin{array}{c}\text { Polymer } \\
\text { Conc. } \\
(\mathrm{ppm})\end{array}$} & \multirow{2}{*}{$\begin{array}{c}\text { TDS } \\
(\mathrm{ppm})\end{array}$} & $\begin{array}{c}\text { Sodium } \\
\text { carbonate } \\
\text { conc. }(\mathrm{ppm})\end{array}$ & \multicolumn{6}{|c|}{ Viscosity at shear rate $(\mathrm{cP})$} \\
\cline { 5 - 10 } & $\begin{array}{c}1 \\
\left(\mathrm{~s}^{-1}\right)\end{array}$ & $\begin{array}{c}4.64 \\
\left(\mathrm{~s}^{-1}\right)\end{array}$ & $\begin{array}{c}10 \\
\left(\mathrm{~s}^{-1}\right)\end{array}$ & $\begin{array}{c}21.5 \\
\left(\mathrm{~s}^{-1}\right)\end{array}$ & $\begin{array}{c}46.4 \\
\left(\mathrm{~s}^{-1}\right)\end{array}$ & $\begin{array}{c}100 \\
\left(\mathrm{~s}^{-1}\right)\end{array}$ \\
\hline $\mathrm{H}-10$ & 1500 & 5267 & - & 6.7 & 6.0 & 5.4 & 4.8 & 4.1 & 3.4 \\
\hline $\mathrm{H}-11$ & 2000 & 5267 & - & 35.1 & 26.0 & 20.7 & 15.9 & 11.8 & 8.7 \\
\hline
\end{tabular}




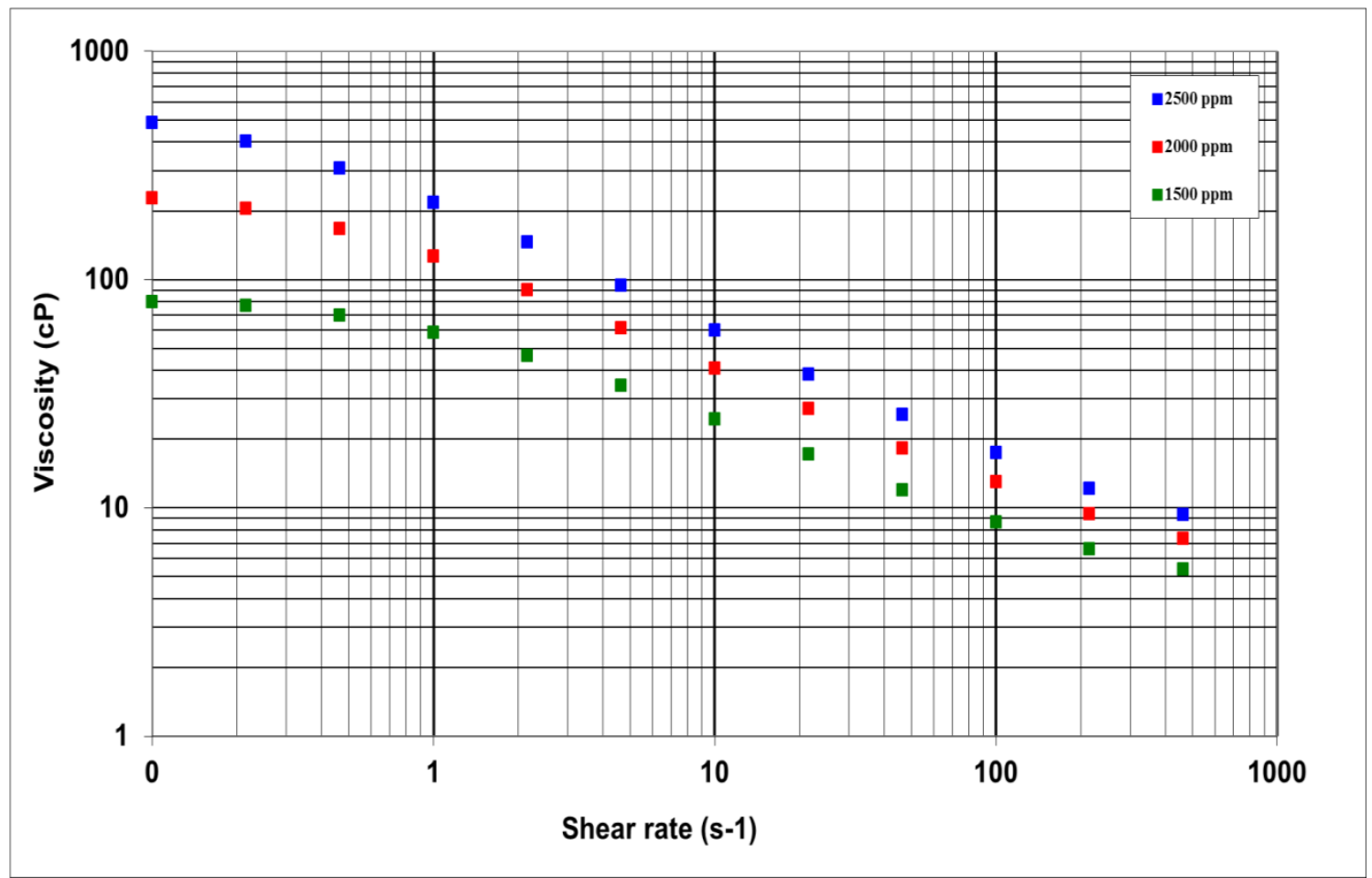

Figure 5.4 Viscosity of FP3630S in Soft Brine-1S at $62{ }^{\circ} \mathrm{C}$

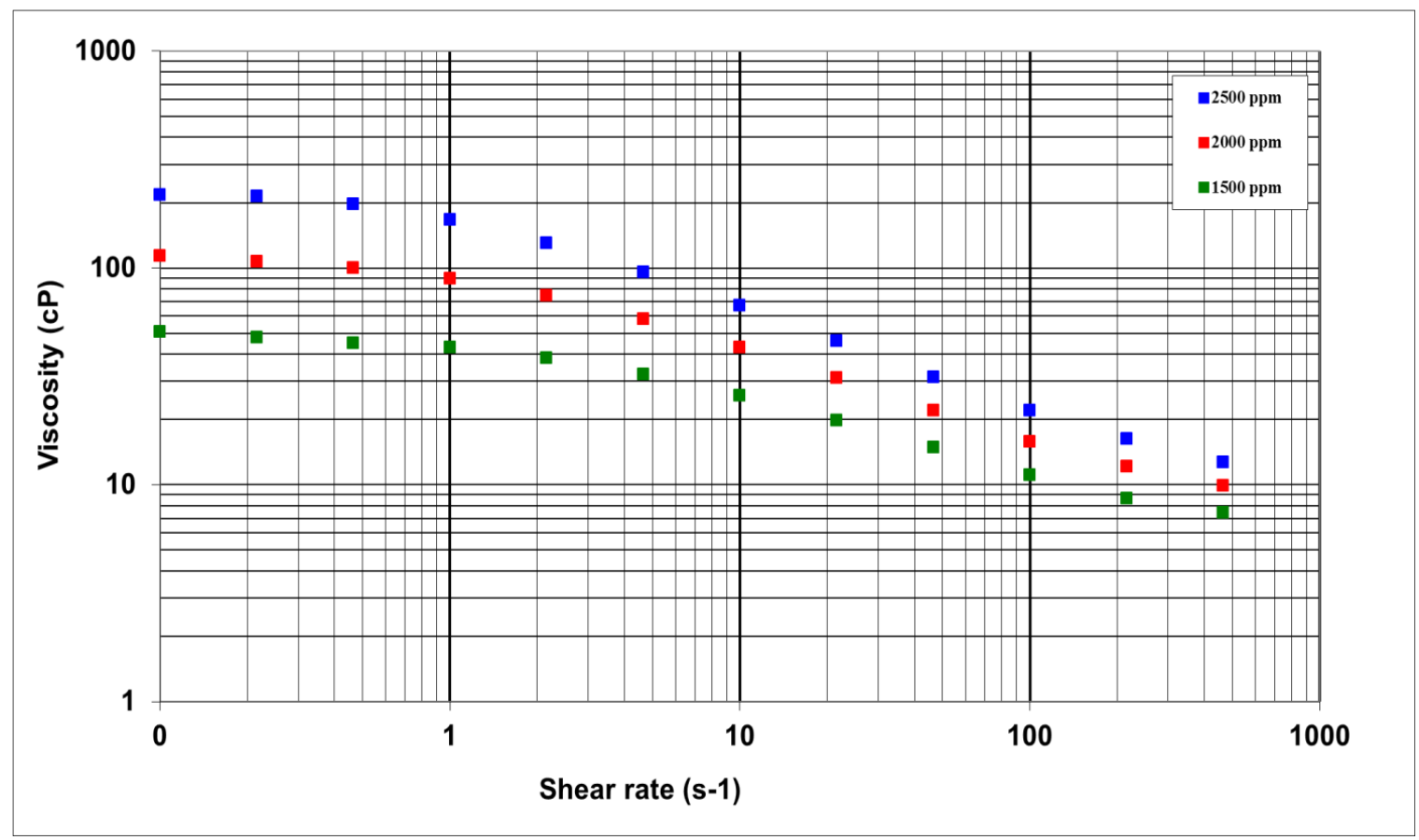

Figure 5.5 Viscosity of FP3630S in Soft Brine-1S + 10,000 ppm Sodium Carbonate at $62^{\circ} \mathrm{C}$ 


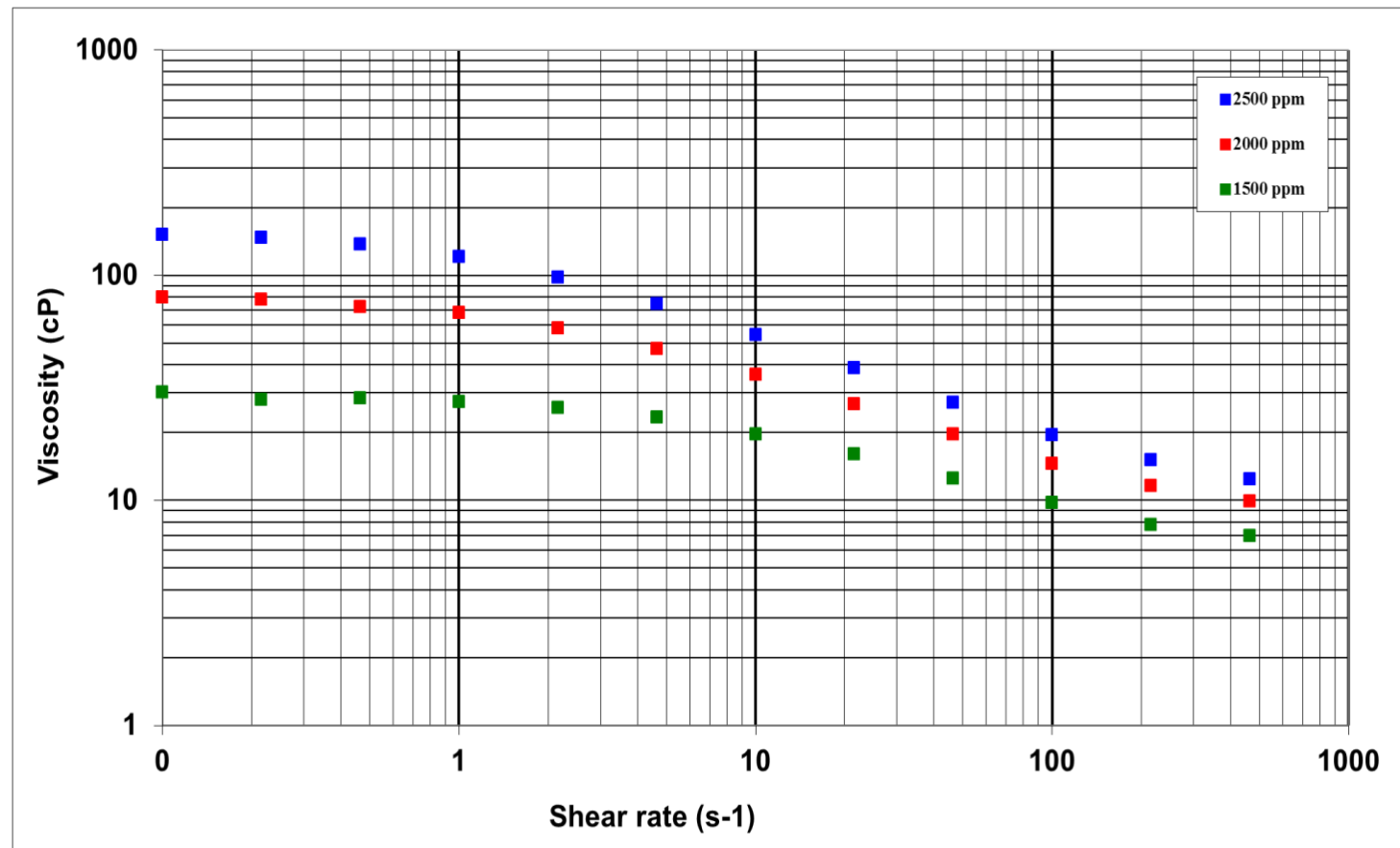

Figure 5.6 Viscosity of FP3630S in Soft Brine-1S + 30,000 ppm Sodium Carbonate at 62 ${ }^{\circ} \mathrm{C}$

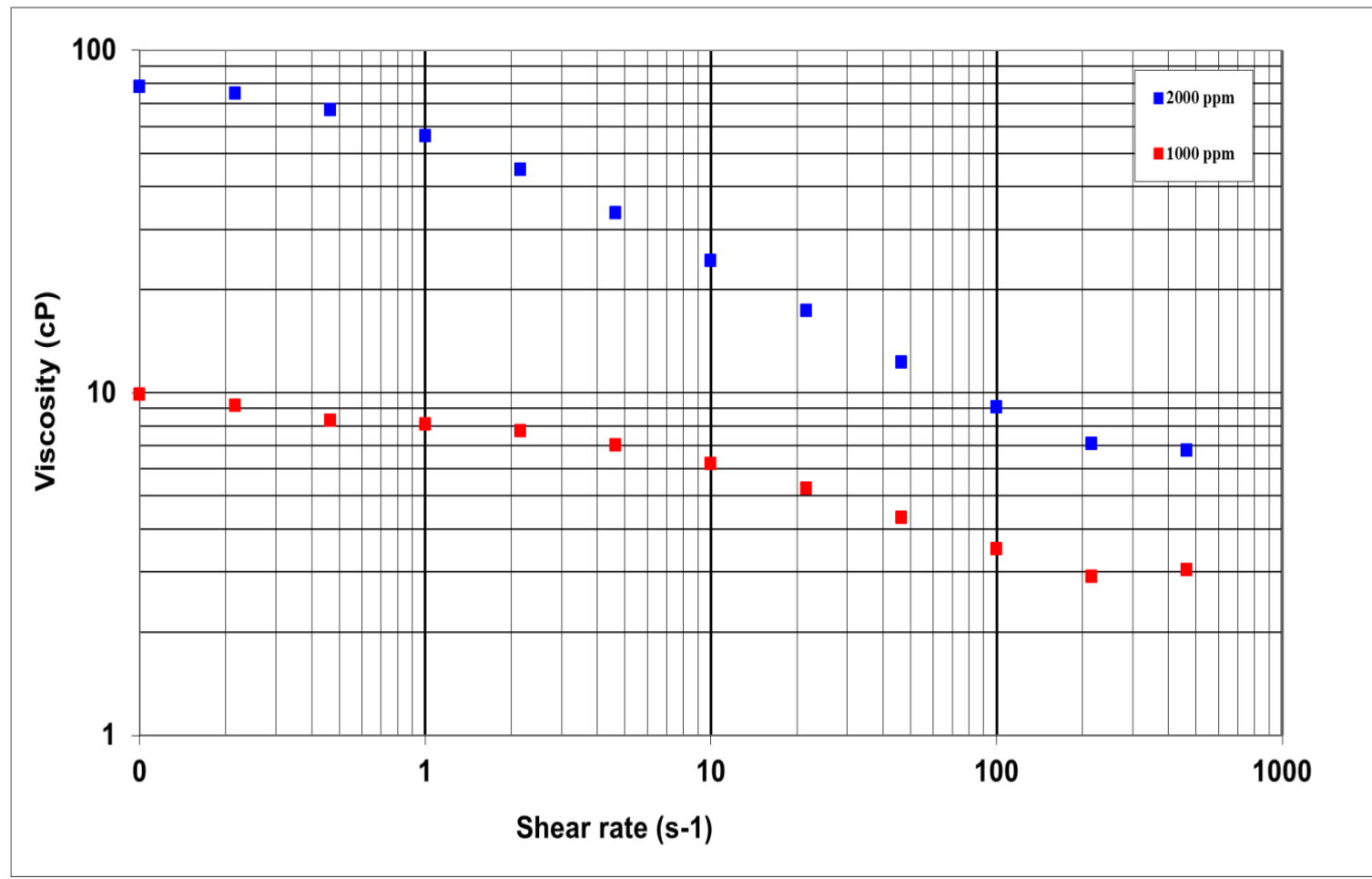

Figure 5.7 Viscosity of FP3630S in Hard Brine- $1 \mathrm{H}$ at $62{ }^{\circ} \mathrm{C}$

100 


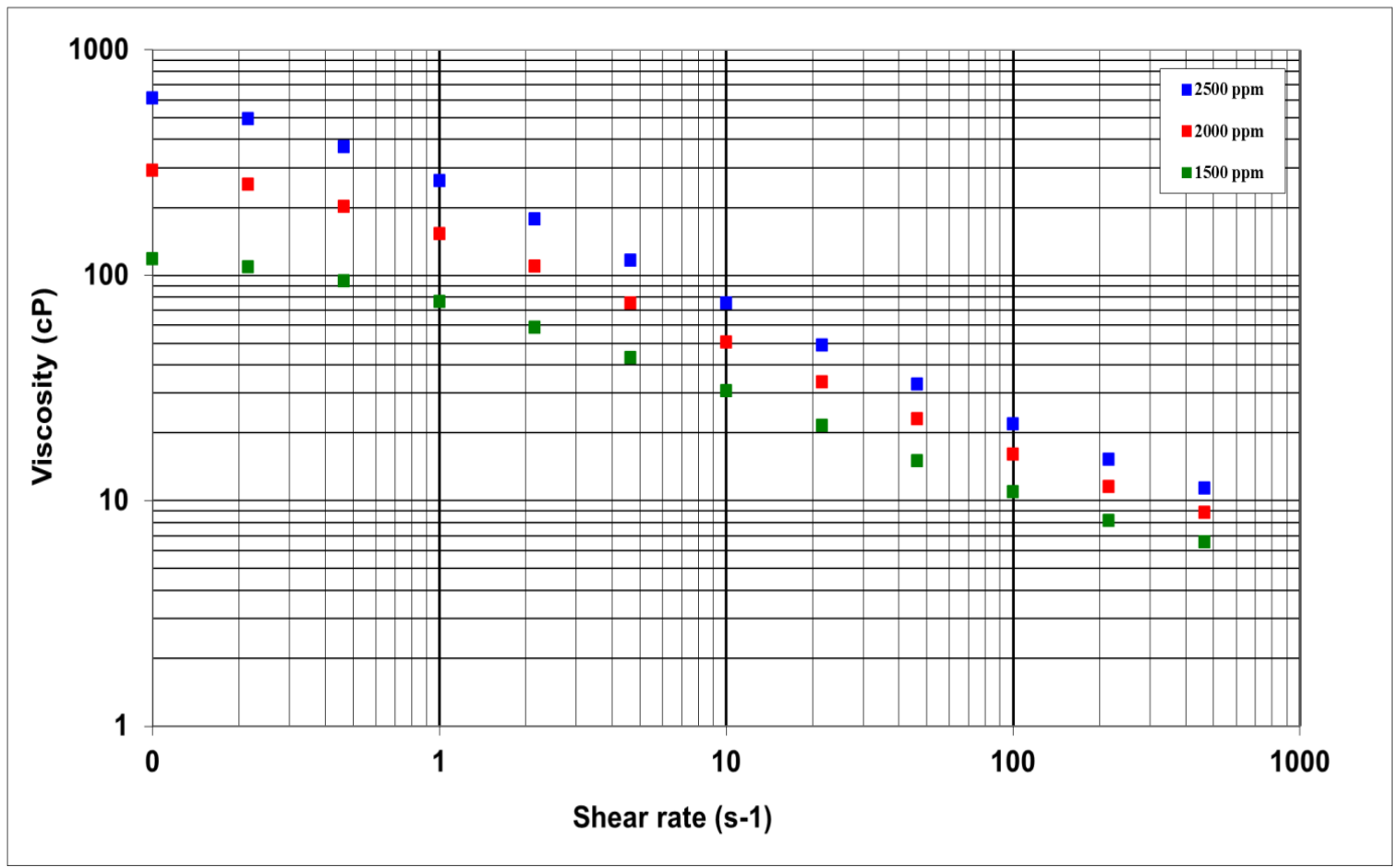

Figure 5.8 Viscosity of H-EOR 63020 in Soft Brine-1S at $62{ }^{\circ} \mathrm{C}$

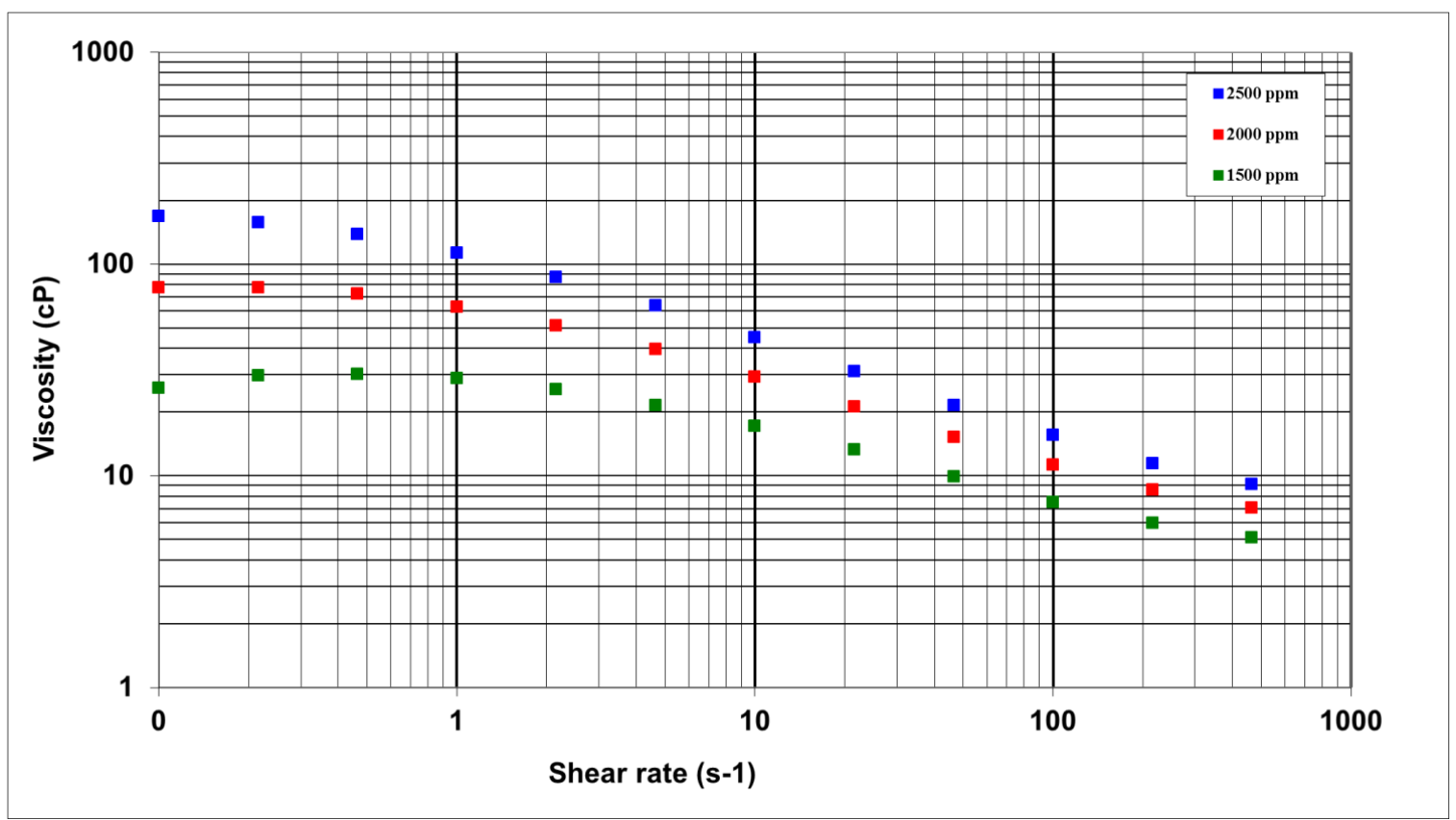

Figure 5.9 Viscosity of H-EOR 63020 in Soft Brine-1S + 10,000 ppm Sodium Carbonate at $62{ }^{\circ} \mathrm{C}$ 


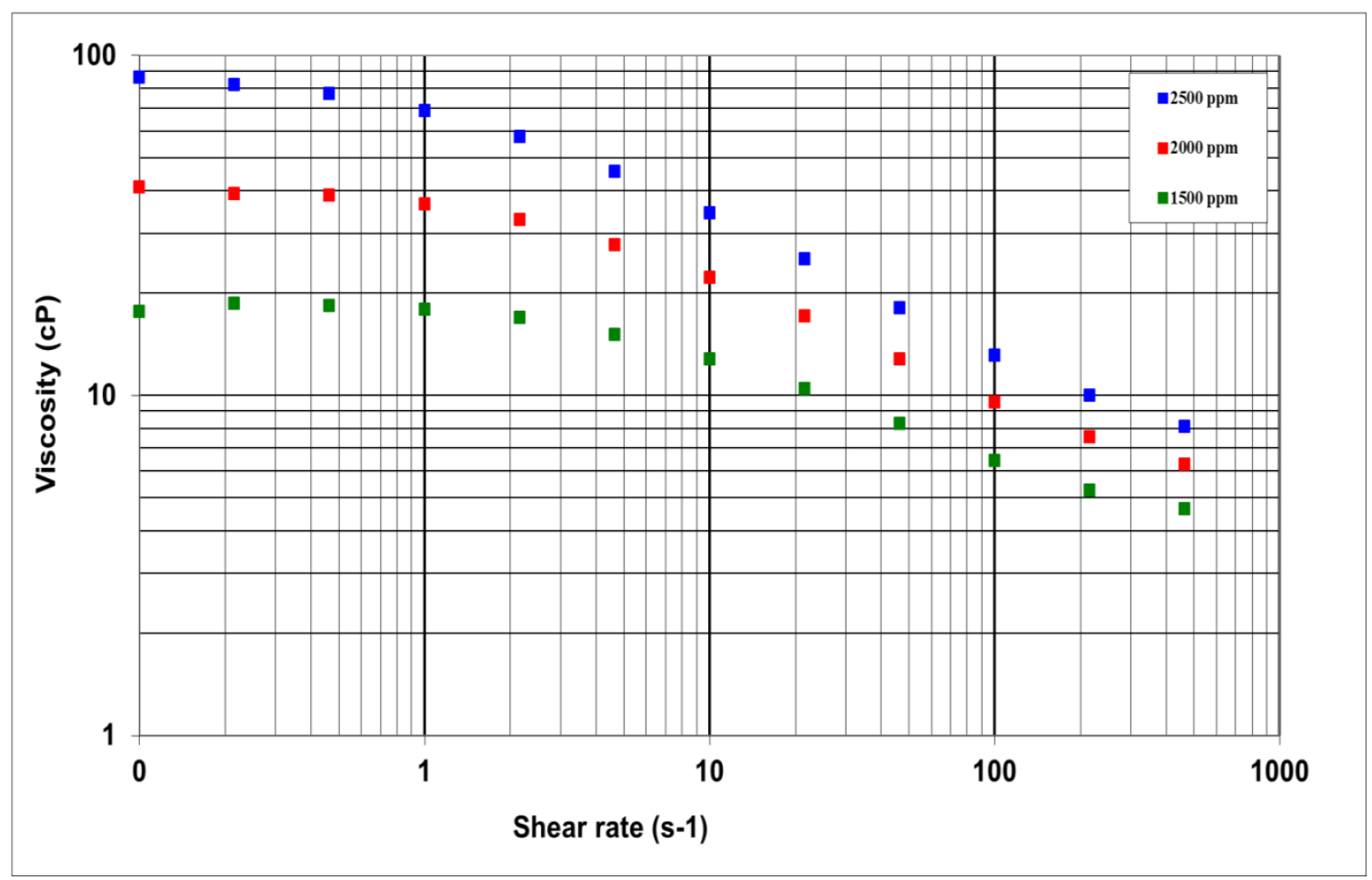

Figure 5.10 Viscosity of H-EOR 63020 in Soft Brine-1S + 30,000 ppm Sodium Carbonate at $62{ }^{\circ} \mathrm{C}$

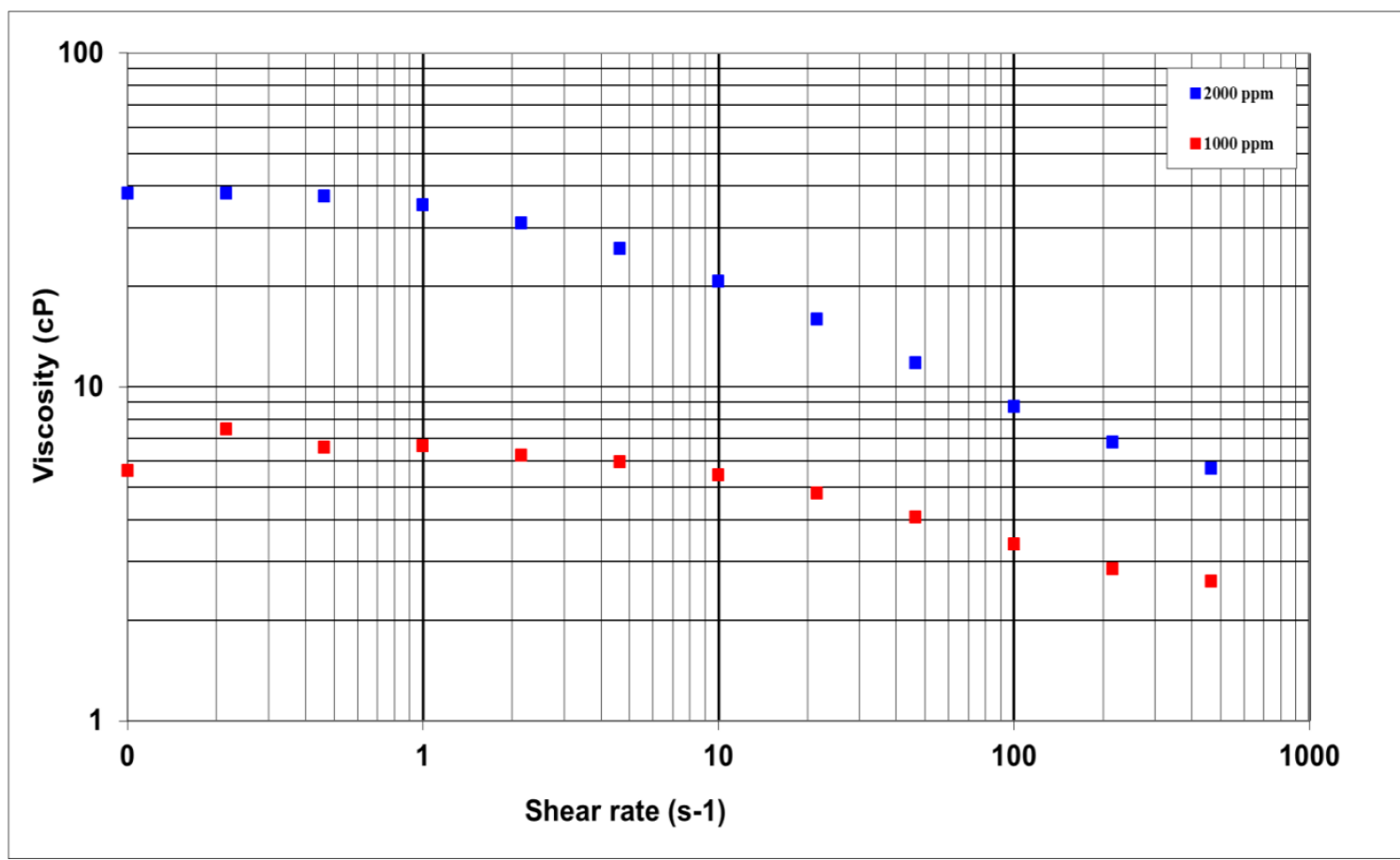

Figure 5.11 Viscosity of H-EOR 63020 in Hard Brine-1H at $62^{\circ} \mathrm{C}$ 


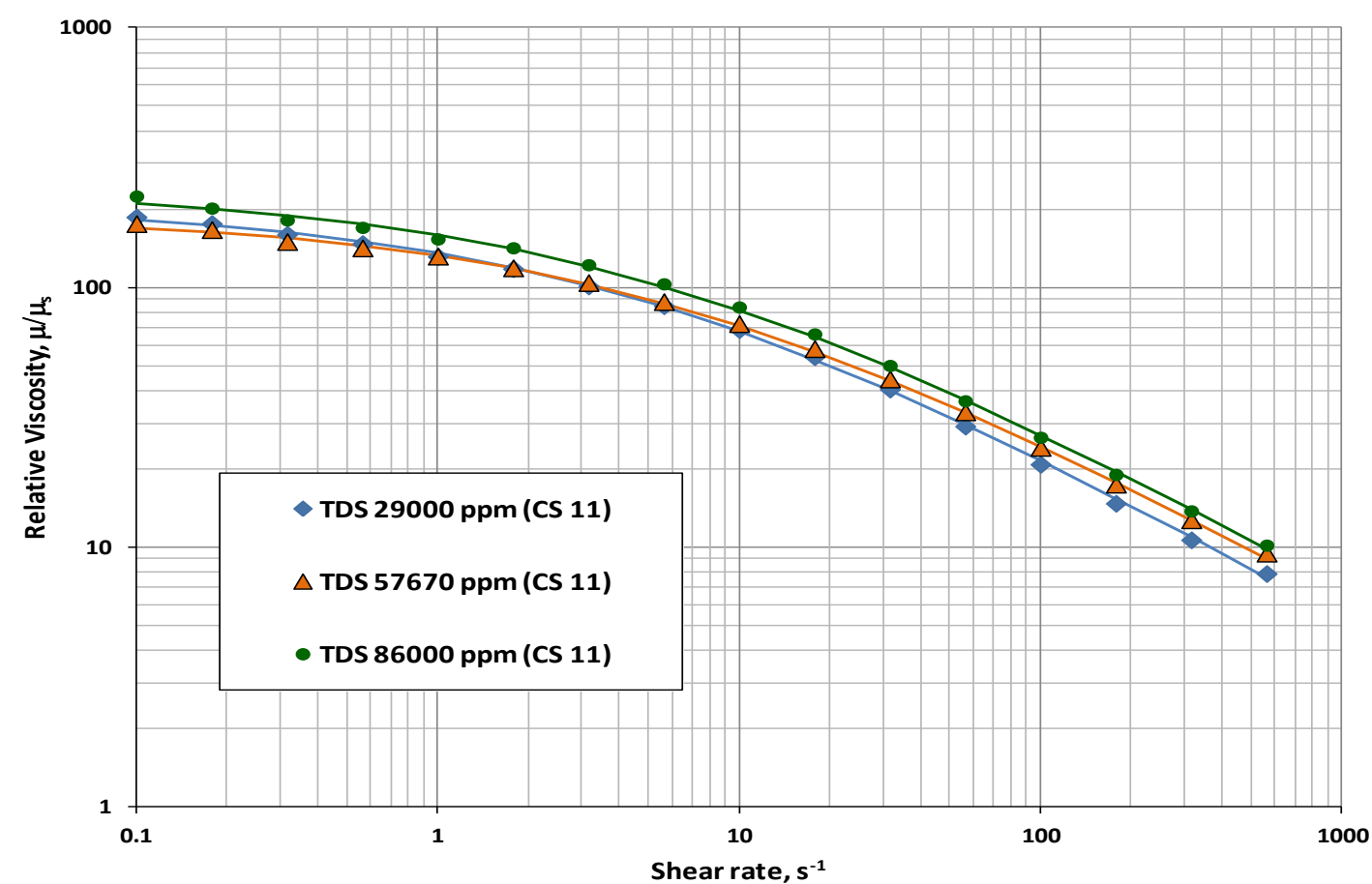

Figure 5.12 Relative viscosity of $1250 \mathrm{ppm}$ scleroglucan at $90{ }^{\circ} \mathrm{C}$

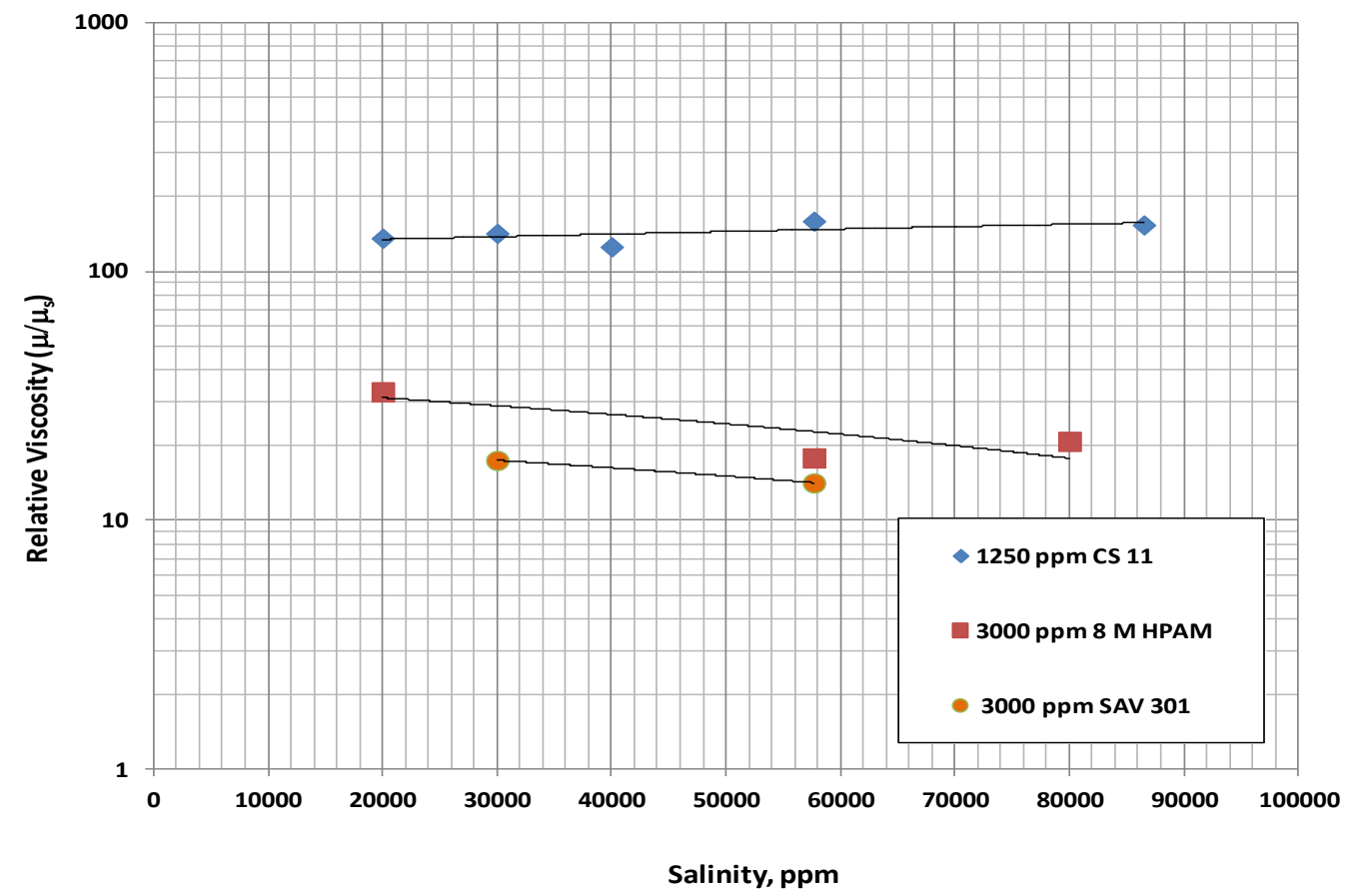

Figure 5.13 Relative viscosity of $1250 \mathrm{ppm}$ scleroglucan and SAV 301 solutions at $90{ }^{\circ} \mathrm{C}$ (shear rate $1 \mathrm{~s}^{-1}$ ). 


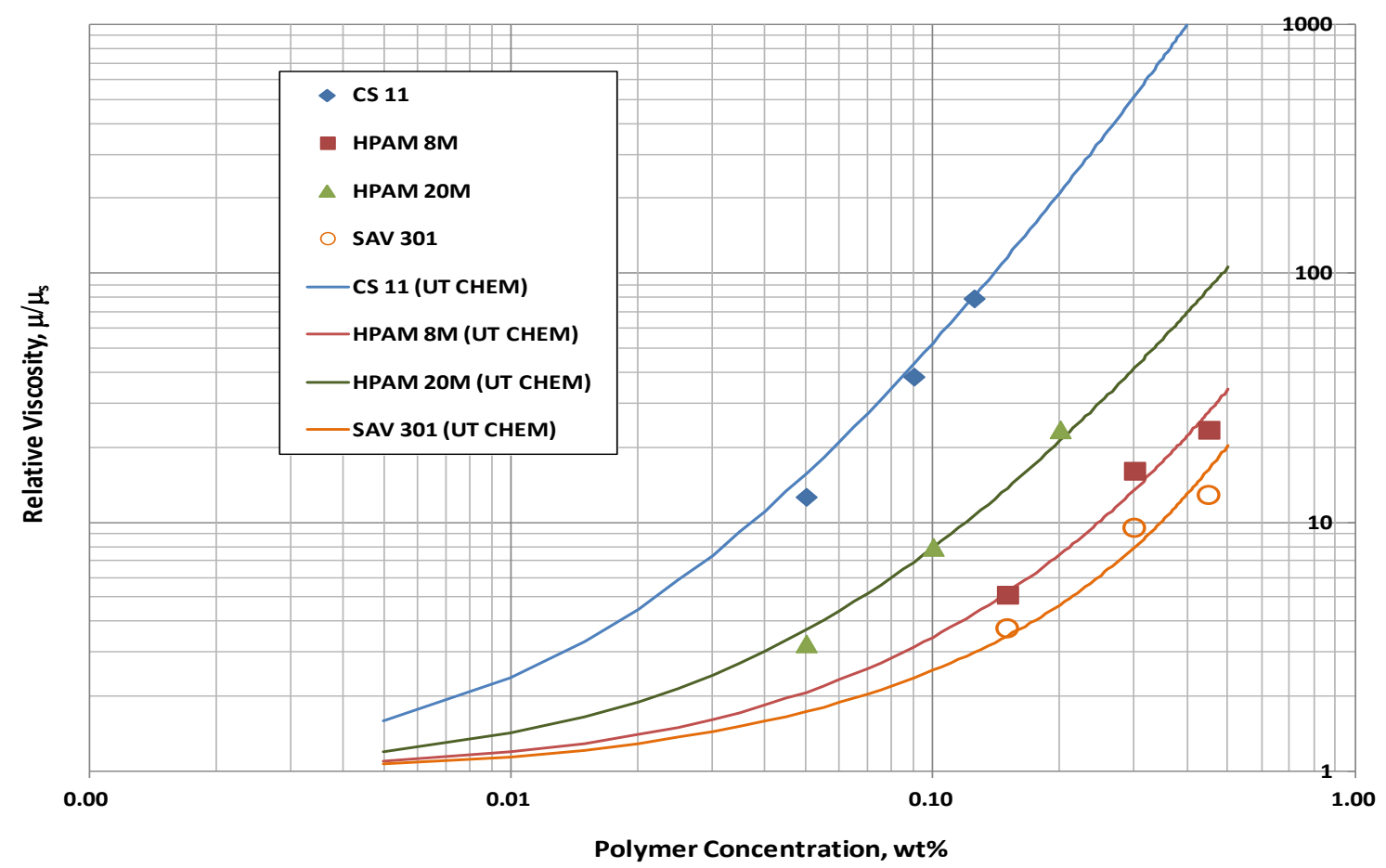

Figure 5.14 Viscosity of different polymers at $90{ }^{\circ} \mathrm{C}$ in synthetic seawater $(57,670 \mathrm{ppm}$ TDS).

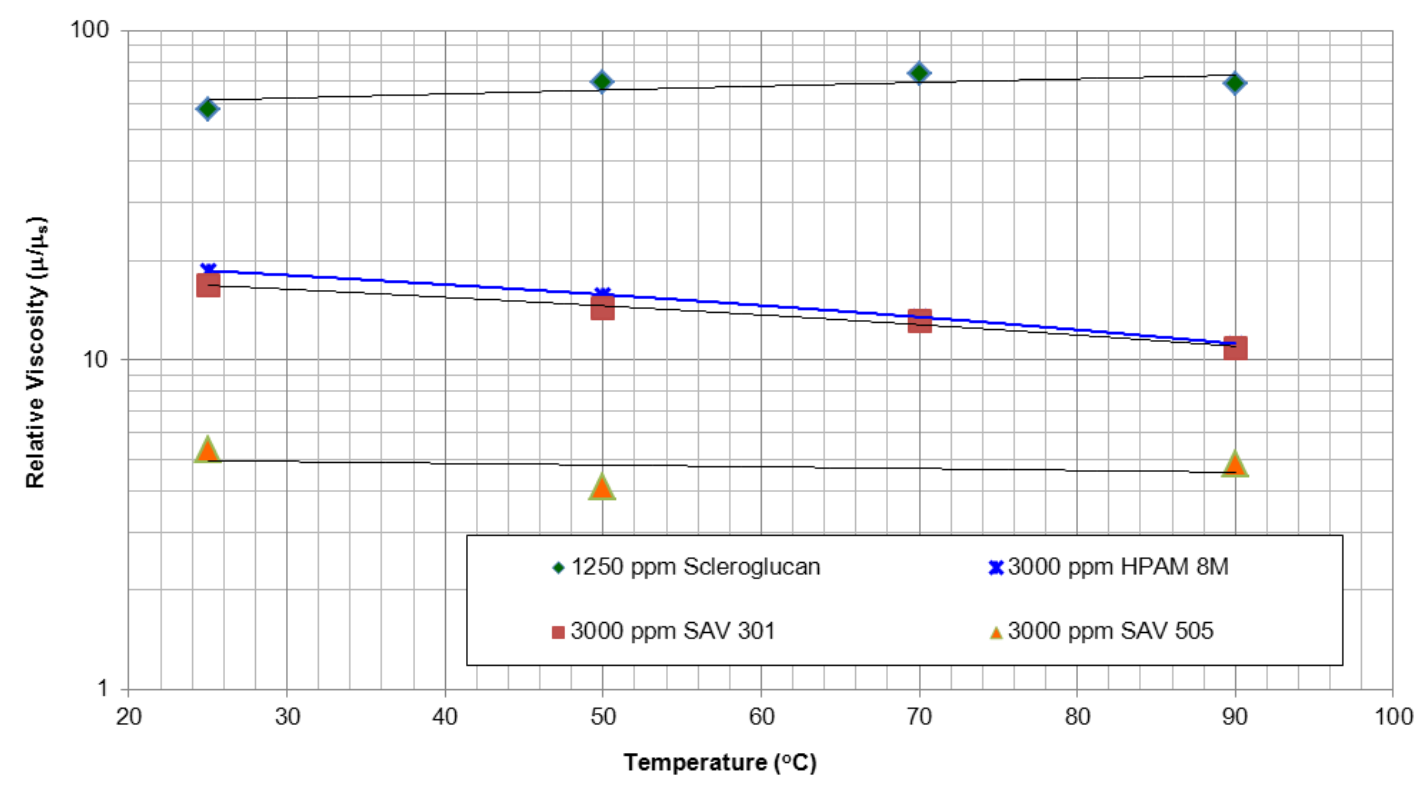

Figure 5.15 Relative viscosities of the polymers in synthetic seawater (30,000 ppm TDS) at a shear rate of $1 \mathrm{~s}^{-1}$ 


\subsubsection{Thermal Stability of Polymers.}

HPAM polymers hydrolyze at high temperature and/or high $\mathrm{pH}$. The molecular weight of the polymer remains relatively the same during hydrolysis and the viscosity of the polymer solution increases in soft brine. Polyacrylamides undergo hydrolysis under both acidic and basic conditions and introduce negative charges into the polymer chain. In hard brines, the interaction between the divalent cations and the negatively charged polymer result in precipitation of polymer molecules, and thereby decrease the viscosity (Ryles, 1988; Doe, 1987; Levitt and Pope, 2008). Incorporation of NVP groups into a polyacrylamide polymer inhibits hydrolysis and consequently such co-polymers can tolerate higher divalent cation concentrations (Hsieh et al., 1992; Vermolen et al., 2011; Gaillard et al., 2010).

The degradation of polyacrylamide backbone via free radicals action has been referred to as thermal degradation. Thermal degradation reduces the average molecular weight of the polymer due to cleavage of the molecules by a free radical mechanism. The lower molecular weight results in decreased viscosity of the polymer solution. Free radicals can form in the presence of oxygen or by decomposition of certain additives used in the polymerization process. Ramsden et al. (1986) suggested that hydroxyl radicals formed in the presence of dissolved oxygen can cause chain scission of the polyacrylamides resulting in a reduction of the molecular weight of the polymer (Ramsden et al., 1986). Seright et al. (2009) reported that polyacrylamides are stable up to $120{ }^{\circ} \mathrm{C}$ for more than 200 days if dissolved oxygen level is below $5 \mathrm{ppb}$. However, with increased dissolved oxygen level ( $>30 \mathrm{ppb})$, viscosity loss is accelerated according to Wu et al. (2009). Wellington (1983) described the combination of iso-propanol alcohol (IPA) and thiourea being able to prevent radical formation and associated degradation. 
Alcohol prevents oxidation of the polymer. Since they can be preferentially oxidized, they act as a sacrificial agent. Thiourea functions as a radical scavenger that can trap the unstable radical species. The synergistic stabilization effect of sacrificial agents and radical scavengers has been exploited to prevent polymer degradation in the presence of oxygen at levels as high as 500 ppb (Gaillard et al., 2010; Kulawardana et al., 2012).

Short term thermal stability of HPAM polymers were investigated in the presence of a commercial additive package containing oxygen scavenger, a corrosion inhibitor and a biocide. The specific additive to be used with each batch of HPAM and their dosage were recommended by the polymer suppliers and is given in Table 5.9.

The thermal stability of FP 3630 S and H-EOR 63020 polymers are summarized in Tables 5.10 and 5.11, respectively. Both HPAM polymers showed signs of hydrolysis at the conditions of the study. Where hydrolysis was prevalent, the retained viscosity for FP 3630 was as high as $187 \%$ of the initial value after 15 days. The same value for H-EOR 63020 was $160 \%$ under same conditions.

Additives seemingly had little effect on the thermal or oxidative stability of the polymers within the timeframe of the study. All except for one polymer sample showed an increase in viscosity, which suggests that thermal degradation did not occur under these conditions. A single sample of H-EOR 63020 with additives showed a drastic decrease in viscosity after 15 days, compared to the one without additives. This was most likely due to oxygen contamination and erroneous dissolved oxygen (DO) measurement. Additives in general showed good compatibility with the HPAM polymers tested. The retained viscosities vs. time plots are given in Figure 5.12 through 5.17. 
Table 5.9 Additive Package used with HPAM in Thermal Stability Experiments

\begin{tabular}{|c|c|c|}
\hline Additive & FP 3630S & H-EOR 63020 \\
\hline Oxygen scavenger & $1.1 \mathrm{ppm}$ (Ammonium bisulfite) & $\begin{array}{c}30 \mathrm{ppm} \text { (Clariant } \\
\text { SCAVTREAT 1000) }\end{array}$ \\
\hline Corrosion inhibitor & $40 \mathrm{ppm}$ (Metalsorb CI) & $\begin{array}{c}7.5 \text { ppm (Clariant } \\
\text { CORRTREAT 5780 ) }\end{array}$ \\
\hline Biocide & $130 \mathrm{ppm}$ (BIOBAN) & $\begin{array}{c}200 \text { ppm (Clariant } \\
\text { BIOTREAT 5475) }\end{array}$ \\
\hline
\end{tabular}

Table 5.10 Retained Viscosity of FP $3630 \mathrm{~S}$ at $62^{\circ} \mathrm{C}$

\begin{tabular}{|c|c|c|c|c|c|c|c|c|c|c|c|c|c|c|c|}
\hline \multirow[b]{2}{*}{ Sample \# } & \multirow{2}{*}{$\begin{array}{c}\text { Polymer } \\
\text { Conc. } \\
\text { (ppm) }\end{array}$} & \multirow{2}{*}{$\begin{array}{c}\text { Additive } \\
\text { Package } \\
(\mathrm{Y} / \mathrm{N})\end{array}$} & \multirow{2}{*}{$\begin{array}{c}\text { Salinity } \\
\text { (ppm) } \\
\text { TDS }\end{array}$} & \multirow{2}{*}{$\begin{array}{c}\text { Brine } \\
\text { hardness } \\
(\mathrm{ppm})\end{array}$} & \multirow{2}{*}{$\begin{array}{l}\text { Alkali } \\
\text { Conc. } \\
\text { (ppm) }\end{array}$} & \multicolumn{10}{|c|}{ Viscosity at $24^{\circ} \mathrm{C}$ and $10 \mathrm{~s}^{-1} /$ Retained Viscosity Ratio } \\
\hline & & & & & & Day 0 & $\begin{array}{c}\mathrm{DO} \\
(\mathrm{ppb})\end{array}$ & Day 1 & $\begin{array}{c}\mathrm{DO} \\
(\mathrm{ppb})\end{array}$ & Day 2 & $\begin{array}{c}\mathrm{DO} \\
(\mathrm{ppb})\end{array}$ & Day 7 & $\begin{array}{c}\mathrm{DO} \\
(\mathrm{ppb})\end{array}$ & Day 15 & $\begin{array}{c}\text { DO } \\
\text { (ppb) }\end{array}$ \\
\hline 1 & 2500 & $\mathrm{~N}$ & 5425 & 0 & 0 & 1.00 & 0.0 & 1.01 & 0.0 & 1.07 & 0.0 & 1.04 & 0.0 & 1.87 & 0.0 \\
\hline 2 & 2500 & $\mathrm{Y}$ & 5425 & 0 & 0 & 1.00 & 0.0 & 0.92 & 0.0 & 0.91 & 0.0 & 0.75 & 20.0 & 1.27 & 0.0 \\
\hline 3 & 2500 & $\mathrm{~N}$ & 15425 & 0 & 10000 & 1.00 & 0.0 & 1.37 & 0.0 & 1.41 & 0.0 & 1.47 & 0.0 & 1.46 & 0.0 \\
\hline 4 & 2500 & $\mathrm{Y}$ & 15425 & 0 & 10000 & 1.00 & 0.0 & 1.33 & 0.0 & 1.43 & 0.0 & 1.41 & 0.0 & 1.47 & 0.0 \\
\hline 5 & 2000 & $\mathrm{~N}$ & 5267 & 342 & 0 & 1.00 & 0.0 & 0.95 & 0.0 & 0.98 & 0.0 & 1.00 & 0.0 & 1.67 & 0.0 \\
\hline 6 & 2000 & Y & 5267 & 342 & 0 & 1.00 & 0.0 & 0.98 & 0.0 & 0.90 & 0.0 & 0.81 & 0.0 & 1.61 & 0.0 \\
\hline
\end{tabular}

Table 5.11 Retained Viscosity of H-EOR 63020 at $62{ }^{\circ} \mathrm{C}$

\begin{tabular}{|c|c|c|c|c|c|c|c|c|c|c|c|c|c|c|c|}
\hline \multirow{2}{*}{ Sample \# } & \multirow{2}{*}{\begin{tabular}{|c} 
Polymer \\
Conc. \\
(ppm)
\end{tabular}} & \multirow{2}{*}{$\begin{array}{c}\text { Additive } \\
\text { Package } \\
(\mathrm{Y} / \mathrm{N})\end{array}$} & \multirow{2}{*}{$\begin{array}{c}\text { Salinity } \\
\text { (ppm) } \\
\text { TDS }\end{array}$} & \multirow{2}{*}{$\begin{array}{c}\text { Brine } \\
\text { hardness } \\
(\mathrm{ppm})\end{array}$} & \multirow{2}{*}{$\begin{array}{l}\text { Alkali } \\
\text { Conc. } \\
\text { (ppm) }\end{array}$} & \multicolumn{10}{|c|}{ Viscosity at $24^{\circ} \mathrm{C}$ and $10 \mathrm{~s}^{-1} /$ Retained Viscosity Ratio } \\
\hline & & & & & & Day 0 & $\begin{array}{c}\mathrm{DO} \\
(\mathrm{ppb})\end{array}$ & Day 1 & $\begin{array}{c}\mathrm{DO} \\
(\mathrm{ppb})\end{array}$ & Day 2 & $\begin{array}{c}\mathrm{DO} \\
(\mathrm{ppb})\end{array}$ & Day 7 & $\begin{array}{c}\mathrm{DO} \\
(\mathrm{ppb})\end{array}$ & Day 15 & $\begin{array}{c}\text { DO } \\
\text { (ppb) }\end{array}$ \\
\hline 1 & 2500 & $\mathrm{~N}$ & 5425 & 0 & 0 & 1.00 & 0.0 & 0.95 & 0.0 & 0.93 & 0.0 & 0.94 & 0.0 & 0.97 & 0.0 \\
\hline 2 & 2500 & $\mathrm{Y}$ & 5425 & 0 & 0 & 1.00 & 0.0 & 0.79 & 0.0 & 0.43 & 10.0 & 0.54 & 10.0 & 0.18 & 10.0 \\
\hline 3 & 2500 & $\mathrm{~N}$ & 15425 & 0 & 10000 & 1.00 & 0.0 & 1.17 & 0.0 & 1.17 & 0.0 & 1.18 & 10.0 & 1.08 & 0.0 \\
\hline 4 & 2500 & Y & 15425 & 0 & 10000 & 1.00 & 0.0 & 1.08 & 0.0 & 1.19 & 0.0 & 1.16 & 0.0 & 1.26 & 10.0 \\
\hline 5 & 2000 & $\mathrm{~N}$ & 5267 & 342 & 0 & 1.00 & 0.0 & 0.94 & 0.0 & 0.90 & 0.0 & 0.96 & 10.0 & 1.60 & 0.0 \\
\hline 6 & 2000 & Y & 5267 & 342 & 0 & 1.00 & 0.0 & 0.85 & 0.0 & 0.91 & 0.0 & - & 40.0 & 1.19 & 10.0 \\
\hline
\end{tabular}




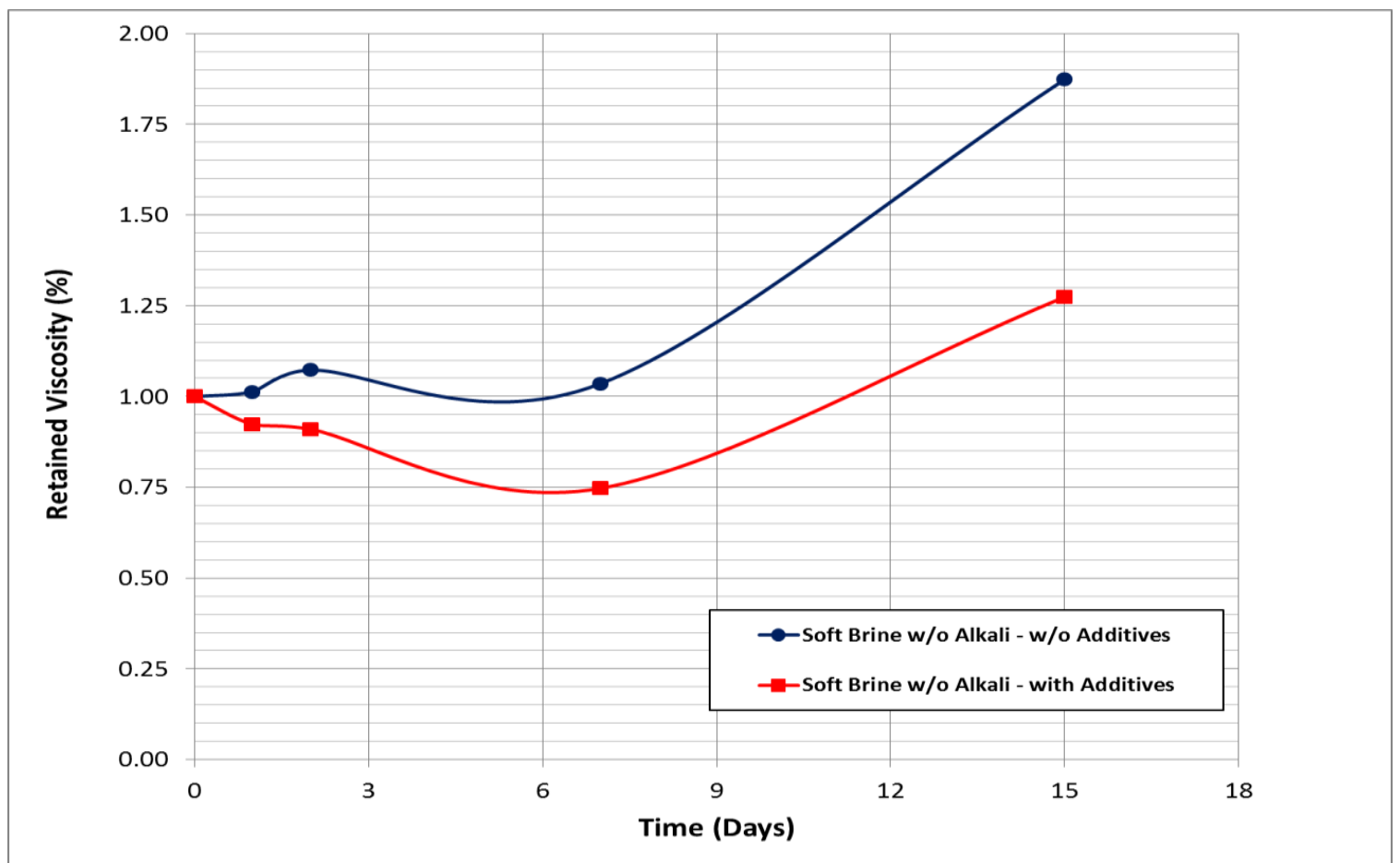

Figure 5.16 Retained Viscosity of FP 3630S in Soft Brine-1S at $62{ }^{\circ} \mathrm{C}$

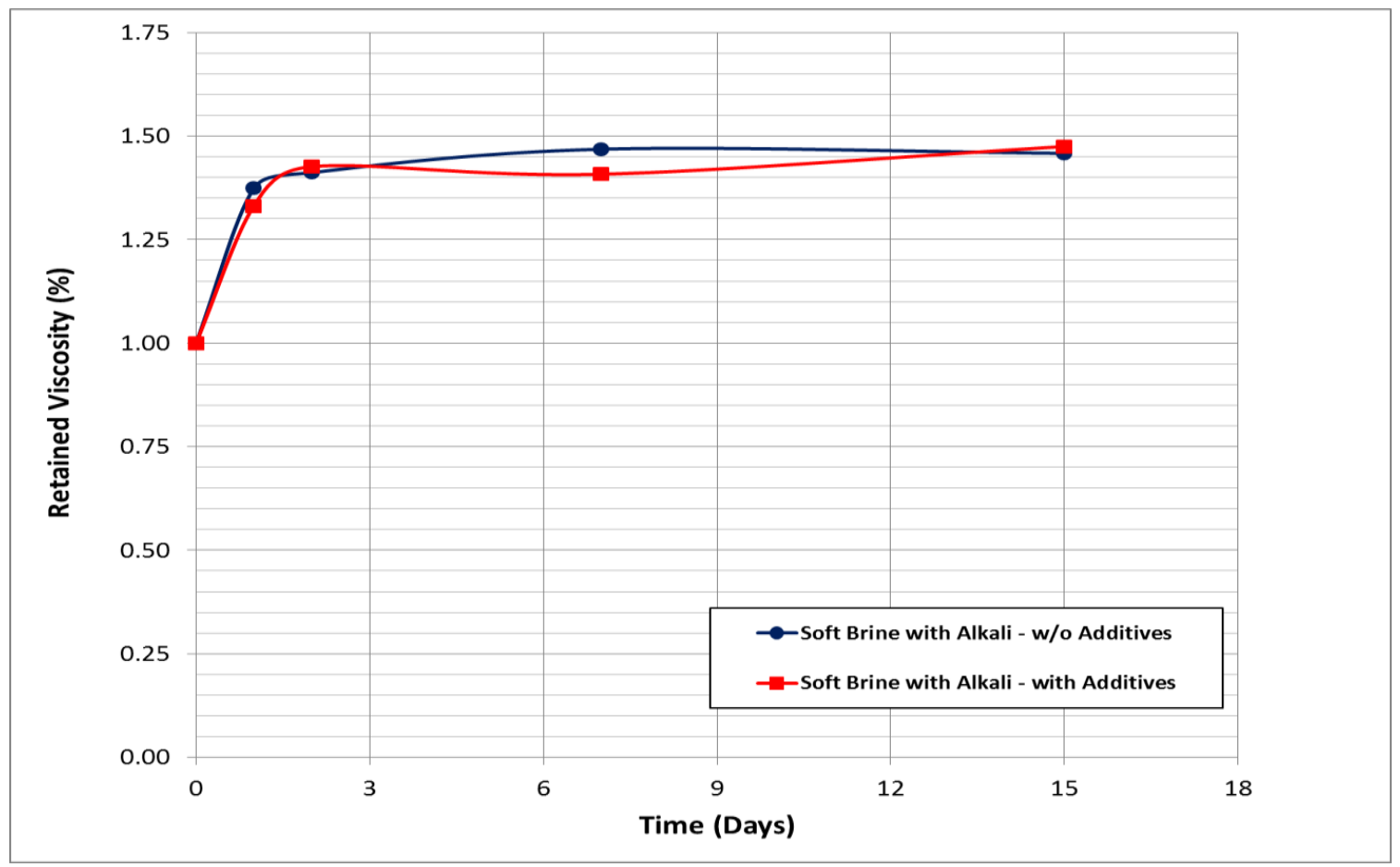

Figure 5.17 Retained Viscosity of FP 3630S in Soft Brine-1S with 10,000 ppm $\mathrm{Na}_{2} \mathrm{CO}_{3}$ at $62{ }^{\circ} \mathrm{C}$ 


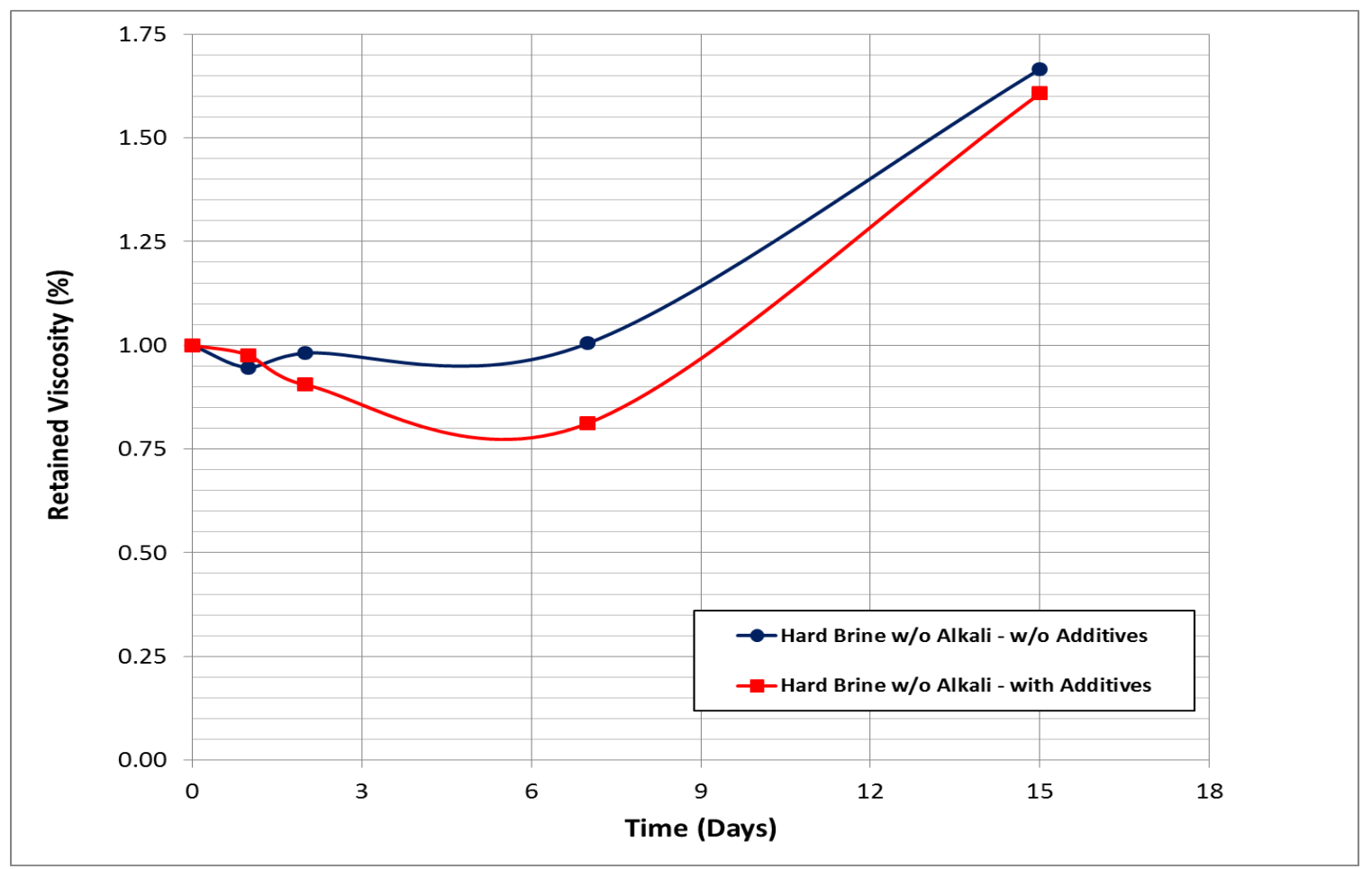

Figure 5.18: Retained Viscosity of FP 3630S in Hard Brine-1H at $62{ }^{\circ} \mathrm{C}$

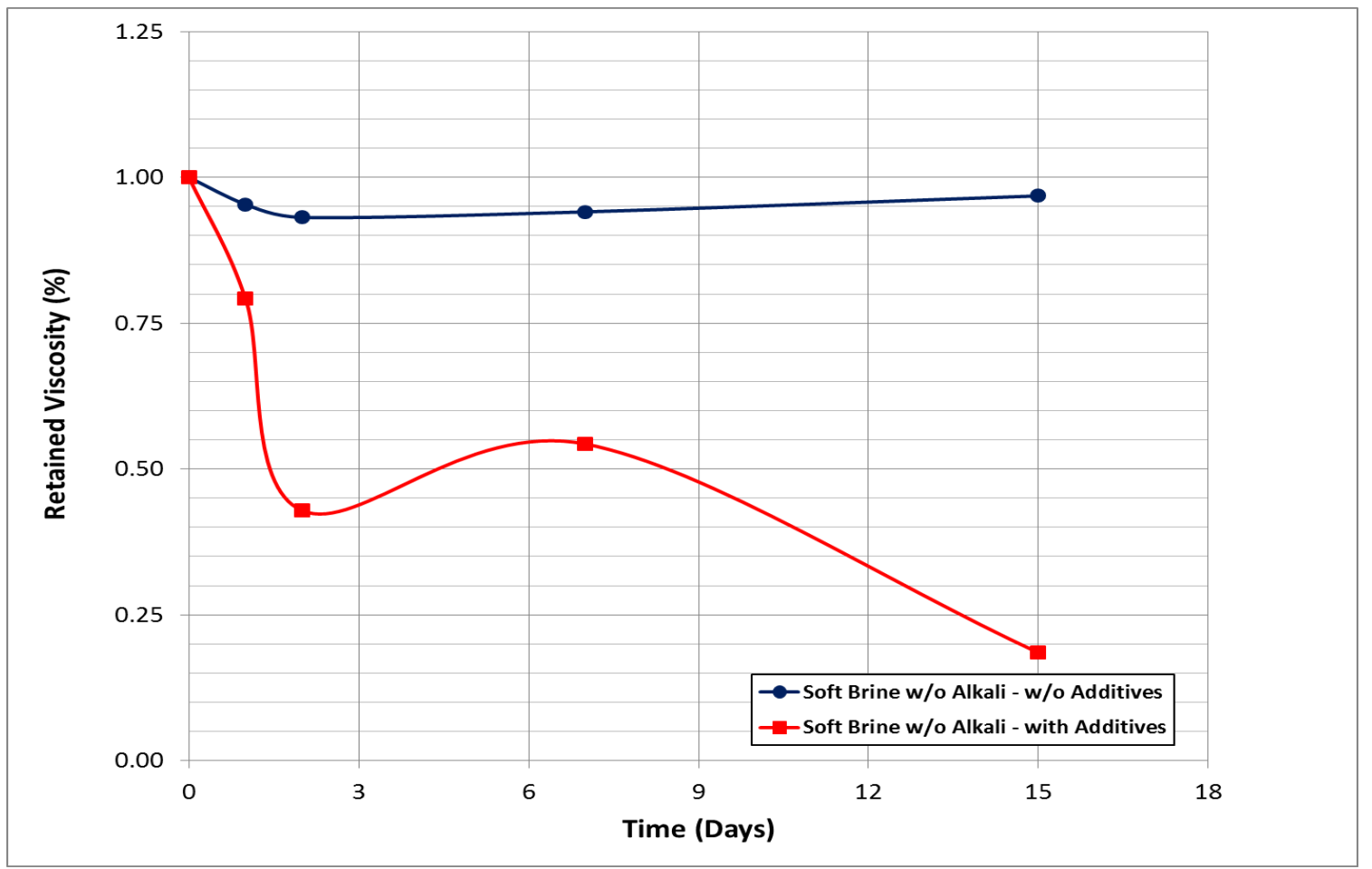

Figure 5.19 Retained Viscosity of H-EOR 63020 in Soft Brine-1S at $62{ }^{\circ} \mathrm{C}$ 


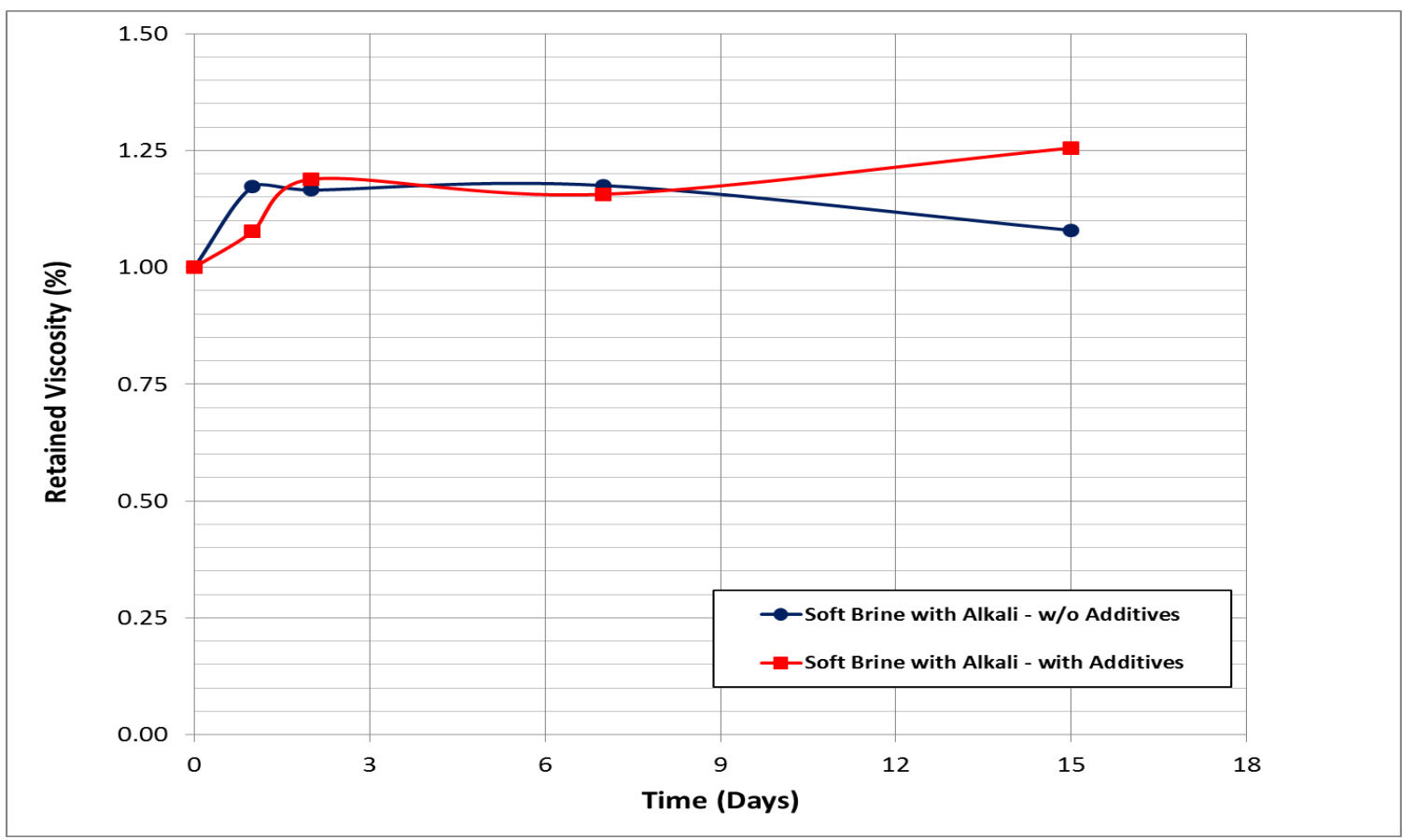

Figure 5.20 Retained Viscosity of H-EOR 63020 in Soft Brine-1S with 10,000 ppm $\mathrm{Na}_{2} \mathrm{CO}_{3}$ at $62{ }^{\circ} \mathrm{C}$

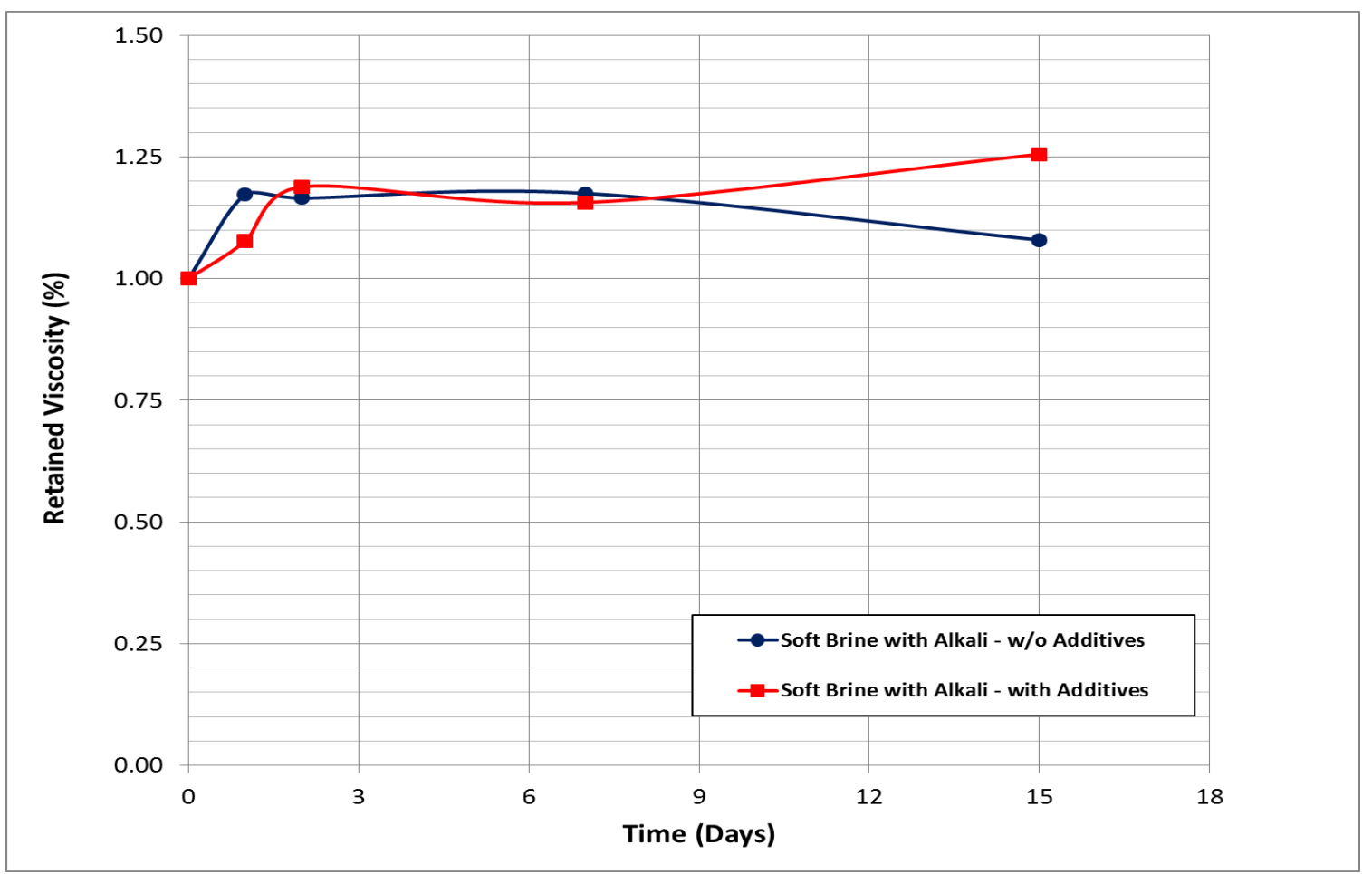

Figure 5.21 Retained Viscosity of H-EOR 63020 in Hard Brine-1H at $62{ }^{\circ} \mathrm{C}$ 


\section{Thermal Stability of SAV Polymers.}

Incorporation of NVP monomers into polyacrylamides effectively protects the amide groups against hydrolysis (Gaillard et. al., 2010, Vermolen et. al., 2011). The triple helix nature of the scleroglucan makes it more resistant to thermal degradation (Kalpacki, et. al., 1990; Rivenq et. al., 1992). However, the performance of biopolymers may deviate from one another according to their source of generation (Ryles, 1983).

Thermal stability tests were done for both scleroglucan and NVP polymers under a low oxygen $(<40 \mathrm{ppb})$ concentration with a protection package consisting of radical scavengers, isopropyl alcohol (IPA) and thiourea (TU) in the mass ratio of polymer:IPA:TU of 10: 4: 2. The same procedure described under HPAM was used to remove oxygen from polymer solutions before preparing the samples. The final polymer concentration of the samples was $3000 \mathrm{ppm}$ and the brine salinity was $57,670 \mathrm{ppm}(2,760$ ppm of total divalent cations). The samples were stored in thick walled ampules as described before and were aged at $100{ }^{\circ} \mathrm{C}$. The dissolved oxygen concentration and the viscosities were measured after cooling the samples to room temperature using a Contraves LS-30 viscometer at a shear rate $11 \mathrm{~s}^{-1}$ at $25^{\circ} \mathrm{C}$.

Figure 5.22 shows the normalized viscosity versus time. Superpusher SAV 505 containing higher levels of NVP showed approximately 95\% normalized viscosity after 30 days at $100{ }^{\circ} \mathrm{C}$ and, $\sim 85 \%$ normalized viscosity after 120 days. Superpusher SAV 522, which contained lower levels of NVP compared to SAV 505 showed inferior results with $77 \%$ normalized viscosity after 30 days and $~ 70 \%$ after 120 days. The results show that higher NVP levels result in higher stability under these harsh conditions. 


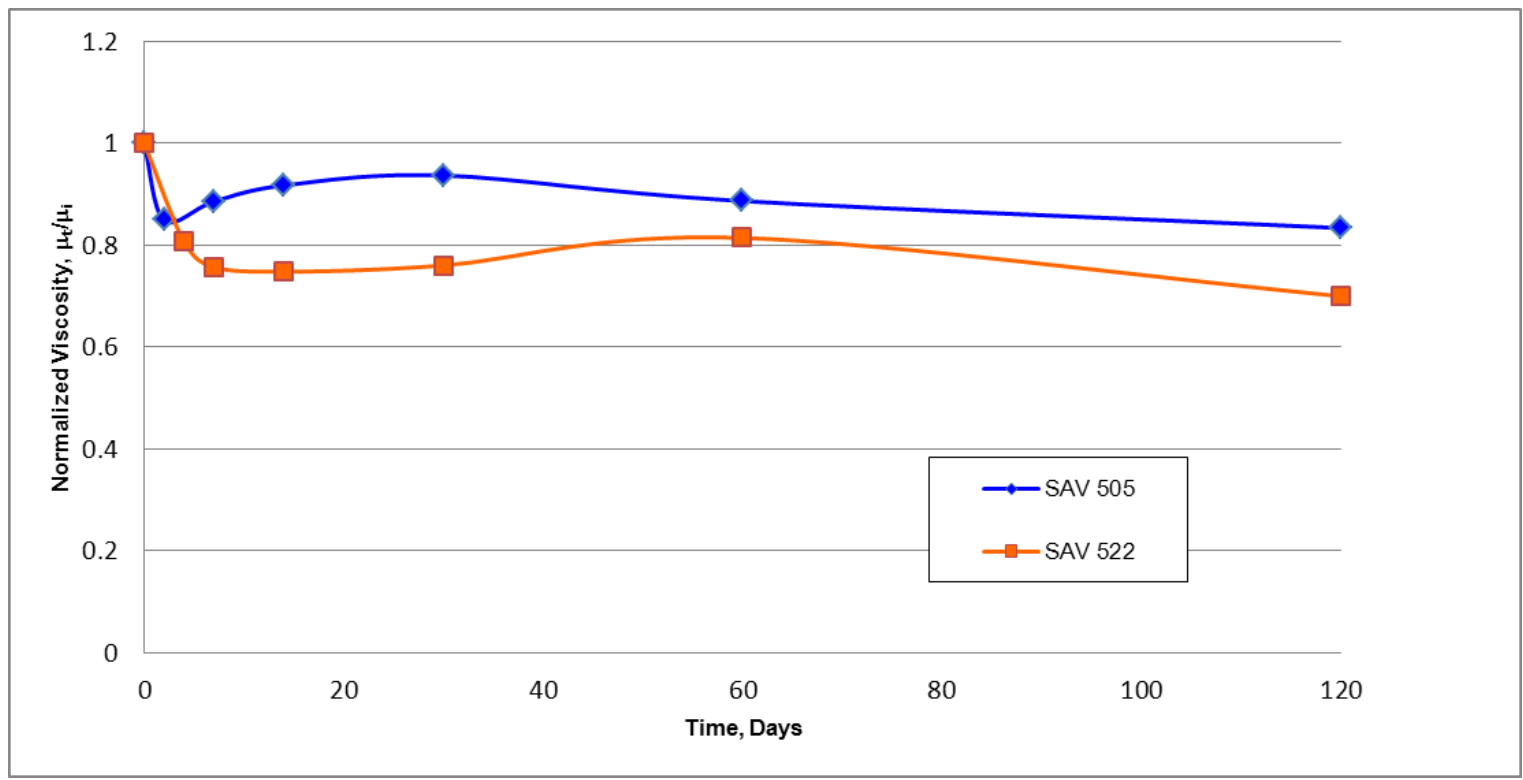

Figure 5.22 Thermal Stability of NVP polymers. Polymer Concentration- 3000 ppm, Additives: IPA $=1200 \mathrm{ppm}, \mathrm{TU}=600 \mathrm{ppm}$. Aged at $100{ }^{\circ} \mathrm{C}$ in synthetic seawater. (Viscosity was measured at shear rate $11 \mathrm{~s}^{-1}$ at $25^{\circ} \mathrm{C}$ ). $\mu_{\mathrm{t}}=$ viscosity of the polymer at day $\mathrm{t}$, and $\mu_{\mathrm{i}}=$ viscosity of the polymer before aging.

\subsubsection{Polymer Transport and Retention.}

A series of single-phase corefloods were performed to study the transport properties of the polymers.

HPAM polymer floods were conducted at $62{ }^{\circ} \mathrm{C}$ in 12 inch long by 1.5 inch diameter Bentheimer sandstone cores. Initially the cores were saturated and flooded with hard Brine- $1 \mathrm{H}$. The brine permeabilities were in the range of 1500 to $1750 \mathrm{mD}$. The properties of the injected polymer solutions are given in Table 5.12 and the coreflood properties are given in Table 5.13. $1500 \mathrm{ppm}$ polymer solutions were prepared in soft Brine-1S according to standard protocol. The filtration ratios for FP $3630 \mathrm{~S}$ and H-EOR 63020 through 1.2 micron filter under 15 psi argon pressure were 1.1 and 1.15, respectively. The viscosities of FP 3630S and H-EOR 63020 are given in Figures 5.23 
and 5.24, respectively. Both HPAM polymers showed excellent transport behavior in Bentheimer cores with steady state pressure drops reaching steady state after approximately 2 PV of injection, as shown by Figure 5.26 and 5.27 for FP 3630S and HEOR 6320, respectively. Approximately 23 total pore volumes of FP 3630 S and 16 pore volumes of H-EOR63020 polymer were injected at multiple flow rates without any signs of plugging in the cores.

The effluent viscosity vs shear rate for FP 3630 S and H-EOR 63020 solutions after $2 \mathrm{PV}$ are given in Figures 5.28 and 5.29, respectively. The $\mathrm{pH}$ and normalized effluent viscosity of the effluent are given in Figures 5.30 and 5.31. The dynamic polymer retention was not measured, but both the effluent viscosity and pressure drop data indicate it was low. 
Table 5.12 Polymer Properties for HPAM Polymers in Bentheimer Cores

\begin{tabular}{|c|c|c|}
\hline Polymer & FP3630S & H-EOR63020 \\
\hline Batch \# & 3477 & $100907-1$ \\
\hline Polymer Producer & SNF Floerger & Beijing Hengju \\
\hline Polymer Concentration (ppm) & 1,500 & 1,500 \\
\hline Polymer solution Salinity, (ppm) TDS & 5425 & 5425 \\
\hline $\begin{array}{c}\text { Total Divalent Cation Concentration } \\
\text { (ppm) }\end{array}$ & 0 & 0 \\
\hline $\begin{array}{c}\text { Filtration Ratio } \\
\text { Filter Membrane Pore Size (micron) }\end{array}$ & 1.1 & 1.15 \\
\hline $\begin{array}{c}\text { Pressure Drop Across Filter (psi) } \\
\text { Polymer Slug Viscosity, cP before } \\
\text { filtration at 10s }{ }^{-1} \text { and 62 }{ }^{\circ} \mathrm{C}\end{array}$ & 15 & 1.2 \\
\hline $\begin{array}{c}\text { Polymer Slug Viscosity, cP after } \\
\text { filtration at 10s }{ }^{-1} \text { and 62 }{ }^{\circ} \mathrm{C}\end{array}$ & 26 & 29 \\
\hline Polymer Slug pH & 7.5 & 29 \\
\hline Flow Rate (mL/min) & 0.075 & 7.5 \\
\hline $\begin{array}{c}\text { Frontal Velocity (ft/day) } \\
\text { Total PV Injected }\end{array}$ & 22 & 0.069 \\
\hline
\end{tabular}


Table 5.13 Polymer Flood Properties for HPAM Polymers in Bentheimer Cores

\begin{tabular}{|c|c|c|}
\hline Core & PF-FP3630-1 & PF-HEOR-1 \\
\hline Rock Type & $\begin{array}{c}\text { Bentheimer } \\
\text { sandstone }\end{array}$ & Bentheimer sandstone \\
\hline Length $(\mathrm{cm})$ & 30.48 & 30.48 \\
\hline Diameter $(\mathrm{cm})$ & 3.76 & 3.76 \\
\hline Area $\left(\mathrm{cm}^{2}\right)$ & 11.1 & 11.1 \\
\hline Mass $(\mathrm{g})$ & 686.9 & 689.4 \\
\hline Pore Volume (mL) & 54.0 & 50.0 \\
\hline Porosity & 0.2 & 0.1 \\
\hline Length to Tap $1(\mathrm{~cm})$ & 7.6 & 7.6 \\
\hline Length to Tap $2(\mathrm{~cm})$ & 15.3 & 15.3 \\
\hline Length to Tap $3(\mathrm{~cm})$ & 22.9 & 22.9 \\
\hline Length to Outlet $(\mathrm{cm})$ & 30.5 & 30.5 \\
\hline Temperature $\left({ }^{\circ} \mathrm{C}\right)$ & 62.0 & 62.0 \\
\hline Brine permeability (mD) & 1752 & 1502 \\
\hline Polymer Permeability (mD) & 1728 & 1476 \\
\hline
\end{tabular}


Table 5.14 Polymer Flood Analysis for HPAM Polymers in Bentheimer Cores

\begin{tabular}{|c|c|c|c|c|c|c|c|c|}
\hline Polvmer Flond \# & \multicolumn{3}{|c|}{ PF-FP3630-1 } & \multicolumn{5}{|c|}{ PF-HEOR-1 } \\
\hline $\begin{array}{l}\text { Polymer and } \\
\text { Batch\# }\end{array}$ & \multicolumn{3}{|c|}{ FP3630S / 3477} & \multicolumn{5}{|c|}{ H-EOR63020 / 100907-1 } \\
\hline $\begin{array}{l}\text { Flow Rate } \\
\text { (mL/min) }\end{array}$ & 0.075 & 0.1875 & 0.2625 & 0.375 & 0.0695 & 0.1735 & 0.243 & 0.345 \\
\hline $\begin{array}{l}\text { Pressure Drop } \\
\text { (psi) }\end{array}$ & 1.46 & 2.54 & 3.47 & 4.89 & 1.68 & 3.35 & 4.5 & 6.13 \\
\hline $\begin{array}{l}\text { Apparent } \\
\text { viscosity }(\mathrm{cP})\end{array}$ & 50.7 & 35.3 & 34.4 & 34.0 & 2.5 & 6.2 & 8.7 & 12.5 \\
\hline $\begin{array}{l}\text { Relative } \\
\text { Viscosity }\end{array}$ & 105.6 & 73.5 & 71.7 & 70.7 & 50.0 & 39.9 & 38.3 & 36.5 \\
\hline $\begin{array}{l}\text { Resistance } \\
\text { Factor }\end{array}$ & 104.7 & 72.8 & 71.1 & 70.1 & 104.2 & 83.1 & 79.7 & 76.0 \\
\hline $\begin{array}{l}\text { Equivalent } \\
\text { Shear Rate }\left(\mathrm{s}^{-1}\right)\end{array}$ & 2.4 & 6 & 8.4 & 12 & 101.8 & 81.2 & 77.9 & 74.3 \\
\hline
\end{tabular}

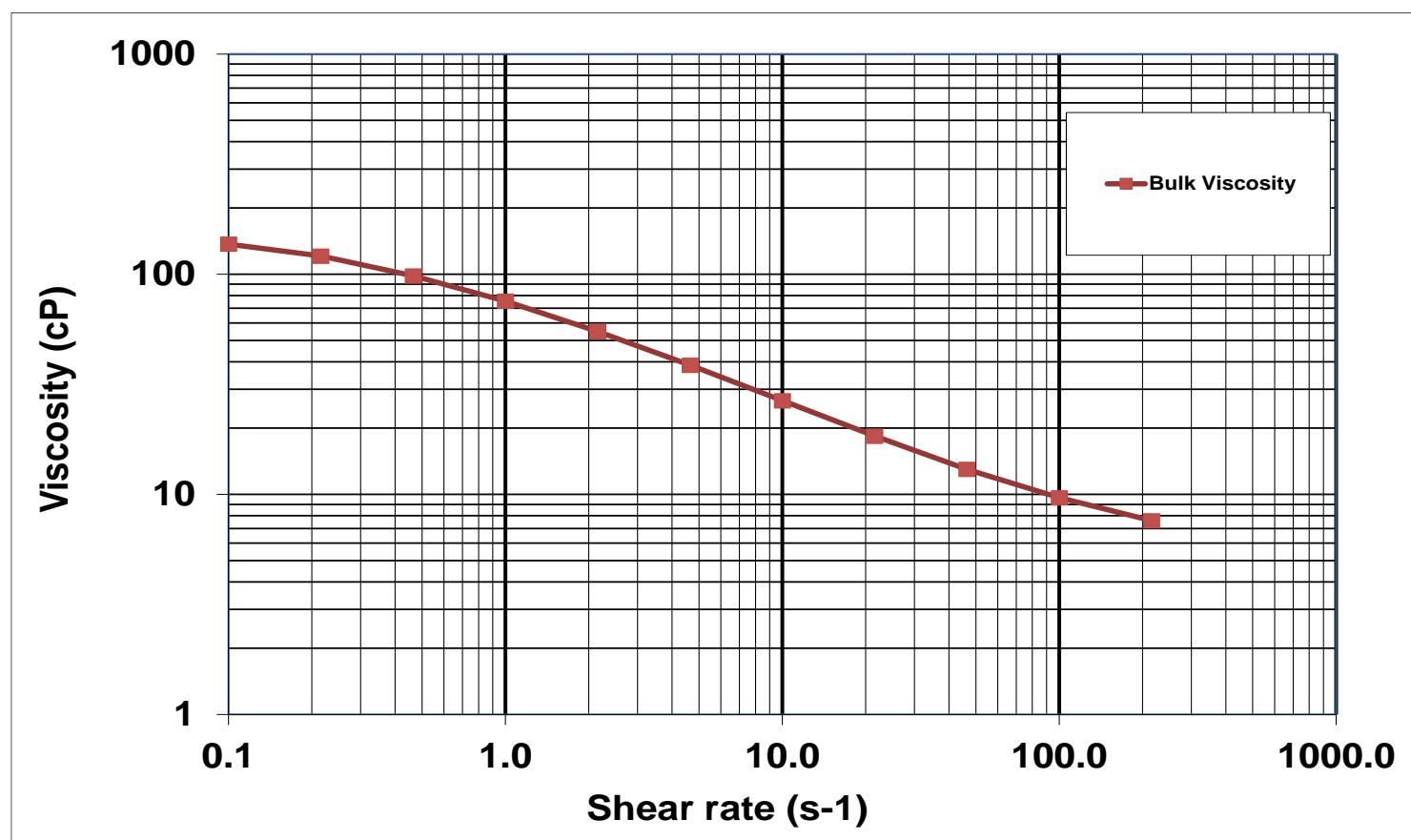

Figure 5.23 Viscosity of 1,500 ppm FP $3630 \mathrm{~S}$ in soft Brine-1S (5,425 ppm) at $62^{\circ} \mathrm{C}$. 


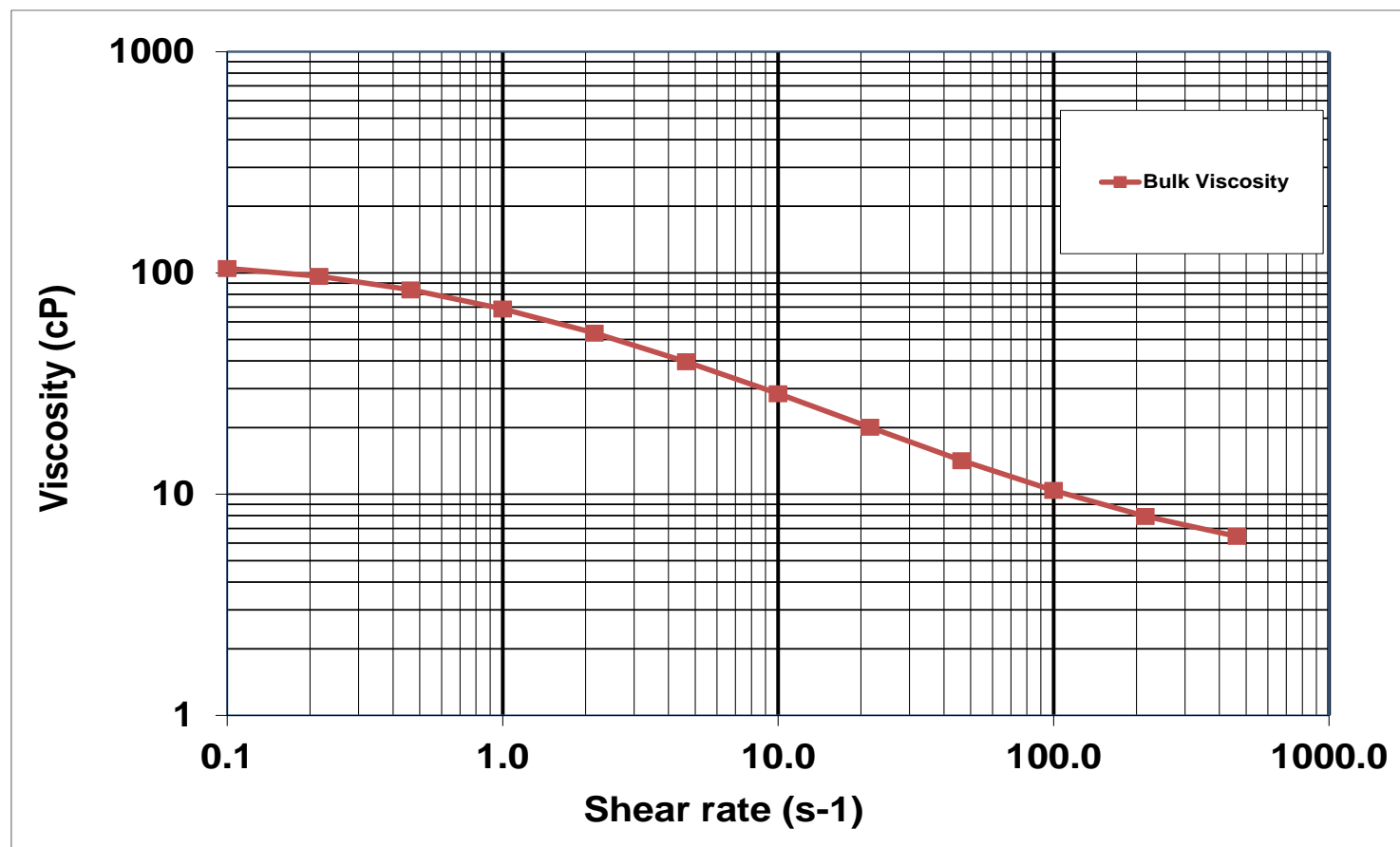

Figure 5.24 Viscosity of 1,500 ppm H-EOR 63020 in soft Brine-1S (5,425 ppm) at 62 ${ }^{\circ} \mathrm{C}$.

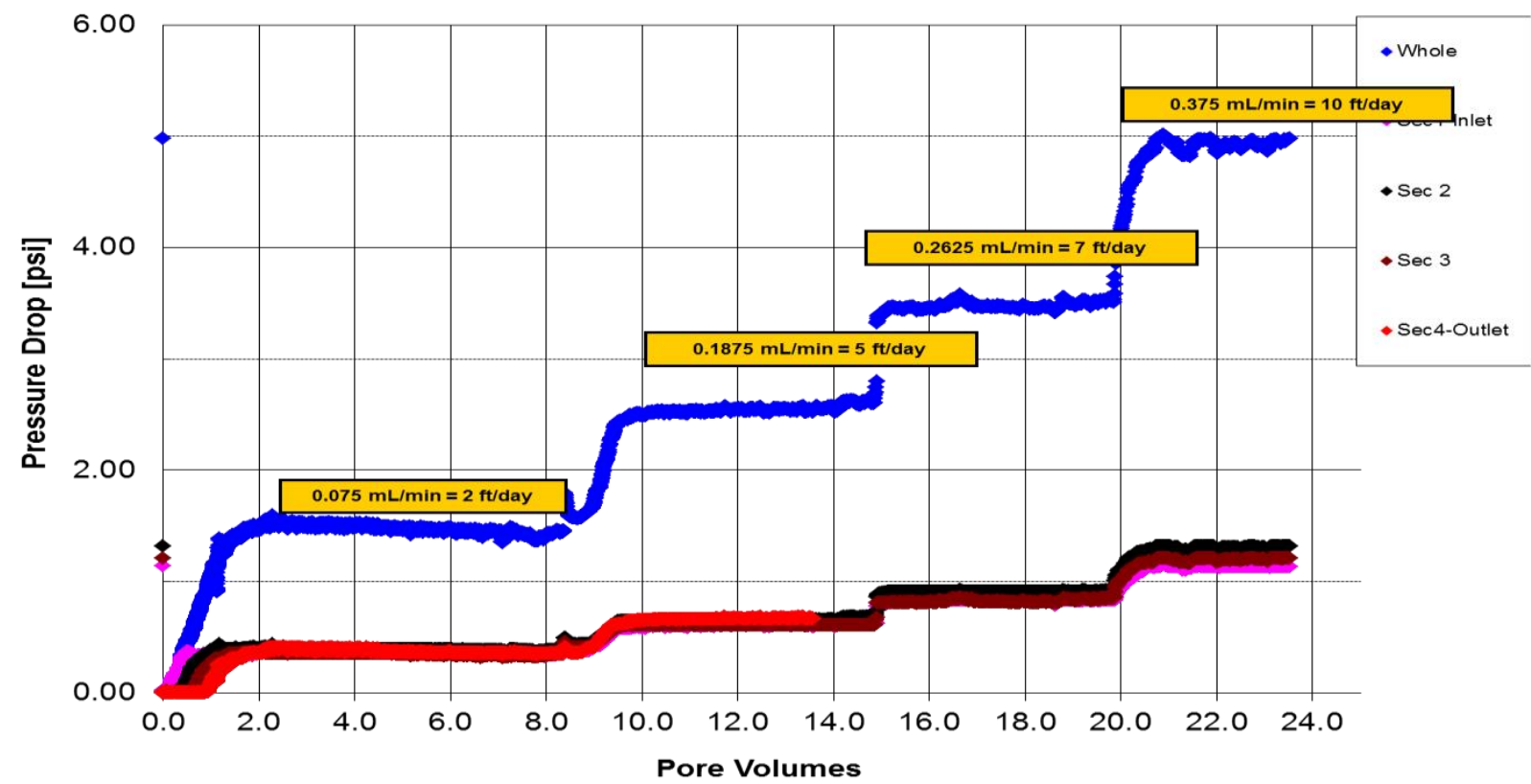

Figure 5.25 Pressure Drop across Bentheimer Core During FP 3630S injection at $62{ }^{\circ} \mathrm{C}$ at Multiple Flow Rates. 


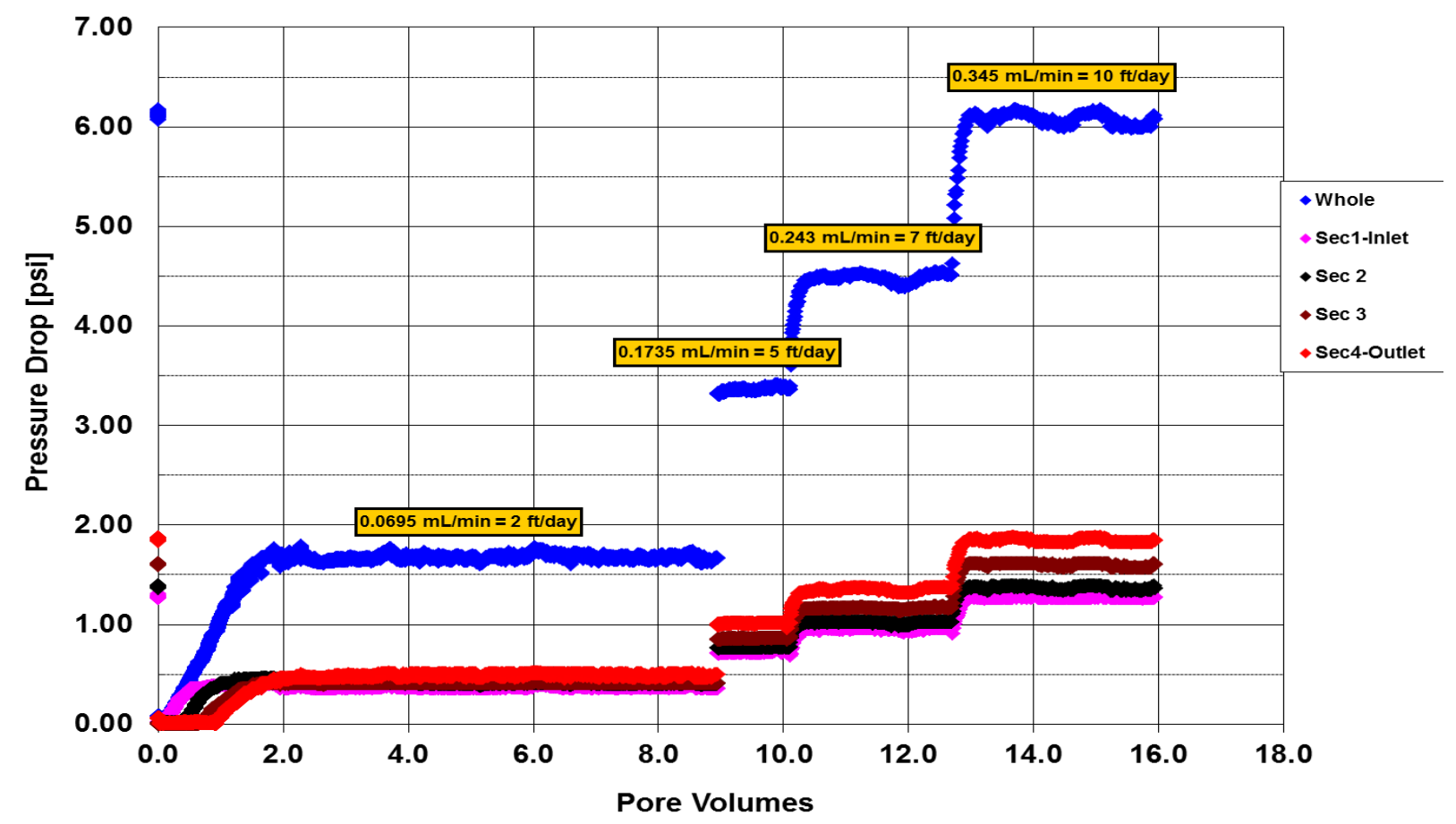

Figure 5.26 Pressure Drop across Bentheimer Core During injection of H-EOR 63020 at $62{ }^{\circ} \mathrm{C}$ at Multiple Flow Rates.

The resistance factor was in excellent agreement with the normalized viscosity at all injection rates. The permeability reduction factor was 1 for both polymers within the experimental uncertainty of the measurements. The effective shear rate of polymer flowing in the core was calculated as follows:

$$
\dot{\gamma}_{e q}=C\left(\frac{3 n+1}{4 n}\right)^{\frac{n}{n-1}} \frac{4 u_{p}}{\sqrt{8 k k_{r w} s_{w} \phi}}
$$

where $\gamma_{e q}$ is the equivalent shear rate $(1 / \mathrm{s}), u$ is $\mathrm{q} / \mathrm{A}$ in $(\mathrm{cm} / \mathrm{s}), \phi$ is the porosity, $k$ is the brine permeability $\left(\mathrm{cm}^{2}\right), k_{r w}$ is the water relative permeability, $S_{w}$ is the water saturation in the core, and the value of the power law exponent $\mathrm{n}$ is the slope of a log-log plot of viscosity vs. shear rate. 
To determine the value of $\mathrm{C}$, it was necessary to measure the rheology of the polymer solutions both in a rheometer as shown in Figures 5.23 and 5.24, and in a core at several shear rates as shown in Figures 5.25 and 5.26. The power law exponent was calculated from the bulk viscosity.

From the pressure drop data and the flow rate, an apparent viscosity was calculated using Darcy's law. An uncorrected shear rate was calculated using equation 5.2 with $\mathrm{C}=1$. Figure 5.27 is a plot of the apparent viscosity vs. uncorrected shear rate.

Table 5.10 Raw data from FP 3630S polymer flood

\begin{tabular}{|l|c|c|c|c|}
\hline Flow rate (ml/min) & 0.075 & 0.188 & 0.263 & 0.375 \\
\hline Frontal Advance (ft/day) & 2 & 5 & 7 & 10 \\
\hline Pressure Drop (psi) & 1.46 & 2.6 & 3.45 & 4.8 \\
\hline Uncorrected Shear rate & 2.27 & 5.68 & 7.95 & 11.36 \\
\hline Apperent viscosity (cP) & 50.7 & 36.1 & 34.2 & 33.3 \\
\hline
\end{tabular}

Table 5.11 Raw data from FP 3630S polymer flood

\begin{tabular}{|l|c|c|c|c|}
\hline Flow rate (ml/min) & 0.075 & 0.173 & 0.243 & 0.345 \\
\hline Frontal Advance (ft/day) & 2 & 5 & 7 & 10 \\
\hline Pressure Drop (psi) & 1.46 & 2.6 & 3.45 & 4.8 \\
\hline Uncorrected Shear rate (s-1) & 2.27 & 5.68 & 7.95 & 11.04 \\
\hline Apparent viscosity (cP) & 50.7 & 36.1 & 34.2 & 33.3 \\
\hline
\end{tabular}




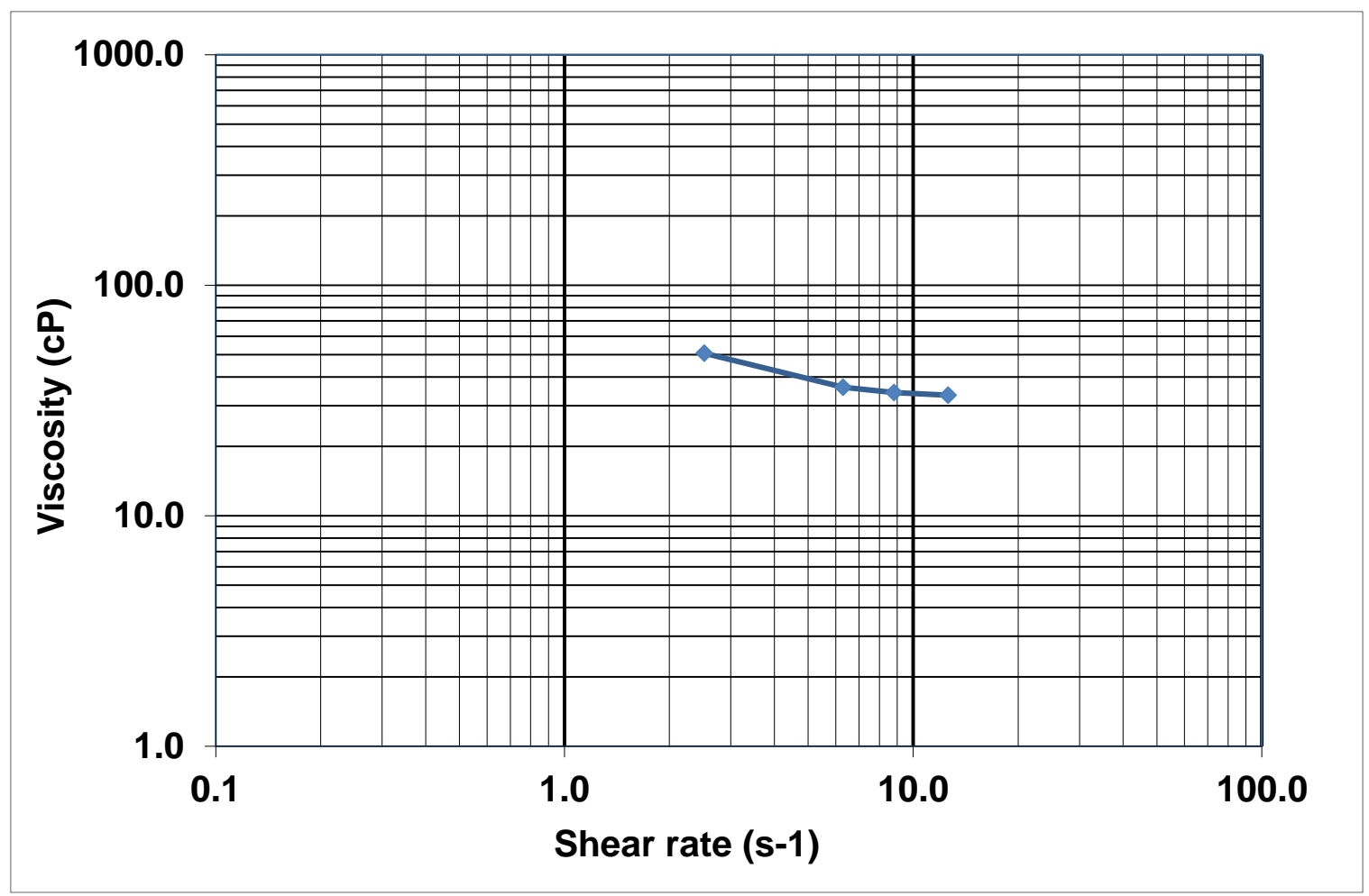

Figure 5.27: Apparent viscosity vs. uncorrected shear rate for FP 3630S polymer flood

After several iterations, $\mathrm{C}=1.1$ was estimated for both FP $3630 \mathrm{~S}$ polymer and H-EOR 63020 polymer using this procedure. This is a much smaller correction favor than most of the values in the literature and is almost negligible and almost 1 within experimental uncertainty. A shear correction factor of 1.1 decreases the calculated apparent viscosity by only about $4 \%$. 


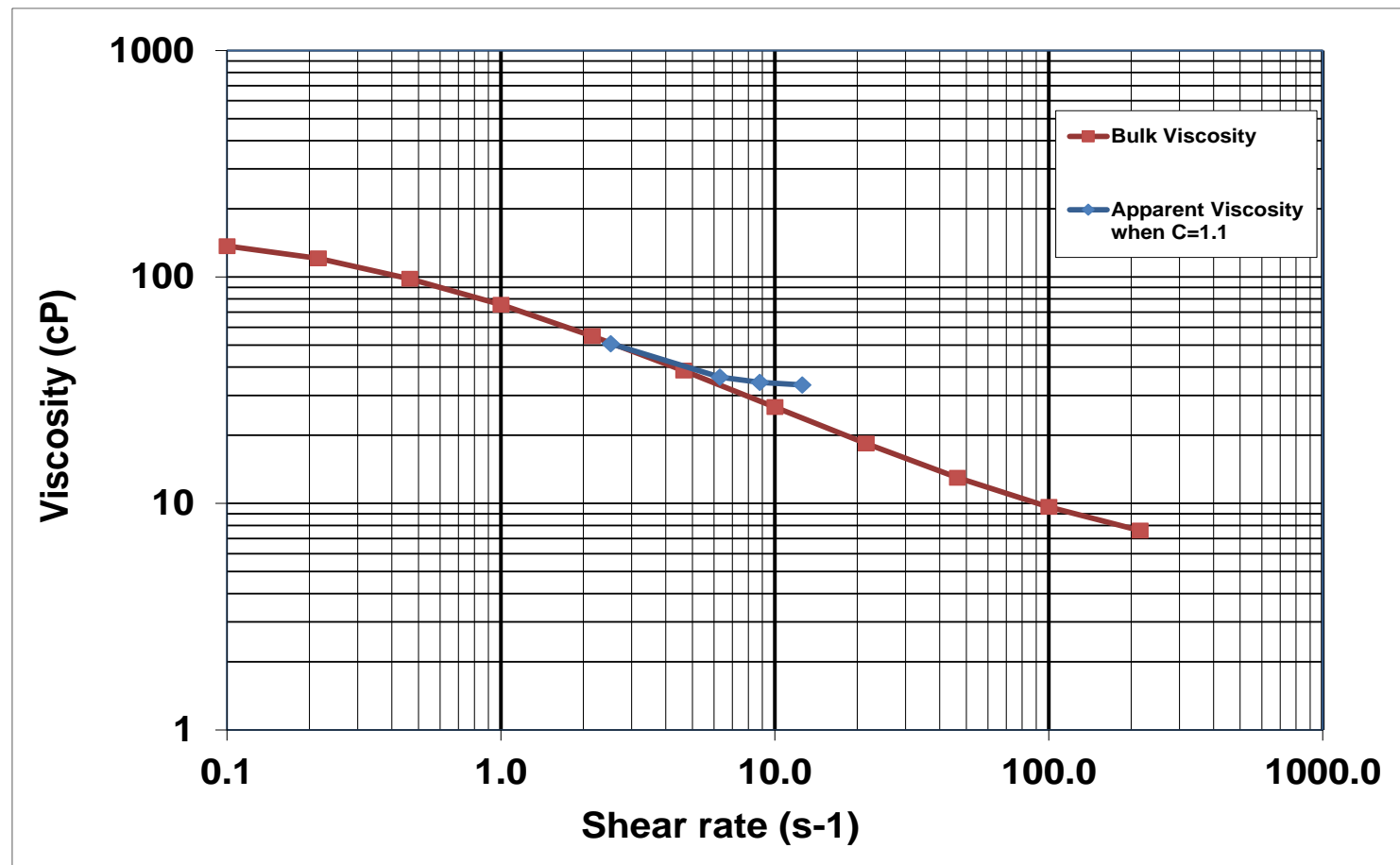

Figure 5.28 Comparison of bulk viscosity and apparent viscosity with corrected shear rate for FP 3630 S polymer flood

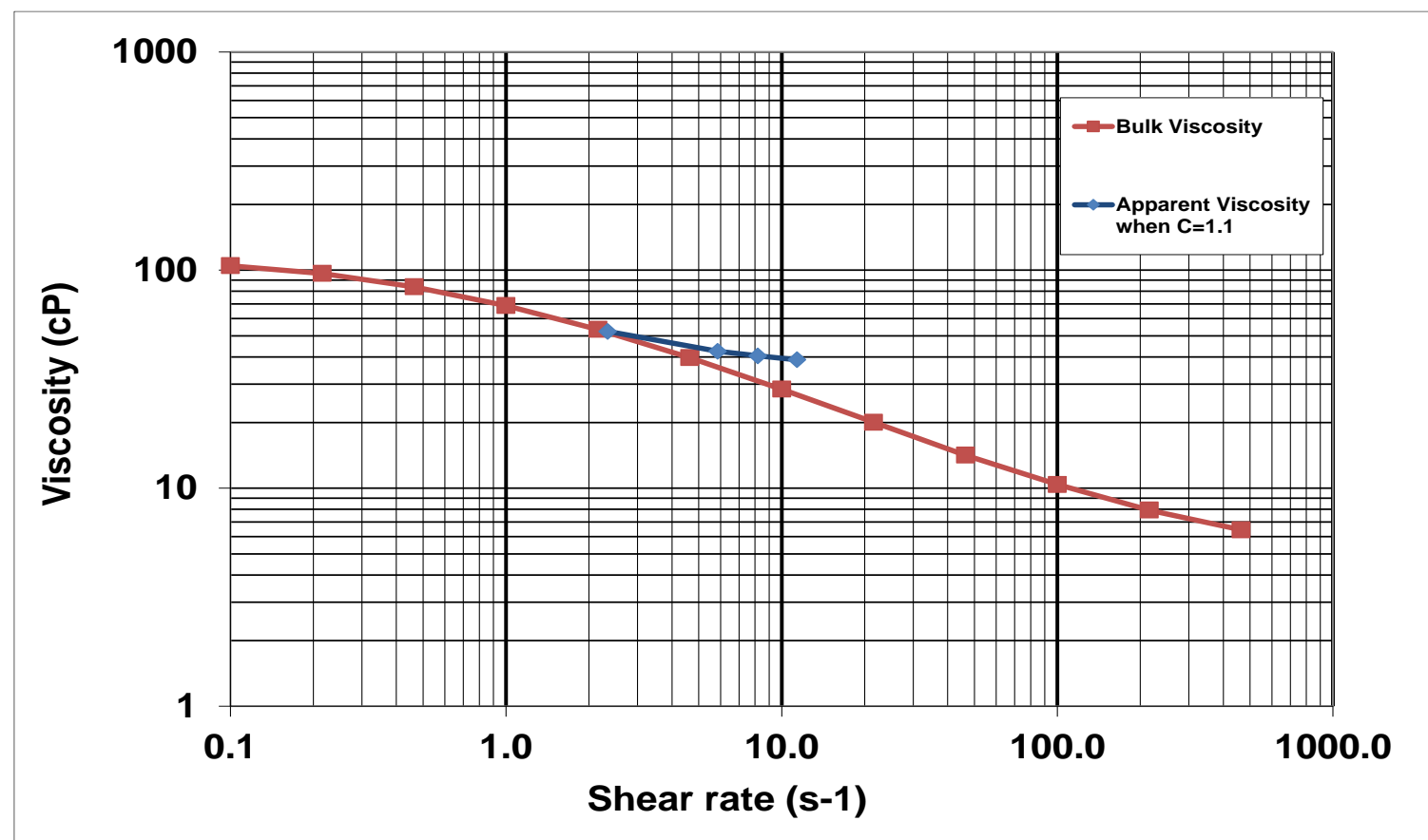

Figure 5.29 Comparison of bulk viscosity and apparent viscosity with corrected shear rate for H-EOR 63020 polymer flood 


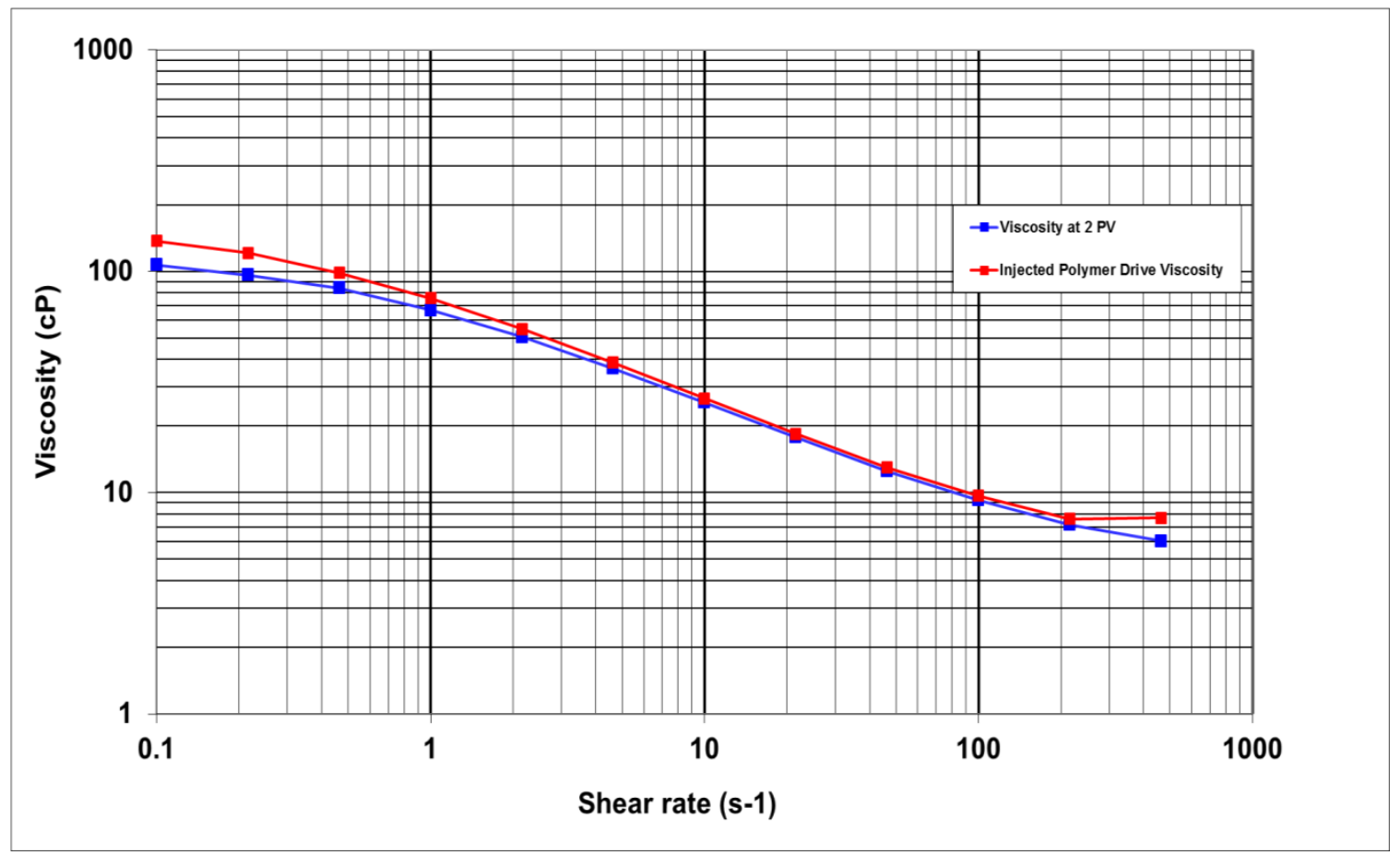

Figure 5.30 Injected and effluent polymer viscosity for FP $3630 \mathrm{~S}$ coreflood at $62{ }^{\circ} \mathrm{C}$.

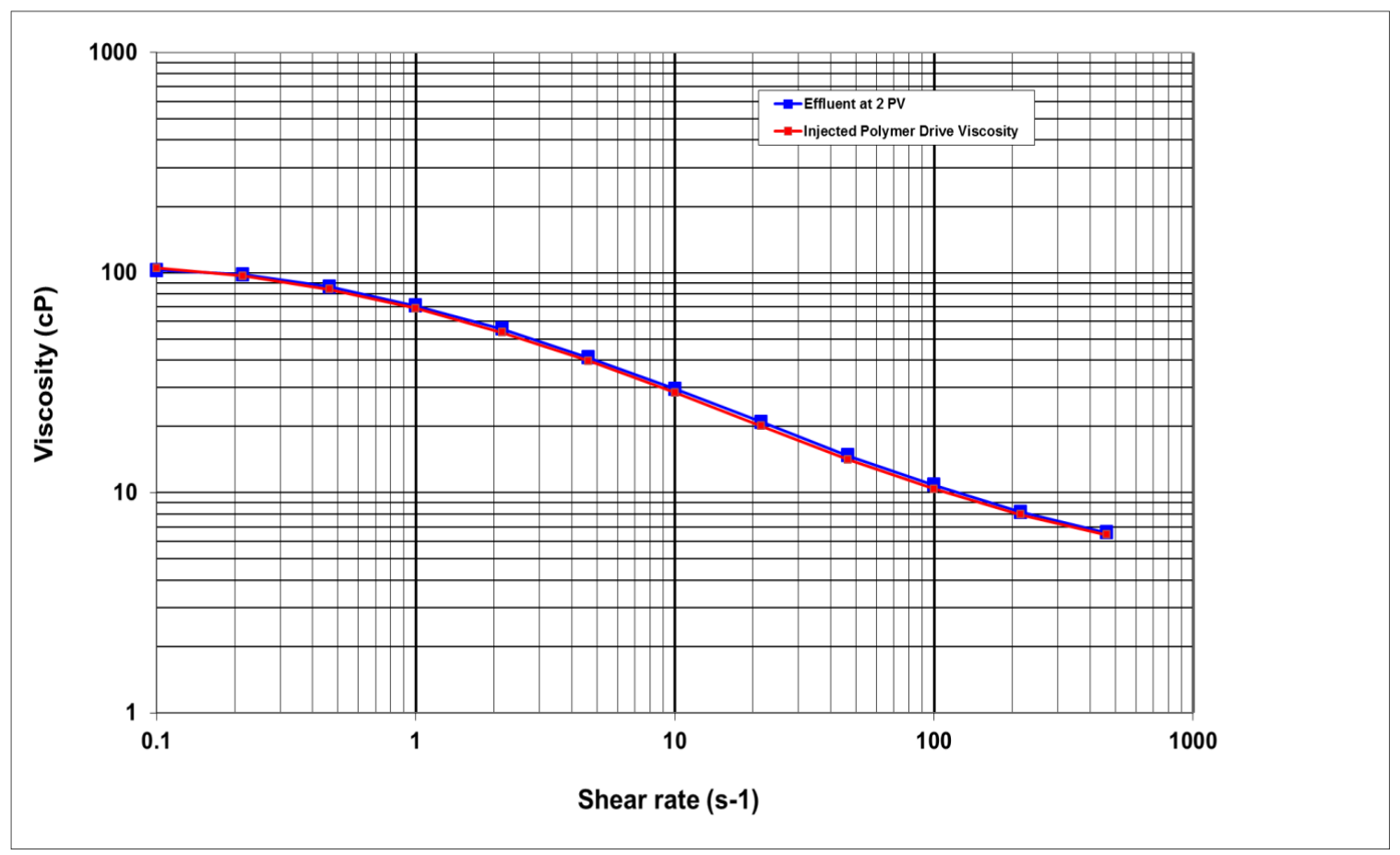

Figure 5.31 Injected and effluent polymer viscosity for H-EOR 63020 coreflood at $62{ }^{\circ} \mathrm{C}$. 


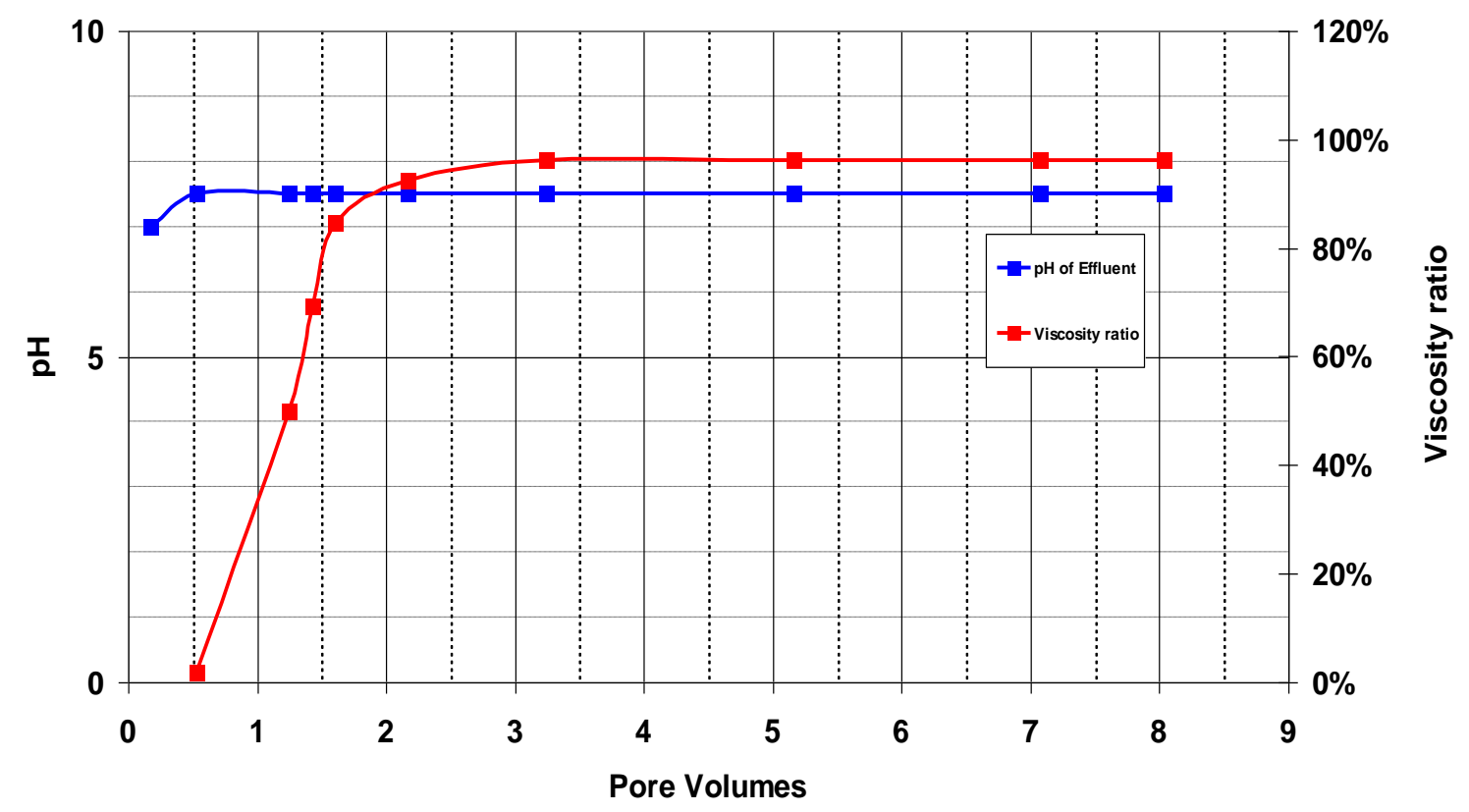

Figure 5.32 The normalized viscosity at $10 \mathrm{~s}^{-1}$ and $\mathrm{pH}$ profile for FP $3630 \mathrm{~S}$ at $62{ }^{\circ} \mathrm{C}$.

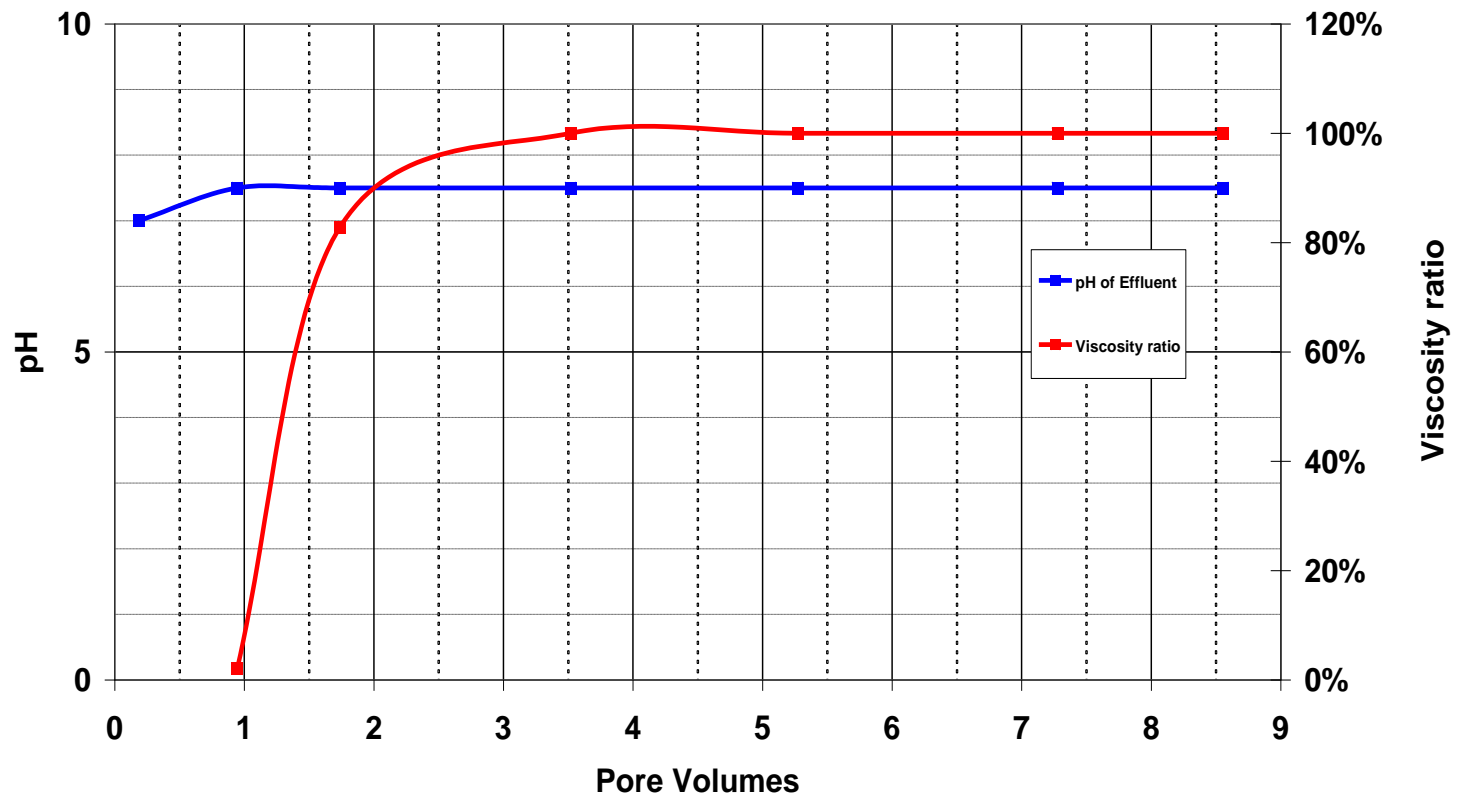

Figure 5.33 The normalized viscosity at $10 \mathrm{~s}^{-1}$ and $\mathrm{pH}$ data for H-EOR 63020 at $62{ }^{\circ} \mathrm{C}$. 
Scleroglucan Transport in Sandstone. Berea sandstone cores with an initial permeability 150 to $300 \mathrm{mD}$ were used for scleroglucan corefloods. A series of polymer corefloods were performed at different temperatures using the polymers filtered by two methods described above. In each polymer flood, the polymer concentration (1250 ppm) and the brine salinity (20,185 ppm TDS, $966 \mathrm{ppm} \mathrm{Ca}^{2+}$ and $\mathrm{Mg}^{2+}$ ) were kept constant. In all cases, the injection polymer solution passed the standard filtration test with filtration ratio of unity.

Figure 5.34 shows the pressure drop during polymer injection in Berea cores at different temperatures. At all temperatures, the pressure drop reached a steady state (around 5 PV), which ultimately indicated good transport behavior and absence of plugging in the core. However, the normalized viscosity of the effluent (by injection polymer viscosity) indicated that polymer breakthrough was drastically different for different temperatures. Figure 5.35 shows the relative viscosity of the effluent samples at different temperatures $\left(25,50,85\right.$ and $\left.100{ }^{\circ} \mathrm{C}\right)$. The polymer breakthroughs at lower temperatures were delayed indicating higher retention in the rock. For example, the polymer breakthrough at $25{ }^{\circ} \mathrm{C}$ was approximately $5 \mathrm{PV}$ compared to $3.5 \mathrm{PV}$ at $100{ }^{\circ} \mathrm{C}$. This trend of lower scleroglucan retention at higher temperatures is consistent with the observations reported in Rivenq et al. (1992). It is also noteworthy that final viscosity retention was above $95 \%$ for all cases, which again indicates the importance of good filterability of polymer solutions to understand and scale the polymer injection process. Cannella et al. (1988) reported that $\mathrm{C}=4$ (using the definition of $\mathrm{C}$ in this work) for xanthan gum polymer in Berea sandstone. The values of $\mathrm{C}$ for scleroglucan were estimated to be 2 to 3.1 , as given in Table 5.15. 
Table 5.15 Scleroglucan Transport in Berea Sandstone Cores

\begin{tabular}{|c|c|c|c|c|c|c|c|c|c|c|c|c|}
\hline \multirow{2}{*}{ Flood No. } & $\mathrm{T}$ & \multirow{2}{*}{$\begin{array}{l}\text { Clarificatio } \\
n \text { method* }\end{array}$} & \multirow{2}{*}{$\mathrm{kw}$} & Q & geff & \multirow{2}{*}{$\mathrm{C}$} & minjected & \multirow{2}{*}{$\mu \mathrm{p} / \mu \mathrm{w}$} & \multirow{2}{*}{$\mathrm{Rf}$} & \multirow{2}{*}{$\begin{array}{c}\text { FReffluen } \\
\mathrm{t}\end{array}$} & $\begin{array}{c}\text { Adsorpti } \\
\text { on }\end{array}$ & \multirow{2}{*}{$\frac{\text { PV }}{\text { injected }}$} \\
\hline & (oC) & & & (ft/day) & $(\mathrm{s}-1)$ & & $(\mathrm{ToC})$ & & & & $(\mu \mathrm{g} / \mathrm{g})$ & \\
\hline 1 & 25 & 1 & 200 & 10 & 14 & 2 & $42(25)$ & 42 & 42.14 & - & 235 & 9 \\
\hline $2 * *$ & 50 & 1 & 278 & 2 & 10 & 2.3 & $23(50)$ & 20.48 & 19.3 & - & 211 & 10 \\
\hline 3 & 85 & 2 & 244 & 10 & 53 & 2.2 & $61(25)$ & 28.75 & 36.3 & 1.2 & 110 & 20 \\
\hline 4 & 100 & 2 & 155 & 10 & 96 & 3.1 & $47.5(25)$ & 24.84 & 23.9 & 1 & 24 & 8 \\
\hline \multicolumn{13}{|c|}{ * Clarification method is the filtration method used in the experiments. 1- Four consecutive high pressure filtrations and 2- filtration after heat treatment. } \\
\hline
\end{tabular}

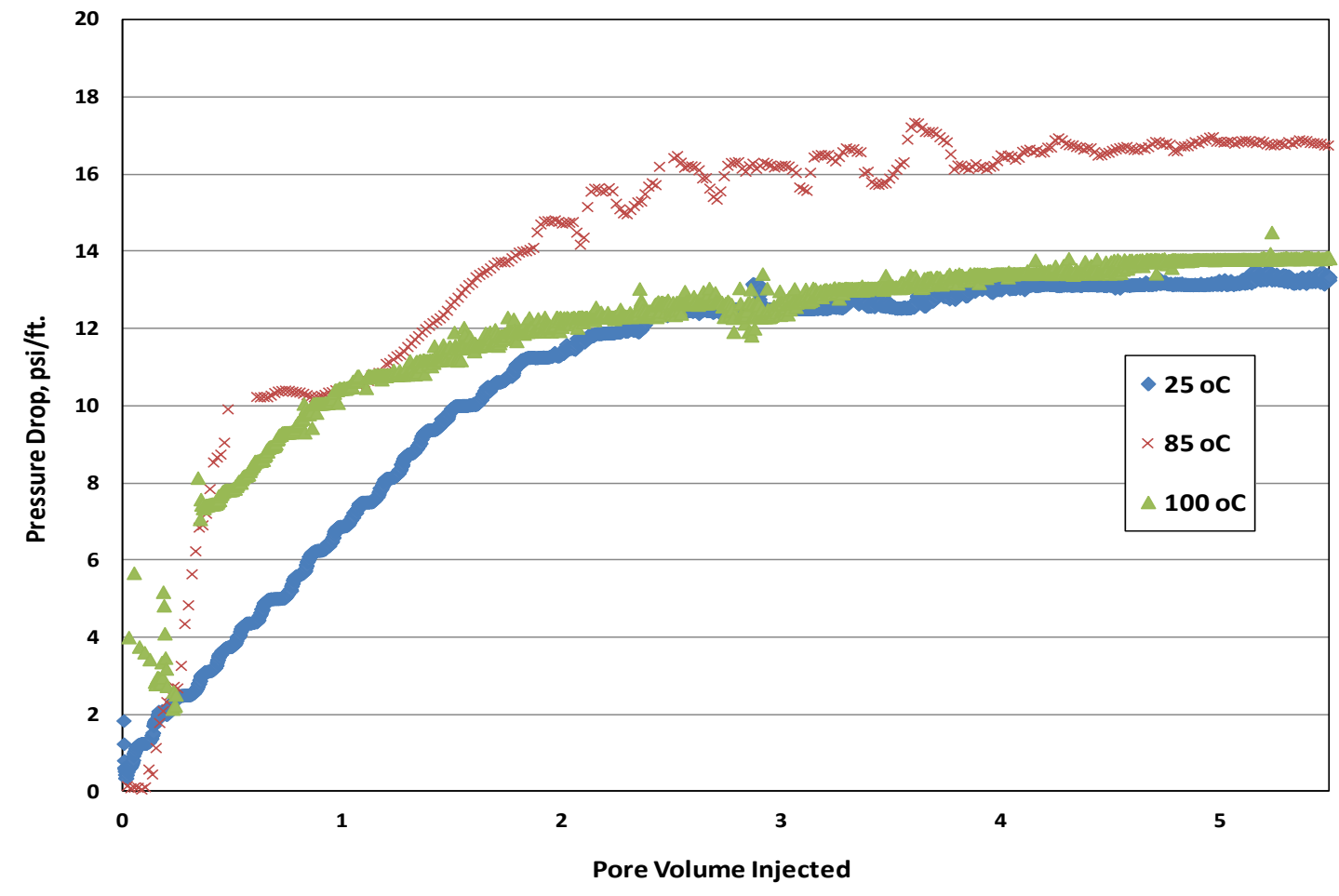

Figure 5.34 Pressure Drop in Berea Sandstone Cores at Different Temperatures at 9 $\mathrm{ft} /$ day injection of $1,250 \mathrm{ppm}$ scleroglucan in 20,185 ppm TDS Brine. 


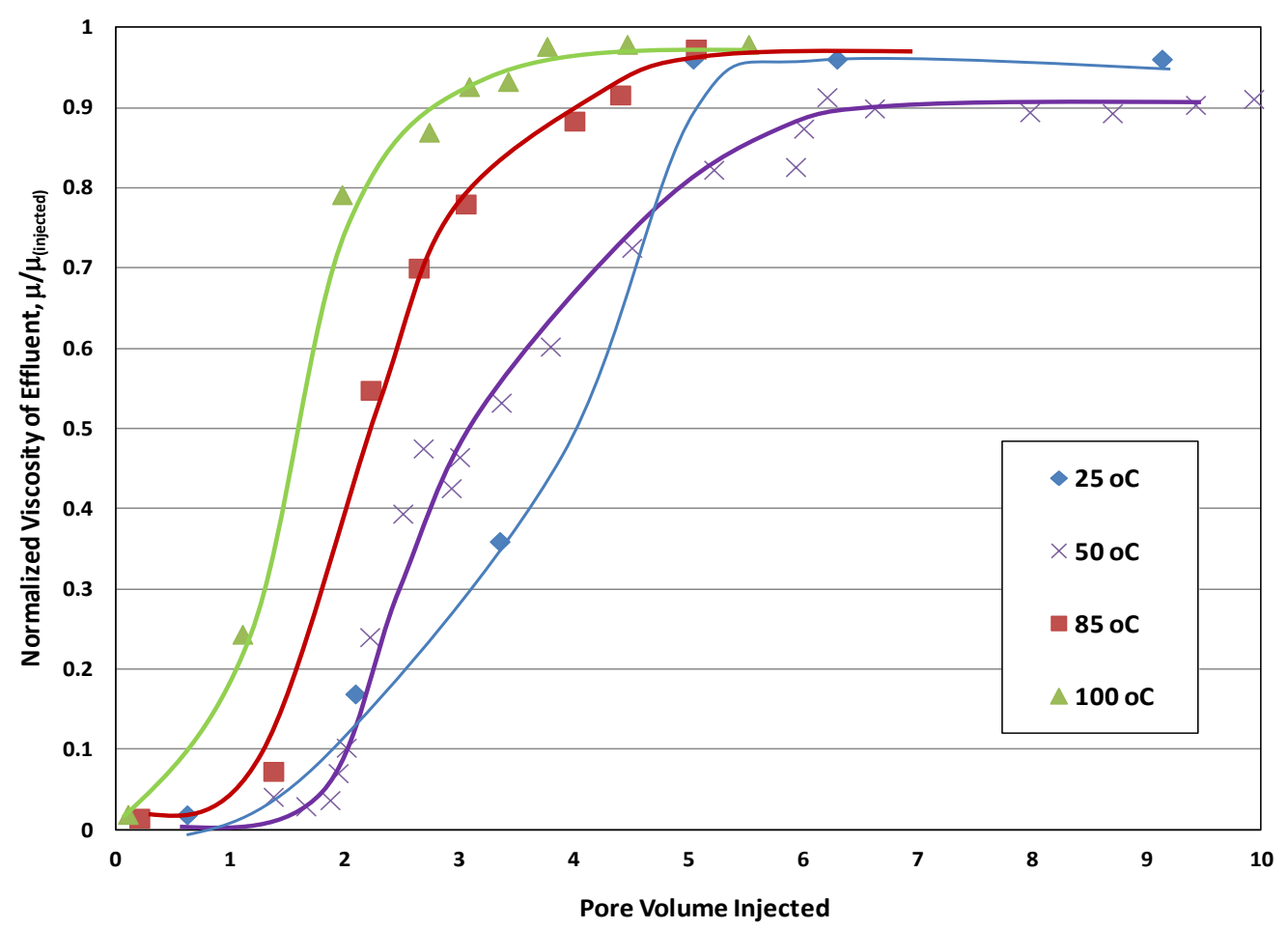

Figure 5.35 Normalized Viscosity of Effluent Samples during Scleroglucan Floods at Different Temperatures. 1,250 ppm scleroglucan in 20,185 ppm TDS Brine.

The transport of SAV 301 polymer in synthetic seawater was studied in an Estillades limestone core. Table 5.16 gives the results of the coreflood. Figure 5.36 shows the pressure drop data. The steady state was reached approximately after 1.5 pore volumes of injection, which indicates good transport of the polymer. Figure 5.37 shows the normalized viscosity of effluent samples approached the injected value after three pore volumes. The $\mathrm{C}$ value for SAV 301 in Bentheimer cores is about 2 in Table 5.16. The dynamic polymer retention in the Estillades core was estimated using the effluent polymer viscosity data and the delay in the polymer breakthrough. This method is subject to several uncertainties, but polymer concentration data were not measured so it was used as a rough estimate of retention. Figure 5.37 shows the polymer retention results for SAV 
301 in the Estillades limestone core at $83{ }^{\circ} \mathrm{C}$. The retention of SAV 301 polymer seems to be lower than for scleroglucan.

Table 5.16 SAV-301 Transport Summary in Estillades Limestone Core

\begin{tabular}{|c|c|c|c|c|c|c|c|c|c|c|}
\hline \multirow{2}{*}{ Flood No. } & $\mathrm{T}$ & \multirow{2}{*}{$\mathrm{k}_{\mathrm{w}}$} & Q & $\mathrm{g}_{\text {eff }}$ & \multirow{2}{*}{$\mathrm{C}$} & $\mathrm{m}_{\text {injected }}$ & \multirow{2}{*}{$\mu_{\mathrm{p}} / \mu_{\mathrm{w}}$} & \multirow{2}{*}{$\mathrm{R}_{\mathrm{f}}$} & $\begin{array}{c}\text { Adsorpti } \\
\text { on }\end{array}$ & $\mathrm{PV}$ \\
\hline & $\left({ }^{\circ} \mathrm{C}\right)$ & & (ft/day) & $\left(\mathrm{s}^{-1}\right)$ & & $\left(\mathrm{T}^{\circ} \mathrm{C}\right)$ & & & $(\mu \mathrm{g} / \mathrm{g})$ & injected \\
\hline 1 & 83 & 150 & 1.45 & 14 & 2 & $10.8(25)$ & 11.25 & 11.78 & 35 & 3.5 \\
\hline
\end{tabular}

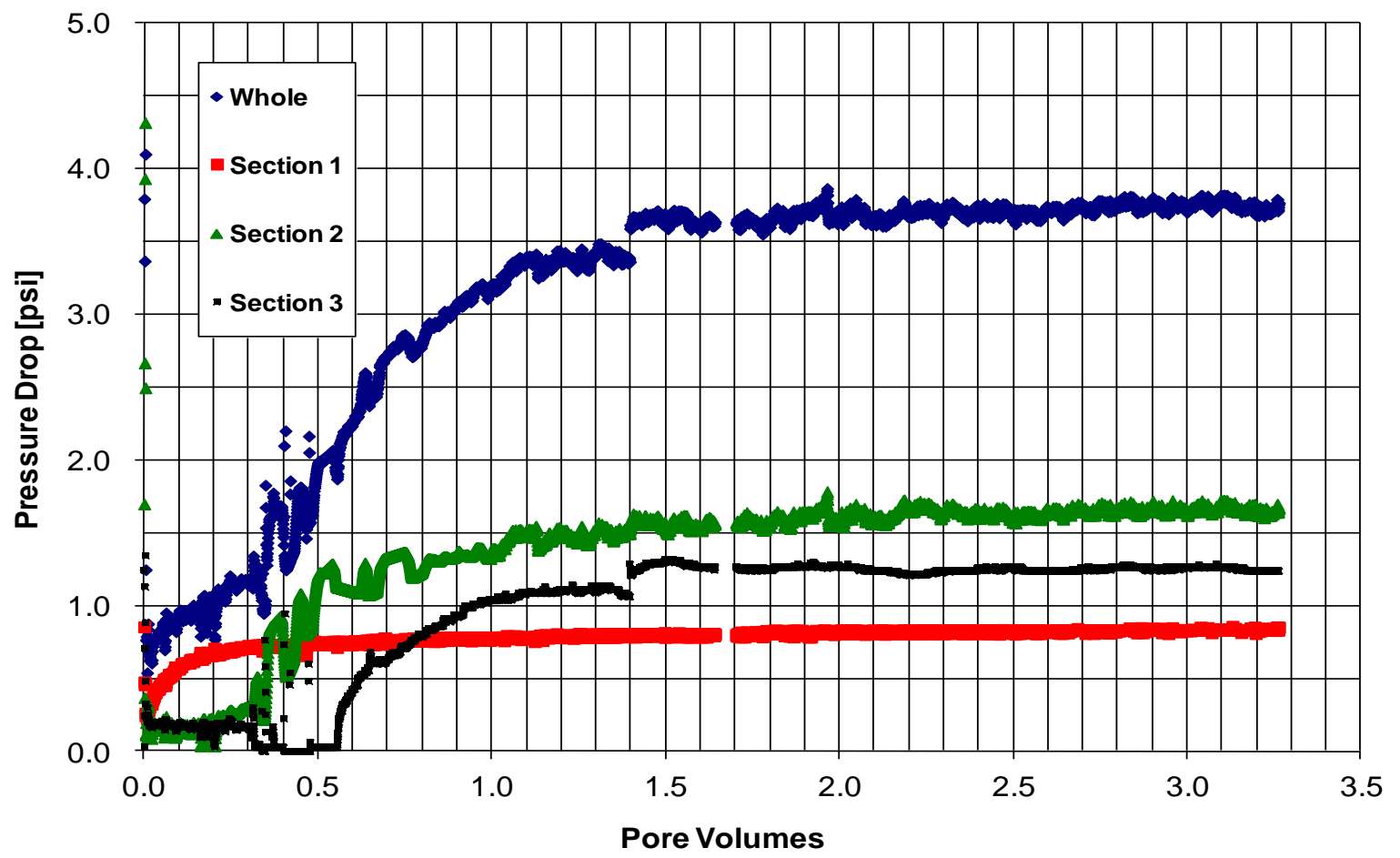

Figure 5.36 Pressure drop for SAV-301 polymer flood in Estillades Limestone core.

Polymer Concentration $=3000$ ppm, Salinity $=29000$ ppm TDS, velocity $=2$ $\mathrm{ft} /$ day. 


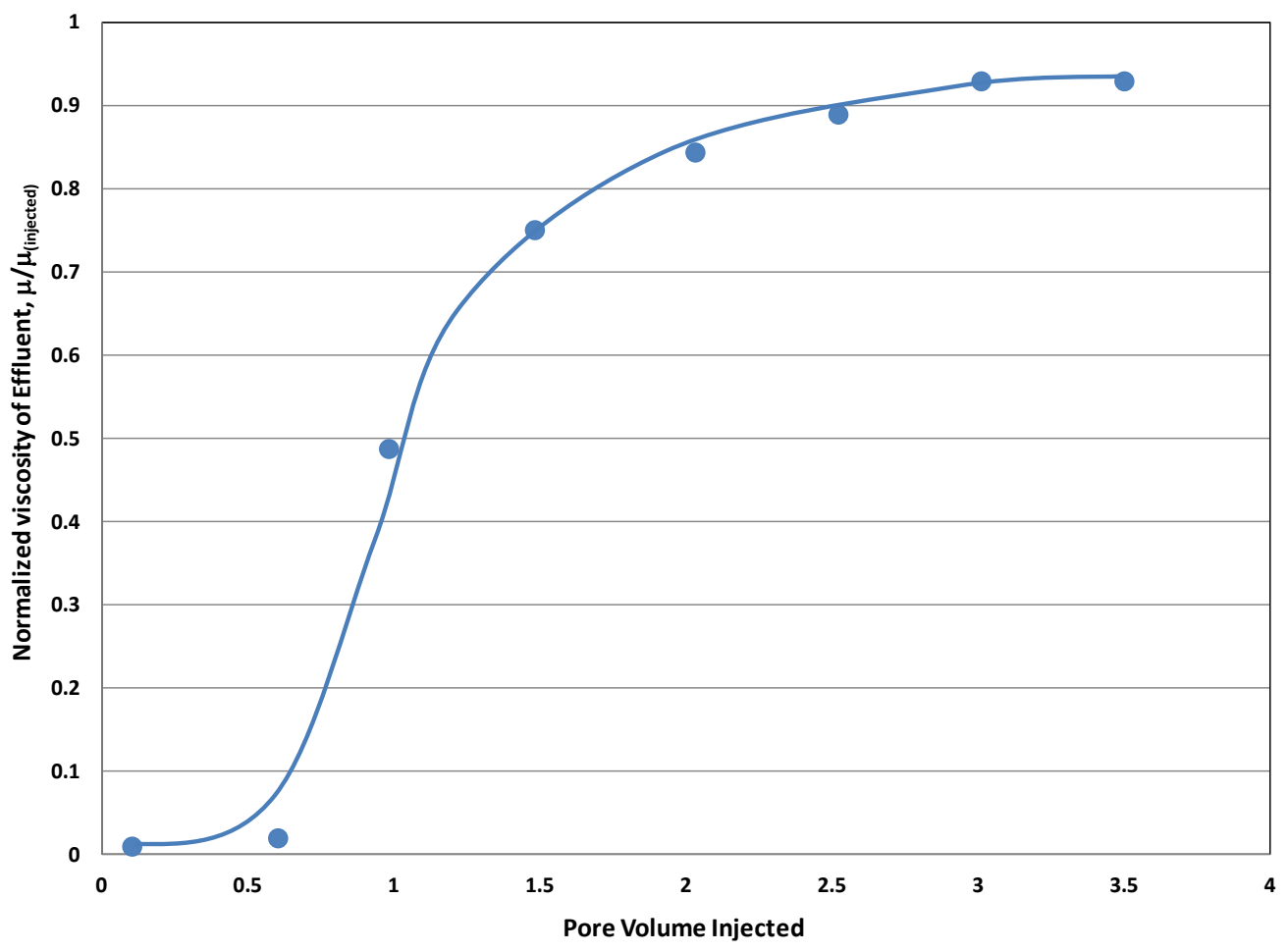

Figure 5.37 SAV 301 viscosity retention. Polymer concentration=3000 ppm, salinity= 29000 ppm TDS 


\subsubsection{Viscoelastic Behavior of High Molecular Weight Polymers in Porous Media}

The effect of increasing water viscosity on oil recovery has been studied for decades. An important characteristic of all synthetic and biopolymers used for enhanced oil recovery is that their viscosity decreases with increase in shear rate (a.k.a. shearthinning). This behavior has been exploited in improving the injectivity of these viscous polymer solutions. It also implies that in high permeability zones in the reservoir, the viscosity of the injected solution would be lower than in the low permeability zones, hence decreasing the desired sweep efficiency. Another key characteristic of HPAM polymer solutions is that after a certain critical flow velocity (or apparent shear rate), the apparent viscosity of the solution begins to increase (shear thickening). This shearthickening behavior has been attributed to the viscoelastic nature of the polymers. In principle, this could be exploited for better sweep efficiency in the high permeability zones of the reservoir, since the apparent viscosity of the solution would increase with increase in flow velocity. This advantage of shear thickening caused by viscoelasticity of polymer solution has been shown in laboratory experiments by Jones (1980). The viscoelastic property of HPAM has also been reported to reduce residual oil saturation (Wang et al., 2001).

\section{Experimental data and analysis}

Magbagbeola (2008) performed the laboratory experiments to estimate relaxation times of polymer solutions and to obtain the apparent viscosities of polymer solutions flowing through porous media. He applied a unified apparent viscosity model (UVM) (Delshad et al., 2008) to fit the experimental data. The UVM is expressed as follows:.

$$
\mu_{\text {app }}=\mu_{\infty}+\left(\mu_{p}^{0}-\mu_{\infty}\right)\left[1+\left(\lambda \dot{\gamma}_{e q}\right)^{\alpha}\right]^{(n-1) / \alpha}+\mu_{\max }\left[1-\exp \left(-\left(\lambda_{2} \tau_{r} \dot{\gamma}_{e q}\right)^{n_{2}-1}\right)\right]
$$


where $\mu_{\infty}, \mu_{\mathrm{p}}^{\circ}, \lambda, \mathrm{n}$ and $\alpha$ are empirical constants that will be obtained from the polymer's bulk viscosity data. The other parameters in the UVM are:

$\mu_{\max }$ : A plateau value of viscosity beyond which shear degradation will occur.

$\lambda_{2}$ : A proposed 'universal' constant that is independent of data being matched.

$\tau_{\mathrm{r}}$ : Relaxation time of injected fluid. It is obtained from the dynamic frequency sweep test.

$\mathrm{n}_{2}$ : Shear-thickening index. It indicates the extent of polymer 'dilatancy'.

These are the fitting parameters required for the application of the unified apparent viscosity model.

Magbagbeola (2008) measured bulk viscosity and relaxation time for several polymer solutions and conducted polymer corefloods at several injection rates to measure apparent viscosity in the Berea sandstone.

Table 5.17 Polymer solutions used for corefloods

\begin{tabular}{|c|c|c|c|c|c|}
\hline Parameter & BV-1 & BV-2 & BV-3 & BV-4 & BV-5 \\
\hline Polymer & FP 3630S & AN-125 & HJ 63020 & HJ 63020 & HJ 63026 \\
\hline $\begin{array}{c}\text { M.W. } \\
\text { (Daltons) }\end{array}$ & $20 \times 10^{6}$ & $8 \times 10^{6}$ & $20 \times 10^{6}$ & $20 \times 10^{6}$ & $26 \times 10^{6}$ \\
\hline $\begin{array}{c}\text { Salinity of } \\
\text { Brine }\end{array}$ & $\begin{array}{c}2 \% \text { hard } \\
\text { brine }\end{array}$ & $\begin{array}{c}2 \% \text { hard } \\
\text { brine }\end{array}$ & $\begin{array}{c}2 \% \text { hard } \\
\text { brine }\end{array}$ & $\begin{array}{c}1.5 \% \text { soften } \\
\text { brine w/ 20 } \\
\text { ppm Ca }\end{array}$ & $\begin{array}{c}1.5 \% \text { soften } \\
\text { brine w/ } 20 \\
\mathrm{ppm} \mathrm{Ca}^{2+}\end{array}$ \\
\hline D.H & $30 \%$ & $20-30 \%$ & $28-30 \%$ & $28-30 \%$ & $28-30 \%$ \\
\hline $\begin{array}{c}\text { Polymer } \\
\text { concentration } \\
\text { (ppm) }\end{array}$ & 1500 & 1500 & 1500 & 1500 & 1500 \\
\hline $\begin{array}{c}\text { Relaxation } \\
\text { time (s) }\end{array}$ & 0.0859 & 0.0605 & 0.045 & 0.0671 & 0.136 \\
\hline
\end{tabular}


Table 5.18 Summary of core properties

\begin{tabular}{|c|c|c|c|c|c|}
\hline Property & BV-1 & BV-2 & BV-3 & BV-4 & BV-5 \\
\hline Mass (g) & 211.14 & 207.4 & 208.79 & 207.0 & 206.6 \\
\hline Porosity & 0.23 & 0.24 & 0.235 & 0.24 & 0.243 \\
\hline Length $(\mathrm{cm})$ & 20.32 & 20.32 & 20.32 & 20.32 & 20.32 \\
\hline Diameter $(\mathrm{cm})$ & 2.54 & 2.54 & 2.54 & 2.54 & 2.54 \\
\hline Area $\left(\mathrm{cm}^{2}\right)$ & 5.07 & 5.07 & 5.07 & 5.07 & 5.07 \\
\hline Pore Volume & 24.86 & 24.26 & 24.66 & 24.7 & 23.06 \\
\hline Brine Permeability (mD) & 647 & 578 & 552 & 372 & 260 \\
\hline
\end{tabular}

Table 5.19 Apparent viscosity of FP3630S in experiment BV-1

\begin{tabular}{|c|c|c|c|}
\hline Flow rate, (ml/min) & Pressure drop, (psi) & $\begin{array}{c}\text { Apparent } \\
\text { viscosity, (cp) }\end{array}$ & Velocity, (ft/day) \\
\hline 0.44 & 10.60 & 15.86 & 18.1 \\
\hline 0.94 & 27.50 & 19.27 & 38.7 \\
\hline 2.00 & 144.07 & 47.44 & 82 \\
\hline
\end{tabular}

Based on Magbagbeola's coreflood data and the UVM model, apparent viscosity profile for entire range of velocity was generated as shown in Figures 5.38 and 5.39. 


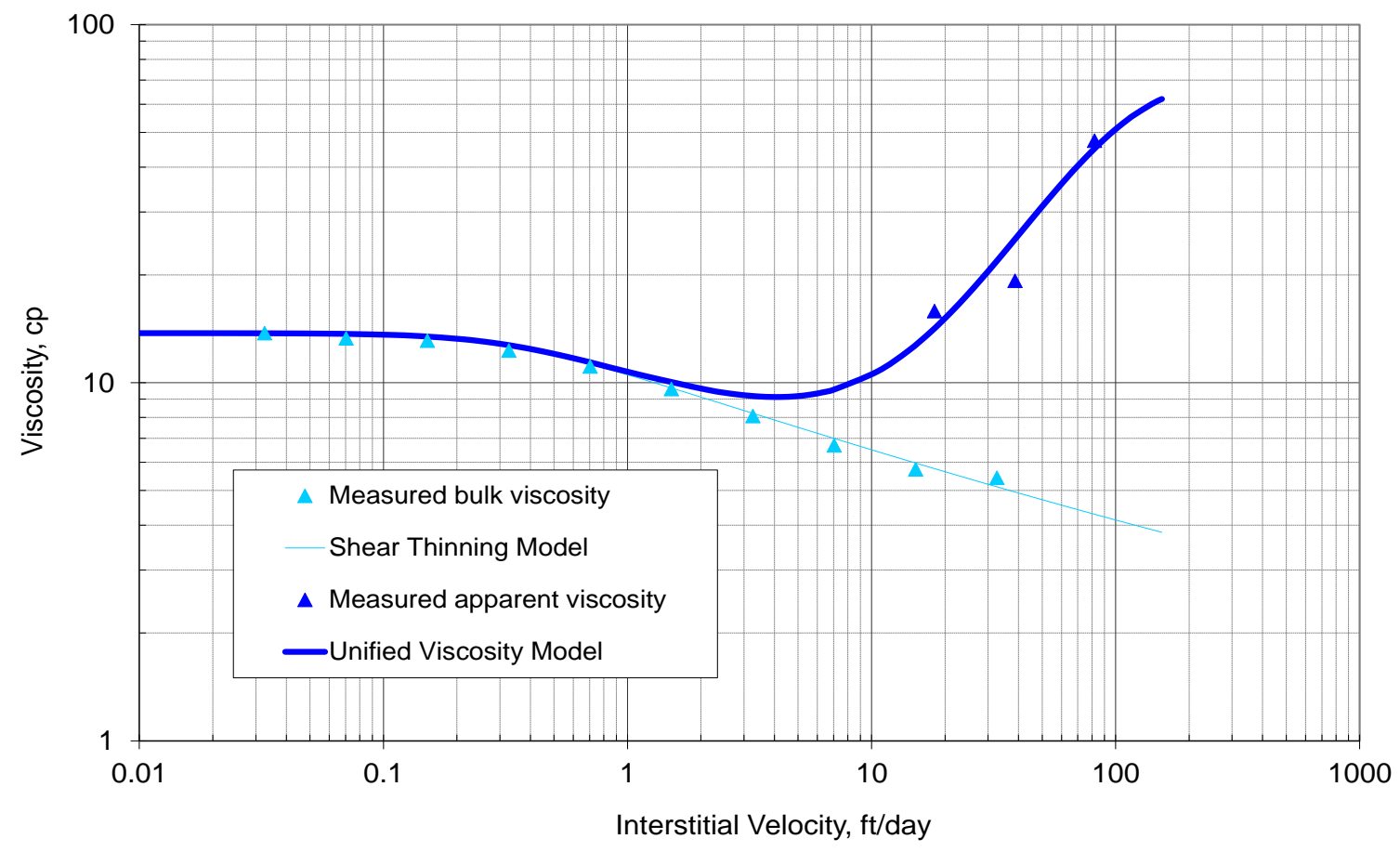

Figure 5.38 Unified apparent viscosity model fit for 1500 ppm FP 3630 S in $2 \%$ soften brine with velocity

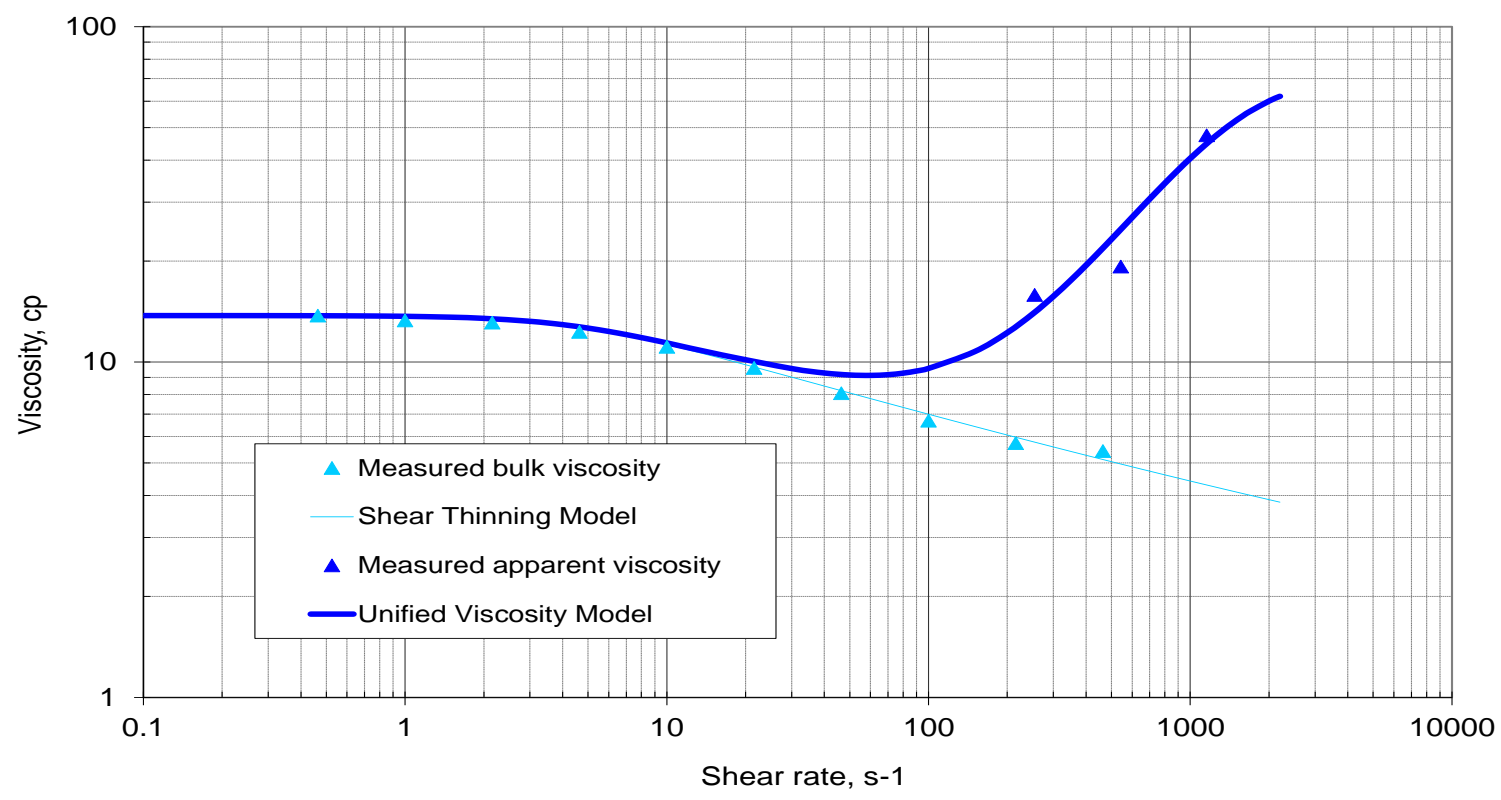

Figure 5.39 Unified apparent viscosity model fit for 1500 ppm FP 3630S in $2 \%$ soften brine with shear rate 
The critical velocity to start showing shear thickening behavior is $4 \mathrm{ft} / \mathrm{day}$ and. the equivalent shear rate at this velocity is $62 \mathrm{~s}^{-1}$.

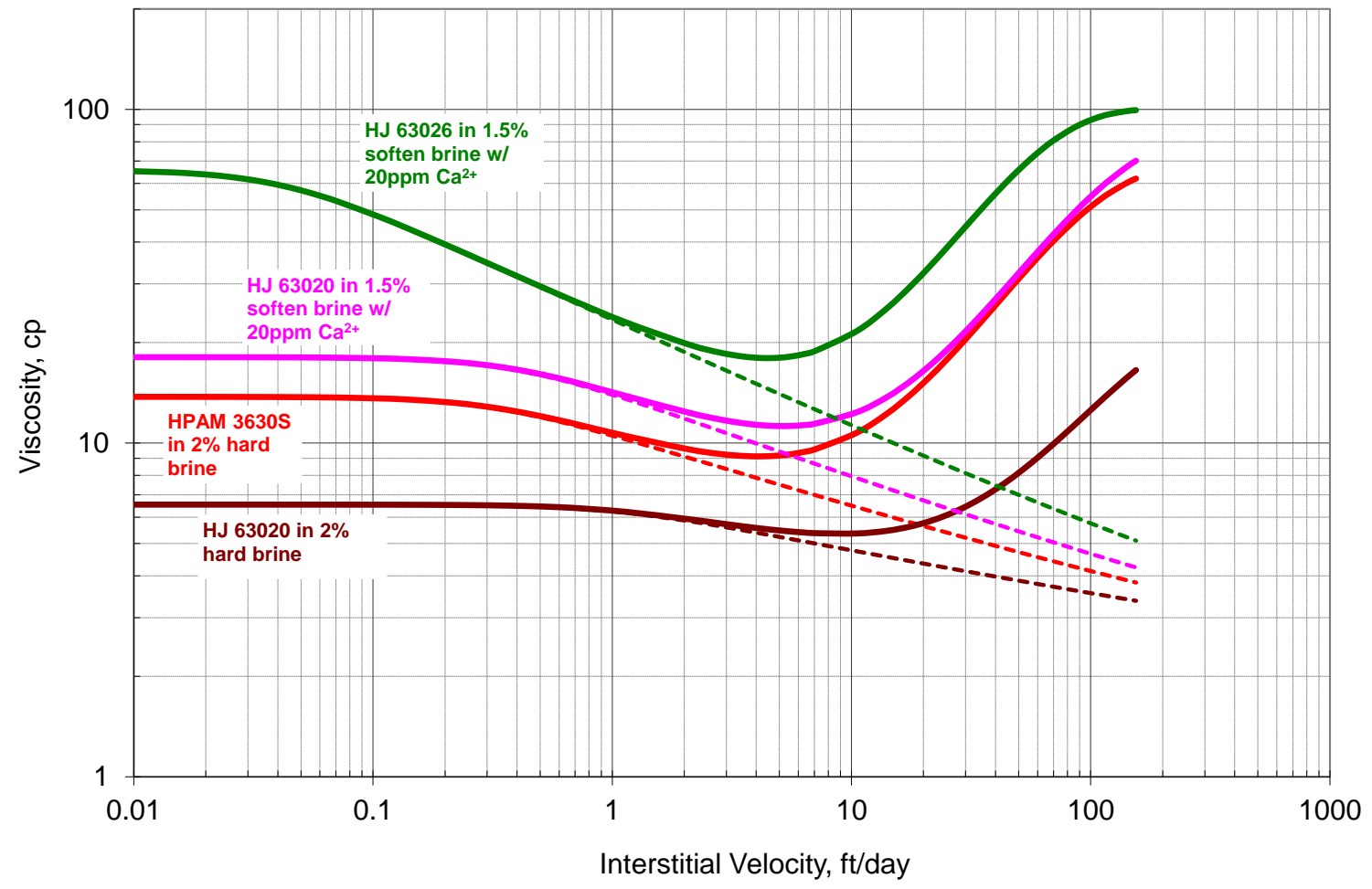

Figure 5.40 Comparison of the unified apparent viscosity model fit for all four polymers.

Figure 5.40 shows apparent viscosity for each polymer solution. In Figure 5.40, FP 3630 S and HJ 63026 showed the lowest critical velocity for shear thickening of about $4 \mathrm{ft} /$ day. HJ 63020 in $2 \%$ hard brine showed the highest value of the critical velocity of about $10 \mathrm{ft} /$ day. From Equation 4.3, The Deborah number has also been expressed as:

$$
\mathrm{De}=\lambda \dot{\gamma}_{e q}
$$

The Deborah number was calculated by using Equation 5.3 where $\lambda$ is the relaxation time and the equivalent shear rate is calculated using Equation 5.1 with $\mathrm{C}=6$. In Table 5.20, the range of Deborah number at the critical velocity for each polymer coreflood is between 4 and 6.3 . 
Table 5.20 Deborah number for core floods

\begin{tabular}{|c|c|c|c|c|}
\hline Parameter & BV-1 & BV-3 & BV-4 & BV-5 \\
\hline Polymer & FP 3630S & HJ 63020 & HJ 63020 & HJ 63026 \\
\hline $\begin{array}{c}\text { M.W. } \\
\text { (Daltons) }\end{array}$ & $20 \times 10^{6}$ & $20 \times 10^{6}$ & $20 \times 10^{6}$ & $26 \times 10^{6}$ \\
\hline $\begin{array}{c}\text { Salinity of } \\
\text { Brine }\end{array}$ & $2 \%$ hard brine & $2 \%$ hard brine & $\begin{array}{c}1.5 \% \text { soften } \\
\text { brine w/ 20 } \\
\mathrm{ppm} \mathrm{Ca}^{2+}\end{array}$ & $\begin{array}{c}1.5 \% \text { soften } \\
\text { brine w/ 20 } \\
\mathrm{ppm} \mathrm{Ca}^{2+}\end{array}$ \\
\hline $\begin{array}{c}\text { Polymer } \\
\text { concentration } \\
\text { (ppm) }\end{array}$ & 1500 & 1500 & 1500 & 1500 \\
\hline $\begin{array}{c}\text { Relaxation } \\
\text { time (s) }\end{array}$ & 0.0626 & 0.05 & 0.051 & 0.073 \\
\hline $\begin{array}{c}\text { Critical } \\
\text { velocity } \\
\text { (ft/day) }\end{array}$ & 4 & 10 & 62 & 65 \\
\hline $\begin{array}{c}\text { Equivalent } \\
\text { shear rate (s }{ }^{-1} \text { ) } \\
\text { @ critical } \\
\text { velocity }\end{array}$ & 65 & 6.3 & 4.1 & 4.7 \\
\hline $\begin{array}{c}\text { Deborah } \\
\text { number @ } \\
\text { critical } \\
\text { velocity }\end{array}$ & 4 & 126 & 62 & 4 \\
\hline
\end{tabular}

\subsection{SUMMARY AND CONCLUSIONS}

The behavior of HPAM polymers, scleroglucan biopolymer and N-

vinylpyrrolidone (NVP)- polyacrylamide (AM) co-polymer were studied and compared..

The results can be summarized as follows:

1. Scleroglucan can withstand harsh reservoir conditions such as high temperature and high salinity/hardness while retaining its viscosity. But insoluble impurities must first be removed before it can be injected into reservoirs for enhanced oil recovery. Two filtration methods were developed 
in the lab to remove insoluble impurities. The first method consists of four consecutive high pressure filtrations with decreasing filter pore sizes $(5-10,5$, 3, 1.2 microns) at a high pressure of $50 \mathrm{psi}$. The second method was to filter scleroglucan solutions at a high temperature $\left(85{ }^{\circ} \mathrm{C}\right)$ through a 1.2 micron filter at 50 psi. After filtration, polymer solutions resulting from both methods passed a standard filtration test routinely used in the laboratory. The second method followed by a filtration step through a Berea core was evidently the most cost-effective method to filter larger volumes. Additional refinement of these methods would be justified. Scleroglucan that passed the standard filtration test with filtration ratios less than 1.2 showed good transport behavior in $150-300 \mathrm{mD}$ Berea cores with no evident plugging. The retention of scleroglucan decreased as the temperature increased.

2. The NVP-AM polymers tested in this study had very good thermal stability at $100{ }^{\circ} \mathrm{C}$ in synthetic seawater and good transport in cores with low retention, but have lower intrinsic viscosity than scleroglucan.

3. Two high molecular weight HPAM polymers (FP 3630S and H-EOR 63020) showed excellent transport behavior in Bentheimer cores with steady state pressure drops across each section of the core. The resistance factor was in excellent agreement with the normalized viscosity and the permeability reduction factor was 1 . The shear rate correction factor was very small for both polymers (about 1.1).

4. The critical velocity for the onset of shear thickening was between $4 \mathrm{ft} / \mathrm{day}$ and $10 \mathrm{ft} /$ day. The corresponding range of Deborah number is between 4 and 6.3 . 


\section{Chapter 6. Oil Recovery Experiments}

\subsection{INTRODUCTION}

Several polymer coreflood experiments have been done using both sandpacks and reservoir cores starting at different water cuts to measure the effect of polymer on the residual oil saturation. In some cases, it would be more accurate to refer to the oil saturation at the end of the flood as remaining oil saturation rather than residual oil saturation.

Nine experiments have been carried out in high permeability sandpacks and three experiments in low permeability reservoir cores. Three sandpacks were made in glass columns and three sandpacks were made in a stainless core holder with 2000 psi confining pressure. Tracer data indicated that the sand packs were nearly homogeneous whereas a tail was observed in the tracer data for the reservoir cores showing they were not homogeneous. The experiments were conducted at reservoir temperature.

Three polymers were used in the transport experiments: FP 3330S and FP 3630S supplied by SNF Floeger and ChemPam 8177 supplied by ChemPam. The molecular weight of FP 3330S is $8 \mathrm{M}$ and the molecular weight of FP $3630 \mathrm{~S}$ is $18 \mathrm{M}$. FP $3330 \mathrm{~S}$ and FP3630S are co-polymers of acrylamide (65\%) and acrylic acid (35\%) (Sheng, 2010; Thomas et al., 2012). ChemPam 8177 is a hydrolyzed polyacrylamide with a molecular weight of $18 \mathrm{M}$. Five different crude oils were used for the corefloods.

\subsection{COREFLOOD EXPERIMENTAL SETUP AND BACKGROUND}

\subsubsection{Coreflood Experimental Setup}

This section describes the details of the set-up of the coreflood experiments carried out to study the transport of polymer solutions for chemical EOR applications. 
Brine flooding experiments were performed to determine the petrophysical properties of the core. A schematic of the coreflood setup is shown in Figure 6.1. It consists of a syringe pump, fluid reservoir, core-holder, pressure transducers, tubing and fraction collectors. Using the pump, the brine or polymer solution in the fluid reservoir is injected into the core which is contained in the core-holder. The pressure difference between sections of the core is measured by the pressure transducers. Effluent samples are collected with the fraction collector and their viscosities measured to ensure consistency with the injected fluid.

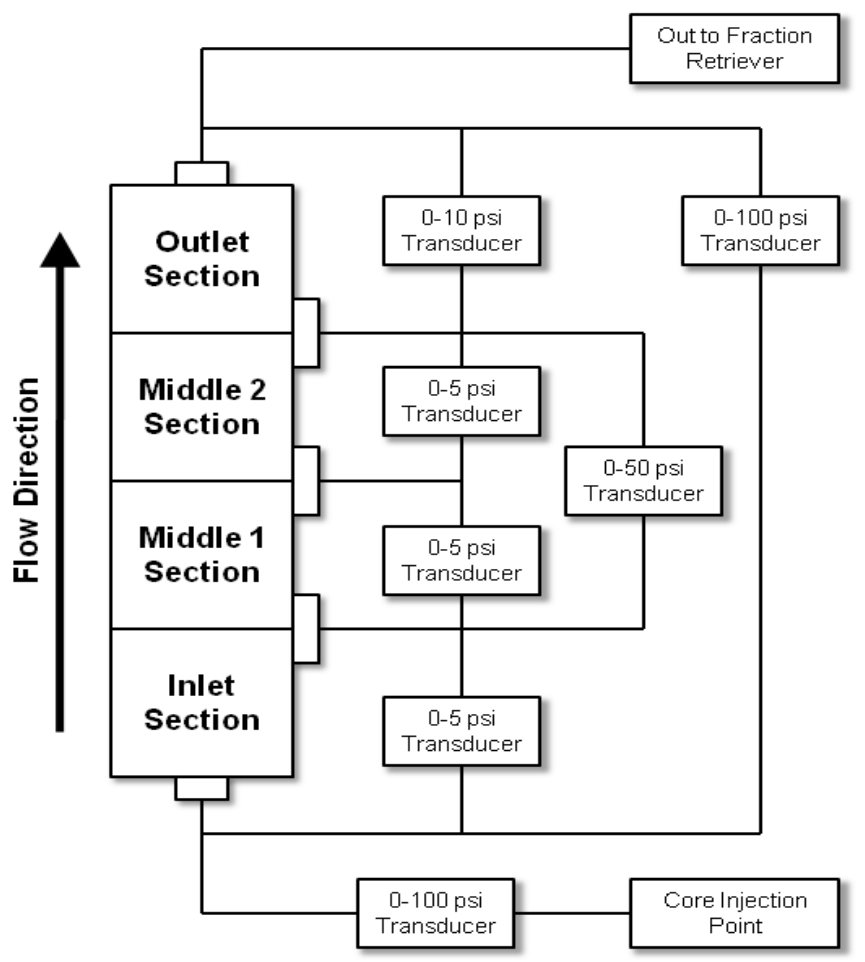

Figure 6.1 Schematic coreflood setup 


\subsubsection{Backgound}

\section{Mechanism of Oil Displacement}

The mobility ratio $(\mathrm{M})$ and the capillary number $(\mathrm{Nc})$ affect the oil remaining after a reservoir displacement process such as a water flood or polymer flood. The mobility ratio is defined as the ratio of the mobility of the displacing fluid (e.g., water or gas) to the mobility of the displaced fluid (e.g., oil), $\mu$ is the viscosity of the fluid, and $\mathrm{k}$ is the effective permeability. To achieve a favorable mobility ratio $(\mathrm{M} \leq 1)$, a polymer can be added to the water to increase the viscosity of the displacing fluid. The mobility ratio affects both the displacement sweep efficiency (microscopic sweep efficiency) and the volumetric sweep efficiency (two and three-dimensional reservoir sweep efficiency).

The capillary number is defined as the ratio of viscous to capillary forces and is a special case of the trapping number (Pope et al., 2000). The most effective way of increasing the capillary number is by reducing the interfacial tension. The true residual oil saturation is the oil trapped by capillary forces at high interfacial tension and is primarily a function of capillary number.

Residual oil saturation is usually assumed to be the same for both water floods and polymer floods if the flood is done at the same capillary number, but for viscous oil the remaining oil saturation is often much less for polymer flooding than for water flooding since only a few pore volumes can be injected in a reservoir under most circumstances. Under at least some conditions, viscoelastic polymers appear to reduce the residual oil saturation compared to water (Huh and Pope, 2008), but whether it is a reduction in residual oil or remaining oil saturation is uncertain. There are several ways to use polymers to improve oil recovery. The water mobility is reduced by increasing its viscosity and under some conditions by reducing its permeability. Permeability reduction 
is less important than viscosity increase. The HPAM polymers used in this research had negligible permeability reduction in the sandpacks and reservoir cores tested.

\section{Mobility Ratio}

The ultimate goal of EOR processes is to increase the oil production, which is a function of both the microscopic sweep efficiency and the volumetric (macroscopic) sweep efficiency. Microscopic efficiency refers to the displacement or mobilization of oil at the pore scale and measures the effectiveness of the displacing fluid in moving the oil at those places in the rock where the displacing fluid contacts the oil (Green and Willhite, 1998). Volumetric displacement efficiency refers to the effectiveness of the displacing fluid(s) in contacting the reservoir in a volumetric sense.

Volumetric sweep efficiency, also known as reservoir conformance, indicates the effectiveness of the displacing fluid in contacting the oil in the reservoir, both areally and vertically, as well as how effectively it displaces the oil toward production wells (Green and Willhite, 1998). Figure 6.2 presents a schematic of sweep efficiencies: microscopic and macroscopic (areal sweep and vertical sweep). 


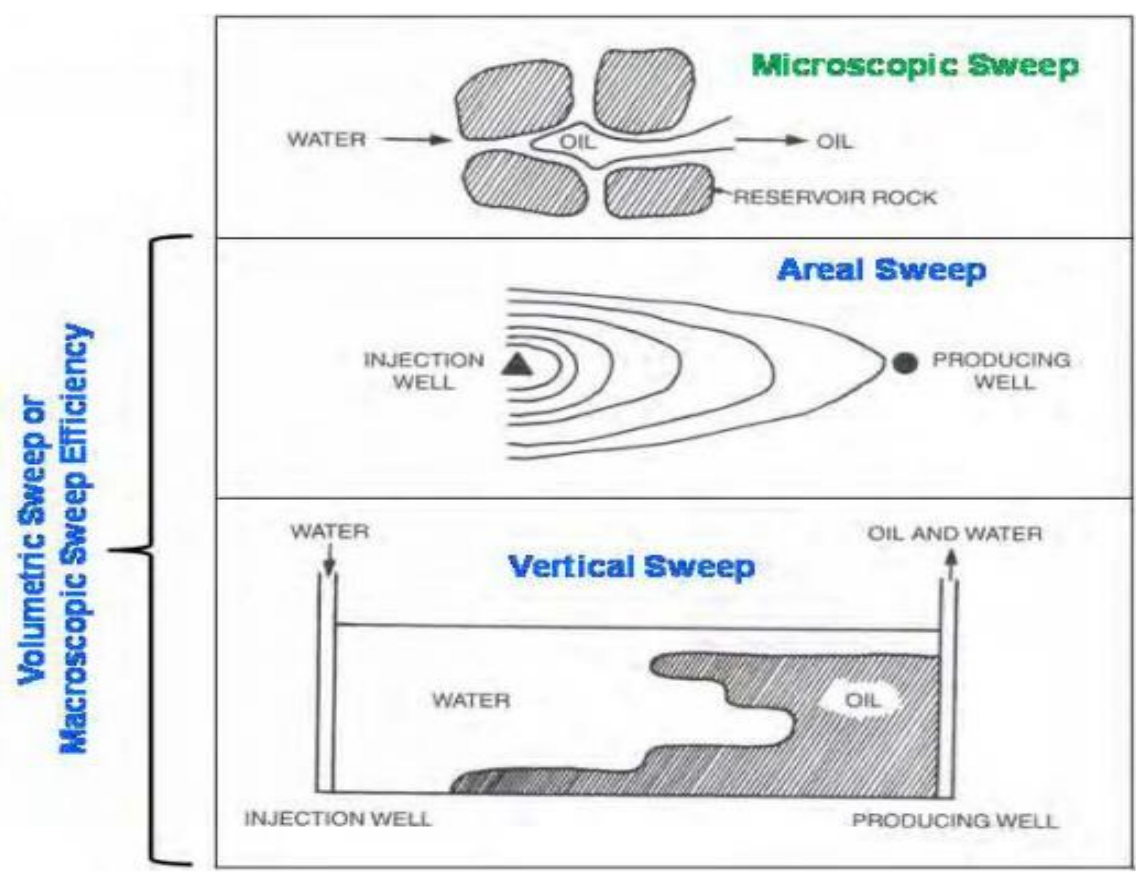

Figure 6.2 Schematic of microscopic and macroscopic sweep efficiencies (Lyons and Plisga, 2005)

The mobility ratio, $\mathrm{M}$, for a waterflood is given by the following expression:

$$
\mathrm{M}=\frac{\text { Mobility of displacing fluid }}{\text { Mobility of displaced fluid }}=\frac{\lambda_{w}}{\lambda_{o}}=\frac{k_{r w} / \mu_{w}}{k_{r o} / \mu_{o}}
$$

The end-point mobility ratio is the mobility ratio using the end-point values of the relative permeability as follows:

$$
\mathrm{M}^{o}=\frac{k_{r w}^{o} / \mu_{w}}{k_{r o}^{o} / \mu_{o}}
$$

where $\lambda_{w}$ and $\lambda_{o}$ are water and oil mobilities, respectively, in md/cp; krw and kro are relative permeabilities to water and oil, respectively, $\mu_{o}$ is oil viscosity and $\mu_{w}$ is water viscosity. 
Volumetric sweep efficiency increases as $M$ decreases, therefore mobility ratio is an indication of the stability of a displacement process, with flow becoming unstable (non-uniform displacement front or viscous fingering) when the mobility ratio is unfavorable $(M>1.0)$. Capillary forces can attenuate fingers even for mobility ratios slightly above one, but eventually fingering will occur if $\mathrm{M}$ is much greater than one. Bypassing of the oil due to heterogeneity is called channeling (Chang et al., 1994). Lowering the mobility ratio attenuates the negative impact of channeling. .

Both the displacement and volumetric sweep efficiencies continue to increase even for values of $\mathrm{M}$ less than 1. Thus, there are several benefits to adding polymer to water to reduce its mobility in a reservoir. The impact of mobility ratio on the

displacement sweep efficiency can be calculated using fractional flow theory (Pope, 1980). Fractional flow theory was applied to the coreflood experiments shown later in this chapter.

\section{Capillary Number}

Typically the pressure gradient in oil reservoirs is much too low to cause the capillary number to exceed the critical capillary number where the residual oil saturation starts to decrease. However, there are exceptions. One exception is close to injection wells where the pressure gradient is much higher than over $99 \%$ of the reservoir. The near wellbore region is insignificant from the point of view of oil recovery, so it is appropriate to do the coreflood experiments at a low pressure gradient representative of most of the reservoir. Another possible exception is for carbonate formations with a very wide pore size distribution. Even a modest increase in the local pressure gradient can cause a reduction in the residual oil saturation in the largest pores of a carbonate rock. However, this investigation is focused entirely on sands and sandstones, so this is not 
relevant to the analysis of these coreflood experiments. A large increase in viscosity due to adding polymer to the water does not itself increase the capillary number for a fixed pressure gradient. This can be inferred from the following definition of capillary number in terms of pressure gradient for a linear displacement:

$$
N_{C}=\frac{k(\Delta p / L)}{\sigma}
$$

The permeability in Equation (6.3) is the brine permeability at $\mathrm{Sw}=1$ (the so called absolute permeability), not the effective water permeability in the presence of oil. The pressure gradient was kept low in all of the coreflood experiments so that the critical capillary number would not be exceeded. The capillary numbers for these corefloods ranged from $7.1 \times 10^{-8}$ to $7.5 \times 10^{-6}$. Even the highest value of capillary number is less than a typical value for the critical capillary number of sands and sandstones such as shown in Figure 6.3. 

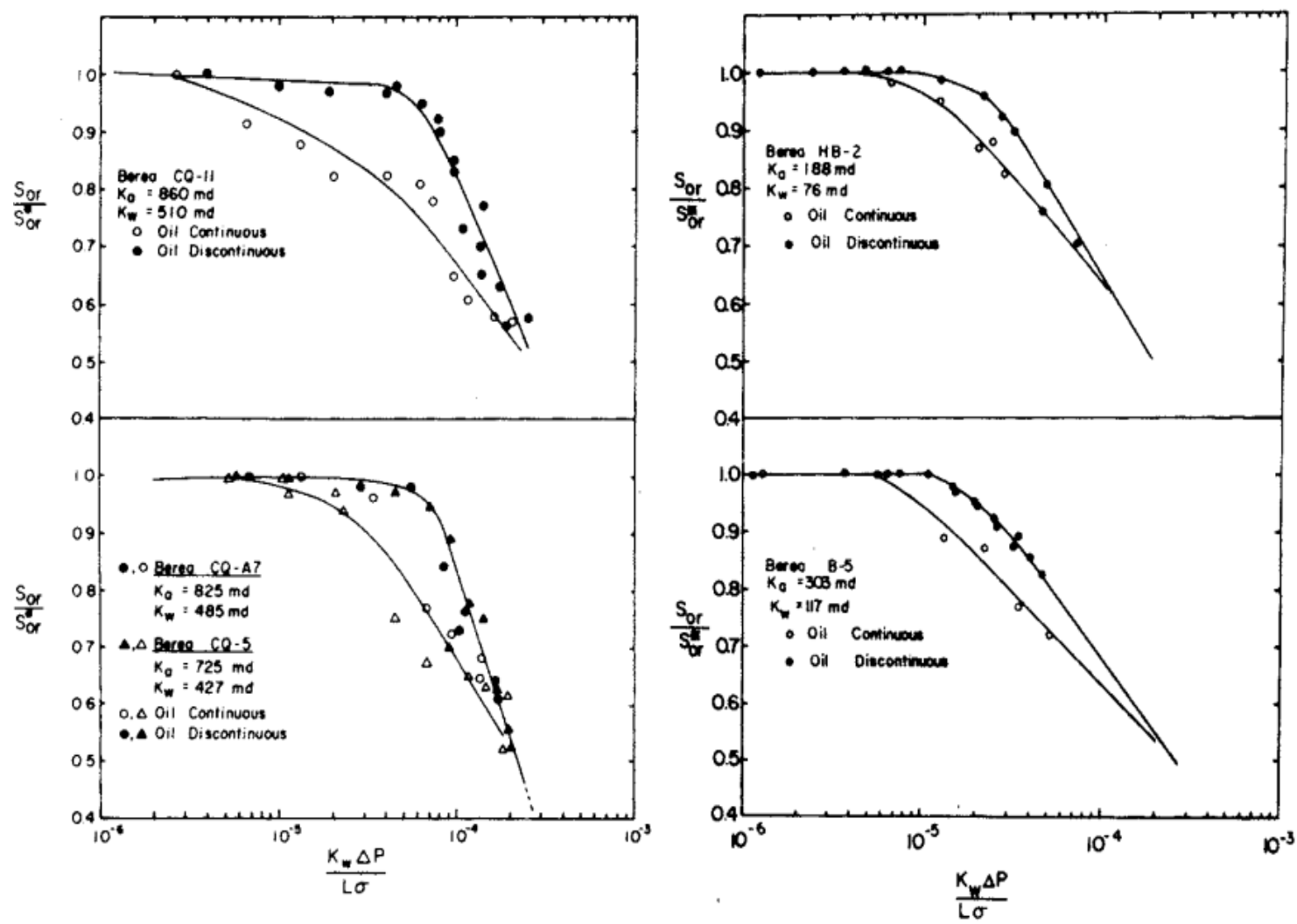

Figure 6.3 Capillary number curves for various sandstones (Chatzis and Morrow, 1984)

\subsection{EXPERIMENT RESULTS USING HPAM POLYMER}

\subsubsection{Coreflood experiments}

Table 6.1 summarizes seven of the corefloods performed as part of this study. The capillary numbers for each experiment were calculated using the definition of capillary number in terms of pressure gradient as shown in equation 6.3. The equivalent shear rates for each experiment were calculated using equation 6.7. The mobility ratios were calculated using Equation 6.2. The Deborah numbers were calculated using the measured relaxation times given in Table 6.1 and Equation 5.3. All saturations are average values based on material balance. 
Table 6.1 Summary of Coreflood Results

\begin{tabular}{|c|c|c|c|c|c|c|c|c|c|}
\hline Experiment & \#1 & \#2 & \#3 & \#4 & \#5 & \#6 & $\# 7$ & \#8 & \#9 \\
\hline Crude Oil & $\mathrm{K}-1$ & $\mathrm{~K}-2$ & K-3 & $\mathrm{L}-1$ & C-1 & $V-1$ & B-4 & $\mathrm{T}-1$ & $\mathrm{~T}-2$ \\
\hline $\begin{array}{l}\text { Porous } \\
\text { Medium }\end{array}$ & \begin{tabular}{|l|} 
Ottawa \\
Sand
\end{tabular} & $\begin{array}{l}\text { Ottawa } \\
\text { Sand }\end{array}$ & \begin{tabular}{|c|} 
Ottawa \\
Sand
\end{tabular} & $\begin{array}{c}\text { Reservoir } \\
\text { Rock }\end{array}$ & $\begin{array}{c}\text { Reservoir } \\
\text { Rock }\end{array}$ & $\begin{array}{c}\text { Reservoir } \\
\text { Rock }\end{array}$ & $\begin{array}{l}\text { Ottawa } \\
\text { Sand }\end{array}$ & $\begin{array}{c}\text { Reservoir } \\
\text { Rock }\end{array}$ & $\begin{array}{c}\text { Reservoir } \\
\text { Rock }\end{array}$ \\
\hline \begin{tabular}{|c|} 
Brine \\
Permeability \\
$(\mathrm{md})$
\end{tabular} & 7900 & 6650 & 7311 & 227 & 151 & 22 & 6078 & 2302 & 2204 \\
\hline \begin{tabular}{|c|}
$\begin{array}{c}\text { Oil Viscosity } \\
(\mathrm{cp})\end{array}$ \\
\end{tabular} & 80 & 120 & 250 & 72 & 3 & 8 & 1050 & 3.5 & 3.5 \\
\hline \begin{tabular}{|c|}
$\begin{array}{c}\text { Temperature } \\
\left({ }^{\circ} \mathrm{C}\right)\end{array}$ \\
\end{tabular} & 65 & 65 & 65 & 55 & 55 & 60 & 40 & 73 & 73 \\
\hline Porosity & 0.35 & 0.36 & 0.37 & 0.28 & 0.2 & 0.17 & 0.39 & 0.23 & 0.26 \\
\hline \multirow{2}{*}{$\begin{array}{l}\text { Velocity } \\
(w / p), f t / D\end{array}$} & 13 & 14 & 14 & 3.3 & 7 & 5.6 & 5 & NA & 1 \\
\hline & 1 & 1 & 1 & 1 & 1 & 1 & 1 & 1 & 1 \\
\hline Polymer & FP3630S & FP3630S & FP3630S & FP3330S & FP3330S & FP3330S & FP3630S & FP3630S & FP3630S \\
\hline $\begin{array}{c}\text { Polymer } \\
\text { conc. (ppm) }\end{array}$ & 1,200 & 1,300 & 2,450 & 2,000 & 1,000 & 2,000 & 3,500 & 800 & 800 \\
\hline $\begin{array}{l}\text { Capillary } \\
\text { Number }\end{array}$ & $6.0 \times 10^{-7}$ & $1.7 \times 10^{-6}$ & $4.1 \times 10^{-6}$ & $5.5 \times 10^{-7}$ & $7.1 \times 10^{-8}$ & $7.5 \times 10^{-6}$ & $2.9 \times 10^{-6}$ & $1.6 \times 10^{-7}$ & $1.9 \times 10^{-7}$ \\
\hline $\begin{array}{c}\begin{array}{c}\text { Equivalent } \\
\text { Shear Rate } \\
\left(\mathrm{s}^{-1}\right)\end{array} \\
\end{array}$ & 10.1 & 10.7 & 9.8 & 130 & 80 & 336 & 52 & 8 & 9 \\
\hline$\mu_{p} \quad(c p)$ & 16 & 28 & 90 & 9 & 4.4 & 2 & 39 & 14.4 & 13.8 \\
\hline \begin{tabular}{|c|} 
End-point \\
Mobility Ratio \\
for polymer
\end{tabular} & 1.2 & 0.9 & 0.8 & 1.1 & 0.1 & 0.5 & 10 & 0.2 & 0.2 \\
\hline $\begin{array}{c}\text { Relaxation } \\
\text { time }(\mathrm{s})\end{array}$ & 0.32 & 0.39 & 1.69 & 0.05 & 0.05 & NA & 0.06 & 0.05 & 0.05 \\
\hline $\begin{array}{l}\text { Deborah } \\
\text { Number }\end{array}$ & 3.2 & 4.2 & 16 & 6.5 & 4.0 & NA & 0.4 & 0.4 & 0.45 \\
\hline $\begin{array}{l}\text { Initial oil } \\
\text { saturation }\end{array}$ & $\begin{array}{l}0.87 \\
0.85 \\
\end{array}$ & $\begin{array}{l}0.88 \\
0.87 \\
\end{array}$ & $\begin{array}{l}0.88 \\
0.88 \\
\end{array}$ & $\begin{array}{l}0.68 \\
0.69 \\
\end{array}$ & 0.77 & 0.70 & 0.89 & 0.69 & 0.65 \\
\hline $\begin{array}{c}\text { Oil saturation } \\
\text { at end of } \\
\text { water flood }\end{array}$ & 0.38 & 0.38 & 0.47 & 0.37 & 0.36 & 0.41 & 0.61 & NA & 0.32 \\
\hline \begin{tabular}{|l|} 
Oil saturation \\
after polymer
\end{tabular} & 0.26 & 0.24 & 0.23 & 0.24 & 0.36 & 0.31 & 0.26 & 0.255 & 0.275 \\
\hline$\Delta \mathrm{S}_{\mathrm{o}}$ & 0.12 & 0.14 & 0.24 & 0.13 & 0 & 0.10 & 0.34 & NA & 0.045 \\
\hline
\end{tabular}


Table 6.2 Core Properties for experiments \#1, \#2 and \#3

\begin{tabular}{|c|ccc|}
\hline Experiment & $\# 1$ & $\# 2$ & $\# 3$ \\
\hline Sand & Ottawa F95 & Ottawa F95 & Ottawa F95 \\
\hline Porosity & 0.35 & 0.36 & 0.37 \\
\hline Length $(\mathrm{cm})$ & 27.94 & 29 & 31.5 \\
\hline Diameter $(\mathrm{cm})$ & 2.54 & 4.8 & 2.54 \\
\hline Area $(\mathrm{cm} 2)$ & 5.07 & 18.01 & 5.07 \\
\hline Temperature $(\mathrm{oC})$ & 65 & 65 & 65 \\
\hline Brine permeability $(\mathrm{md})$ & 7900 & 6650 & 7311 \\
\hline Pore Volume $(\mathrm{ml})$ & 55 & 192 & 60 \\
\hline
\end{tabular}

For experiments \#1, \#2 and \#3, the cores were saturated with $4000 \mathrm{ppm} \mathrm{NaCl}$ and then flooded with the same brine to measure brine permeability at $6 \mathrm{~mL} / \mathrm{min}$. The salinity was then reduced to $2000 \mathrm{ppm} \mathrm{NaCl}$ at $25{ }^{\circ} \mathrm{C}$. The reduction in salinity was used as a conservative tracer at $100 \%$ water saturation. The tracer data were used to verify the core did not have any significant heterogeneity and to independently determine the pore volume. After the tracer test, $2000 \mathrm{ppm} \mathrm{NaCl}$ brine was injected at $68{ }^{\circ} \mathrm{C}$. Figure 6.4 shows the salinity profile.

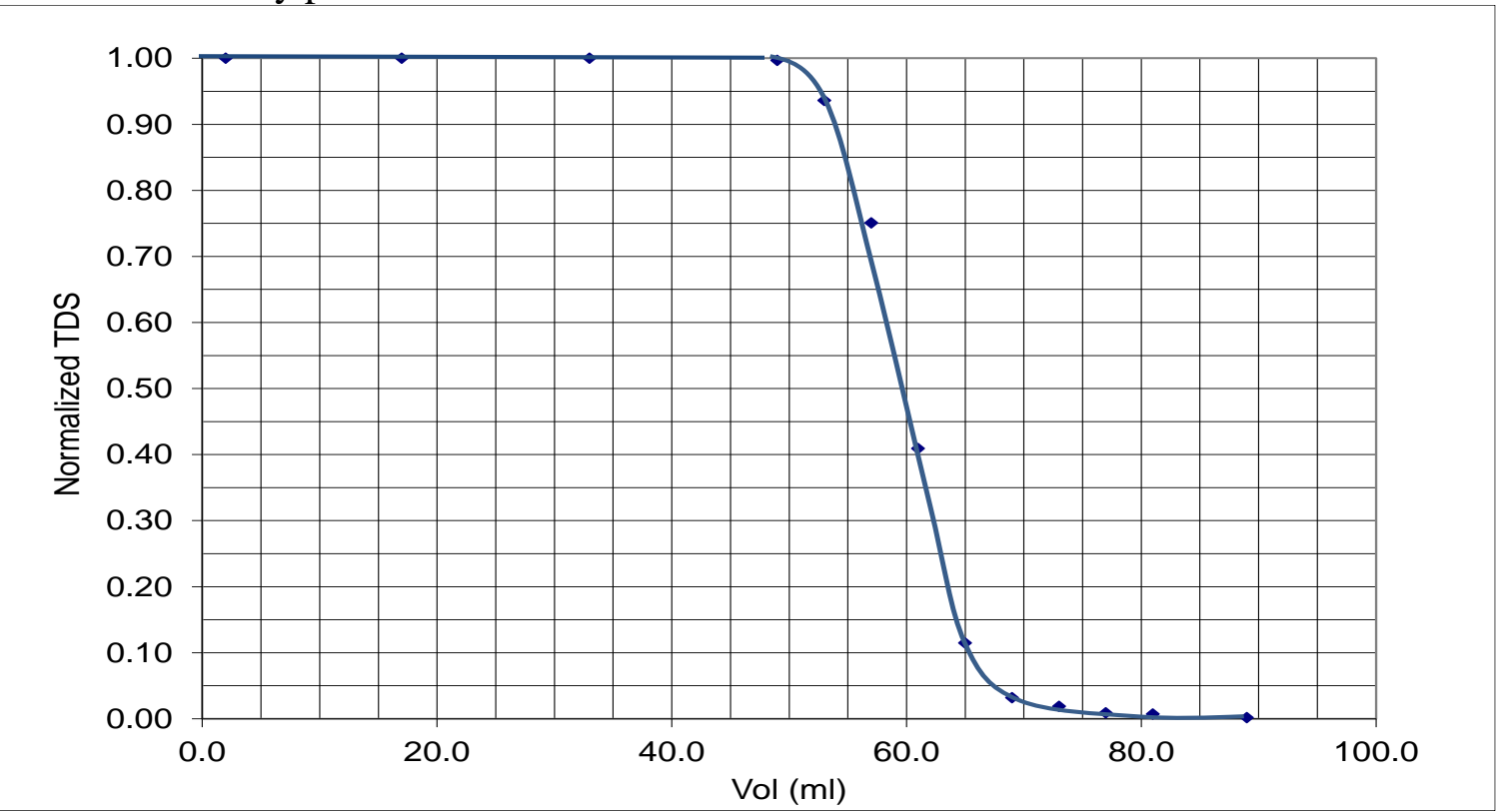

Figure 6.4 Experiment \#3 tracer test data 
The pore volume was estimated to be $60 \mathrm{~mL}$ by integrating below the curve. The water floods and polymer floods were done at the capillary numbers and mobility ratios shown in Table 6.1. Each water flood and each polymer flood was initiated at residual water saturation following an oil flood to establish the initial oil saturation.

The oil viscosities under reservoir conditions were $80 \mathrm{cp}, 120 \mathrm{cp}$ and $250 \mathrm{cp}$ for experiments \#1, \#2 and \#3, respectively. The same brine was used for all of three experiments. The brine salinity was $2000 \mathrm{ppm}(0.2 \mathrm{wt} \% \mathrm{NaCl})$. Figure 6.5 shows oil saturation after water flood for experiments \#1, \#2, and \#3.

For experiment \#1, the core was flooded with $2000 \mathrm{ppm} \mathrm{NaCl}$ brine at a flow rate of $0.5 \mathrm{ml} / \mathrm{min}(\sim 13 \mathrm{ft} /$ day $)$. The residual oil saturation to water was 0.38 . The water permeability was $596 \mathrm{mD}$ and $\mathrm{k}_{\mathrm{rw}}$ was 0.075 after $3.75 \mathrm{PV}$ of water injection. Figures 6.7 and 6.8 show the oil recovery and the water flood pressure drop data, respectively. Table 6.2 lists the properties of the sandpacks.

For experiment \#2, the core was flooded with $2000 \mathrm{ppm} \mathrm{NaCl}$ brine at a flow rate of $2 \mathrm{ml} / \mathrm{min}$ ( $\sim 14 \mathrm{ft} / \mathrm{day})$. The residual oil saturation was 0.38 , the water permeability was $538 \mathrm{mD}$ and $\mathrm{k}_{\mathrm{rw}}$ was 0.081 after $6.5 \mathrm{PV}$ of water injection. Figures 6.9 and 6.10 show the oil recovery and pressure drop data. Table 6.2 lists the properties of the sandpack after the water flood.

For experiment \#3, the core was water flooded with $2000 \mathrm{ppm} \mathrm{NaCl}$ brine at a flow rate of $0.55 \mathrm{ml} / \mathrm{min}(\sim 14 \mathrm{ft} / \mathrm{day})$. The residual oil saturation was 0.48 , the water permeability was $596 \mathrm{mD}$ and $\mathrm{k}_{\mathrm{rw}}$ was 0.092 after $6 \mathrm{PV}$ of water injection with an oil cut less than $1 \%$. Figures 6.11 and 6.12 show the oil recovery and pressure drop data. Table 6.2 lists the properties of the sandpack after the water flood. 
After each water flood, the cores were re-saturated with the same oil. The $2^{\text {nd }}$ oil saturation for experiments \#1, \#2, and \#3 are shown in Table 6.1. The second oil flood showed consistent values of oil saturation and oil permeability with the first oil flood. FP $3630 \mathrm{~S}$ polymer in the same brine was used in all three of these sandpack experiments. The target viscosity was chosen to give an end point mobility ratio equal to 1 calculated using the definition given in equation 5.1 and the polymer viscosity values shown in Figure 6.6 at the equivalent shear rate calculated using equation 5.2.

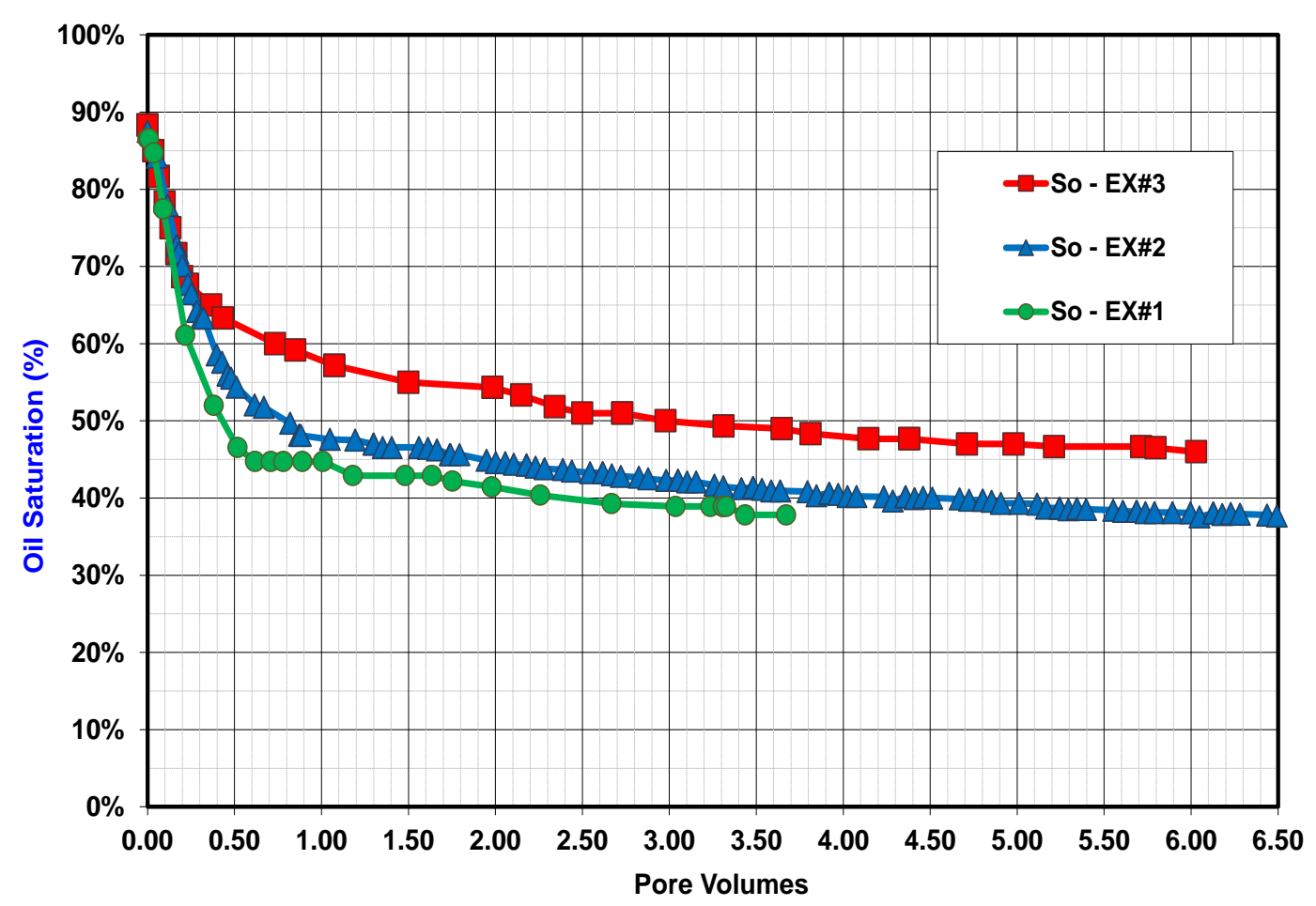

Figure 6.5 Oil saturations for experiments \#1, \#2 and \#3 waterfloods 


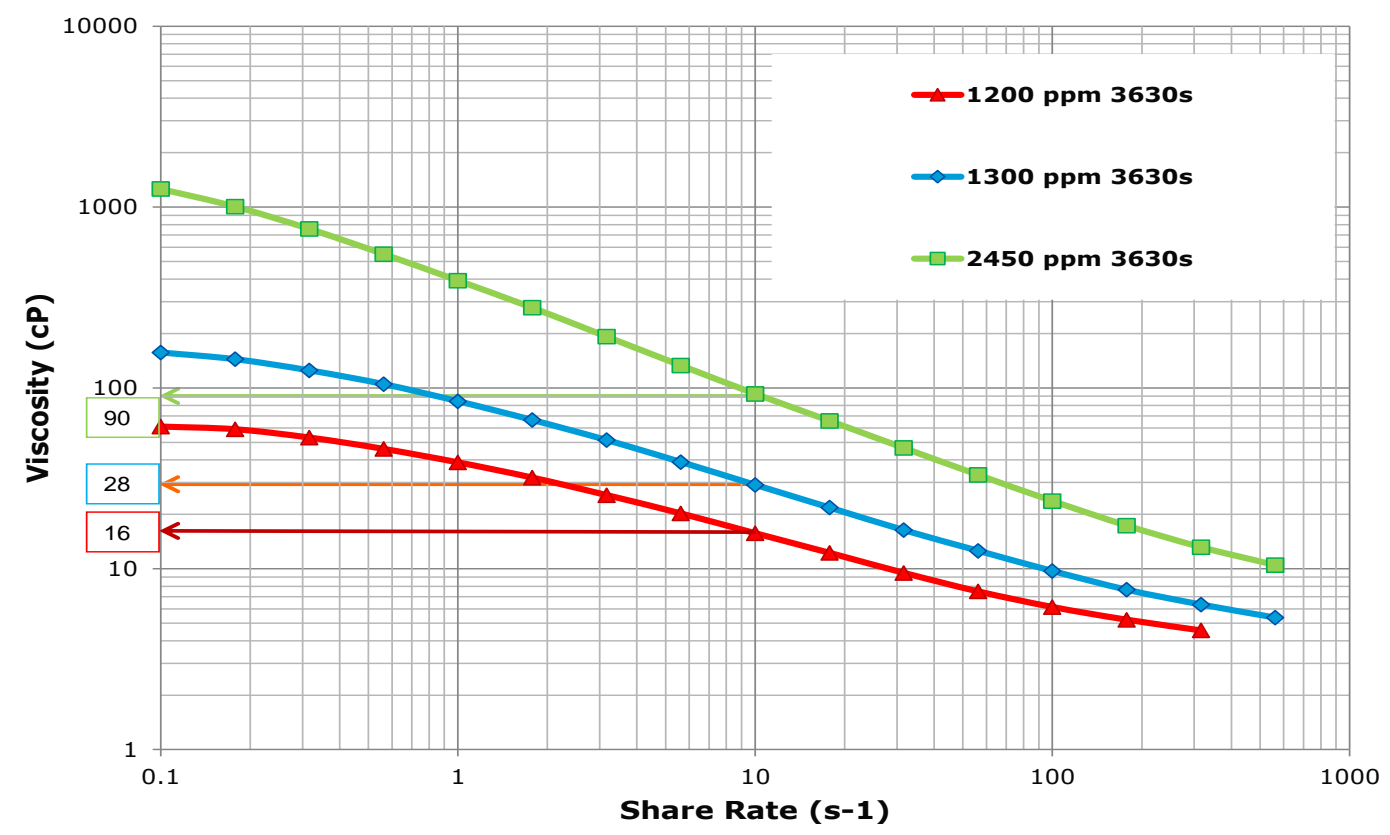

Figure 6.6 Viscosity of FP 3630s in 2000 ppm NaCl Brine at 68oC

The remaining oil saturation for these coreflood experiments is the value measured by material balance. The volume of oil collected in each tube is recorded until it is too small to be measured within the experimental uncertainty of the volume measurements. With the current water floods a viscous oil is displaced with an adverse mobility ratio. Therefore, this may not be the "true" residual oil saturation. The sandpacks are not perfectly homogeneous and they are not identical among other factors that complicate the interpretation of such data. The trend in the oil saturation shown in Figure 6.7, Figure 6.9 and Figure 6.11 suggests that the water flood might have further reduced the oil saturation if it had been continued for an even longer time than $6 \mathrm{PV}$. However, this is a very large number of pore volumes to inject in a reservoir flood and it would take a very long time to inject $6 \mathrm{PV}$, so it would rarely be practical to inject such as large volume of water. 
For experiment \#1, the residual oil saturation for the secondary polymer flood was 0.12 less than for the water flood. For experiment \#2, the residual oil saturation for the secondary polymer flood was 0.14 less than for the water flood. For experiment \#3, the residual oil saturation for the secondary polymer flood was 0.24 less than for the water flood. The oil viscosity and the water flood residual oil saturation were both much higher for experiment \#3 than for experiments \#1 and \#2. This suggests that the water flood did not efficiently displace the more viscous oil even though the mobility ratio is less than one. As shown below, this result can be explained using fractional flow calculations.

Unfortunately it is not possible to draw any conclusions about the effect of viscoelasticity from these three experiments because there is no way to distinguish the different causes of the decrease in residual oil saturation. One approach to sorting out the different effects would be to repeat the same experiments using a Newtonian fluid with the same viscosity. 


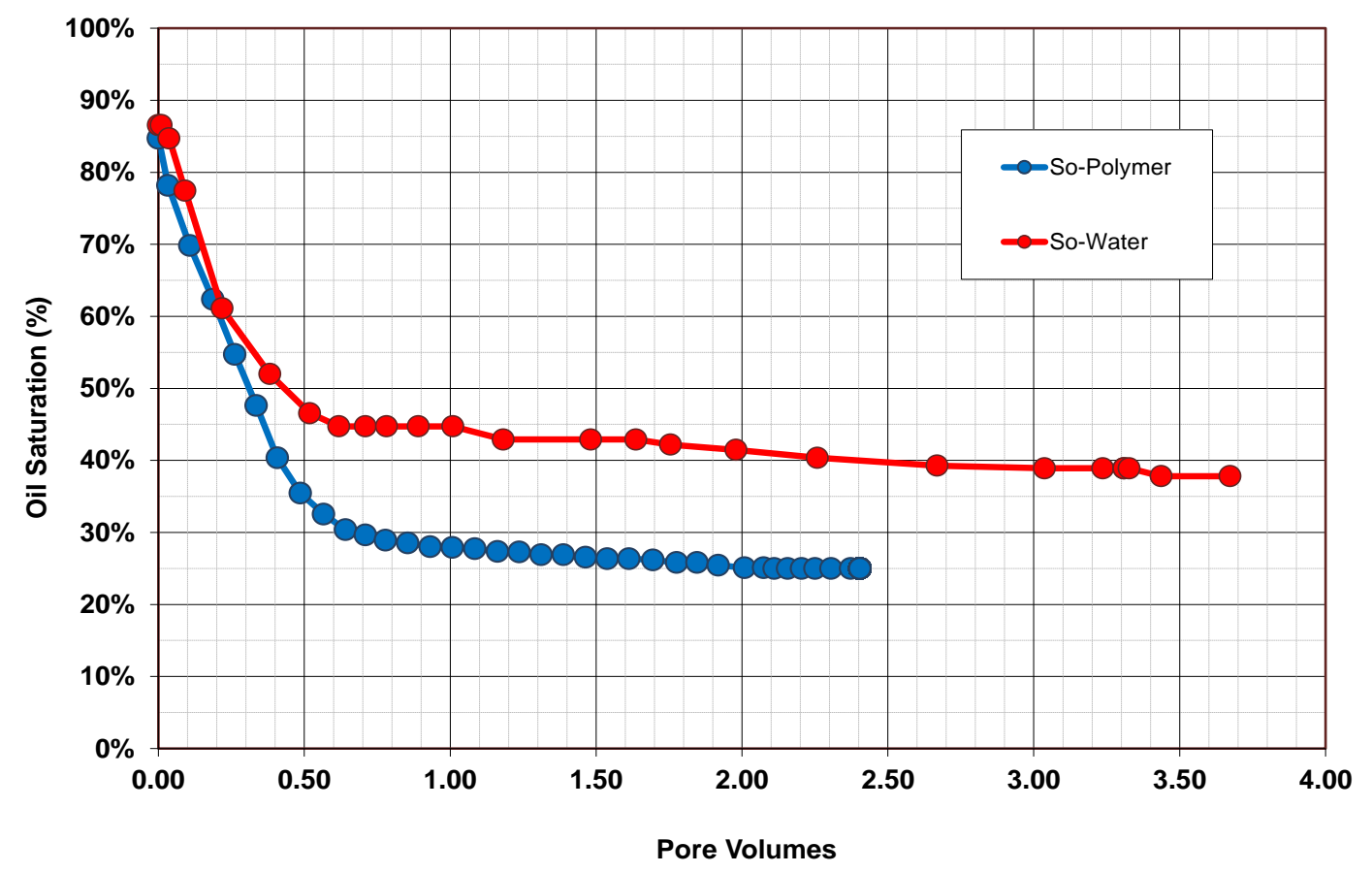

Figure 6.7 Oil Saturations for experiment \#1

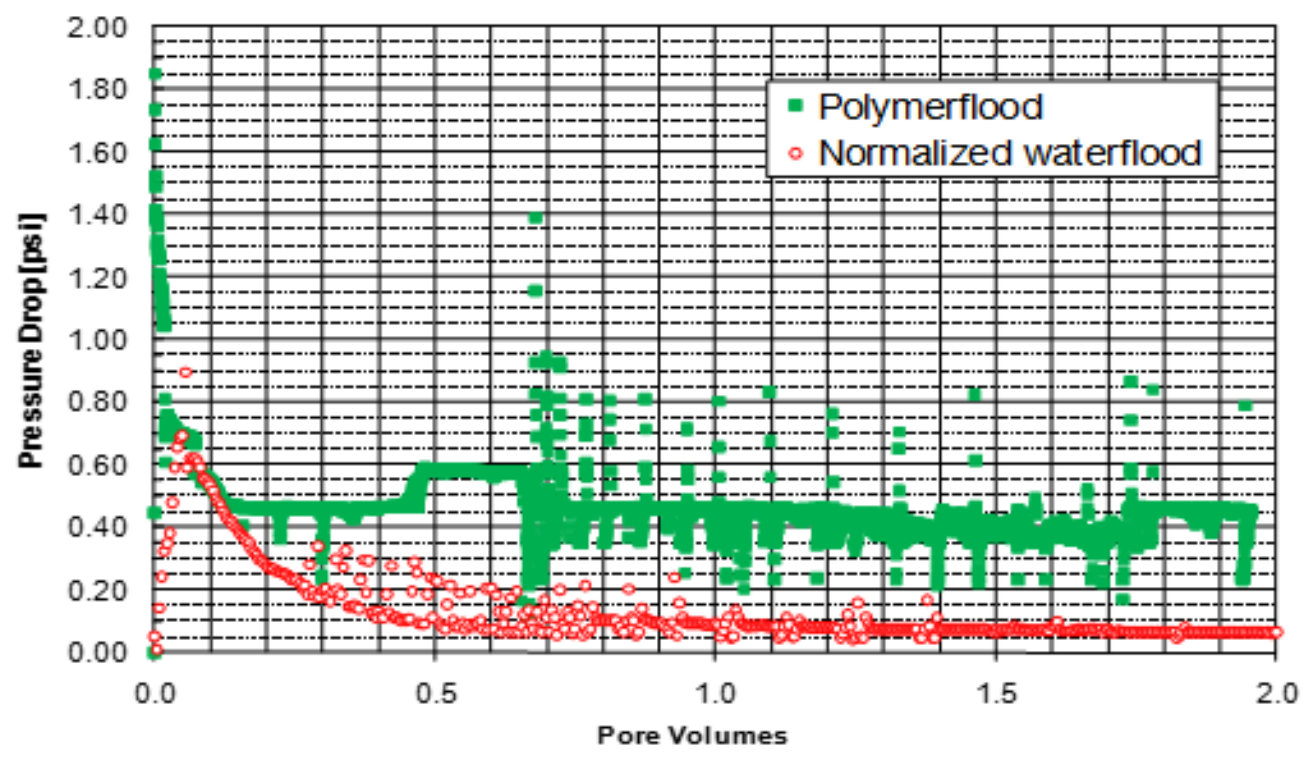

Figure 6.8 Pressure drop data for experiment \#1 


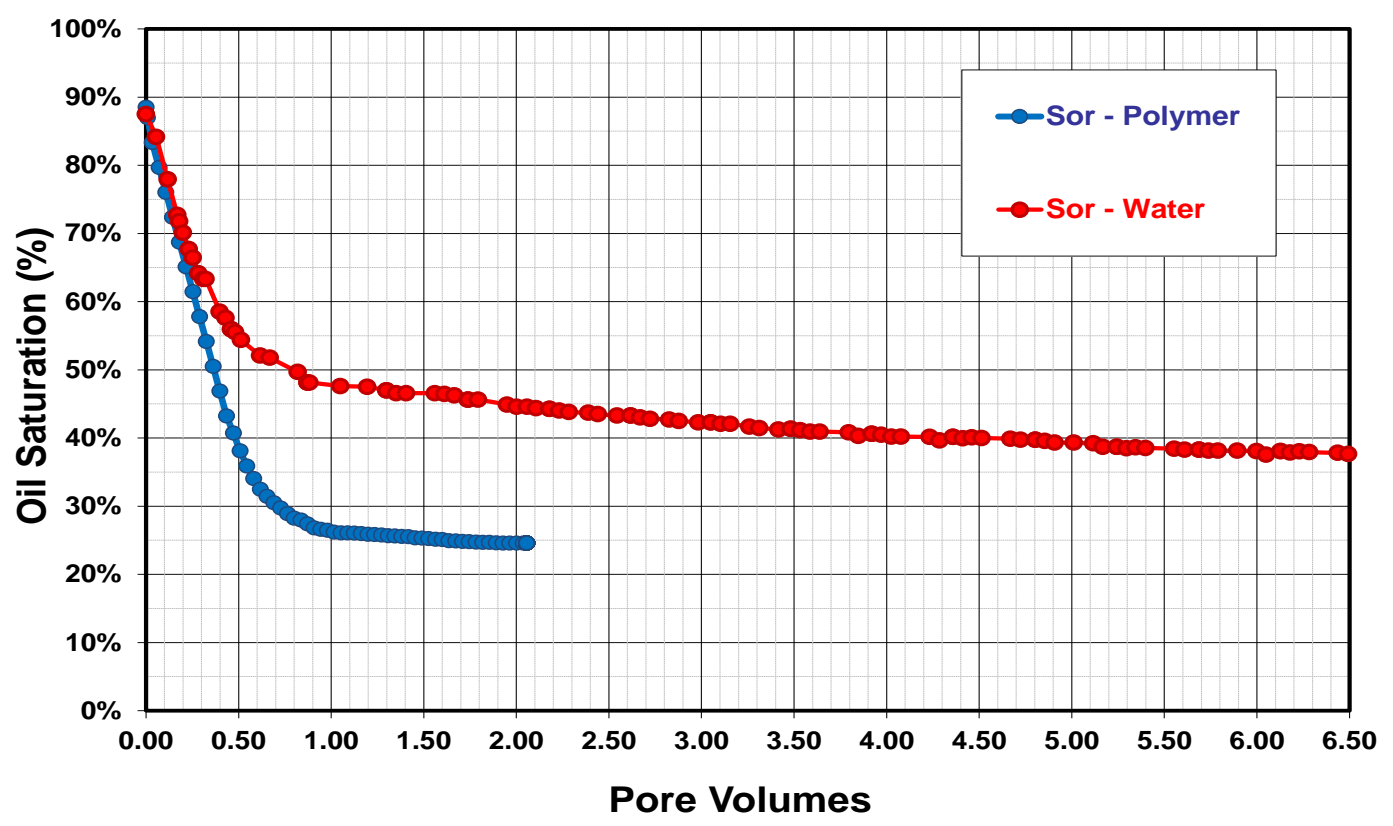

Figure 6.9 Oil Saturations for experiment \#2

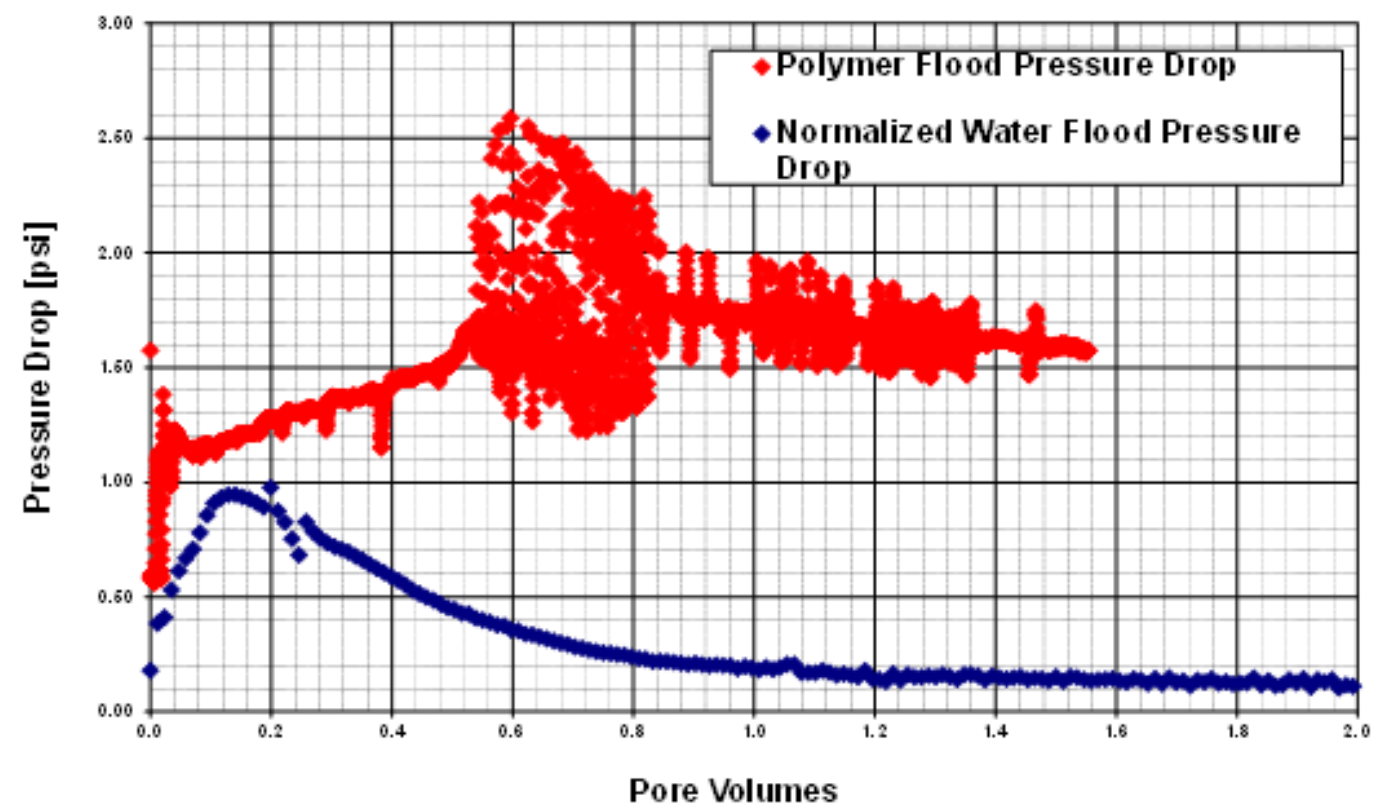

Figure 6.10 Pressure drop data for experiment \#2 


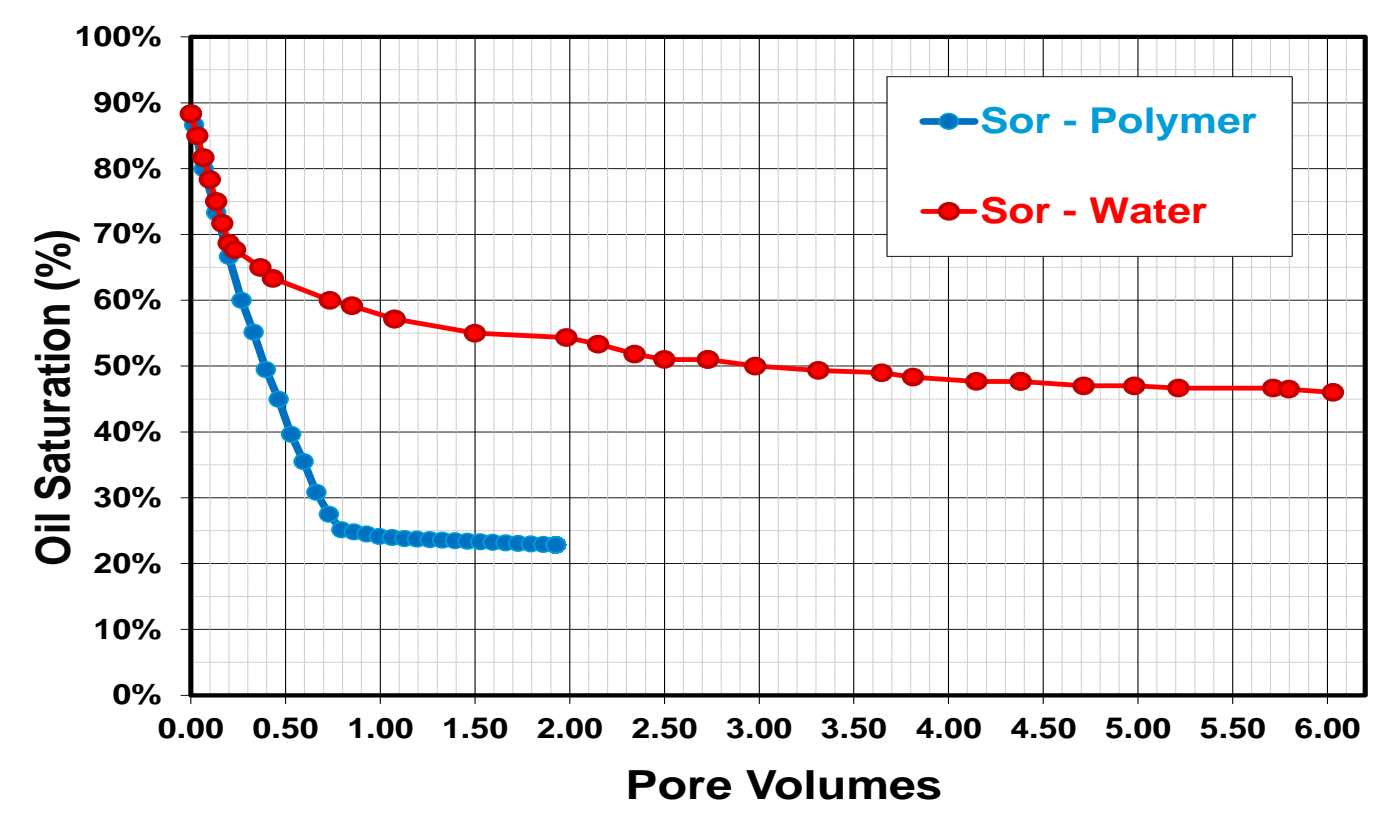

Figure 6.11 Oil Saturations for experiment \#3

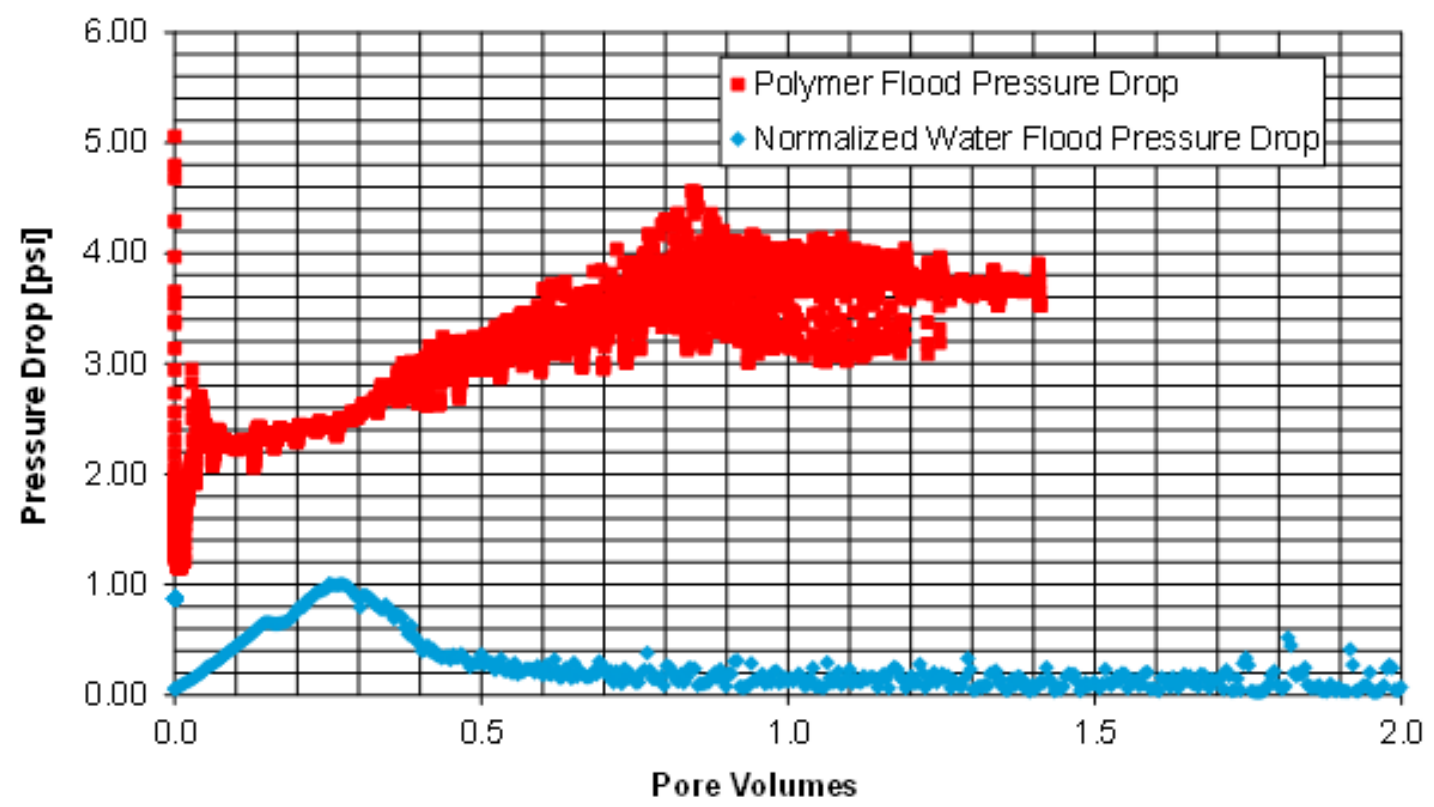

Figure 6.12 Pressure drop data for experiment \#3 


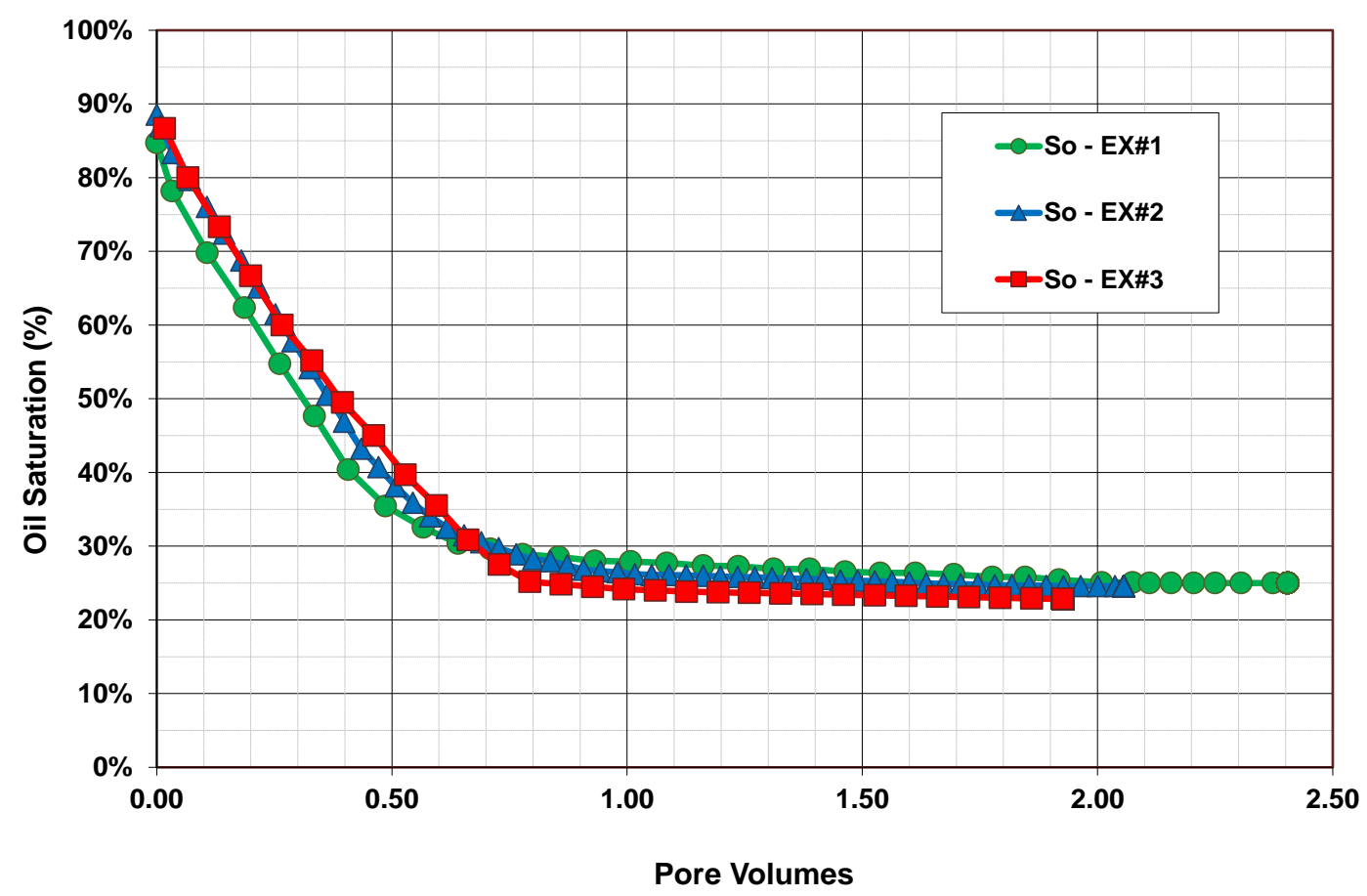

Figure 6.13 Oil Saturations during polymer flood for experiments \#1,\#2 and \#3

Reservoir cores were used for the next series of experiments (experiments \#4 to experiment \#6). In experiment \#4, the incremental oil recovery from a tertiary polymer flood was measured for comparison with a water flood. The salinity of brine was 25,878 ppm. The brine permeability of the reservoir core was $227 \mathrm{md}$. The core was initially vacuum saturated with $51,756 \mathrm{ppm}$ of brine, which is twice the salinity of the hard synthetic brine. Then, a salinity tracer test was conducted with $25,878 \mathrm{ppm}$ brine at 25 ${ }^{\circ} \mathrm{C}$. To put the core in a sufficiently reduced state with oxidation-reduction potential $(\mathrm{ORP})<-200 \mathrm{mV}, 1000 \mathrm{ppm}$ sodium dithionite was injected for $10 \mathrm{PV}$. The injection rate was $2 \mathrm{ml} / \mathrm{min}$ ( $33 \mathrm{ft} / \mathrm{day}$ ). When the ORP of the effluent water was consistently less than - $200 \mathrm{mV}$, the core was considered to be adequately reduced. The final composition of 
brine used in the brine flood was made up of 1,000 ppm sodium dithionite in brine, ORP $\sim-350 \mathrm{mV}$. The ORP of the final brine flood effluent was about-100 mV.

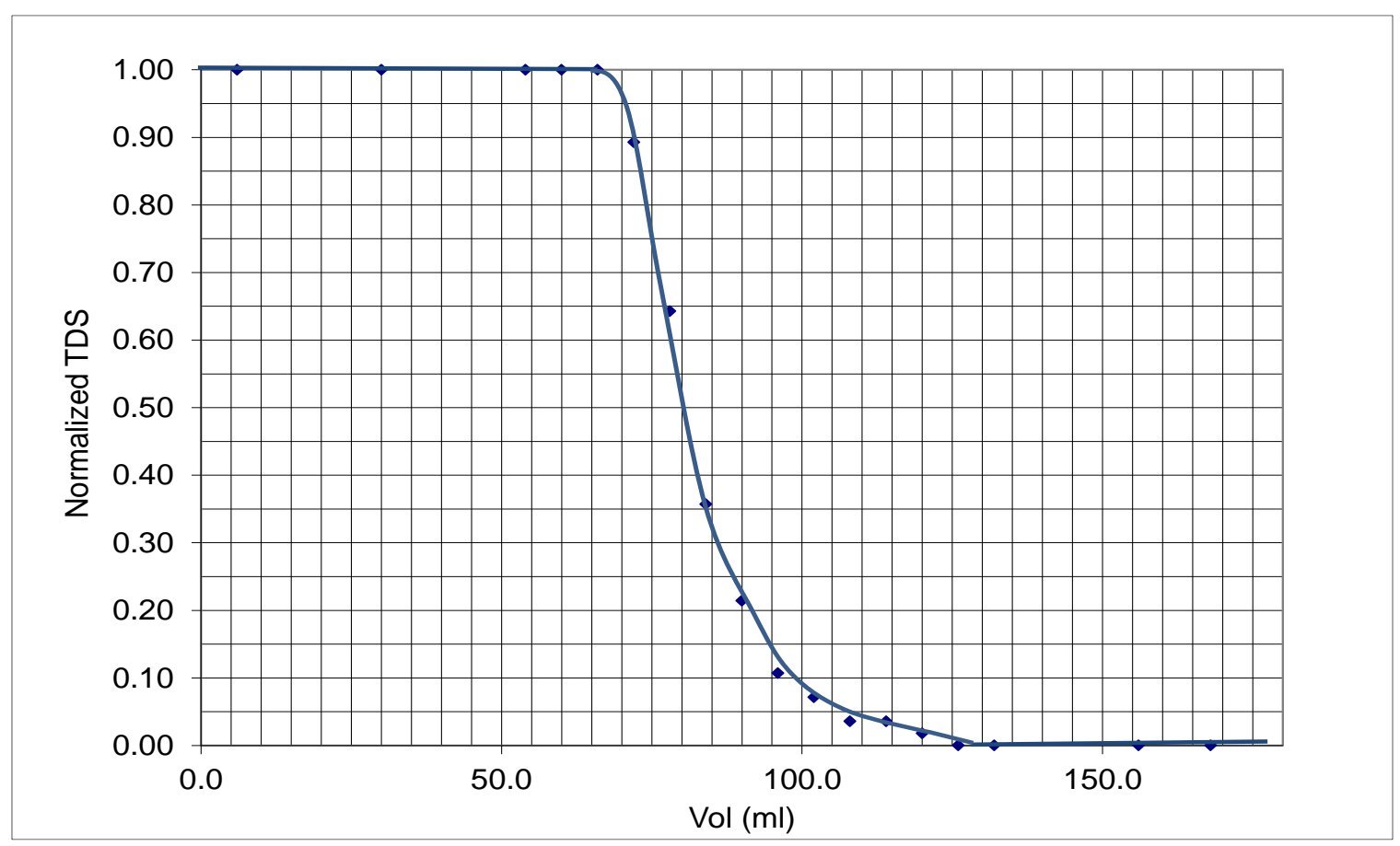

Figure 6.14 Experiment \#3 tracer test 


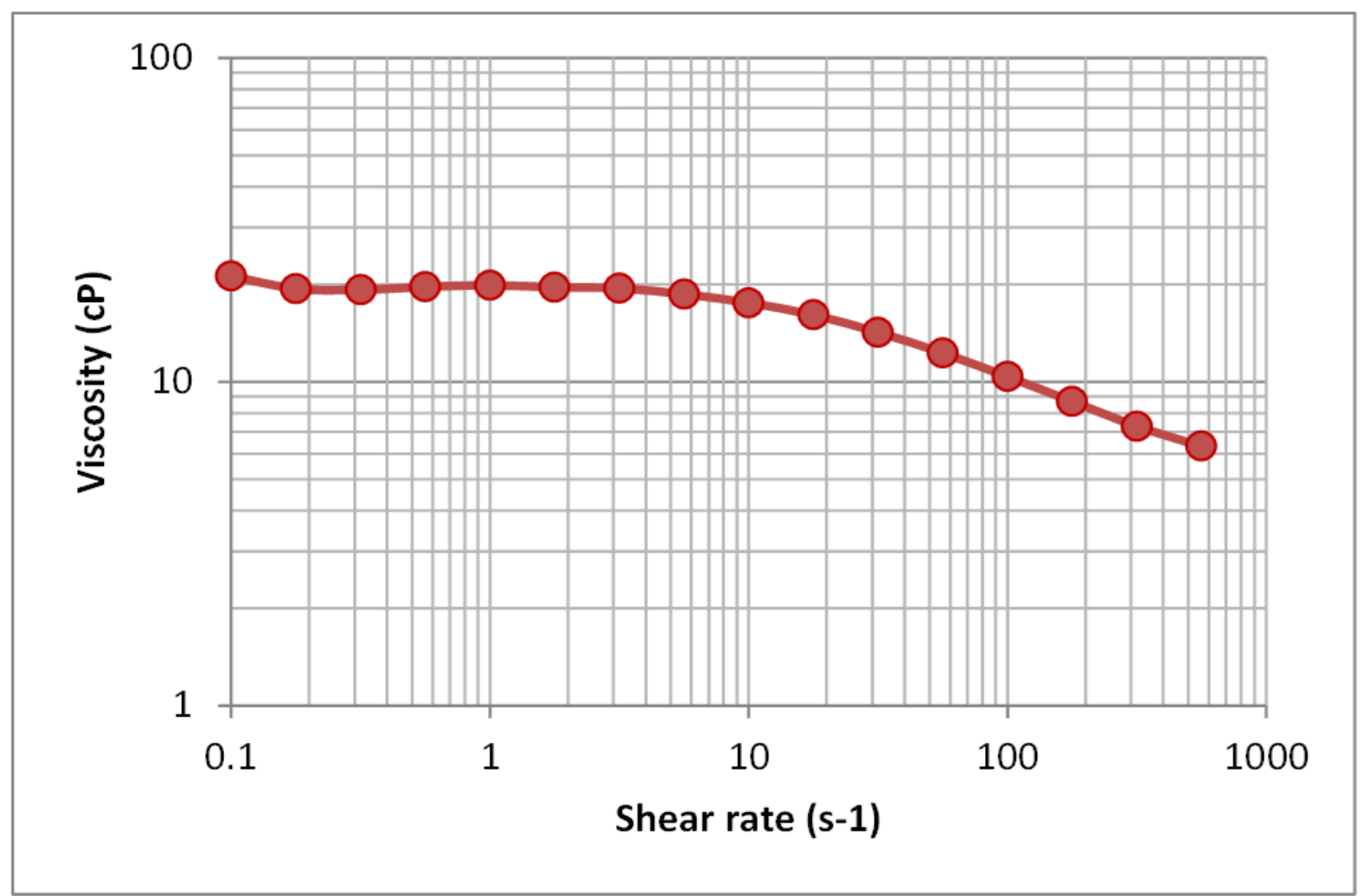

Figure 6.15 Viscosity of 2000 ppm FP 3330S

The tracer data show that the reservoir core was more heterogeneous than the sandpacks used for experiments \#1 to \#3. The core was saturated with a crude oil with a viscosity of $72 \mathrm{cp}$ at the reservoir temperature of $55^{\circ} \mathrm{C}$. Then, the core was flooded with $25,878 \mathrm{ppm}$ hard synthetic brine at a flow rate of $0.2 \mathrm{ml} / \mathrm{min}(\sim 3.3 \mathrm{ft} / \mathrm{day})$. The residual oil saturation was 0.37 at a water cut greater than $99 \%$ after 2 PV of water flooding. The water permeability was $15 \mathrm{md}$ and $\mathrm{k}_{\mathrm{rw}}$ was 0.065 . Next the reservoir core was flooded with oil to re-establish initial oil saturation and then water was again injected until the oil cut decreased to $5 \%$ followed by injection of FP 3330S polymer solution. 2000 ppm FP3330S polymer in brine was injected at a frontal (interstitial) velocity of $1 \mathrm{ft} / \mathrm{D}(0.06$ $\mathrm{ml} / \mathrm{min}$ ) until the oil cut was less than $1 \%$. The equivalent shear rate corresponding to 1 $\mathrm{ft} / \mathrm{D}$ frontal velocity was calculated to be $86 \mathrm{~s}^{-1}$. The value of $\mathrm{C}$ was 4 used for this 
calculation. The target viscosity needed to achieve a unit mobility ratio at this shear rate is $10 \mathrm{cP}$ at $86 \mathrm{~s}^{-1}$.

The residual oil saturation after 3.7 PV of polymer flooding at greater than $99 \%$ water cut was 0.24 and $\mathrm{k}_{\mathrm{rw}}$ was 0.138 . The oil recovery from the polymer flood was $43 \%$ of the remaining oil after the water flood stopped at $95 \%$ water cut. Table 6.3 shows the results for the first water flood and polymer flood with the second water flood. Figure 6.17 shows that the polymer flood produced an oil bank. This is expected from fractional flow theory, which will be discussed in detail later.

Table 6.3 Relative water permeability for experiment \#4

\begin{tabular}{|c|c|c|c|}
\hline & $1^{\text {st }}$ water flood & $2^{\text {st }}$ water flood & Polymer flood \\
\hline $\mathrm{S}_{\mathrm{o}}$ & 0.37 & 0.43 & 0.24 \\
\hline $\mathrm{S}_{\mathrm{wr}}$ & 0.63 & 0.56 & 0.76 \\
\hline $\mathrm{k}_{\text {water }}$ & $15 \mathrm{md}$ & $8 \mathrm{md}$ & $31 \mathrm{md}$ \\
\hline $\mathrm{k}_{\mathrm{rw}}$ & 0.065 & 0.03 & 0.135 \\
\hline
\end{tabular}




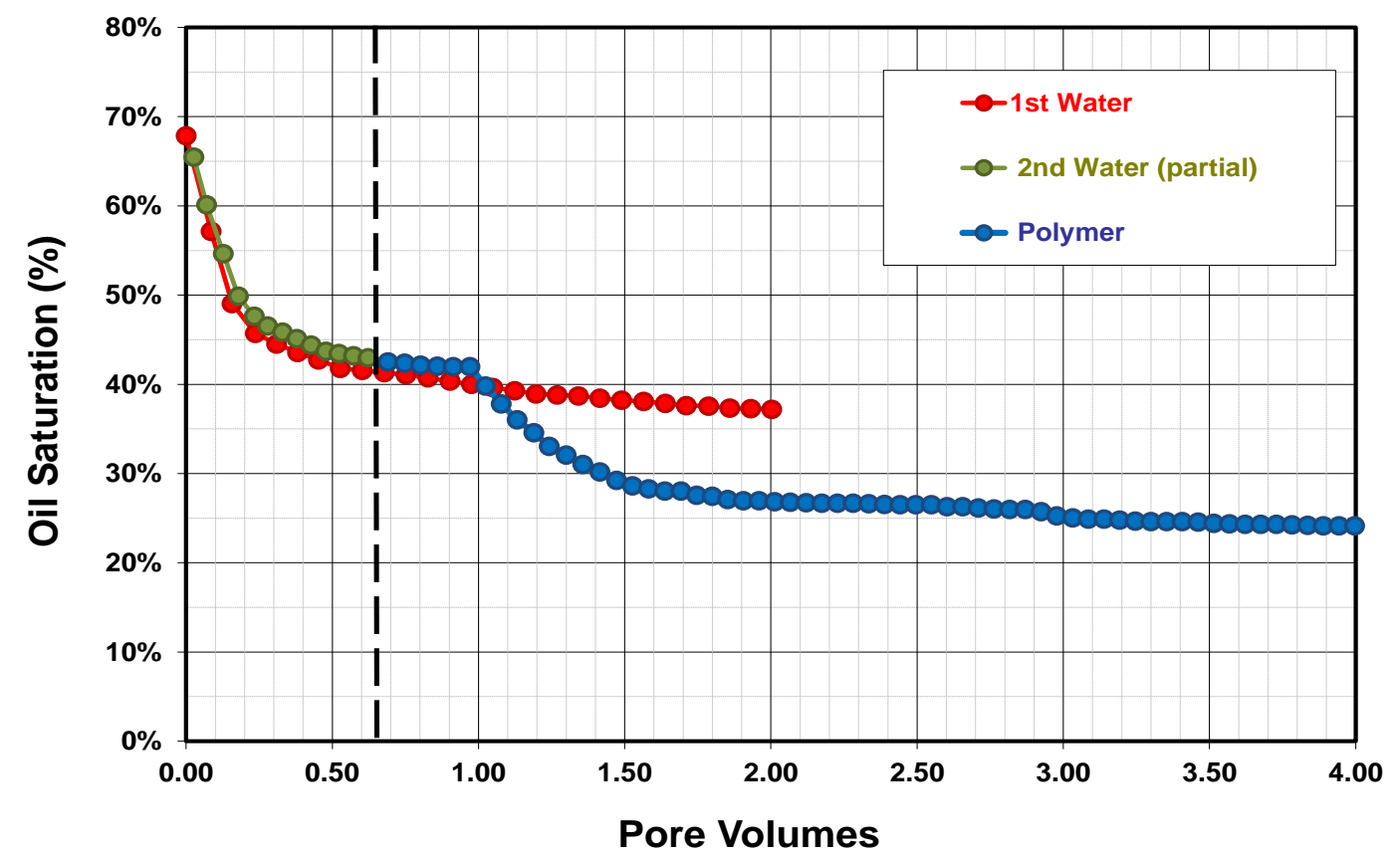

Figure 6.16 Oil Saturation for experiment \#4

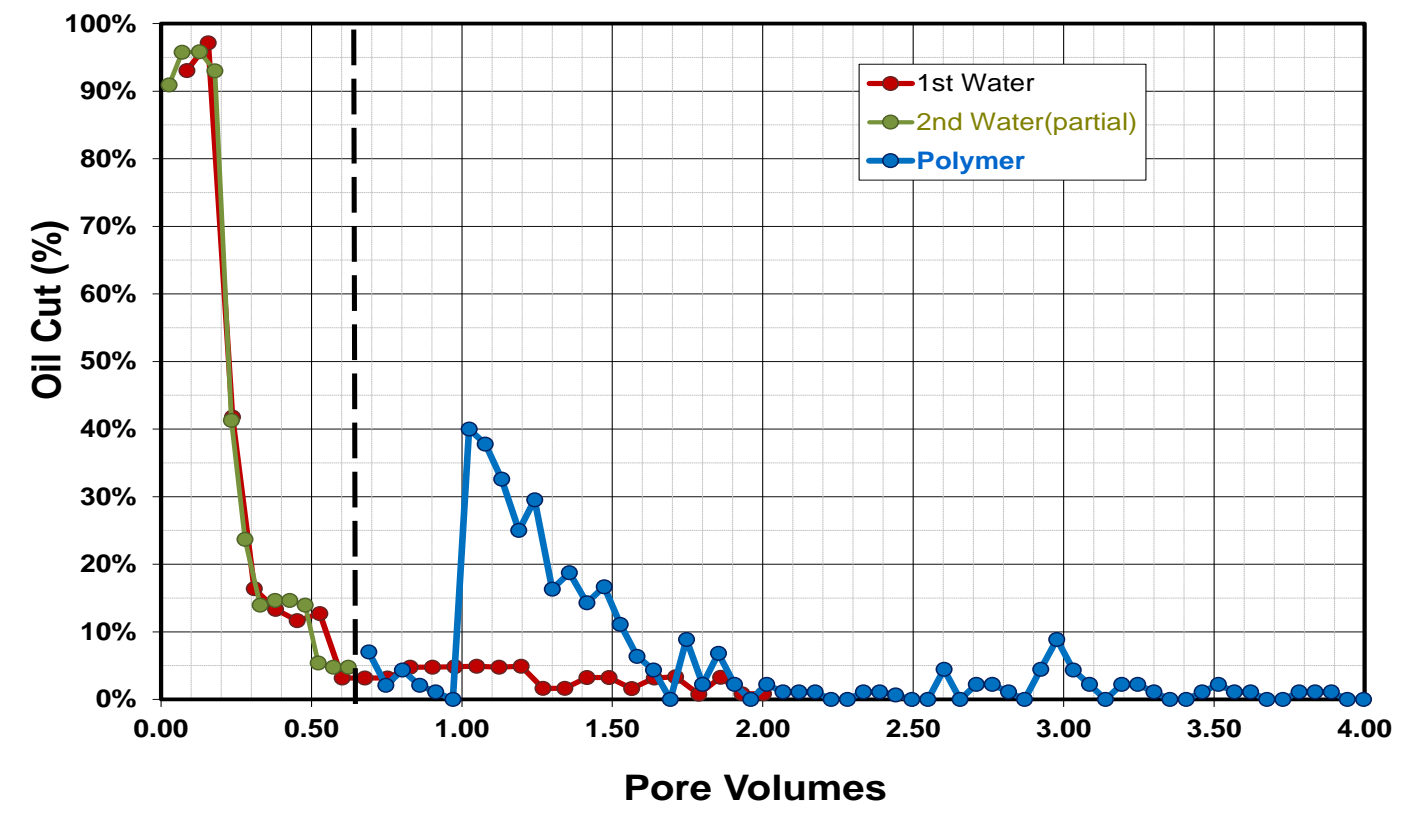

Figure 6.17 Oil Cut for experiment \#4 
Experiment \#5 was done to compare the residual oil saturation for a water flood and a tertiary polymer flood. An unconsolidated reservoir core was used for this experiment. The oil viscosity was $3 \mathrm{cp}$ at $55{ }^{\circ} \mathrm{C}$. Water was injected at $7 \mathrm{ft} / \mathrm{D}$ until no more oil was produced and then FP 3330S polymer was injected at $1 \mathrm{ft} / \mathrm{D}$. The water flood residual oil saturation was 0.36 . Unlike the previous secondary polymer floods, the polymer flood did not reduce the residual oil saturation.

Experiment \#6 was done with a low permeability reservoir core with a brine permeability $22 \mathrm{md}$. The oil viscosity was $8 \mathrm{cp}$ at $60{ }^{\circ} \mathrm{C}$. The core was water flooded with $55,624 \mathrm{ppm}$ of hard brine at a flow rate of $0.2 \mathrm{ml} / \mathrm{min}(\sim 5.6 \mathrm{ft} /$ day $)$ until the water cut reached $90 \%$, which occurred at $\sim 0.43 \mathrm{PV}$. At the end of the water flood, the average oil saturation was 0.41 , the water permeability was $1.3 \mathrm{md}$ and $\mathrm{k}_{\mathrm{rw}}$ was 0.03 . Then $2000 \mathrm{ppm}$ FP $3330 \mathrm{~S}$ polymer in $4 \mathrm{wt} \% \mathrm{NaCl}$ brine was injected at $1 \mathrm{ft} / \mathrm{D}$. The permeability was thought to be too low to inject high molecular weight FP 3330S polymer without the risk of plugging the core. Therefore, the polymer solution was sheared for $70 \mathrm{~s}$ in a Waring blender at low speed to reduce its viscosity to about $2 \mathrm{cp}$ (Figure 6.18). 


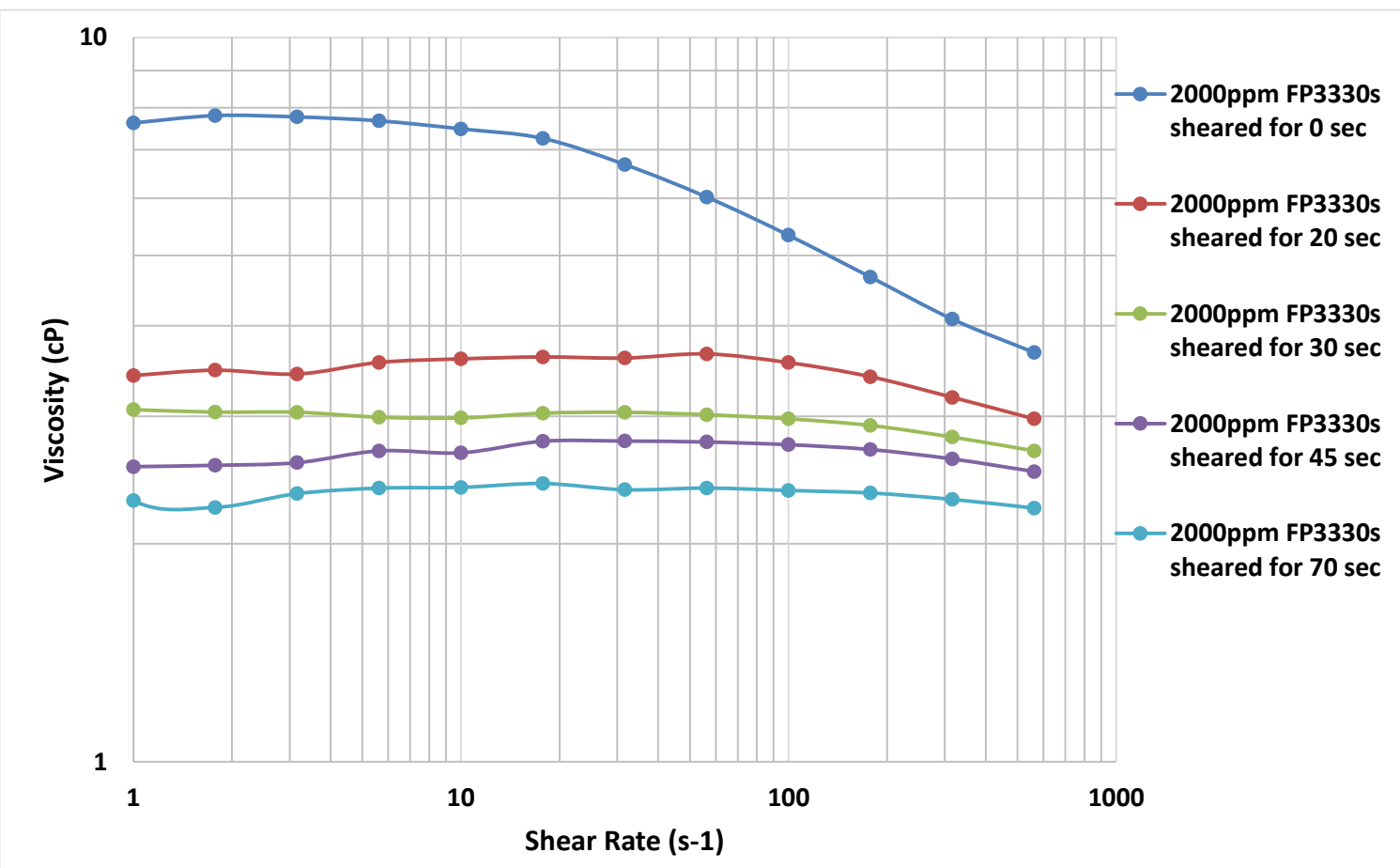

Figure 6.18 Viscosity of FP $3330 \mathrm{~S}$ in $4 \% \mathrm{NaCl}$ at $60 \mathrm{oC}$

The average oil saturation versus pore volumes injected is shown in Figure 6.19 and the oil cut is shown in Figure 6.20. No oil bank was formed as shown in Figure 6.20. The residual oil saturation following the polymer flood was 0.31 , which is a typical water flood residual oil saturation. 


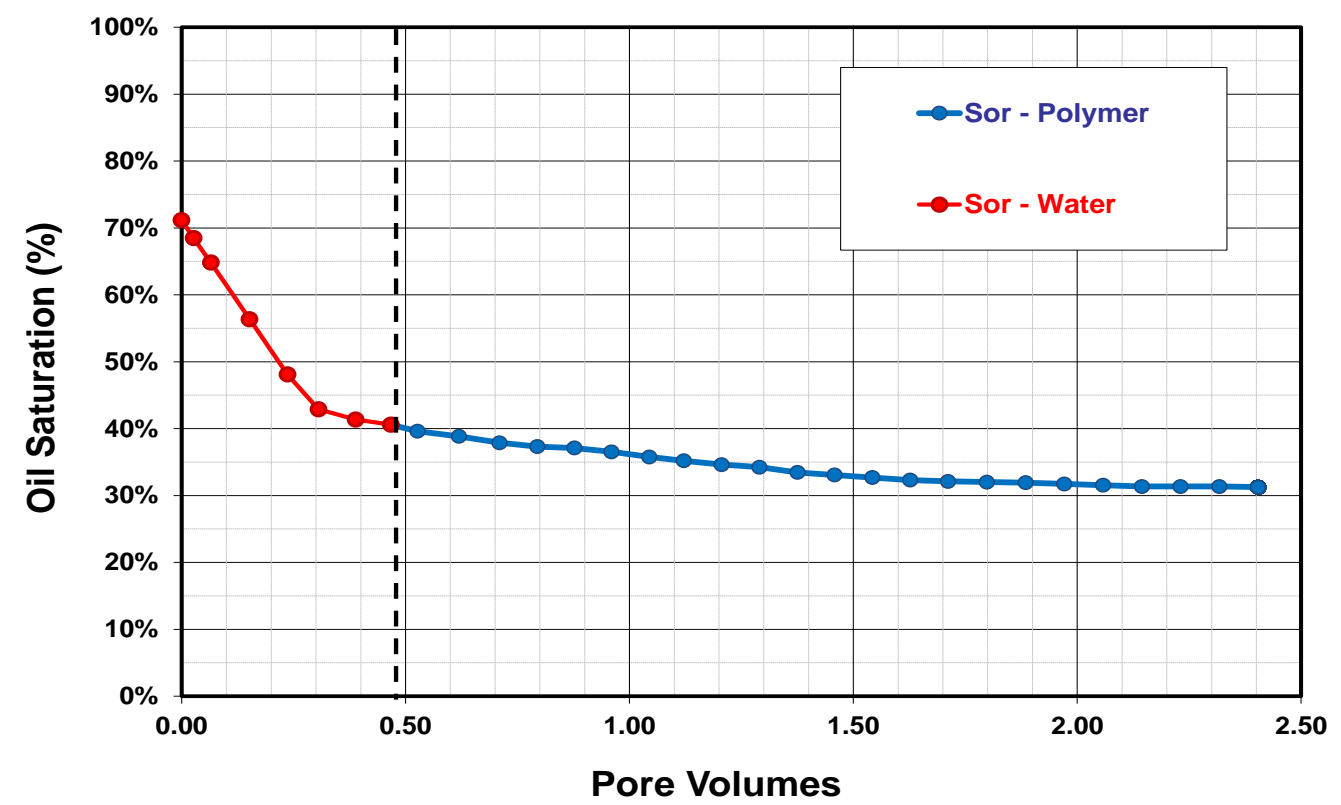

Figure 6.19 Oil Saturation for experiment \#6

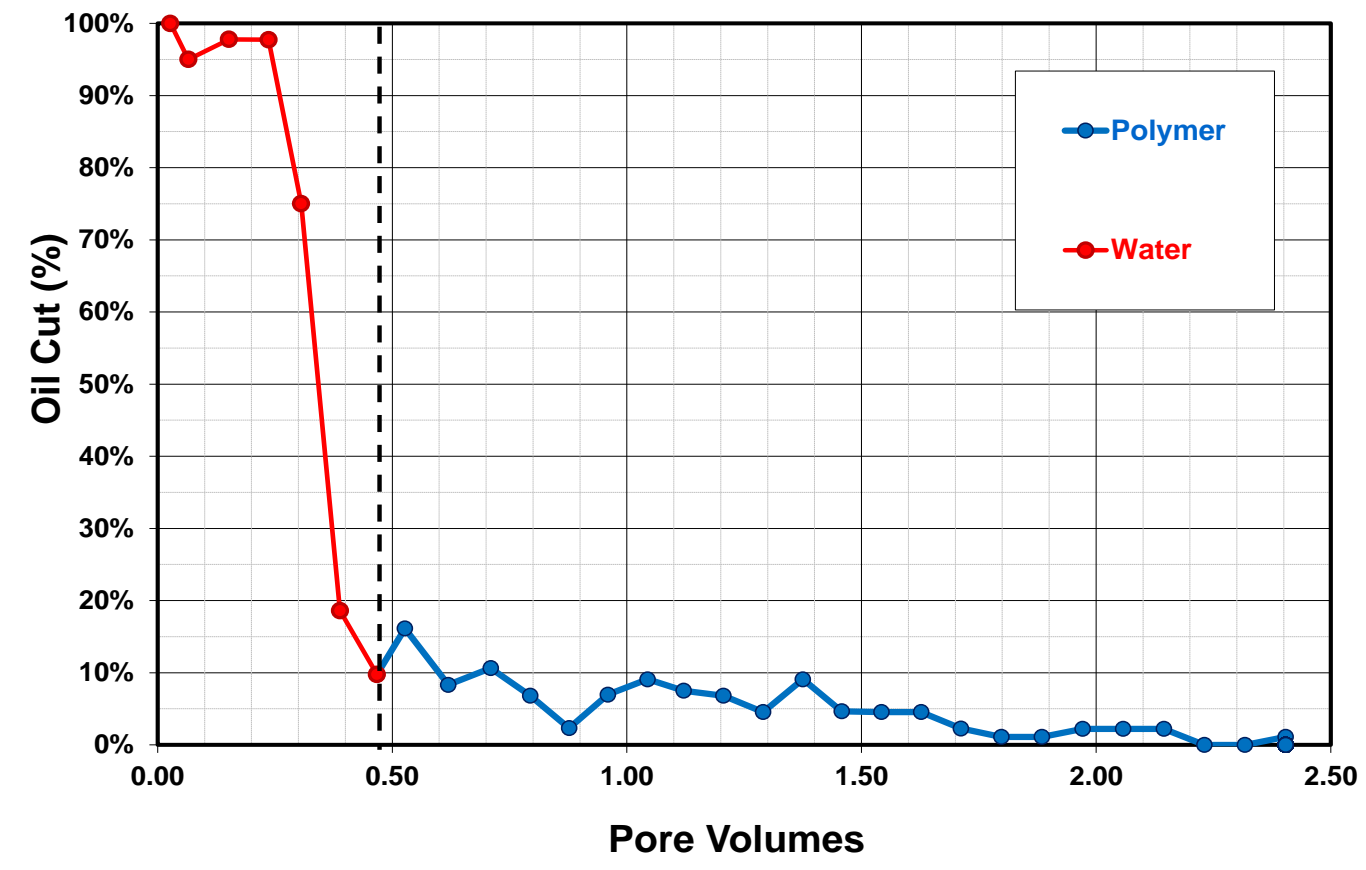

Figure 6.20 Oil Cut for experiment \#6 
Experiment \#7 was done with a sandpack in a 1.5 inch ID stainless steel core holder with pressure taps. A confining pressure of 2000 psi was applied to the outside of the core with an ISCO syringe pump. The purpose of this experiment was to measure the incremental oil recovery from a tertiary polymer flood using FP 3630s polymer and a viscous crude oil of $1000 \mathrm{cp}$. In experiment \#7, the water flood stopped at $99 \%$ water cut. The water flood remaining oil saturation decreased from 0.61 at $99 \%$ water cut to 0.26 after 4 pore volumes for the tertiary polymer flood (Figure 6.21). The oil cut is shown in Figure 6.22. The results are summarized in Table 6.1. The polymer retention was determined to be $43 \mu \mathrm{g} / \mathrm{g}$ of sand. The pressure gradient at the end of the polymer flood was about 1 psi/ft (Figure 6.23).

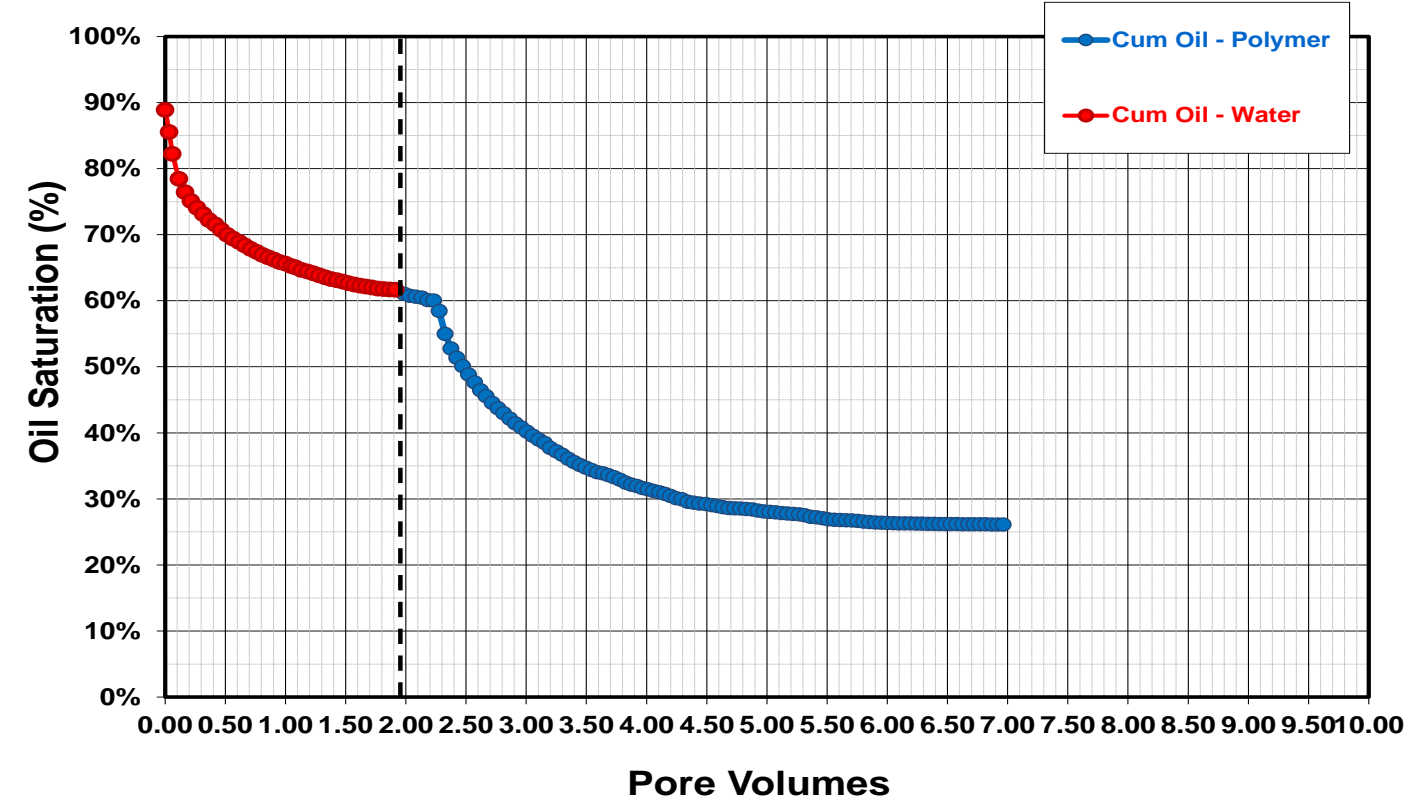

Figure 6.21 Oil Saturation for experiment \#7 


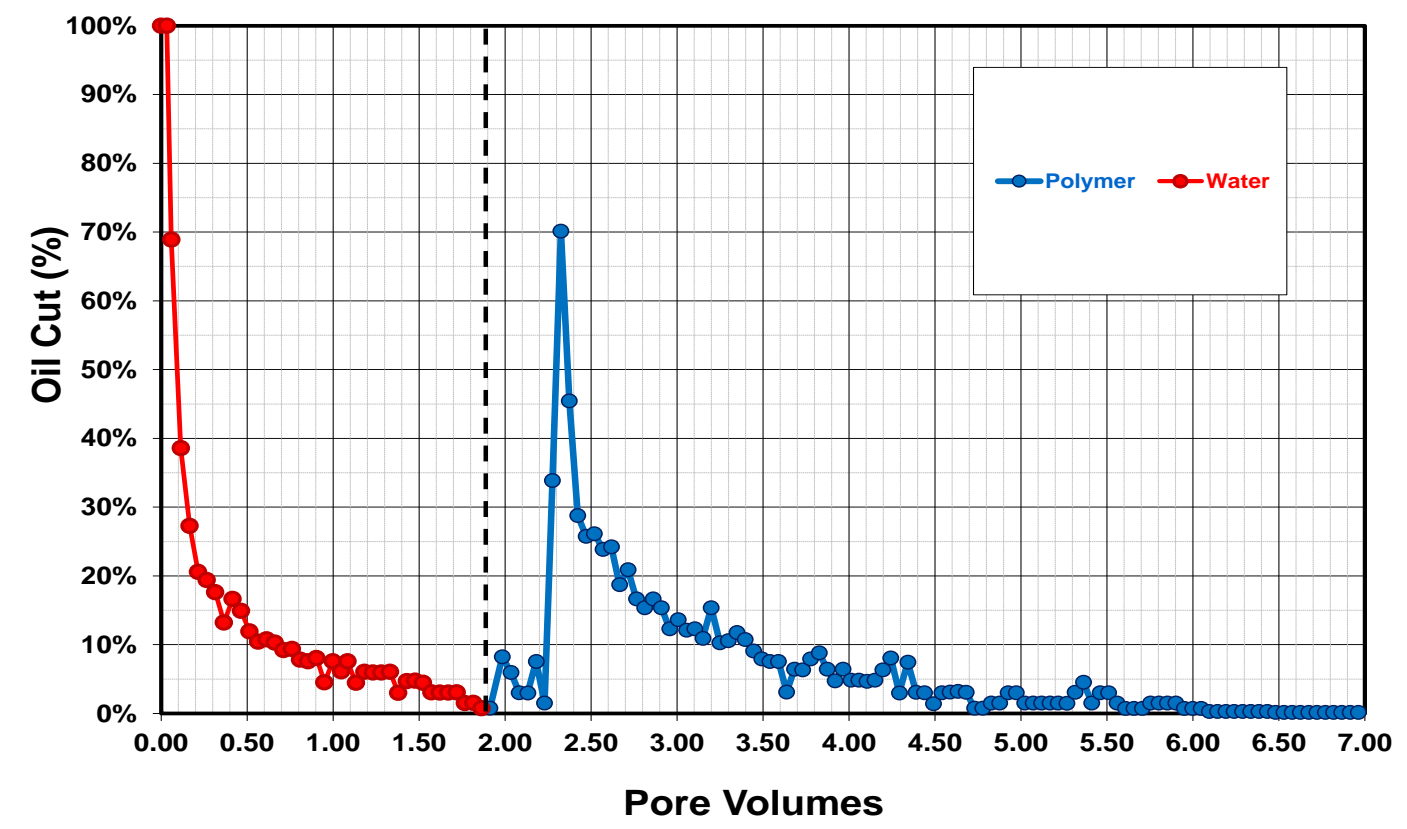

Figure 6.22 Oil Cut for experiment \#7

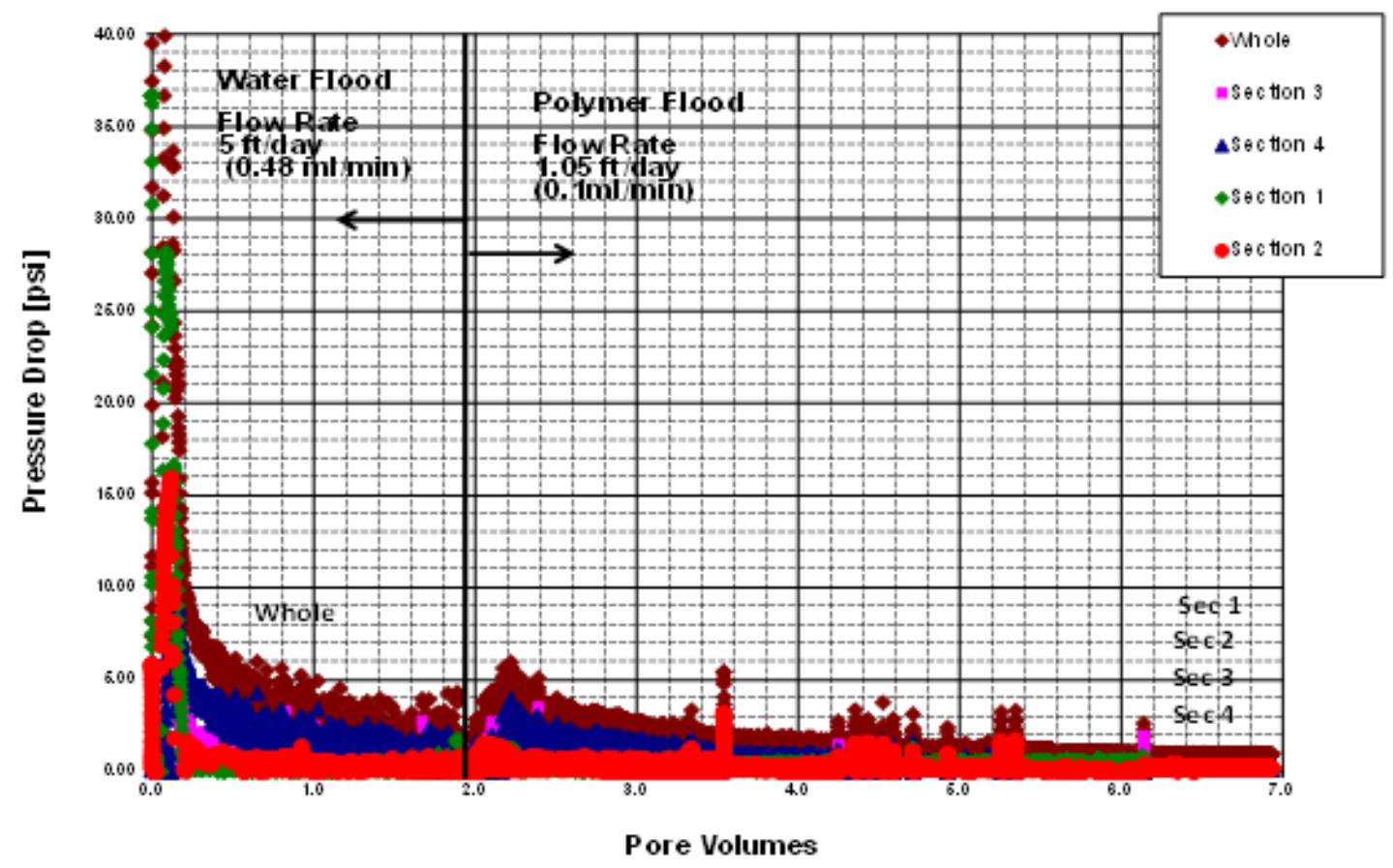

Figure 6.23 Pressure drop for experiment \#7 
Experiments \#8 and \#9 were performed to compare the polymer flood performance of secondary and tertiary polymer floods using the same reservoir core and crude oil. FP 3630S was used for both experiments. Sandpacks for each experiment were made by using reservoir sand in a 1.5 inch ID stainless steel core holder with pressure taps. A confining pressure of 1000 psi was applied to the core with an ISCO syringe pump. Each sandpack was vacuum saturated with formation brine whose composition is given in Table 6.4. The sand packs were placed in the $73^{\circ} \mathrm{C}$ oven and flushed with formation brine under a back pressure of at least 20 psi to remove any possible remaining air. A salinity tracer test was used to estimate the pore volume of the sand packs. Then formation brine was injected to measure the pressure drop across the sand pack to determine the brine permeability. Next, crude oil was injected until no more water was produced. The core was aged for 14 days and then at least another pore volume of oil was injected to measure any additional water production caused by a shift in the wettability after aging. The pressure drop versus flow rate was measured to determine the oil permeability at residual water saturation.

Table 6.4 Brine Compositions

\begin{tabular}{|c|c|c|c|}
\hline Ion & & Lake Water & Formation Brine \\
\hline $\mathrm{Ca}^{2+}$ & ppm & 9 & 170 \\
\hline $\mathrm{Mg}^{2+}$ & ppm & 23 & 44 \\
\hline $\mathrm{Na}^{+}$ & ppm & 49 & 1954 \\
\hline $\mathrm{K}^{+}$ & $\mathrm{ppm}$ & 49 & 334 \\
\hline $\mathrm{Cl}^{-}$ & $\mathrm{ppm}$ & 195 & 3739 \\
\hline $\mathrm{SO}_{4}^{-}$ & $\mathrm{ppm}$ & 11 & 11 \\
\hline $\mathrm{TDS}$ & $\mathrm{ppm}$ & 336 & 6038 \\
\hline
\end{tabular}

For experiment $\# 8$, a solution of 800 ppm polymer in Lake Water was injected at $1 \mathrm{ft} /$ day $(0.053 \mathrm{~mL} / \mathrm{min})$ until no more oil was produced. The residual oil saturation at the 
end of the polymer flood was 0.26 . The oil recovery is shown in Figure 6.25. The pressure drop is shown in Figure 6.26.

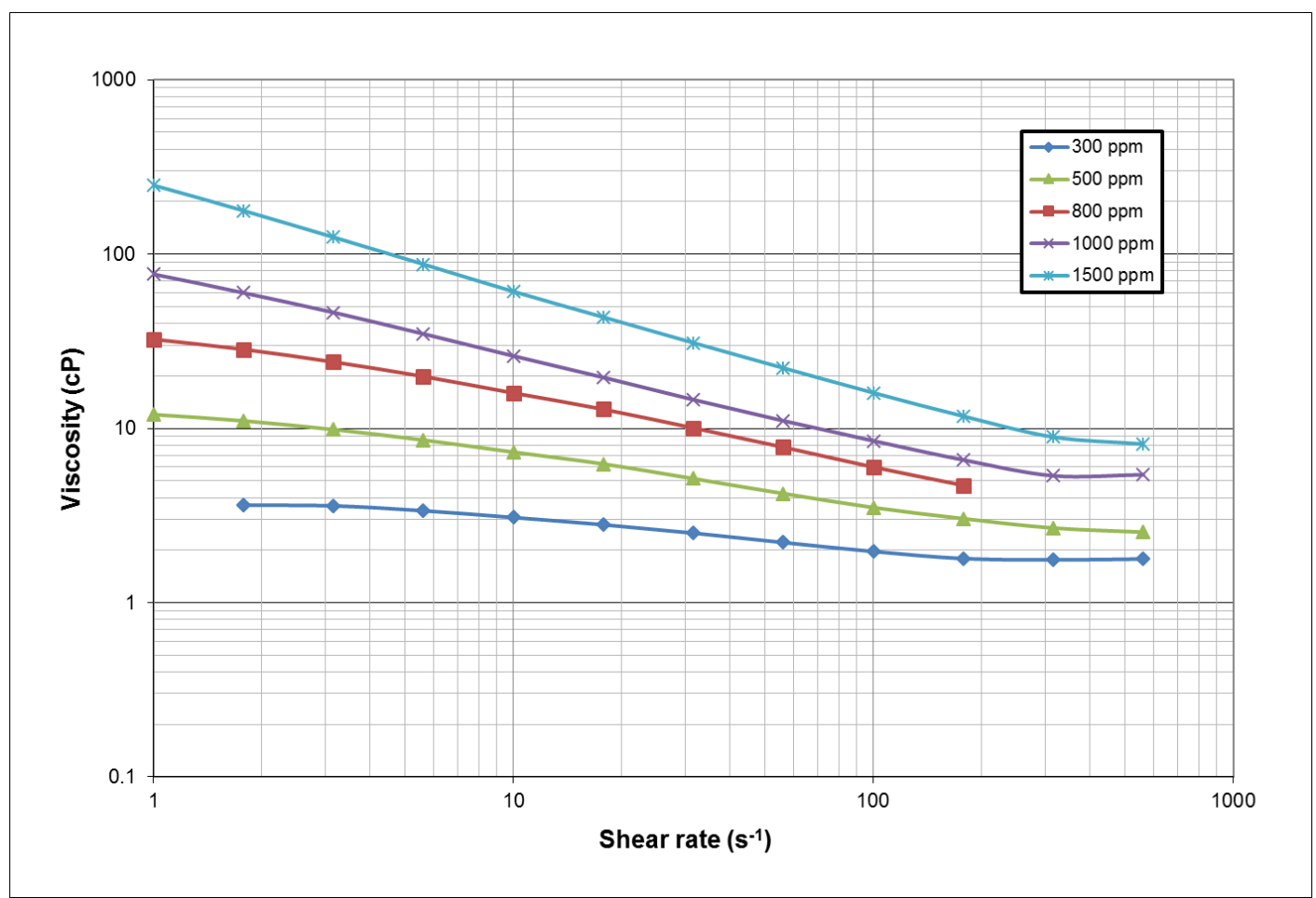

Figure 6.24 Viscosity of FP $3630 \mathrm{~S}$ polymer solutions in Lake Water at $73^{\circ} \mathrm{C}$ 


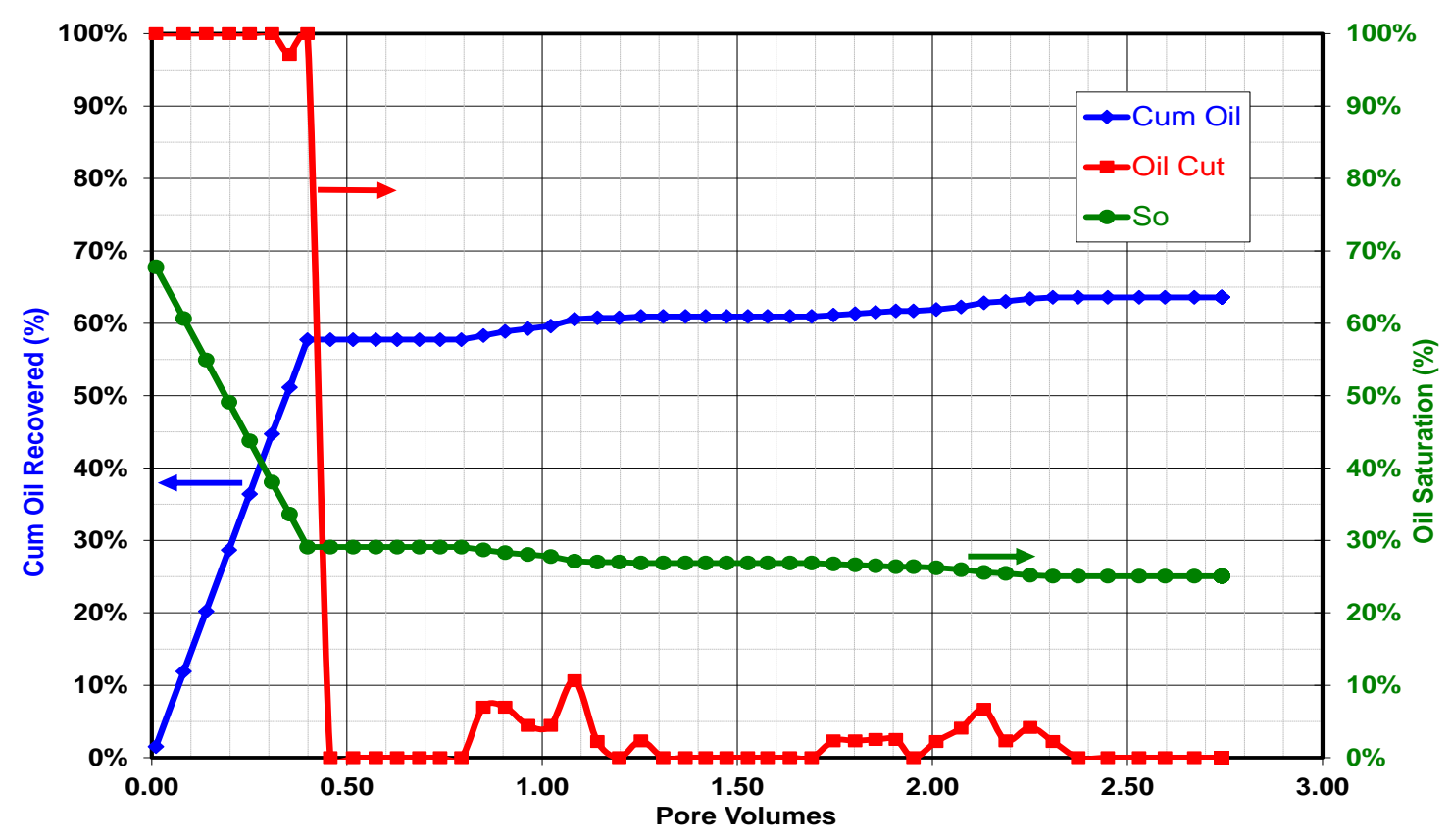

Figure 6.25 Oil Recovery for experiment \#8

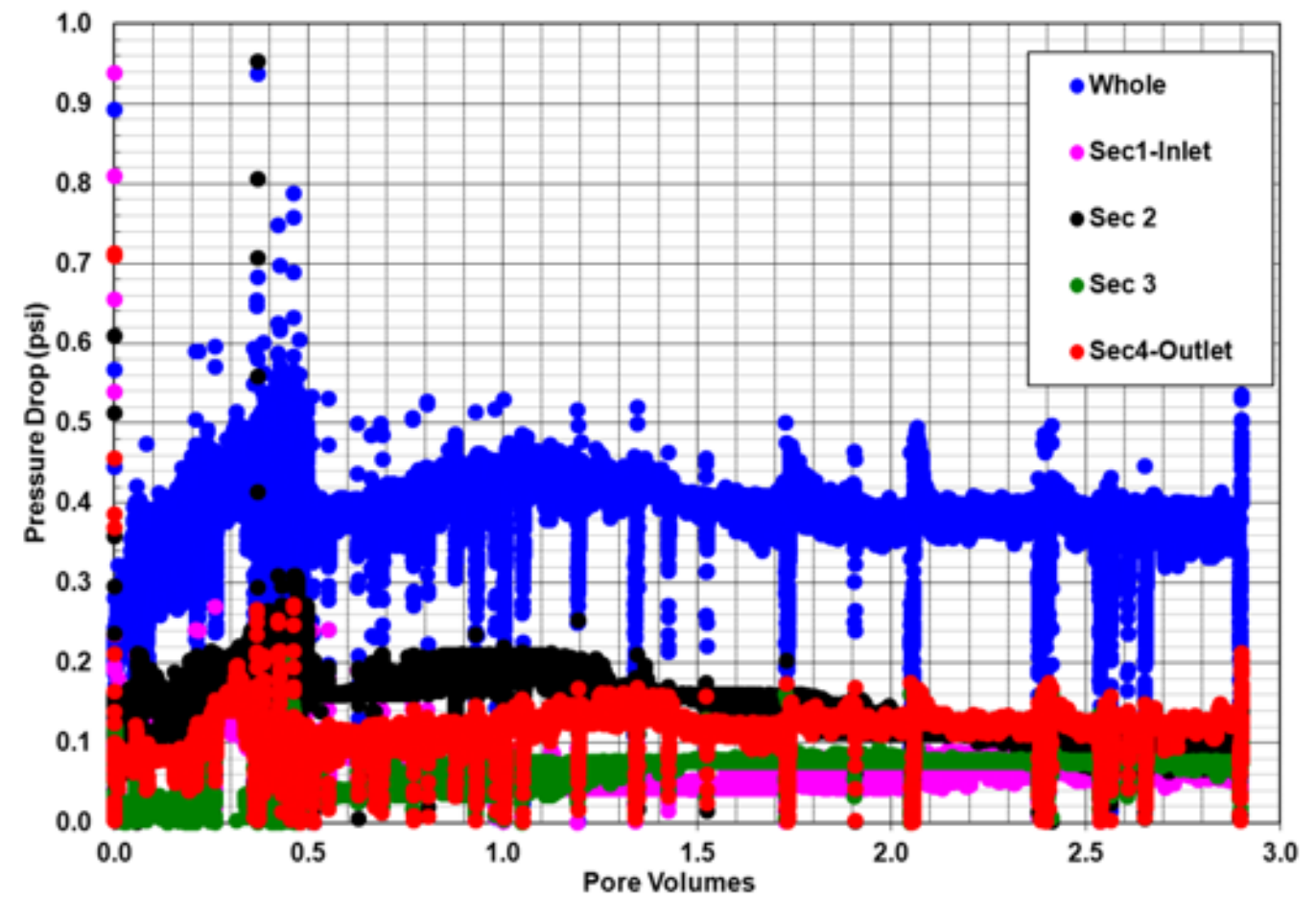

Figure 6.26 Pressure drop data for experiment \#8 
For experiment $\# 9$, Lake Water was injected at $1 \mathrm{ft} /$ day $(0.065 \mathrm{~mL} / \mathrm{min})$ until the water cut was $99 \%$. The remaining oil saturation at the end of the water flood was 0.32 . Following the water flood, a solution of $800 \mathrm{ppm}$ polymer in lake water was injected at 1 $\mathrm{ft} /$ day $(0.065 \mathrm{~mL} / \mathrm{min})$ until no more oil was produced. The oil recovery is shown in Figure 6.27. The pressure drop is shown in Figure 6.28. The residual oil saturation at the end of the polymer flood was 0.28 , which is 0.04 less than the water flood remaining oil saturation (Figure 6.27) and 0.02 higher the polymer flood shown in Figure 6.25 (experiment \#8).

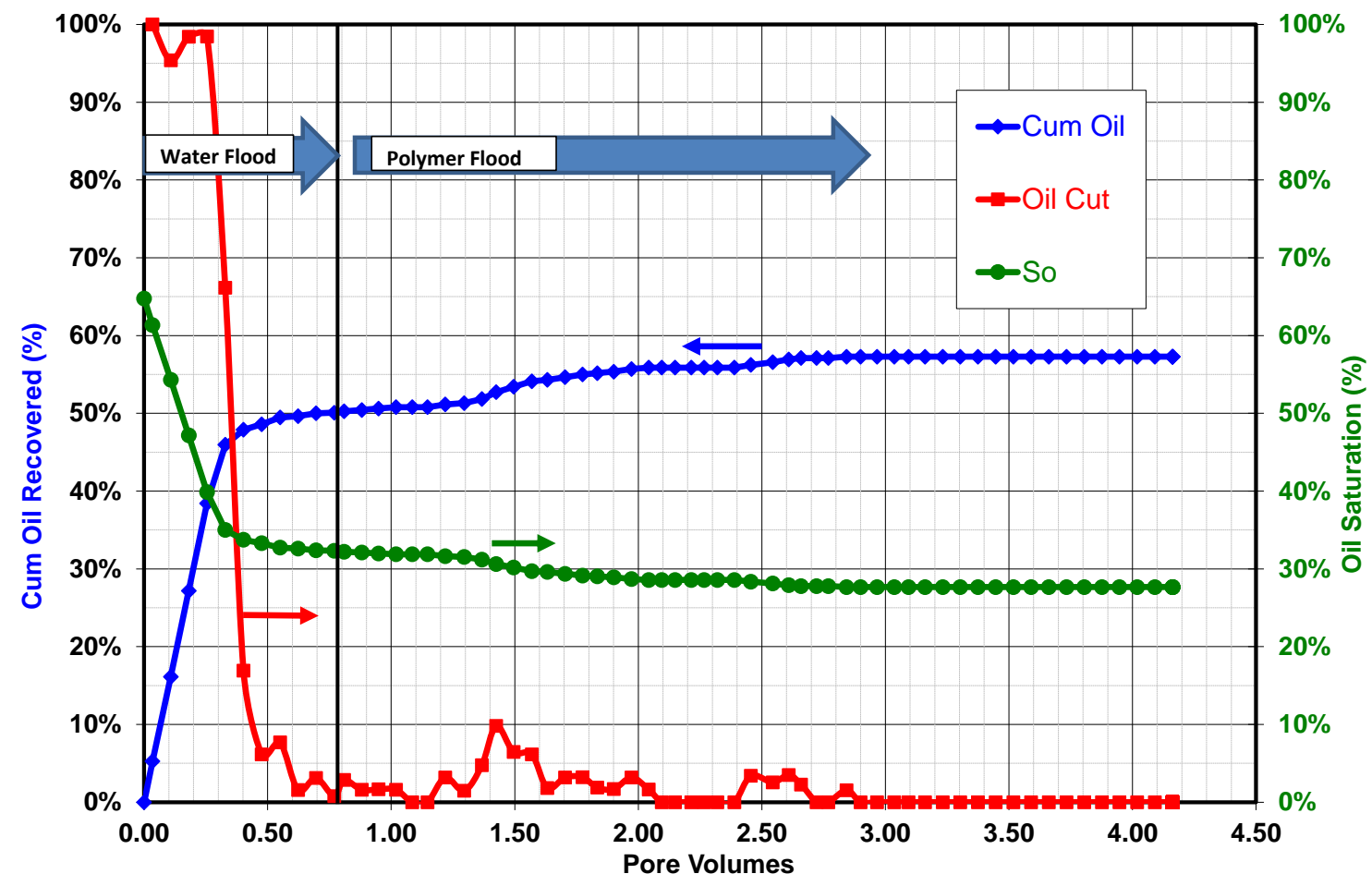

Figure 6.27 Oil Recovery for experiment \#9 


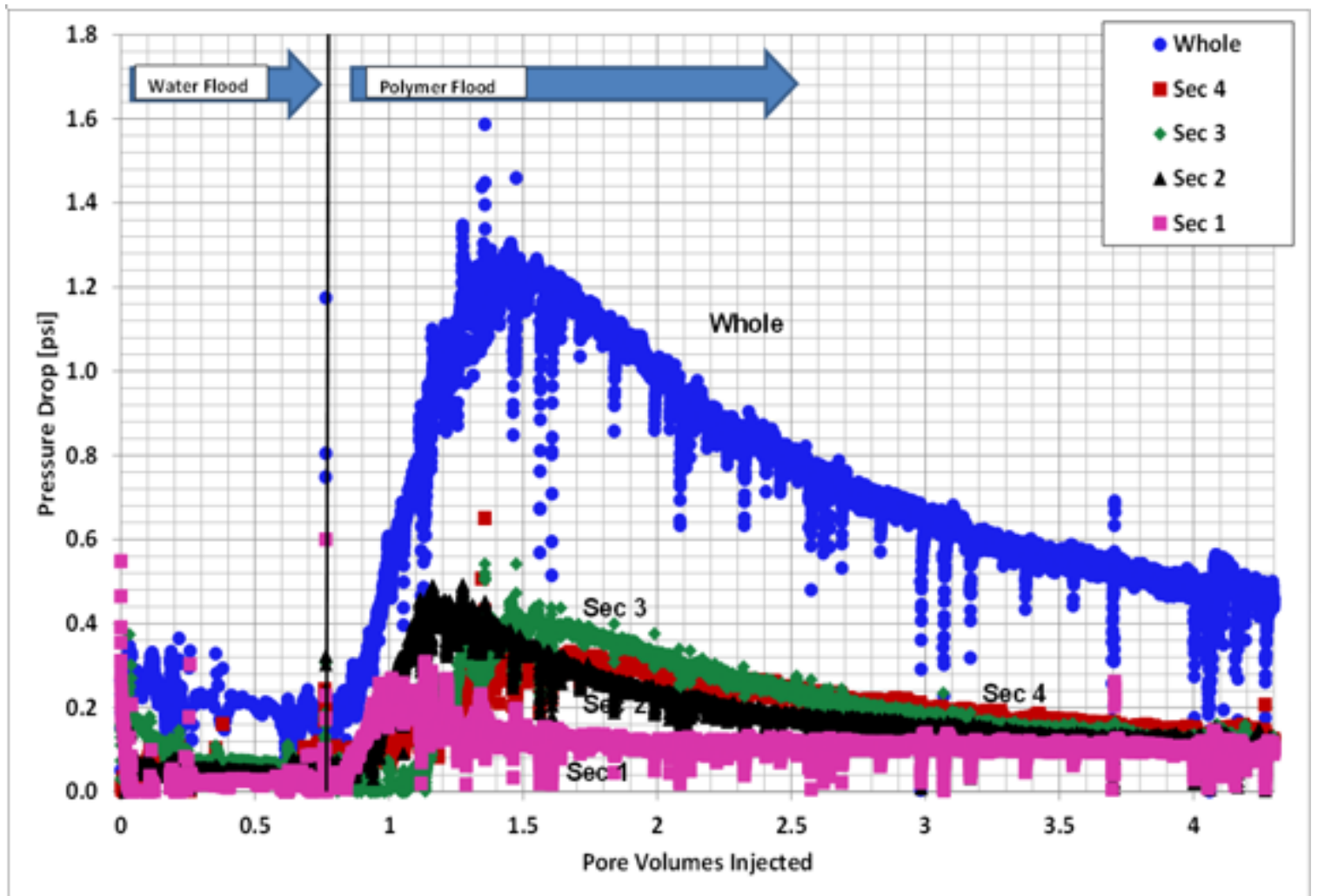

Figure 6.28 Pressure drop data for experiment \#9

\subsubsection{Analysis of Coreflood Results}

The initial core properties for experiments \#1, \#2, \#3 and \#7 were similar since F95 Ottawa sand with the properties shown in Table 6.2 was used for all four of these experiments. Oil viscosities for each experiment are shown in Table 6.1. The water flood data are summarized in Table 6.5. The oil saturations versus pore volumes of injected water are shown in Figure 6.29. The oil saturations up to $99 \%$ water cut are shown in Figure 6.30. 


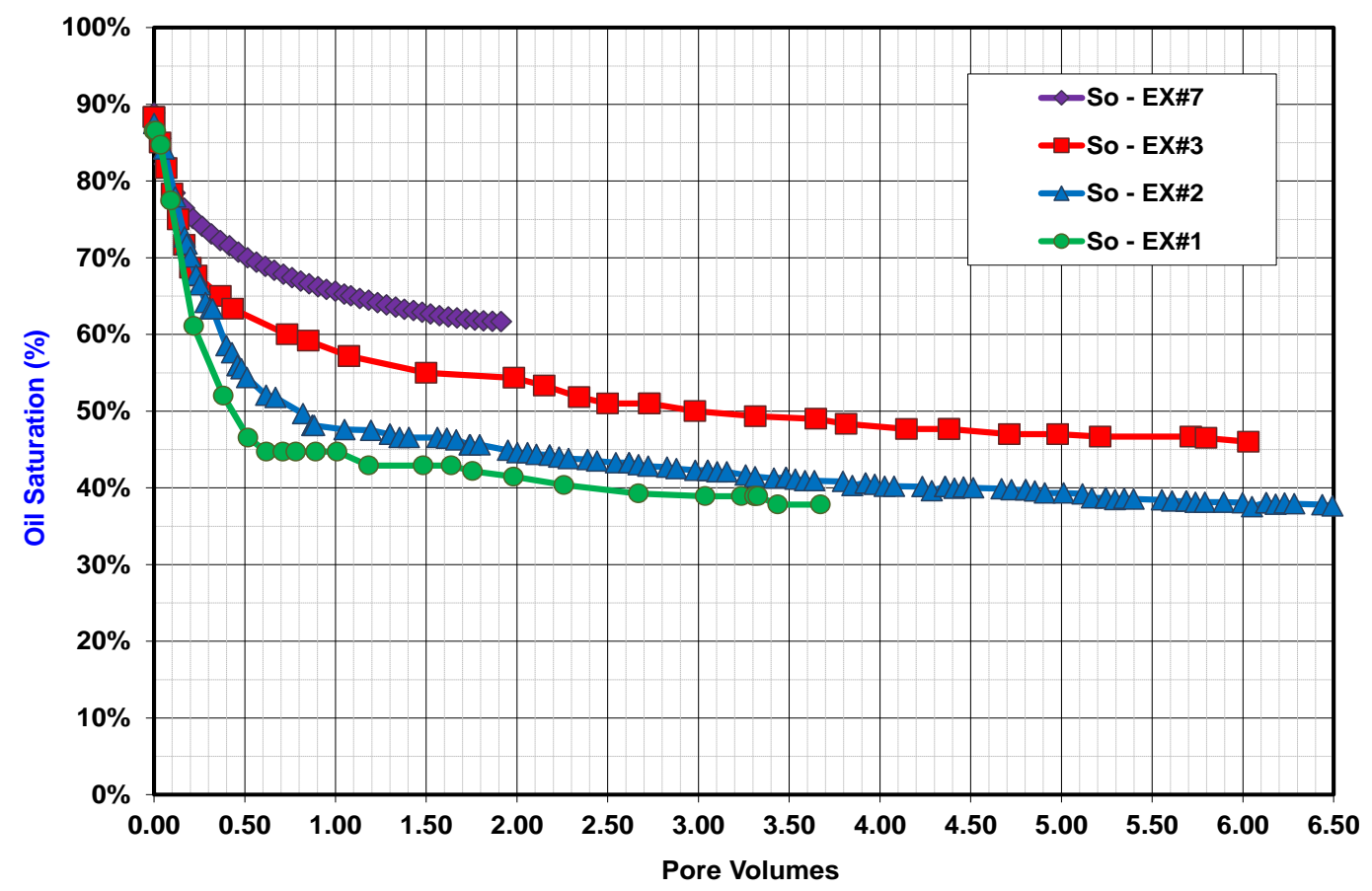

Figure 6.29 Comparison of oil saturations for experiments \#1, \#2, \#3 and \#7 water floods

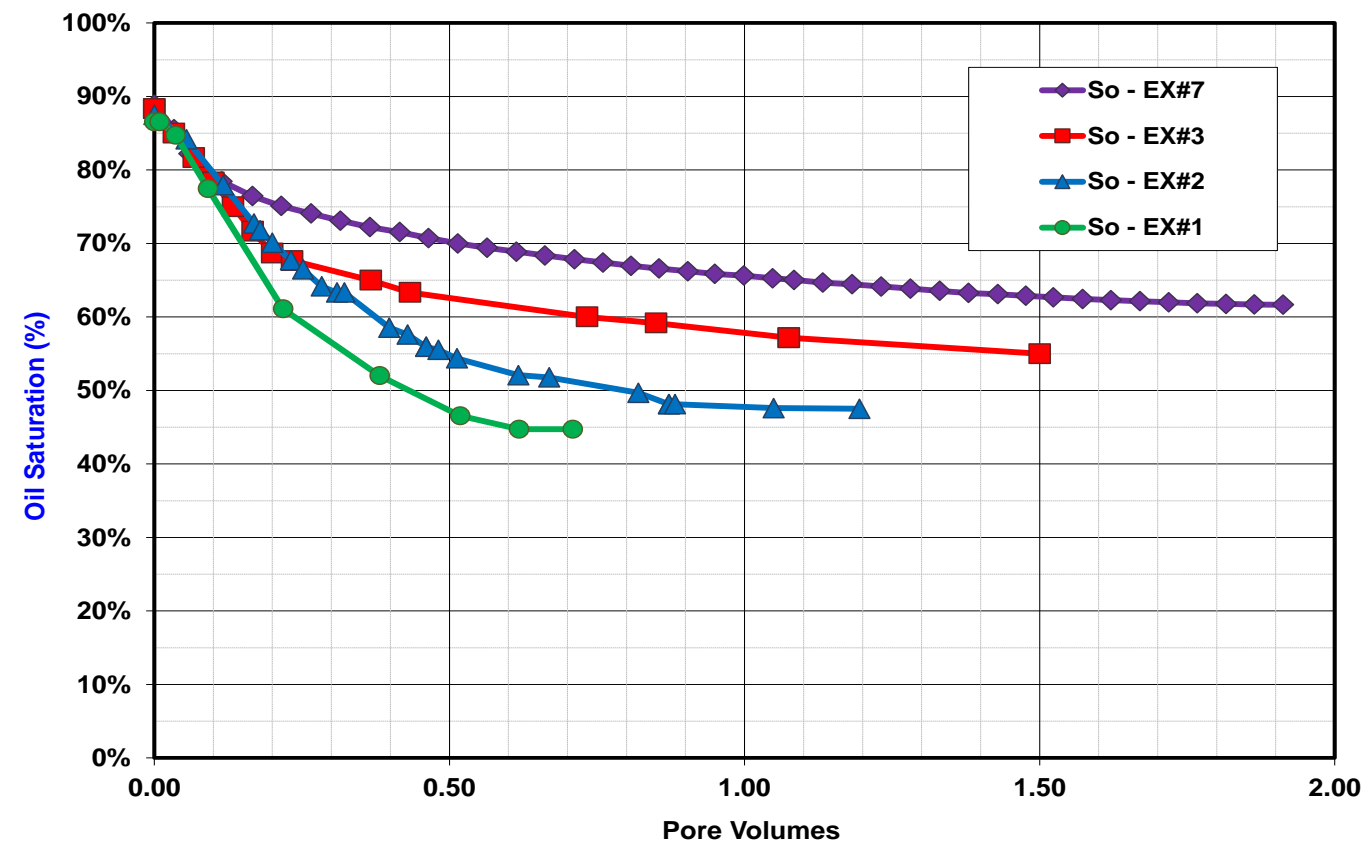

Figure 6.30 Comparison of oil saturation for experiments \#1, \#2, \#3 and \#7 water floods up to $99 \%$ water cut 
Table 6.5 Water flood results for experiments \#1, \#2, \#3 and \#7

\begin{tabular}{|c|c|c|c|c|}
\hline Experiment & $\# 1$ & $\# 2$ & $\# 3$ & $\# 7$ \\
\hline Water viscosity & 0.48 & 0.48 & 0.48 & 0.66 \\
\hline $\begin{array}{c}\text { oil/water } \\
\text { viscosity }\end{array}$ & 166 & 250 & 520 & 1590 \\
\hline $\begin{array}{c}\text { krw } \\
\text { at 1\% oil cut }\end{array}$ & 0.045 & 0.024 & 0.055 & 0.017 \\
\hline $\begin{array}{c}\text { krw at end of } \\
\text { water flood }\end{array}$ & 0.075 & 0.081 & 0.092 & 0.1 \\
\hline $\begin{array}{c}\text { End-point kro } \\
\text { Mobility Ratio }\end{array}$ & 12.5 & 0.84 & 0.814 & 1.17 \\
\hline $\begin{array}{c}\text { Initial oil } \\
\text { saturation }\end{array}$ & 0.87 & 0.88 & 0.88 & 0.89 \\
\hline $\begin{array}{c}\text { Oil saturation at } \\
\text { end of water } \\
\text { flood }\end{array}$ & 0.38 & 0.38 & 0.47 & 0.61 \\
\hline
\end{tabular}

There is a significant difference in the Deborah numbers for experiments \#1, 2 and 3. The Deborah number for experiment \#3 was 16, which is $4 \sim 5$ times higher than that of experiments $\# 1$ and $\# 2$, but the residual oil saturation of experiment $\# 3$ of 0.23 is only slightly less than the residual oil saturation of experiments \#1 and \#2 (0.26 and 0.24). As discussed in Chapter 4, 2450 ppm FP 3630S in $0.2 \% \mathrm{NaCl}$ brine has a higher relaxation time than the other polymer solutions. Most of oil was produced before polymer breakthrough and just 2 3\% additional oil was produced after polymer breakthrough indicating a very efficient displacement of the oil by the polymer solutions.

While experiments \#1, \#2 and \#3 were polymer floods in secondary mode, experiment \#7 was conducted in tertiary mode. It is interesting that the remaining oil saturation of 0.26 is the same as the secondary polymer flood with a mobility ratio less 
than 1 even though oil/polymer viscosity ratio was 1591 and mobility ratio was 10 . However, the oil recovery profile for experiment \#7 was different than for experiments $\# 1, \# 2$ and \#3. For experiment \#7, the additional oil recovery after polymer breakthrough was $28 \%$ of the initial oil saturation, or $82 \%$ of the additional oil recovery by polymer injection. This means the mechanism of oil recovery is different from the previous experiments. In this case, since it is tertiary polymer flood, the displacement after polymer breakthrough seems to be dominated by improved sweep along existing water/polymer channels as fingers are widened and coalesce to water channel by polymer flood. The oil mobilized by the improved sweep by the polymer result in the high oil cut after polymer breakthrough in Figure 6.22. This can be explained by polymer shear thinning behavior in porous media combining with the fraction flow theory below.

\section{Polymer Retention}

In polymer retention experiments, a polymer solution of known concentration is flooded through a core and the effluent polymer concentration is measured. In this manner, a concentration history as shown in Figure 6.31 is obtained. The polymer retention is then calculated from a mass balance on the polymer. The amount of polymer that is retained in the porous medium is related to the shaded area in Fig 6.31 minus the pore volume.

The pore volume is the effective pore volume that is filled with polymer solution. 


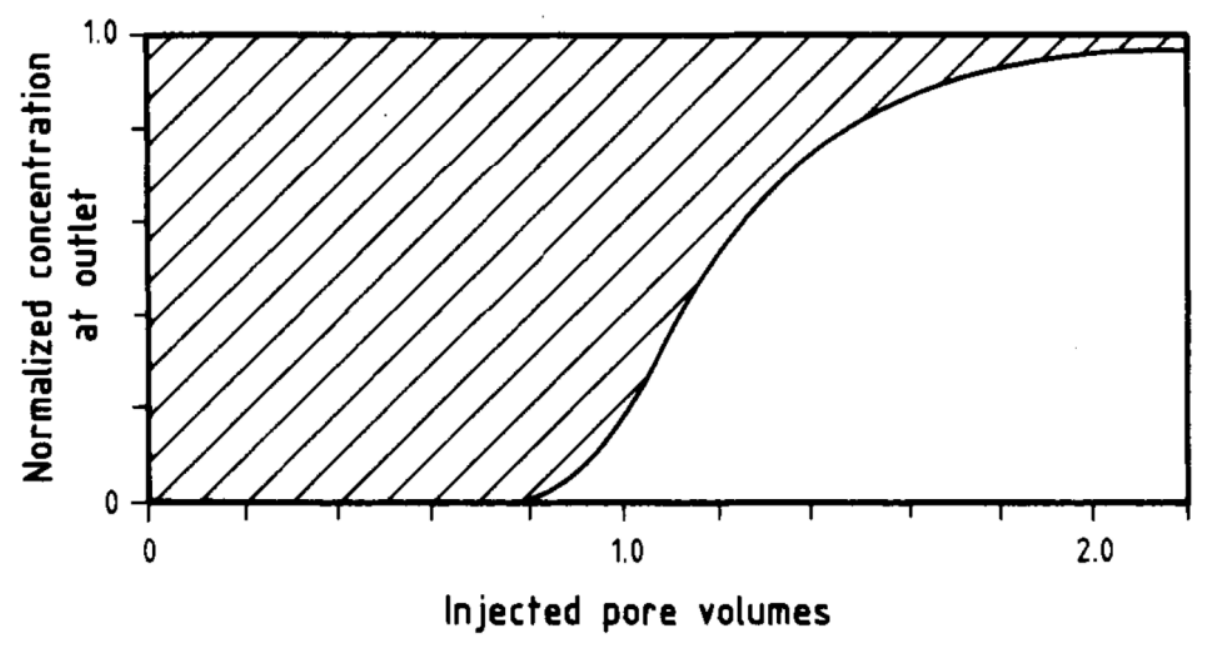

Figure 6.31: Concentration profile at outlet in a core flood experiment.

\section{Determination of polymer concentration to calculate polymer retention}

Several methods have been used to measure concentration of polyacrylamide polymers: Size exclusion chromatography (SCE), fluorescence spectrophotometry, polarography, electron spin resonance spectrophotometry (ESR), radioactive labeling, calorimetry, viscosity, organic carbon content, ion chromatography (IC), UV-visible spectrophotometry, refractive index, precipitation, colloid titration and turbidimetry are some of the methods that have been reported in the literature. Each of these methods have their own advantages and disadvantages. For example using the viscosity to determine the polymer concentration, much easier method, but the non-Newtonian properties and their sensitivity to ionic strength, the measurement becomes much more complicated.

Turbidimetry is a well-known method to determine the polymer concentration. Herein, acrylamide co-polymer is subjected to react with compounds that can produce an insoluble complex. The formed colloidal complex can be quantitatively measured by the light scattering using a turbidimeter or a spectrophotometer. Hyamine 1622, Tannic acid, Superfloc 310 and bleach are some of the chemicals reported in the literature.

The turbidimetric method with bleach was used in this study. Polyacrylamides react with sodium hypochlorite (bleach) under acidic conditions to produce an insoluble 
product (chloramide) giving a colloidal suspension. Figure 6.32 shows a schematic reaction of formation of chloramide. The turbidity produced by this reaction is measured by a spectrophotometer at two wave lengths $470 \mathrm{~nm}$ and $520 \mathrm{~nm}$.

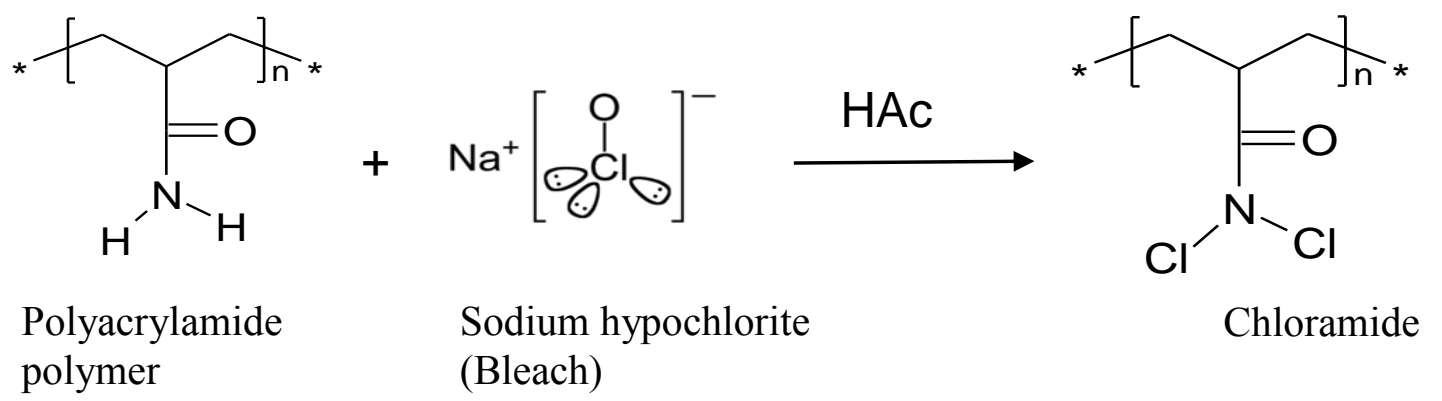

Figure 6.32 Polyacrylamide reaction with bleach to produce chloramide insoluble suspension.

\section{Experimental Method}

\section{Materials}

5000 ppm FP 3330, FP 3630 in brine stock solutions as polyacrylamide, Bleach (25\% "Clorox") as Sodium hypochlorite and 5N Acetic acid were used.

1. Polymer Preparation for the calibration plot

Dilutions of the stock polymer solution with appropriate brine solution to prepare standard solutions 50, 100, 150, 200, 250, 300, 350, 400, 450, and $500 \mathrm{ppm}$.

2. Sample polymer solution preparation

Effluent samples were diluted 10 fold to 20 fold depending on the injected polymer concentration. Every other sample of the core flood effluents were used for concentration measurements.

3. Sample preparation for the measurements

A volume of $3 \mathrm{~mL}$ of the standard solution or the sample solution was added to a vial and $3 \mathrm{~mL}$ of $5 \mathrm{~N}$ acetic acid was added to the vial. The solution was stirred for 2-4 hours to mix. The bleach must be added just before starting the measurements. $3 \mathrm{~mL}$ of bleach 
solution was added, stirred gently for 5 minutes and transfer about $4 \mathrm{~mL}$ to a quartz cell and measure the UV-Vis absorbance at $470 \mathrm{~nm}$ and $520 \mathrm{~nm}$.

Figure 6.33 shows the absorbance spectrum of the polymers with different concentrations and the Figure 6.34 show the calibration plot for different polymer concentrations at 520 $\mathrm{nm}$.

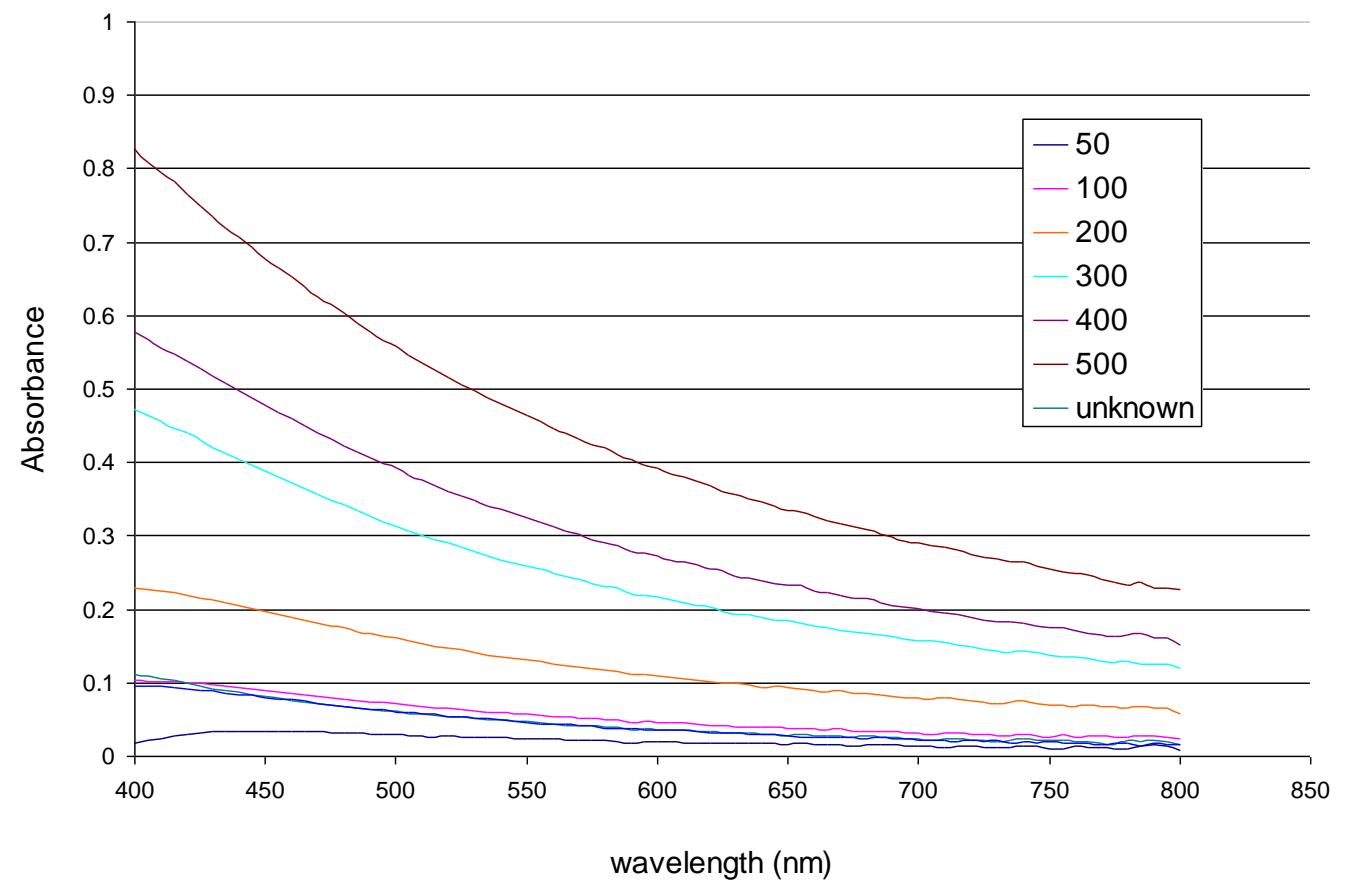

Figure 6.33: UV-visible spectra for different concentrations of FP $3330 \mathrm{~S}$ in $3 \% \mathrm{NaCl}$.

From the calibration plot we can estimate the polymer concentration in the unknown polymer sample. 


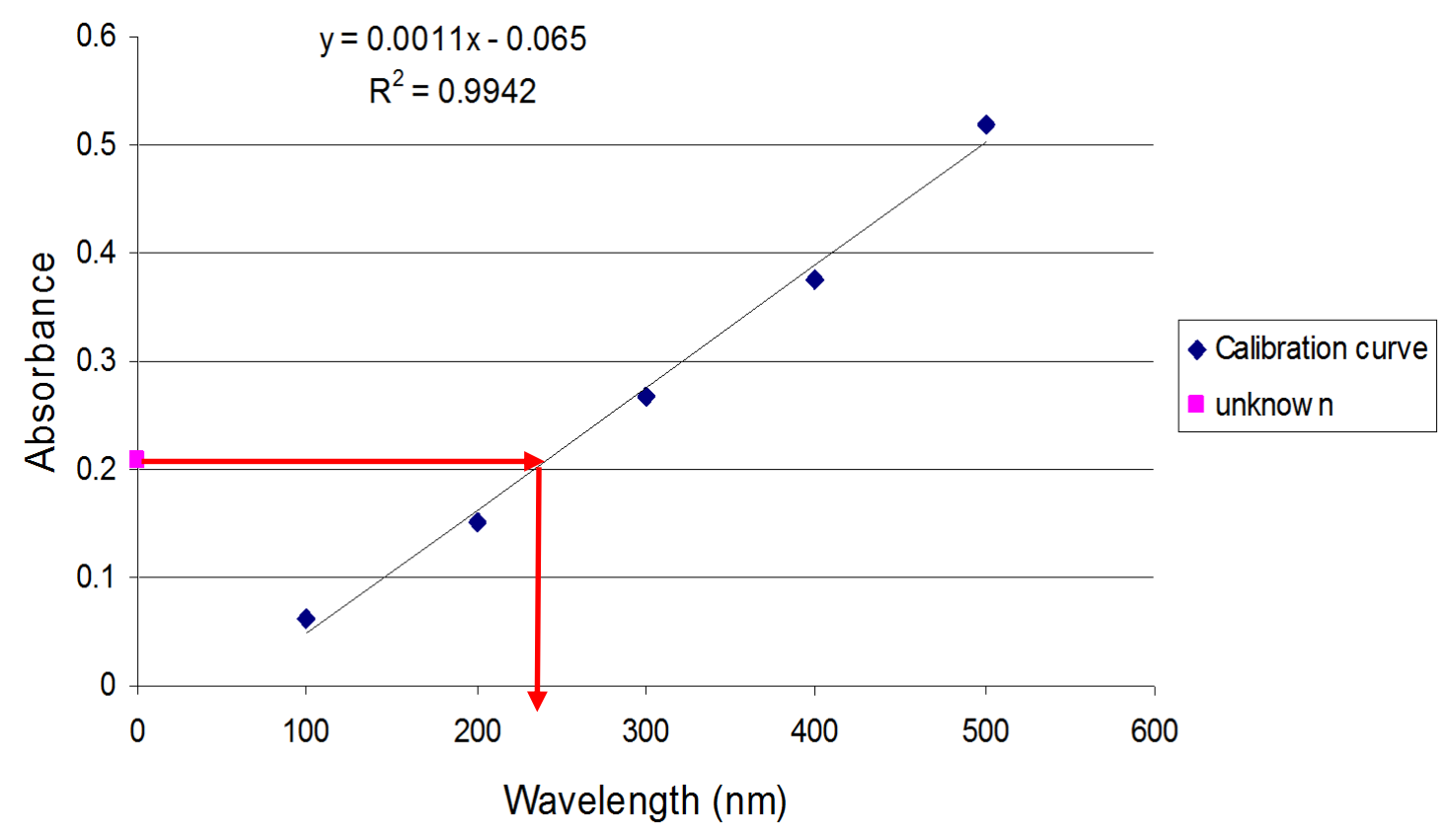

Figure 6.34 Calibration curve for FP 3330 polymer in 3\% $\mathrm{NaCl}$.

\section{Polymer concentration measurements for corefloods \#7 and \#10}

The effluent samples were diluted and the polymer concentration measured according to the turbidity method. Core flood \#7 was done using FP 3630S and \#10 was done using ChemPam 8177. Figure 6.35 is the calibration plot for the FP 3630S in high salinity brine (51000 ppm TDS) and the Figure 6.36 is the calibration plot for the ChemPam 8177 in high salinity brine (51000 ppm TDS). 


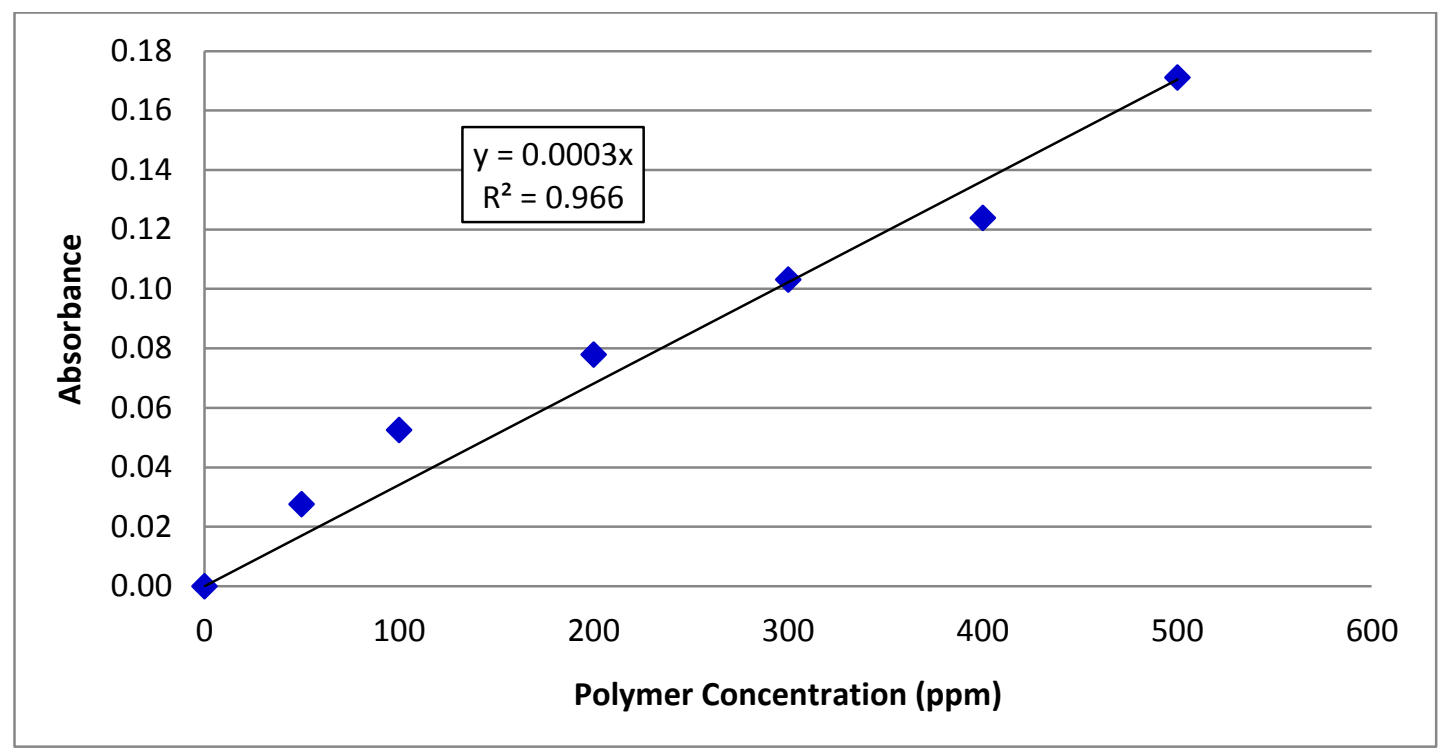

Figure 6.35 Calibration curve for FP 3630S polymer standard solutions in high salinity brine (56000 ppm TDS) at $520 \mathrm{~nm}$.

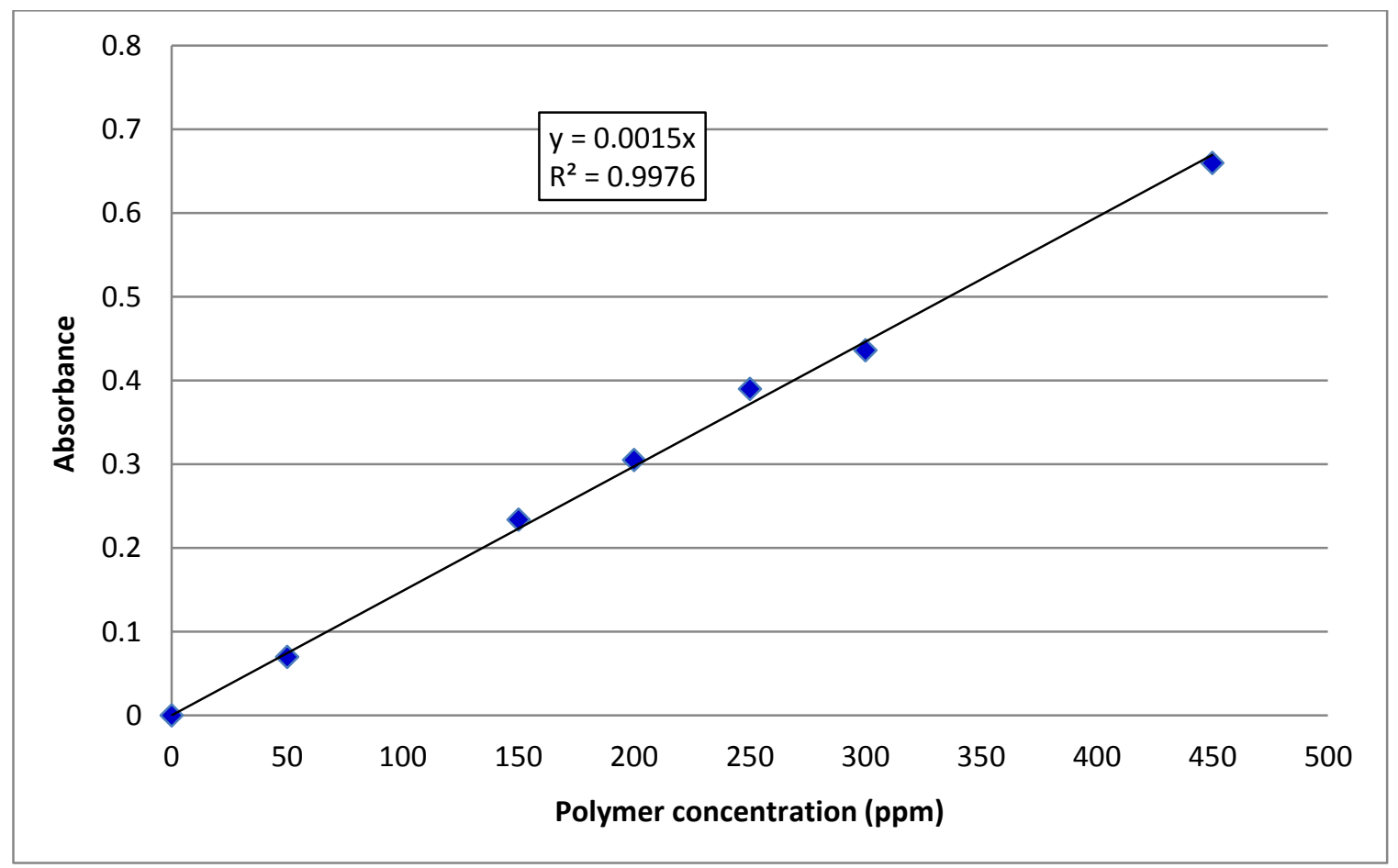

Figure 6.36 Calibration curve for ChemPam 8177 polymer standard solutions in high salinity brine (56000 ppm TDS) at $520 \mathrm{~nm}$. 
By using the calibration curve, the effluent polymer concentrations were estimated. Figures 6.37 and 6.38 show the effluent concentrations. The polymer retention for experiment \#7 was $43 \mu \mathrm{g} / \mathrm{g}$ of rock and for coreflood \#10 it was $574 \mu \mathrm{g} / \mathrm{g}$ of rock. Also by looking at the viscosity curve, we can see its complexity towards estimating the concentration. The viscosities were measured at $11 \mathrm{~s}^{-1}$ shear rate. And also the viscosity reached its maximum after 3 PVs for experiment $\# 7$ and was not reached even after 6 PVs for the core flood \#10. Therefore the polymer concentration method is a better way to estimate the polymer retention than the viscosity method.

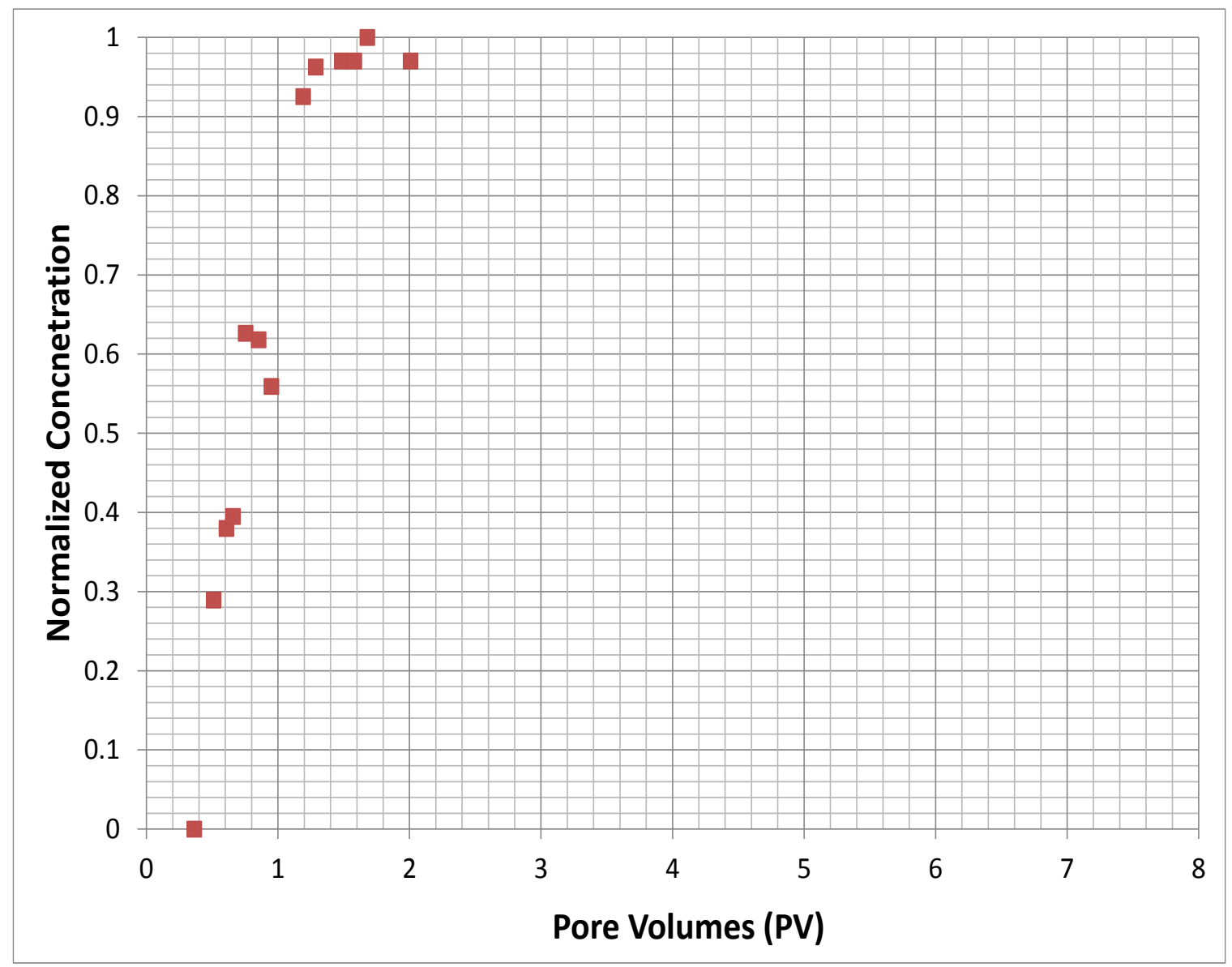

Figure 6.37 Effluent polymer concentration for experiment \#7. 


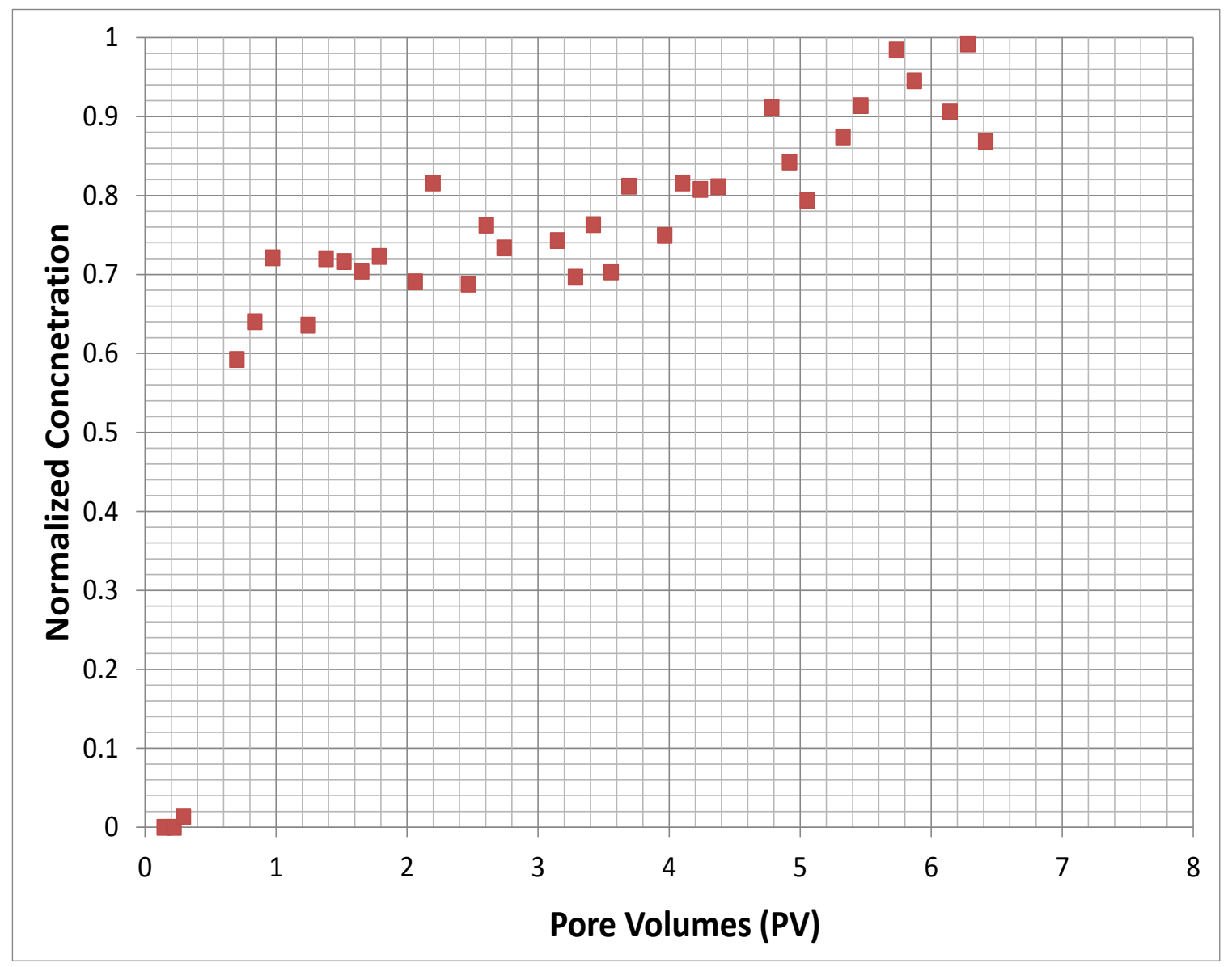

Figure 6.38 Effluent polymer concentration for experiment \#10. 


\section{Fractional Flow Analysis}

Each of the coreflood experiments has been analyzed using fractional flow theory. Fractional flow theory can be used to calculate the displacement sweep efficiency for both water floods and polymer floods. Only first order effects are modeled using fractional flow theory. In other words, capillary pressure, dispersion and compressibility are neglected. However, it is still useful to understand the effect of polymer on displacement sweep efficiency for both water floods and polymer floods.

In corefloods, improvement in displacement sweep efficiency is usually the primary cause for higher oil recovery. However, the more heterogeneous the core, the greater the impact of the polymer on the volumetric sweep efficiency, although the impact is much greater on a reservoir scale. The reduction in residual oil saturation is often small and depends on many factors that are still difficult to quantify after many years of research. The effect of polymer viscoelasticity on the residual oil saturation was reviewed in Chapter 2.

The motivation in analyzing each of the polymer corefloods using fractional flow theory was to determine how much of the improved oil recovery could be explained by displacement sweep efficiency. Since the cores used in this study were nearly homogeneous, the effect of volumetric sweep efficiency was assumed to be small. The reduction in the true residual oil saturation is unknown. However, the approach was taken that if the residual oil saturation was assumed to be the same for polymer as for water and the fractional flow calculation was in good agreement with the oil recovery data, then it could be inferred that the reduction in true residual oil saturation is either zero or negligible under the specific conditions of these corefloods. The capillary number was 
maintained around $10^{-7}$ and the in situ equivalent shear rate for the polymer drive was between 8 and $80 \mathrm{~s}^{-1}$.

It is very important to distinguish between residual oil saturation and remaining oil saturation. When the oil is viscous as in most of these corefloods or more precisely when the mobility ratio is high, then a few pore volumes of water are insufficient to reduce the oil saturation to its true residual value, so the oil saturation at the end of water injection is called the remaining oil saturation. However, it is not appropriate to use the remaining oil saturation in the fractional flow equations. This simple insight proved to be very helpful in the fractional flow analysis results discussed below.

Polymer corefloods are typically done at a constant flow rate in linear cores with a constant cross-sectional area (A) and uniform or nearly uniform porosity. Therefore, the frontal velocity $(\mathrm{q} / \mathrm{A} \phi)$ would be constant for single phase flow under these assumptions. However, for two-phase flow of water (or aqueous phase) and oil, the velocity is not constant since the water saturation is changing. This in turn means the shear rate is not constant and the polymer viscosity depends on shear rate, so it is also not constant. In most simplified applications of fractional flow theory, the polymer solution viscosity is assumed to be constant, but in this analysis the effect of shear rate was taken into account as shown below.

The major assumptions for fractional flow theory are summarized below (Pope, 1980):

1. Dispersion and capillary pressure are neglected

2. Uniform adsorption of polymer on rock

3. One-dimensional linear flow

4. $\phi, \rho_{\mathrm{w}}, \rho_{\mathrm{p}}, \mu_{\mathrm{w}}$ constant 
5. Continuous injection of polymer at constant concentration

6. There are no chemical or biological reactions.

8. Fluids are incompressible

10. Isothermal reservoir

11. Local equilibrium

The governing differential equation for mass conservation in 1D linear flow is,

$$
\varnothing \frac{\partial S_{w}}{\partial t}+u \frac{\partial f_{w}}{\partial t}=0
$$

For water flood, the fractional flow neglecting gravity is

$$
f_{w}=\frac{1}{1+\frac{k_{r o} \mu_{w}}{k_{r w} \mu_{o}}}
$$

For polymer flood, the fractional flow neglecting gravity is

$$
f_{p}=\frac{1}{1+\frac{k_{r o} \mu_{p}}{k_{r p} \mu_{o}}}
$$

The polymer solution was modeled as a power law fluid. Polymer solutions obey shear thinning rheology at moderate shear rates corresponding to the velocities of the coreflood experiments analyzed below. 


$$
\begin{gathered}
\mu_{p}=K_{p l}\left(\dot{\gamma}_{a p p}\right)^{n-1} \\
\dot{\gamma}_{a p p}=C\left(\frac{3 n+1}{4 n}\right)^{\frac{n}{n-1}} \frac{4 u f_{p}}{\sqrt{8 k k_{r p} s_{w} \phi}}
\end{gathered}
$$

Substitution of the non-Newtonian fluid viscosity equation in terms of an apparent or equivalent shear rate into the definition of $f_{p}$ yields,

$$
f_{p}=\frac{1}{1+\frac{k_{r o} K_{p l}}{k_{r w} \mu_{o}}\left\{C\left(\frac{3 n+1}{4 n}\right)^{\frac{n}{n-1}} \frac{4 u f_{p}}{\sqrt{8 k k_{r w} s_{w} \phi}}\right\}^{n-1}}
$$

Equation 6.9 is a non-linear equation for the fractional flow that must be solved by iteration.

Experiment \#3 was modeled using the fractional flow equations shown above. The relative permeability parameters are given in Table 6.6. As mentioned above, the true residual oil saturation for water and polymer were assumed to be the same. The only adjustable parameters were the Corey exponents. 
Table 6.6 Experimental data and adjusted Corey exponents for experiment \#3

\begin{tabular}{|c|c|c|}
\hline & Water flooding & Polymer flooding \\
\hline$k_{r w}{ }^{\circ}$ & 0.257 & 0.257 \\
\hline$k_{r o}{ }^{o}$ & 0.814 & 0.814 \\
\hline$n_{w}$ & 3.1 & 3.1 \\
\hline$n_{o}$ & 1.3 & 1.3 \\
\hline$S_{w r}$ & 0.12 & 0.12 \\
\hline$S_{w i}$ & 0.12 & 0.12 \\
\hline$S_{o r}$ & 0.23 & 0.23 \\
\hline$\mu_{w}(\mathrm{cP})$ & 0.48 & 108 \\
\hline$\mu_{o}(\mathrm{cP})$ & 250 & 250 \\
\hline
\end{tabular}

Numerous investigators have reported large permeability reduction factors for HPAM polymers. However, as shown in Table 5.13, the permeability reduction measured for FP 3630S polymer in Bentheimer sandstone was 1 (no reduction). All of the oil recovery coreflood experiments were modeled assuming no permeability reduction due to the polymer.

The retardation factor $\left(D_{p}\right)$ is needed for the fractional flow calculations shown below. A retardation factor of 0.05 was used for all of the experiments analyzed using fractional flow calculations. 


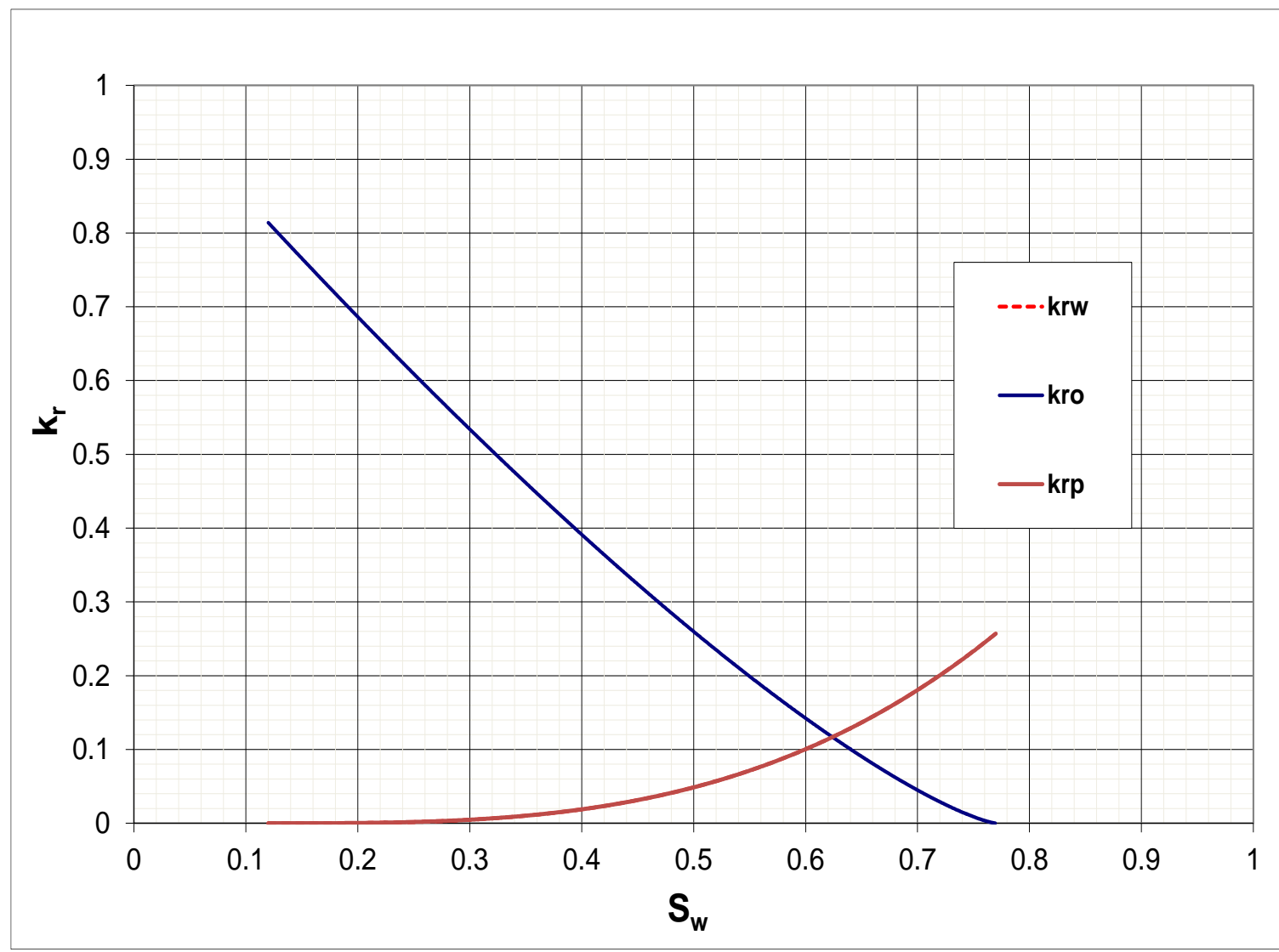

Figure 6.39 Relative permeability curve for experiment \#3

Based on the assumptions and experimental data in Table 6.6, the relative permeability curve was generated by using Corey-type model equations. The fractional flow curves shown in Figure 6.40 were generated based on the relative permeability curves shown in Figure 6.39. 


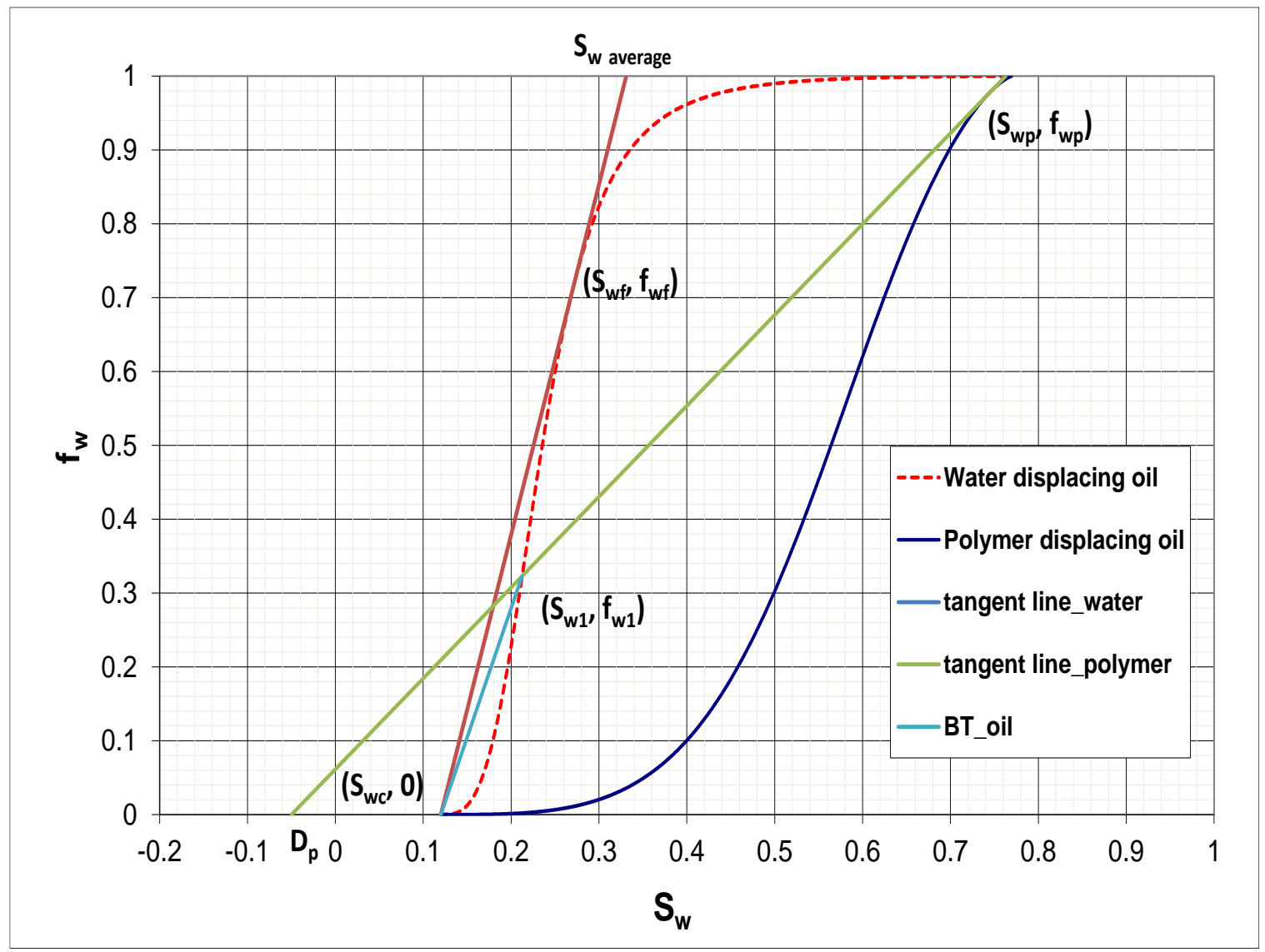

Figure 6.40 Fractional flow curve for experiment \#3

In Figure 6.40, all of fractional flow curves and tangent lines were calculated using equations 6.5 and 6.6. With this fractional flow curve, oil breakthrough time, water breakthrough time, polymer breakthrough time and oil cut were calculated.

It is known that the velocity and the saturations at the water front are found by equating the shock velocity to the Buckely-Leverett velocity for water/oil flow

$$
\left(\frac{\partial f_{w}}{\partial S_{w}}\right)_{S_{w f}}=\frac{f_{w f}}{S_{w f}-S_{w c}}
$$


where $S_{w c}$ is the connate (initial) water saturation. $S_{w f}$ is the water saturation at the polymer front.

This is shown graphically in Figure 6.40. Since $S_{w f}$ moves at the specific velocity of $f_{w f}^{\prime}=\left(\frac{\partial f_{w}}{\partial S_{w}}\right)_{S_{w f}}$, then water breakthrough time in pore volumes is $t_{D b t}=\frac{1}{f_{w f}^{\prime}}$ at $X_{D}=1$.

From experiment \#3, water breakthrough occurred at $0.2 \mathrm{PV}$. The average water saturation at water breakthrough was 0.31 . These values agree with the fractional flow calculations. Oil cut was calculated from oil fractional flow in Figure 6.44. For the polymer flood, both the experimental and calculated water breakthrough times were 0.27 PV. The shape of polymer-oil fractional flow curve shown in Figure 6.40 indicates the polymer displaces the oil with only a very small Buckley-Leverett tail, i.e., the displacement is nearly piston-like. The calculated polymer breakthrough time based on Figure 6.40 was $0.8 \mathrm{PV}$. The pressure drop data shown in Figure 6.42 show the pressure drop did not increase after this time. 


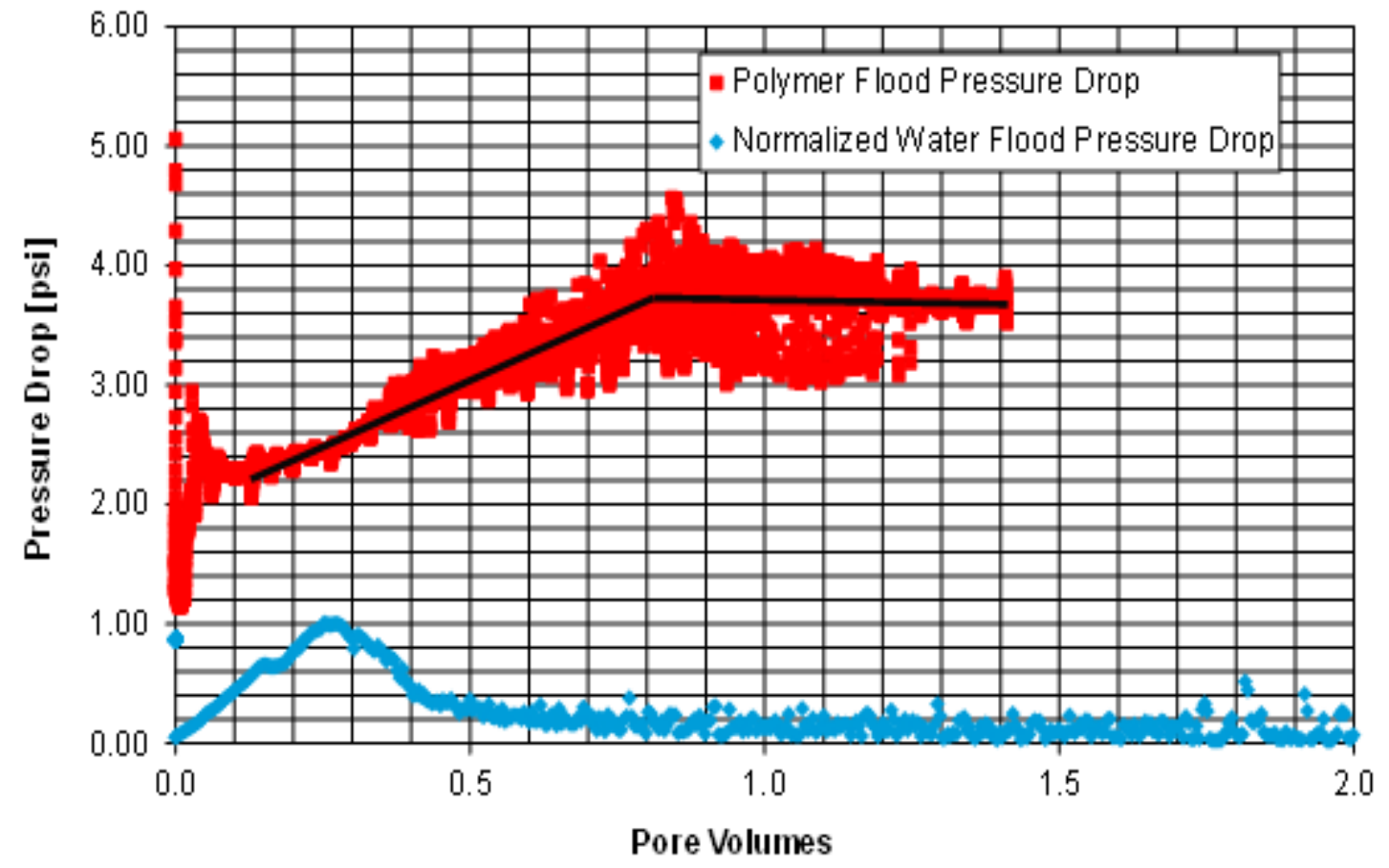

Figure 6.41 Pressure drop data for experiment \#3

Oil recovery is related to the average water saturation change as shown in Figure 6.42 and Figure 6.43.

$$
\text { Oil recovery }(\%)=\frac{\bar{S}_{w}-S_{w c}}{1-S_{w c}}
$$

where $\bar{S}_{w}$ is the average water saturation. 


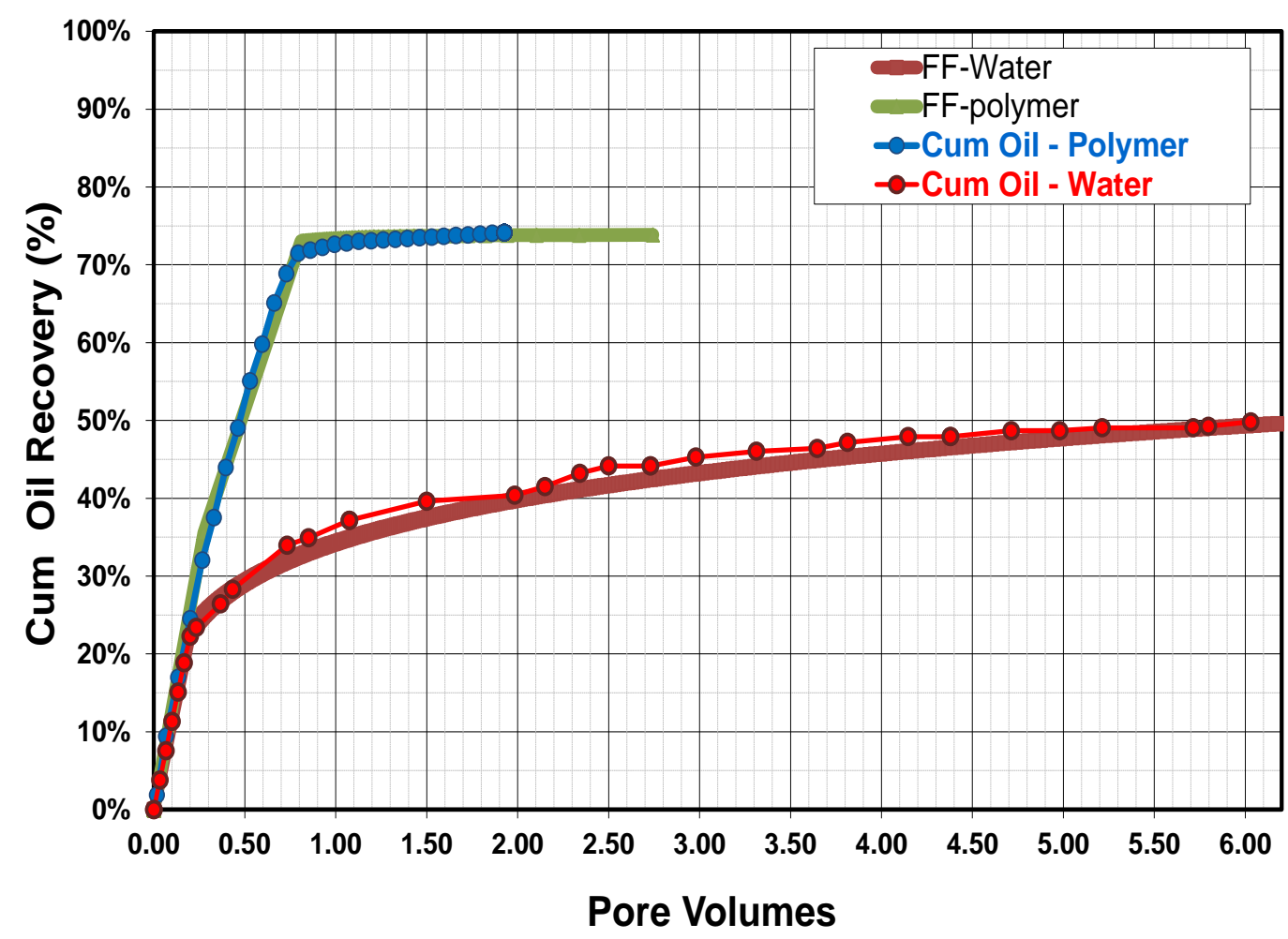

Figure 6.42 Oil recovery by secondary polymer flood for experiment \#3 


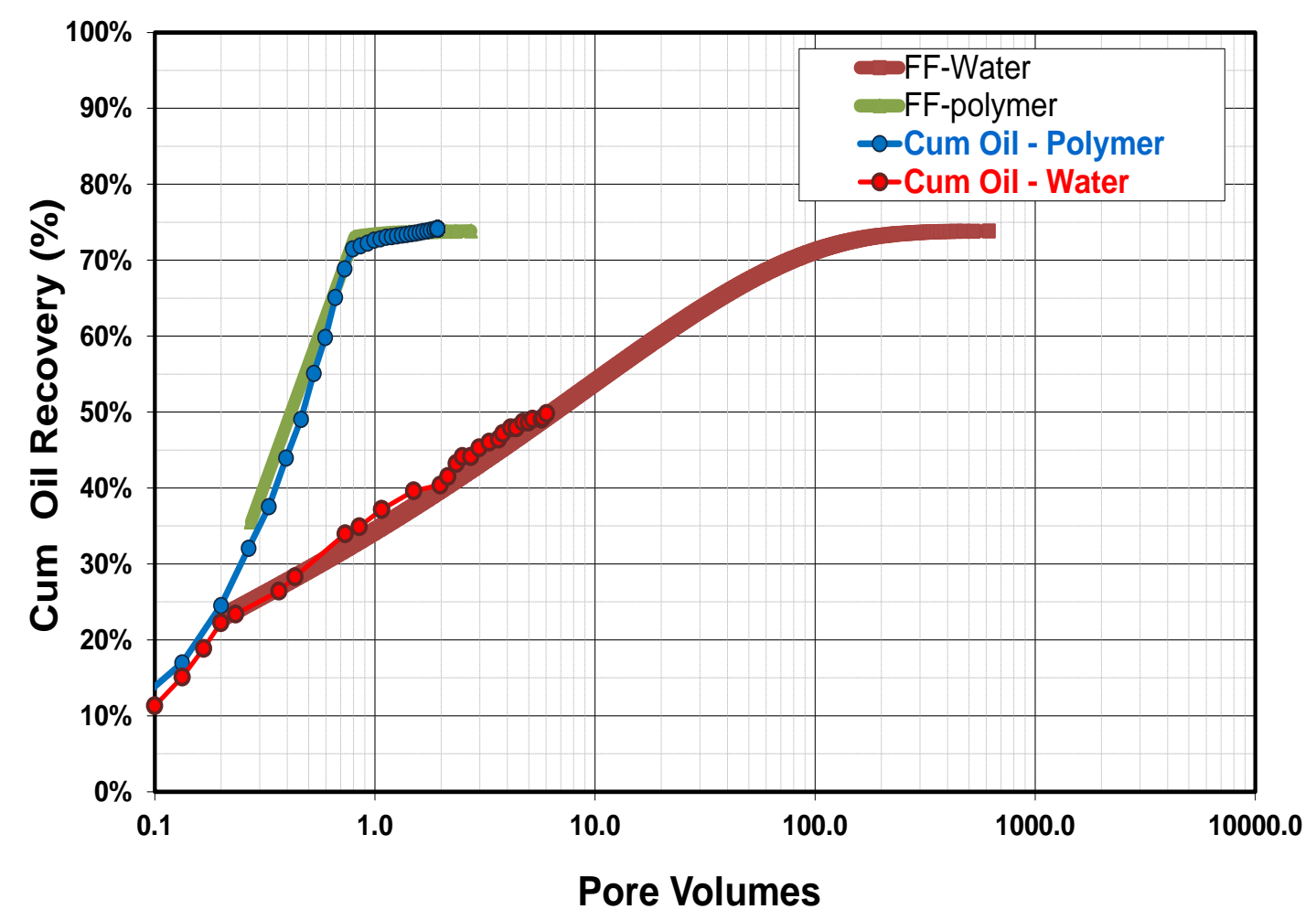

Figure 6.43 Oil recovery versus log of pore volumes for experiment \#3

At the end of the water flood, the relative permeability was 0.067 at the remaining oil saturation of $0.46\left(S_{\mathrm{w}}=0.54\right)$ using a relative permeability curve with the same endpoint as the relative permeability measured at the end of the polymer flood. From Figure 6.39 , the water relative permeability at a water saturation of 0.54 is 0.069 . The oil and water Corey exponents were adjusted to match the experimental data. The results also depend on the value used for the shear correction factor. Figures 6.39 to 6.44 were generated using a shear rate correction factor of 4 . Different values of the shear correction factors besides 4 were also tried. The best match for this as well as all other experiments was when the shear correction factor was 4 . 


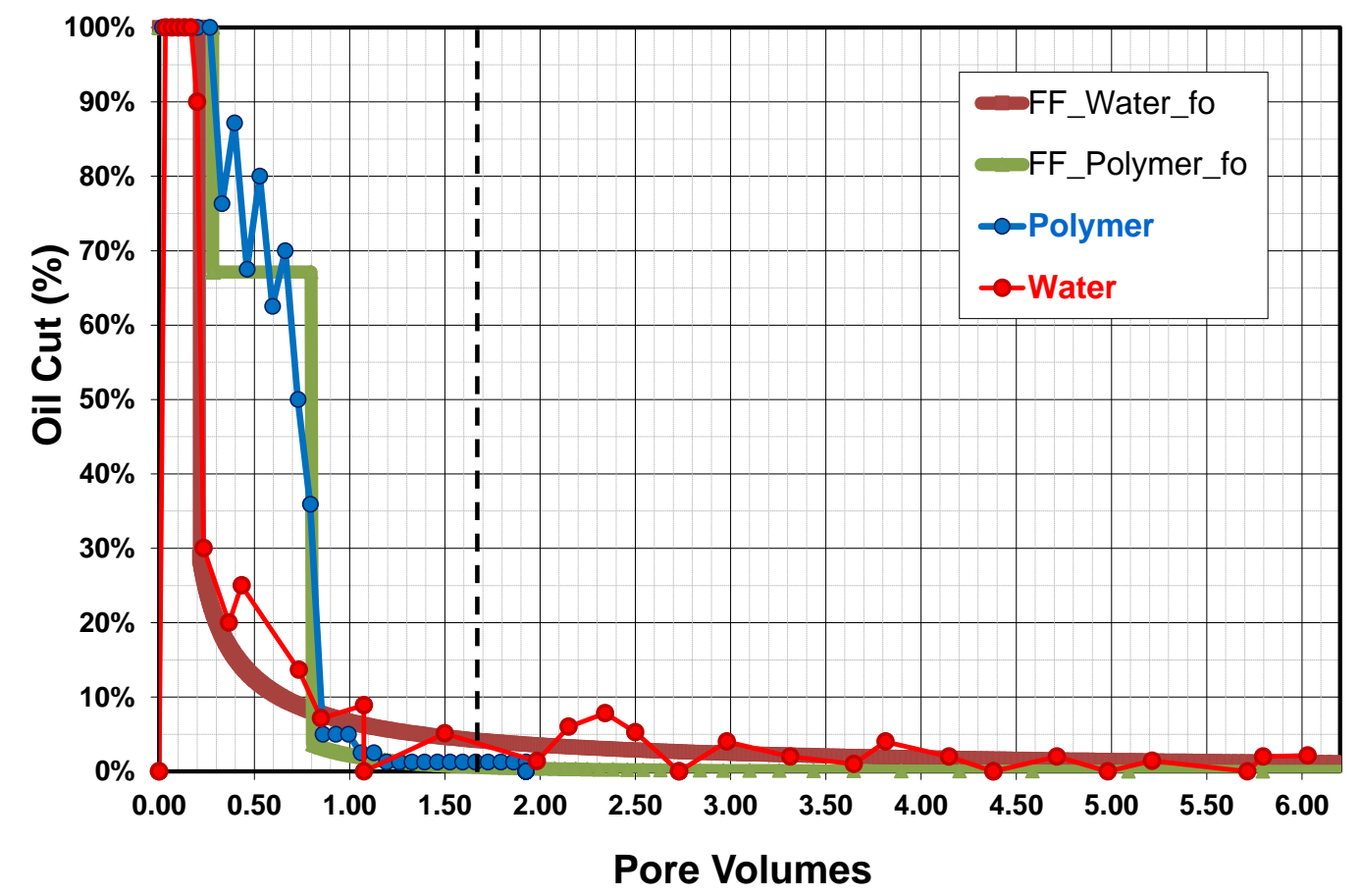

Figure 6.44 Oil cut for experiment \#3

The same analysis was done for experiment \#4 as was done for experiment \#3. Figure 6.45 shows the pressure data for experiment \#4. Since experiment \#4 is a tertiary polymer flood experiment, the initial water saturation for the polymer flood was 0.56 . The experimental oil cut, oil breakthrough time, water breakthrough time and, polymer breakthrough time were all matched by adjusting only the water and oil Corey exponents. Based on the assumptions and experimental data in Table 6.7, the relative permeability curve was generated by using Corey type model equations and then the fractional flow curve was generated based on the relative permeability curve in Figure 6.46.

The experimental oil breakthrough time was $0.3 \mathrm{PV}$ as shown in Figure 6.17. The calculated oil breakthrough time for the polymer flood, based on equations 6.12 and 6.13 as illustrated graphically in Figure 6.47 was 0.35 PV. 


$$
\begin{gathered}
v_{o b}=\frac{f_{w i}-f_{w 1}}{S_{w i}-S_{w 1}} \\
t_{o B T}=\frac{1}{v_{o b}}=\frac{S_{w i}-S_{w 1}}{f_{w i}-f_{w 1}}
\end{gathered}
$$

The calculated polymer breakthrough time by the fractional flow curves in Figure 6.47 was $0.74 \mathrm{PV}$. The polymer flood pressure drop in Figure 6.45 shows a decrease starting at $0.75 \mathrm{PV}$.

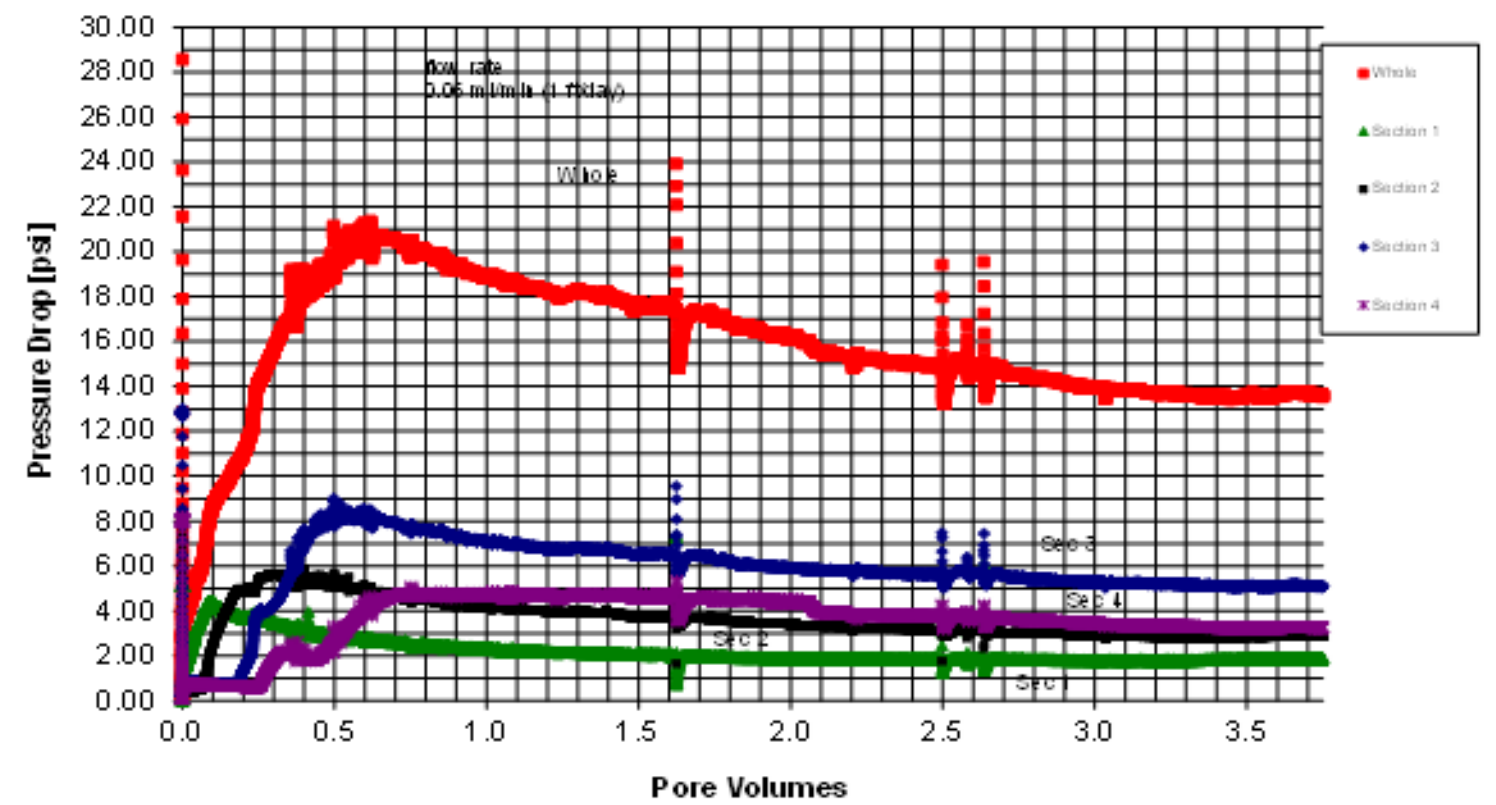

Figure 6.45 Polymer flood pressure drop for experiment \#4

A shear rate correction factor of 4 was chosen for the best match with oil cut and oil, water and polymer breakthrough times (Figures 6.48 and 6.49). When the water flood was stopped at $5 \%$ oil cut, the relative permeability was 0.03 and the water saturation was 0.56 . From Figure 6.46, the relative permeability is 0.032 at a water saturation of 0.56 . 
The measured relative permeability at the end of the water flood showed good agreement with the relative permeability curve used to model the polymer flood.

Table 6.7 Experiment data and adjusted Corey exponents for experiment \#4

\begin{tabular}{|c|c|c|}
\hline & Water flooding & Polymer flooding \\
\hline$k_{r w}{ }^{\circ}$ & 0.15 & 0.15 \\
\hline$k_{r o}{ }^{o}$ & 1.12 & 1.12 \\
\hline$n_{w}$ & 2.7 & 2.7 \\
\hline$n_{o}$ & 2.4 & 2.4 \\
\hline$S_{w r}$ & 0.32 & 0.32 \\
\hline$S_{w i}$ & 0.32 & 0.56 \\
\hline$S_{o r}$ & 0.24 & 0.24 \\
\hline$\mu_{w}(\mathrm{cP})$ & 0.5 & 12 \\
\hline$\mu_{o}(\mathrm{cP})$ & 72 & 72 \\
\hline
\end{tabular}




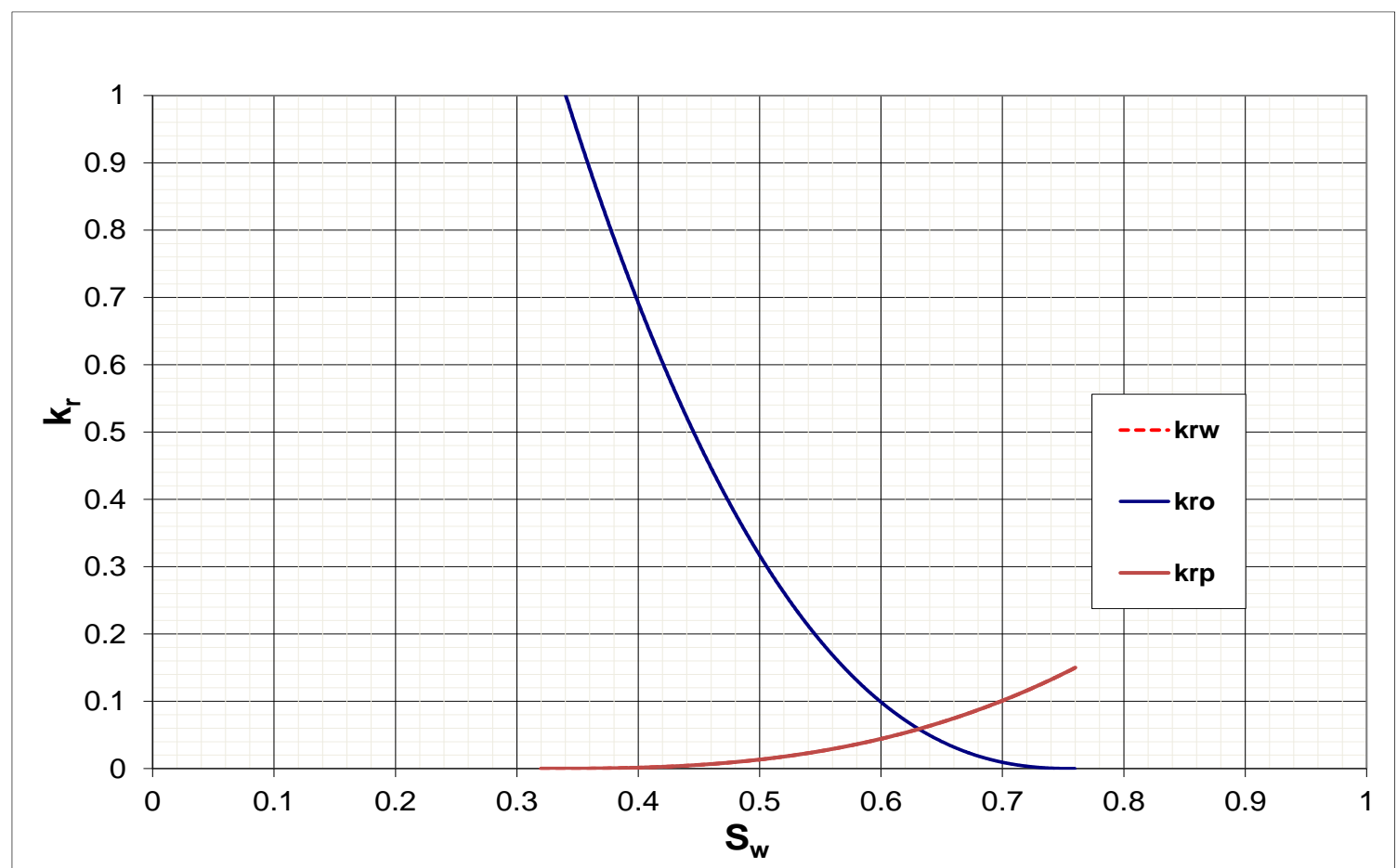

Figure 6.46 Relative permeability curve for experiment \#4

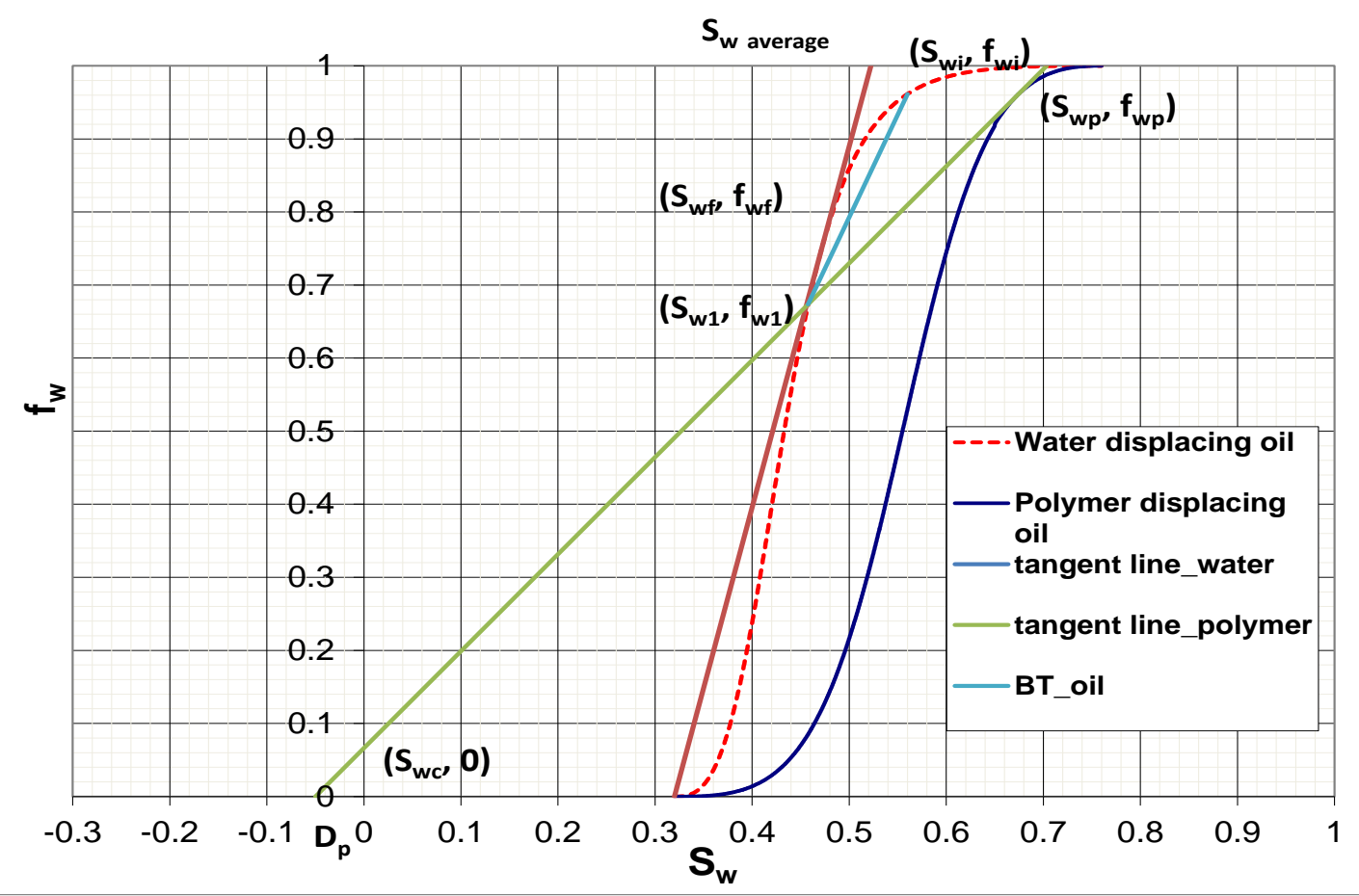

Figure 6.47 Fractional flow curve for experiment \#4 


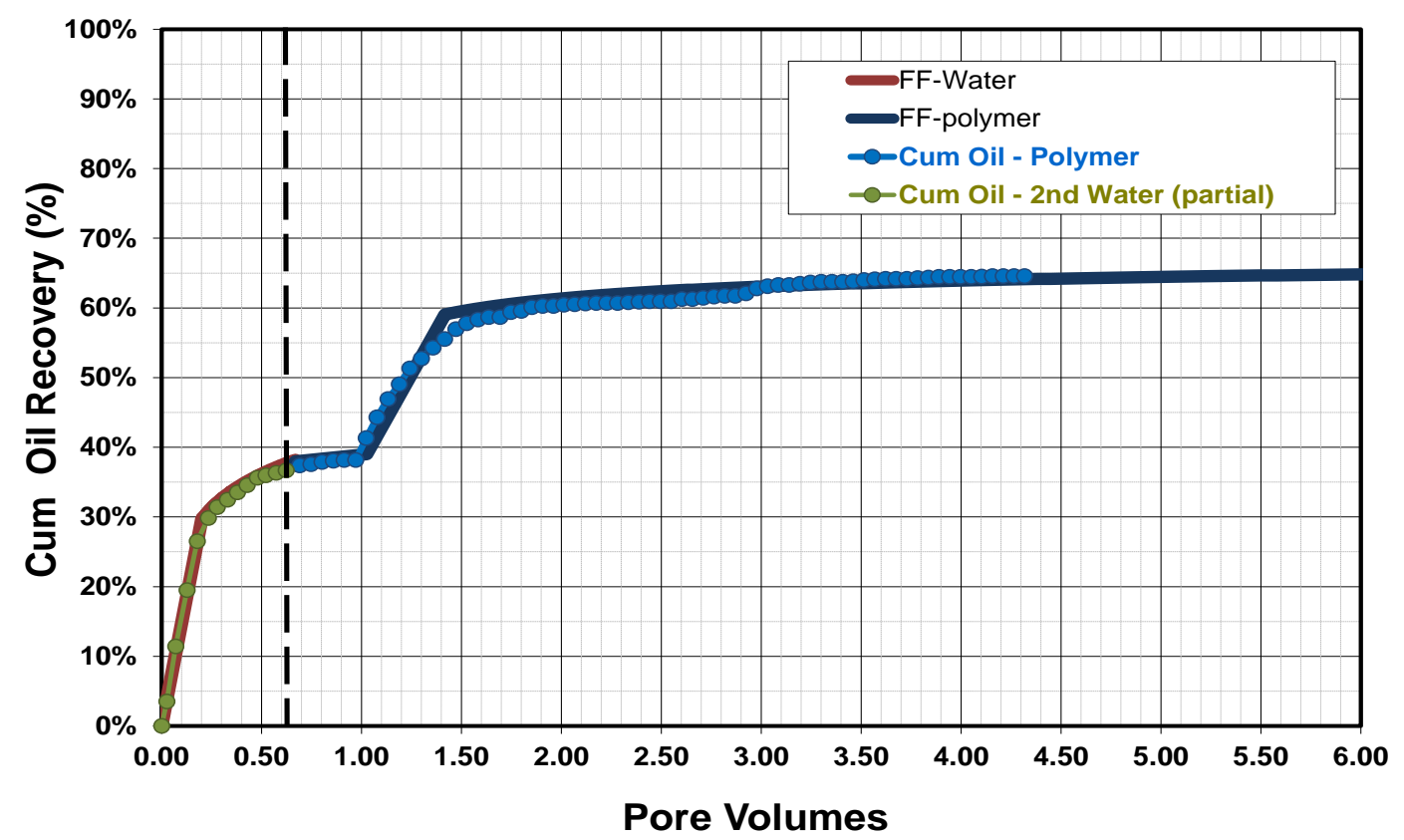

Figure 6.48 Oil recovery for tertiary polymer flood experiment \#4

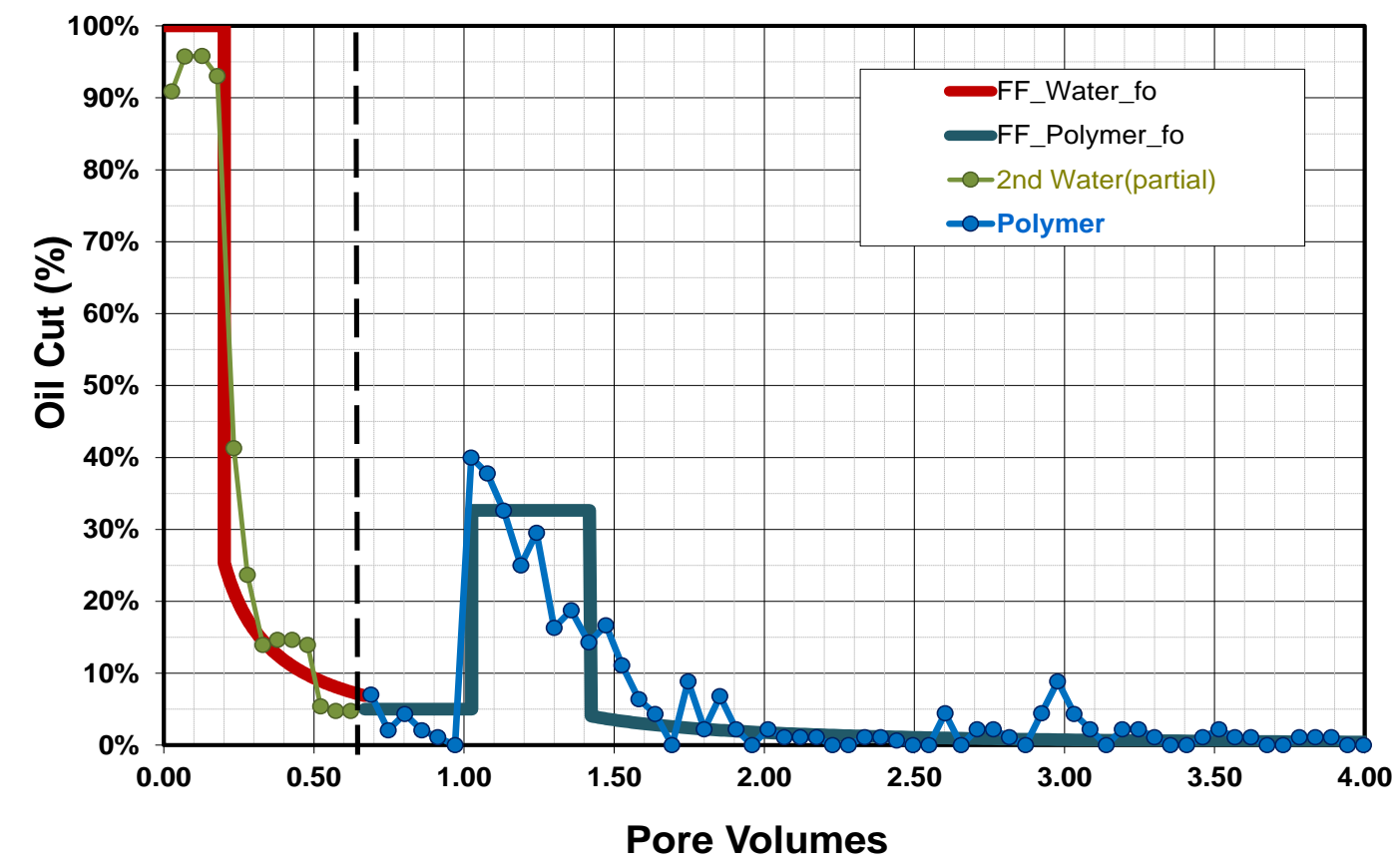

Figure 6.49 Oil cut for experiment \#4 
Analysis of experiment \#7 was performed by using equation 6.9 instead of 6.6. When a constant polymer viscosity was used in the fractional flow equation, the experimental data such as oil breakthough time, oil bank size and polymer breakthrough time could not be matched. As mentioned earlier, for two-phase flow, the shear rate is not constant since the water saturation and relative permeability change during the displacement. Therefore, the polymer viscosity is not constant since the polymer viscosity depends on shear rate. Especially for polymer floods of viscous oil, the effect of variable shear rate and viscosity is significant after polymer breakthrough. The difference in the fractional flow curves is shown in Figure 6.53. Even for a tertiary polymer flood, the polymer flood shows earlier polymer breakthrough than that for less viscous oil. Comparing the oil cut and oil recovery for experiment \#4 in Figure 6.49 and Figure 6.50 with the oil cut and oil recovery for experiment \#7 in Figure 6.54 and Figure 6. 56 shows $83 \%$ of the oil from polymer flood experiment \#4 was produced in the oil bank before polymer breakthrough while only $18 \%$ of the oil from polymer flood experiment \#7 was produced in the oil bank before polymer breakthrough and $82 \%$ of the additional oil recovery was produced after polymer breakthrough. As discussed earlier, oil recovery after polymer breakthrough depends on the polymer viscosity, which depends on the shear rate, which depends on the variable water saturation.. Therefore, in this analysis of experiment \#7, the effect of shear rate was taken into account as shown below.

When the effect of shear rate on the oil cut after polymer breakthrough is significant, the shear rate correction factor $\mathrm{C}$ in Equation 6.8 has to be considered to match the oil cut after polymer breakthrough. 
Table 6.8 Experiment data and adjusted parameters for Corey correlation

\begin{tabular}{|c|c|c|}
\hline & Water flooding & Polymer flooding \\
\hline$k_{r w}{ }^{o}$ & 0.54 & 0.54 \\
\hline$k_{r o}{ }^{o}$ & 1.17 & 1.17 \\
\hline$n_{w}$ & 3.2 & 3.2 \\
\hline$n_{o}$ & 1 & 1 \\
\hline$S_{w r}$ & 0.11 & 0.11 \\
\hline$S_{w i}$ & 0.11 & 0.38 \\
\hline$S_{o r}$ & 0.26 & 0.26 \\
\hline$\mu_{w}(\mathrm{cP})$ & 0.66 & 46 \\
\hline$\mu_{o}(\mathrm{cP})$ & 1050 & 1050 \\
\hline
\end{tabular}

In Figure 6.54, the orange line for the oil cut calculated using a constant polymer solution viscosity is lower than the experimental oil cut data. For the best match of the experimental results such as oil breakthrough time, water/polymer breakthrough time, and oil cut, the $\mathrm{C}$ value was chosen as 4 . If a constant viscosity based on the shear rate at the end of the polymer flood is used for the fractional flow calculation, the dashed fractional curve in Figure 6.53 predicts later polymer breakthrough, lower oil cut after polymer breakthrough and an oil bank that is too large. The most obvious thing was the worse match of the polymer breakthrough time. Figure 6.50 and Figure 6.51 show the change in equivalent shear rate and viscosity after polymer breakthrough. 


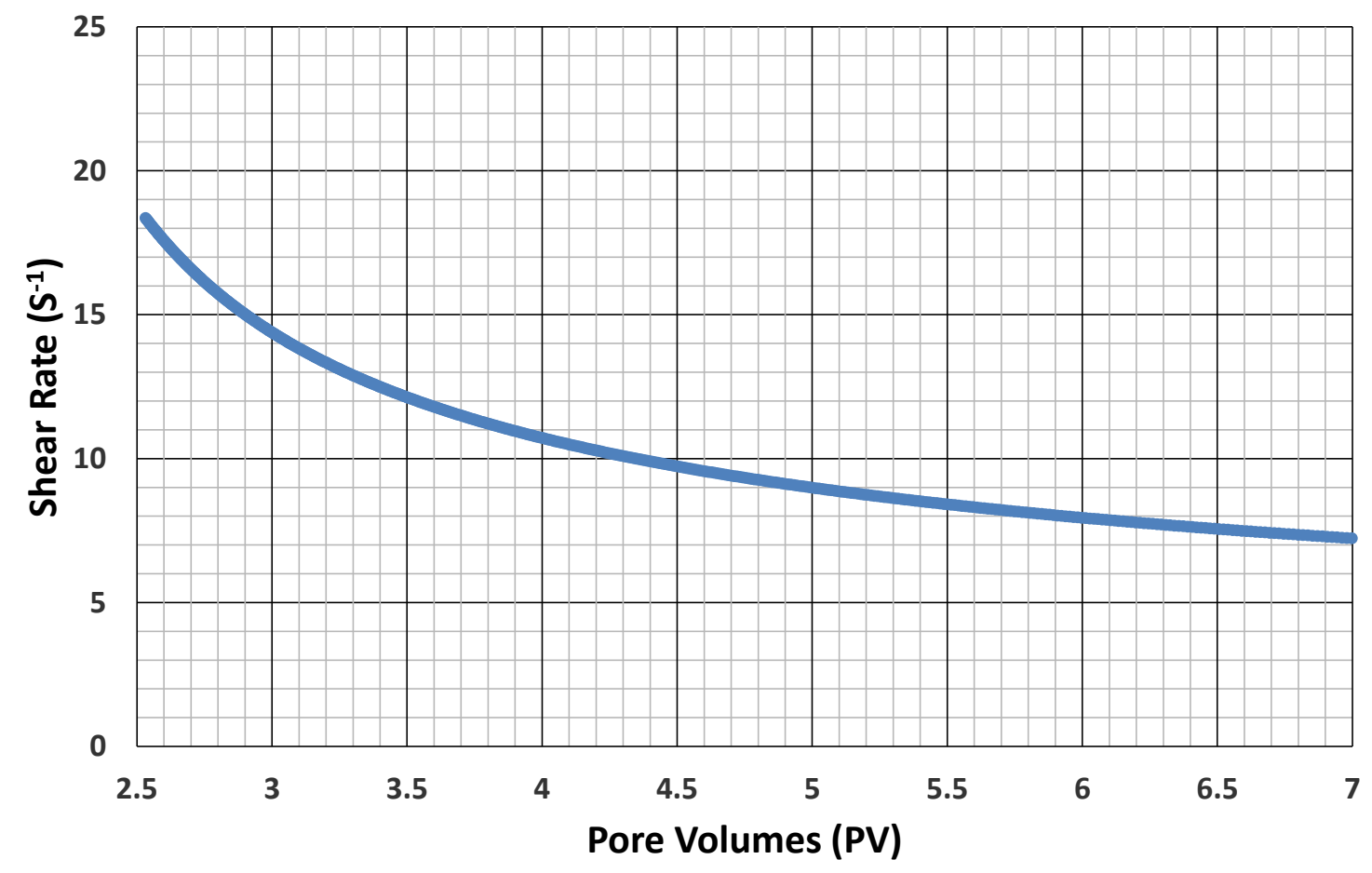

Figure 6.50 Equivalent shear rates after polymer breakthrough for experiment \#7

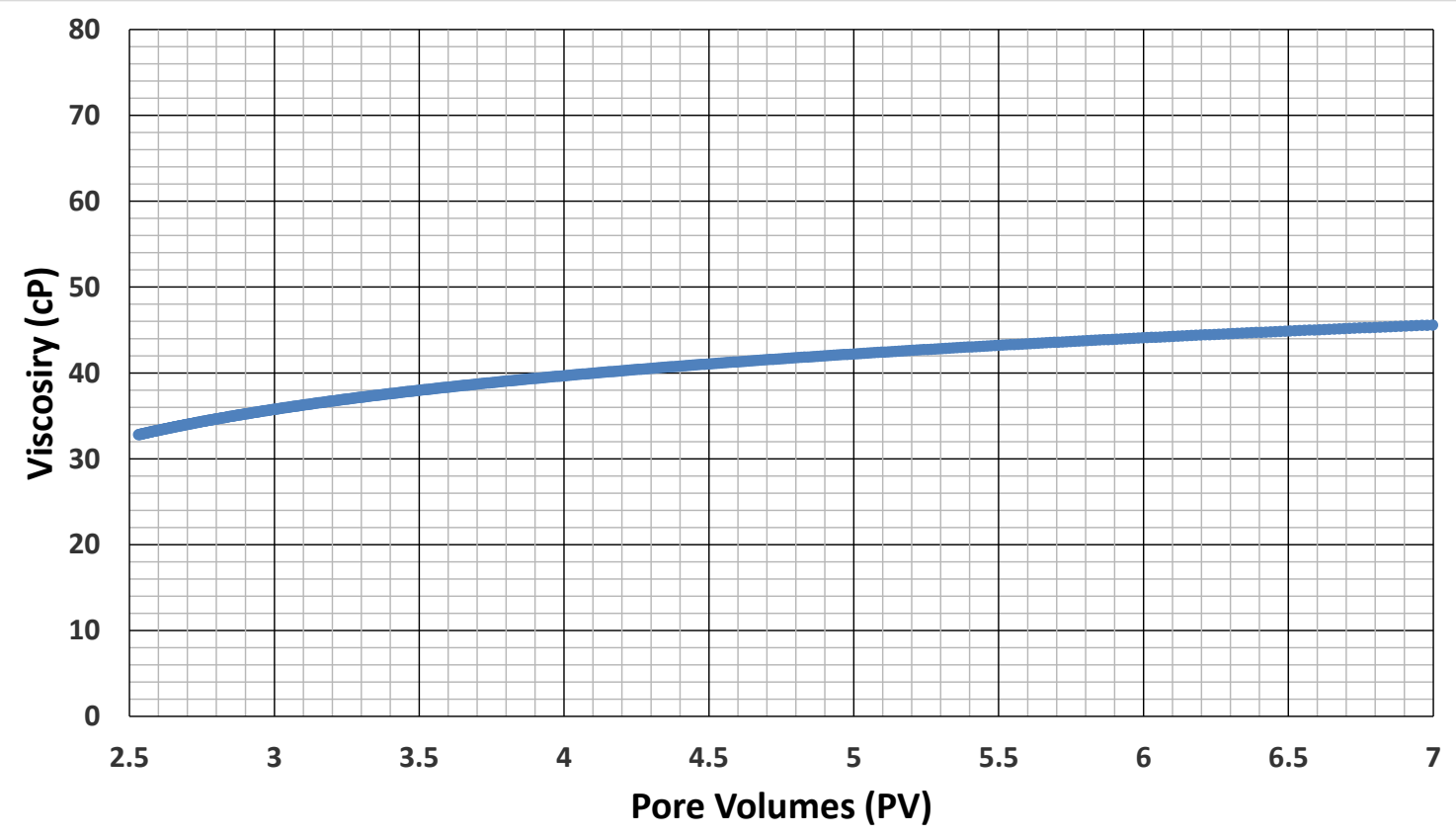

Figure 6.51 Viscosity after polymer breakthrough for experiment \#7 based on shear rates shown in Figure 6.51 


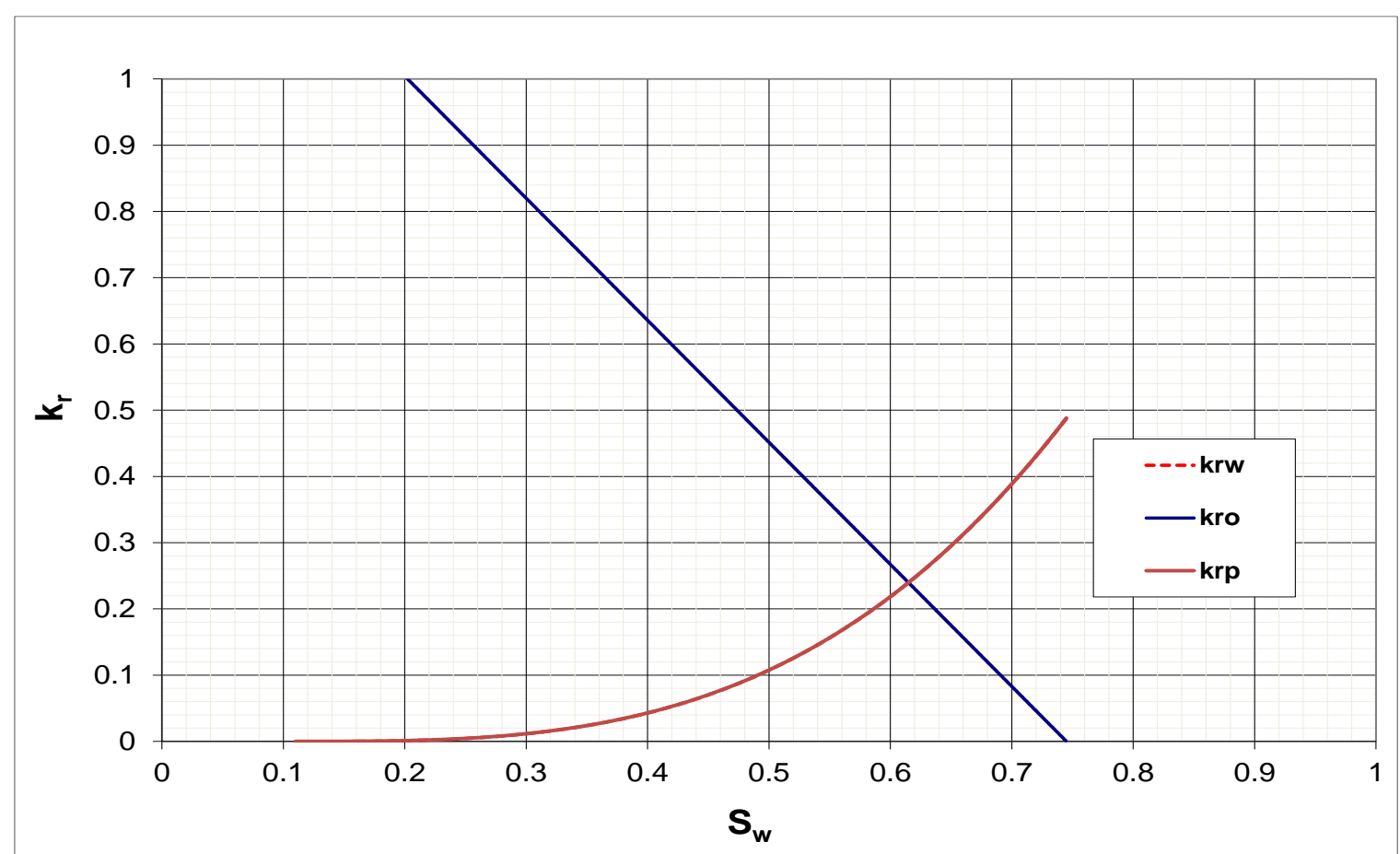

Figure 6.52 Relative permeability curve for experiment \#7

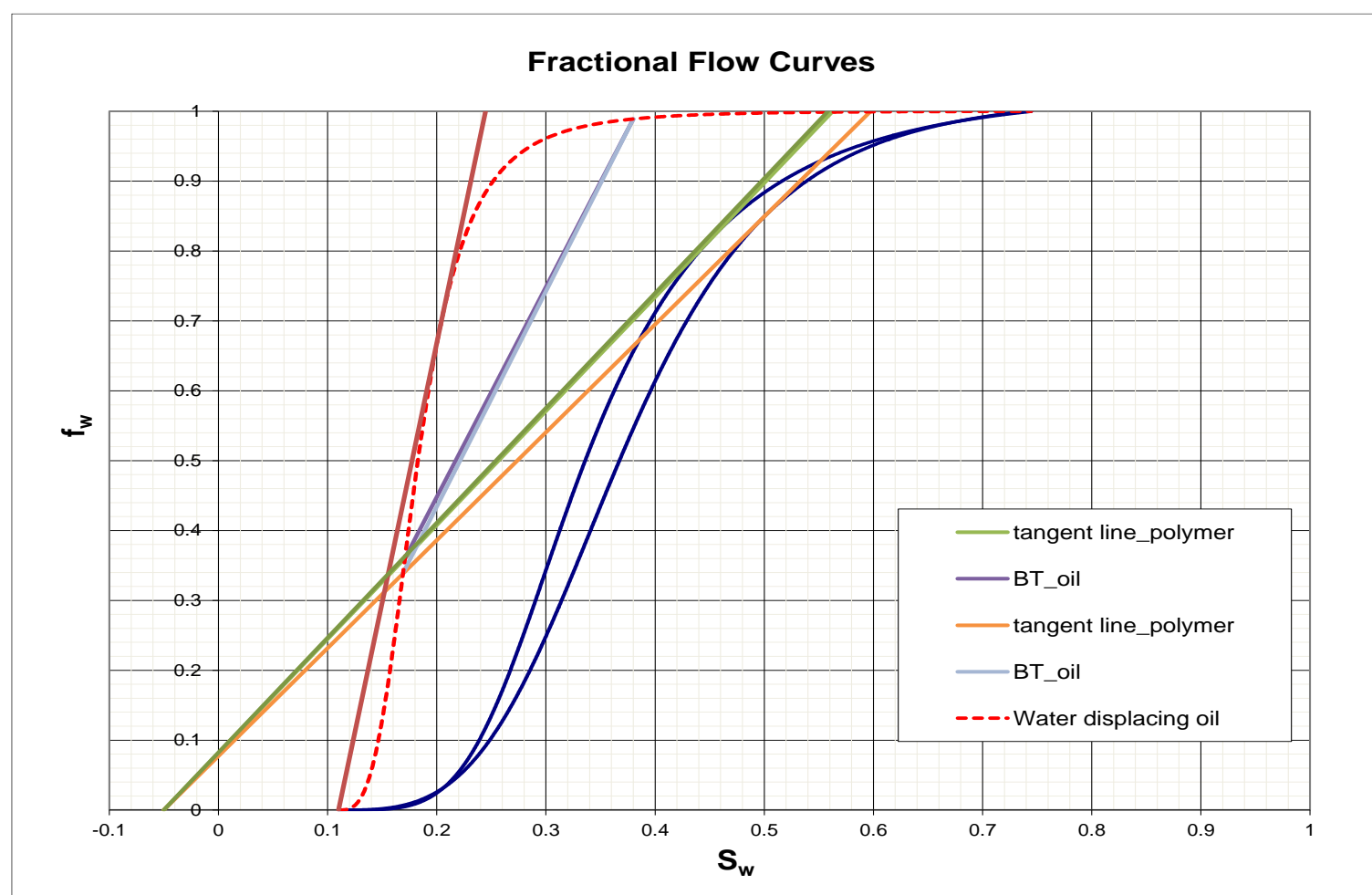

Figure 6.53 Fractional flow curve for experiment \#7 


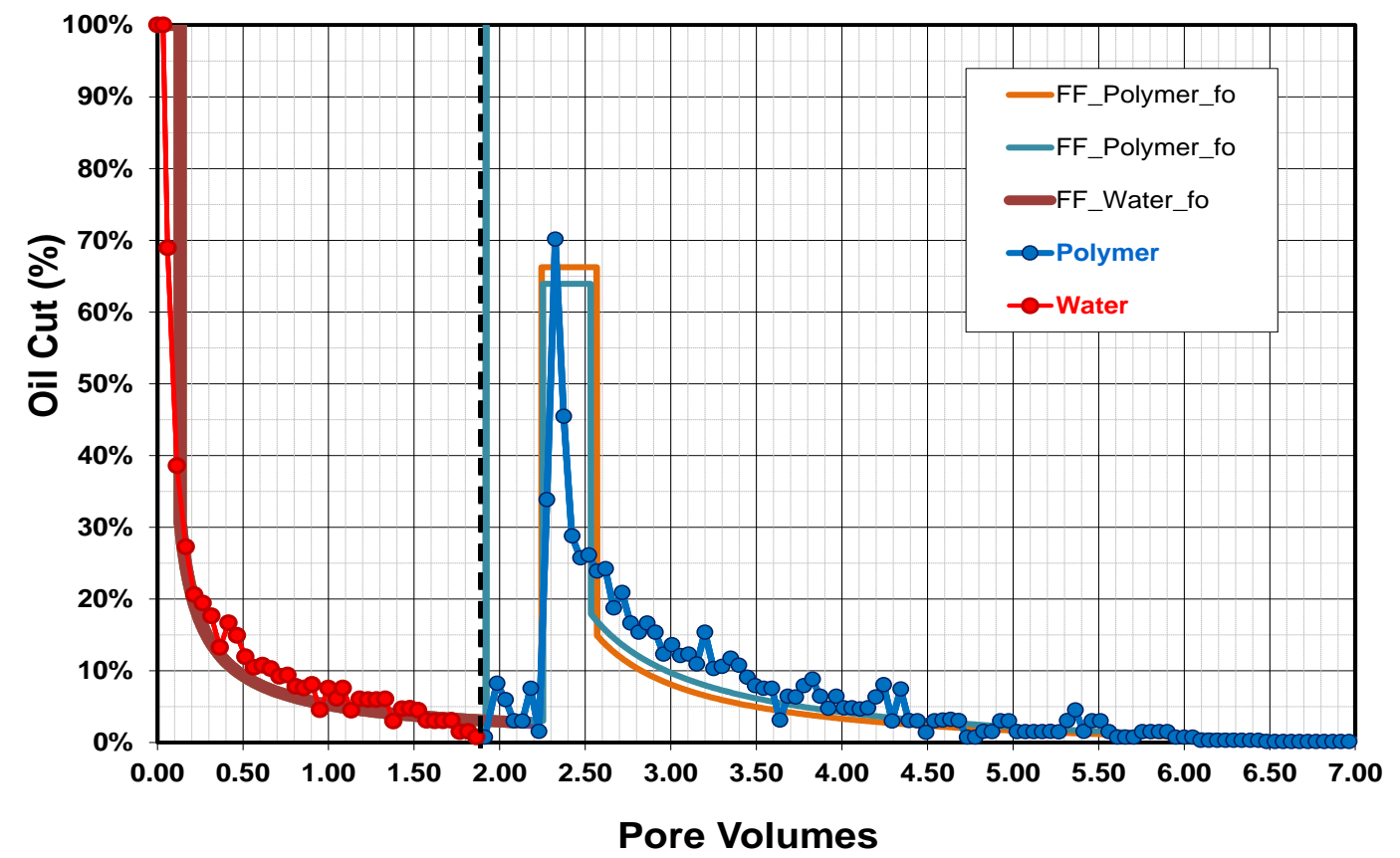

Figure 6.54 Oil cut for experiment \#7

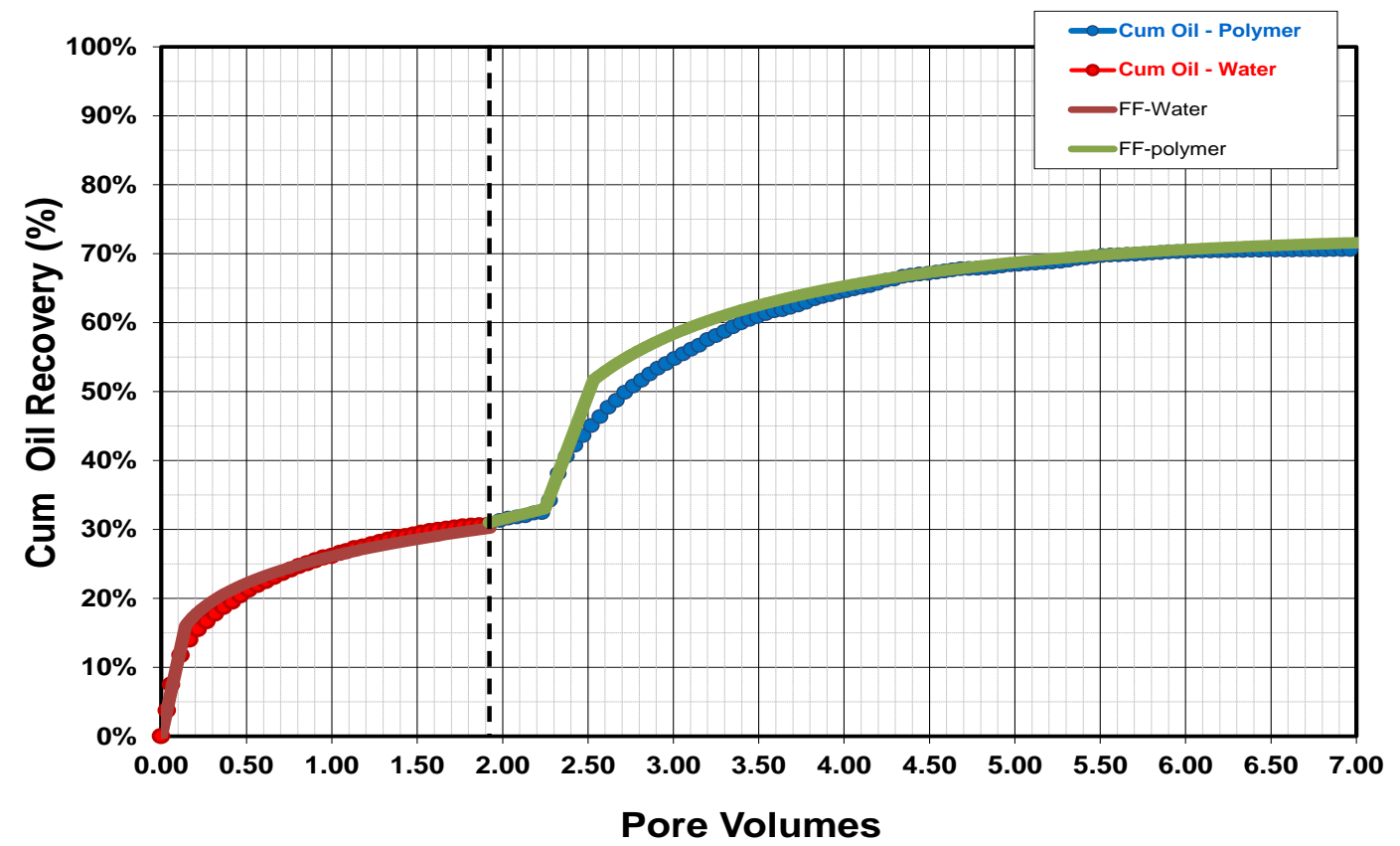

Figure 6.55 Oil recovery by tertiary polymer flood for experiment \#7 
Fractional flow curves for experiment \#8 were generated using the experimental data shown in Table 6.9. The cumulative oil recovery and oil cut were matched based on the fractional flow curve. Polymer breakthrough times from the fractional flow curve matched the experimental data. Average water saturation at polymer breakthrough is 0.74 calculated from the fractional flow curve compared with the experimental value of 0.72 . Table 6.9 Experiment data and adjusted Corey exponents for experiment \#8

\begin{tabular}{|c|c|c|}
\hline & Water flooding & Polymer flooding \\
\hline$k_{r w}{ }^{o}$ & 0.56 & 0.56 \\
\hline$k_{r o}{ }^{o}$ & 0.89 & 0.89 \\
\hline$n_{w}$ & 3.6 & 3.6 \\
\hline$n_{o}$ & 1.7 & 1.7 \\
\hline$S_{w r}$ & 0.31 & 0.31 \\
\hline$S_{w i}$ & 0.31 & 0.31 \\
\hline$S_{o r}$ & 0.255 & 0.255 \\
\hline$\mu_{w}(\mathrm{cP})$ & 0.4 & 14 \\
\hline$\mu_{o}(\mathrm{cP})$ & 3.5 & 3.5 \\
\hline
\end{tabular}




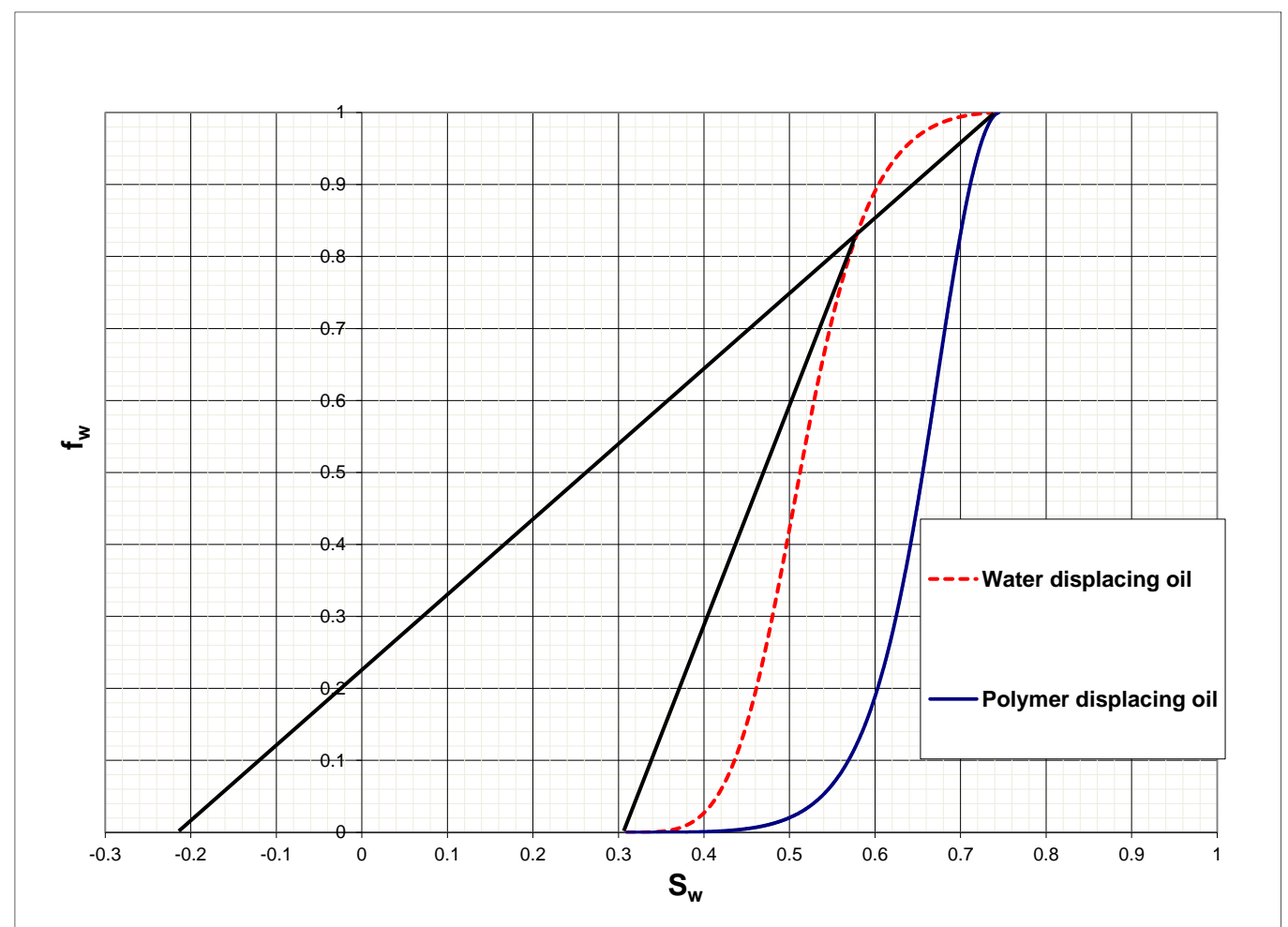

Figure 6.56 Fractional flow curve for experiment \#8

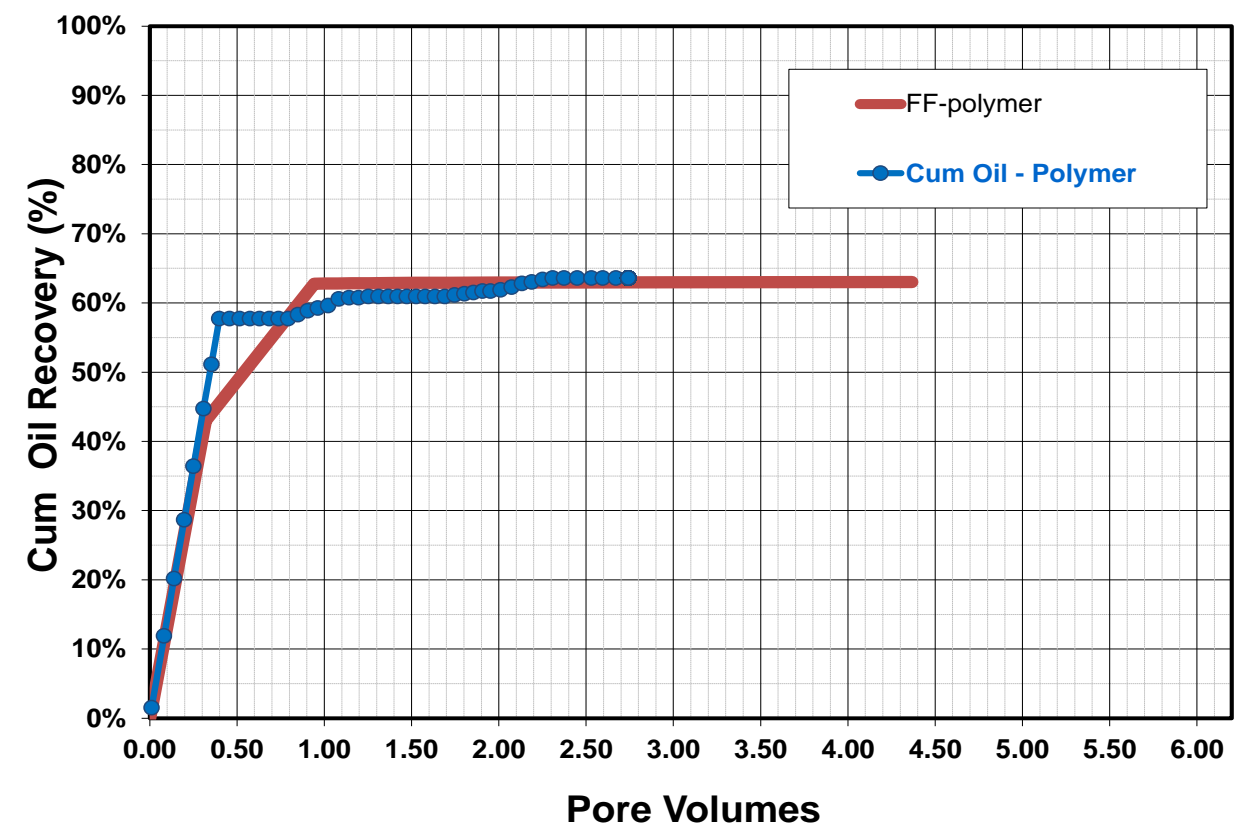

Figure 6.57: Oil recovery for experiment \#8 


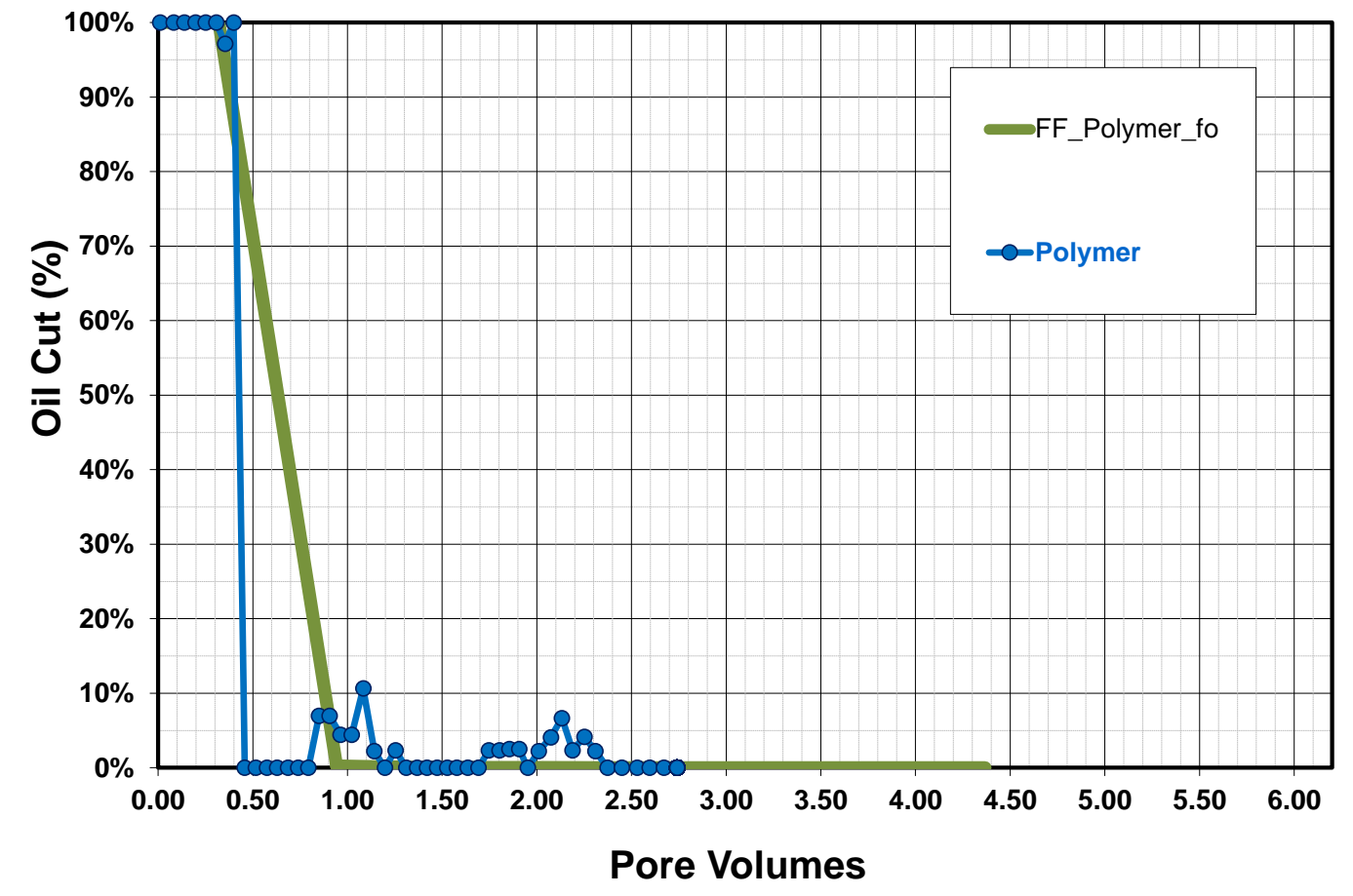

Figure 6.58 Oil cut for experiment \#8

Fractional flow curves for experiment \#9 were generated using the experimental data shown in Table 6.10. The cumulative oil recovery and oil cut were matched based using the fractional flow curve. Water breakthrough time, oil cut, oil breakthrough time and polymer breakthrough time calculated from the fractional flow curve are in good agreement with the experimental data. For example, the computed water breakthrough time is $0.26 \mathrm{PV}$ and the actual water breakthrough time is $0.25 \mathrm{PV}$. Average water saturation at water breakthrough is 0.62 calculated from the fractional flow curve compared to the experimental value of 0.60 . 
Table 6.10 Experiment data and adjusted Corey exponents for experiment \#9

\begin{tabular}{|c|c|c|}
\hline & Water flooding & Polymer flooding \\
\hline$k_{r w}{ }^{o}$ & 0.54 & 0.54 \\
\hline$k_{r o}{ }^{o}$ & 0.72 & 0.72 \\
\hline$n_{w}$ & 3.6 & 3.6 \\
\hline$n_{o}$ & 1.7 & 1.7 \\
\hline$S_{w r}$ & 0.35 & 0.35 \\
\hline$S_{w i}$ & 0.35 & 0.68 \\
\hline$S_{o r}$ & 0.275 & 0.275 \\
\hline$\mu_{w}(\mathrm{cP})$ & 0.4 & 14 \\
\hline$\mu_{o}(\mathrm{cP})$ & 3.5 & 3.5 \\
\hline
\end{tabular}

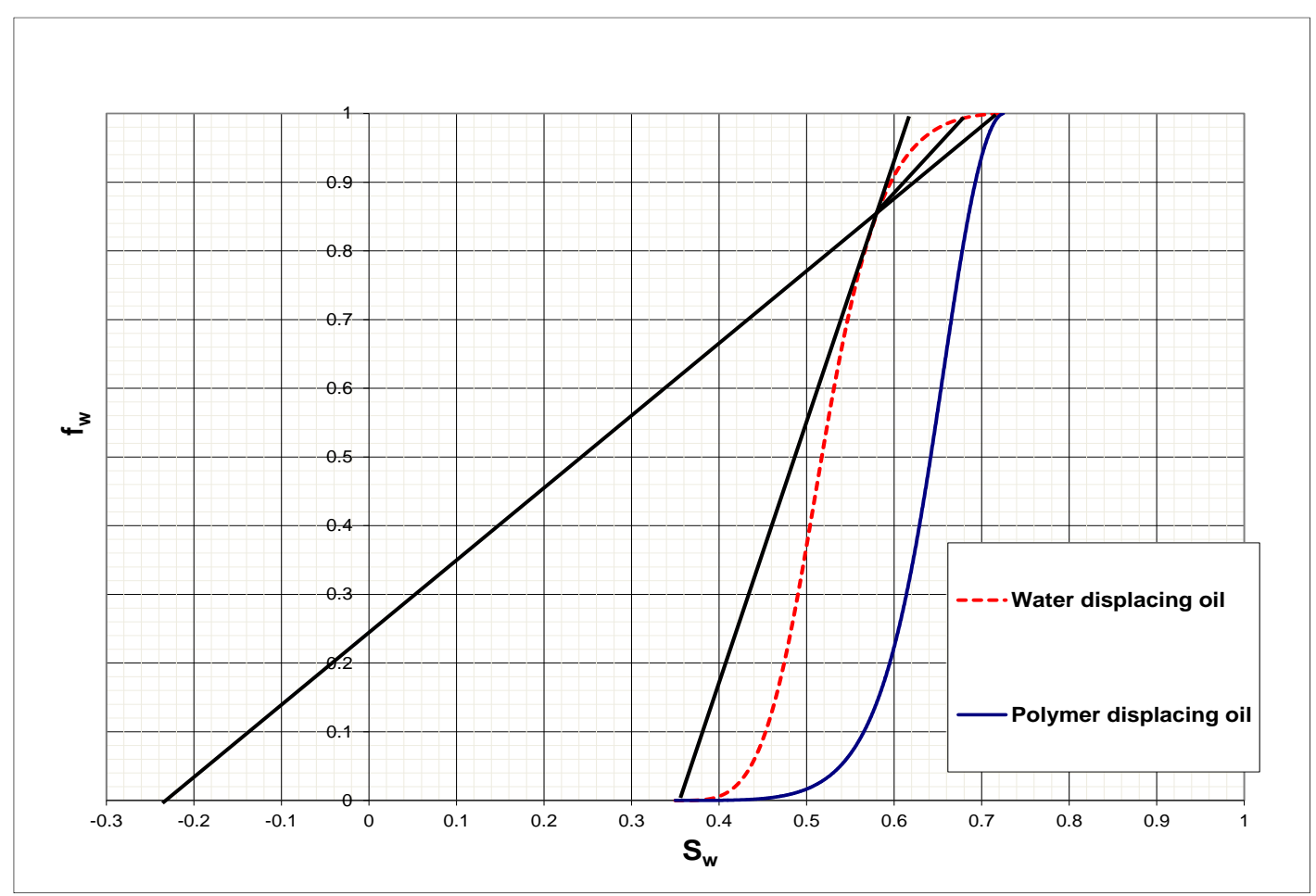

Figure 6.59 Fractional flow curves for experiment \#9 


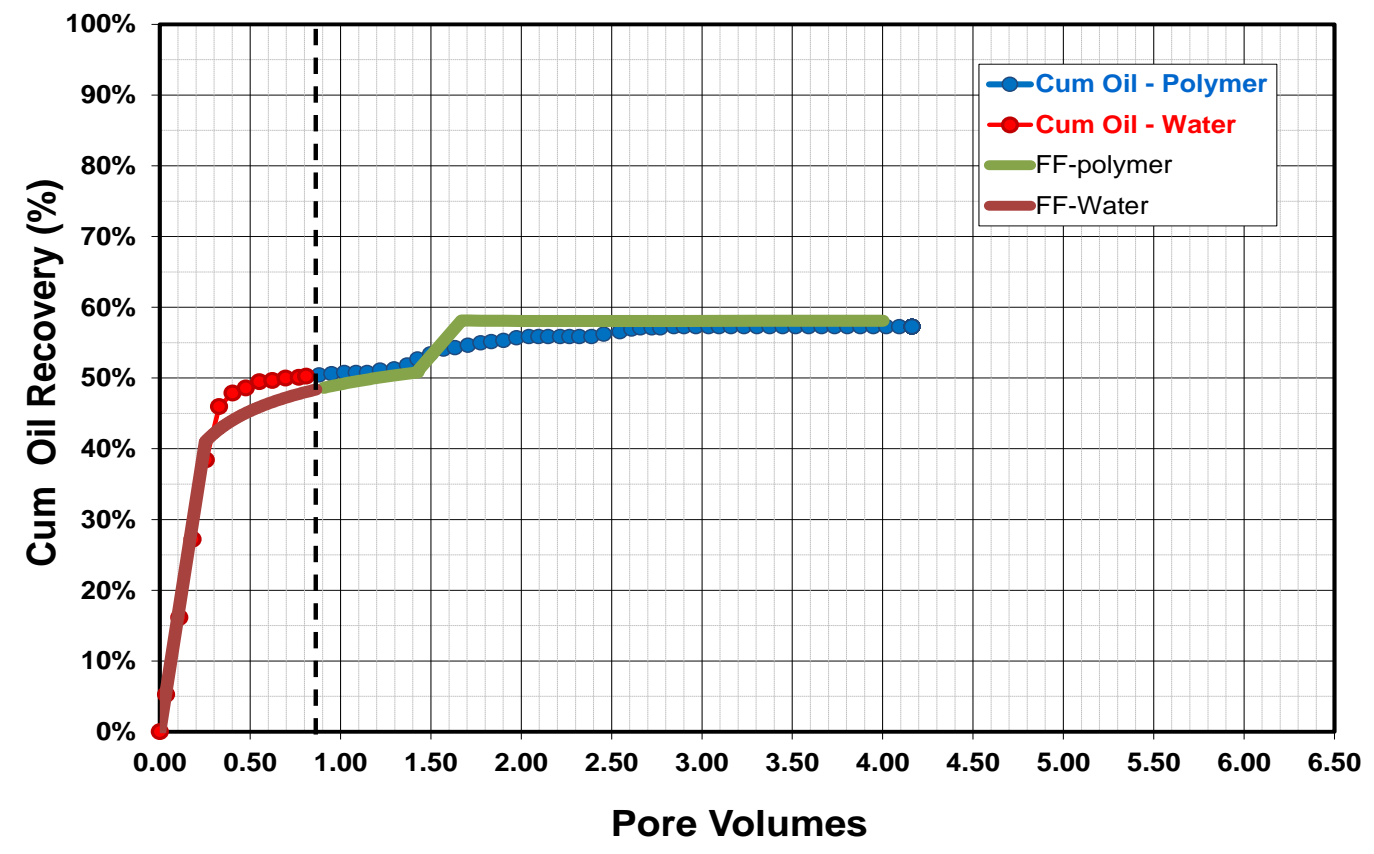

Figure 6.60 Oil recovery for experiment \#9

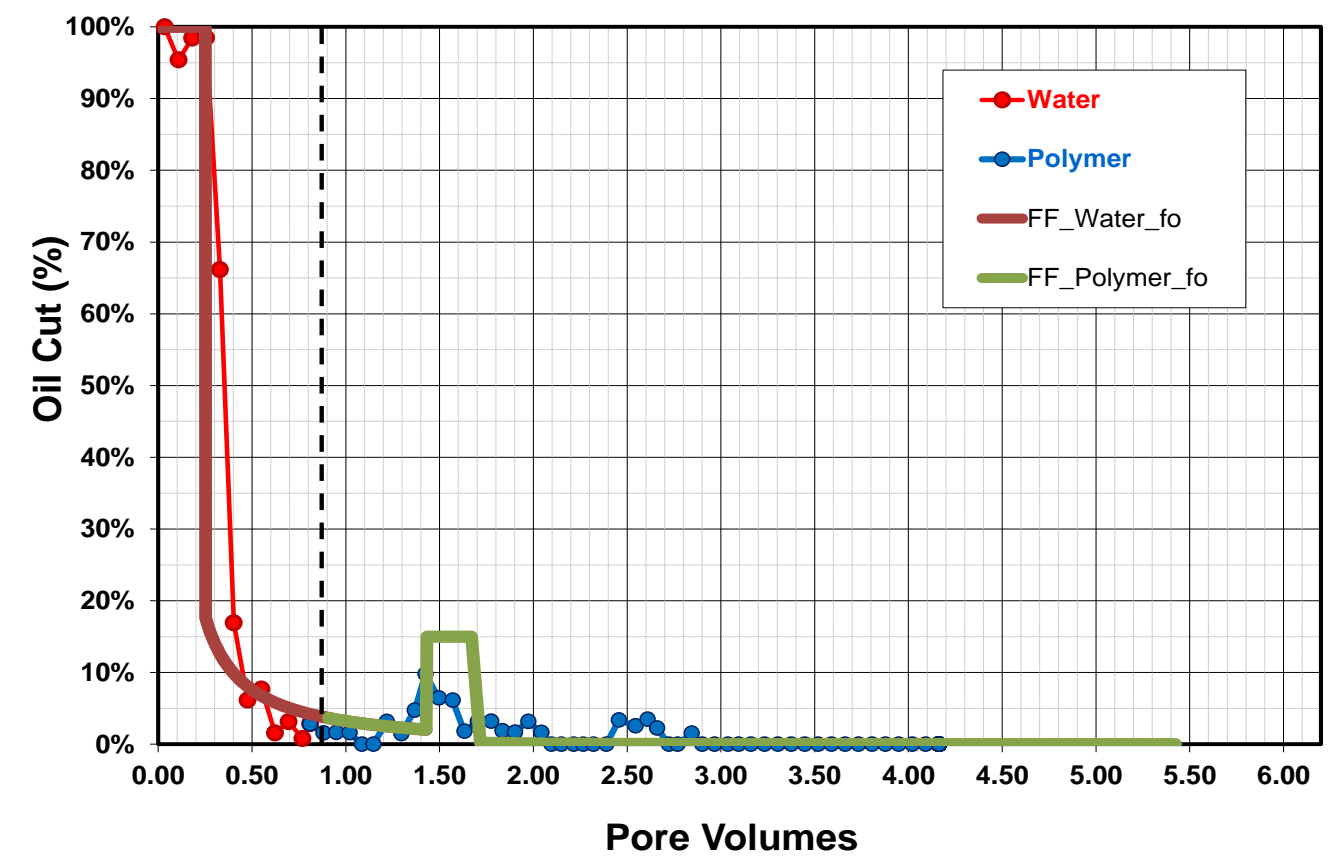

Figure 6.61 Oil cut for experiment \#9 


\section{The shear correction factor}

Wreath (1989) used his experimental data as well as coreflood data from the literature to calculate the shear rate correction factor for HPAM polymers. As shown in Figure 6.62, the shear rate correction factor decreased from about 500 to 1 as the product of the effective permeability and porosity $(\mathrm{k} \phi)$ increased. Except for the value of $\mathrm{C}=1$ from a 40 Darcy bead pack, all of the $\mathrm{C}$ values were in the range of 10 to 500. Even for cores with a permeability on the order of 1 Darcy, C ranged between 10 and 100.

Figure 6.63 shows the values of $\mathrm{C}$ for xanthan gum polymer reported by several investigators. These values are generally much lower with a range of about 3 to 9 . For a 1 Darcy core, most of the values are about 4 . The definition of the shear correction factor used by Cannella et al. (1988) is slightly different than the definition used in this work. The value in this work must be multiplied by the square of 2 for comparison with their values. For example, $\mathrm{C}=4$ becomes 5.64 using their definition and their typical value was 6. 


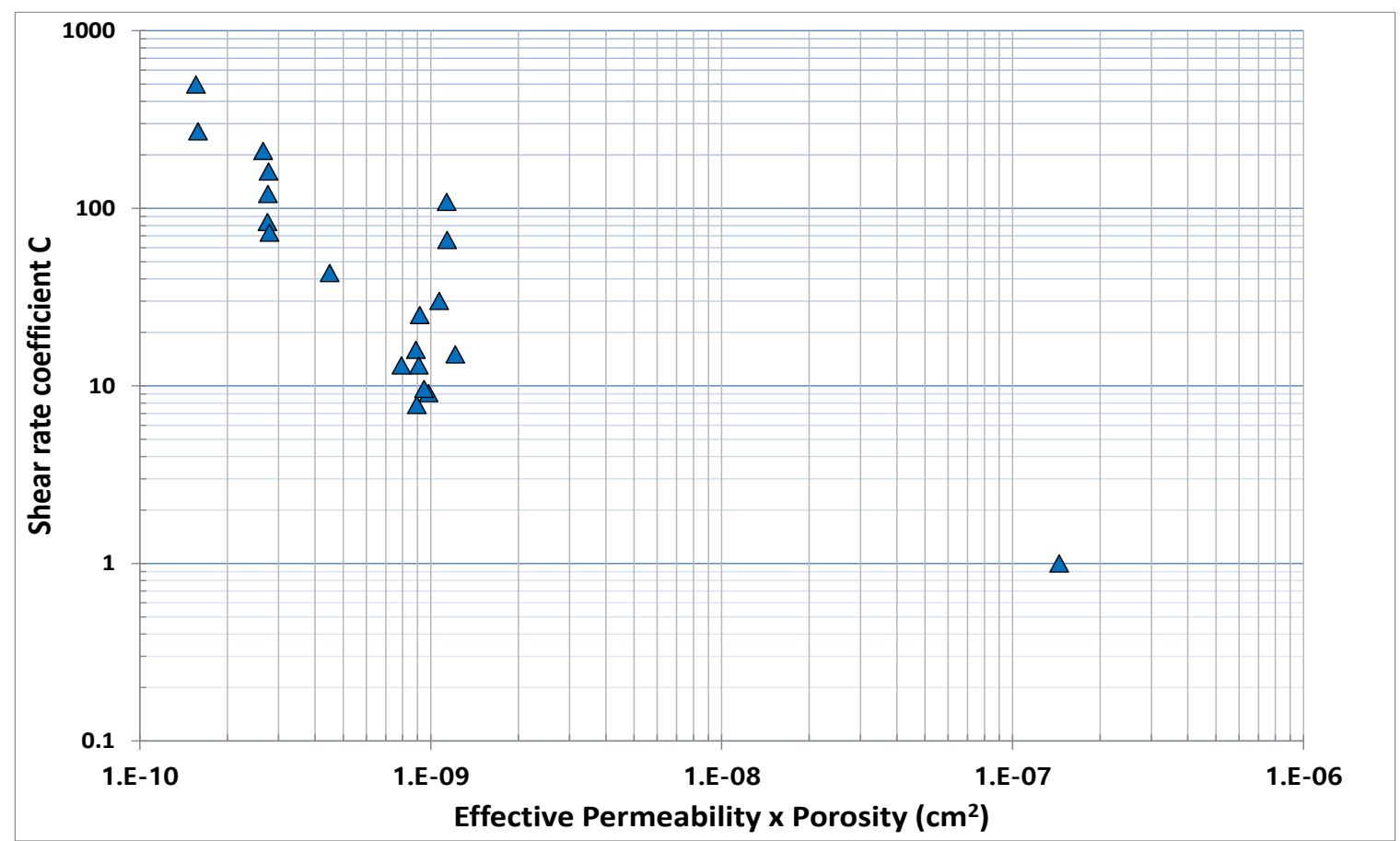

Figure 6.62 Shear rate correction factor for HPAM polymers taken from Wreath (1989)

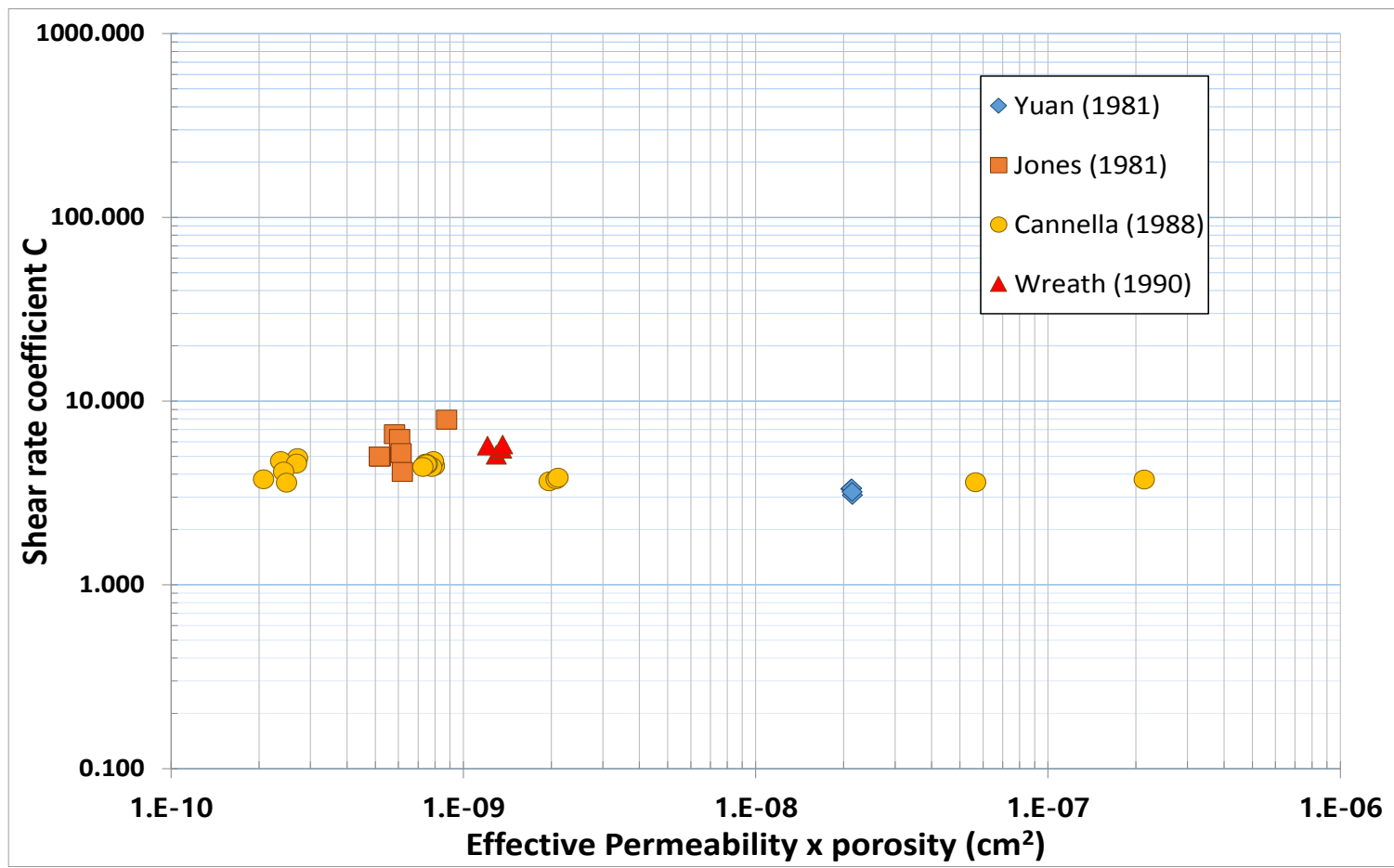

Figure 6.63 Shear rate correction factor for xanthan gum polymer 


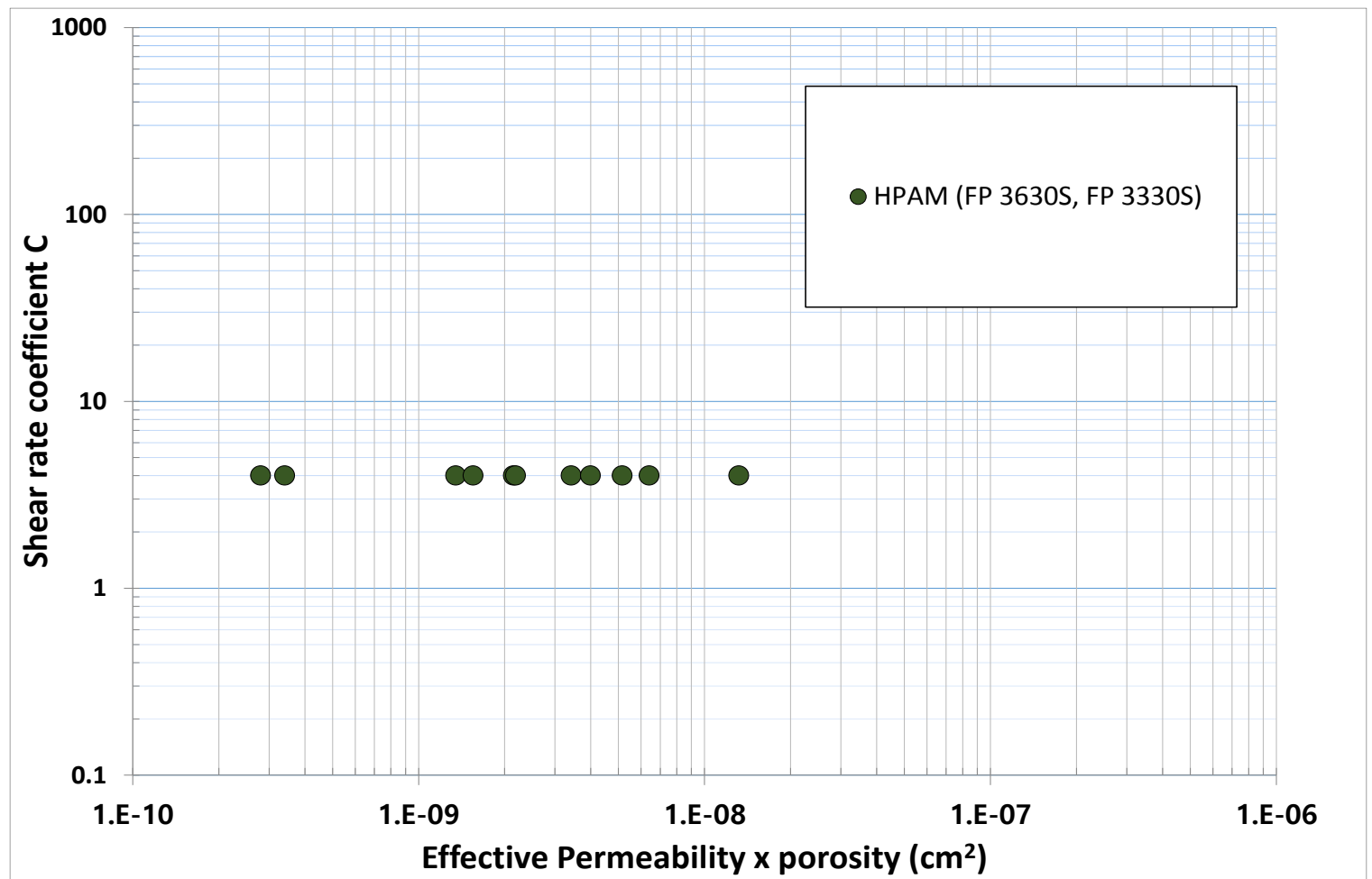

Figure 6.64 Shear rate correction factor calculated from experiments using HPAM

After history matching by fractional flow analysis, it was found that a shear correction factor of 4 showed the best match for all of the oil displacement experiments using HPAM polymer. The value of shear rate correction factor $\mathrm{C}$ versus the effective permeability porosity product is shown in Figure 6.64. The result showed a constant $\mathrm{C}$ value of 4 over the entire range of effective permeability. This result is in sharp contrast to Wreath (1989) as shown in Figure 6.62.

There are many factors such as slip at the pore walls, polymer retention and tortuosity that may account for the need for a shear correction factor to match the measured apparent viscosity in cores and the differences among various experimental results in addition to the fundamental limitations of the capillary tube model of the porous medium. One of these factors is polymer retention. Under ideal conditions, polymer 
retention is due mainly to adsorption and is very low, but in other cases the polymer solution may have microgels that cause pore plugging and high retention as well as possibly in-depth filtration of the polymer, which may not reach a true steady state in short cores. The microgels might explain the observed permeability reduction in some experiments but not in others using HPAM polymers with similar molecular weight. When permeability reduction occurs but is not accounted for, the estimated apparent viscosity could be higher than the actual viscosity value (Sheng, 2011).

The polymers used in this study are of a higher quality than many of the polymers studied between the 1960's and 1990's. The preparation and filtration of the polymers is also extremely important. Long cores such as used in this study are more likely to produce steady state results that apply to longer transport distances in oil reservoirs than short cores most often reported in the literature and the use of pressure taps along the core is absolutely essential for proper interpretation of the data. Nevertheless, it is remarkable how significant the differences are between most of the older experiments and the results of this study.

The value of the shear correction factor $\mathrm{C}$ was 1.1 for HPAM polymer solutions performed in cores without oil and 2 3 for scleroglucan polymer solutions performed in cores without oil. This may be related to the higher shear thinning behavior of scleroglucan compared to HPAM. The higher values obtained from the oil recovery experiments may be due to the higher tortuosity for two-phase flow compared to single phase flow at $100 \%$ water saturation.

Another source of in-situ polymer viscosity data are alkaline-surfactant-polymer (ASP) corefloods. The shear correction factor for HPAM polymer was determined from the steady state pressure drop data at the completion of several ASP floods that produced 
essentially all of the water flood residual oil. The following is a typical example of one such coreflood. The brine permeability of the Berea sandstone used for this ASP coreflood was 356 md. A 0.3 PV ASP slug with 2000 ppm FP 3630S polymer concentration was injected at $1 \mathrm{ft} / \mathrm{d}$ followed by a polymer drive with $1150 \mathrm{ppm}$ FP 3630 S polymer concentration at the same flow rate. Figure 6.66 shows the pressure drop data across the whole core, section 1 (inlet), section 2 and 3, and section 4 (outlet) versus the pore volumes injected. The oil breakthrough occurred at $0.31 \mathrm{PV}$ and the emulsion breakthrough occurred at $0.84 \mathrm{PV}$. The total oil recovery was $97.6 \%$ of the water flood residual oil and the final residual oil saturation was 0.008 (Figure 6.65). Figure 6.67 shows the viscosity of the polymer drive versus shear rate.

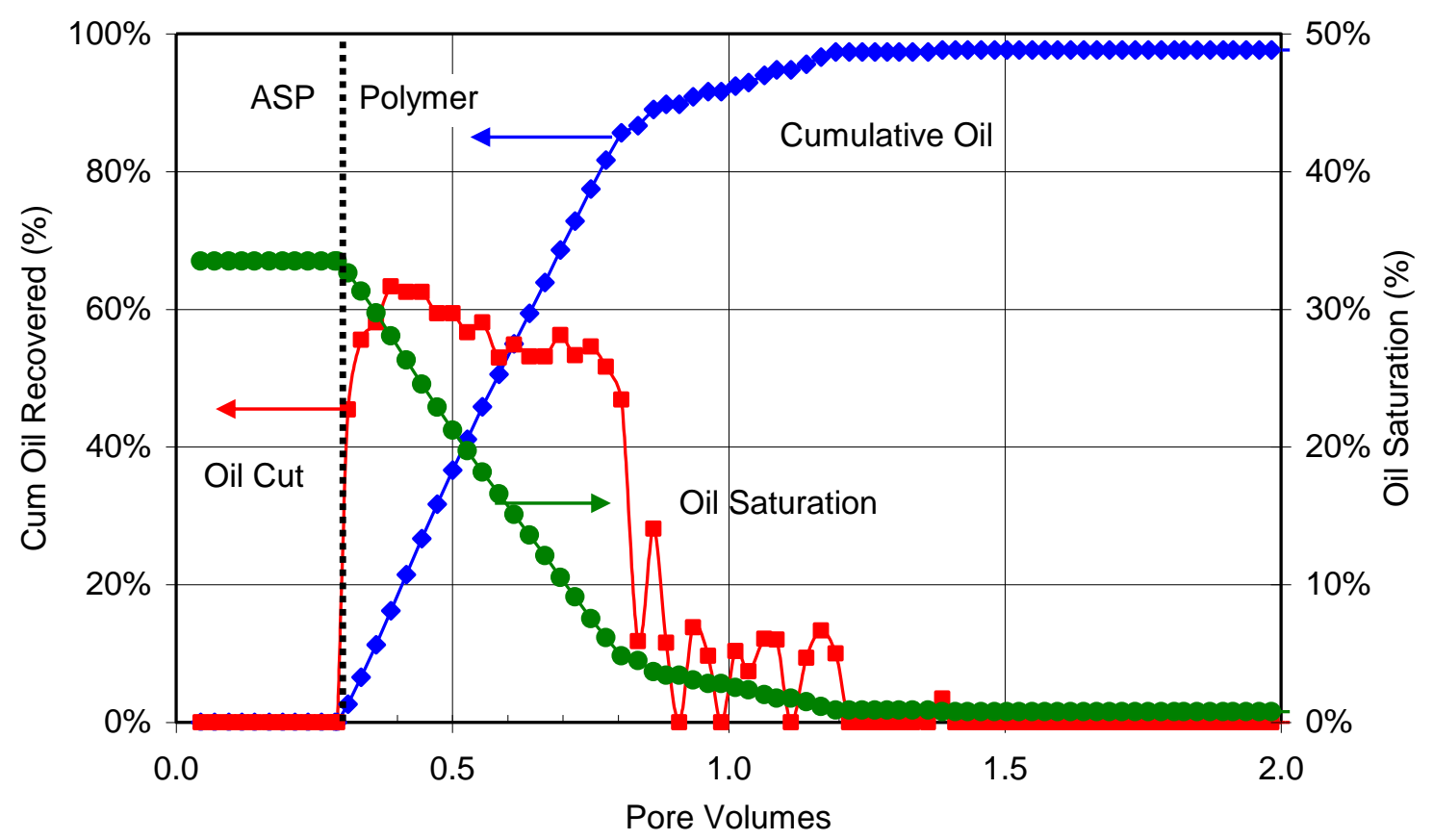

Figure 6.65 Oil Recovery for ASP experiment 


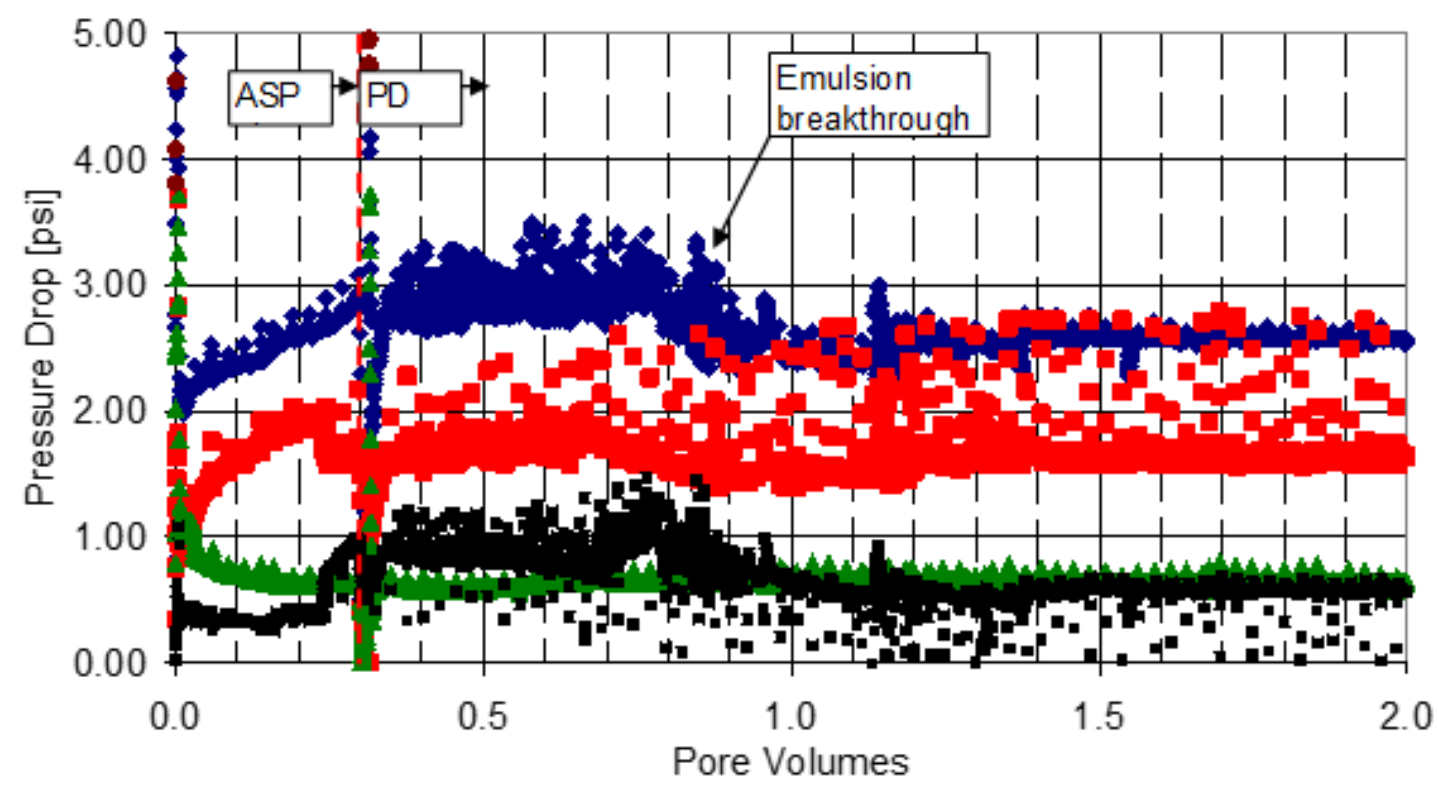

-Whole $\square$ Sec2+3 $\star$ Sec1 $\cdot$ Sec4 $\bullet$ back pressure

Figure 6.66 Pressure drop data for ASP experiment

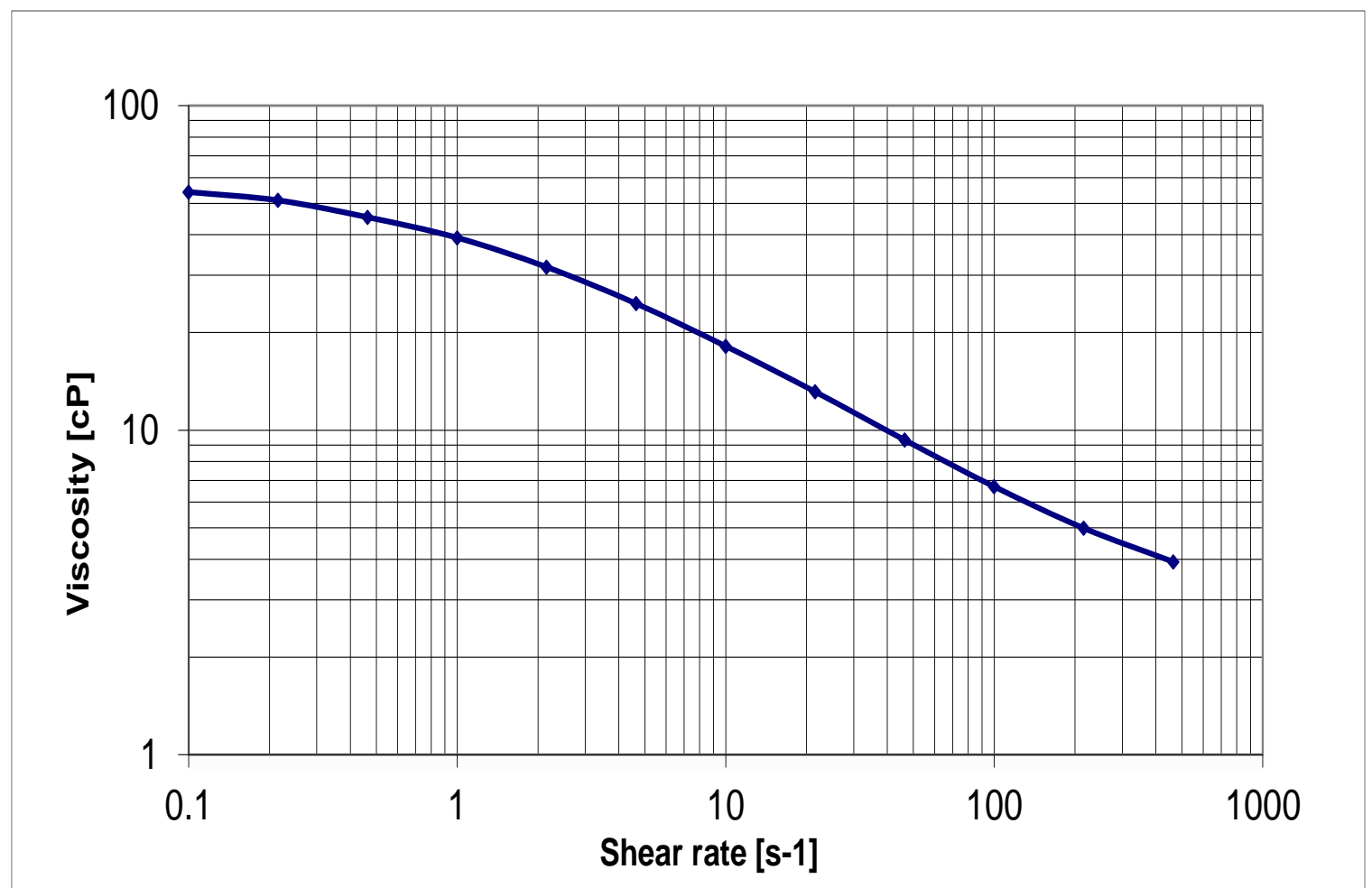

Figure 6.67 Viscosity of polymer drive 
Assuming the water relative permeability is 1 since the final water saturation was nearly 1 and assuming the permeability reduction factor is 1 , a shear correction factor of 1.14 was calculated to match the apparent viscosity based on the steady state pressure drop at the end of the polymer flood. This value is in agreement with the values for the polymer flood experiments without oil presented in Chapter 5.

Fortenberry (2013) measured the shear correction factor for HPAM polymer drive in a Bentheimer sandstone with a brine permeability of $2000 \mathrm{mD}$ after completion of an alkaline-co-solvent-polymer (ACP) flood with $\sim 100 \%$ oil recovery. He reported the shear correction factor as 1.33. Ehrenfried (2013) reported a shear correction factor of 2.06 for single phase flow of HPAM polymer in Bentheimer sandstone. All of these values indicate that when the same polymer is used and the measurements are done using the same experimental protocols and interpretation methods then the results are indeed consistent. However, until now there were only a few isolated examples of such experiments.

The shear correction factors for HPAM polymer solutions are different for experiments with (Chapter 6) and without oil (Chapter 5). For polymer flooding without oil, the shear correction factor $\mathrm{C}$ is only about 1.1, whereas the value of the shear correction factor $\mathrm{C}$ for two phase flow is 4 . A simple explanation is the higher tortuosity and thus higher shear rate for two phases compared to one phase. The shear rate is proportional to the square root of the tortuosity. It would be interesting to measure the tortuosity or estimate it from resistivity measurements to determine if it could be used to quantitatively explain the shear correction factor. 
The shear correction factor for xanthan gum and scleroglucan biopolymers is higher than that for HPAM polymers studied in this work. Biopolymer has relatively well-arranged molecules at low shear rate due to its rigid molecular structure and shows strong shear thinning, whereas HPAM shows relatively stronger viscoelasticity with a flexible coil molecular structure. These properties may also be related to the difference of the shear correction factor between the biopolymers and HPAM. 


\subsection{EXPERIMENT RESULTS USING MODIFIED HPAM POLYMER}

Experiments \#10, \#11 and \#12 were done using a new polymer called ChemPam 8177, which is a hydrophobically modified polyacrylamide polymer from ChemPam. This polymer did not pass through a 1.2 micron Millipore cellulose acetate filter at $15 \mathrm{psi}$, but it did pass the filtration ratio test using a 5 micron filter paper. Both FP 3330 S and $3630 \mathrm{~S}$ did pass the 1.2 micron filtration test. None of these polymers reduce the interfacial tension between the oil and water. The capillary numbers for all of the polymer floods were less than $10^{-5}$. A crude oil with a viscosity of about $1200 \mathrm{cp}$ at $40^{\circ} \mathrm{C}$ was used in all three experiments. Ottawa F-95 sand was packed in a glass column for experiment \#10 and in a core holder for experiments \#11 and \#12. Table 6.11 shows the properties of the three cores used in these experiments.

Table 6.11 Coreflood data

\begin{tabular}{|c|c|c|c|}
\hline Experiment & $\# 10$ & $\# 11$ & $\# 12$ \\
\hline Sand & Ottawa F95 & Ottawa F95 & Ottawa F95 \\
\hline Porosity & 0.37 & 0.33 & 0.34 \\
\hline Length $(\mathrm{cm})$ & 31 & 30 & 30 \\
\hline Diameter $(\mathrm{cm})$ & 2.54 & 3.81 & 3.81 \\
\hline Area $\left(\mathrm{cm}^{2}\right)$ & 5.07 & 11.4 & 11.4 \\
\hline Temperature $\left({ }^{\circ} \mathrm{C}\right)$ & 40 & 40 & 40 \\
\hline Brine permeability $(\mathrm{mD})$ & 6918 & 4916 & 5319 \\
\hline Pore Volume $(\mathrm{mL})$ & 59 & 115 & 122 \\
\hline
\end{tabular}

In experiment \#10, water was injected at $5 \mathrm{ft} / \mathrm{D}$ until the oil cut decreased to $10 \%$ and then polymer solution was injected at $1 \mathrm{ft} / \mathrm{D}$. Figure 6.68 shows the oil cut, oil saturation and cumulative oil recovery versus pore volumes. The oil saturation was 0.66 after $0.7 \mathrm{PV}$ of water flood in part due to the high oil viscosity and in part due to the incomplete water flood. The oil saturation decreased to 0.03 after about $6 \mathrm{PV}$ of polymer 
injection. This is a very surprising result and the reason for the almost zero oil saturation is not yet understand. The resistance factor at the end of the polymer flood was 2600, which is more than 40 times the ratio of the polymer viscosity to the water viscosity. This indicates the polymer was probably plugging pore throats. No intermediate pressure measurements could be made on the glass column, so the variation of the pressure gradient is not known.

One possible explanation for the low oil saturation was experimental error due to formation of a water in oil emulsion in this very heavy oil. However, the oil was dewatered using salt and a correction made for the water in the oil. The oil contained about $4 \%$ water. The final oil saturation of 0.02 is the value after this correction was made.

In the next two experiments, a core holder was used so that the pressure gradient could be measured across four sections of the core. Experiment \#11 was a repeat of \#10 except that the velocity of the polymer flood was reduced from $1 \mathrm{ft} / \mathrm{D}$ to $0.5 \mathrm{ft} / \mathrm{D}$ since the pressure drop for experiment \#10 was too high (26 psi/ft). Also, the polymer concentration in the effluent was measured for experiment \#11. The retention was 600 $\mu \mathrm{g} / \mathrm{g}$ of sand. This value is more than 10 times higher than that measured for HPAM polymers in a similar high permeability clean sand.

In experiment \#12, water was injected at $5 \mathrm{ft} / \mathrm{D}$ until the oil cut decreased to $1 \%$, then followed by injection of ChemPam 8177 polymer solution at $1 \mathrm{ft} / \mathrm{D}$ (Figure 6.69). The oil saturation was 0.6 after 1.9 PV of water flood mainly due to the high oil viscosity. The oil saturation decreased to 0.06 after about $4.3 \mathrm{PV}$ of polymer injection. The resistance factor was 1018, which is about 20 times higher than the polymer/water viscosity ratio. The polymer retention was determined to be $574 \mu \mathrm{g} / \mathrm{g}$ of rock, which is a 
very high value similar to the value measured for experiment \#11. This large retention and high resistance factor both indicate at least partial plugging of pore throats. Figures 6.70 and 6.71 compare the oil recovery results for experiment \#12 with the results for experiment \#7, which used a conventional HPAM polymer. Figures 6.72 and 6.73 compare the pressure drop data for experiments \#12 and \#7, respectively. Experiment \#12 shows a markedly different behavior compared with the conventional HPAM polymer. The mobility reduction using the modified HPAM is significantly higher than the viscosity of the injected solution and its retention is extremely high.

Experiment \#12 was done to measure the incremental oil recovery from a tertiary polymer flood using ChemPAM 8177 polymer and compare the results with experiments \#10 and \#11 using the same polymer viscosity, but for polymer injection starting at a higher oil cut $(10 \%)$. Experiment \#10 showed significant incremental oil recovery, however the pressure drop was too high and there were no pressure taps to measure pressure drop across different sections of the core. For experiment \#11, pressure drop data were measured using a stainless core holder with pressure taps. Also, the confining pressure was 2000 psi.

Experimental data for experiments \#10,\#11 and \#12 are summarized in Table 6.12. For experiment $\# 12$, the water flood stopped at $99 \%$ water cut compared with $90 \%$ water cut in the two previous experiments. The effluent concentration of polymer was measured. A fourth difference was that the polymer solution was injected at $1 \mathrm{ft} / \mathrm{D}$. The remaining oil saturation decreased from 0.61 for the water flood at $99 \%$ water cut to 0.056 after 4.5 pore volumes for the tertiary polymer flood. The peak pressure gradient was about $15 \mathrm{psi} / \mathrm{ft}$ (Figure 6.72) in the $5319 \mathrm{mD}$ sandpack. The polymer retention was determined to be $574 \mu \mathrm{g} / \mathrm{g}$ of sand. The polymer concentration did not reach its injected 
value until about 6 PV (Figure 6.75) and the viscosity was only about $60 \%$ of its injected value at $6 \mathrm{PV}$ even though the polymer was stable. These values indicate poor transport of the polymer as was the case with experiments \#10 and \#11.

Table 6.12 Summary of core flood results

\begin{tabular}{|c|c|c|c|c|}
\hline Experiment & \#7 & \#10 & $\# 11$ & \#12 \\
\hline Crude Oil & B-4 & $B-1$ & B-2 & B-3 \\
\hline Sand & Ottawa Sand & Ottawa Sand & Ottawa Sand & Ottawa Sand \\
\hline Permeability (md) & 6078 & 6913 & 4916 & 5319 \\
\hline Oil Viscosity (cp) & 1050 & 1230 & 1111 & 1290 \\
\hline Temperature $\left({ }^{\circ} \mathrm{C}\right)$ & 40 & 40 & 40 & 40 \\
\hline Porosity & 0.39 & 0.37 & 0.33 & 0.34 \\
\hline \multirow{2}{*}{ Velocity (w/p), ft/D } & 5 & 5 & 5 & 5 \\
\hline & 1 & 1 & 1 & 1 \\
\hline Polymer & FP3630S & ChemPam 8177 & ChemPam 8177 & ChemPam 8177 \\
\hline Polymer conc. (ppm) & 3,500 & 3,500 & 3,500 & 3,500 \\
\hline Capillary Number & $2.9 \times 10^{-6}$ & $8.1 \times 10^{-6}$ & $2.9 \times 10^{-6}$ & $2.9 \times 10^{-6}$ \\
\hline \begin{tabular}{|c|}
$\begin{array}{c}\text { Equivalent Shear Rate } \\
(\mathrm{s}-1)\end{array}$ \\
\end{tabular} & 52 & 38 & 19 & 31 \\
\hline \begin{tabular}{|c|} 
Polymer Viscosity $\mu p$ \\
$(\mathrm{cp})$
\end{tabular} & 39 & 28 & 40 & 32 \\
\hline Mobility Ratio & 10 & 0.7 & 0.7 & 1.5 \\
\hline Relaxation time (s) & 0.06 & 0.06 & 0.06 & 0.06 \\
\hline Deborah Number & 0.6 & 2.3 & 1.1 & 1.9 \\
\hline Initial oil saturation & 0.89 & 0.85 & 0.82 & 0.88 \\
\hline \begin{tabular}{|c|}
$\begin{array}{c}\text { Oil saturation at end } \\
\text { of water flood }\end{array}$ \\
\end{tabular} & 0.61 & 0.66 & 0.64 & 0.60 \\
\hline $\begin{array}{c}\text { Oil saturation after } \\
\text { polymer }\end{array}$ & 0.26 & 0.03 & 0.05 & 0.06 \\
\hline$\Delta$ So & 0.34 & 0.63 & 0.59 & 0.54 \\
\hline
\end{tabular}


The fractional flow curves for experiment \#12 are plotted in Figure 6.74. The injected ChemPam 8177 polymer concentration was $3500 \mathrm{ppm}$. The retardation factor $\left(D_{p}\right)$ calculated from the polymer retention and the injected polymer concentration is 0.76. The oil bank breakthrough time and oil cut are in rough agreement when using the observed final oil saturation, but the fractional flow does not explain why the final oil saturation is so low. However, it does clearly illustrate why the remaining oil saturation is much higher for water than for polymer when the oil is highly viscous (1200 cp in this case) even if the water flood is carried out to more than $99 \%$ water cut.

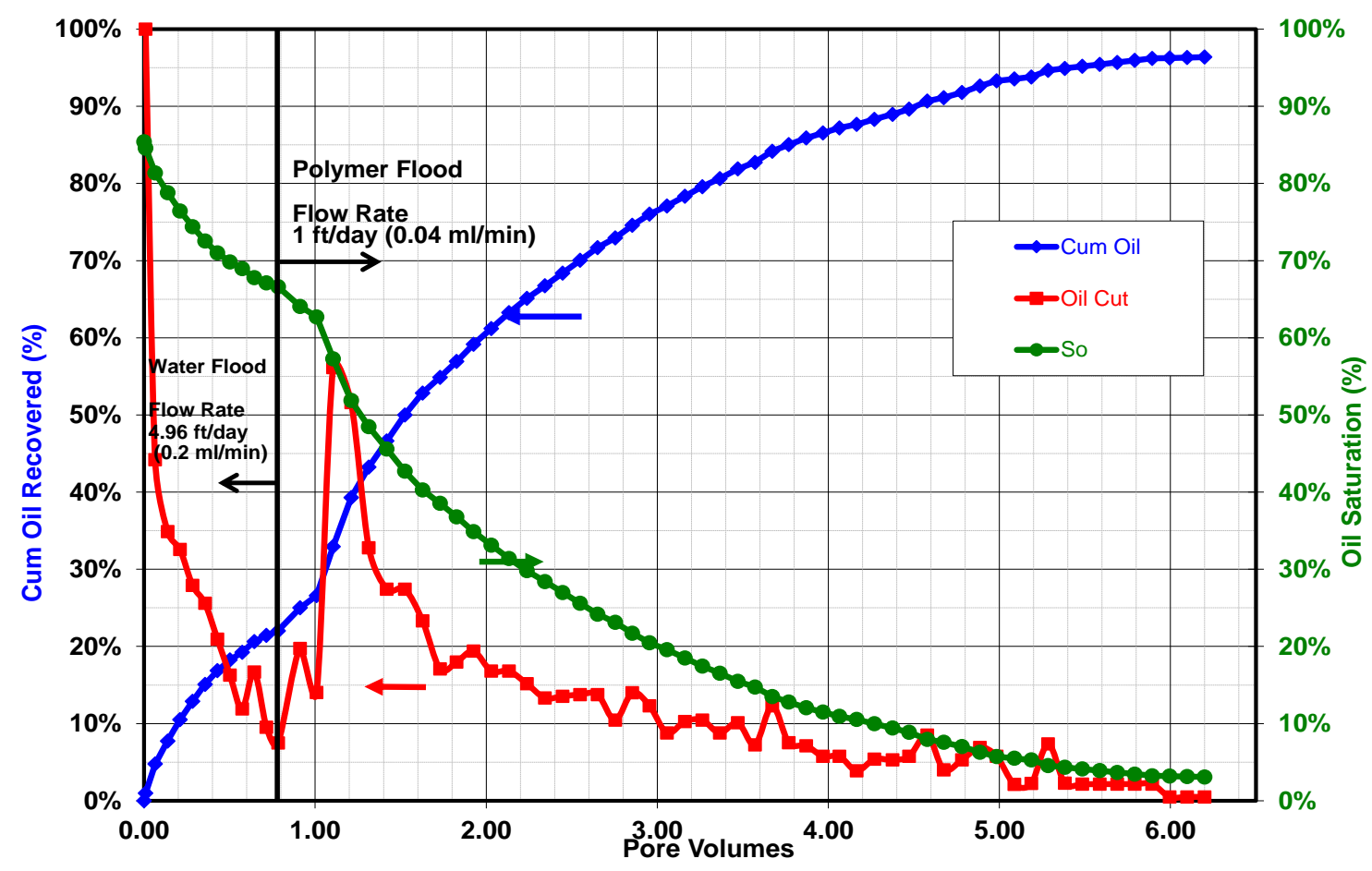

Figure 6.68 Cumulative Oil Recovery, Oil Saturation and Oil Cut for Experiment \#10 


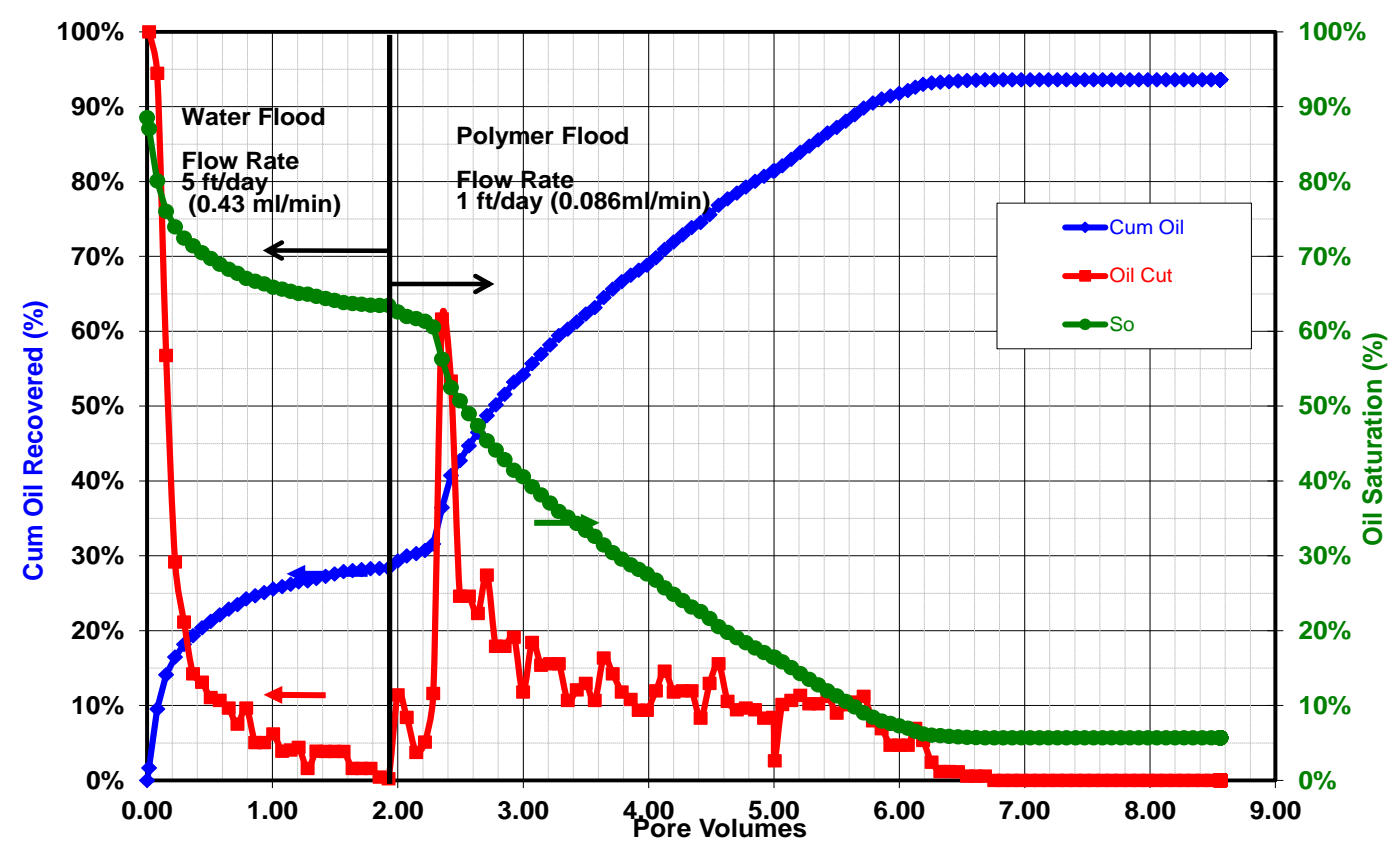

Figure 6.69 Oil Recovery, Oil Saturation and Oil Cut for Experiment \#12 using ChemPam 8177 polymer

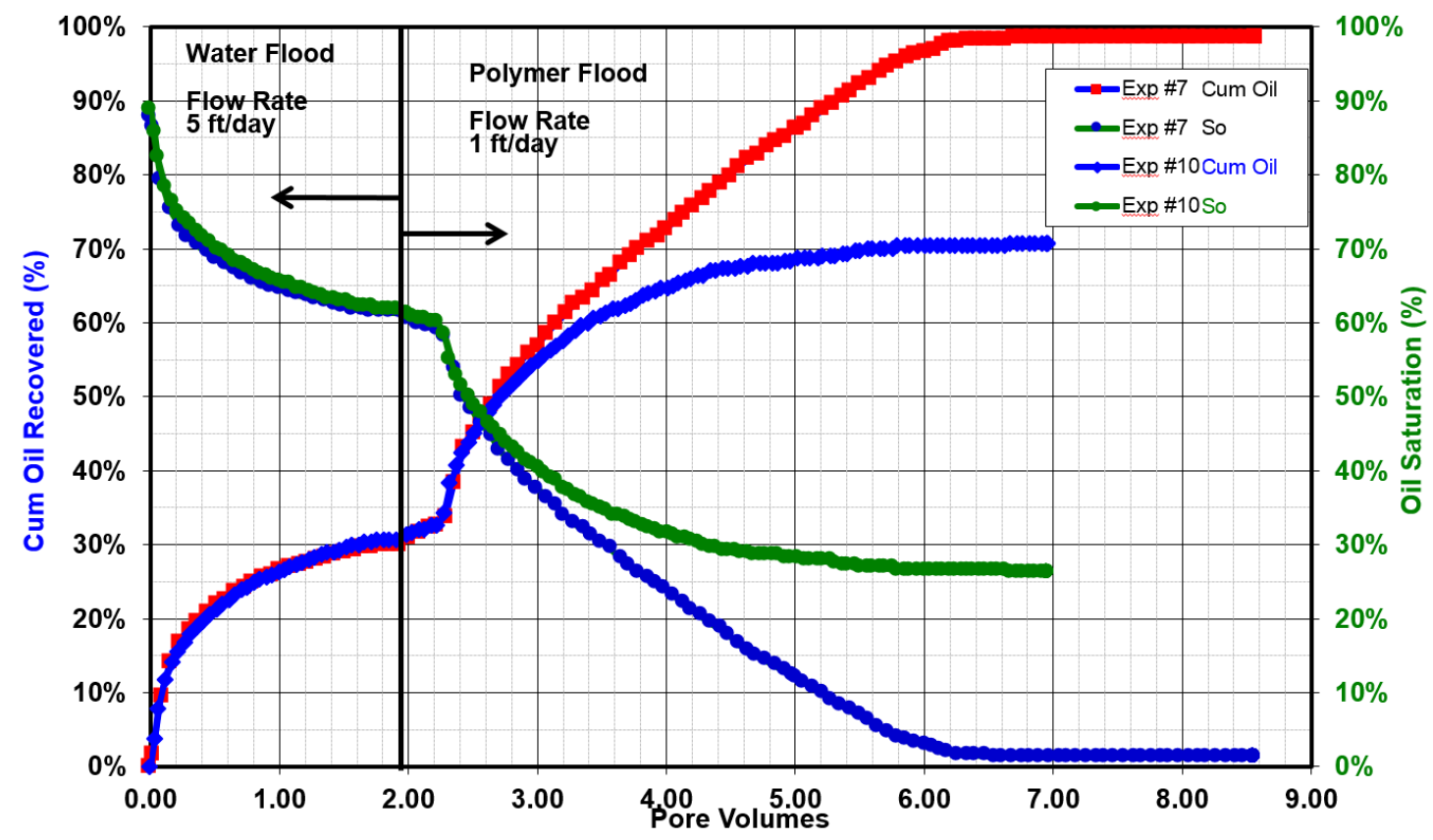

Figure 6.70 Comparison for cumulative oil recovery and oil saturation for experiments \#7 and \#12 


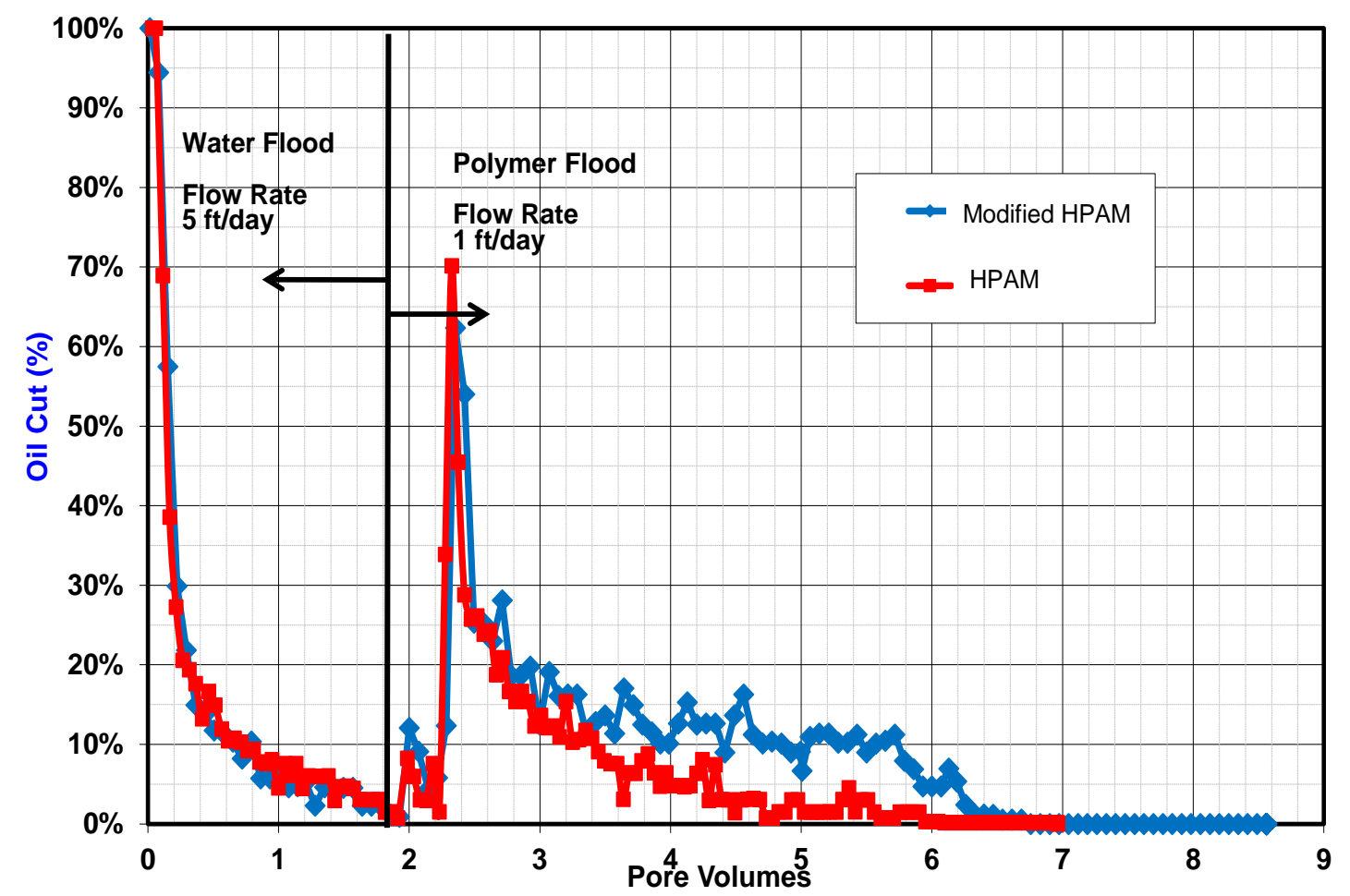

Figure 6.71 Comparison of oil cuts for experiment \#7 and \#12

For experiment \#12, long term injections of diluted solutions of this modified HPAM into porous media under monophasic conditions reveal markedly different behavior compared with the conventional HPAM in Figure 6.72 and Figure 6.73. The mobility reductions reached with modified HPAM are significantly higher than the viscosity of the injected solution. 


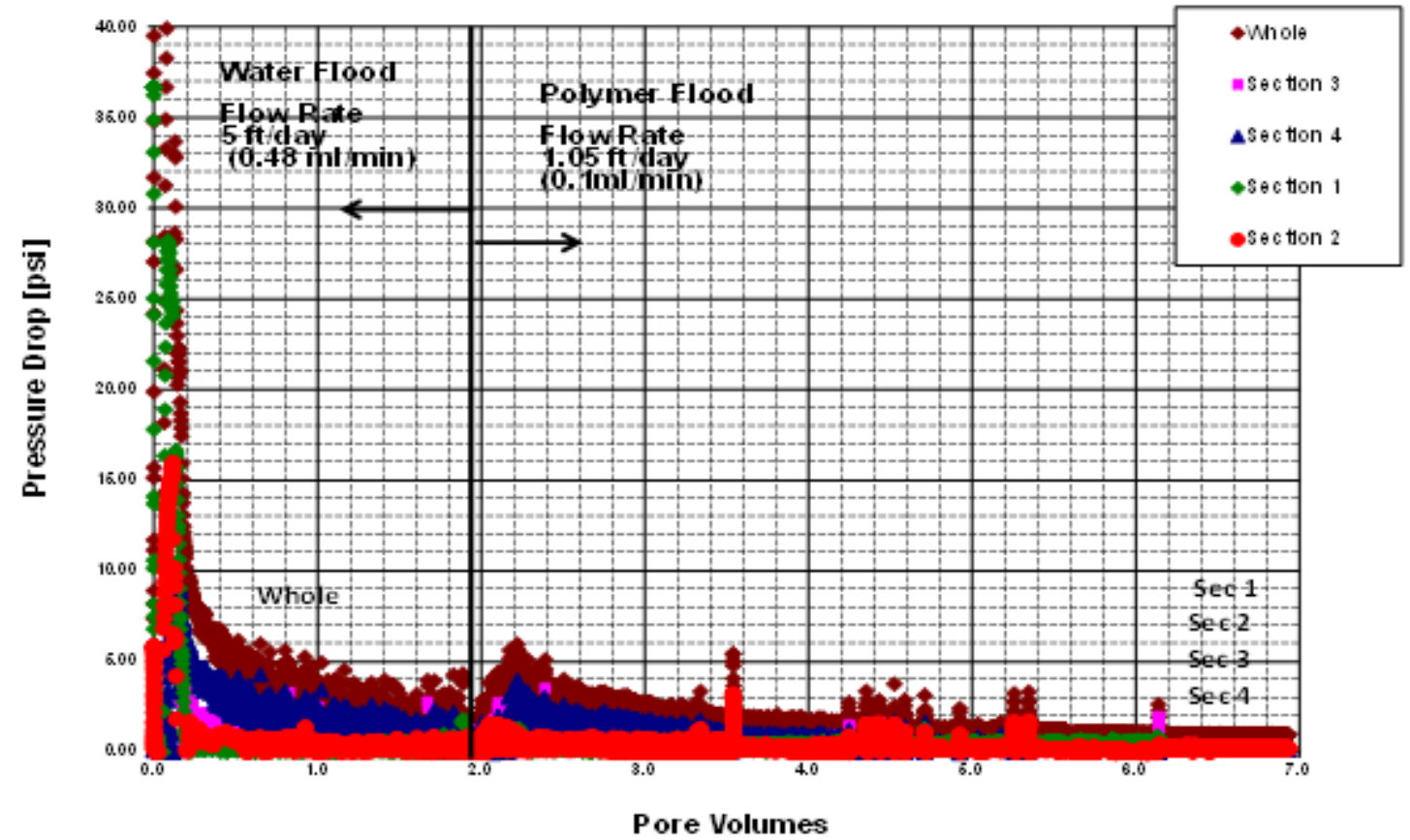

Figure 6.72 Pressure drop data for experiment \#12 using ChemPam 8177 polymer

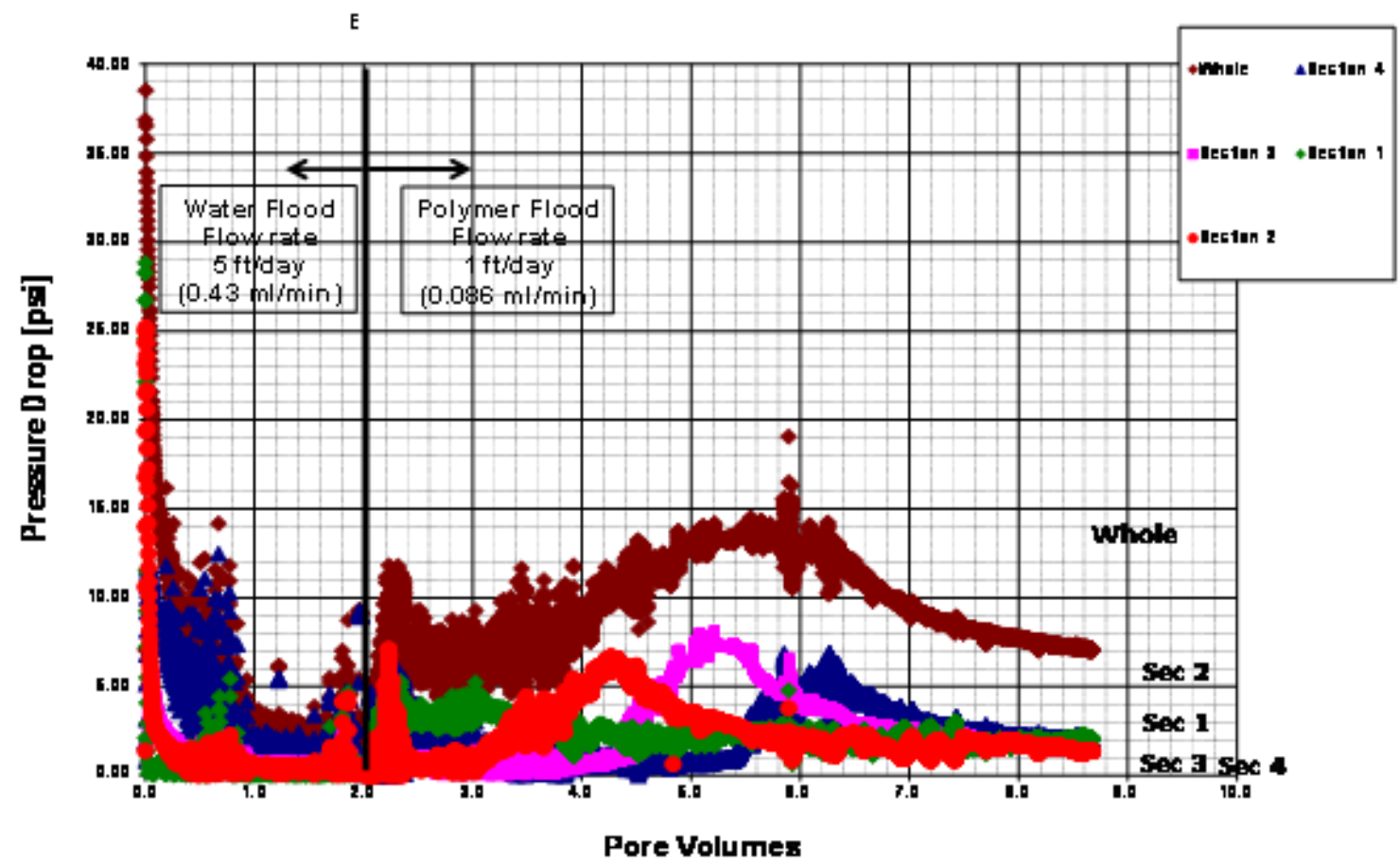

Figure 6.73 Pressure drop for experiment \#7 
Figure 6.75 shows an extremely high polymer retardation for ChemPam 8177 polymer. The high mobility reduction observed with modified HPAM are because of the hydrophobic component in the polymer. Modified HPAM coreflood results indicate huge permeability reduction, which could be linked to the formation of thick layers of physical gel adsorbed on the pore throats. Figures $6.76,6.77$ and 6.78 show the oil recovery for modified HPAM injection after HPAM polymer flooding. The modified HPAM was injected after aging the core for one week after finishing HPAM polymer flooding. After 3.5 PV of modified HPAM injection, the remaining oil after HPAM polymer flood started to move and $8 \%$ addition oil was produced after $6 \mathrm{PV}$.

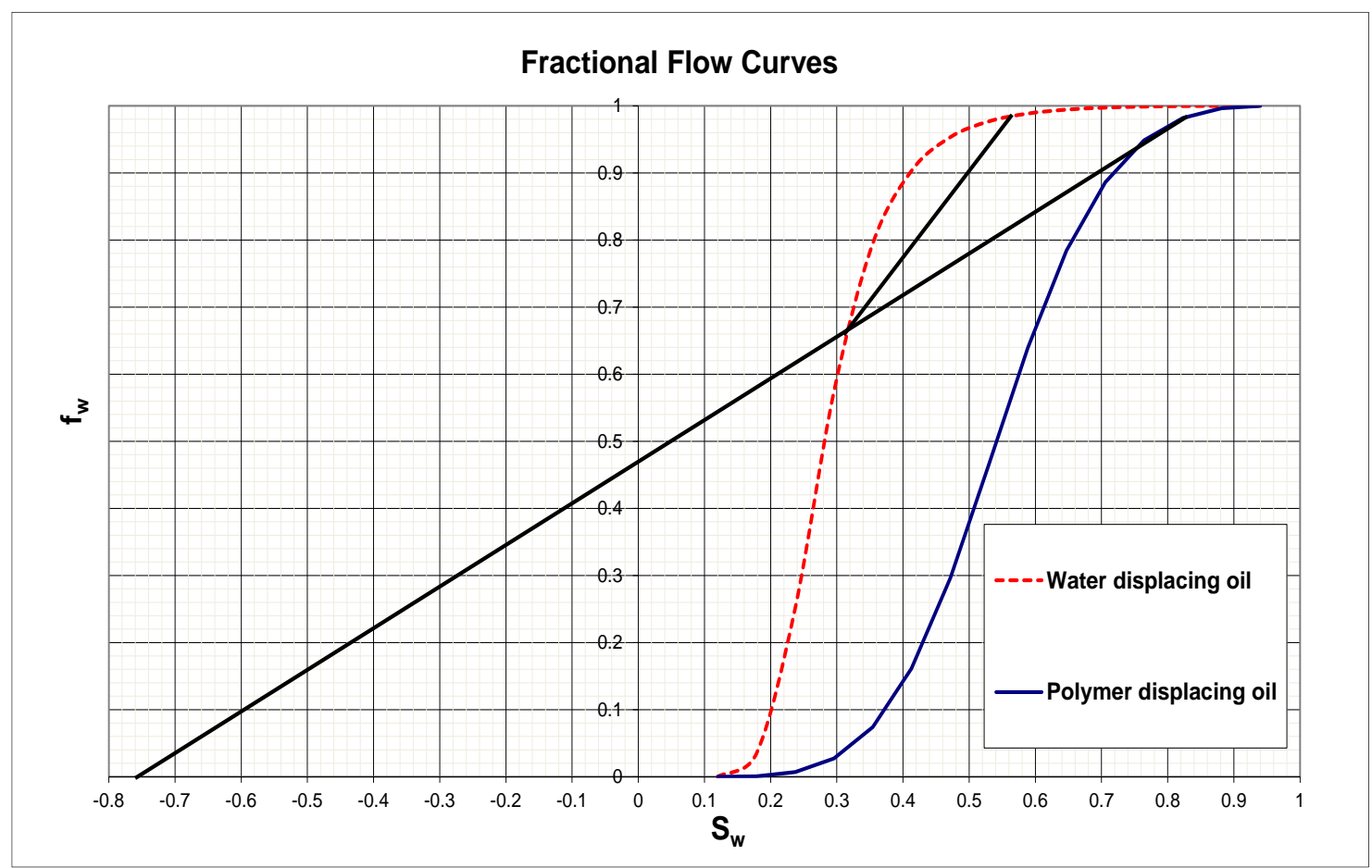

Figure 6.74 Fractional flow curve for Experiment \#10 


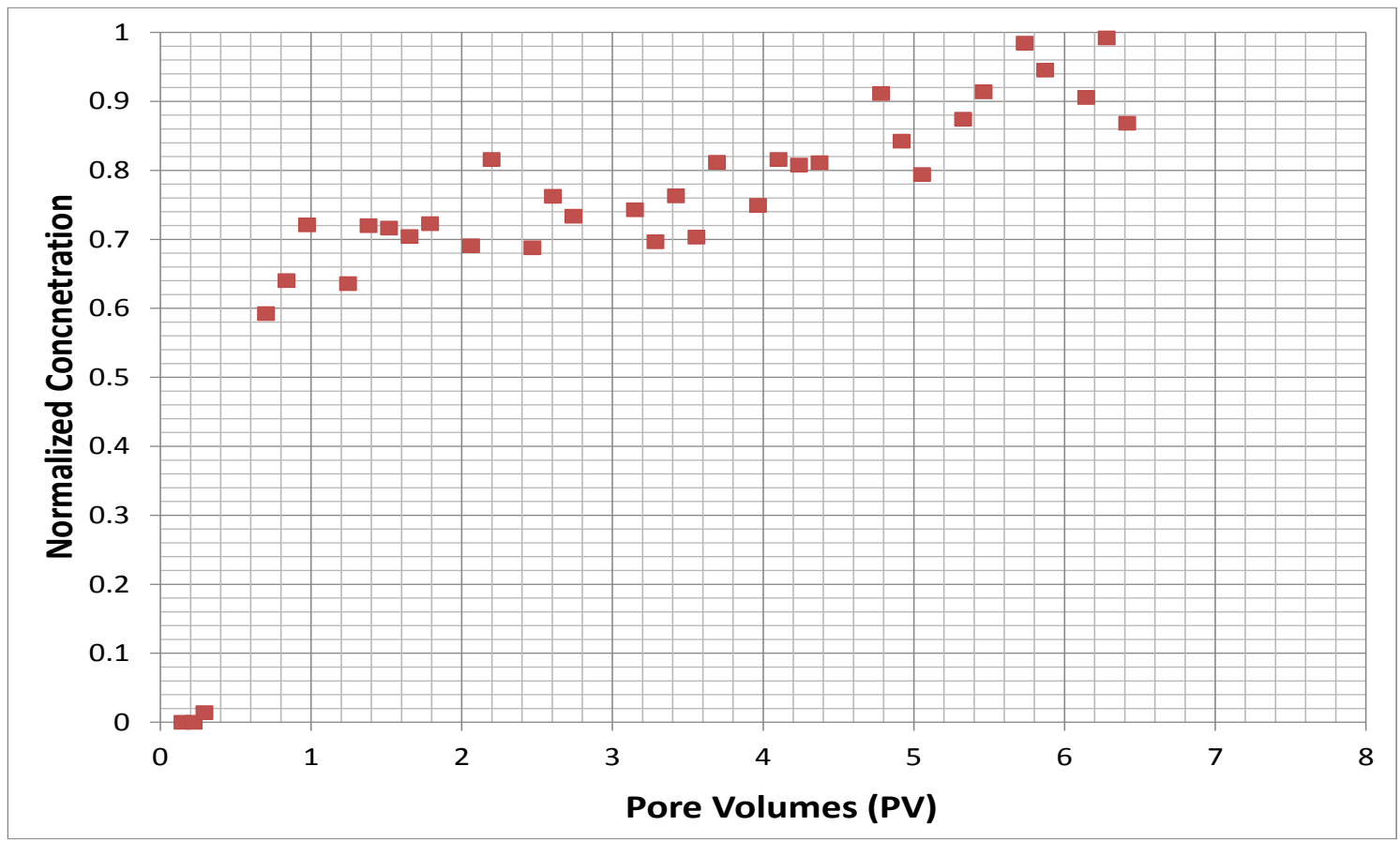

Figure 6.75 Effluent polymer concentration for experiment \#12 using ChemPam 8177 polymer

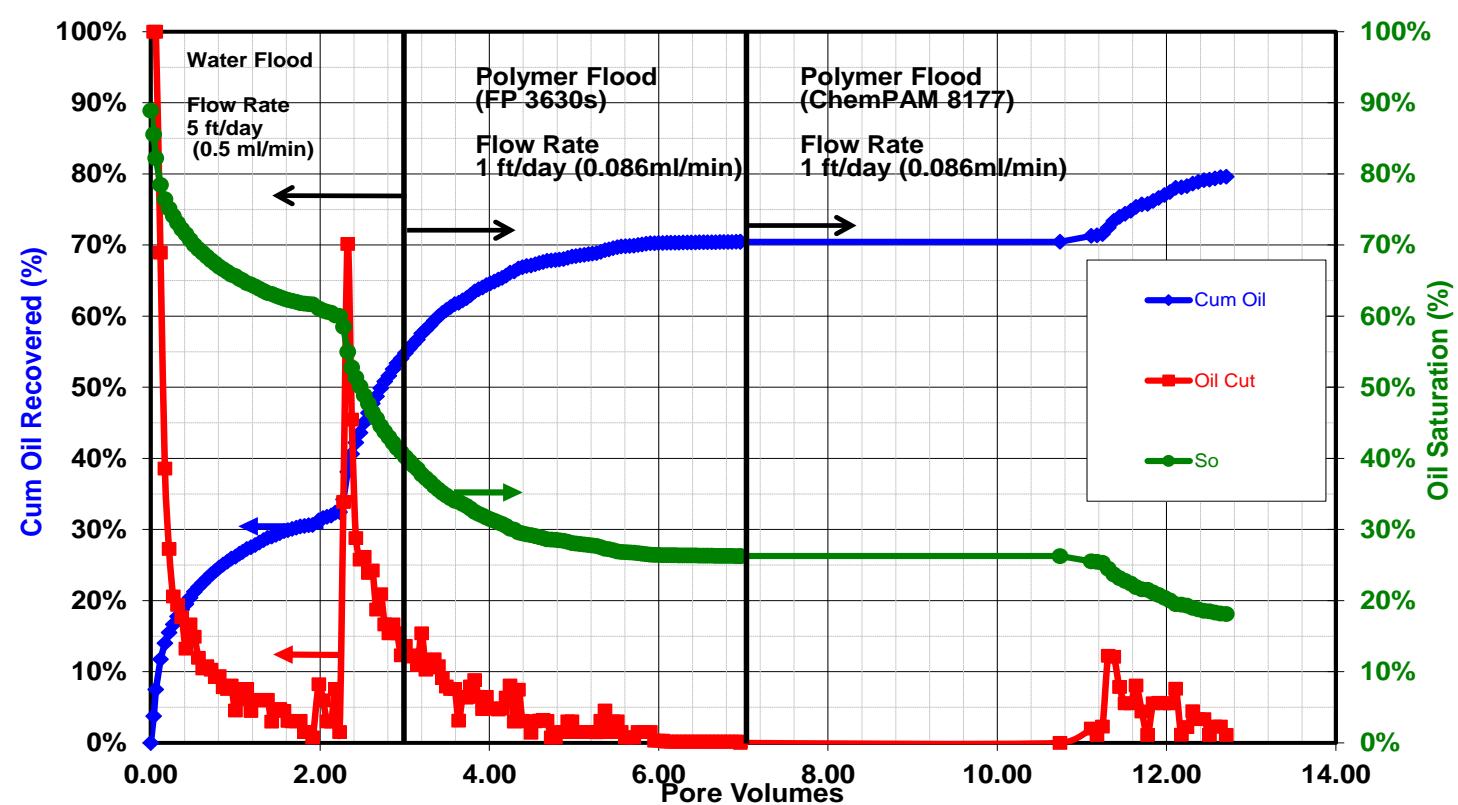

Figure 6.76 Oil Recovery, Oil Saturation and Oil Cut for Experiment \#12 


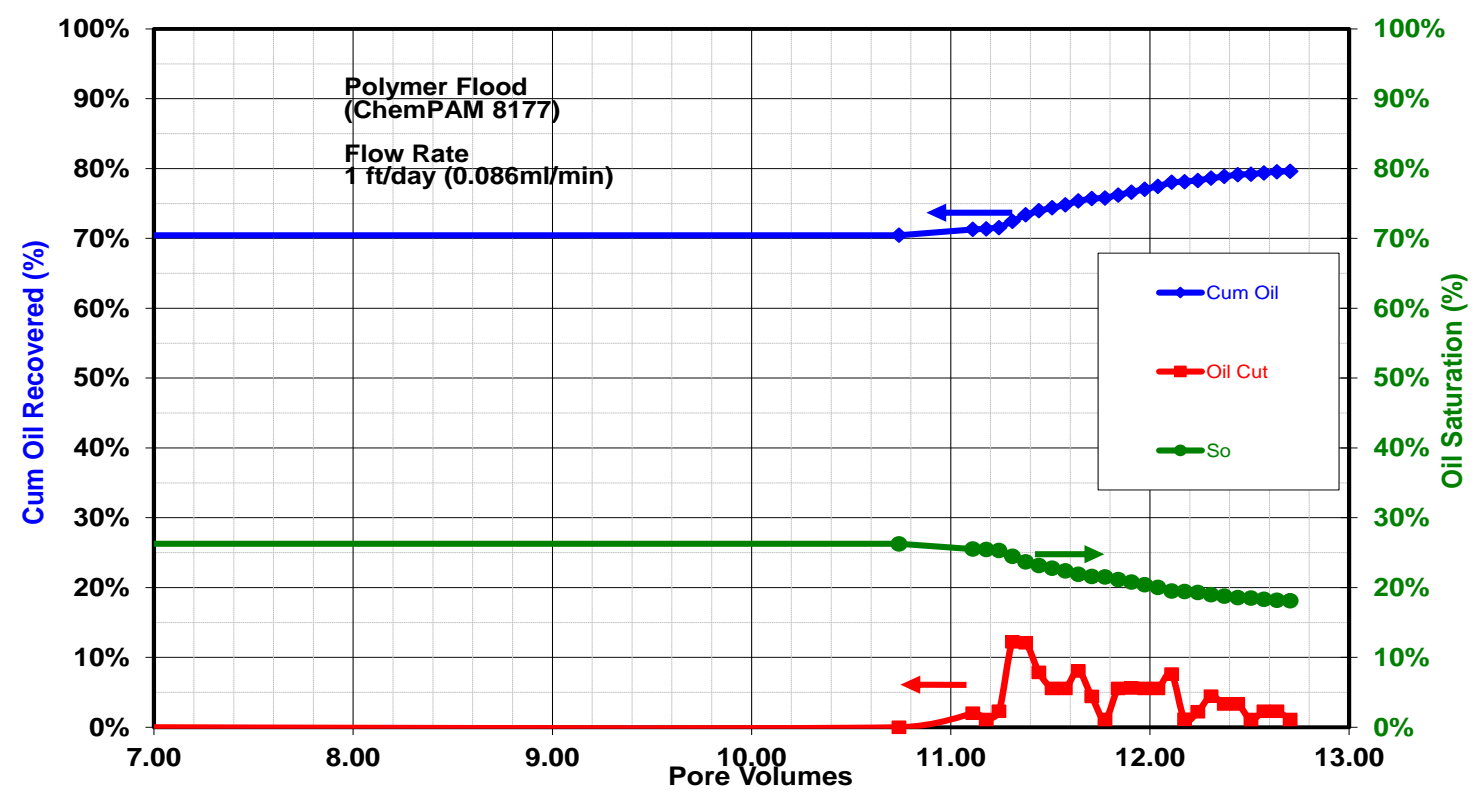

Figure 6.77 Oil Recovery, Oil Saturation and Oil Cut for Experiment \#12

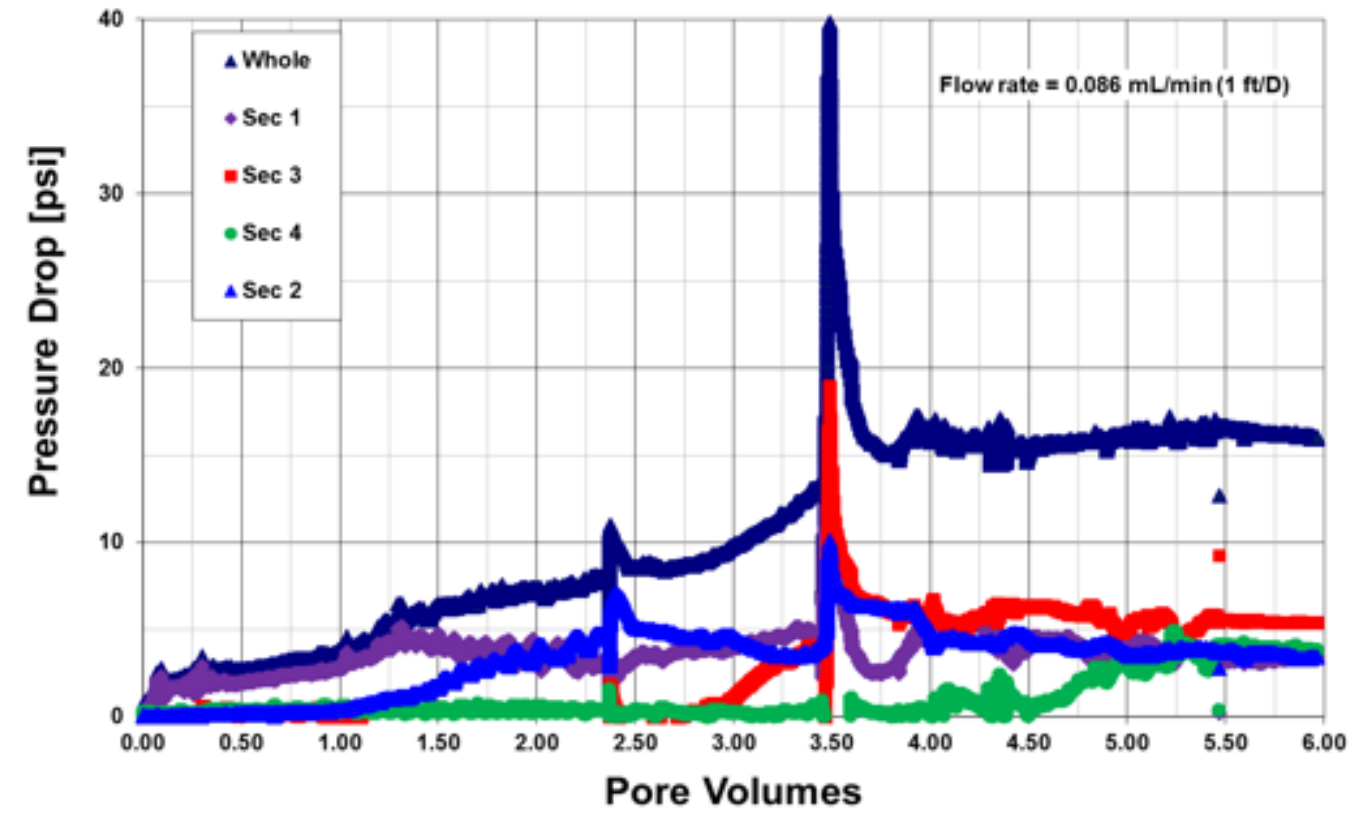

Figure $\overline{6} . \overline{78}$ Pressure dirop data for experiment \#12 


\section{Discussion}

Two oil recovery mechanisms can be considered in polymer flooding of viscous oil. One is the pushing force acting on crude oil before polymer breakthrough and the other is widening water path by the friction acting on oil films after polymer breakthrough. Mechanism of the pushing effect on oil is the same for water flooding and polymer flooding. Because the pushing force acting on oil is the main driving power for displacing oil and polymer cannot decrease interfacial tension in this situation, the effect is the same for both polymer flooding and water flooding. However, in mechanism after polymer breakthrough, the friction force between polymer molecules and oil molecules can be different from the force between water and oil as illustrated in Figure 6.79. As shown in Figure 6.69, the oil recovery and oil cut for experiment \#7 and experiment \#12 before polymer breakthrough is almost the same. This means that mechanism of the pushing effect on oil is the same for HPAM and modified HPAM polymer drive. However, the additional oil recovery of modified HPAM flood is $29 \%$ higher than HPAM flood after polymer breakthrough.

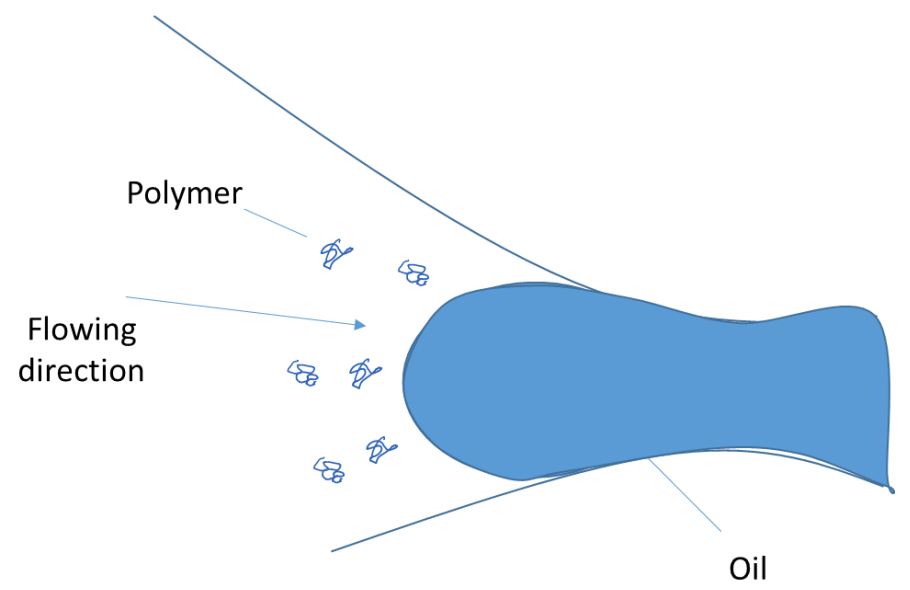

Figure 6.79 Schematic diagram of pushing force 


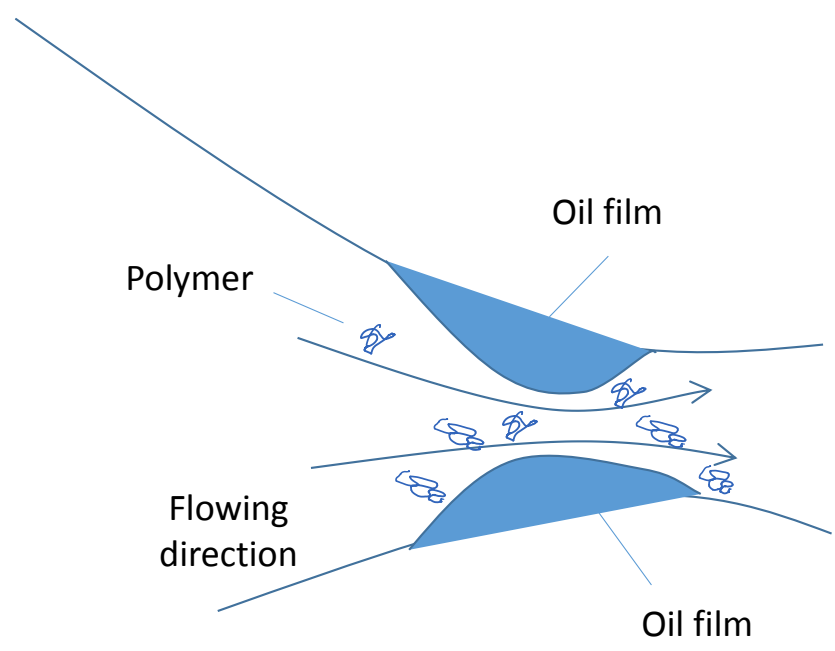

Figure 6.80 Schematic diagram of friction force

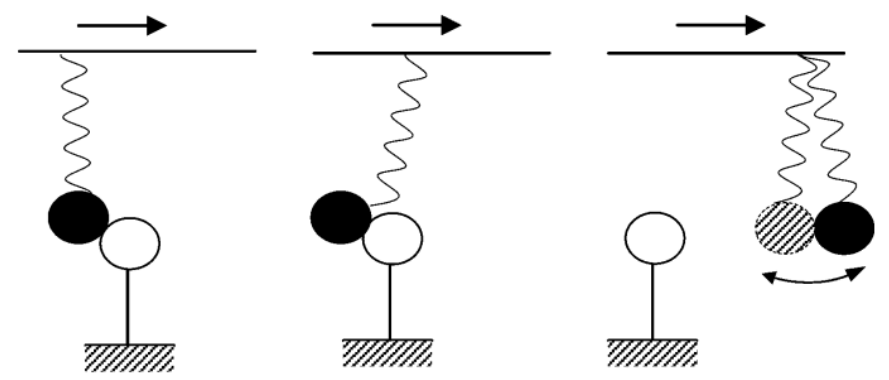

Figure 6.81 Tomlinson atom motion model

Zhang et al. (2008) tried to prove that polymer flooding can enlarge displacement efficiency and thus enhance ultimate oil recovery efficiency by using microscopic theoretical analysis. According to the theory of molecular motion, the macro pressure results from motions and interactions of molecules. The process of water or polymer flooding is the course of collision and oscillation between molecules of injected fluid and oil molecules. Between polymer molecules and surface oil molecules can be described by the Tomlinson atom motion model (Tomlinson, 1929), in which friction is considered as resulting from collision, oscillating and adhesion between atoms in Figure 6.81. 
For macro solid friction, the frictional force is directly proportional to the normal load

$$
\mathrm{F}=\mu \mathrm{P}
$$

Where $\mu$ is the coefficient of friction.

For interface friction at adhesion contact surface, Bowden and Tabor (1966) presented,

$$
\mathrm{F}=\tau_{c} A
$$

Where $A$ is the real area of contact, and $\tau_{c}$ is the shear strength. The shear strength can be composed of three parts

$$
\tau_{c}=\tau_{c 1}+\tau_{c 2}+\tau_{c 3}
$$

Where $\tau_{c 1}$ is shear strength resulted from interface forces of the two contact surfaces, $\tau_{c 2}$ is the shear strength from applied load, $\tau_{c 3}$ is the shear strength from elastic deformation (Homola et al., 1989). For viscoelastic fluids, shear strength can be

$$
\tau_{c}(t)=G \cdot \dot{\gamma}(t)
$$

Where $\mathrm{G}$ is the viscoelastic modulus and $\dot{\gamma}(t)$ is the shear stress as a function of angular frequency. For polymer solution, friction force is

$$
\mathrm{F}=G \cdot \dot{\gamma}(t) A
$$

The larger the viscoelastic energy of polymer molecule is, the greater the force acting on oil molecules is. Viscoelasticity makes surface atoms and oil molecules collide with greater acting force than that of water flooding under the same flow velocity. With the flow velocity of polymer solution increasing, the collision that polymer molecules act on oil molecules intensives, so does the friction. More oil molecules are detached from oil film and come into the flowing of injected fluid. Thus, polymer flooding can improve the displacement efficiency.

Even though the frictional energy is dissipated in plastically deforming material asperities at the interface during shear, there is some cases where sliding with friction 
does occur without wear (McClelland, 1989). McClelland claimed that the two surfaces can slide across each other without dissipating energy when the sliding velocity is slow. The fact that the AFM can image surfaces with atomic resolution through the perpendicular repulsive force between a tip and a surface is evidence that sliding can occur without wear.

Hydrophobically modified HPAM contains a small number of hydrophobic groups attached directly to the polymer backbone. In aqueous solution, the hydrophobic groups of theses polymers can associate to minimize their exposure to the solvent. This association results in an increase in the hydrodynamic size of the polymer and because of the existence of hydrophobic groups between molecular chain and intramolecular, the modified HPAM molecular chain tended to form network structure.

In the case of sandstones, the rock surface is negatively charged thus introducing anionic charges in the polymer reduces the affinity of the polymer to the rock surface thereby reducing the amount of adsorbed polymer. In the case of the estelada, the rock surface is positively charged, so the presence of anionic charges along the polymer backbone increases the affinity of the polymer to the rock thus the amount of adsorbed polymer.

A comparison between the adsorption behavior of the hydrophobically modified HPAM with that of the corresponding HPAM showed that the hydrophobic moieties play an important role in the adsorption process. Adsorption for the hydrophobically modified polyacrylamide shows an unusual shape, characterized by the absence of a plateau region and a continual increase in the adsorbed amount with polymer concentration in the bulk. This particular behavior was explained by the formation of multilayers through hydrophobic associations, the adsorbed layers being formed in part by some chains that 
are not directly in contact with the surface. Figure 6.82 shows schematically the probable structures of the adsorbed layers formed by HPAM and hydrophobically modified HPAM. The HPAM forms a classical layer. In the case of the modified HPAM, a large part of the train segments is formed by the hydrophobic blocks. Inside the first layer, hydrophobic domains are formed by intra and intermolecular hydrophobic associations. This formation leads to a reorganization of the adsorbed layer and to a higher chain density. At the same time, polymer chains can adsorb by hydrophobic interaction (multilayer formation). The continuously increasing amount is due to a chain reorganization in the adsorbed layer and to a successive formation of additional polymer layers by hydrophobic association (Volpert et al., 1998).
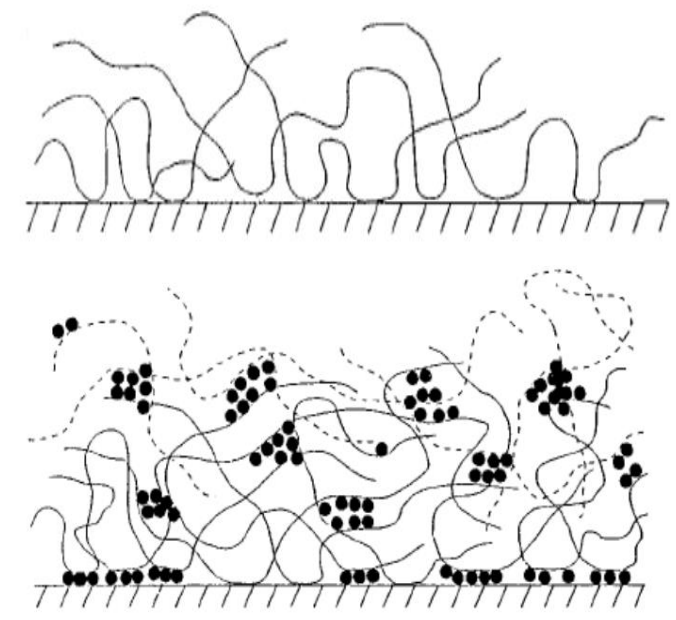

Figure 6.82 Schematic illustration of the probale structures of the adsorbed layer for HAPM (top) and hydrophobically modified HPAM (bottom) (Volpert et al., 1998)

Figure 6.75 shows an extremely high polymer retention value for experiment \#12. The polymer retention was determined to be $574 \mu \mathrm{g} / \mathrm{g}$ of rock, which is extremely high value. This means the core contains extra $2500 \mathrm{ppm}$ of polymer in the pores besides of 
injection of $3500 \mathrm{ppm}$ of polymer drive. Total $6000 \mathrm{ppm}$ of polymer concentration would generated high friction force on oil film and ganglia by high viscoelasticity of modified HPAM. Also, Huh and Pope (2008) proved that as the elastic modulus of the fluid increases, the growth rate for the interfacial disturbance decreases by using a simplified model for oil ganglia generation. This means polymer solution which has high elastic modulus delay the oil column breakage into oil ganglia, thereby poltentially decerasing the residaul oil saturation. This mechanism could help to explain the long oil cut tail for modified HPAM polymer flooding.

As flow continues, new polymer solution is introduced, and more molecules will come into contact with the retained layer, which causes the growth of the layer and thereby reduces the permeability. Even though Modified HPAM showed remarkable permeability reductions, pressure drop had stabilization trend after oil cut became nearly $0 \%$ at $5 \sim 6 \mathrm{PV}$ of injection and it didn't show core plugging in figure 6.73 and 6.78 . This modified HPAM did not passed through 1.2 and $3 \mu \mathrm{m}$ Millipore cellulose acetate filter at 15 psi, but it did pass the filtration ratio test using a $5 \mu \mathrm{m}$ filter paper. Experiment \#7 \#10 was performed with 6 7 Darcy sand pack and corresponding to average pore size $\mathrm{r}_{\mathrm{p}}, 13 \sim 14 \mu \mathrm{m}$. This result consents with observation of Dupuis et al. (2011). Dupuis performed coreflood tests of hydrophobically modified HPAM on sand packs with high permeability in range of $1 \sim 11$ Darcy. 


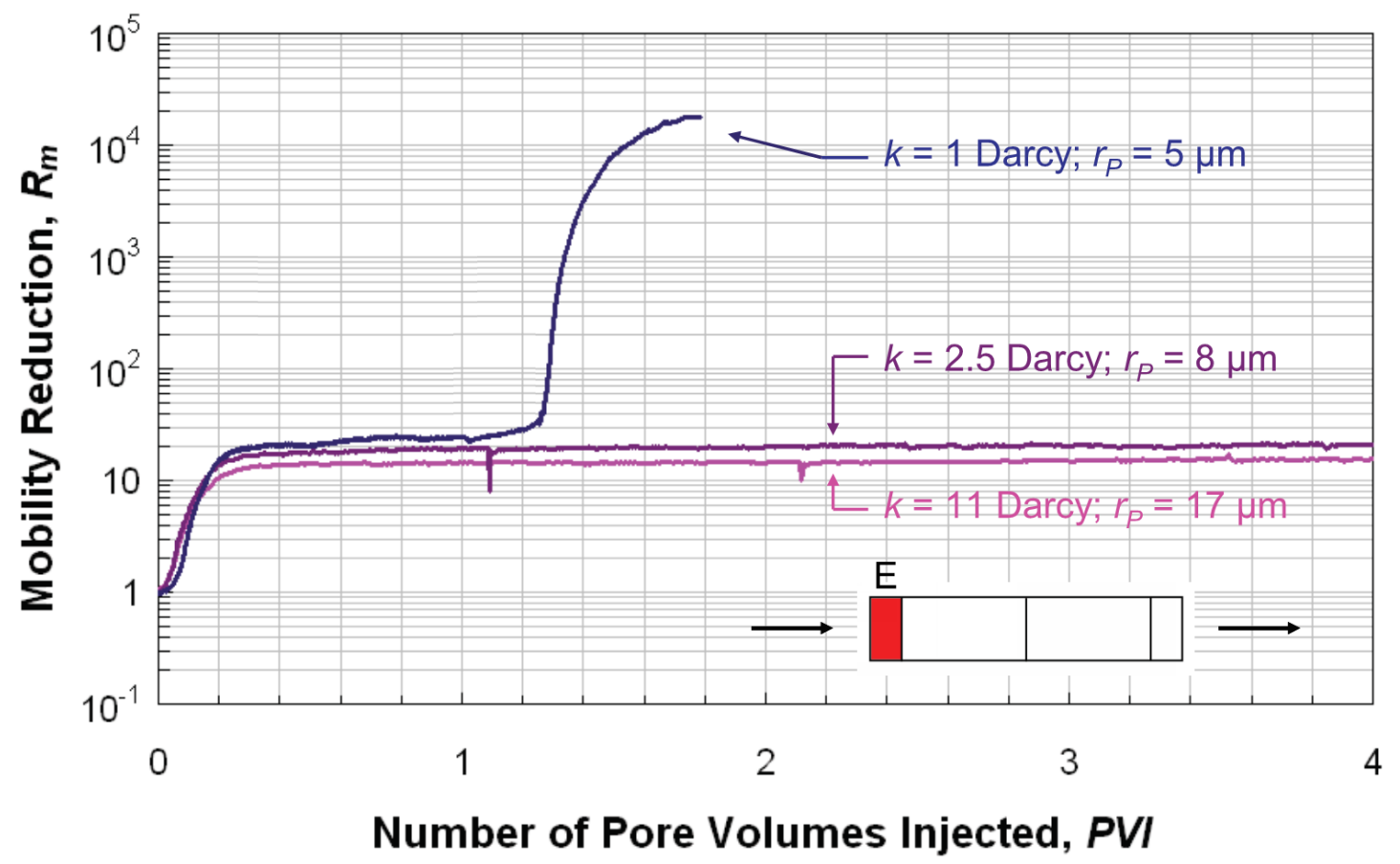

Figure 6.83 Mobility reduction on section $\mathrm{E}$ for injection of the modified HPAM solution in 1,2.5 and 11 Darcy sand packs (Dupuis et al., 2011)

Figure 6.84 shows that the mobility reduction remains stable for 2.5 and 11 Darcy cores whereas plugging behavior appeared by gel formation of polymer. These results indicate that the permeability of a formation is a critical criterion for determining where modified HPAM polymer is able to be applied for polymer flooding.

Cheng et al. (2010) observed that the behavior of remaining oil distribution for polymer flooding is similar to water flooding, which is mainly controlled by capillary force. This is a result of the fact that some oil becomes disconnected and will not mobilize even with a higher gradient. Similarly, a tertiary flood with a favorable viscosity ratio following a secondary flood with a less favorable ratio recovers less oil than the favorable viscosity front flooding in secondary mode. In tertiary polymer flood \#7 after polymer breakthough with relatively small oil bank oil recovery profile is similar to water 
flood oil production for heavy oil. Water flood for heavy oil showed small oil bank and long oil production tail. However shear thinning effect of polymer reduce the reduction of oil cut after oil bank. Figures 6.76, 6.77 and 6.78 show the oil recovery for modified HPAM injection after HPAM polymer flooding. The modified HPAM was injected after core aging with 1 week after finishing HPAM polymer flooding. After $3.5 \mathrm{PV}$ of modified HPAM injection, the remaining oil after HPAM polymer flood started to move and $8 \%$ addition oil was produced after $6 \mathrm{PV}$. The pressure build up for each section looks as similar as the case of experiment \#12 in Figure 6.78 which modified HPAM polymer drive followed water flood. It looks like remaining oil after HPAM polymer flood broke into oil ganglia and settled down during core aging for 1 week. Then, modified HPAM polymer solution generated a small oil bank among oil ganglia or oil cluster after 3.5 PV of injection. The presence of high amount of adsorbed polymer at pore may play a role in overcoming the capillary pressure necessary to mobilized trapped oil.

\subsection{SUMMARY AND CONCLUSIONS}

Several polymer coreflood experiments have been done using both sandpacks and reservoir cores starting at different water cuts to measure the effect of polymer on secondary and tertiary oil recovery.

1. Conventional polymers such as HPAM are well understood and when properly prepared show excellent transport and low retention in laboratory corefloods. Secondary and tertiary polymer floods were performed in various cores using polymer solutions with different rheological properties. 
2. Fractional flow analysis was used to help interpret the experimental oil recovery results. The polymer floods were well explained and matched by fractional flow analysis.

3. The dependence of shear rate on water saturation was included in the fractional flow calculations using an iterative procedure to solve the equations. This procedure improved the match with the experimental data.

4. All of the oil recovery experiments could be matched using a shear correction factor of 4 .

5. Hydrophobically modified HPAM polymer behaved in a radically different way than HPAM. It had 10 times higher polymer retention and 8 times higher resistance factor than HPAM under the same conditions. Oil recovery was $27 \%$ higher and remaining oil saturation was 0.06 . However, almost nothing is known about how associative polymers will behave on a reservoir scale i.e. how to predict their behavior in a reservoir from their behavior in a lab scale coreflood. 


\section{Chapter 7. Summary, Conclusions and Recommendations}

\subsection{Rheological Properties of Polymer Solutions}

Two types of polymer were used in the rheological study presented in Chapter 4: two partially hydrolyzed polyacrylamide (HPAM) polymers with different molecular weights and a co-polymer of acrylamido-methyl-propane sulfonate (AMPS) and acrylamide. The rheological measurements were made to characterize the steady state and oscillatory viscosity of these polymers as a function of polymer concentration, salinity, hardness, and temperature. The results can be summarized as follows:

1. Both $\mathrm{G}^{\prime}$ and $\mathrm{G}^{\prime \prime}$ increase with polymer concentration.

2. Both $\mathrm{G}^{\prime}$ and $\mathrm{G}^{\prime \prime}$ decrease with salinity and hardness.

3. There is a critical polymer concentration and a critical salinity above which the relaxation time increases from its minimum value.

4. The effect of $\mathrm{Ca}^{2+}$ ions on the relaxation time for FP $3630 \mathrm{~S}$ polymer solution is about 800 times higher than the effect of $\mathrm{Na}^{+}$ions. The relaxation time for 3000 ppm FP 3630 S solution in $0.1 \% \mathrm{NaCl}$ and $500 \mathrm{ppm} \mathrm{Ca}^{2+}$ is about the same as it is for $500 \mathrm{ppm}$ FP 3630S solution in $0.5 \% \mathrm{NaCl}$ brine.

5. The relaxation time correlates well with the product of polymer concentration and the hydrodynamic volume of the polymer.

\subsection{Transport of Polymer Solution in Cores}

The behavior of HPAM polymers, scleroglucan biopolymer and Nvinylpyrrolidone (NVP)- polyacrylamide (AM) co-polymer were studied and compared. These results are presented in Chapter 5 and can be summarized as follows: 
1. Scleroglucan can withstand harsh reservoir conditions such as high temperature and high salinity/hardness while retaining its viscosity. But insoluble impurities must first be removed before it can be injected into reservoirs for enhanced oil recovery. Two filtration methods were developed in the lab to remove insoluble impurities. The first method consists of four consecutive high pressure filtrations with decreasing filter pore sizes $(5-10,5$, 3, 1.2 microns) at a high pressure of 50 psi. The second method was to filter scleroglucan solutions at a high temperature $\left(85{ }^{\circ} \mathrm{C}\right)$ through a 1.2 micron filter at 50 psi. After filtration, polymer solutions resulting from both methods passed a standard filtration test routinely used in the laboratory. The second method followed by a filtration step through a Berea core was evidently the most cost-effective method to filter larger volumes. Additional refinement of these methods would be justified. Scleroglucan that passed the standard filtration test with filtration ratios less than 1.2 showed good transport behavior in $150-300 \mathrm{mD}$ Berea cores with no evident plugging. The retention of scleroglucan decreased as the temperature increased.

2. The NVP-AM polymers tested in this study had very good thermal stability at $100{ }^{\circ} \mathrm{C}$ in synthetic seawater and good transport in cores with low retention, but have lower intrinsic viscosity than scleroglucan.

3. Two high molecular weight HPAM polymers (FP 3630S and H-EOR 63020) showed excellent transport behavior in Bentheimer cores with steady state pressure drops across each section of the core. The resistance factor was in excellent agreement with the normalized viscosity and the permeability 
reduction factor was 1 . The shear rate correction factor was very small for both polymers (about 1.1).

4. The critical velocity for the onset of shear thickening was between $4 \mathrm{ft} / \mathrm{day}$ and $10 \mathrm{ft} /$ day. The corresponding range of Deborah number is between 4 and 6.3 .

\subsection{OIL RECOVERY EXPERIMENTS}

Several polymer coreflood experiments were done using both sandpacks and reservoir cores starting at different water cuts to measure the effect of polymer on secondary and tertiary oil recovery. The results of these experiments are presented in Chapter 6 and can be summarized as follows:

1. Conventional HPAM polymers when properly prepared show excellent transport and low retention in laboratory corefloods. Secondary and tertiary polymer floods were performed in various cores using polymer solutions with different rheological properties.

2. Fractional flow analysis was used to help interpret the experimental oil recovery results. The polymer floods were well explained and matched by fractional flow analysis assuming the residual oil saturation and the end-point water relative permeability were identical for the water floods and the polymer floods.

3. The dependence of shear rate on water saturation was included in the fractional flow calculations using an iterative procedure to solve the equations. This procedure is novel and improved the match with the experimental data. 
4. All of the oil recovery experiments could be matched using a shear correction factor of 4. This value is higher than for the experiments without oil, but lower than most values reported in the literature for similar polymers.

5. A hydrophobically modified polyacrylamide polymer behaved in a radically different way than conventional HPAM. It had 10 times higher polymer retention and 8 times higher resistance factor than conventional HPAM polymers under the same conditions. The remaining oil saturation after injecting several pore volumes of polymer solution was nearly zero. However, almost nothing is known about how it will behave on a reservoir scale i.e. how to predict their behavior in a reservoir from the behavior in a laboratory scale coreflood.

\subsection{Conclusions About SHEAR CoRrection FACTOR}

Fundamental limitations of the capillary tube model of a porous medium may account for the need for a shear correction factor in the model of apparent viscosity. Several other factors may also contribute to the non-ideal transport behavior observed in cores. One of these factors is polymer retention. Under ideal conditions, polymer retention is due mainly to adsorption and is very low, but in other cases the polymer solution may have microgels that cause pore plugging and high retention as well as possibly in-depth filtration of the polymer, which may not reach a true steady state in short cores. The microgels might explain the observed permeability reduction in some experiments but not in others using HPAM polymers with similar molecular weight.

The polymers used in this study are of a higher quality than many of the polymers studied between the 1960's and 1990's. The preparation and filtration of the polymers is also extremely important. Long cores such as used in this study are more likely to 
produce steady state results that apply to longer transport distances in oil reservoirs than short cores most often reported in the literature. The use of pressure taps along the core is absolutely essential for proper interpretation of the data. Nevertheless, it is remarkable how significant the differences are between most of the older experiments and the results of this study.

The value of the shear correction factor $\mathrm{C}$ was about 1.1 for HPAM polymer solutions performed in cores without oil and between 2 and 3 for scleroglucan polymer solutions performed in cores without oil. This may be related to the higher shear thinning behavior of scleroglucan compared to HPAM polymers.

The shear correction factors for HPAM polymer solutions are different for experiments with (Chapter 6) and without oil (Chapter 5). For single phase polymer flooding, the shear correction factor $\mathrm{C}$ was very small, whereas the value of the shear correction factor $\mathrm{C}$ for two-phase flow is 4 . A simple explanation is the higher tortuosity and thus higher shear rate for two phases compared to one phase. The shear rate is proportional to the square root of the tortuosity. It would be interesting to measure the tortuosity or estimate it from resistivity measurements to determine if it could be used to quantitatively explain the shear correction factor.

The flow of polymers in porous media is complex and even after many decades of research much remains to be learned, however this work has contributed significantly to the understanding using both conventional HPAM polymers and less conventional polymers. In particular, it is now clear that high-quality HPAM polymers show little if any permeability reduction at least in moderate to high permeability cores corresponding to the range investigate in this work. It is also interesting that the shear correction factor is very small for single phase flow compared to older results in the literature. The 
results of this research show that it is also not very large even for two phase flow. Low values are favorable because they correspond to higher values of apparent viscosity in the porous medium.

\subsection{Conclusions About Residual OIL SATURATION}

One of the original goals of this research was to better understand the role of viscoelasticity on residual oil saturation. As with much research, the complexity of the subject eventually resulted in a different focus in the research that resulted in raising as many questions as it answered. Many of the polymer floods were done using viscous oils. It became apparent that the oil saturation following water injection was not the true residual oil saturation, but rather the remaining oil saturation even when the cores were nearly homogeneous and large pore volumes of water were injected. Both the secondary and tertiary polymer floods in the same cores strongly indicated the dominant mechanism for improved oil recovery was the increase in displacement sweep efficiency. A slightly modified fractional flow analysis was applied to interpret these oil recovery experiments taking into account the change in shear rate and its effect on the polymer viscosity with changing water saturation.

This fractional flow analysis demonstrated that the much smaller remaining oil saturation for polymer floods compared to water floods presented in this study could be explained quantitatively without assuming any difference in the residual oil saturation between water and polymer floods. However, under other flow conditions the residual oil saturation may be lower for polymer floods compared to water floods. In particular, data in the literature suggests that the residual oil saturation may be lower when a viscoelastic polymer is used at high Deborah number. The oil recovery experiments performed in this work were done at low Deborah number. Even for high molecular weight HPAM 
polymers such as FP $3630 \mathrm{~S}$, high pressure gradients of at least $10 \mathrm{psi} / \mathrm{ft}$ are needed to produce high Deborah numbers, but the pressure gradient in reservoirs with a typical well spacing is on the order of only $1 \mathrm{psi} / \mathrm{ft}$. High pressure gradients eventually lead to lower residual oil saturation due to high capillary numbers even with no reduction of interfacial tension, so it is difficult to clearly distinguish these two different phenomena using data from most previous experiments.

The excellent agreement between the experimental oil recovery and the fractional flow calculations is remarkable given the simplifications of fractional flow theory. In particular, the porous medium is assumed to be homogeneous. Thus, the displacement sweep efficiency calculated from the fractional flow equations does not and cannot account for heterogeneity. Furthermore, the cores used in this study were nearly homogeneous based on tracer data and other observations. The oil recovery experiments performed in this study were all done in vertical cores with injection from the bottom so there would be no effect of gravity segregation. The gravity term in the fractional flow equations was neglected since it is very small compared to the viscous pressure gradient in all cases. Capillary pressure effects were also neglected but are very small in these experiments compared to the viscous pressure gradient and would certainly not account for the large differences between the water floods and polymer floods. All of the experiments were done at high interfacial tension and at low flow rates so that the capillary number would be less than its critical value for desaturation based on literature data for homogenous sandstones and sandpacks.

Fractional flow theory does account for the effects of viscosity and relative permeability on displacement sweep efficiency. Adding polymer to the water increases the aqueous viscosity, which is the whole point of polymer flooding. The permeability 
reduction factor was assumed to be one (no permeability reduction due to the polymer). A shear correction factor of 4 resulted in an apparent viscosity consistent with the pressure drop in all of the experiments. In this study, the end-point water relative permeability was measured and the same value was used in the fractional flow equations for both the water floods and polymer floods. However, the Corey exponents for both the water flood and the polymer flood were adjusted to fit the oil production data.

The displacement sweep efficiency calculated from fractional flow theory takes into account the effect of relative permeability on a microscopic level. In other words, in general it takes more than one pore volume to displace oil locally even in a homogeneous porous medium. As evident from the shape of the fractional flow curve, this is true even when the end-point mobility ratio is 1 . However, it is much more pronounced for higher mobility ratios corresponding to the water floods of the viscous oils compared to the polymer floods of the same oils in the same cores. Fractional flow theory does not account for fingering. However, when the water flood mobility ratios are high, the Corey exponents are low reflecting the fact that the flow is at least partially segregated due to the fingers.

The Corey exponents for the water phase were about 3 for both the water floods and the polymer floods even when the mobility ratio was high. On the other hand, the Corey exponents for the oil phase varied from 1 to 2.4 depending on the mobility ratio. This implies that some of the displacements were unstable. The highest water flood mobility ratio was 136 , so fingering almost certainly did occur under those conditions. This does not invalid the use of fractional flow theory or the interpretation of the experiments results. 


\subsection{RECOMMENDATIONS FOR FUTURE RESEARCH}

Based on the results of this study, additional research on HPAM polymers, biopolymers such as scleroglucan and modified HPAM polymers would be highly desirable. The results shown for the modified HPAM in Chapter 6 are intriguing but not well understood and there is no basis for scaling up those results. Much more research will need to be done with modified polymers to better understand their behavior and determine under what reservoir conditions they might be appropriately used. On the other

hand, scleroglucan biopolymer has been studied for a long time and is much closer to being a viable candidate for field use based on this and previous work. However, more experiments are also needed to better understand its limitations, for example, its transport in low permeability rocks. More oil recovery experiments to better understand the effect of HPAM on residual oil saturation could be designed based on the measured relaxation times and the correlation of those data given in Chapter 4. It would be highly desirable to do these corefloods using $\mathrm{CT}$ imaging to determine the oil saturation distribution in the cores. 


\section{Appendix A}

\section{Standard Protocol for Polymer Handling}

\section{Polymer Stock Solution Preparation}

This standard protocol was used to mix $500 \mathrm{~g}$ of polymer stock solution containing polymer in Soften Mangala brine (SSTMB ). Subsequently, this stock solution will be filtered and diluted to desired concentrations to be used in the latter stages of testing.

1. Prepare appropriate make up water, and filter through $0.45 \mu$ Millipore nitrocellulose membrane filter.

2. Weigh exactly $497.5 \mathrm{~g}$ of brine into $700 \mathrm{~mL}$ shallow polycarbonate bottle.

3. Place the mixing bottle on a magnetic stir plate and equip with $5.5 \mathrm{~cm}$ long magnetic stirrer bar and create a water vortex while maintaining an Argon blanket (stir speed 300-440 rpm).

4. Weigh exactly $2.5 \mathrm{~g}$ of powder polymer FP3630S (lot\#3477).

5. Sprinkle the polymer gently on the side of the water vortex, ensuring that no agglomerates are formed. If any agglomerates do form, discard the contents of the beaker and start again (the polymer should be added in a period of 1 minute; if it is added too fast then lumps will be formed and if it is added too slow the solution will get too viscous for the last amount of polymer to go into solution).

6. Stir at full vortex for 5 minutes until the polymer is well-dispersed. The stirrer speed to maintain full vortex depends on the fluid viscosity and the ingress of air into the solution. The latter should be prevented, while the increased fluid viscosity can result in the loss of contact between the magnetic stirrer and the stirrer bar at high stirrer 
speeds. This implies that the stirrer speed will probably be lower than the initial $300-$ $400 \mathrm{rpm}$.

7. Reduce the stirrer speed to $250 \mathrm{rpm}(\sim$ the surface of the liquid is just moving). Stir at this speed for 48 hours at room temperature with bottle closed while maintaining an Argon blanket.

\section{Polymer Filtration Procedure}

For the filtration of $5000 \mathrm{ppm}$ polymer stock solution, a stainless steel filter press apparatus was used with 5.0 $\mu$ Millipore nitrocellulose membrane filter with a diameter of $90 \mathrm{~mm}$

1. Pour $220 \mathrm{ml}$ of the prepared polymer solution into the upside down top part of the filtration set-up; place the nitrocellulose membrane filter on top and insert the bottom part and close (Refer to the figure 50).

2. Set the nitrogen pressure at 15 psi $(\sim 1$ bar $)$ and measure the filtrate volume as a function of time. Stop the filtration process when a filtrate volume of $200 \mathrm{ml}$ has been collected.

3. Calculate the filtration ratio with:

$\mathrm{FR}=(\mathrm{t} 200 \mathrm{ml}-\mathrm{t} 180 \mathrm{ml}) /(\mathrm{t} 80 \mathrm{ml}-\mathrm{t} 60 \mathrm{ml})$

4. Disassemble the apparatus and observe the filter for any agglomerates on the filter.

\section{Diluting Polymer Stock Solution}

Above prepared polymer stock solution will be further diluted in appropriate brines to prepare polymer solutions with target concentration and salinity. 
Polymer solution 1: 250g of 2500 ppm FP3630S in SSTMB

1. Weigh exactly $125 \mathrm{~g}$ of above prepared stock solution into polycarbonate mixing bottle.

2. Equip with a $5.5 \mathrm{~cm}$ long magnetic stirrer bar and place on a stir plate.

3. Stir at an rpm of $\sim 200-300$

4. $\quad$ Add $125 \mathrm{~g}$ of SSTMB.

5. Stir for additional two hours at $\sim 200-300 \mathrm{rpm}$ and under Argon blanket before filtration.

6. Separate $10 \mathrm{~mL}$ of the un-filtered polymer solution and measure the viscosity.

7. Filter the polymer solution according to the above given procedure.

8. Take $10 \mathrm{~mL}$ of the filtered polymer solution and measure the viscosity; compare the value with that of the unfiltered polymer solution to determine if there is any viscosity loss.

9. Repeat the procedure to verify the reproducibility of the result

Polymer solution 1: 250g of 2000 ppm FP3630S in STMB

1. Weigh exactly $100 \mathrm{~g}$ of above prepared stock solution into polycarbonate mixing bottle.

2. Equip with a $5.5 \mathrm{~cm}$ long magnetic stirrer bar and place on a stir plate.

3. Stir at an rpm of $\sim 200-300$ 
4. Add $150 \mathrm{~g}$ of appropriate brine (with the divalent cations). The final mixture is to have the composition of the Synthetic Thumbli Mangala Brine (STMB) .

5. Stir for additional two hours at $\sim 200-300 \mathrm{rpm}$ and under Argon blanket before filtration.

6. Separate $10 \mathrm{~mL}$ of the un-filtered polymer solution and measure the viscosity.

7. Filter the polymer solution according to the above given procedure.

8. Take $10 \mathrm{~mL}$ of the filtered polymer solution and measure the viscosity; compare the value with that of the unfiltered polymer solution to determine if there is any viscosity loss.

9. Repeat the procedure to verify the reproducibility of the result

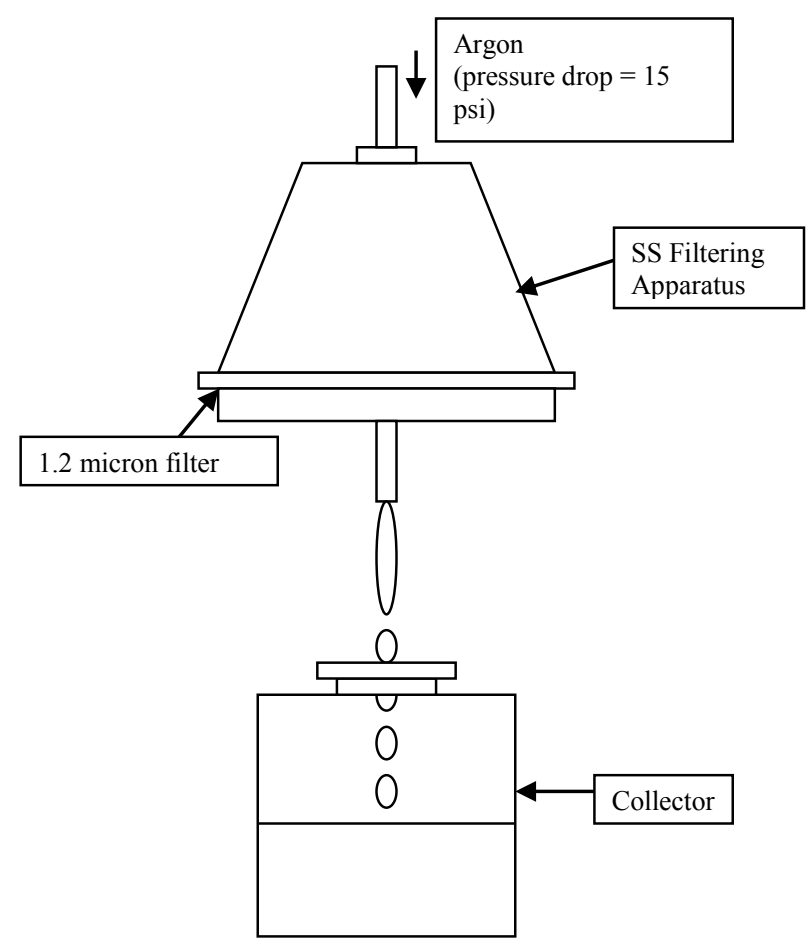

Figure A.1 Filtration apparatus 


\section{Short-Term Thermal Stability Protocol}

Equipment:

Mass balances: All experiments were conducted on a mass basis. Used an Ohaus Discovery mass balance with a precision of $0.0001 \mathrm{~g}$ for quantities smaller than 10 grams, and a Denver Instruments mass balance with a precision of $0.01 \mathrm{~g}$ was used for larger quantities.

Water Deionizer: Deionized water (DI) was used for all experiments. A NanopureTM filter system was used to deionize the water. Distilled water was fed into the system and deionized water was obtained at the outlet.

Stir plates: Corning PC-420D magnetic stirring hot plates along with a Teflon coated magnetic stir bars were used to mix the brine and polymer solutions.

Dissolved Oxygen (DO): Chemets ${ }^{\circledR}$ Self-Filling Ampoules (R-7510) ampules were used to measure dissolved oxygen levels.

Experimental Procedure

1. Followed the standard protocol of "Polymer Stock Solution Preparation Procedure" to prepare the entire above mentioned polymer solutions.

2. Prepared polymer solution was placed in a $500 \mathrm{ml}$ round bottom flask equipped with a stir bar (Fisherbrand Egg-shaped magnetic stirring bar, Cat \# 1451354) and covered using a rubber stopper. Two nylon tubings (Newage Industries Nvlotube $12.058 * 0.12565831 / 12)$ were attached to the rubber stopper for the Argon (Ar) inlet and Ar outlet. The setup (Figure 53) was placed on a magnetic stir plate at $200 \mathrm{rpm}$ and bubbled with Argon for $1 \mathrm{~h}$. 


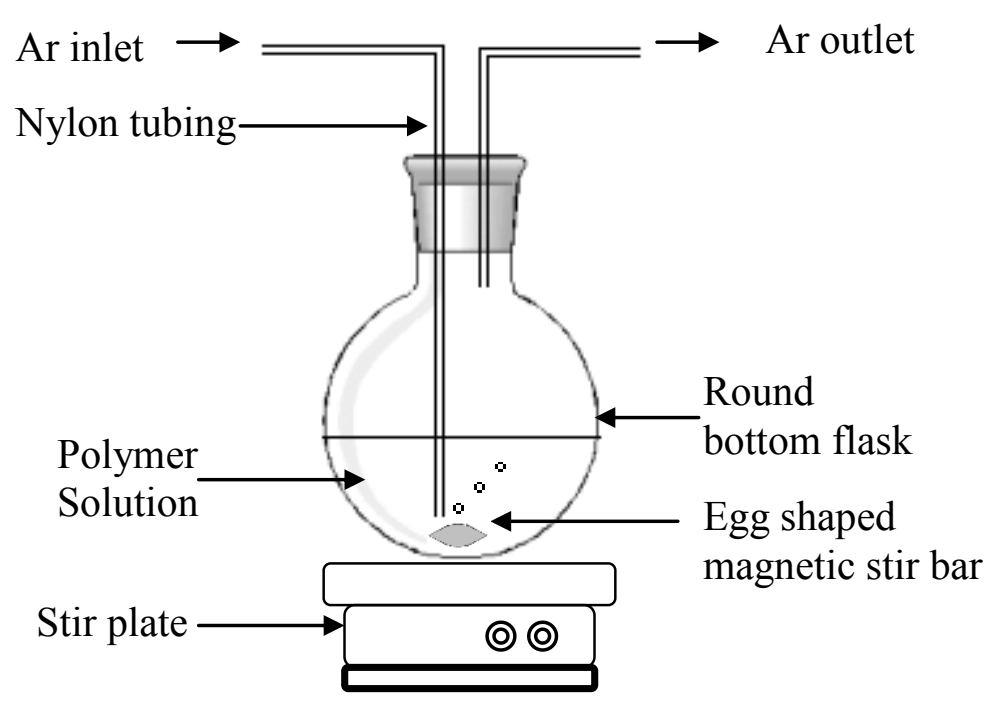

Figure A.2 The Setup Used for Polymer Degassing.

3. Used a similar setup to degas the brine solution. The brine solution degassed for $1 \mathrm{~h}$ to get rid of any trace amounts of Oxygen. At this stage three additives (oxygen scavenger, corrosion inhibitor and biocide) were introduced to the brine solution and degassed for another $1 \mathrm{~h}$. The solution with additives was transferred to the polymer using Ar pressure through a Nylon tube. This final solution mixed for another $1 \mathrm{~h}$.

4. While the polymer was mixing, custom-made $20 \mathrm{~mL}$ borosilicate ampules were prepared with the nylon fitting and brown o-ring and set in an Ar chamber. An Ar chamber was prepared using a $1000 \mathrm{ml}$ beaker equipped with an Ar line and sealed with Parafilm.

5. Using the Ar pressure, polymer solution was pushed into the borosilicate ampule through the Ar-outlet which is now dipped in the polymer solution (Figure 54). This was difficult, as it must be done slowly as not to shear the polymer. Once the 
ampule is filled with polymer solution to 1 inch below neck, Ar outlet must pull out from the polymer solution.

6. The ampule was transferred to the manifold immediately and quickly screwed into the manifold. The valve was opened for a few seconds to evacuate head space then closed.

7. Step 4 and 5 was repeated for the next six ampules. When the manifold was full, all the valves were opened while still under vacuum. A good vacuum was achieved when the pressure gauge read $30 \mathrm{in} \mathrm{Hg.} \mathrm{The} \mathrm{manifold} \mathrm{was} \mathrm{then} \mathrm{switched} \mathrm{to} \mathrm{argon} \mathrm{until}$ the pressure gauge read 10 psi. These vacuum-Argon cycles were repeated for two more times (for a total of 3 cycles). With the manifold still on argon, all valves were closed and the manifold was switched to vacuum.

8. Using a methane-oxygen torch, an area $1 / 2$ inch below the plastic fitting was gently heated evenly around the ampule. When the area was completely heated around the ampule, gently pulled down until the neck was completely broken away. Care must be taken to not heat the polymer solution. This is repeated for the rest of the ampules on the 
manifold.

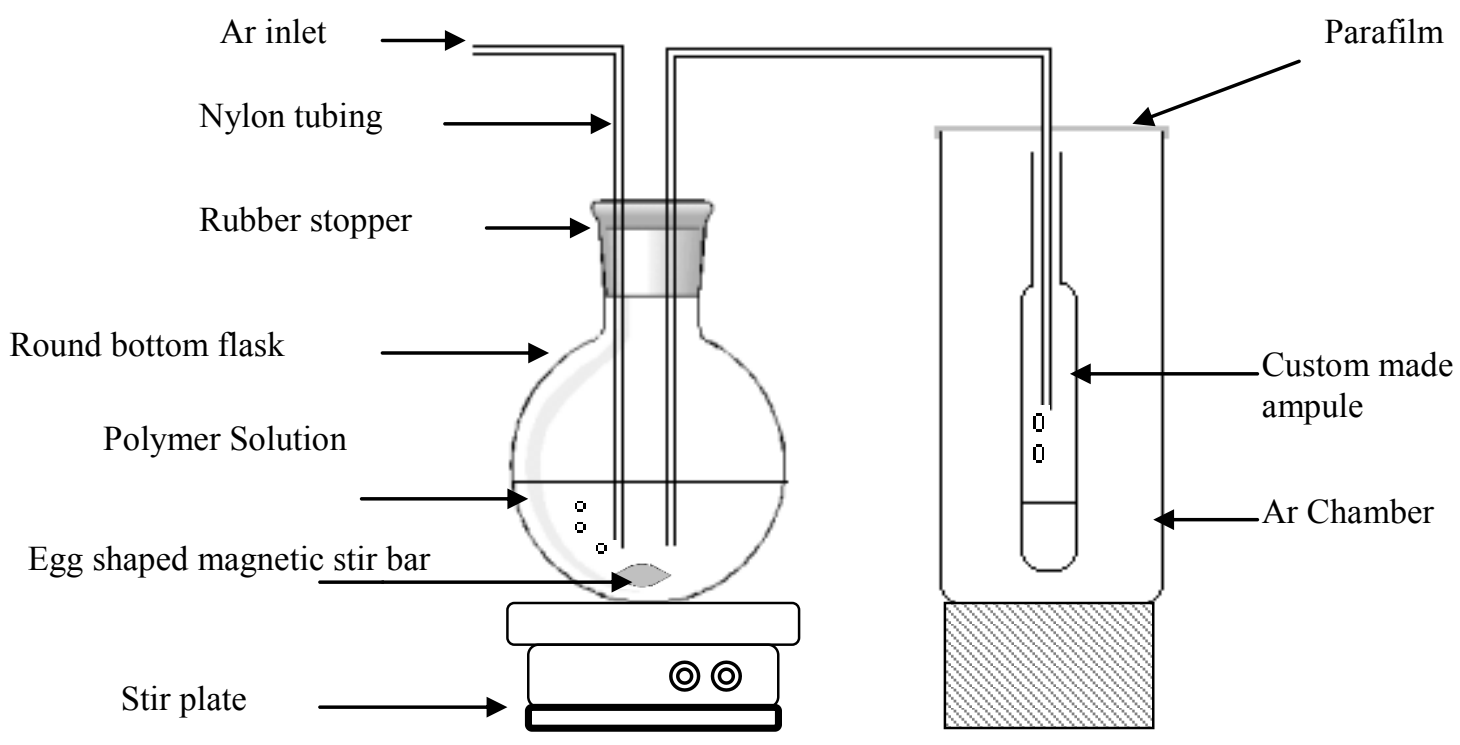

Figure A.3 Polymer Transfer Setup

9. The remaining polymer solution in the round bottom flask was used to measure viscosity and DO. These measurements were recorded as initial values (Day 0).

10. Ampules were then labeled and placed in the oven at $62 \mathrm{C}$ to age and be removed after $1,2,7$, and 15 days.

11. Ampules were removed after an appropriate time from the oven and allowed to cool to room temperature.

12. They were cracked open and measured for the solution viscosity and DO. It was extremely necessary to record DO measurements as soon as possible before oxygen was allowed to affect the polymer sample. The ampoule was sealed with Parafilm under an Ar blanket and moved to record the viscosity of the solution. (Chang, 2010) 


\section{References}

Abidin, a. Z., Puspasari, T., Nugroho, W. a., 2012. Polymers for Enhanced Oil Recovery Technology. Procedia Chem. 4, 11-16. doi:10.1016/j.proche.2012.06.002

Abrams, A., 1975. The influence of fluid viscosity, interfacial tension, and flow velocity on residual oil saturation left by waterflood. SPE J.

Afsharpoor, A., Balhoff, M.T., 2013. Static and Dynamic CFD Modeling of Viscoelastic Polymer: Trapped Oil Displacement and Deformation at the Pore-Level. SPE Annu. Tech. Conf. .... doi:10.2118/166114-MS

Afsharpoor, A., Ma, K., Duboin, A., Mateen, K., Jouenne, S., Cordelier, P., 2014. MicroScale Experiment and CFD Modeling of Viscoelastic Polymer; Trapped Oil Displacement and Deformation at the Dead-End. SPE Improv. Oil Recover. Symp. doi:10.2118/169037-MS

Baijal, S.K., 1981. Polymer Molecules Interact With Porous Matrix. Oil Gas J. 106-107.

Balhoff, M.T., Thompson, K.E., 2006. A macroscopic model for shear-thinning flow in packed beds based on network modeling. Chem. Eng. Sci. 61, 698-719. doi:10.1016/j.ces.2005.04.030

Bondor, P.L., Hirasaki, G.J., Tham, M.J., 1972. Mathematical Simulation of Polymer Flooding in Complex Reservoirs. Soc. Pet. Eng. J. 12, 369-382. doi:10.2118/3524PA

Bowden, F.P., Tabor, D., 1966. Friction, lubrication and wear: a survey of work during the last decade. Birt. J. Appl. Phys. 17, 1521-1544.

Cannella, W.J., Huh, C., Seright, R.S., 1988. Prediction of Xanthan Rheology in Porous Media. Proc. SPE Annu. Tech. Conf. Exhib. doi:10.2523/18089-MS

Carman, P.C., 1937. Fluid flow through granular beds. Trans. Inst. Chem. Eng. 15, 150166. doi:10.1016/S0263-8762(97)80003-2

Chang, G.S., 2010. Large Amplitude Oscillatory Shear Flow Behavior of Viscoelastic Polymer Solutions. Pusan National University.

Chang, H.L., ZHANG, Z.Q., WANG, Q.M., XU, Z.S., GUO, Z.D., SUN, H.Q., CAO, X.L., QIAO, Q., 2006. Advances in polymer flooding and 
alkaline/surfactant/polymer processes as developed and applied in the People's Republic of China. J. Pet. Technol. 58, 84-89.

Chatzis, I., Morrow, N., 1984. Correlation of capillary number relationships for sandstone. Soc. Pet. Eng. J. 555-562.

Chauveteau, G., Kohler, N., 1974. Polymer flooding: The essential elements for laboratory evaluation. SPE Improv. Oil Recover. Symp.

Cheng, J., Wei, J., Song, K., Han, P., 2010. Study on Remaining Oil Distribution after Polymer Injection. SPE Annu. Tech. Conf. Exhib.

Christopher, R., Middleman, S., 1965. Power-law flow through a packed tube. Ind. Eng. Chem. Fundam. 3-7.

Davison, P., Mentzer, E., 1982. Polymer flooding in North Sea reservoirs. Soc. Pet. Eng. J. 353-362.

Delamaide, E., Corlay, P., Demin, W., 1994. Daqing oil field: The success of two pilots initiates first extension of polymer injection in a giant oil field. SPE/DOE Improv. Oil Recover. Symp.

Delamaide, E., Zaitoun, A., Renard, G., Tabary, R., 2013. Pelican Lake Field: First Successful Application of Polymer Flooding in a Heavy Oil Reservoir, in: SPE Enhanced Oil Recovery Conference.

Delaplace, P., Delamaide, E., Roggero, F., Renard, G., 2013. History Matching of a Successful Polymer Flood Pilot in the Pelican Lake Heavy Oil Field (Canada). SPE Annu. Tech. Conf. Exhib. SPE 166256.

Delshad, M., Kim, D., Magbagbeola, O., Huh, C., Pope, G., Tarahhom, F., 2008. Mechanistic Interpretation and Utilization of Viscoelastic Behavior of Polymer Solutions for Improved Polymer-Flood Efficiency. Proc. SPE/DOE Symp. Improv. Oil Recover. doi:10.2118/113620-MS

Denys, K.F., 2003. Flow of polymer solutions through porous media. Dissertation. Delft Univerity. doi:10.1021/ie00106a028

Doe, P.H., Co, P.P., Moradi-araghi, A., Shaw, J.E., Stahl, G.A., 1987. Development and Evaluation of EOR Polymers Suitable for Hostile ofVinylpyrrolidone and Aerylamide. SPE Reserv. Eng. 461-467. 
Doi, M., Edwards, S., 1986. The Theory of Polymer Dynamics. Oxford University Press, New York.

Dominguez, J.G., Willhite, G.P., 1977. Retention and Flow Characteristics of Polymer Solutions in Porous Media. Soc. Pet. Eng. J. 17, 111-121. doi:10.2118/5835-PA

Dupuis, G., Rousseau, D., 2010. How to Get the Best Out of Hydrophobically Associative Polymers for IOR? New Experimental Insights. SPE Improv. Oil Recover. Symp.

Dupuis, G., Rousseau, D., Tabary, R., Grassi, B., 2011. Injectivity of Hydrophobically Modified Water Soluble Polymers for IOR: Controlled Resistance Factors vs . Flow-Induced Gelation. SPE Int. Symp. Oilf. Chem. SPE 140779, 1-13.

Fernandez, I., 2005. Evaluation of Cationic Water-Soluble Polymers with Improved Thermal Stability. SPE Int. Symp. Oilf. Chem.

Flory, P.J., 1953. Principles of Polymer chemistry. Cornell University Press.

Gaillard, N., Giovannetti, B., Favero, C., Caritey, J.-P., Dupuis, G., Zaitoun, a., 2014. New Water Soluble Anionic NVP Acrylamide Terpolymers for Use in Harsh EOR Conditions. SPE Improv. Oil Recover. Symp. doi:10.2118/169108-MS

Gaillard, N., Sanders, D., Favero, C., 2010. Improved oil recovery using thermally and chemically protected compositions based on co-and ter-polymers containing acrylamide. SPE Improv. Oil Recover. Symp.

Gogarty, W.B., 1967. Mobility Control With Polymer Solutions. Soc. Pet. Eng. J. 7, 161173. doi:10.2118/1566-B

Graessley, W., 2004. Polymeric Liquids and Networks: Structure and Properties. Taylor and Francis Group, INC, New York.

Gramain, P., Myard, P., 1981. Adsorption studies of polyacrylamides in porous media. J. Colloid Interface Sci. 84, 114-126.

Green, D.W., Willhite, G.P., 1998. Enhanced oil recovery. Richardson, Tex. : Henry L. Doherty Memorial Fund of AIME, Society of Petroleum Engineers.

Gupta, R., Sridhar, T., 1985. Viscoelastic effects in non-Newtonian flows through porous media. Rheol. acta 151, 148-151. 
Heemskerk, J., Rosmalen, R., 1984. Quantification of viscoelastic effects of polyacrylamide solutions. SPE Enhanc. Oil Recover. Conf.

Hirasaki, G.J.G., Pope, G. a. G., 1974. Analysis of Factors Influencing Mobility and Adsorption in the Flow of Polymer Solution Through Porous Media. Soc. Pet. Eng. J. 14, 337-346. doi:10.2118/4026-PA

Homola, A.M., Israelachvili, J.N., Gee, M.L., McGuiggan, P.M., 1989. Measurements ofand Relation Between the Adhesion and Friction of Two Surfaces Separated by Molecularly Thin Liquid Films. J. Tribol. 111, 675-682.

Huh, C., Lange, E.A., Cannella, W.J., 1990. Polymer Retention in Porous Media. SPE/DOE Improv. Oil Recover. Symp.

Huh, C., Pope, G., 2008. Residual Oil Saturation From Polymer Floods: Laboratory Measurements and Theoretical Interpretation. Proc. SPE/DOE Symp. Improv. Oil Recover. 1-21. doi:10.2118/113417-MS

Jennings, R., Rogers, J., West, T., 1971. Factors influencing mobility control by polymer solutions. J. Pet. Technol.

Jones, D., Walters, K., Williams, P., 1987. On the extensional viscosity of mobile polymer solutions. Rheol. acta 30, 20-30.

Kalpakci, B., Jeans, Y., Magri, N.F., 1990. Thermal stability of scleroglucan at realistic reservoir conditions, in: SPE/DOE Seventh Symposium on Enhanced Oil Recovery. Tulsa, Oklahoma.

Kamaraj, K., Zhang, G., Liu, Y., Seright, R.S., 2011. Effect of Residual Oil Saturation on Recovery Efficiency during Polymer Flooding of Viscous Oils. Proc. OTC Arct. Technol. Conf. doi:10.4043/22040-MS

Kim, D., Lee, S., Ahn, C., Huh, C., Pope, G., 2010. Development of a viscoelastic property database for EOR polymers. SPE Improv. Oil Recover. Symp. 1-16.

Kohler, N., Chauveteau, G., 1981. Xanthan polysaccharide plugging behavior in porous media-preferential use of fermentation broth. J. Pet. Technol. 349-358.

Kulawardana, E.U., Koh, H., Kim, D.H., Liyanage, P.J., Upamali, K., Huh, C., Weerasooriya, U., Pope, G.A., 2012. Rheology and Transport of Improved EOR Polymers under Harsh Reservoir Conditions. SPE Improv. Oil Recover. Symp. 114. doi: $10.2118 / 154294-\mathrm{MS}$ 
Lakatos, I., Lakatos-Szabo, J., 2001. Effect of IOR/EOR Chemicals on Interfacial Rheological Properties of Crude Oil/Water Systems. Proc. SPE Int. Symp. Oilf. Chem. doi:10.2523/65391-MS

Lake, L., 1989. Enhanced oil recovery. Prentice Hall, Inc, Englewood Cliffs, New Jersey.

Lake, L.W., Johns, R.T., Rossen, W.R., Pope, G.A., 2014. Fundamentals of Enhanced Oil Recovery. Society of Petroleum Engineers, Richardson, TX.

Landoll, L., 1982. Nonionic polymer surfactants. J. Polym. Sci. Polym. Chem. ... 20, 443-455.

Lee, S., Kim, D., Huh, C., Pope, G., 2009. Development of a comprehensive rheological property database for EOR polymers, in: SPE Annual Technical Conference and Exhibition.

Leonhardt, B., Ernst, B., Reimann, S., Steigerwald, A., 2014. Field Testing The Polysaccharide Schizophyllan: Results of The First Year. SPE Improv. Oil Recover. Symp.

Levitt, D., Bondino, I., Santanach-Carreras, E., Jounne, S., Bourrel, M., 2013. Polymer Flooding of Heavy Oil Under Adverse Mobility Conditions, in: SPE Enhanced Oil Recovery Conference. Kuala Lumpur, Malaysia, pp. 2-4.

Levitt, D., Bourrel, M., Bondino, I., 2011. The interpretation of polymer coreflood results for heavy oil. SPE Heavy Oil Conf. Exhib.

Levitt, D., Pope, G., 2008. Selection and screening of polymers for enhanced-oil recovery. SPE Symp. Improv. Oil Recover.

Lipatov, Y.S., Sergrrva, L.M., Kondor, R., Slutzkin, D., 1974. Adsorption of Polymers. Wiley, New York.

Littmann, W., 1988. Polymer flooding. Elsevier Inc.

Lu, Y., 1994. A Study of Residual Oil Saturation in Heterogeneous Sandstone. Thesis, The Univeristy of Texas at Austin.

Magbagbeola, O.A., 2008. Quantification of the Viscoelastic Behavior of High Molecular Weight Polymers used for Chemical Enhanced Oil Recovery. The University of Texas at Austin. 
McClelland, G.M., 1989. Friction at Weakly Interacting Interfaces. Adhes. Frict. 17, 115.

Metzner, A., White, J., Denn, M., 1966. Constitutive equations for viscoelastic fluids for short deformation periods and for rapidly changing flows: significance of the Deborah number. AIChE J. 12, 863-866.

Moradi-Araghi, A., Cleveland, D.H., Westerman, I.J., 1995. Development and Evaluation of EOR Polymers Suitable for Hostile Environments: II-Copolymers of Acrylamide and Sodium AMPS. SPE Int. Symp. Oilfiled Chem.

Mungan, N., 1969. Rheology and Adsorption of Aqueous Polymer Solutions. J. Can. Pet. Technol. 8, 45-50. doi:10.2118/69-02-01

Mungan, N., Smith, F.W., Thompson, J.L., 1966. Some Aspects of Polymer Floods. J. Pet. Technol. 18, 1143-1150. doi:10.2118/1628-PA

Noik, C., Audibert, A., Delaplace, P., 1994. Injectivity of Sulfonated Polymers Under North Sea Field Conditions. SPE/DOE Improv. Oil Recover. Symp.

Prasad, D., Pandey, A., Kumar, M.S., Koduru, N., 2014. Pilot to Full-field Polymer Application in One of the Largest Onshore Field in India, in: SPE Improved Oil Recovery Symposium. Tulsa, Oklahoma, p. SPE-169146-MS.

Reichenbach-Klinke, R., Langlotz, B., 2011. Hydrophobic associative copolymer with favorable properties for the application in polymer flooding. SPE Pap. 141107 ....

Rivenq, R.C., Donche, A., Noik, C., 1992. Improved scleroglucan for polymer flooding under harsh reservoir conditions. SPE Reserv. Eng. 7, 15-20.

Sandiford, B., 1964. Laboratory and field studies of water floods using polymer solutions to increase oil recoveries. J. Pet. Technol.

Sau, A.C., Landoll, L.M., 1989. Synthesis and solution properties of hydrophobically modified (hydroxyethyl) cellulose. Adv. Chem. 343-363.

Schneider, F., Owens, W., 1982. Steady-state measurements of relative permeability for polymer/oil systems. Old SPE J. 79-86.

Seright, R.S., 2010. Potential for polymer flooding reservoirs with viscous oils. SPE Reserv. Eval. Eng. 24-28. 
Seright, R.S., ZHANG, G., AKANNI, O.O., WANG, D., 2012. A Comparison of Polymer Flooding With In-Depth Profile Modification. J. Can. Pet. Technol. 51, 393-402.

Sheng, J., 2010. Modern Chemical Enhanced Oil Recovery: Theory and Practice. Elsevier.

Skauge, A., Ormehaug, P., Gurhoh, T., 2012. 2-D Visualisation of Unstable Waterflood and Polymer Flood for Displacement of Heavy Oil. SPE Improv. Oil Recover. ....

Slaughter, W., 2010. Stability of Polymers used for Enhanced Oil Recovery. The University of Texas at Austin.

Smith, F.W., 1970. The Behavior of Partially Hydrolyzed Polyacrylamide Solutions in Porous Media. J. Pet. Technol. 22, 148-156. doi:10.2118/2422-PA

Sorbie, K.S., 1991. Polymer-Improved Oil Recovery, Glasgow and London: Blackie and Son Ltd. CRC Press, Inc.

Stavland, A., Jonsbroten, H., Lohne, A., Moen, A., 2010. Polymer Flooding-Flow Properties in Porous Media Versus Rheological Parameters. SPE Eur. Annu. Conf. Exhib.

Stegemeier, G., 1974. Relationship of trapped oil saturation to petrophysical properties of porous media. SPE Improv. Oil Recover. Symp.

Szabo, M., 1975. Laboratory investigations of factors influencing polymer flood performance. Soc. Pet. Eng. J.

Szabo, M.T., 1979. An Evaluation of Water-Soluble Polymers For Secondary Oil Recovery - Parts 1 and 2. J. Pet. Technol. 31, 553-570. doi:10.2118/6601-PA

Tanford, C., 1961. Physical Chmistry of Macromolecules. John Wiley and Sons, New York.

Taylor, K.C., Nasr-El-Din, H. a., 1998. Water-soluble hydrophobically associating polymers for improved oil recovery: A literature review. J. Pet. Sci. Eng. 19, 265280. doi:10.1016/S0920-4105(97)00048-X

Thomas, A., Gaillard, N., Favero, C., 2012. Some Key Features to Consider When Studying Acrylamide-Based Polymers for Chemical Enhanced Oil Recovery. OGST 67. doi:10.2516/ogst2012065 
Thurston, G.B., Pope, G.A., 1981. Shear rate dependence of the viscoelasticity of polymer solutions. J. Nonnewton. Fluid Mech. 9, 69-78. doi:10.1016/03770257(87)87007-6

Tomlinson, G.A., 1929. A Molecular Theory of Friction. Philos. Mag. J. Sci. 7, 905-939.

Vermolen, E., Haasterecht, Masalmeh, S.K., Faber, M.J., Boersma, D.M., Gruenenfelder, M., 2011. Pushing the envelope for polymer flooding towards high-temperature and high-salinity reservoirs with polyacrylamide based ter-polymers. SPE Middle East Oil Gas Show Conf.

Volpert, E., Selb, J., Candau, F., Green, N., 1998. Adsorption of hydrophobically associating polyacrylamides on clay. Langmuir 7463, 1870-1879.

Wang, D., Cheng, J., Yang, Q., Gong, W., 2000. Viscous-elastic polymer can increase microscale displacement efficiency in cores. SPE Annu. Tech. Conf. Exhib.

Wang, D., Jiecheng, C., Qun, L., 2001. First Ultra-Low Interfacial Tension Foam Flood Field Test Is Successful. SPE Annu. Tech. ....

Wang, D., Xia, H., Liu, Z., Yang, Q., 2001. Study of the Mechanism of Polymer Solution With Visco-Elastic Behavior Increasing Microscopic Oil Displacement Efficiency and the Forming of Steady "Oil Thread" Flow Channels. Proc. SPE Asia Pacific Oil Gas Conf. Exhib. 1-9. doi:10.2523/68723-MS

Wang, M., 1995. Laboratory investigation of factors affecting residual oil saturation by polymerflooding. The University of Texas at Austin.

Wreath, D., 1989. A study of polymer flooding and residual oil saturation. The University of Texas at Austin.

Wreath, D., Pope, G.A., Sepehrnoori, K., 1990. Dependence of polymer apparent viscosity on the permeable media and flow conditions. Situ 14, 263-284.

Wu, W., Wang, D., Jiang, H., 2007. Effect of the visco-elasticity of displacing fluids on the relationship of capillary number and displacement efficiency in weak oil-wet cores. Asia Pacific Oil Gas Conf. Exhib. 1-5.

Xia, H., Wang, D., Wu, J., Kong, F., 2004. Elasticity of HPAM Solutions Increases Displacement Efficiency under Mixed Wettability Conditions. Proc. SPE Asia Pacific Oil Gas Conf. Exhib. 1-8. doi:10.2523/88456-MS 
Yanaki, T., Kojima, T., Norisuye, T., 1981. Triple helix of scleroglucan in dilute aqueous sodium hydroxide. Polym. J. 13, 1135-1143.

Yerramilli, R.C., Zitha, P.L.J., Yerramilli, S.S., Bedrikovetsky, P., 2013. A Novel Water Injectivity Model and Experimental Validation using CT Scanned Core-Floods. SPE Eur. Form. Damage Conf. Exhib. doi:10.2118/165194-MS

Yin, H., Wang, D., Zhong, H., 2006. Study on flow behavoirs of viscoelastic polymer solution in micropore with dead end. SPE Annu. Tech. Conf. Exhib.

Zaitoun, a., Chauveteau, G., 1998. Effect of Pore Structure and Residual Oil on Polymer Bridging Adsorption. SPE/DOE Improv. Oil Recover. Symp. doi:10.2118/39674MS

Zaitoun, A., Potie, B., 1983. Limiting Conditions for the Use of Hydrolyzed Polyacrylamides in Brines Containing Divalent Ions. SPE Oilf. Geotherm. Chem. Symp. doi:10.2118/11785-MS

Zhang, J.-C., Song, K.-P., Liu, L., 2008. Investigation on mechanisms of polymer enhanced oil recovery by nuclear magnetic resonance and microscopic theoretical analysis. Chinese Phys. Lett. 1750.

Zhang, Z., Li, J., Zhou, J., 2010. Microscopic Roles of "Viscoelasticity" in HPMA polymer flooding for EOR. Transp. Porous Media 86, 199-214. doi:10.1007/s11242-010-9616-6

Zitha, P., Botermans, C., 1996. Bridging-adsorption of flexible polymers in low permeability porous media. SPE Annu. Tech. Conf. 15-20.

Zitha, P., Chauveteau, G., Zaitoun, A., 1995. Permeability-dependent propagation of polyacrylamides under near-wellbore flow conditions. Int. Symp. Oilf. Chem. 